Synthesis of Novel Hexahydroindoles from the Dearomatization of Indoline Using a Tungsten $\pi$-Base

\author{
Brianna Laurie MacLeod \\ Portage, Michigan \\ Chemistry B.A. \\ Wellesley College, 2011
}

\begin{abstract}
A Dissertation Presented to the Graduate Faculty of the University of Virginia in Candidacy for the Degree of

Doctor of Philosophy
\end{abstract}

Department of Chemistry

University of Virginia

May, 2016

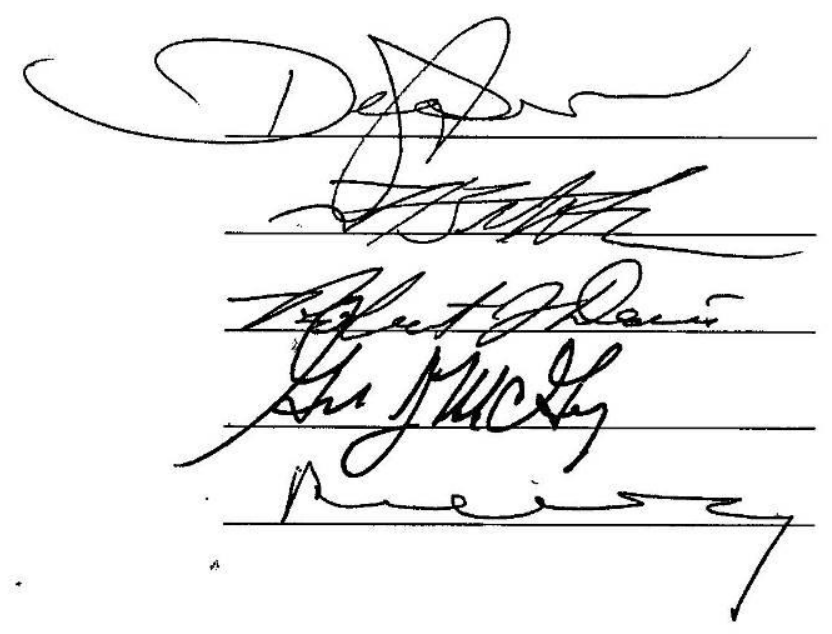




\begin{abstract}
Chapter 1 explores the reactivity of aromatic molecules in traditional organic chemistry. Due to their innate stability, arenes require harsh forcing conditions for substitution products and rarely are dearomatized. Through the use of transition metal complexes, this dearomatization is now possible. The use of electron deficient and electron rich metal systems are explored, though the main focus of this chapter is on the $\left\{\mathrm{TpW}(\mathrm{NO})\left(\mathrm{PMe}_{3}\right)\right\}$ metal fragment. This system has the ability to $\eta^{2}$-coordination many arenes, but coordination of $N, N$-dimethylaniline and derivatives are of great importance. Through this coordination, these systems have synthesized multiple novel small molecules
\end{abstract}

Chapter 2 describes previous work completed towards the synthesis of naturally found alkaloids which contain indoles, indolines and perhydroindoles. The ability to expand the synthesis of biologically interesting molecules away from aromatic molecules and into fully saturated cores broadens the potential of compounds available for biological testing. This chapter elaborates on methods of how organic chemists are synthesizing the perhydroindoles synthetic core in various alkaloids and the biological interest of these alkaloids.

Chapter 3 explores the coordination of larger alkaloid like aromatics to the $\left\{\mathrm{TpW}(\mathrm{NO})\left(\mathrm{PMe}_{3}\right)\right\}$ metal system. These include $N$-alkylindoline and 1-methyl-1,2,3,4tetrahydroquinoline. Through an acid trapping type synthesis, these alkaloids are bound through an $\eta^{2}$-bond. The protonation of these systems in either an ortho vs para position is explored, as is protonation anti vs syn to the metal complex. The initial reactivity of the 
$\mathrm{N}$-ethylindoline complex is investigated with $\mathrm{H}^{+}$as an electrophile. Preliminary testing of 1,6-dimethyl-1,2,3,4-tetrahydroquinoline as a ligand, and subsequent reactivity, is explored.

Chapter 4 elaborates on the reactivity of the $N$-ethylindoline complex with various electrophiles, including isocyanates, mCPBA and halides. The reduction of the iminium bond is explored successfully, allowing the formation of multiple new complexes which have much lower reduction potentials. The oxidation of these systems generates novel hexahydroindoles with broad functionality.

Chapter 5 focuses on the exploration of new reactivity pathways for both the $N$ ethylindoline and $\mathrm{N}, \mathrm{N}$-dimethyaniline systems. The addition of NCS as an electrophile leads to the concept of possible double nucleophilic addition reactions. A ring turn product allows for the activation of a new position of the aniline and indoline systems through the addition of new electrophiles. The isolation of a new organic hexahydroindoles from this ring turn system is explored. 


\section{Copyright Information}

Chapters 3 and $\mathbf{4}$ are compilations of two published works and have been reproduced in accordance with Section II.1 or American Chemical society Journal Publishing Agreement. Chapter 5 is an outline for future papers. Proper citations for Chapters 3 and $\mathbf{4}$ are given collaborative efforts described the work in chapter 5. All chapters are presented as individual pieces.

\section{Chapter 3 and Chapter 4:}

MacLeod, B. L.; Pienkos, J. A.; Myers, J. T.; Sabat, M.; Myers, W. H.; Harman, W. D. Organometallics 2014, 33, 6286.

MacLeod, B. L.; Pienkos, J. A.; Wilson, K. B.; Sabat, M.; Myers, W. H.; Harman, W. D. Organometallics 2016.

\section{Chapter 5:}

Brianna L. MacLeod, Katy B. Wilson, Jared A. Pienkos, William H. Myers, and W. Dean Harman 
This is dedicated to my family. Thanks for believing I could do this and reminding me that Pooh is always right... Love you all!

You're braver than you believe, Stronger than you seem, And smarter than you think.

-Winnie the Pooh 
Table of Contents

\begin{tabular}{lc} 
Abstract & ii \\
\hline Copyright information & iv \\
\hline Dedication & $\mathbf{v}$ \\
\hline Table of Contents & vi \\
\hline List of Figures & $\mathbf{x}$ \\
\hline List of Schemes & xii \\
\hline List of Tables & xvii \\
\hline List of Abbreviations & xviii
\end{tabular}

Chapter 1 - An Introduction of Aromatic Molecules and Their Dearomatization

$\begin{array}{ll}\text { 1.1 Overall Goal of Dearomatization } & 2\end{array}$

1.2 Traditional Organic Transformations for Arenes 3

$\begin{array}{ll}\text { 1.3 Organic Based Dearomatization } & 5\end{array}$

$\begin{array}{ll}\text { 1.4 Catalytic Methods } & 7\end{array}$

1.5 Dearomatization with Stoichiometric Metal Fragments $\quad 8$

$\begin{array}{ll}1.6 \pi \text {-Acidic Metal Fragments } & 9\end{array}$

$\begin{array}{lr}1.7 \pi \text {-Basic Metal Fragments } & 10\end{array}$

1.8 Dearomatization with Metal Fragment $\left\{\mathrm{Os}\left(\mathrm{NH}_{3}\right)_{5}\right\}^{+2}$

1.9 Dearomatization with $\{\operatorname{TpRe}(\mathrm{CO})(\mathrm{L})\}$ and $\{\operatorname{TpMo}(\mathrm{NO})(\mathrm{L})\} \quad 14$

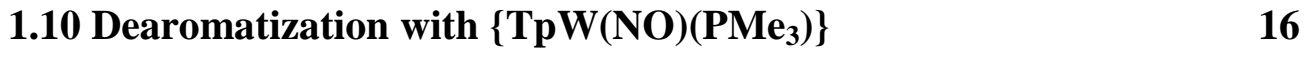


1.11 Reactivity of $\left[\mathrm{TpW}(\mathrm{NO})\left(\mathrm{PMe}_{3}\right)\left(\eta^{2}-N, N\right.\right.$-dimethylanilinium] 17 and derivatives

$\begin{array}{ll}\text { 1.12 Reference } & 23\end{array}$

Chapter 2 - A Brief Overview of Perhydroindole Alkaloid Chemistry

$\begin{array}{ll}2.1 \text { Indoles and Indoline } & 27\end{array}$

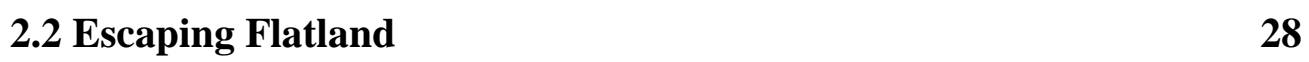

$\begin{array}{ll}\text { 2.3 Perhydroindoles } & 31\end{array}$

2.4 Stemona Alkaloids $\quad 32$

$\begin{array}{ll}\text { 2.4.1 Stenine Synthesis } & 34\end{array}$

2.4.2 Tuberostemonine Synthesis $\quad 36$

$\begin{array}{ll}2.5 & \text { Amaryllidaceae Alkaloids } \\ \end{array}$

$\begin{array}{ll}\text { 2.5.1 Lycorine Synthesis } & 38\end{array}$

$\begin{array}{ll}\text { 2.5.2 Crinine Synthesis } & 40\end{array}$

2.6 Sceletium Alkaloids / Mesembrine Synthesis 40

2.7 Aeruginosin Alkaloids $\quad 42$

2.7.1 Aeruginosin 298-A Synthesis 43

2.7.2 Dysinosin A Synthesis $\quad 44$

2.8 Dearomatization of Indoles 45

$\begin{array}{ll}2.9 \text { Conclusion } & 46\end{array}$

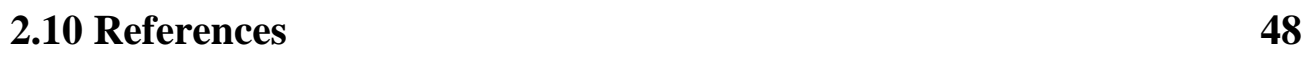

Chapter 3 - Isolation and Initial Reactivity of $N$-Ethylindolinium Complex 3.1 Introduction $\quad 52$ 
3.2 Isolation of $\mathbf{N}$-Alkylindolinium and $\mathrm{N}$-Alkylquinolinium

Complexes

3.2.1 Para vs Ortho Protonation 57

3.2.2 Anti vs Syn Protonation

$\begin{array}{lc}\text { 3.3 Preliminary Reactivity } & 60\end{array}$

$\begin{array}{lr}\text { 3.4 Quinoline Isomerization } & 66\end{array}$

3.4.1 Para and Meta-Toluidine Isomerization 67

$\begin{array}{ll}\text { 3.4.2 Para Methyl Quinoline Complexation } & 68\end{array}$

3.5 Quinolinium and Para-Methyl Anilinium Initial Reactivity 69

$\begin{array}{ll}\text { 3.6 Conclusion } & 71\end{array}$

$\begin{array}{ll}\text { 3.7 Experimental Section } & 72\end{array}$

$\begin{array}{lr}3.8 \text { References } & 101\end{array}$

Chapter 4- Further Reactivity Testing of $N$-Ethylindolinium Complex and Isolation of Novel Hexahydroindoles.

$\begin{array}{lr}\text { 4.1 Introduction } & 105\end{array}$

$\begin{array}{ll}\text { 4.2 Exploration of Electrophiles } & 105\end{array}$

4.2.1 Carbon Based Electrophiles $\quad 106$

4.2.2 Fluorination of Indolinium $\quad 108$

4.2.3 Halogenation of Indolinium 110

4.2.4 Hydroxylation of Indolinium with mCPBA 113

$\begin{array}{ll}4.3 \text { Reduction of Iminium } & 116\end{array}$

4.4 Oxidation and Isolation of Hexahydroindole Organics 121

$\begin{array}{lr}4.5 \text { Conclusion } & 124\end{array}$ 
$\begin{array}{lr}\text { 4.6 Experimental Section } & 125\end{array}$

$\begin{array}{lr}\text { 4.7 References } & 170\end{array}$

Chapter 5 - New Branches of Reactivity with $N$-Ethylindolinium and $N, N$ Dimethylanilinium Systems

$\begin{array}{lc}5.1 \text { Introduction } & 173\end{array}$

$\begin{array}{ll}\text { 5.2 NCS/NBS Reactivity Expansion } & 173\end{array}$

$\begin{array}{ll}\text { 5.2.1 Elimination of Halide } & 175\end{array}$

5.2.2 Reactivity of Elimination Product $\quad 178$

$\begin{array}{ll}\text { 5.3 Additions to the Reduced Indoline Complex } & 184\end{array}$

$\begin{array}{ll}5.4 \text { Ring Turn Reactivity } & 186\end{array}$

$\begin{array}{ll}\text { 5.5 Further Aniline Exploration } & 190\end{array}$

5.5.1 Isolation of Novel Aniline Organics 192

5.5.2 New Allyl Generation from Aniline Complex 195

$\begin{array}{lr}5.6 \text { Conclusion } & 198\end{array}$

$\begin{array}{ll}5.7 \text { Experimental Section } & 199\end{array}$

$\begin{array}{lr}5.8 \text { References } & \mathbf{2 2 0}\end{array}$

$\begin{array}{lr}\text { Appendix } & 222\end{array}$ 


\section{List of Figures}

Figure 1.1. Energy diagram of varied levels of unsaturation of 2 functionalization

Figure 1.2. Reactivity of arene bound of $\pi$-acidic and $\pi$-basic

metal systems

Figure 1.3. $\eta^{2}$-Coordination stabilization via $\pi$-backbonding $\quad 11$

Figure 1.4. Structure of $\operatorname{TpRe}(\mathrm{CO})(\mathrm{MeIm})\left(\eta^{2}\right.$-naphthalene $) \quad 14$

Figure 2.1. Natural products containing indole and indoline cores 27

Figure 2.2. Isomers of dimethyl pyridine and dimethylpiperidine 30

Figure 2.3. Naturally found cores of Stemona alkaloids 33

$\begin{array}{ll}\text { Figure 2.4. Naturally found stenine alkaloids } & 34\end{array}$

Figure 2.5. General structure of Aeruginosin family 42

Figure 2.6. Full structure of aeruoginosin 298-A 43

Figure 2.7. Retrosynthesis of dysinosin A 45

Figure 3.1. Examples of natural products with a perhydroindole $\quad 52$

and decahydroquinoline cores

Figure 3.2. Dearomatization of indoline and tetrahydroquinoline 53

Figure 3.3: Crystal structure of N-Methyl-Quinoline (4B) 55

Figure 3.4. The dihapto-coordinated indoline intermediate, 58 stereochemistry of protonation, and numbering system

Figure 3.5: Crystal structure of compound 8-iPr 63

Figure 3.6. NOE interactions supporting stereochemical assignments 66 in $\mathbf{8 - 2 0}$ 
Figure 4.1. Crystal structure of fluorinated tetrahydroindolium complex 31

Figure 4.2: Crystal structure of compound $\mathbf{5 2}$

Figure 4.3. Dearomatization as part of creating a diverse chemical library 124

Figure 5.1. Structures of (-)-Conduramine-D1 and Ketamine 195

Figure 5.2. Comparison of known allyl $\mathbf{1 0 5}$ and $\mathbf{1 0 4} 198$ 


\section{List of Schemes}

Scheme 1.1. Bromination of benzene and cyclohexene 3

Scheme 1.2. General Friedel-Crafts alkylation of benzene 4

Scheme 1.3. General acylation of pyridine 5

Scheme 1.4. Examples of enzymatic dearomatization for synthesis 5

of natural products

Scheme 1.5. Hydrogenation and Birch reduction of benzene 6

Scheme 1.6. Common organic dearomatization reactions 7

Scheme 1.7. Cycloaddition reactions with benzene and anisole 7

Scheme 1.8. Dearomatization of benzene with $\mathrm{OsO}_{4}$ and palladium 8

Scheme 1.9. Dearomatization and reactivity of benzene with $\left[\mathrm{Mn}(\mathrm{CO})_{3}\right]^{+} 9$

Scheme 1.10. Dearomatization and reactivity of benzene coordinated 10 to $\mathrm{CrCO}_{3}$

Scheme 1.11. Reaction scheme of an anisole derivative using 11 $\left\{\mathrm{Os}\left(\mathrm{NH}_{3}\right)_{5}\right\}^{+2}$

Scheme 1.12. Diels-Alder reactivity of the osmium pyrrole system 12

Scheme 1.13. Osmium coordination of $N, N$-dimethylaniline and 13 subsequent reactivity

Scheme 1.14. Reactivity of rhenium bound naphthalene with acid and MTDA

Scheme 1.15. Dearomatization of naphthalene and formal catalytic cycle with $\{\mathrm{TpMo}(\mathrm{NO})(\mathrm{MeIm})\}$

Scheme 1.16. Coordination of Aniline and $N, N$-dimethylaniline to $\left\{\mathrm{TpW}(\mathrm{NO})\left(\mathrm{PMe}_{3}\right)\right\}$ 
Scheme 1.17. Reactivity of $\left[\mathrm{TpW}(\mathrm{NO})\left(\mathrm{PMe}_{3}\right)\right.$

$$
\left(\eta^{2}-N, N\right. \text {-dimethylanilinium][OTf] }
$$

Scheme 1.18. Cycloproponation of $N, N$-dimethylaniline and subsequent ring opening

Scheme 1.19. Formation of $\alpha, \beta$-unsaturated ketone organic products from oxidation

Scheme 1.20. Ring turn of aniline system with further reactivity scheme 21

Scheme 1.21. Reactivity of 4-dimethylaminopyridine and 2-(dimethylamino)pyrimidine complexes

Scheme 1.22. Synthesis of N-Alkylindolinium complex and possible final organic products

Scheme 2.1. Retrosynthetic process of stenine beginning with L-tyrosine 34

Scheme 2.2. Retrosynthetic process of stenine through an intramolecular Diels-Alder

Scheme 2.3. Stenine synthesis through a domino synthesis

Scheme 2.4. Tuberostemonine synthesis from the Wipf group 37

Scheme 2.5. (+)-Lycorine retrosynthetic thought of Schultz 38

Scheme 2.6. Key step in synthesis of (-)-lycorine 39

Scheme 2.7. Simplest retrosynthetic thought for lycorine 39

Scheme 2.8. Synthetic thought for the synthesis of Crinine 40

Scheme 2.9. Retrosynthetic thought of mesembrine by Taber 41

Scheme 2.10. Synthesis of mesembrine through Pd-mediated reaction $\quad 42$

Scheme 2.11. Synthesis of perhydroindoline core in aeruoginosin 298-A 43 
Scheme 3.1: Protonation of Dihapto-coordinated Indoline and

Tetrahydroquinoline Complexes Leading to Ortho and Para Isomers

Scheme 3.2. In situ protonation of $\eta^{2}$-coordinated $N$-alkylated indoline complexes and associated protonation ratios;

$$
[\mathrm{W}]=\mathrm{TpW}(\mathrm{NO})\left(\mathrm{PMe}_{3}\right)
$$

Scheme 3.3. Overall reaction scheme for perhydroindole synthesis;

$$
[\mathrm{W}]=\mathrm{TpW}(\mathrm{NO})\left(\mathrm{PMe}_{3}\right)
$$

Scheme 3.4: Functionalization of 5 with $\mathrm{E}^{+}=\mathrm{H}^{+}$

Scheme 3.5. Preparation of tetrahydroindolium complexes 7-19

Scheme 3.6. Isolation of methyl-toluidine derivative complexes

Scheme 3.7. Complexation of 6-methyl-1-methyl-

\section{1,2,3,4-tetrahydroquinoline}

Scheme 3.8. Tandem addition of HOTf and 2-methylfuran with 21

Scheme 3.9. Addition of HOTf and 2-methylfuran to $\mathbf{2 3}$

Scheme 4.1. Reactivity of indolinium complex 5 with isocyanates 106

Scheme 4.2. Simmons-Smith Cyclopropanation of 5 107

Scheme 4.3. Fluorination of the indolinium complex 5 108

Scheme 4.4. Bromination tandem addition of 5

Scheme 4.5. Chlorination of the indolinium complex 5

Scheme 4.6. Substitution of iodine with the indolinium complex 5

Scheme 4.7. Hydroxylation of the indolinium complex 5

Scheme 4.8. The formation of cyclic imidates from the hydroxylation product $\mathbf{4 3}$ 
Scheme 4.10. Reduction of the iminium bond in the tetrahydroindolium 118 Complexes

Scheme 4.11. Displacement of triazole with hydride of $\mathbf{1 6}$

Scheme 4.12. Decomplexation of the final hexahydroindole products

Scheme 4.13. Examples of further elaboration to perhydroindoles

Scheme 5.2. Double nucleophilic addition of aniline with anthracene

Scheme 5.3. Elimination of $\mathbf{3 4}$ with propyl amine

Scheme 5.4. Elimination of $\mathbf{3 5}, \mathbf{3 7}$ and $\mathbf{3 9}$ with propyl amine

Scheme 5.5. Scheme of reactivity for elimination products

Scheme 5.6. Exchange of pyrazole for thiophenol

Scheme 5.7. Double nucleophilic addition of propyl amine onto 34

Scheme 5.8. Long term reactivity of $\mathbf{3 5}$ and $\mathbf{3 9}$ with propyl amine

Scheme 5.9. Proposed scheme of intramolecular double nucleophilic addition with ethylenediamine

Scheme 5.10. Iodolactonization of MTDA addition on naphthalene

Scheme 5.11. Addition of MTDA to reduced ethylindoline complex

Scheme 5.12. Ring turn of $\mathbf{5 4}$ to yield $\mathbf{8 0}$

Scheme 5.13. Ring turn scheme of $N, N$-dimethylaniline complex

Scheme 5.14. Ring turn elimination and addition of allyl bromide

Scheme 5.15. Reduction of 80 iminium with $\mathrm{NaCNBH}_{3}$

Scheme 5.16. Reduction of iminium in $\mathbf{8 2}$ to yield cis ring juncture 
Scheme 5.18. Reduction of the iminium bond in aniline addition products $(\mathbf{8 6 - 9 2})$

$\begin{array}{ll}\text { Scheme 5.19. Oxidation to yield novel aminocyclohexenes } & 193\end{array}$

Scheme 5.20. Novel organic isolation with protonation of amine 193

Scheme 5.21. Reaction scheme for new cyclohexene organic products 196

Scheme 5.22. Allyl 104 Creation from 98 with DPhAT 197 


\section{List of Tables}

Table 5.1. Nucleophiles and their corresponding $\mathrm{pKa}$ 's 


\section{List of Abbreviations}

\begin{tabular}{|c|c|}
\hline $\mathrm{aq}$ & Aqueous \\
\hline br & Broad \\
\hline CAN & Ceric Ammonium nitrate \\
\hline $\mathrm{Cbz}$ & Carboxybenzyl \\
\hline COSY & Correlation Spectroscopy \\
\hline $\mathrm{CV}$ & Cyclic voltammetry \\
\hline DCM & Dichloromethane \\
\hline DDQ & 2,3-dichloro-5,6-dicyanoquinone \\
\hline DFT & Density functional theory \\
\hline DiPAT & Diisopropyl ammonium triflate \\
\hline DMA & Dimethylacetamide \\
\hline DME & 1,2-Dimethyoxyethane \\
\hline DPhAT & Diphenyl ammonium triflate \\
\hline EtOAC & Ethyl Acetate \\
\hline HATR & Horizonatal Attenuated Total Reflectance \\
\hline HMBS & Heteronuclear Multiple Bond Coherence Spectroscopy \\
\hline HRMS & High Resolution Mass Spectroscopy \\
\hline HSQC & Hetereonuclear Single Quantum Correlation Spectrscopy \\
\hline $\mathrm{Hz}$ & Hertz \\
\hline IR & Intrared \\
\hline KHMDS & Potassium bis(trimethylsilyl)amide \\
\hline
\end{tabular}




\begin{tabular}{|c|c|}
\hline $\mathrm{LAH} / \mathrm{LiAlH}_{4}$ & Lithium Aluminium Hydride \\
\hline LiDMM & Lithium dimethyl malonate \\
\hline LRMS & Low Resolution Mass Spectroscopy \\
\hline $\mathrm{mCPBA}$ & $m$-Chloroperbenzoic acid \\
\hline MeIM & $N$-Methylimidazole \\
\hline MTDA & Methyl trimethylsilyl dimethylketene acetal \\
\hline NBS & $N$-Bromosuccinimide \\
\hline NCS & $\mathrm{N}$-Chlorosuccinimide \\
\hline NHE & Normal Hydrogen Electrode \\
\hline NIS & $N$-Iodosuccinimide \\
\hline NMR & Nuclear Magnetic Resonance \\
\hline NOE & Nuclear Overhauser Effect \\
\hline NOESY & Nuclear Overhauser Effect Spectroscopy \\
\hline $\mathrm{NOPF}_{6}$ & Nitrosonium Hexafluorophosphate \\
\hline ORTEP & Oak Ridge Thermal Ellipsoid Program \\
\hline OTf & Trifluoromethanesulfonate or triflate \\
\hline $\mathrm{PMe}_{3}$ & Trimethylphosphine \\
\hline Ppm & Parts Per Million \\
\hline sat'd & Saturated \\
\hline TBAH & Tetrabutylammonium Hexafluorophosphate \\
\hline TEA & Triethylamine \\
\hline THF & Tetrahydrofuran \\
\hline TLC & Thin layer chromatography \\
\hline
\end{tabular}


TMS

$\mathrm{Tp}$

UV
Tetramethylsilane

Hydridotris(pyrazolyl)borate

Ultraviolet 


\title{
Chapter 1:
}

\section{An Introduction of Aromatic Molecules}

\author{
and Their Dearomatization
}




\subsection{Overall Goal of Dearomatization}

There is a disconnect in the world of synthetic chemistry between what is easily made and what is both biologically and synthetically interesting. Some of the most common compounds, aromatics, have a low degree of functionalization and a limitation in reactivity. As described later in chapter 2, they have a lower probability of being biologically active than their saturated analogs. The inability to easily go from these aromatic compounds to the fully saturated, alicyclic systems is a difficult problem to solve.

The ability to use aromatics as a scaffold for further elaboration would be invaluable for the discovery of new novel small organic compounds. This elaboration can be seen in Figure 1.1. The range of compounds that could be synthesized from a ring of 6 unsaturated carbons through controlled addition reactions would be widely variable. Sites of functionalization could be added selectively around to the ringed structure. The diversity of new cyclohexanes can be broadened by the range of substituted aromatic molecules found in nature.
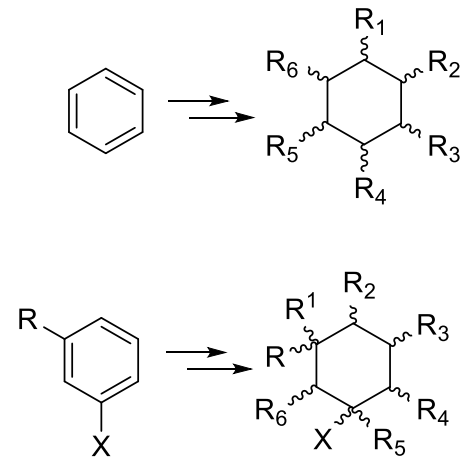

Figure 1.1. Use of benzene derivatives as scaffolds for functionalization 
Aromatic molecules have an innate stability. This has been realized through the identification of resonance stabilization energy of aromatic molecules. For benzene, this is $36 \mathrm{kcal} / \mathrm{mol}$ compared to theoretical non-aromatic $1,3,5$-cyclohexatriene. ${ }^{1}$ This stabilization does not allow for stepwise addition reactions to aromatic rings, but rather causes the inert nature of these systems.

\subsection{Traditional Organic Transformations for Arenes}

Due to their prevalence and diversity in nature, aromatic molecules represent an ideal starting material for derivatization. They have 6 positions of unsaturation that are ideal for functionalization. The problem is that due to their increased stabilization, aromatic molecules are resistant to chemical reactions that would disrupt their $\pi$ systems, leading to a specific type of reactivity. The products of many reactions with aromatic molecules retain their aromatic nature, where non-aromatic unsaturated molecules could create addition products under the same conditions. An example of this can be seen by comparing the products resulting from the bromination of benzene and cyclohexene (Scheme 1.1). Benzene yields a substituted aromatic product while the cyclohexene yields a fully saturated cyclohexane through the addition of bromine.

Scheme 1.1. Bromination of benzene and cyclohexene

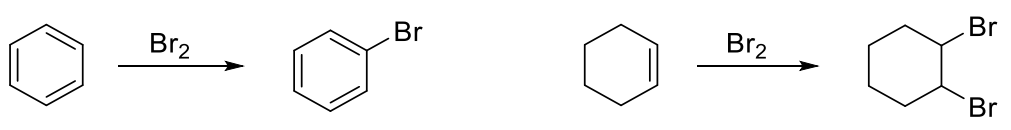


This means that reactions frequently produce substitution products or they require harsh reaction conditions (i.e., electrophilic substitution reactions or Birch reductions). Electrophilic substitution reactions generally require harsh reagents and have little control in the number of substitutions which occur. For instance, Friedel-Crafts alkylation, which is promoted by the Lewis acids $\mathrm{AlCl}_{3}$ or $\mathrm{FeCl}_{3}$, yields substituted alkylated benzene products (Scheme 1.2). ${ }^{2}$ A major problem with this reaction stems from the lack of control over the products formed. Upon a single substitution of the alkyl group, the aromatic ring becomes activated towards further electrophiles. This leads to multisubstituted products that retain their aromatic nature.,3

Scheme 1.2. General Friedel-Crafts alkylation of benzene

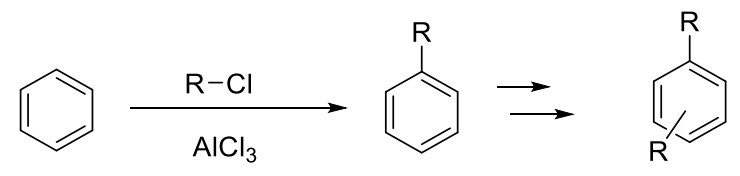

Heterocycles often offer reactivity with the lone pair outside of the aromatic core; however, substitution reactions within this core are subject to the same downfall as their hydrocarbon counterparts. For instance, pyridine rings are more electron poor than benzene, but the lone pair on the nitrogen can react with electrophiles to yield pyridiniums under traditional organic modifications (Scheme 1.3). This produces compounds which have a positive charge, but maintain the aromatic nature. Substituents on the aromatic ring, such as in anisole and aniline, have the ability to activate the aromatic ring towards these substitutions. ${ }^{2}$ 
Scheme 1.3. General acylation of pyridine

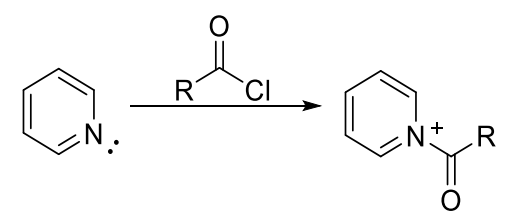

\subsection{Organic Based Dearomatization}

There has been surge in the investigation of dearomatization of aromatic compounds in the organic chemistry realm. The ability to use aromatic molecules as starting scaffolds for the synthesis of saturated natural products would be invaluable in the synthetic simplicity. In nature, dearomatiziation occurs most often enzymatically as seen in Scheme 1.4. This has been harnessed for natural product synthesis, for example (+)-hexacyclinol. ${ }^{4}$ Beginning with iodobenzene, using enzymatic dihydroxylation, the system is dearomatized and further reacted onto the final desired natural product. Benzoic acid can also be enzymatically dearomatized via a dihydroxylation, both stereo and regioselectively This dearomatized, functionalized system is elaborated further onto the natural product (-)-deoxycycline. ${ }^{4}$

Scheme 1.4. Examples of enzymatic dearomatization for synthesis of natural products

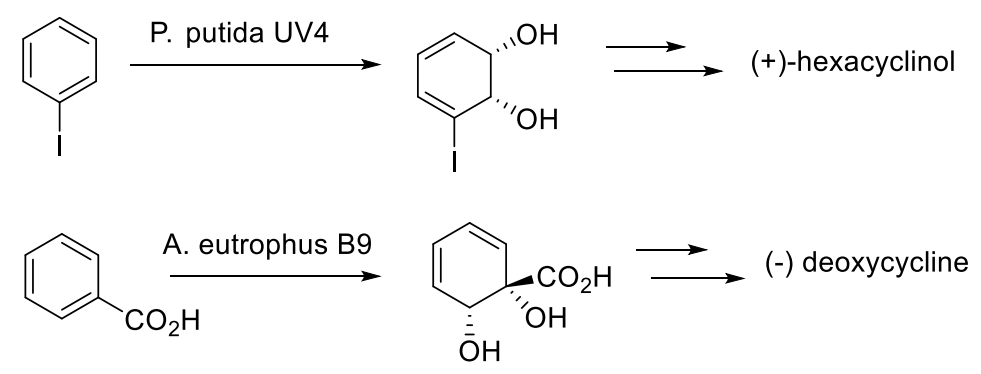


While limited, there are organic based dearomatization reactions. The most common are the Birch reduction and hydrogenation. The Birch reduction requires harsh reaction conditions for dearomatization, requiring $\mathrm{Na}^{0} / \mathrm{NH}_{3}$ in a protic solvent in order to dearomatize the ring (Scheme 1.5). ${ }^{5}$ These conditions do not allow for many functional groups prior to dearomatization. This system is unique however and rather than fully saturating the cyclic ring, there are remaining sites of unsaturation to elaborate on further and increase functionalization. Hydrogenation also requires harsh conditions: high pressure and heat. This creates a fully saturated cyclohexane ring.

Scheme 1.5. Hydrogenation and Birch reduction of benzene

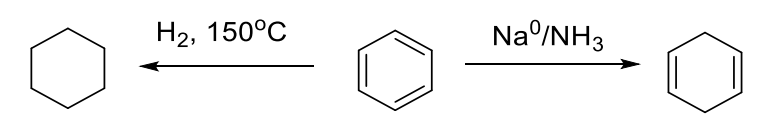

More simple reagents, such as mCPBA, have the ability to directly dearomatize polycyclic aromatic hydrocarbons. PAH's overall resonance energy is less than the same number of isolated benzenes, making them more reactive (Scheme 1.6). ${ }^{6}$ The use of hypervalent iodine reagents in total synthesis has been used by the Wipf group for the synthesis of many natural products, such as those discussed further in chapter $2{ }^{7}$ 
Scheme 1.6. Common organic dearomatization reactions

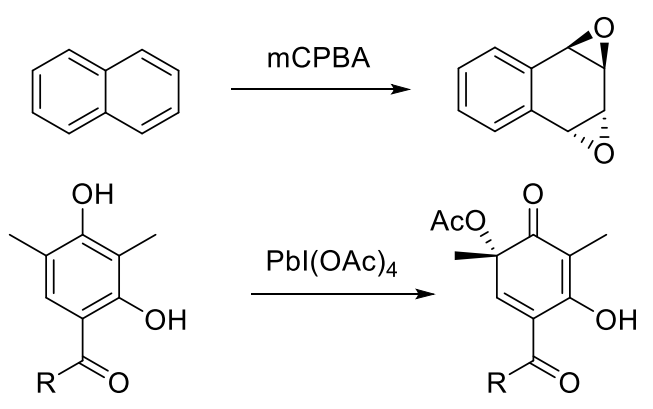

Diels-Alder reactions with furan or pyrrole are common ways to dearomatize smaller aromatic systems. Benzene itself cannot react in a [4+2] reaction as a diene unless under high temperatures and in the presence of reactive dienophiles, such as dicyanoacetylene. Even then, low yields plague the reaction. Under photolytic conditions, $[2+2]$ cycloaddition reactions are possible, such as shown in Scheme 1.7 with anisole. ${ }^{8,9}$

Scheme 1.7. Cycloaddition reactions with benzene and anisole

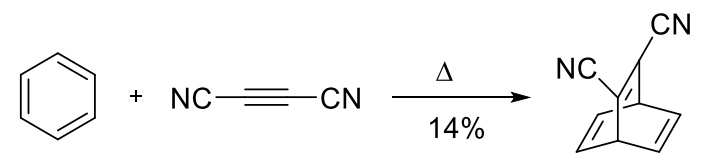

$[2+2]$

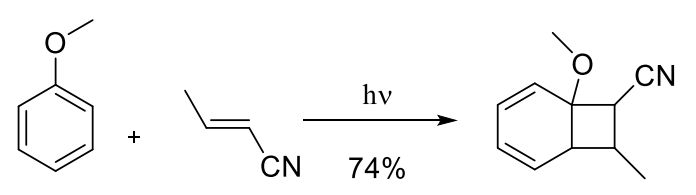

\subsection{Catalytic Methods}

Catalytic methods of dearomatization would be preferable to stoichiometric metal-based dearomatization due to the small amounts of starting catalyst needed and the 
ability of catalysts to lower the energy barrier for dearomatization. An example of this is with $\mathrm{OsO}_{4}$ with benzene to generate a fully saturated acetoxy derivatized cyclohexane. ${ }^{10}$ Photochemical stimulation can frequently assist in catalytic dearomatization methods. Another example of this work is with palladium. Palladium catalyzed dearomatization is less generalizable. As seen in Scheme 1.8, this dearomatization is limited by the necessity of removing a halide to create a new unsaturated site. ${ }^{11}$

Scheme 1.8. Dearomatization of benzene with $\mathrm{OsO}_{4}$ and palladium

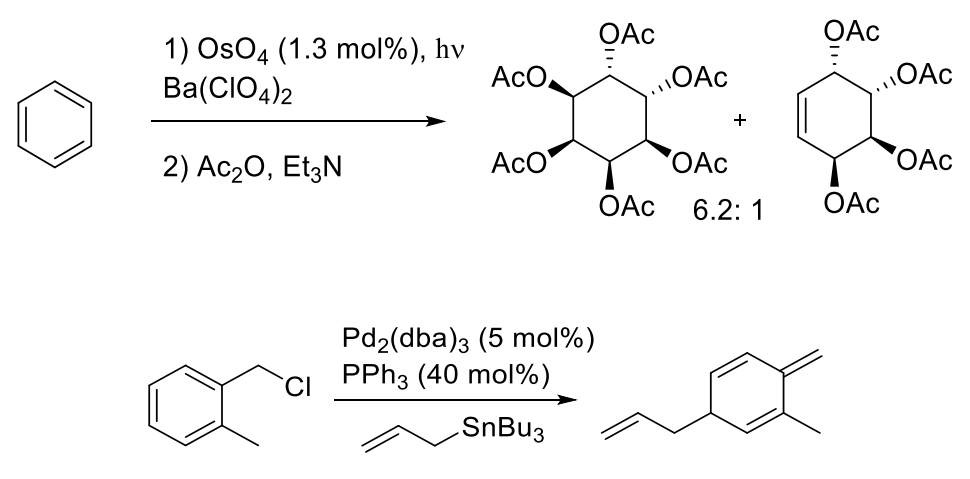

\subsection{Dearomatization with Stoichiometric Metal Fragments}

While catalytic dearomatization has many advantages, the ability to stoichiometrically bind arenes to a metal fragment expands the possibility of future elaboration of the unsaturated core. The arene is dearomatized upcoordination to the metal system, allowing the metal to influence further reactivity. With a $\pi$-acidic metal, the electron poor metal complex draws electron density from the arene, leading to increased reactivity of the ligand towards nucleophiles. In a $\pi$-basic system, the aromatic 
ligand is more electron rich, now donating electron density into the arene, leading to an increased reactivity towards electrophiles (Figure 1.2).

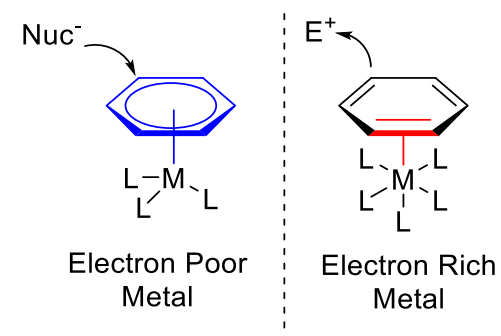

Figure 1.2. Reactivity of arene bound of $\pi$-acidic and $\pi$-basic metal systems

\section{$1.6 \pi$-Acidic Metal Fragments}

In a $\pi$-acidic metal case, such as $\left[\mathrm{Mn}(\mathrm{CO})_{3}\right]^{+}$, the aromatic ligand coordinates in a $\eta^{6}$ fashion, forming a "piano-stool” complex with benzene. These systems are electron deficient and have the ability to be reduced via lithium aluminum hydride (LAH), followed by a secondary nucleophilic addition (Scheme 1.9). ${ }^{12,13}$ This scheme is similar to the $\left[\mathrm{Ru}^{+} \mathrm{Cp}\right]$ fragment, which has been used to make natural products such as spirolactams. ${ }^{14,15}$

Scheme 1.9. Dearomatization and reactivity of benzene with $\left[\mathrm{Mn}(\mathrm{CO})_{3}\right]^{+}$

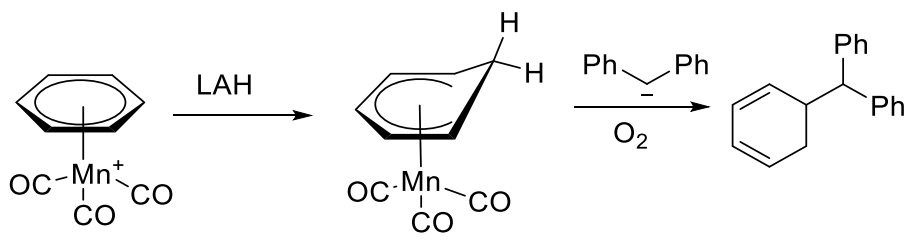


$\mathrm{A}\left[\mathrm{CrCO}_{3}\right]$ fragment has similar reactivity, though this system has more interesting regioselectivity (Scheme 1.10). There have been studies involving the regioselectivity of additions to these bound arenes, where the $\mathrm{R}$ group dictates the further electrophilic sites. ${ }^{13}$ This is a dearomatization agent that has been used to synthesize natural products, such as (-)-acetoxytubipofuran. ${ }^{16}$

Scheme 1.10. Dearomatization and reactivity of benzene coordinated to $\mathrm{CrCO}_{3}$
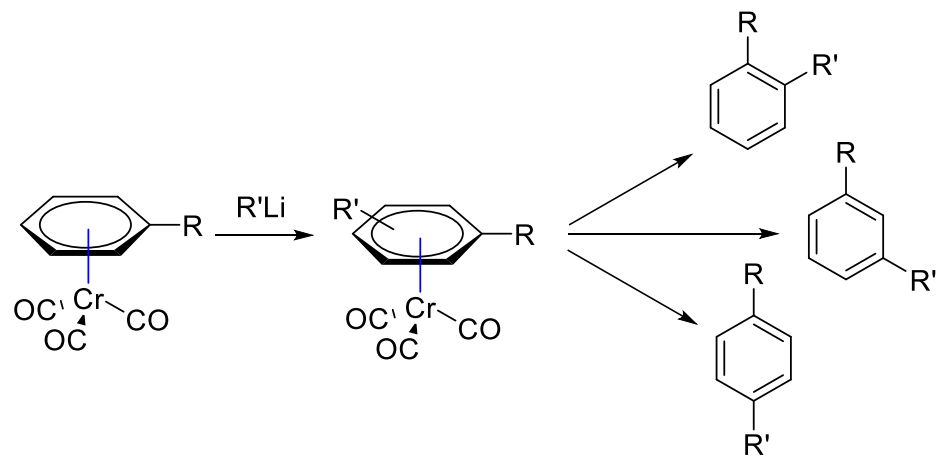

\section{$1.7 \pi$-Basic Metal Fragments}

While the $\pi$-acidic fragments increase the arene's reactivity towards nucleophiles, $\pi$-basic metal fragments increase the arene's affinity towards electrophiles. The metal system has the ability to back donate from its filled $\mathrm{d}_{\pi}$ orbitals into the empty $\pi^{*}$ orbital of the bound arene (Figure 1.3). ${ }^{17}$ This $\eta^{2}$-bond protects the now isolated double bond from reactivity and allows the remainder of the ligand to react similarly to a diene, a more reactive and predictable motif. The research in this field has been concentrated over 4 dearomatization agents: $\left\{\mathrm{Os}\left(\mathrm{NH}_{3}\right)_{5}\right\}^{+2},{ }^{18}\{\mathrm{TpRe}(\mathrm{CO})(\mathrm{L})\}^{+18,19}\{\mathrm{TpMo}(\mathrm{NO})(\mathrm{L})\},,^{20,21}$ and $\{\mathrm{TpW}(\mathrm{NO})(\mathrm{L})\}^{22}$ fragments. 


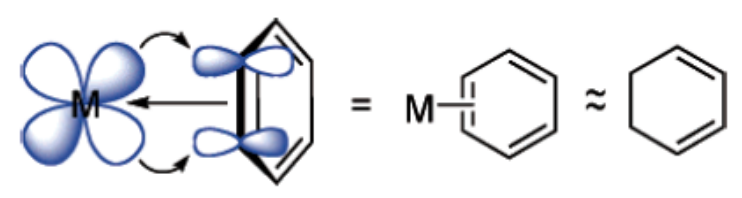

Figure 1.3. $\eta^{2}$-Coordination stabilization via $\pi$-backbonding

\subsection{Dearomatization with Metal Fragment $\left\{\mathrm{Os}\left(\mathrm{NH}_{3}\right)_{5}\right\}^{+2}$}

The pivotal pentaamineosmium (II) system has the ability to bind various arenes including benzene, substituted benzenes, furans, pyrroles and naphthalene. ${ }^{23}$ All of these systems, once bound $\eta^{2}$, are shown to have enhanced reactivity towards electrophiles. The first report of this complex showed the ability to selectively hydrogenate benzene into cyclohexene. ${ }^{24}$ Further investigation showed reactivity with other electrophiles, such as $\mathrm{H}^{+}$, acetals, ketals, Michael acceptors, and anhydrides. ${ }^{25}$ An example of a stepwise dearomatization and functionalization of anisole once, bound to $\left\{\mathrm{Os}\left(\mathrm{NH}_{3}\right)_{5}\right\}$, through the addition of electrophiles and nucleophiles can be seen in Scheme 1.11.

Scheme 1.11. Reaction scheme of an anisole derivative using $\left\{\mathrm{Os}\left(\mathrm{NH}_{3}\right)_{5}\right\}^{+2}$

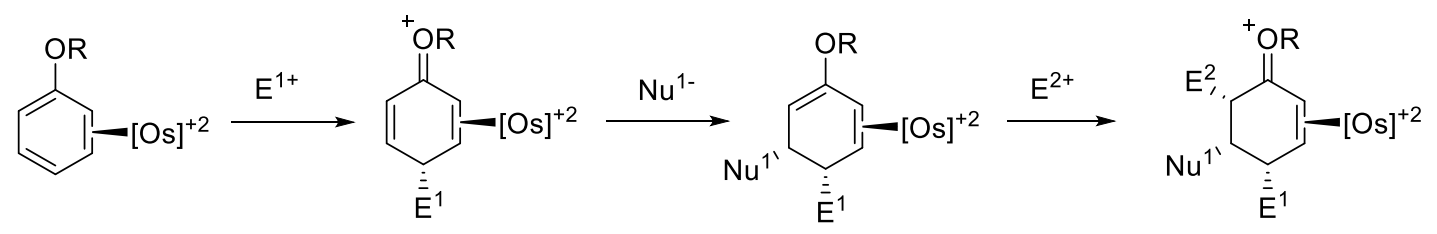

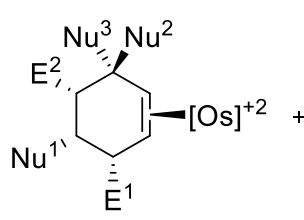

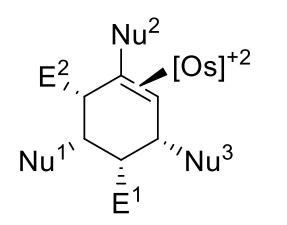<smiles>N#CC#N</smiles>

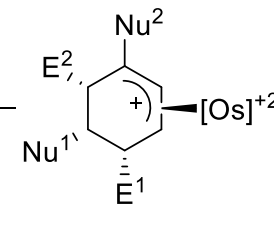

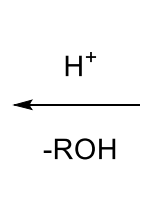

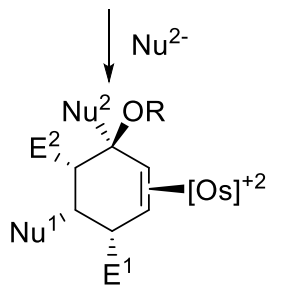


This pentaamineosmium (II) system has the ability to release the new organic molecules via an oxidation of the metal center. Through these oxidations there have been various natural products synthesized. Spirolactones have been synthesized from aniline and various pyrrolizidines and epibatidine-derivatives were synthesized through DielsAlder reactions with the pyrrole complex (Scheme 1.12).

Scheme 1.12. Diels-Alder reactivity of the osmium pyrrole system.

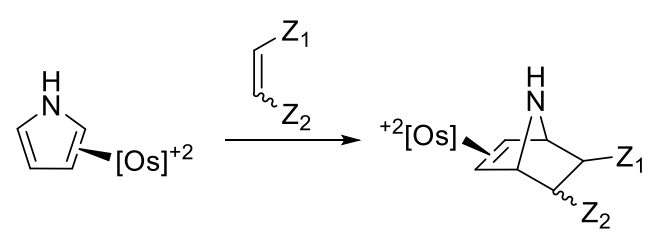

This metal fragment had the ability to bind aniline molecules. The reactivity was explored and it was shown to react with Michael acceptors following treatment with acid to form substituted anilines (A, Scheme 1.13). ${ }^{26,27}$ Further reactivity occurs with a reduction of the iminium followed by acid and oxidation to yield an almost fully saturated aniline derivative $(\mathrm{B}$, Scheme 1.13$) .^{30}$ 
Scheme 1.13. Osmium coordination of $N, N$-dimethylaniline and subsequent reactivity

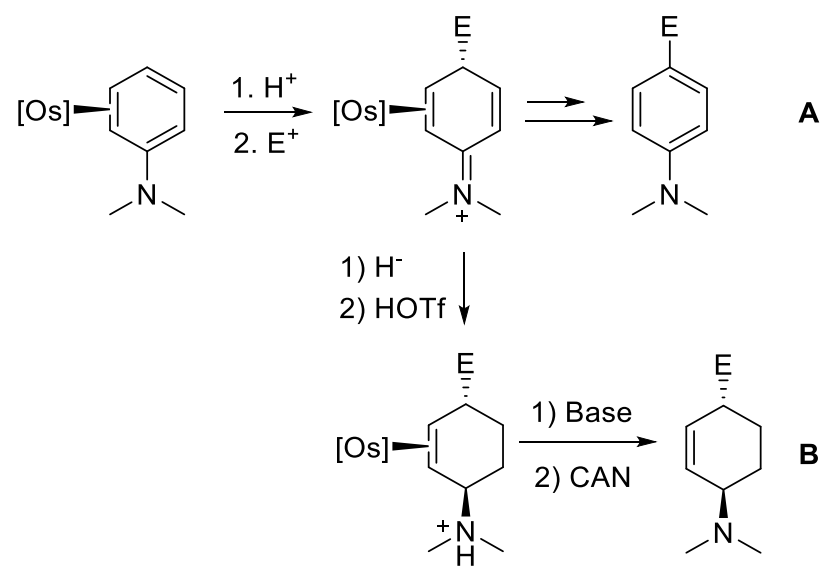

The downfall of the pentaamineosmium (II) system is that it is achiral. It is not possible to exchange an amine ligand for a different $\pi$-acid because the metal can no longer bind the aromatics. The reduction potential $\left(\mathrm{E}_{\mathrm{p}, \mathrm{a}}\right)$ for the system must remain close to $0.00 \mathrm{~V}$ NHE to bind the aromatics. This achiral center makes additions to the ligand stereoselective relative to the metal, but final organics are enantiomers. The osmium complex is recyclable, but still overall expensive. Overall, it was determined that a new metal complex would be necessary to make this type of reactivity viable for expansion. 


\subsection{Dearomatization with $\{\operatorname{TpRe}(\mathrm{CO})(\mathrm{L})\}$ and $\{\mathrm{TpMo}(\mathrm{NO})(\mathrm{L})\}$}

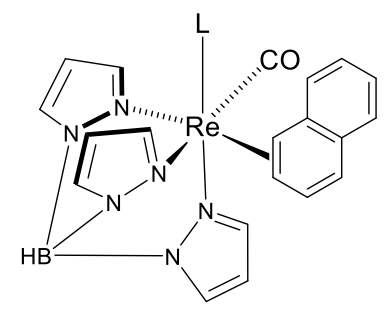

Figure 1.4. Structure of $\mathrm{TpRe}(\mathrm{CO})(\mathrm{MeIm})\left(\eta^{2}\right.$-naphthalene $)$

When synthesizing a new generation of dearomatization agents, the electronics of the new metal complexes were important. If the metal is too electron rich, oxidative addition becomes favored. If the metal is too electron poor, the back-bonding ability of the metal is hindered, making the binding of the aromatic more difficult. When transitioning to rhenium, the trispyrazolylborate (Tp) ligand was introduced along with an ancillary ligand (L). A strong $\pi$-acid was necessary to reduce the electron density on the more electron rich metal center, compared to the $\left\{\mathrm{Os}\left(\mathrm{NH}_{3}\right)_{5}\right\}$ fragment. For this complex, a $\mathrm{CO}$ ligand was used to reduced the electron density on rhenium, lowering the reduction potential back to the desired $\approx 0.00 \mathrm{~V}$ vs NHE. This was found to be the desired potential for binding the aromatic ligands with the osmium (II) system. The ancillary ligand can be exchanged for various ligands depending on how the electronics of the metal system needed to be altered (Figure 1.4).

Similar to the osmium (II) system, the rhenium (I) complex has the ability to bind benzene and other aromatics, including naphthalene, furan and thiophene. Using naphthalene as an example, there is a unique reaction scheme once bound to the metal 
system. Through the addition of strong acid, the transient allylic species is made, which is stabilized via back-bonding from the metal system (Scheme 1.14). ${ }^{28}$ With naphthalene the ligand rearranges, which allows weaker nucleophiles to be added in a 1,4electrophilic, nucleophilic manner. The tandem addition product can be removed from the metal via oxidation with AgOTf to regenerate a Re(II) species and the novel small molecule. ${ }^{17}$ Due to the chirality of the metal center, the rhenium complex is synthesized in a racemic mixture of $\mathrm{R}$ and $\mathrm{S}$ hands of the metal, leading to the same problem as osmium eventually synthesizing racemic mixtures of organic molecules. Using a sacrificial chiral ligand, $\alpha$-pinene, rhenium has the ability to be made enantioselectively. ${ }^{17}$ Unfortunately, the high cost and low scalability of $\{\operatorname{TpRe}(\mathrm{NO})(\mathrm{L})\}$ makes it not viable.

Scheme 1.14. Reactivity of rhenium bound naphthalene with acid and MTDA

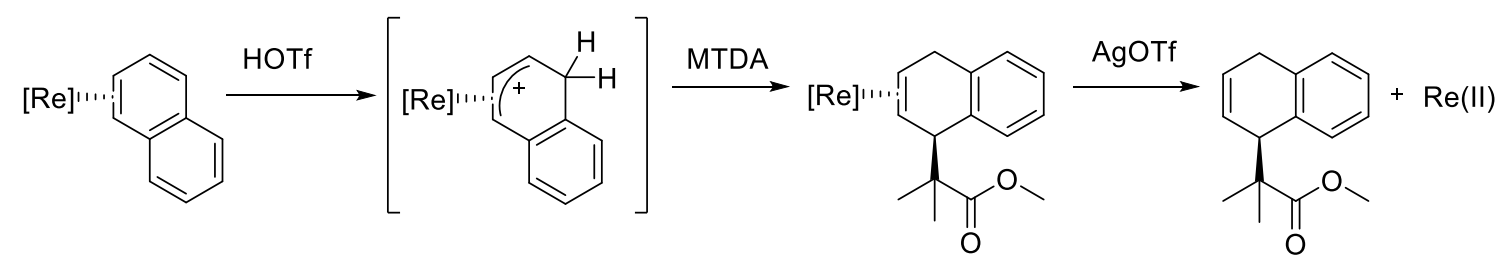

In order to make the metal complex less expensive, but retain the reactivity and recyclability, molybdenum was investigated. For this system it was necessary to use a NO ligand as the $\pi$-acidic ligand in order to allow the metal to bind the aromatics, but it still maintains the tunable auxiliary ligand. The $\{\mathrm{TpMo}(\mathrm{NO})(\mathrm{L})\}$ system is a weaker $\pi$ base than the rhenium (I) analog and subsequently cannot bind benzene and many of its derivatives. ${ }^{17,21}$ This metal complex is also sensitive to harsh reagents, such as strong acids or oxidants. Naphthalene has been one of the most successful ligands for this 
complex. Similar to $\operatorname{Re}(\mathrm{I}), \mathrm{Mo}(0)$ can be regenerated through the use of $\mathrm{I}_{2}$ as an oxidant (Scheme 1.15). ${ }^{29}$ It is interesting to note the now 1,2-tandem addition product.

The enantioenrichment of this Molybdenum complex is currently being investigated. Using the same $\alpha$-pinene concept as with rhenium, two hands of the metal can be isolated, but upon exchange of the ligand to an aromatic, the metal epimerizes. This problem of epimerization is currently being explored.

Scheme 1.15. Dearomatization of naphthalene and formal catalytic cycle with $\{\mathrm{TpMo}(\mathrm{NO})(\mathrm{MeIm})\}$

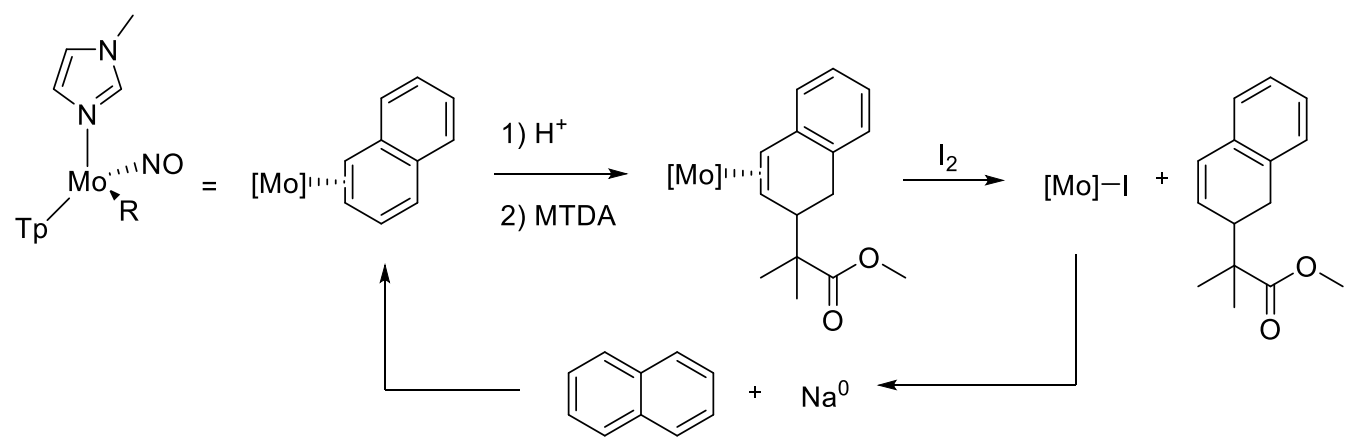

\subsection{Dearomatization with $\left\{\mathrm{TpW}(\mathrm{NO})\left(\mathrm{PMe}_{3}\right)\right\}$}

Due to the scalability and stability towards reaction conditions, the $\left\{\mathrm{TpW}(\mathrm{NO})\left(\mathrm{PMe}_{3}\right)\right\}$ metal fragment has been adopted as an important dearomatization agent. $^{30}$ This system is unique from the previous metal complexes for a few reasons. The $\mathrm{PMe}_{3}$ ancillary ligand cannot be exchanged without detrimental changes in yield and scalability, unlike the $\operatorname{Mo}(0)$ and $\operatorname{Re}(\mathrm{I})$ systems which would allow for various ancillary ligands. ${ }^{31}$ The $\mathrm{W}(0)$ is also the most $\pi$-basic of all the metal fragments, which increases 
the stability of the complexes towards many reaction conditions. ${ }^{22}$ Due to this electronic increase, the $\left\{\mathrm{TpW}(\mathrm{NO})\left(\mathrm{PMe}_{3}\right)\right\}$ fragment can $\eta^{2}$-bond the largest range of aromatics, including benzene. The $\eta^{2}$-benzene complex allows for the exchange of benzene for other arenes easily, so it can be used as a generalized starting material. ${ }^{22}$ Frequently these exchanges occur by saturating the benzene complex with another arene, such as furan, naphthalene and 1,3-dimethyoxybenzene, but this is not always possible.

\subsection{Reactivity of $\left[\mathrm{TpW}(\mathrm{NO})\left(\mathrm{PMe}_{3}\right)\left(\eta^{2}-N, N\right.\right.$-dimethylanilinium] and derivatives}

Aniline is an aromatic which does not allow for this simple exchange process. Firstly, the N-H bond of aniline will react before the aromatic ring, leading to metal insertion into the N-H bond (Scheme 1.16). This means that a protected aniline is necessary, such as $N, N$-dimethylaniline. Secondly, aniline is already electron rich, so the metal complex cannot easily donate electrons through back-bonding into the arene to break the aromaticity. This means that a simple exchange of $N, N$-dimethylaniline with the $\mathrm{TpW}(\mathrm{NO})\left(\mathrm{PMe}_{3}\right)\left(\eta^{2}\right.$-benzene) yields only decomposition. In order to stabilize this complex, the addition of diisopropylammonium triflate (DiPAT) to the exchange leads to protonation of the $\mathrm{N}, \mathrm{N}$-dimethylaniline following coordination to produce the conjugate acid (Scheme 1.16). ${ }^{32}$ 
Scheme 1.16. Coordination of Aniline and $N, N$-dimethylaniline to $\left\{\mathrm{TpW}(\mathrm{NO})\left(\mathrm{PMe}_{3}\right)\right\}$

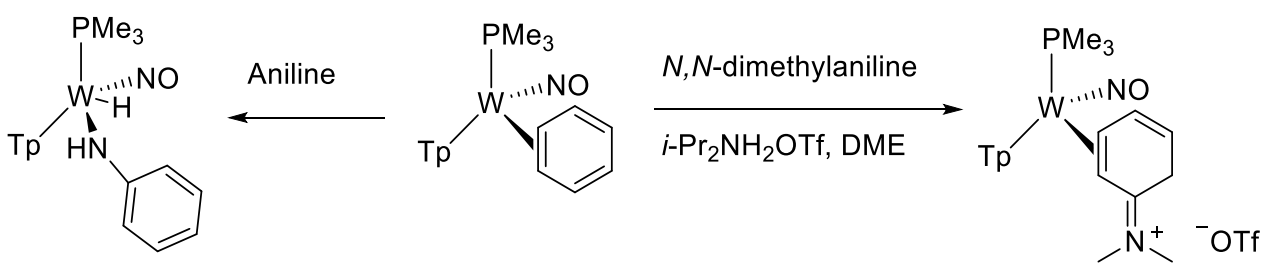

The $\left[\mathrm{TpW}(\mathrm{NO})\left(\mathrm{PMe}_{3}\right)\left(\eta^{2}-N, N\right.\right.$-dimethylanilinium $\left.)\right]$ species was hoped to synthesize novel small aniline derivatives. This complex has unique stability towards many reaction conditions due to the iminium bond stabilization. The iminium acts as an "electron sink", allowing the metal to donate heavily into the positively charged iminium, creating a complex with reduction potentials around $1.3 \mathrm{~V}$ vs NHE. Upon protonation with a strong acid, the anilinium complex quantitatively generates a stable double cationic allylic species. This stable species can then react with fairly weak nucleophiles. This reaction scheme works similarly with other electrophiles, such as Selectfluor ${ }^{\circledR}$ or mCPBA, which react with methanol to yield a tandem addition product. In addition to this, the anilinium complex can be deprotonated in situ with a strong base and the rearomatized ligand can react with alkyl bromides (Scheme 1.17). ${ }^{32,33}$ 
Scheme 1.17. Reactivity of $\left[\mathrm{TpW}(\mathrm{NO})\left(\mathrm{PMe}_{3}\right)\left(\eta^{2}-N, N\right.\right.$-dimethylanilinium] [OTf]

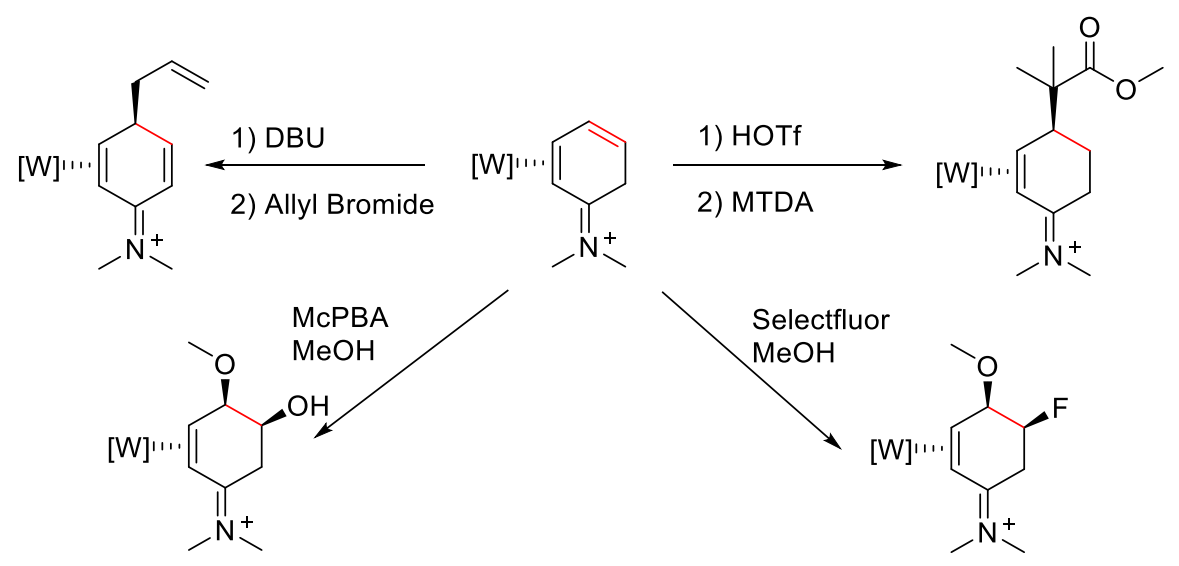

This complex can be cycloproponated via a Simmons-Smith cycloproponation reaction. This new ring system can be ring opened with a strong acid, creating an allylic species which can react with weak nucleophiles (Scheme 1.18). This creates two new stereocenters in contrast to the acid protonation previously reported. ${ }^{34}$

Scheme 1.18. Cycloproponation of $N, N$-dimethylaniline and subsequent ring opening

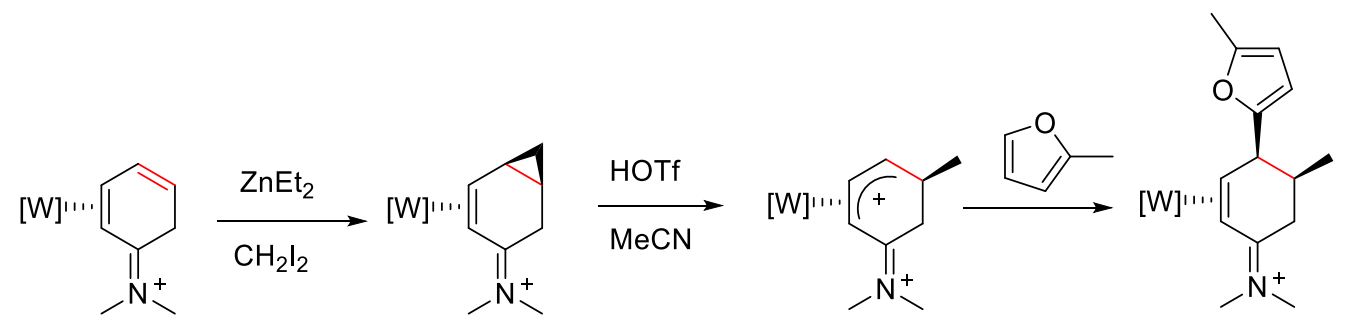

While this system is predictable and reactive, the ability to remove these new organic molecules was found to be difficult. With the reduction potential of these products close to $1.5 \mathrm{~V}$, it is difficult to oxidize the metal systems to release the organics. It was determined that using ceric ammonium nitrate (CAN) as an oxidant could oxidize the system. Unfortunately, CAN requires an aqueous workup which hydrolyzes the 
iminium bond. This transforms the aniline derivatives into enone derivatives. ${ }^{33,34}$ This can be seen in Scheme 1.19 with a 1,3-dimethoxybenzene addition and a cyclopropane opening product. While these are synthetically interesting molecules, they are not accepted into biological libraries for testing because of the $\alpha, \beta$-unsaturated ketone. These are susceptible to reacting with cysteine and DNA nucleobases.

Scheme 1.19. Formation of $\alpha, \beta$-unsaturated ketone organic products from oxidation
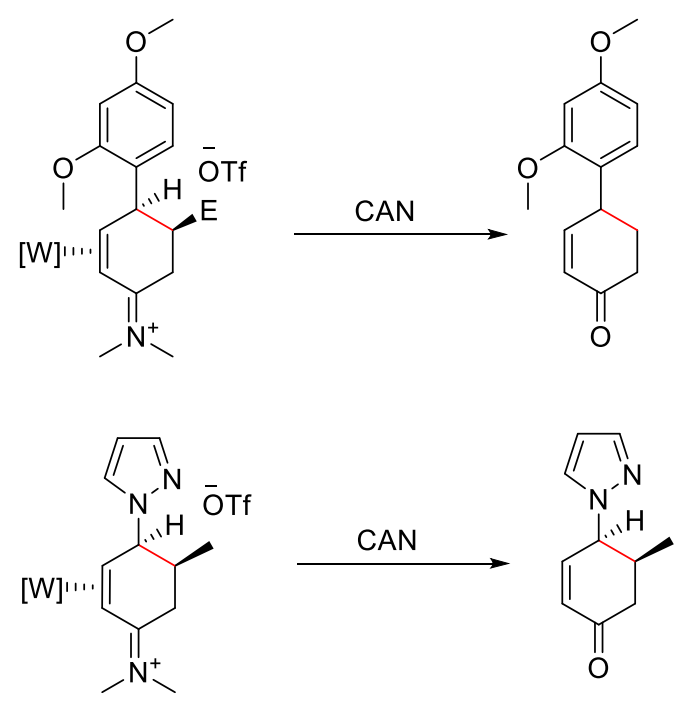

Attempts were made to reduce the iminium bond after tandem additions occurred, though this was unsuccessful. Only the starting anilinium complex was found to be reduced by $\mathrm{NaBH}_{4}\left(\right.$ Scheme 1.20). ${ }^{35}$ Upon reduction of the iminium bond, the electrochemistry shows an $\mathrm{E}_{\mathrm{p}, \mathrm{a}} \approx 0.40 \mathrm{~V}$ vs NHE. This means that weaker oxidants would be viable for removing the organic ligand from the metal system. Attempts to further functionalize this system through tandem addition reactions were unsuccessful. It was however found that the reduced aniline complex would ring turn and reform the iminium upon addition of diphenyl ammonium triflate (DPhAT). Through the addition of the base, 
potassium hexamethyldisilazide (KHMDS), followed by an electrophile, a new position of the aniline complex can be activated, though additions were not generally clean. The most successful addition was with allyl bromide as an electrophile. Isolation of this novel small molecule was never attempted.

Scheme 1.20. Ring turn of aniline system with further reactivity scheme

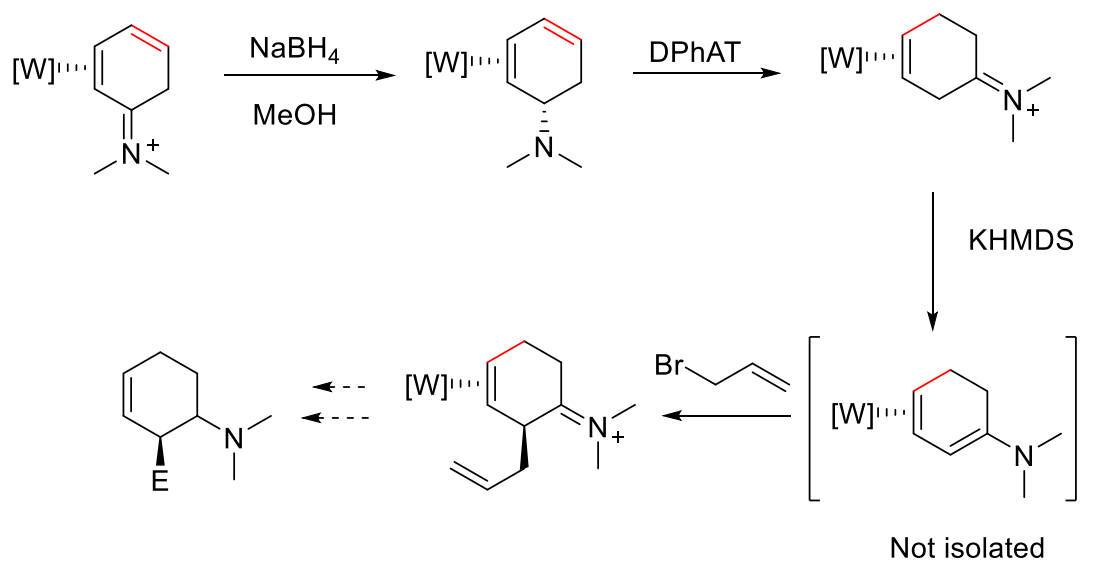

In an attempt to find ways to synthesize the aniline based organic molecules, 2(dimethylamino)pyridine (2-DMAP) and 2-(dimethylamino)pyrimidine were bound to the metal using the same protonation technique as with the $N, N$-dimethylaniline system. ${ }^{36}$ The pyrimidine complex could not be protonated a second time at the desired carbon, but rather protonated at the bound nitrogen. The 2-DMAP complex could be protonated with strong acid and thiophene could be added as a nucleophile. Upon decomplexation with CAN, the iminium was found to remain intact (Scheme 1.21). 
Scheme 1.21. Reactivity of 4-dimethylaminopyridine and 2-(dimethylamino)pyrimidine complexes
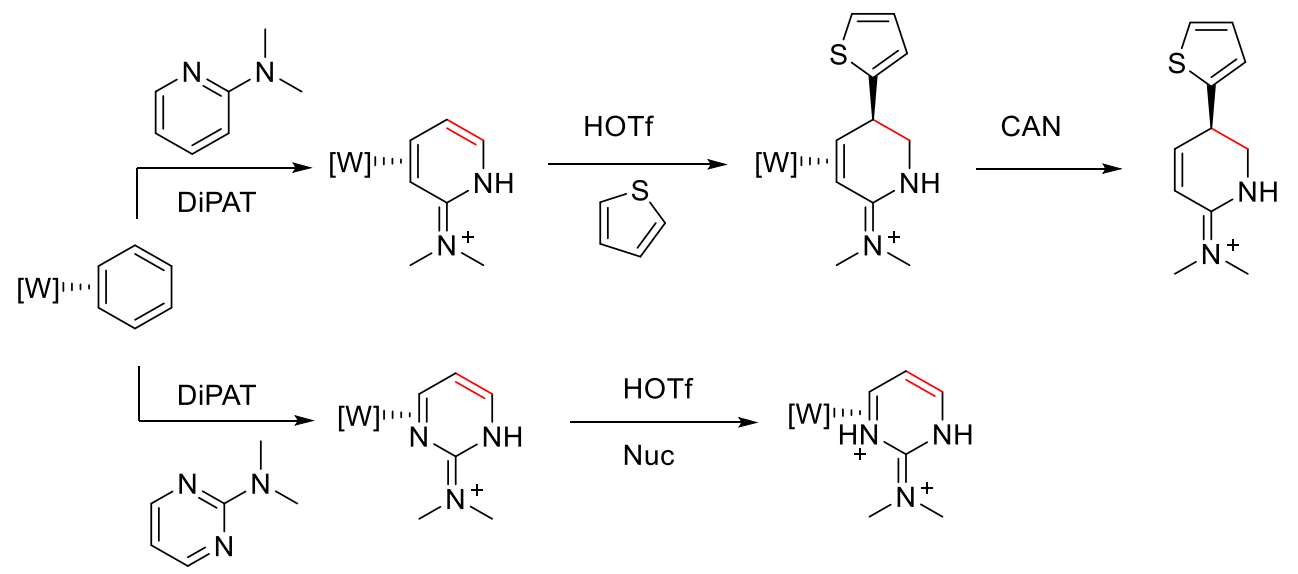

Using the aniline complex as a starting point, a generalization procedure for the synthesis of novel small molecules with alkaloid cores could be imagined. By branching into other with the aniline core, but have saturated rings containing that nitrogen, such as indoline or 1,2,3,4-tetrahydroquinoline, new complexes could be synthesized. For the remaining chapters of this work, the focus will be on research surrounding the indoline system (Scheme 1.22). Through the addition of a bottom 5-membered saturated ring, new chemical space can be explored. Never before has the synthesis of perhydroindoles been explored from indoline itself and through the use of the $\left\{\mathrm{TpW}(\mathrm{NO})\left(\mathrm{PMe}_{3}\right)\right\}$ dearomatization agent this becomes possible.

Scheme 1.22. Synthesis of N-Alkylindolinium complex and possible final organic products

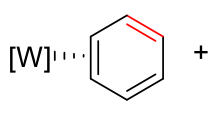<smiles>CC(C)NC(C)C</smiles>
(DiPAT)

1

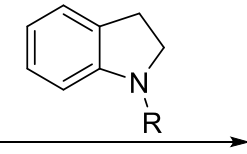<smiles>C1CCCCC1</smiles>

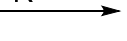

$[\mathrm{W}]$<smiles>[R]N1C=C2C=CC=CC2CC1</smiles>
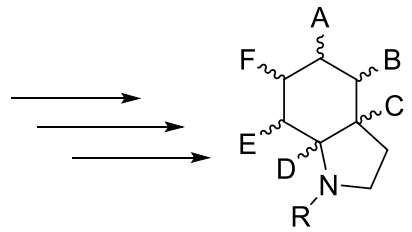


\subsection{References:}

(1) Schleyer, P. v. R.; Pühlhofer, F. Organic Letters 2002, 4, 2873.

(2) Carey, F. A.; Sundberg, R. J. Advanced Organic Chemistry Part B: Reactions and Synthesis; 5th ed.; Springer: New York, 2007.

(3) Carey, F. A.; Sundberg, R. J. Advanced Organic Chemistry Part A: Structure and Mechanisms; Springer: New York, 2007.

(4) Roche, S. P.; Porco, J. A. Angewandte Chemie International Edition 2011, 50, 4068.

(5) Rabideau, P. W. Tetrahedron 1989, 45, 1579.

(6) Ishikawa, K.; Griffin, G. W. Angewandte Chemie International Edition in English 1977, 16, 171.

(7) Wipf, P.; Kim, Y.; Goldstein, D. M. Journal of the American Chemical Society 1995, 117, 11106.

(8) Ohashi, M.; Tanaka, Y.; Yamada, S. Tetrahedron Letters 1977, 18, 3629.

(9) Mortier, J. Arene Chemistry: Reaction Mechanisms and Methods for Aromatic Compounds; 1st ed.; John Wiley \& Sons: New York, 2016.

(10) Motherwell, W. B.; Williams, A. S. Angewandte Chemie International Edition in English 1995, 34, 2031.

(11) Bao, M.; Nakamura, H.; Yamamoto, Y. Journal of the American Chemical Society 2001, 123, 759.

(12) Roell, B. C.; McDaniel, K. F.; Vaughan, W. S.; Macy, T. S. Organometallics 1993, 12, 224.

(13) Pape, A. R.; Kaliappan, K. P.; Kündig, E. P. Chemical Reviews 2000, 100, 2917. 
(14) Pigge, F. C.; Dhanya, R.; Hoefgen, E. R. Angewandte Chemie International Edition 2007, 46, 2887.

(15) Pigge, F. C.; Coniglio, J. J.; Dalvi, R. Journal of the American Chemical Society 2006, 128,3498 .

(16) Kündig, E. P.; Cannas, R.; Laxmisha, M.; Ronggang, L.; Tchertchian, S. Journal of the American Chemical Society 2003, 125, 5642.

(17) Keane, J. M.; Harman, W. D. Organometallics 2005, 24, 1786.

(18) Brooks, B. C.; Brent Gunnoe, T.; Dean Harman, W. Coordination Chemistry Reviews 2000, 206-207, 3.

(19) Meiere, S. H.; Brooks, B. C.; Gunnoe, T. B.; Carrig, E. H.; Sabat, M.; Harman, W. D. Organometallics 2001, 20, 3661.

(20) Meiere, S. H.; Keane, J. M.; Gunnoe, T. B.; Sabat, M.; Harman, W. D. Journal of the American Chemical Society 2003, 125, 2024.

(21) Mocella, C. J.; Delafuente, D. A.; Keane, J. M.; Warner, G. R.; Friedman, L. A.; Sabat, M.; Harman, W. D. Organometallics 2004, 23, 3772.

(22) Graham, P. M.; Meiere, S. H.; Sabat, M.; Harman, W. D. Organometallics 2003, 22,4364 .

(23) Harman, W. D. Chemical Reviews 1997, 97, 1953.

(24) Harman, W. D.; Taube, H. Journal of the American Chemical Society 1988, 110, 7906.

(25) Smith, P. L.; Chordia, M. D.; Dean Harman, W. Tetrahedron 2001, 57, 8203.

(26) Kopach, M. E.; Gonzalez, J.; Harman, W. D. Journal of the American Chemical Society 1991, 113, 8972.

(27) Kolis, S. P.; Gonzalez, J.; Bright, L. M.; Harman, W. D. Organometallics 1996, $15,245$. 
(28) Keane, J. M.; Chordia, M. D.; Mocella, C. J.; Sabat, M.; Trindle, C. O.; Harman, W. D. Journal of the American Chemical Society 2004, 126, 6806.

(29) Myers, J. T.; Shivokevich, P. J.; Pienkos, J. A.; Sabat, M.; Myers, W. H.;

Harman, W. D. Organometallics 2015, 34, 3648.

(30) Welch, K. D.; Harrison, D. P.; Lis, E. C.; Liu, W.; Salomon, R. J.; Harman, W. D.; Myers, W. H. Organometallics 2007, 26, 2791.

(31) Ha, Y.; Dilsky, S.; Graham, P. M.; Liu, W.; Reichart, T. M.; Sabat, M.; Keane, J. M.; Harman, W. D. Organometallics 2006, 25, 5184.

(32) Salomon, R. J.; Todd, M. A.; Sabat, M.; Myers, W. H.; Harman, W. D. Organometallics 2010, 29, 707.

(33) Pienkos, J. A.; Zottig, V. E.; Iovan, D. A.; Li, M.; Harrison, D. P.; Sabat, M.; Salomon, R. J.; Strausberg, L.; Teran, V. A.; Myers, W. H.; Harman, W. D. Organometallics 2013, 32, 691.

(34) Pienkos, J. A.; Knisely, A. T.; Liebov, B. K.; Teran, V.; Zottig, V. E.; Sabat, M.; Myers, W. H.; Harman, W. D. Organometallics 2014, 33, 267.

(35) Salomon, R. J., University of Virginia, 2010.

(36) Pienkos, J. A.; Knisely, A. T.; MacLeod, B. L.; Myers, J. T.; Shivokevich, P. J.; Teran, V.; Sabat, M.; Myers, W. H.; Harman, W. D. Organometallics 2014, 33, 5464. 


\section{Chapter 2:}

\section{A Brief Overview of Perhydroindole}

Alkaloid Chemistry 


\subsection{Indoles and Indoline}
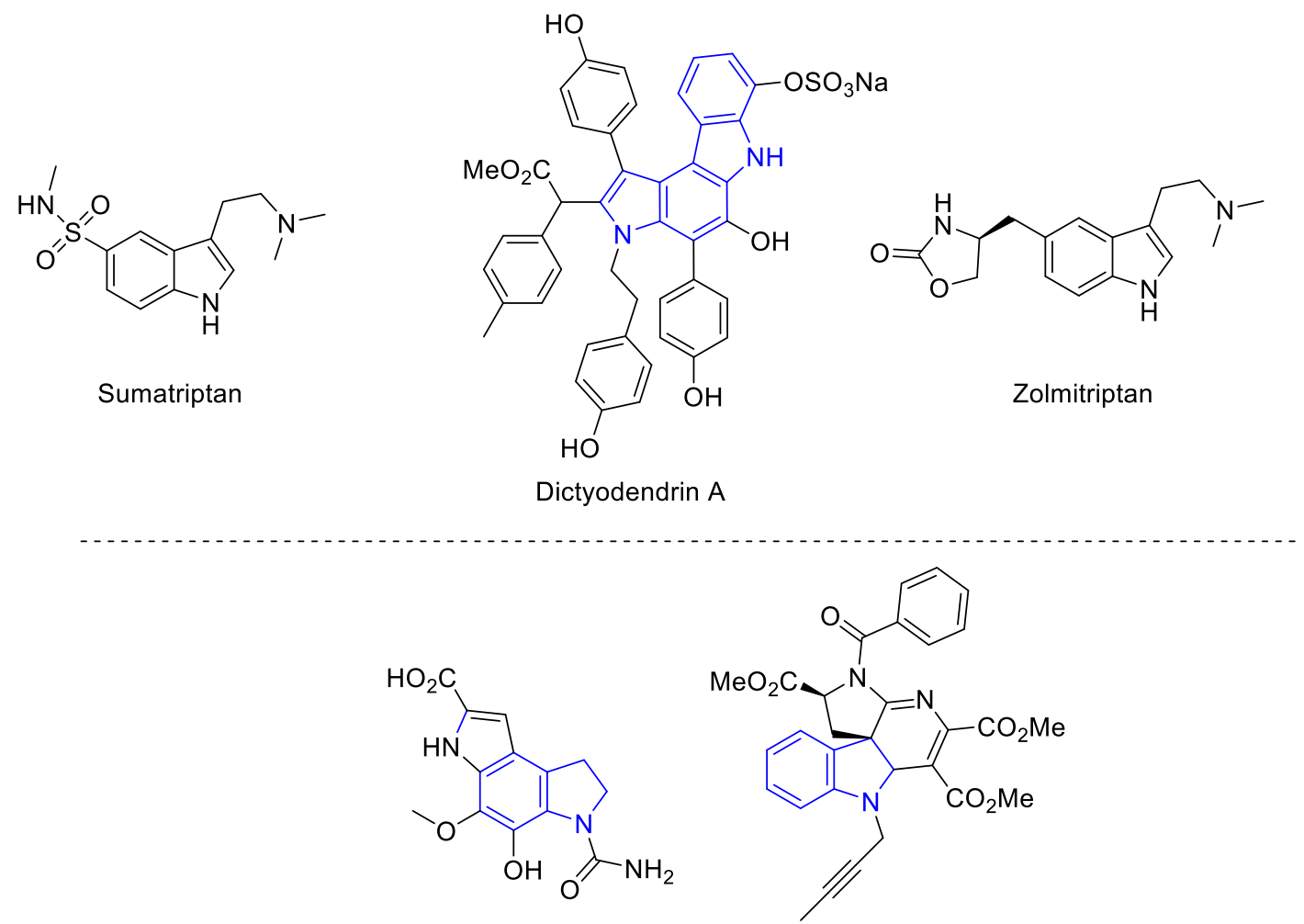

PDE-I

CMLDBU3402

Figure 2.1. Natural products containing indole and indoline cores.

The bicyclic indole structure is common in nature and also in synthetic processes.

This family has 3 subsets: the fully aromatized indole, the partially hydrogenated indoline, and the fully dearomatized perhydroindole. Of these, the aromatized systems are much simpler to synthesize, due to their innate stability. Indoles, specifically, have known biological activity. They are believed to have a large effect in the central nervous system. ${ }^{1}$ An example of this would be the triptan family. Examples of these are sumatriptan (Imitrex) from Glaxco and zolmitriptan (Zomig) from AstraZeneca, both migraine medications (Figure 2.1). They are serotonin receptor agonists. More complex 
systems also are used medicinally, for example dictyodendrin A and B are telomerase inhibitors that are being investigated as potential cancer chemotherapy agents. ${ }^{2}$ The number of novel structures are limited however by the aromatic core. There are only a limited number of substitutions around that ring and the aromatic core restricts addition reactions. There is also no stereochemistry surrounding the central core.

Partially hydrogenated indolines are the structural component of several pharmaceutical compounds that are ACE inhibitors and antihypertensive drugs. ${ }^{3,4}$ PDE-I and PDE II are inhibitors of cyclic adenosine-3',5'-monophosphate phosphodiesterase, an important messaging system in cells (Figure 2.1). ${ }^{5}$ CMLDBU3402 is a potential treatment for non-segmented negative-strand (NNS) RNA viruses. ${ }^{6}$ These NNS viruses include Ebola, rabies and measles. While these systems have promising biological activity, they are limited in the fact that they have few stereocenters around the indole core due to their maintained aromaticity.

\subsection{Escaping Flatland}

Since Lipinski's rule of 5 was introduced in 1997, the way drug candidates have been evaluated has changed. Lipinski and his colleagues analyzed thousands of drugs and drug candidates to determine what common characteristics they contain. These rules included a molecular weight below 500, a factor for water solubility called $\log \mathrm{P}$, and restrictions on the number of hydrogen bond donors and acceptors. ${ }^{7}$ These rules were intended to help ensure that drug candidates could break through cell membranes and be bioavailable. This system is not perfect however. Over time, the Lipinski rule of 5 has 
morphed into a system for organic chemists to evaluate lead molecules, which is not what it was designed for. Some chemists believe the concept of "molecular obesity" stemmed from chemists' goal to make compounds fit into Lipinski's rules by increasing the lipophilicity of drugs. ${ }^{8}$

In recent years there has been a push for the creation of a new system of lead molecule identification. Hopkins designed a mathematical study that provided a continuous quantitative estimate of drug-likeness (QED) on a scale of what was most drug-like. ${ }^{9}$ This system never fully integrated itself into the medicinal chemistry lexicon. A system which has become more popular is the absorption, distribution, metabolism, excretion and toxicity (ADMET) screening process. ${ }^{10}$ As the name implies, this system is able to determine if a compound will be absorbed by the body, how it will distribute itself throughout the body, how it will be broken down and removed, and also how those metabolites will affect the body. In one particular study, this system was applied along with a parallel structure-activity relationship (SAR) study during the lead generation. ${ }^{11}$ The conclusion from this study introduces the idea of molecular topology being included into this classification, calling into question the idea of aromatic molecules being used as the backbone for drug candidates. Using this system, a new area of drug candidates can be explored.

Another fact which drew into sharp focus the use of aromatics in medicinal chemistry was the discovery that aromatic molecules actually have toxic side effects after being processed by the metabolism. This toxicity is most frequently seen specifically in reference to aniline-based systems. These must be deactivated towards metabolism via 
the incorporation of electron-withdrawing groups; otherwise the metabolites formed are toxic. Some heterocycles are found to be susceptible to nucleophilic attack (pyridine) and oxidation (thiophene), leading to side reactions in the body. ${ }^{12}$ These systems have a low bioavailability due to low solubility in aqueous medium. As mentioned previously with indole, there is also a limitation of the number of compounds available for synthesis when using an aromatic.

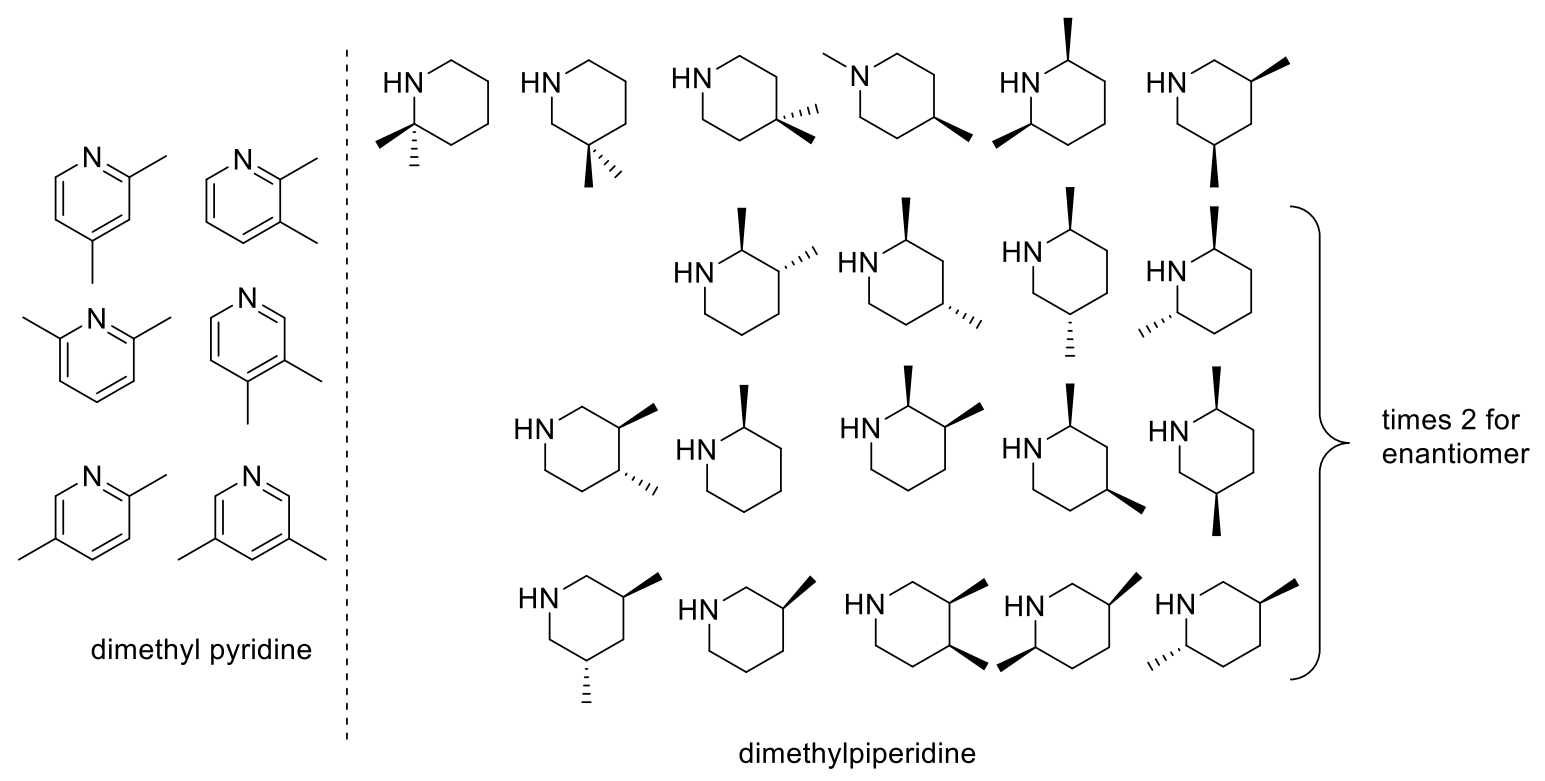

Figure 2.2. Isomers of dimethyl pyridine and dimethylpiperidine.

A new system which includes factors such as the fraction of $\mathrm{sp}^{3}$-hybridized carbons (Fsp3) and chiral atom counts, now suggests that a more three-dimensional structure is more likely to be biologically active. This concept of having chemists "Escape from Flatland" 13 was explored more in depth by directly exploring the increased saturation of compounds as an approach to improving clinical success. A simple example of this concept can be seen when looking at the number of isomers available to dimethyl pyridine and saturated analogues of dimethylpiperidine (Figure 2.2). For 6 isomers of 
dimethyl pyridine, there are 34 isomers of dimethylpiperidine. These all have similar molecular weights, but the number of saturated carbons increases the ability to design the molecule for specific moieties. This is a simple example, but it shows the effect of saturating a ring for allowing a much broader area of exploration. For a more clinical example, Lovering and coworkers explored the GVK BIO database and examined the Fsp3 for various stages of drug development. They determined that as drug development stages progressed, it was statistically significant that the Fsp3 increases, along with the number of chiral centers. This means that chirality is important when biologically interesting molecules are explored. This means that the saturated counterparts are more biologically active overall, not only because there are more options.

This need for chirality and saturated carbons is reflected in alkaloid chemistry. While some unsaturated rings are naturally found, such as ergoline rings including an indole, many systems have almost fully saturated cores. One example would be perhydroindoles. Perhydroindoles can be found in numerous alkaloid systems, including Stemona, Amaryllidaceae, Sceletium and Aeruginonsin alkaloids. Each of these systems will be explored further, both in their biological uses and their synthetic processes.

\subsection{Perhydroindoles}

In comparison to the aromatic analog, the synthesis of perhydroindoles are much more difficult due the sensitivity of the final product. These systems are sensitive to acid, base, and heat. This limits the number of synthetic pathways in order to make novel compounds of this nature. The chemical pathways that are necessary to synthesize these 
are very complex and most work related to perhydroindoles is focused on making natural products, rather than analogs. There are few examples of non-biologically focused work with this system, but one example shows how the perhydroindole skeleton can be used as a ligand for catalytic asymmetric transformations or in reversible organic hydrogen storage liquids for hydrogen-powered fuel cells. ${ }^{14}$

\subsection{Stemona Alkaloids}

Stemona alkaloids are derived from the Stemonacae family. The Stemona family represents a class of polycyclic alkaloids with complex structures that were originally used in Chinese and Japanese folk medicine for the treatment of respiratory diseases. ${ }^{15}$ They are claimed to have antituberculosis, antibacterial, antifungal and anthelmintic properties. This system has over 130 alkaloids which have been found so far. They have been split up into 8 groups: stenine (I), stemoamide (II), tuberostemospironine (III), stemonamine (IV), parvistemoline (V), stemofoline (VI), stemocurtisine (VII) and a miscellaneous group (Figure 2.3). ${ }^{16}$ The stenine core consists of a perhydroindole core. 

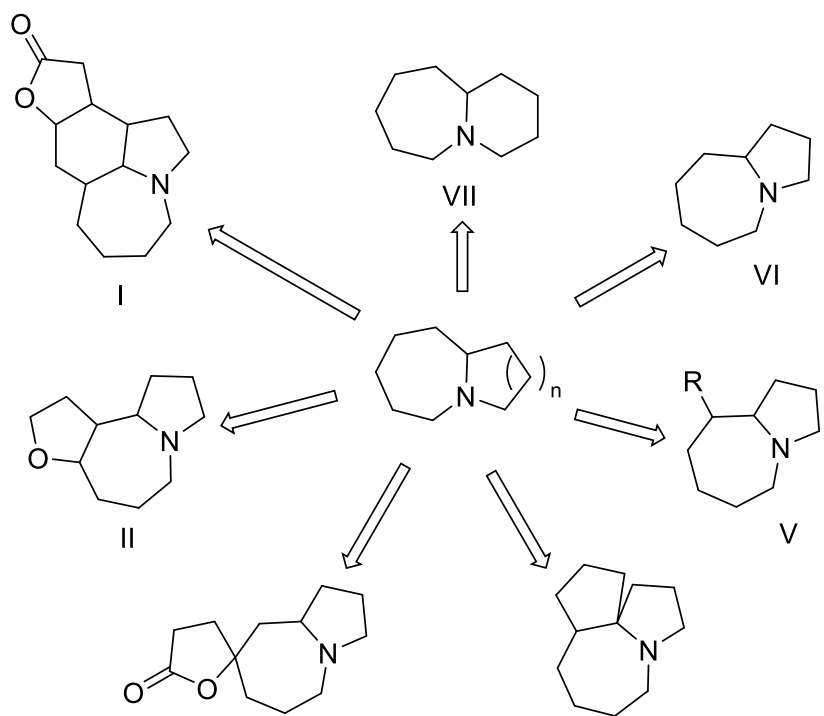

III

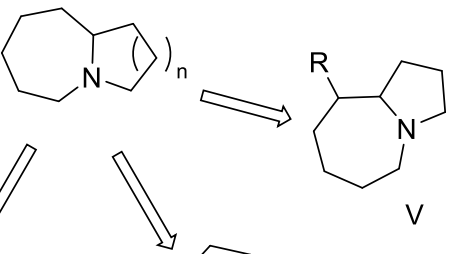

Figure 2.3. Naturally found cores of Stemona alkaloids.

The stenine group itself is comprised of 21 compounds. The compounds which most closely resemble perhydroindoles are stenine and tuberostemonine (Figure 2.4). These have fully saturated central cores with nitrogen next to a bridgehead in the 5membered ring. Everything in this family has been identified using ${ }^{1} \mathrm{H}$ and ${ }^{13} \mathrm{C}$ NMR. ${ }^{16}$ All compounds in this group have very specific stereochemistry. A trans-linked ring juncture is prevalent, though cis-fused ring systems are being discovered as well. Recently there has been a resurgence of research towards synthesizing this scaffold due to the discovery that neostenine, a stereoisomer of stenine, has antitussive activity comparable to codine. ${ }^{17}$ 


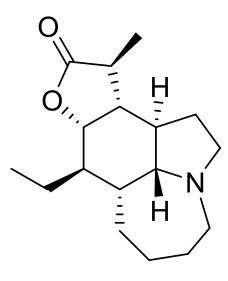

Stenine

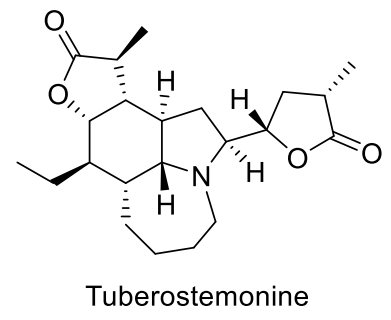

Figure 2.4. Naturally found stenine alkaloids

\subsubsection{Stenine Synthesis}

Wipf and coworkers ${ }^{18}$ developed an elegant synthesis of (-)-stenine, one of the two stereoisomers of stenine. They were the first to develop a method to asymmetrically synthesize the system. Their retrosynthetic thought process can be seen in Scheme 2.1, showing their work beginning with the amino acid L-tyrosine. Tyrosine is cyclized, using a $\mathrm{PdI}(\mathrm{OAc})_{2}$ catalyst, creating a cis-linked junction with one position having a hydroxyl group instead of the desired trans-linked hydrogen bridgehead. Using a $\mathrm{Pd}_{2}(\mathrm{dba})_{3} \cdot \mathrm{CHCl}_{3}$ catalyst, this hydroxyl is exchanged for hydrogen, creating the trans-linked junction that is necessary for the stenine core.

Scheme 2.1. Retrosynthetic process of stenine beginning with L-tyrosine

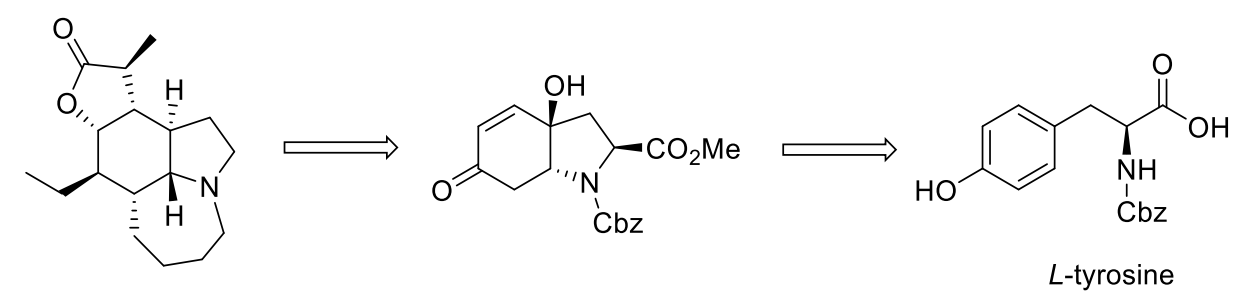

Almost simultaneously Morimoto and associates ${ }^{19}$ developed another asymmetric synthesis of (-)-stenine. Their retrosynthetic thought can be seen in Scheme 2.2. Their synthesis required an intramolecular asymmetric Diels-Alder reaction to create a tricyclic 
core, which is then rearranged using $m$-chloroperpenzoic acid followed by subsequent oxidative cleavage of the resulting ketone with orthoperiodic acid. Finally, an in situ iodolactonization forms the central perhydroindole core. The selectivity of the system produces the trans-linked juncture which is important to stenine. This procedure was believed to be applicable later towards tuberostemonine.

Scheme 2.2. Retrosynthetic process of stenine through an intramolecular Diels-Alder
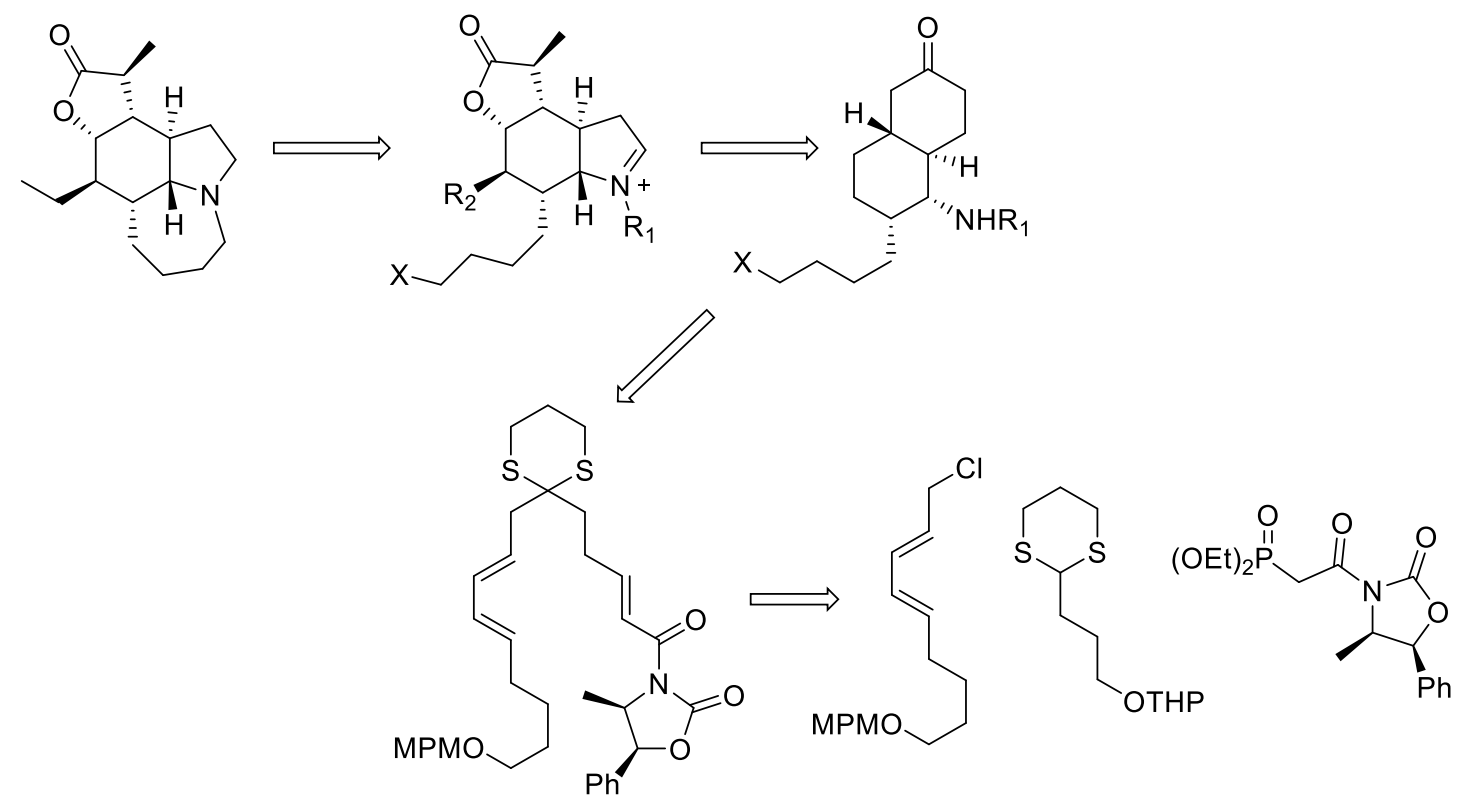

Aube and $\mathrm{Zeng}^{20}$ developed a rapid and efficient route to produce the central core of stenine using a domino reaction combining an intermolecular Diels-Alder reaction with a Schmidt reaction. The key step in this reaction scheme yields the ring juncture, though only in a 3:1 ratio of trans to cis. This can be seen in Scheme 2.3. This system however has almost double the yield of any other overall synthesis to this compound, $14 \%$ verses other syntheses which range from $0.9-7.2 \%$ overall. 
Scheme 2.3. Stenine synthesis through a domino synthesis

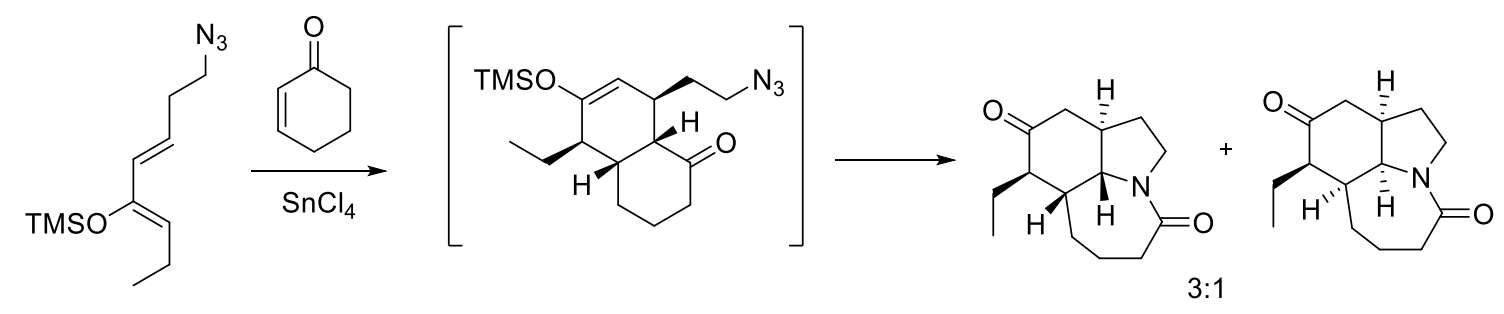

1

\subsubsection{Tuberostemonine Synthesis}

The Wipf group created an asymmetric total synthesis of tuberostemonine and similar derivatives. Using a similar technique as with stenine, using L-tyrosine as the starting material and creating the central trans-linked core using two palladium catalysts, they were able to synthesize tuberostemonine. ${ }^{18,21}$ The main difference is that instead of removing the ester group adjacent to the nitrogen, this was elaborated into a lactone. Tuberostemonine is a more complex system than stenine, so the synthetic methods involved additional steps, but they were successful in isolating tuberostemonine with a 27 step synthesis and a $1 \%$ overall yield from Cbz-L-tyrosine. ${ }^{22}$ They were also able to synthesize didehydrotuberostemonine and 13-epituberostemonine, systems which include a rearomatized pyrrole ring. 
Scheme 2.4. Tuberostemonine synthesis from the Wipf group

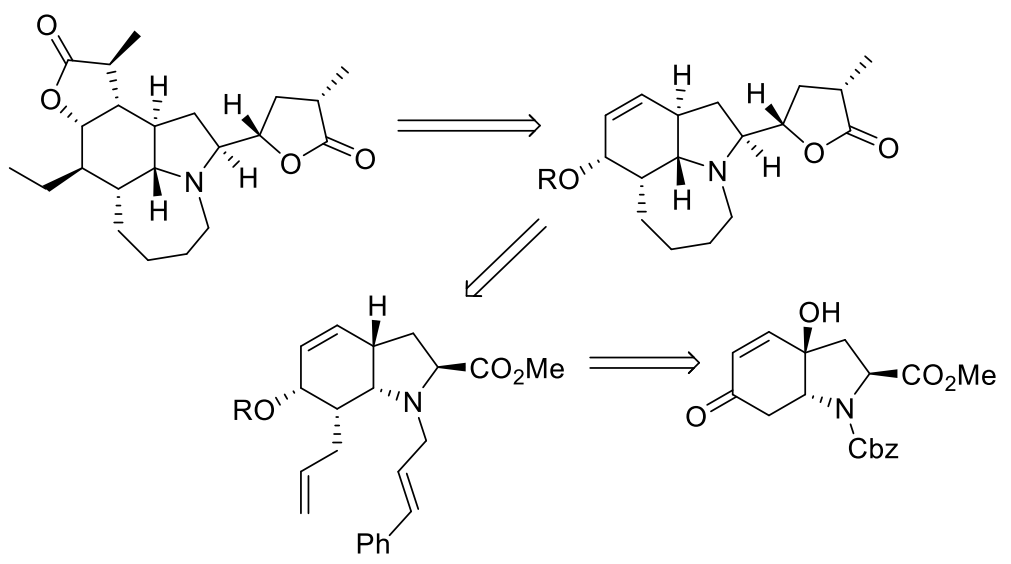

\subsection{Amaryllidaceae Alkaloids}

This type of alkaloid comes from the plants of the Amaryllidaceae family. These plants are well-known ornamental flowering plants, such as amaryllis, daffodils and snowdrops. ${ }^{23}$ These species have long been investigated for their medicinal uses, due to their prevalence in traditional Chinese medicine. Anticancer activity of these extracts were recorded in the first century $\mathrm{AD}$ and even in the Bible there are references to these plants' medicinal uses. ${ }^{24}$ The most famous compound from this family is galanthamine. This compound is currently a commercialized Alzheimer's disease treatment under the name of Razadyne. ${ }^{25}$ Originally, this compound was used in traditional medicine to ease nerve pain and prevent paralysis from polio. The wide biological activity stems from the compound's ability to cross the blood-brain barrier. ${ }^{23}$ Galanthamine does not have the perhydroindoline core, but rather a quinolone core. The second-most common alkaloid from this family, lycorine, has the perhydroindoline core which is relevant to this work. This compound and its subgroup have been investigated for their antitumor activity. They 
were found to induce apoptosis in human leukemia cells. ${ }^{26}$ Other alkaloids from this family show promising anti-cancer effects against human glioblastoma multiforme (GBM) tumors. Non-medicinal uses have also been investigated as insecticide-like compound, to provide plants protection from pests with nervous systems. ${ }^{23}$

\subsubsection{Lycorine Synthesis}

Scheme 2.5. (+)-Lycorine retrosynthetic thought of Schultz

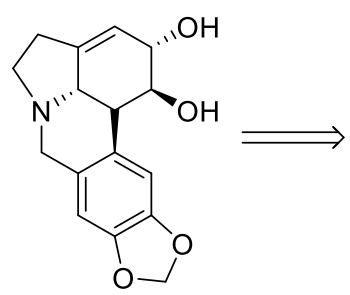

Lycorine
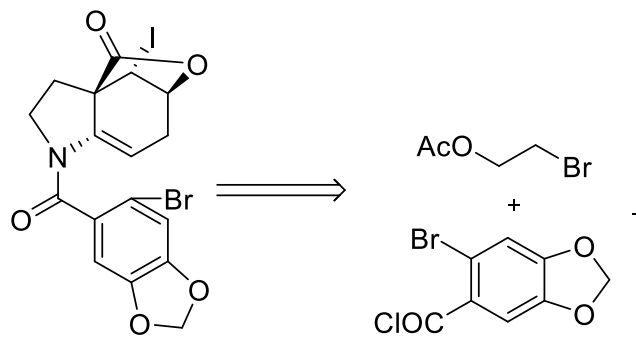

Lycorine was the first alkaloid isolated from the Amaryllidaceae plant family. In recent years there has been a resurgence of work towards the synthesis of Lycorine and its derivatives due to the discovery of its anticancer activity. ${ }^{27,28}$ In 1996 , Schultz and associates $^{29}$ discovered the first asymmetric total synthesis of (+)-lycorine, the unnatural enantiomer. The retrosynthetic thought process can be seen in Scheme 2.5. The key step to making the central multiringed structure comes from a Birch reduction-alkylation. This creates a cyclohexanone, which upon treatment with triphenyphosphine gave an enantiopure immine. This is then isomerized into an enamine to continue the synthesis to reach the multiringed system through a chiral enamide cyclization. This step is interesting due to its inclusion of a perhydroindole intermediate, which is unusual. 
A total synthesis of the naturally found (-)-lycorine enantiomer was reported by Tomioka and co-workers. ${ }^{30}$ Their work depends on a chiral ligand controlled asymmetric cascade conjugate addition reaction. This key step is shown in Scheme 2.6. The chiral ligand mediates an asymmetric conjugate addition which enantioslectively forms the cyclohexane intermediate, which can be transformed into the final 5-ringed system.

Scheme 2.6. Key step in synthesis of (-)-lycorine
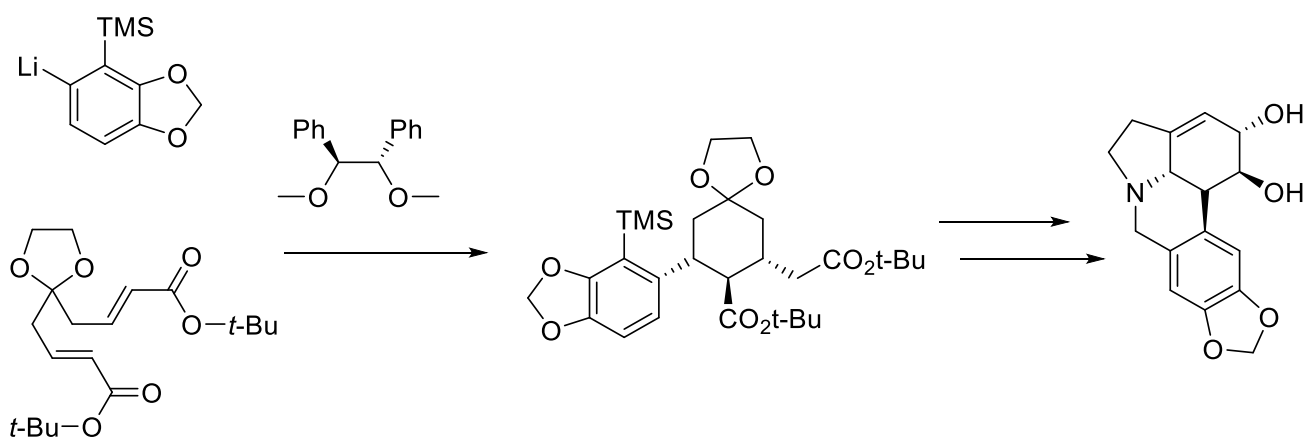

Later that year, Banwell et $a l^{31}$ stereoselectively synthesized the central core of lycorine using chemoenzymatic techniques. They specifically focused on a degradation product, which might lead to further drug-leads. Their synthesis begins with an enzymatically dearomatized bromo-benzene derivative. The entire synthesis depends on the starting material already being dearomatized into a cyclic-diene for further elaboration. The central structure is synthesized in only 6 steps, which is one of the simpler schemes for this compound (Scheme 2.7).

Scheme 2.7. Simplest retrosynthetic thought for lycorine<smiles>O[C@H]1C=C2C3=C[C@H](O)CC(=C1)N(CC3)Cc1cc3c(cc12)OCO3</smiles> 


\subsubsection{Crinine Synthesis}

Crinine is also a common alkaloid from this family. To synthesize this natural product, the Cho group ${ }^{32}$ used a Diel-Alder reaction in order to create a bicyclic core starting from a 3,5-dibromo-2-pyrone as the enophile. This system has been used to make other natural products. This can be seen in pathway A of Scheme 2.8. LeBeuf and coworkers took a very unique approach. They are one of few research programs to focus on the creation of the 6-5 ring system, what they labeled as a azabicyclo[4.3.0]nonane core. This was in order to expand the possible perhydroindoles that could be synthesized. In order to achieve this, they begin with activated arenes and use a "BRAD" method: a Birch reductive alkylation-desymmetrization sequence. ${ }^{33}$ This can be seen in pathway B in Scheme 2.8.

Scheme 2.8. Synthetic thought for the synthesis of Crinine

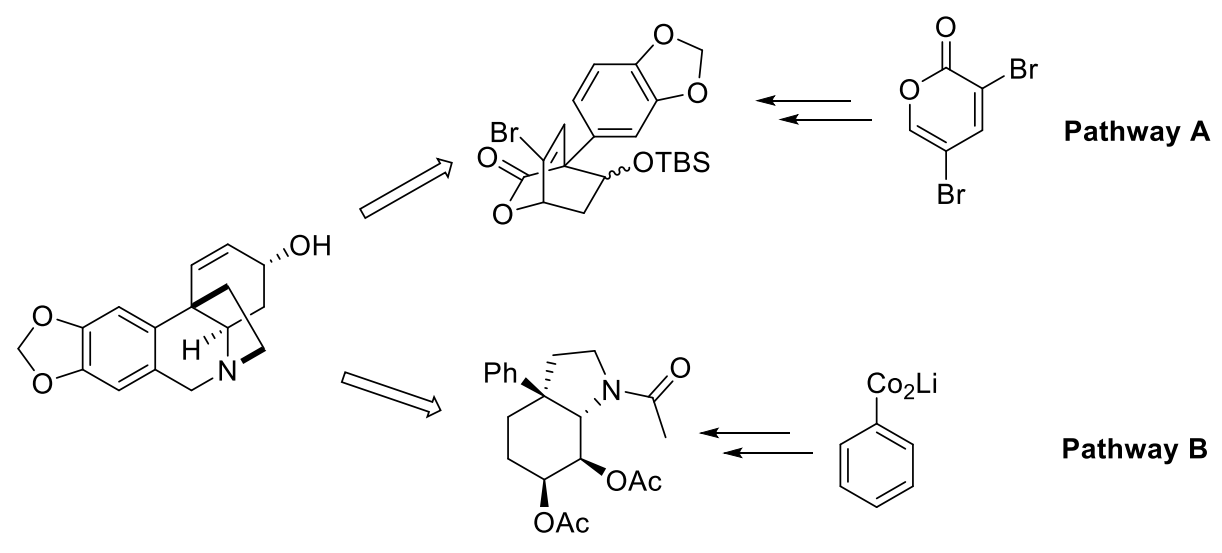

\subsection{Sceletium Alkaloids / Mesembrine Synthesis}

Alkaloids which stem from the Sceletium plant have been shown to have mood elevation and anti-anxiety properties. ${ }^{26}$ These are not as well characterized as several of 
the previous alkaloid families. Most focus has been on the compound mesembrine. This is a serotonin uptake inhibitor (SSRI). The central problem with the synthesis of this compound lies in the sensitivity of the perhydroindole moiety and the quaternary carbon bridgehead.

Taber and Neubert ${ }^{34}$ established the ternary center with high purity using a conjugate addition with a Grignard reagent (Scheme 2.9). This center is then inverted later in the synthesis using a strong base to establish a quaternary carbon. The perhydroindole is not synthesized until the final step though an amination, oxidation and cyclization with methyl amine and magnesium oxide to yield the desired (-)-mesembrine. This is a common theme in the synthesis of this compound: the perhydroindole being synthesized at the last step. ${ }^{35,36}$

Scheme 2.9. Retrosynthetic thought of mesembrine by Taber

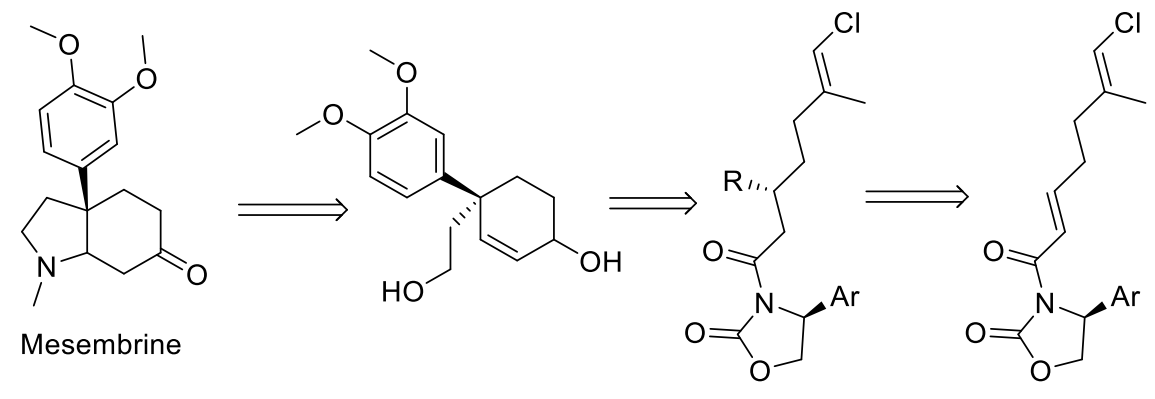

Evans and Geoghegan ${ }^{37}$ use a unique approach to this synthesis from the terpene (s)-(-)-perillyl alcohol. By using a diastereo- and regioselective Pd-mediated intramolecular Heck reaction, a more complex intermediate is formed, which holds the central structure of mesembrine. This can be seen in Scheme 2.10. They also introduce the fully formed perhydroindole prior to the final step, though still at the end of the synthetic scheme. 
Scheme 2.10. Synthesis of mesembrine through $\mathrm{Pd}$-mediated reaction
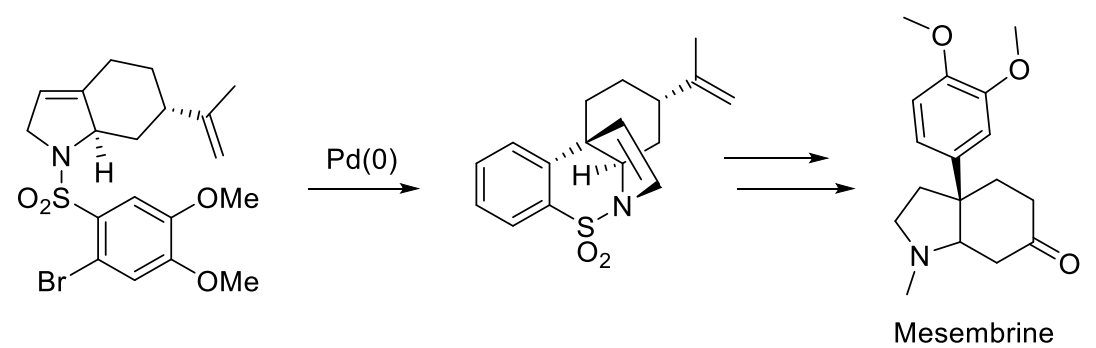

\subsection{Aeruginosin Alkaloids}

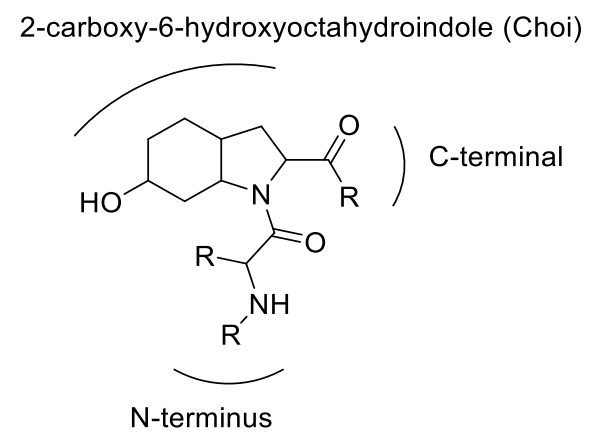

Figure 2.5. General structure of Aeruginosin family

This class of alkaloid is found in both toxic and nontoxic strains of blue-green algae, particularly Microcystis aeruginosa. This family has the largest number of compounds which are closely related to simple perhydroindoles. Nearly all aeruginosins have a central core with a 2-carboxy-6-hydroxyoctahydroindole (Figure 2.5). This is also referred to as the L-Choi amino acid. This family is very interesting from a biological point of view due to the wide variety of inhibitory activity against serine proteases, specifically with respect to blood coagulation. ${ }^{38}$ 


\subsubsection{Aeruginosin 298-A Synthesis}

The most common alkaloid, and first one identified, in this family is aeruginosin 298-A (Figure 2.6). The discovery of this compound introduced a whole new class of peptidic serine protease inhibitors which has led to numerous studies into small molecules for inhibition. Murakami ${ }^{39}$ and associates first published the isolation and structural elucidation of this system in 1994. This identification occurred via 2D NMR techniques including COSY, NOESY and HMBC. Soon after this identification, Bojoch ${ }^{40}$ and coworkers reported the first synthesis of the octahydroindole core, later referred to as perhydroindole (Scheme 2.11). This particular synthesis began with L-tyrosine, followed by a birch reduction. The ring is closed via acid treatment. Though this synthesis created two enantiomers, it allowed for a fused ring core. What is unique about this system is that the perhydroindole core is synthesized early in the scheme, very different than previous alkaloid syntheses.

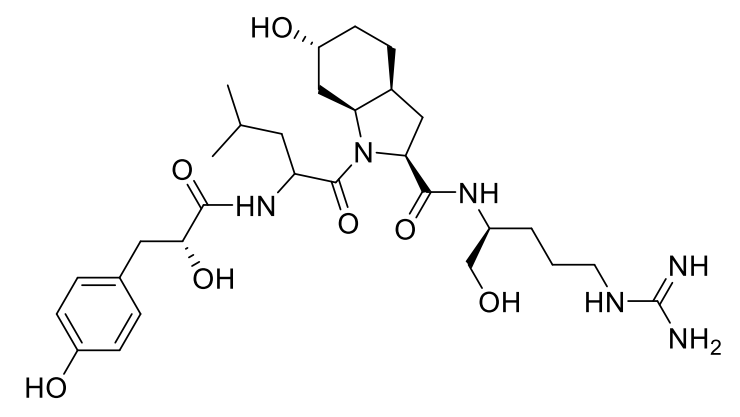

Figure 2.6. Full structure of aeruoginosin $298-\mathrm{A}$

Scheme 2.11. Synthesis of perhydroindoline core in aeruoginosin 298-A

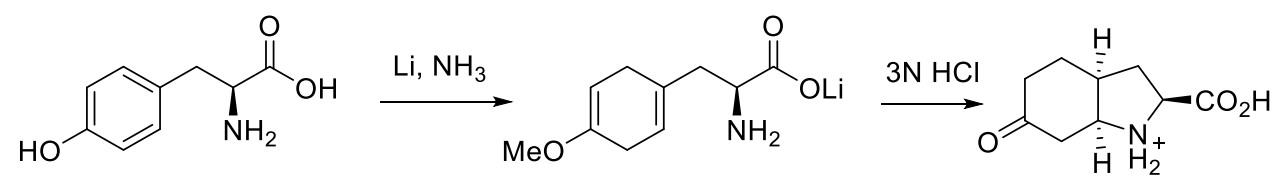


Wipf and Methot $^{41}$ reported a total stereoselective synthesis for (+)-aeruoginosin 298-A, the unnatural version of this alkaloid. Again this work began with a L-tyrosine residue, similar to previous work from Wipf highlighted previously. The key step of the synthesis focuses on the use of the oxidant $\mathrm{PhI}(\mathrm{OAc})_{2}$ to stereoselectively create the fused ring core in a >98:2 dr. This synthesis begins to show the sensitivity of this central perhydroindole structure to reaction conditions. Numerous protecting groups were necessary to complete the synthesis, and yields were moderate at best.

Little work has focused solely on the synthesis of the octahydroindole core. Only recently, Baudoin ${ }^{42}$ and coworkers investigated this type of synthesis via intramolecular C-H alkenylation reactions using a $\mathrm{Pd}(\mathrm{OAc})_{2}$ catalyst. This was a first attempt at isolating such a molecule without making an aromatic indoline core. This was successful and was later applied to the total synthesis of aeruoginosin 298-A.

\subsubsection{Dysinosin A Synthesis}

Dysinosin A is a peptidic thrombin inhibitor. This compound includes a D-Leu residue and an additional hydroxyl group on the octahydroindole core. Most work focused on dysinosin A has come from the Hanessian group. ${ }^{43}$ Their retrosynthetic thought can be seen in Figure 2.7 through breaking the compound in three sections. This shows the starting materials broken down into a perhydroindole core (A), butyrolactone (C), D-leucine and D-mannitol (B). The octahydroindole uniquely is made from Lglutamic acid. Following the synthesis of the pyrrolidine core, the full indole core is synthesized through an elegant use of Grubbs catalyst through an olefin metathesis. This 
allows for the stereoselective ring closing creating the perhydroindole core, in a trans fashion. This scheme is unique due to the creation of the perhydro core prior to the completion of the compound.

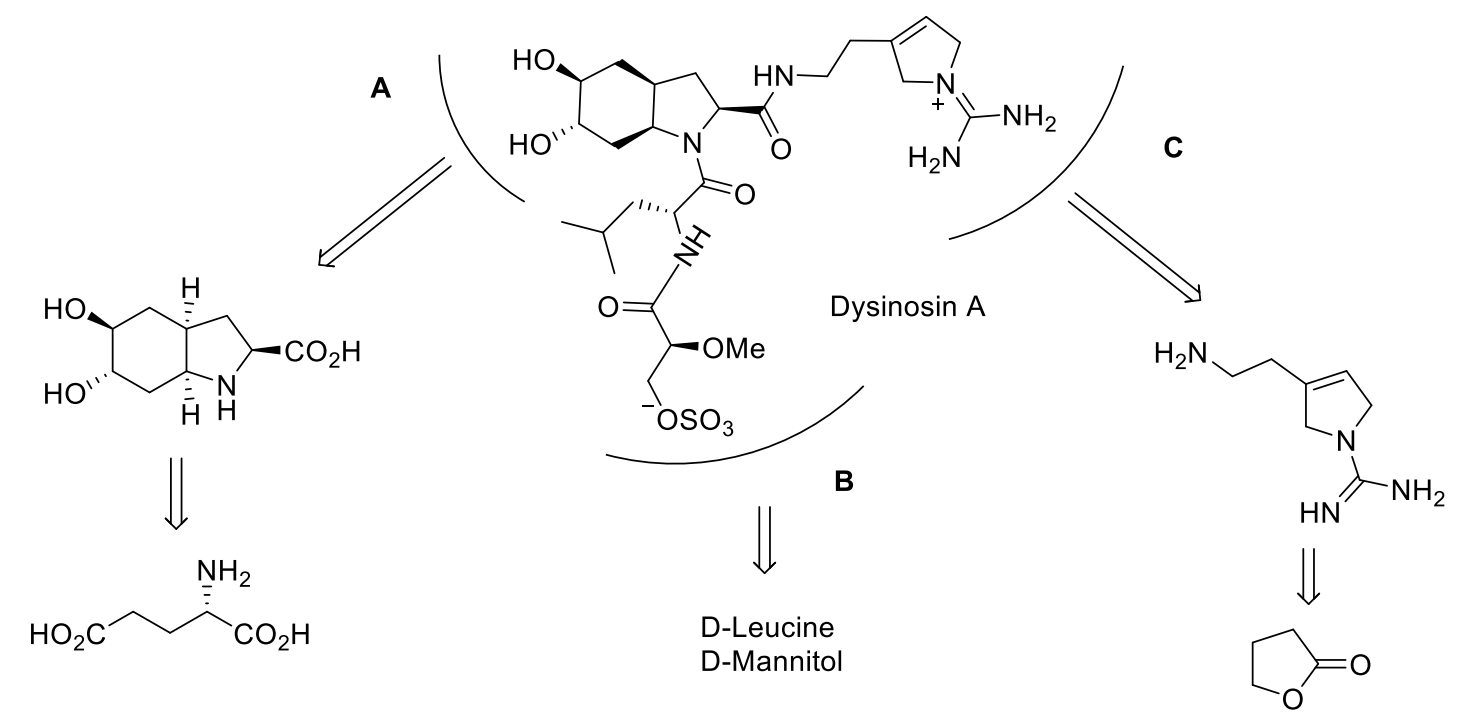

Figure 2.7. Retrosynthesis of dysinosin A

\subsection{Dearomatization of Indoles ${ }^{44}$}

It is important to note that although these syntheses are elegant and well thought out, none of these procedures are able to use indoles or indolines as starting materials. Frequently the final steps of the synthesis show the creation of a perhydroindole, but indole itself cannot be used to make these systems. Rather amino acids and difficult synthetic schemes are necessary to create this core.

In 2011, Porco published a review examining the dearomatization strategies in the synthesis of complex natural products. This focused on everything from arenes to furans and indoles. ${ }^{44}$ There is not a single example of both rings of indole being dearomatized for a perhydroindole core. Only the pyrrole ring is regularly dearomatized to create an 
indoline. Further, there are no examples of using indoline as a starting material through the dearomatization of the benzene ring.

\subsection{Conclusion}

The remaining chapters of this work will elucidate the ability to use indoline as a starting material for small molecules with a hexahydroindole core. The synthetic process for this depends greatly on a dearomatization agent described in the previous chapter. It is hoped that new small molecule analogs of these biologically interesting systems can be synthesized through limited synthetic steps. 


\subsection{References:}

(1) Kochanowska-Karamyan, A. J.; Hamann, M. T. Chemical reviews 2010, $110,4489$.

(2) Okano, K.; Fujiwara, H.; Noji, T.; Fukuyama, T.; Tokuyama, H. Angewandte Chemie International Edition 2010, 49, 5925.

(3) Liu, D.; Zhao, G.; Xiang, L. European Journal of Organic Chemistry 2010, 2010, 3975.

(4) Jana, S.; Rainier, J. D. Organic Letters 2013, 15, 4426.

(5) Okano, K.; Mitsuhashi, N.; Tokuyama, H. Tetrahedron 2013, 69, 10946.

(6) Filone, C. M.; Hodges, E. N.; Honeyman, B.; Bushkin, G. G.; Boyd, K.;

Platt, A.; Ni, F.; Strom, K.; Hensley, L.; Snyder, J. K.; Connor, J. H. Chemistry \& biology 2013, 20, 424.

(7) Leeson, P. Nature 2012, 481, 455.

(8) Hann, M. M. MedChemComm 2011, 2, 349.

(9) Bickerton, G. R.; Paolini, G. V.; Besnard, J.; Muresan, S.; Hopkins, A. L. Nat Chem 2012, 4, 90.

(10) van de Waterbeemd, H.; Gifford, E. Nat Rev Drug Discov 2003, 2, 192.

(11) Yang, Y.; Engkvist, O.; Llinàs, A.; Chen, H. Journal of Medicinal Chemistry 2012, 55, 3667.

(12) Marson, C. M. Chemical Society Reviews 2011, 40, 5514. 
(13) Lovering, F.; Bikker, J.; Humblet, C. Journal of Medicinal Chemistry 2009, 52, 6752 .

(14) Clarisse, D.; Fenet, B.; Fache, F. Organic \& Biomolecular Chemistry 2012, 10, 6587.

(15) Aloise Pilli, R.; da Conceicao Ferreira de Oliveira, M. Natural Product Reports 2000, 17, 117.

(16) Pilli, R. A.; Rosso, G. B.; de Oliveira, M. d. C. F. Natural Product Reports 2010, 27, 1908.

(17) Chung, H.-S.; Hon, P.-M.; Lin, G.; But, P. P.-H.; Dong, H. Planta Med 2003, 69, 914.

(18) Wipf, P.; Kim, Y.; Goldstein, D. M. Journal of the American Chemical Society 1995, 117, 11106.

(19) Morimoto, Y.; Iwahashi, M.; Nishida, K.; Hayashi, Y.; Shirahama, H. Angewandte Chemie International Edition in English 1996, 35, 904.

(20) Zeng, Y.; Aubé, J. Journal of the American Chemical Society 2005, 127, 15712.

(21) Wipf, P.; Rector, S. R.; Takahashi, H. Journal of the American Chemical Society 2002, 124, 14848.

(22) Wipf, P.; Spencer, S. R. Journal of the American Chemical Society 2005, 127, 225.

(23) Takos, A. M.; Rook, F. Int J Mol Sci 2013, 14, 11713.

(24) Evidente, A.; Kornienko, A. Phytochemistry Reviews 2009, 8, 449. 
(25) Lindsley, C. W. ACS Chemical Neuroscience 2012, 3, 804.

(26) Jin, Z. Natural Product Reports 2011, 28, 1126.

(27) Lamoral-Theys, D.; Decaestecker, C.; Mathieu, V.; Dubois, J.; Kornienko, A.; Kiss, R.; Evidente, A.; Pottier, L. Mini-Reviews in Medicinal Chemistry 2010, 10, 41. (28) Lamoral-Theys, D.; Andolfi, A.; Van Goietsenoven, G.; Cimmino, A.; Le Calvé, B.; Wauthoz, N.; Mégalizzi, V.; Gras, T.; Bruyère, C.; Dubois, J.; Mathieu, V.; Kornienko, A.; Kiss, R.; Evidente, A. Journal of Medicinal Chemistry 2009, 52, 6244.

(29) Schultz, A. G.; Holoboski, M. A.; Smyth, M. S. Journal of the American Chemical Society 1996, 118, 6210.

(30) Yamada, K.-i.; Yamashita, M.; Sumiyoshi, T.; Nishimura, K.; Tomioka, K. Organic Letters 2009, 11, 1631.

(31) Jones, M. T.; Schwartz, B. D.; Willis, A. C.; Banwell, M. G. Organic Letters 2009, 11, 3506.

(32) Tam, N. T.; Cho, C.-G. Organic Letters 2008, 10, 601.

(33) Lebeuf, R.; Robert, F.; Schenk, K.; Landais, Y. Organic Letters 2006, 8, 4755.

(34) Taber, D. F.; Neubert, T. D. The Journal of Organic Chemistry 2001, 66, 143.

(35) Chavan, S. P.; Khobragade, D. A.; Pathak, A. B.; Kalkote, U. R. Tetrahedron Letters 2004, 45, 5263.

(36) Ilardi, E. A.; Isaacman, M. J.; Qin, Y.-c.; Shelly, S. A.; Zakarian, A. Tetrahedron 2009, 65, 3261. 
(37) Geoghegan, K.; Evans, P. The Journal of Organic Chemistry 2013, 78, 3410.

(38) Ersmark, K.; Del Valle, J. R.; Hanessian, S. Angewandte Chemie International Edition 2008, 47, 1202.

(39) Murakami, M.; Okita, Y.; Matsuda, H.; Okino, T.; Yamaguchi, K. Tetrahedron Letters 1994, 35, 3129.

(40) Bonjoch, J.; Catena, J.; Isábal, E.; López-Canet, M.; Valls, N. Tetrahedron: Asymmetry 1996, 7, 1899.

(41) Wipf, P.; Methot, J.-L. Organic Letters 2000, 2, 4213.

(42) Sofack-Kreutzer, J.; Martin, N.; Renaudat, A.; Jazzar, R.; Baudoin, O. Angewandte Chemie International Edition 2012, 51, 10399.

(43) Hanessian, S.; Margarita, R.; Hall, A.; Johnstone, S.; Tremblay, M.; Parlanti, L. Journal of the American Chemical Society 2002, 124, 13342.

(44) Roche, S. P.; Porco, J. A. Angewandte Chemie International Edition 2011, $50,4068$. 


\section{Chapter 3:}

\section{Isolation and Initial Reactivity of $N$ -}

\section{Ethylindolinium Complex}




\subsection{Introduction}

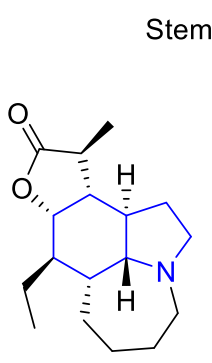

(-) Stenine

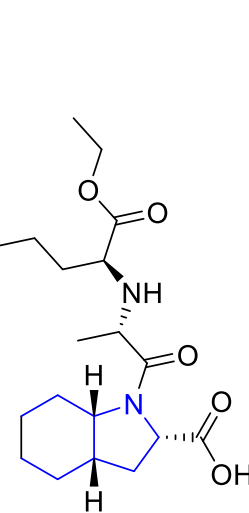

Perindopril

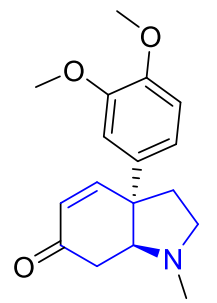

Memembrenone

Sceletium Alkaloids

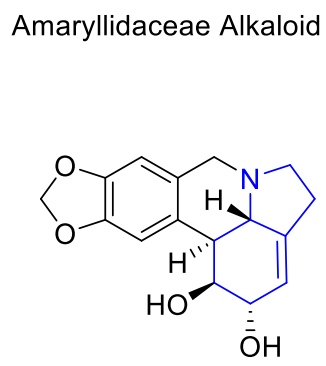

Lycorine

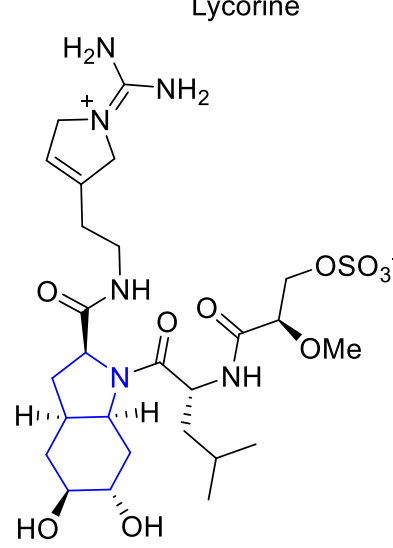

Dysinosin A

Aeruginosin Alkaloid

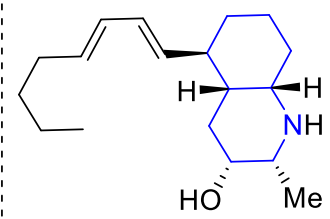

(+)-Lepadin B

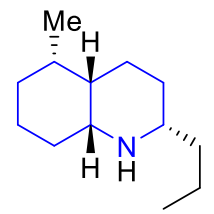

Pumiliotoxin $\mathrm{C}$

Figure 3.1. Examples of natural products with a perhydroindole and decahydroquinoline cores (shown in blue).

The indole skeleton is ubiquitous in nature and one of the most widely investigated cores in medicinal chemistry. ${ }^{1}$ In particular, hexahydro- and perhydroindole cores appear in numerous alkaloids including Stemona, Amaryllidaceae, Aeruginosin and Sceletium alkaloids (Figure 3.1). ${ }^{2-13}$ These species have shown promising beneficial biological activity, acting as ACE inhibitors (perindorpil), serine protease inhibitors (dysinosin A), and in tuberculosis treatments (tuberostemonine). ${ }^{14}$ Fully saturated 
quinolone cores are also naturally found and highly desirable, such as in the nicotinic antagonist pumiliotoxin $\mathrm{C}^{15,16}$ and anti-cancer agent lepadin $\mathrm{B} .{ }^{17,18}$

As seen in chapter 2, the syntheses of these indoline systems involve elaborate synthetic schemes, many stemming from cycloaminations from natural amino acids, such as L-tyrosine ${ }^{8}$ or from other linear alkylamine systems. ${ }^{19-22}$ Multiple steps are required to form the central core of the indoline system, usually with detriment to overall yield. Synthetic approaches that involve a preformed bicyclic system would be attractive alternatives, but are uncommon. ${ }^{23}$ In particular, the ability to use indoline, a common, naturally occurring aromatic, as a starting material for these biologically interesting systems could be advantageous given that every atom of the carbocycle is unsaturated and therefore represents a potential site of elaboration. Other bicyclic systems, such as quinolone, can also be explored with this manner of thought.
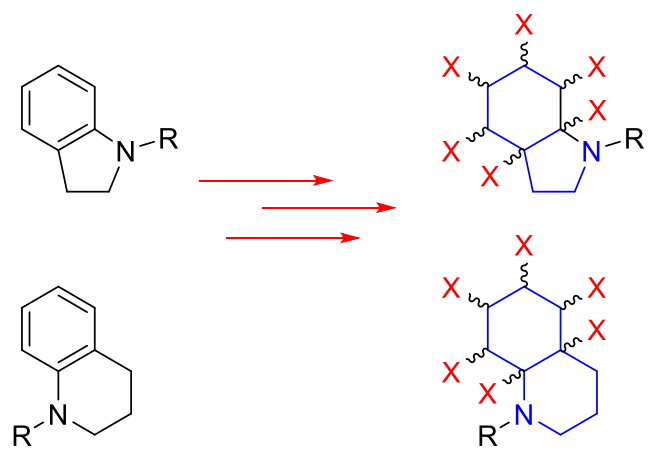

Figure 3.2. Dearomatization of indoline and tetrahydroquinoline.

Previous work has shown that a $N, N$-dimethylanilinium complex (2) can be synthesized from $\mathrm{TpW}(\mathrm{NO})\left(\mathrm{PMe}_{3}\right)\left(\eta^{2}\right.$-benzene) (1), $N, N$-dimethylaniline, and the weak acid diisopropylammonium triflate (DiPAT) in DME. ${ }^{24-26}$ This complex appears in 
solution as a single tautomer, resulting from ortho protonation (Scheme 3.1).

Additionally, the complex precipitates out of solution cleanly to $\mathbf{2 A}$ selectively. Through reactivity discussed in chapter 1 , various cyclohexenone derivatives have been generated from modification of this material, $\left[\mathrm{TpW}(\mathrm{NO})\left(\mathrm{PMe}_{3}\right)(2 \mathrm{H}\right.$-anilinium) $] \mathrm{OTf}^{25,27}$ It was determined that the $\mathrm{A}$ isomer was desired due to this reactivity. The $\mathrm{B}$ isomer was not similarly reactive, so was not desired. In order to branch into work which would have more medicinal relevance and possibly a larger scope of biological activity, the addition of a bottom ring onto the anilinium system was proposed. It was anticipated that by binding an indoline or tetrahydroquinoline to this $\pi$-basic tungsten complex would allow for novel and highly-substituted hydroindole and hydroquinoline systems to be obtained (Figure 3.2).

\subsection{Isolation of $\mathbf{N}$-Alkylindolinium and $\mathbf{N}$-Alkylquinolinium Complexes}

In order to be able to directly compare the $N, N$-dimethylanilinium complex $(\mathbf{2 A})$ to these bicyclic analogs, the A isomer was desired. This would allow the metal mediated reactivity that is desired, unlike the B isomer. When a DME solution of $N$ methylindoline, DiPAT, and TpW(NO) $\left(\mathrm{PMe}_{3}\right)\left(\eta^{2}\right.$-benzene $)$ was prepared and stirred overnight, a salt precipitated from solution in $47 \%$ yield. Unfortunately, analysis of the product mixture showed that along with the desired ortho protonation product (the $3 \mathrm{a} H$ indolinium complex, 3A), its para tautomer ( $5 H$-indolinium 3B) was formed in almost

equal amounts $(\mathbf{3 A}: 3 \mathbf{B} \approx 1.5 / 1)$. The monitoring $\left({ }^{31} \mathrm{P} \mathrm{NMR}\right)$ of a similar reaction with 1 - 
methyl-1,2,3,4-tetrahydroquinoline showed the formation of an analogous cationic complex $\left(J^{183}{ }_{\mathrm{W}-}^{31}{ }_{\mathrm{P}}=286 \mathrm{~Hz}\right.$; cf. $285 \mathrm{~Hz}$ for $\left.\mathbf{3 A}\right)$, however, this compound failed to precipitate from the DME solvent. By repeating the reaction as a heterogeneous mixture of TpW(NO)(PMe $)\left(\eta^{2}\right.$-benzene) (1), DiPAT, and 1-methyl-1,2,3,4-tetrahydroquinoline in hexanes, a quinoline-derived salt could be isolated as a mixture of two isomers, $\mathbf{4 A + 4 B}$, in a ratio of $\sim 1 / 10$. By layering a DCM solution of $\mathbf{4 A + A B}$ with acetone and isooctane, a crystal of $\mathbf{4 B}$ was produced that was suitable for structural analysis by X-ray diffraction. A molecular structure diagram of the cation (Figure 3.3) confirms the assigned structure of $\mathbf{4 B}$ as the para isomer (a $6 H-1,2,3,4,-$ tetrahydroquinolinium complex). This structure is consistent with an $\eta^{2}$-coordinated aromatic. The bound alkene has lengthened to $1.45 \AA$ and the iminium is shorter at $1.31 \AA$.

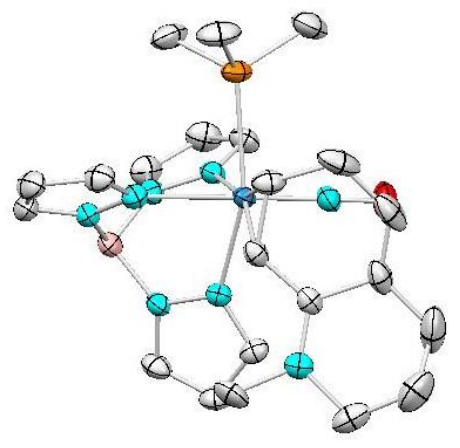

Figure 3.3. Crystal structure of $N$-Methyl-Quinoline (4B) (30\% ellipsoids).

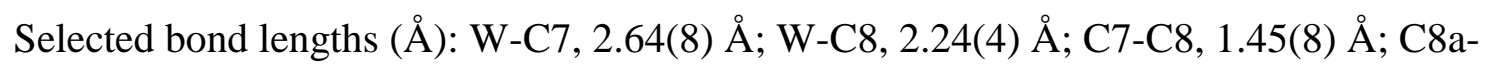
$\mathrm{N}, 1.31(0) \AA$. 
Scheme 3.1: Protonation of Dihapto-coordinated Indoline and Tetrahydroquinoline Complexes Leading to Ortho and Para Isomers. [W] $=\left\{\mathrm{TpW}(\mathrm{NO})\left(\mathrm{PMe}_{3}\right)\right\}$

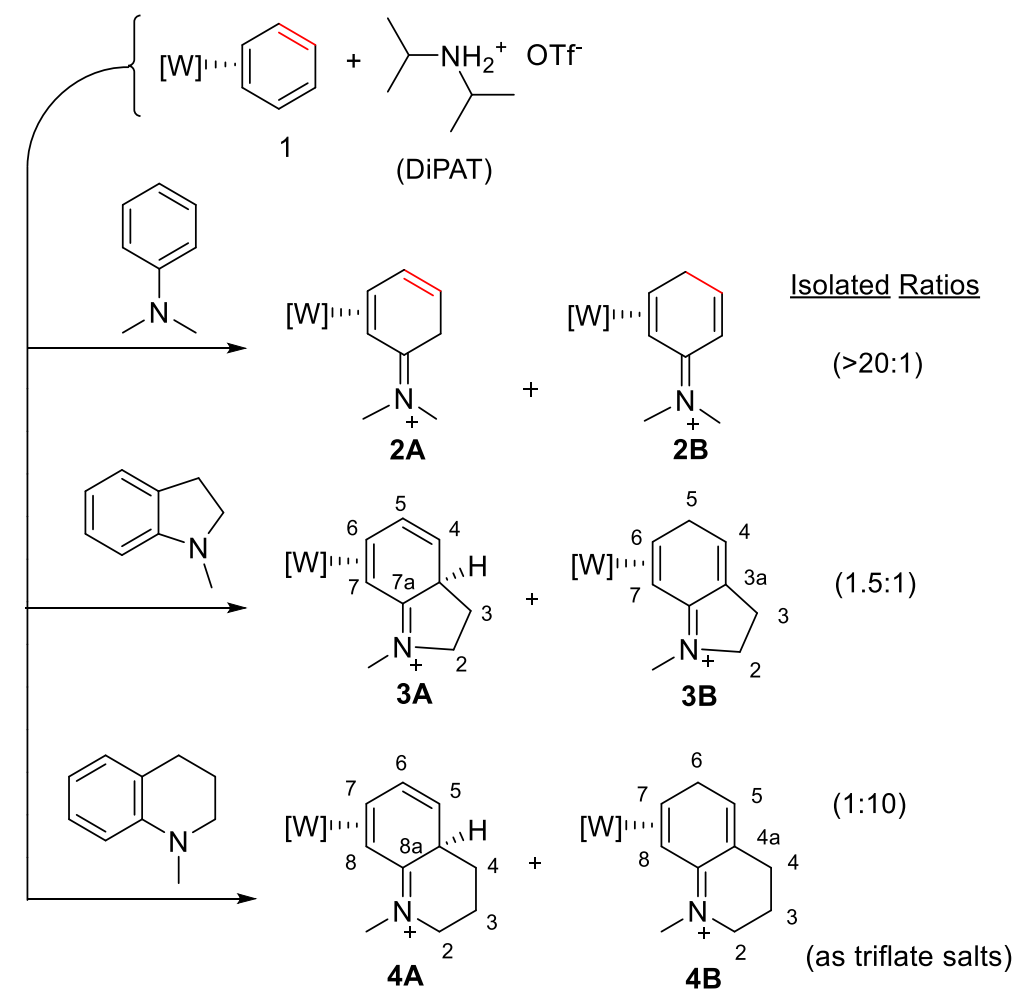

Identification of the para and ortho isomers of both the indoline and quinoline systems were completed primarily by ${ }^{1} \mathrm{H}$ NMR and 2D NMR. Both $\mathbf{3 A}$ and $\mathbf{4 A}$, a doublet of doublets at $4.9 \mathrm{ppm}$ and a multiplet at $6.5 \mathrm{ppm}$, both integrating for 1 proton. These protons correspond to $\mathrm{H} 4$ and $\mathrm{H} 5$ respectively in indoline and $\mathrm{H} 5$ and $\mathrm{H} 6$ of the quinoline systems. The para protonated system has a doublet of doublet at $4.3 \mathrm{ppm}$ for one proton which has a strong COSY interaction with another proton at $3.4 \mathrm{ppm}$. These peaks identify as a $\mathrm{CH}_{2}$ group using HSQC. This geminal set is identified as $\mathrm{H} 5$ in the para protonated indoline species and H6 in the quinoline species. 


\subsubsection{Para vs Ortho Protonation}

For both the indoline $(\mathbf{3 A}+\mathbf{3 B})$ and tetrahydroquinoline $(\mathbf{4 A + 4 B})$ salts, DFT calculations indicated that in contrast to the $N, N$-dimethylanilinium complex (2), para protonation (C5, Figure 3.4) is thermodynamically favored. Details of the DFT calculations can be found in the experimental general methods. Consistent with this, a dilute homogeneous reaction mixture was monitored $\left({ }^{31} \mathrm{P} \mathrm{NMR}\right)$, containing $N$ methylindoline, DiPAT, and TpW(NO) $\left(\mathrm{PMe}_{3}\right)\left(\eta^{2}\right.$-benzene $)$, in order to hinder precipitation from solution. After $1 \mathrm{~h}$ the ratio of $\mathbf{3 A} / \mathbf{3 B}$ was $8 / 1$. After $3 \mathrm{~h}$, however, this ratio had degraded to $4 / 1$. It was determined that the kinetic product is the desired ortho isomer (C3a protonation, Figure 3.4). This isomer has the desired bond orientation to allow for further elaboration using the type of reactivity described herein. This is possibly due to the closer proximity of the electron-donating nitrogen to the 3 a position in the dienamine fragment of the putative aniline intermediate (Figure 3.4). There is no evidence of alternate ortho protonation at $\mathrm{C} 7$, most likely due to the increased stability of the $\mathrm{C} 3 \mathrm{a}$ isomer. This stability stems from the positive charge being stabilized in the anisotropy of the Tp rings. Unfortunately, the ortho form could not be synthesized free of its para isomer (3B). A similar experiment was performed with 1-methyl-1,2,3,4tetrahydroquinoline, which showed that the ratio of $\mathbf{4 A / 4 B}$ changed from $1 / 4$ (30 min) to 1/8 (2.5 h), showing that $\mathbf{4 B}$ is the thermodynamically favored isomer (C6 protonation, Figure 3.4). 

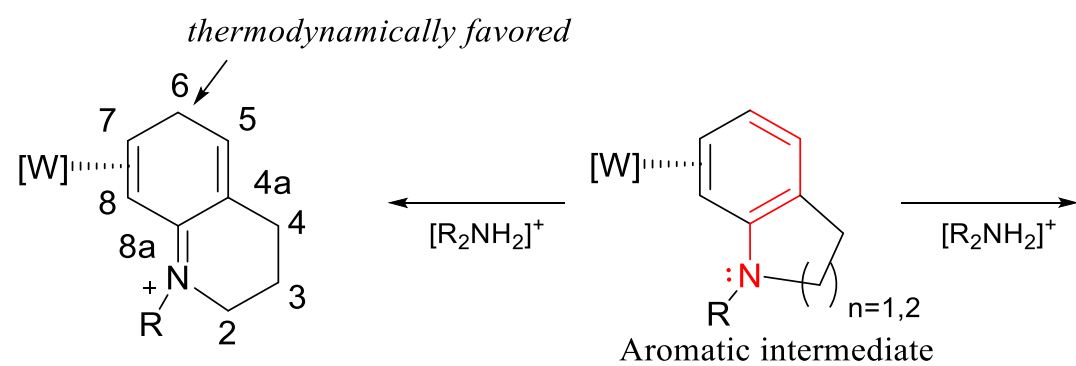

Aromatic intermediate (dienamine fragment in red)

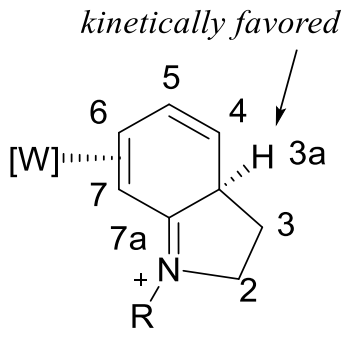

Figure 3.4. The dihapto-coordinated intermediate, stereochemistry of protonation, and numbering systems.

Scheme 3.2. In situ protonation of $\eta^{2}$-coordinated $N$-alkylated indoline complexes and associated protonation ratios; $[\mathrm{W}]=\mathrm{TpW}(\mathrm{NO})\left(\mathrm{PMe}_{3}\right)$.

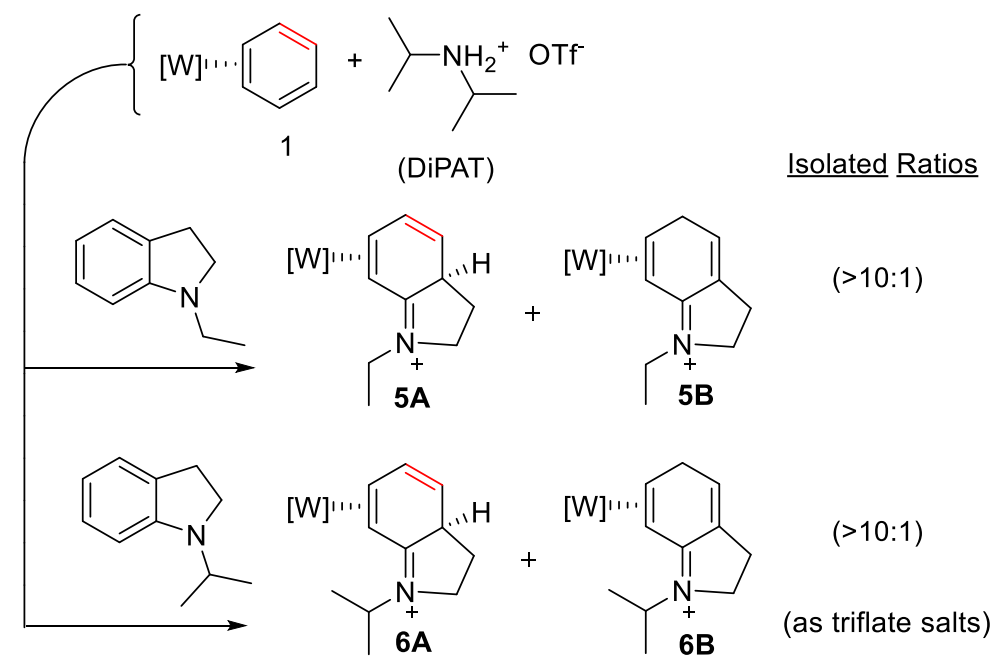


With the hope of generating a higher ratio of the ortho protonation isomers, other $\mathrm{N}$-alkylated indolines and tetrahydroquinolines were synthesized and combined with $\mathrm{TpW}(\mathrm{NO})\left(\mathrm{PMe}_{3}\right)\left(\eta^{2}\right.$-benzene) (1) and DiPAT. For the indoline derivatives, it was found that larger alkyl groups on the nitrogen dramatically enhanced ortho protonation (C3a) over para-protonation (C5) (Scheme 3.2). In the hope of maximizing the formation of the A isomer, two other indoline derivatives were synthesized and protonated. For $N$-ethyl(5) and $N$-isopropyl- (6) variants, it was found that the ratio of $\mathbf{5 A} / \mathbf{5 B}$ and $\mathbf{6 A} / \mathbf{6 B}$ in the isolated product was greater than 10:1, favoring ortho protonation (5A or $\mathbf{6 A})$. Regarding the role that the ethyl or isopropyl group plays in improving this ratio, the larger alkyl substituents appear to reduce the solubility of the ortho isomers, facilitating their removal from solution prior to isomerization. DFT calculations also gave insight into the regioselectivity of protonation. It was determined that the para isomer is thermodynamically favored by $1.6 \mathrm{kcal} / \mathrm{mol}$ for methylindoline $(\mathbf{3 B})$ and $1.4 \mathrm{kcal} / \mathrm{mol}$ for ethylindoline (5B) over the syn-ortho tautomer. Unfortunately, this was not the case for the quinolone-derived system, where the ortho/para ratio was virtually identical for the $\mathrm{N}$ methyl and $N$-ethyl analogs.

\subsubsection{Anti vs Syn Protonation}

For all of these systems, the ortho protonation occurs syn to the metal, which seemed at first counterintuitive, ${ }^{28}$ given that the addition to the ring-face away from the metal appears to be more kinetically accessible at C3a. However, DFT calculations 
indicate that syn-ortho protonation is thermodynamically favored over the anti-ortho protonation by $6.9 \mathrm{kcal} / \mathrm{mol}$ for the methyl indoline derived complex, likely because this action results in the bicyclic framework curving away from the metal (metal located on convex side). This is similar to the ethylindoline derived complex, which favors synortho protonation by $7.0 \mathrm{kcal} / \mathrm{mol}$.

\subsection{Preliminary Reactivity}

Similar to the $N, N$-dimethylaniline complex, it was expected that the indoline system (I) would have the ability to react with electrophiles to give a stable double cationic species (II), which could then react with nucleophiles to form a tetrahydroindolium complex (III). Unlike the anilinum system, it was believed that this system could then be reduced to yield a hexahydroindole with up to four new stereocenters (IV). Each of these additions would be determined by the initial metal stereochemistry. The metal could then be oxidatively cleaved to yield novel hexahydroindoles of the type $\mathbf{V}$. 
Scheme 3.3. Overall reaction scheme for perhydroindole synthesis; [W] = $\mathrm{TpW}(\mathrm{NO})\left(\mathrm{PMe}_{3}\right)$<smiles>[R]N1CCc2ccccc21</smiles>

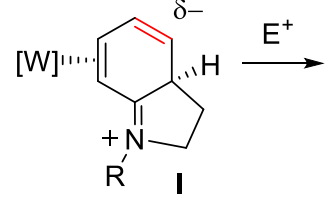

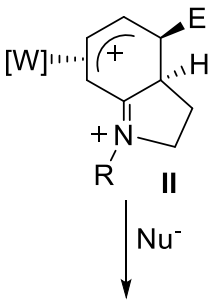

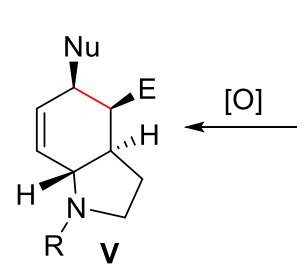

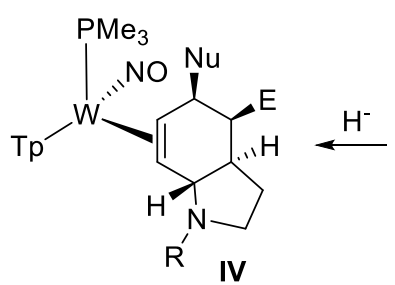

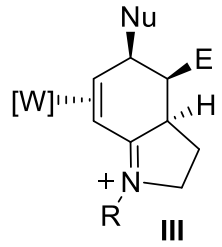

To begin preliminary reactivity testing, the aniline system was used as a model with the full range of nucleophiles possible being investigated and demonstrating the breadth of diversity available by this new methodology.The anilinium complex was able to react with the weak acid, diphenyl ammonium triflate (DPhAT), to yield an allylic species which was reactive with various nucleophiles to yield a Friedel-Crafts like product. $^{25,26,29}$ Most reactions could be done under catalytic acid. It was known that a stronger acid than DiPAT would be necessary due to the conditions under which the complex was synthesized. Preliminary testing showed that with the indoline system, catalytic acid would not fully protonate the system and reactions with DPhAT were slow. An alternate method was determined, similarly to aniline, which using 2 equivalents of HOTf leveled in acetonitrile efficiently yielded the double cationic allylic species which could react further (Scheme 3.4). 
In a typical experiment a sample of compound $\mathbf{5}$ was stirred in an acetonitrile solution of triflic acid $(0.125 \mathrm{M})$ and the reaction was monitored using ${ }^{31} \mathrm{P}$ NMR spectroscopy. A new signal appeared within less than five minutes, which showed a ${ }^{183} \mathrm{~W}-{ }^{31} \mathrm{P}$ coupling constant significantly lower than that of the starting material. Whereas compound 5 has a $J_{\mathrm{W}-\mathrm{P}}$ of $279 \mathrm{~Hz}$, the newly formed species 7 has a $J_{\mathrm{W}-\mathrm{P}}$ shift of $250 \mathrm{~Hz}$. This suggests a decrease in the amount of tungsten "s" character in the W-P bond. Such a decrease is consistent with the expected increase in coordination number as the $\mathrm{W}$-alkene bond in $\mathbf{5}$ is converted into an $\mathrm{W}-\eta^{3}$-allylic bond upon protonation. ${ }^{24}$

Scheme 3.4: Functionalization of 5 with $\mathrm{E}^{+}=\mathrm{H}^{+}$

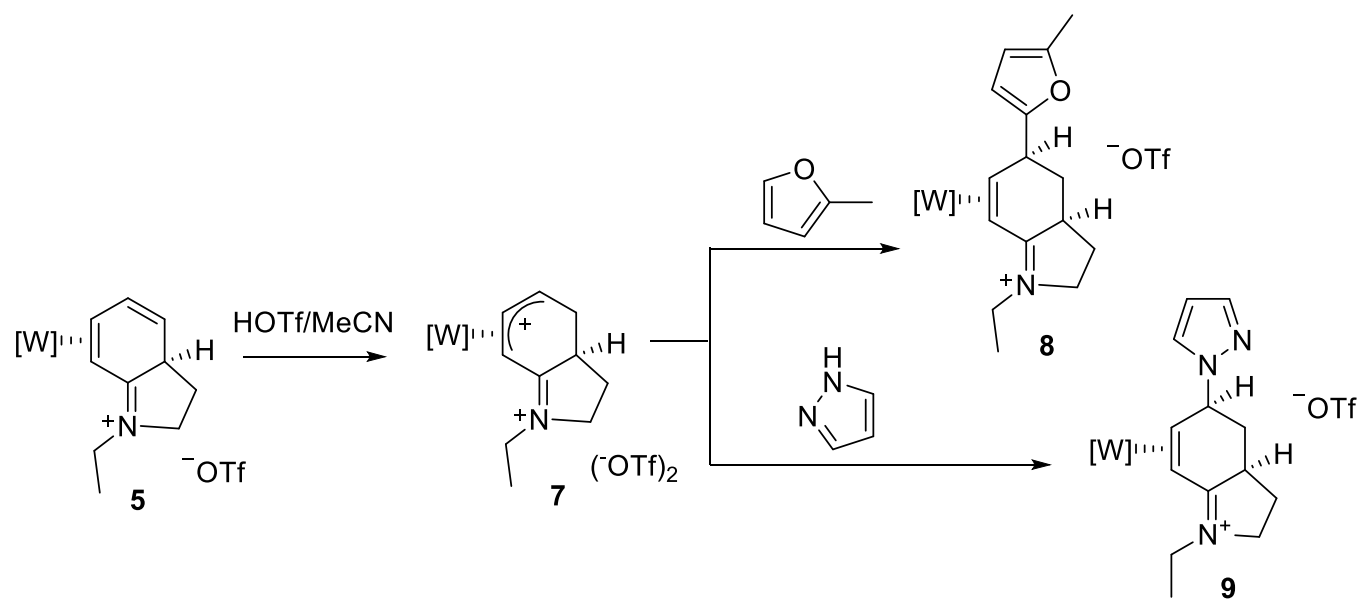

In order to test the reactivity of the new allylic species, two nucleophiles were tested before a full scope of reactivity was attempted. Hydroamination with pyrazole and hydroarylation with 2-methylfuran was attempted with the $N$-ethyl derivative. These nucleophiles were some of the most reactive with the anilinium system and they also have unique properties. Compatibility of the tungsten complex with the nucleophile was of concern as was the unintended deprotonation at $\mathrm{C} 4$. Pyrazole is both nucleophilic and 
basic, showing the susceptibility of the allylic species to deprotonation. The 2-

methylfuran is a fairly weak nucleophile, showing how strong the nucleophiles must be in order to react, but also has an obvious NMR handle to watch the reaction in situ. When $\mathbf{5}$ is treated with HOTf in $\mathrm{CH}_{3} \mathrm{CN}$, the resulting allyl intermediate readily reacts with pyrazole at $\mathrm{C} 5$, to give the salt $\mathbf{9}$. In a similar fashion, compound $\mathbf{5}$ is able to undergo hydroarylation with 2-methylfuran to produce the Friedel-Crafts product $\mathbf{8} .^{30}$ Because of its ease of crystallization, a solid-state analysis of the $N$-isopropyl derivative, 8-iPr, was carried out using X-ray diffraction, which confirms the relative stereochemistry of these addition products (Figure 3.5). This stereochemistry is consistent with expectations with the nucleophile adding anti to the metal center.

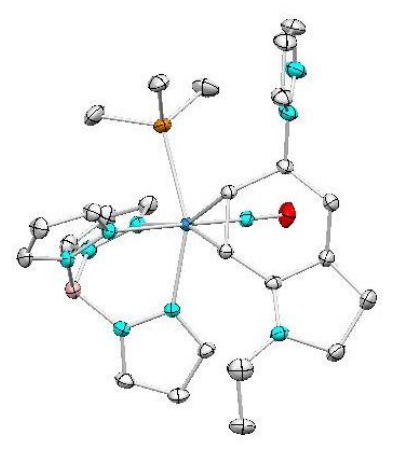

Figure 3.5: Crystal structure of compound 8-iPr (30\% ellipsoids). Selected bond

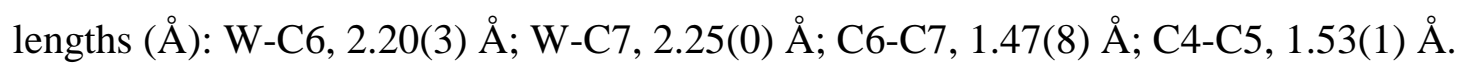

The structure of $\mathbf{8 - i P r}$, along with NOE data for $\mathbf{8}$ and $\mathbf{9}$, indicate that the ortho protonation occurs syn to the metal center, matching what was determined using $2 \mathrm{D}$ NMR and DFT calculations. We attribute this to the steric interaction that the lower ring would have with the metal complex, were protonation to occur anti to the metal. 
Scheme 3.5. Preparation of tetrahydroindolium complexes 8-20 (isolated as triflate salts).

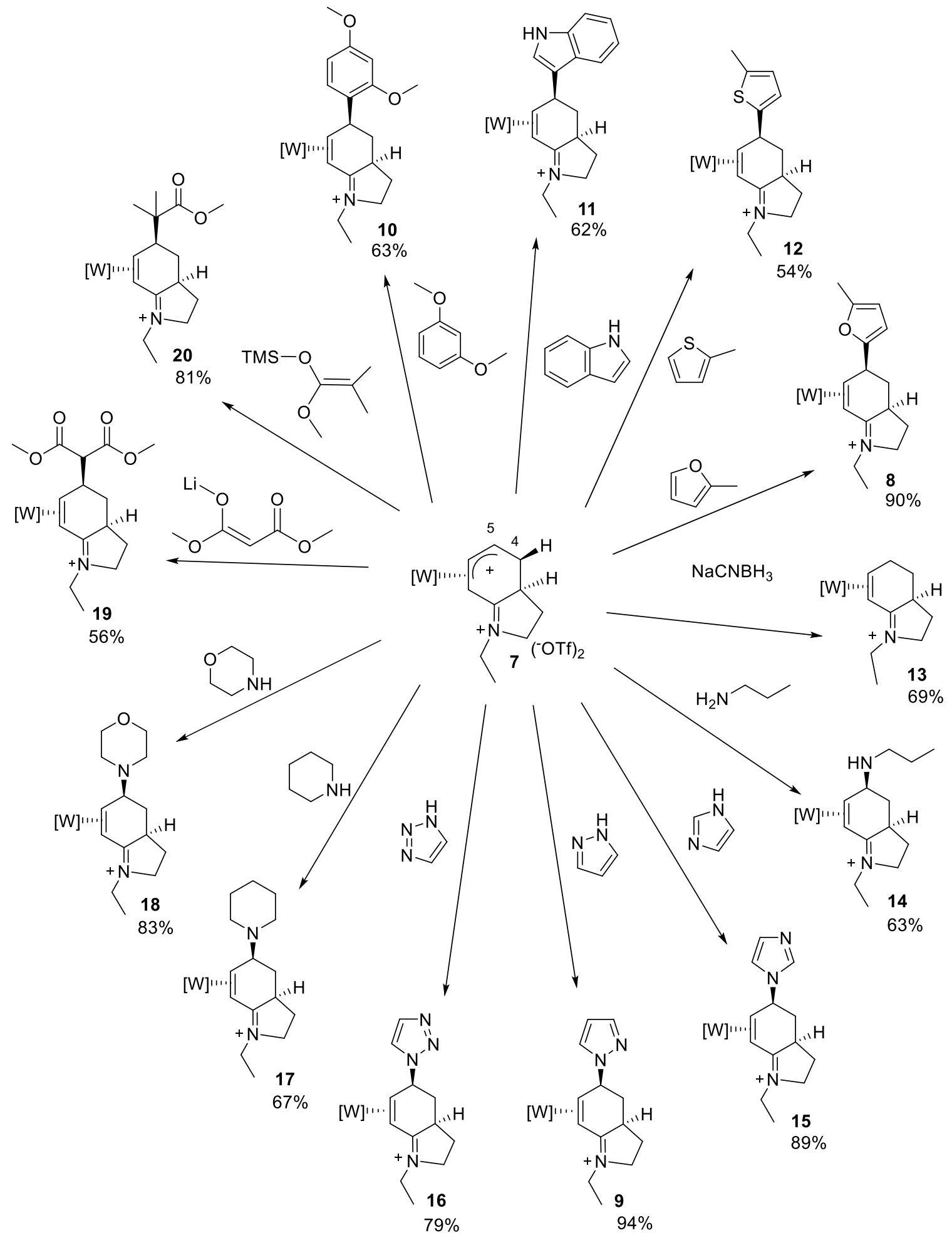


The full scope of reactivity for compound $\mathbf{5}$ was explored with the protonation at C4 followed by the addition of various nucleophiles to C5. Again, compound $\mathbf{5}$ was stirred in an acetonitrile solution of triflic acid $(0.125 \mathrm{M})$ to yield the allylic species $(7)$. To this solution, various nucleophiles were added to yield tetrahydroindolium salts $\mathbf{8 - 2 0}$. Nucleophiles that successfully add to C5 include various aromatics $(\mathbf{8 , 1 0 - 1 2})$, a hydride (13), amines (9, 14-18), and activated alkenes (19-20). These additions were again followed using ${ }^{31} \mathrm{P}$ NMR, which showed the appearance of a new peak with coupling close to $280 \mathrm{~Hz}$, indicating the return to a $\eta^{2}$-coordination of the organic ligand. ${ }^{24}$ As seen previously, all nucleophilic additions were found to be both stereo- and regioselective, occurring at $\mathrm{C} 5$ and anti to the metal center. This assignment was supported by 2D-NMR data, which showed NOE interactions between the H5 proton and both the $\mathrm{PMe}_{3}$ and $\mathrm{H} 3$ a proton (Figure 3.6). The chemical shifts of $\mathrm{H} 5$ in the ${ }^{1} \mathrm{H}$ NMR spectrum of 8-20 span a range of nearly 3 ppm, depending on the type of nucleophile that adds at the C5 carbon. With aromatic nucleophiles, H5 is located between 4.5 and 5 ppm, owing to the ring anisotropy. Carbon nucleophiles that resulted in an $\mathrm{sp}^{3}$ carbon adjacent to the $\mathrm{C} 5$ carbon were more upfield at $3-3.5 \mathrm{ppm}$, and amine substituents resulted in an intermediate chemical shift (4.0-4.5 ppm) for H5. Aromatic amine nucleophiles resulted in products with the most downfield shift ranging from 5.7-6.1 ppm.

${ }^{13} \mathrm{C}$ NMR, cyclic voltammetric, and IR data indicate the iminium remains intact after these addition reactions. ${ }^{13} \mathrm{C}$ NMR specifically shows an iminium peak at approximately $188 \mathrm{ppm}$. For all additions, an anodic wave was seen at $\mathrm{E}_{\mathrm{p}, \mathrm{a}} \approx 1.4 \mathrm{~V}$, indicating the organic ligand is still a strong $\pi$ acid. IR data also show two peaks near 
$1600 \mathrm{~cm}^{-1}$ indicating nitrosyl (NO) and iminium (CN) stretches. Nucleophiles which were unreactive under these conditions included thiophene, anisole and $N$-methylpyrrole. It was determined that these nucleophiles were too weak or gave multiple products. The nucleophiles which resulted in eventual deprotonation at C4 included alcohols and alkyl halides activated by zinc. We suspect that addition of alcohols occurs as well, but that the reaction is reversible and not thermodynamically favored.

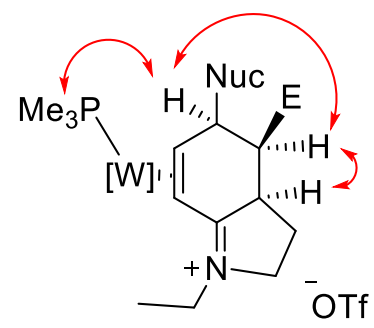

Figure 3.6. NOE interactions supporting stereochemical assignments in 8-20.

\subsection{Quinoline Isomerization}

In an attempt to continue working with both the indoline and quinolone systems and to broaden the scope of reactivity which was available, the problem of the para protonation of quinoline was investigated further. In order to explore this system, model compounds were explored for ease of synthesis. Commercially available methyl substituted $N, N$-dimethylaniline systems were used to test the ability of a methyl group to change the isomer ratios. It was believed that by adding a bulky group on the ring, protonation could be hindered at the para position. 


\subsubsection{Para and Meta-Toluidine Isomerization}

Using para-toluidine in a dilute homogeneous reaction mixture, monitored by ${ }^{31} \mathrm{P}$ NMR, containing the ligand, DiPAT and TpW(NO) $\left(\mathrm{PMe}_{3}\right)\left(\eta^{2}\right.$-benzene $)(\mathbf{1})$ in DME, it was determine that the para-toluidine $(\mathbf{2 1 A} / \mathbf{2 1 B})$ maintained a ratio of $>20 / 1$ over 5 hours. These are the same conditions as the $N, N$-dimethylaniline system, which also has a $>20 / 1$ ratio. Consistent with previous experiments, ortho-toluidine was not able to be protonated, which lead to decomposition. ${ }^{31}$ With meta-toluidine, after 20 min the ratio of 22A/22B) was $1 / 4$ in situ. After 3 hours, this degraded to a ratio of $1 / 2.5$, when followed with ${ }^{31} \mathrm{P}$ NMR. On a larger scale experiment, the isomers of the complexes which precipitate out of DME were fully identified. This ratio was determined with both ${ }^{1} \mathrm{H}$ NMR and 2D NMR. Using N,N-dimethylaniline as a model system, the position of $\mathrm{H} 4$ and $\mathrm{H} 5$ are predictable. In the $\mathrm{A}$ isomer, an umpolong effect occurs. $\mathrm{H} 4$ moves downfield from the partial positive charge and H5 moves upfield due to the partial negative. In the $\mathrm{B}$ isomer, these positions are switched. Using this information, the two isomers were identified and the ratios were determined. This change in the ratio between the isomers is believed to be due to hyperconjugation through the methyl at the meta position. An interesting discovery was that while the meta-toluidine cleanly precipitated from DME, similar to the anilinium species, the para-toluidine did not, which mimics the quinoline species. 
Scheme 3.6. Isolation of methyl-toluidine derivative complexes

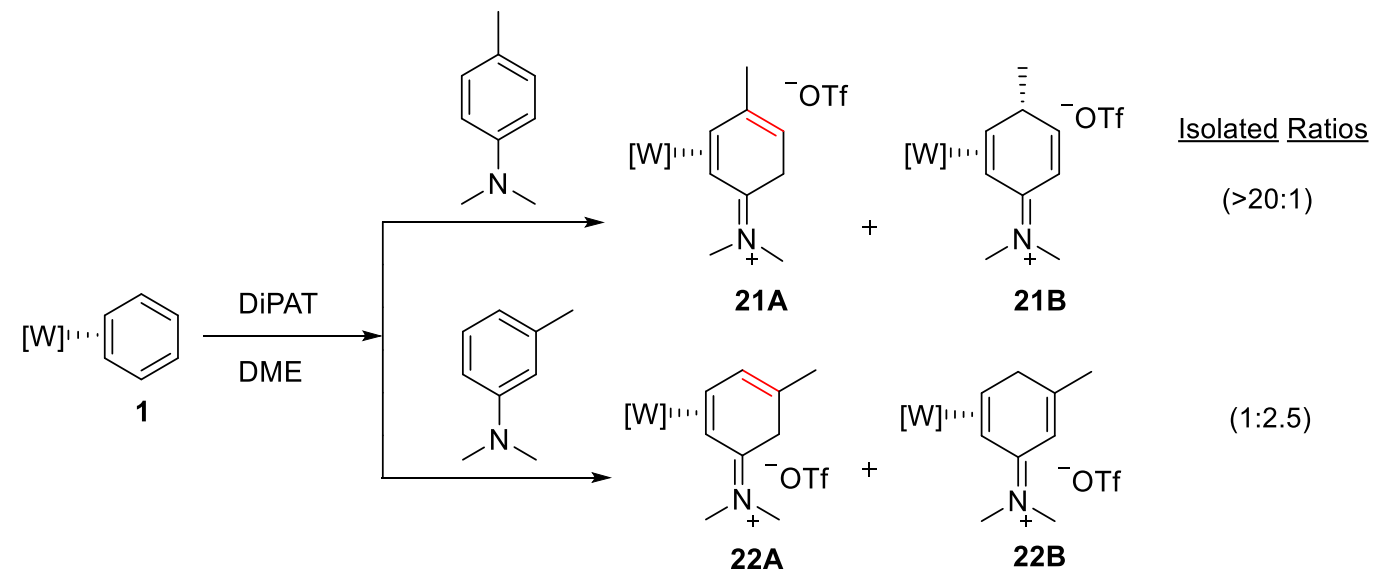

\subsubsection{Para Methyl Quinoline Complexation}

Using a heterogeneous mixture of $\mathrm{TpW}(\mathrm{NO})\left(\mathrm{PMe}_{3}\right)\left(\eta^{2}\right.$-benzene), DiPAT, 6methyl-1-methyl-1,2,3,4-tetrahydroquinoline in hexanes, a quinoline-derived salt could be isolated as a mixture of two isomers, $\mathbf{2 3 A} / \mathbf{2 3 B}$, in a ratio of $\sim 10 / 1$. The addition of a methyl at C6 introduces a new level of steric interference. In the para-protonation form, the protonation occurs anti to the metal, forcing the methyl into the metal complex's $\mathrm{PMe}_{3}$ ancillary ligand. This destabilizes the system.

Scheme 3.7. Complexation of 6-methyl-1-methyl-1,2,3,4-tetrahydroquinoline

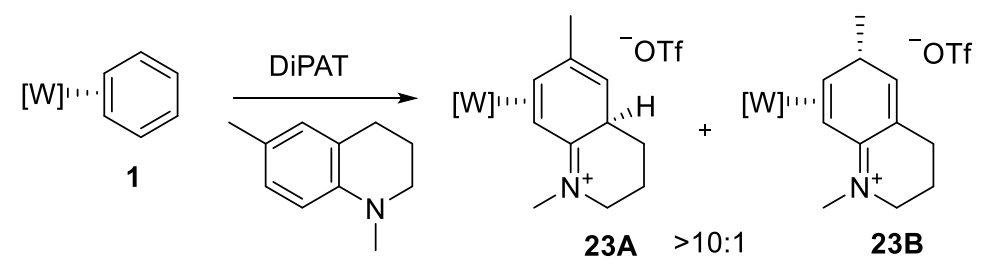




\subsection{Quinolinium and Para-Methyl Anilinium Initial Reactivity}

Due to the expense of the 6-methyl quinoline derivative in comparison to paratoluidine, 21 was explored for reactivity. Upon addition of 2 equivalents of a HOTf solution in acetonitrile $(0.125 \mathrm{M})$, when followed with ${ }^{31} \mathrm{P}$ NMR spectroscopy, shows a new signal with a $J_{\mathrm{W}-\mathrm{P}}$ of $252 \mathrm{~Hz}$ began to appear with the starting material. An additional 2 equivalents were necessary to force the reaction to completion. The addition of 2methylfuran was successful in generating the Friedel-Crafts product (24). Using 2D NMR, it was determined that the nucleophile added anti to the metal center, as expected. The methyl at $\mathrm{C} 4$ has a strong $\mathrm{NOE}$ interaction with the $\mathrm{PMe}_{3}$. This reaction, with both the aniline (2) and indoline (5) complexes, took less than $1 \mathrm{~h}$ (monitored with ${ }^{31} \mathrm{P} N M R$ ). With 21, the addition of the nucleophile took a minimum of 1 day with over 10 equivalents of nucleophile. The slower reaction speed speaks to both stability of the now tertiary carbocation at the para position and also to the sterics upon the addition of the nucleophile. The methyl group is now close to the $\mathrm{PMe}_{3}$ ligand. The addition of 1,3dimethoxybenzene appeared, over a week, to yield a single product as well. The addition of more basic nucleophiles, such as MTDA and pyrazole, yielded starting material. 
Scheme 3.8. Tandem addition of HOTf and 2-methylfuran with 21 (isolated as triflate salts)
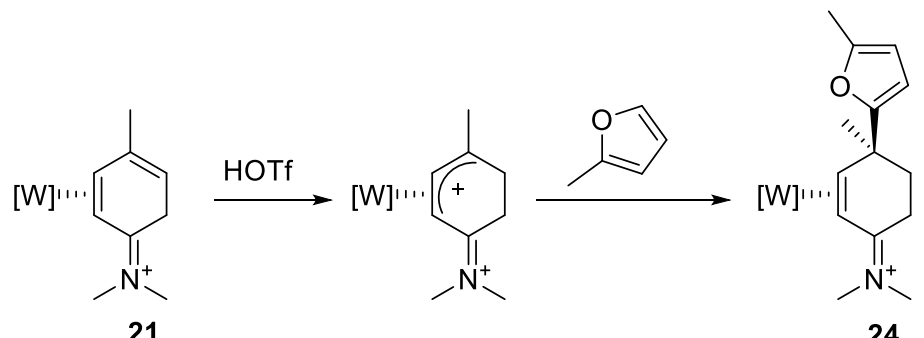

21

24

Using similar conditions to the para-toluidine system, the 6-methyl quinoline system was protonated with a more concentrated solution of HOTf in acetonitrile $(0.250$ M) to yield the allylic species. This was again monitored using ${ }^{31} \mathrm{P}$ NMR, showing a $J_{\mathrm{W}-\mathrm{P}}$ of $250 \mathrm{~Hz}$ for the new species in solution. Upon addition of 2-methylfuran, a new tandem addition product was formed. Again, 2D NMR determined that addition of the nucleophile occurred anti to the metal center. Further reactivity with this system is ongoing. This initial reactivity is very promising.

Scheme 3.9. Addition of HOTf and 2-methylfuran to 23 (isolated as triflate salts)

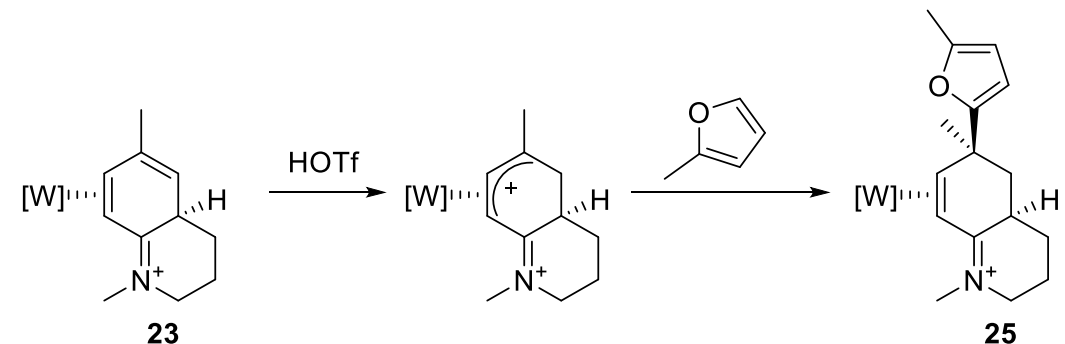




\subsection{Conclusion:}

Using the $N, N$-dimethylanilinium complex as a model system, the indoline complex can be isolated in a high diastereomer ratio and yield. The initial reactivity of this system shows it to be similar to that of the aniline complex, making it a strong candidate for further elaboration which will be discussed in the next chapter. 


\subsection{Experimental Section}

General Methods: NMR spectra were obtained on a 300, 500, 600, or $800 \mathrm{MHz}$ spectrometer. All chemical shifts are reported in ppm and proton and carbon shifts are

referenced to tetramethylsilane (TMS) utilizing residual ${ }^{1} \mathrm{H}$ or ${ }^{13} \mathrm{C}$ signals of the deuterated solvents as an internal standard. Phosphorus NMR signals are referenced to $85 \% \mathrm{H}_{3} \mathrm{PO}_{4}(\delta=0.00)$ using a triphenylphosphate external standard $(\delta=-16.58)$.

Coupling constants $(J)$ are reported in hertz $(\mathrm{Hz})$. Infrared spectra (IR) were recorded as a glaze on a spectrometer fitted with a Horizontal Attenuated Total Reflectance (HATR) accessory, or on a FT-IR spectrometer equipped with a diamond anvil ATR assembly. Electrochemical experiments were performed under a dinitrogen atmosphere using a potentiostat. Cyclic voltammetry data was taken at ambient temperature $\left(\sim 25^{\circ} \mathrm{C}\right)$ at 100 $\mathrm{mV} / \mathrm{s}$ in a standard three-electrode cell with a glassy carbon working electrode, $N, N$ dimethylacetamide (DMA) or acetonitrile (MeCN) solvent (unless otherwise specified), and tetrabutylammonium hexafluorophosphate (TBAH) electrolyte (approx. 0.5 M). All potentials are reported versus NHE (Normal Hydrogen Electrode) using cobaltocenium hexafluorophosphate $\left(E_{1 / 2}=-0.78 \mathrm{~V}\right)$, ferrocene $\left(E_{1 / 2}=+0.55 \mathrm{~V}\right)$, or decamethylferrocene $\left(\mathrm{E}_{1 / 2}=+0.04 \mathrm{~V}\right)$ as an internal standard. The peak-to-peak separation was less than 100 $\mathrm{mV}$ for all reversible couples. High-resolution mass spectra were acquired in ESI mode, from samples dissolved in a 3:1 acetonitrile/water solution containing sodium trifluoroacetate (NaTFA). Mass spectra are reported as $\mathrm{M}^{+}$for monocationic complexes, or as $\left[\mathrm{M}+\mathrm{H}^{+}\right]$or $\left[\mathrm{M}+\mathrm{Na}^{+}\right]$for neutral complexes, using $\left[\mathrm{Na}(\mathrm{NaTFA})_{\mathrm{x}}\right]^{+}$clusters as an internal standard. In all cases, observed isotopic envelopes were consistent with the 
molecular composition reported. For organic products, the monoisotopic ion is reported; for complexes, the major peaks in the isotopic envelope are reported. Unless otherwise noted, all synthetic reactions were performed in a glovebox under a dry nitrogen atmosphere. $\mathrm{CH}_{2} \mathrm{Cl}_{2}$ and benzene were purified by passage through a column packed with activated alumina. Other solvents and liquid reagents were thoroughly purged with dry nitrogen prior to use. Triflate salts of amines were synthesized by addition of an $\mathrm{Et}_{2} \mathrm{O}$ solution of triflic acid to the appropriate conjugate base dissolved in $\mathrm{Et}_{2} \mathrm{O}$. Deuterated solvents were used as received from Cambridge Isotopes. Pyrazole (Pz) protons of the (trispyrazolyl) borate (Tp) ligand were uniquely assigned (eg., "PzB3”) using a combination of 2-dimensional NMR data and phosphorus-proton NOE interactions. $\mathrm{TpW}(\mathrm{NO})\left(\mathrm{PMe}_{3}\right)\left(\eta^{2}\right.$-benzene $)(\mathbf{1})$ and $\left[\mathrm{TpW}(\mathrm{NO})\left(\mathrm{PMe}_{3}\right)\left(\eta^{2}-N, N\right.\right.$-dimethylanilinium $\left.)\right]$ [OTf] were synthesized using previously reported methods. ${ }^{24,26,32,33} \mathrm{BH}$ peaks (around 4$5 \mathrm{ppm}$ ) are not identified due to their quadrupole broadening; IR data is used to confirm the presence of a $\mathrm{BH}$ (around $2500 \mathrm{~cm}^{-1}$ ). $\mathrm{OH}$ and $\mathrm{NH}$ peaks are not always identified due to exchange with water in solvent. Where appropriate, $\mathrm{OH}$ peaks have been confirmed with IR data.

DFT Calculations. Initial structures were built in GAUSSVIEW (5.0.8) and optimized with the PM6 semiempirical method in GAUSSIAN 09. These structures were refined stepwise in Gaussian using B3LYP and a series of basis functions incorporating LANL2 pseudopotentials and associated basis functions provided in the GAUSSIAN package. The most demanding calculations reported here put the LANL2DZ 
pseudopotential and its basis only on the W atom and used the 6-31G(d) basis for all other atoms.

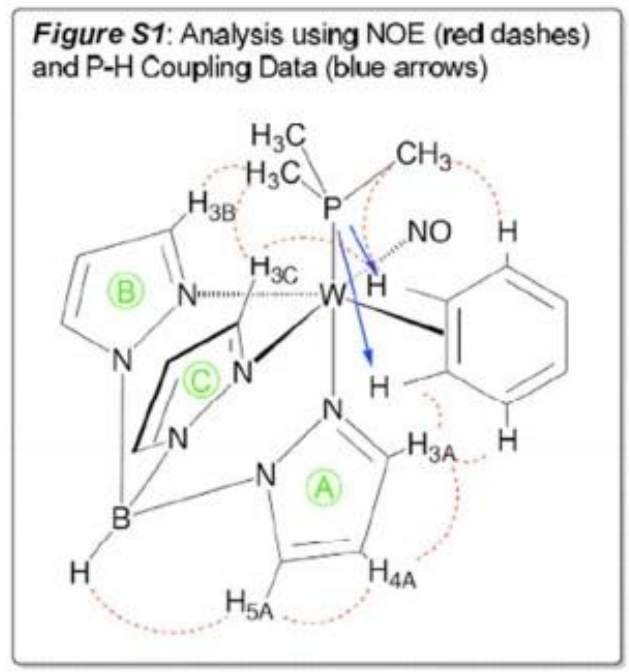

Compound 3: Compound 1 (1.79 g, $3.08 \mathrm{mmol})$ was combined with DiPAT (0.81 g, $3.22 \mathrm{mmol})$. To this heterogeneous mixture was added a DME (6 mL) solution of $N$ methylindoline (4.05 g, $30.41 \mathrm{mmol})$. This dark-yellow and homogeneous solution was stirred overnight $(\sim 14 \mathrm{~h})$, forming a precipitate. The reaction mixture was filtered through a $30 \mathrm{~mL}$ fine-porosity fritted funnel. The collected yellow solid was washed with DME $(2 \times 2 \mathrm{~mL})$, and $\mathrm{Et}_{2} \mathrm{O}(2 \times 50 \mathrm{~mL})$, yielding a mixture of $(\mathbf{3 A}+\mathbf{3 B})(1.14 \mathrm{~g}$, $1.45 \mathrm{mmol}, 47 \%)$. Major Species (Compound 3A) ${ }^{1} \mathrm{H}$ NMR $\left(\mathrm{CD}_{3} \mathrm{CN}, \delta\right): 8.09(\mathrm{~d}, J=$ 2.0, 1H, Pz3/5), 7.98 (d, $J=2.0,1 \mathrm{H}, \mathrm{Pz} 3 / 5), 7.93$ (d, $J=2.0,1 \mathrm{H}, \mathrm{Pz} 3 / 5), 7.84$ (d, $J=2.0$, 1H, Pz3/5), 7.73 (d, J = 2.0, 1H, Pz3/5), 7.39 (d, J=2.0, 1H, Pz3/5), 6.57 (m, 1H, H5), 6.44 (overlapping triplets, $J=2.0,2 \mathrm{H}, \mathrm{Pz} 4), 6.32(\mathrm{t}, J=2.0,1 \mathrm{H}, \mathrm{Pz} 4), 4.92(\mathrm{dd}, J=1.9$, 9.3, 1H, H4), 4.28 (m, 1H, H2x), 3.96 (m, 2H, H6 and H3a), 3.79 (dd, $J=8.9,10.5$, H2y), 2.82 (s, 3H, NMe), 2.54 (m, 1H, H3x), 2.29 (d, J = 8.0, 1H, H7), 1.95 (m, 1H, 
$\mathrm{H} 3 \mathrm{y}), 1.24\left(\mathrm{~d}, J=9.3,9 \mathrm{H}, \mathrm{PMe}_{3}\right) .{ }^{13} \mathrm{C} \mathrm{NMR}\left(\mathrm{CD}_{3} \mathrm{CN}, \delta\right): 191.7(\mathrm{C} 7 \mathrm{a}), 145.6(\mathrm{~d}, J=2.3$, Pz3), 143.4 (Pz3), 142.5 (Pz3), 138.8 (Pz5), 138.7 (Pz5), 138.2 (Pz5), 131.5 (d, J = 3.4, C5), 116.3 (C4), 108.4 (Pz4), 108.0 (Pz4), 107.5 (Pz4), 70.8 (d, J = 12.8, C6), 59.0 (C2), 50.1 (C7), 45.1 (C3a), 35.8 (NMe), 29.2 (C3), 13.5 (d, $\left.J=32.1, \mathrm{PMe}_{3}\right) .{ }^{31} \mathrm{P} \mathrm{NMR}(d-$ acetone, $\delta)$ : $-9.03\left(J_{\mathrm{WP}}=285\right)$. Minor Species $($ Compound 3B $){ }^{1} \mathrm{H} \mathrm{NMR}\left(\mathrm{CD}_{3} \mathrm{CN}, \delta\right)$ : $8.00(\mathrm{~d}, J=2.0,1 \mathrm{H}, \mathrm{Pz} 3 / 5), 7.97(\mathrm{~d}, J=2.0,1 \mathrm{H}, \mathrm{Pz} 3 / 5), 7.91(\mathrm{~d}, J=2.0,1 \mathrm{H}, \mathrm{Pz} 3 / 5)$, $7.89(\mathrm{~d}, J=2.0,1 \mathrm{H}, \mathrm{Pz} 3 / 5), 7.63(\mathrm{~d}, J=2.0,1 \mathrm{H}, \mathrm{Pz} 3 / 5), 7.38(\mathrm{~d}, J=2.0,1 \mathrm{H}, \mathrm{Pz} 3 / 5)$, $6.43(\mathrm{t}, J=2.0,1 \mathrm{H}, \mathrm{Pz} 4), 6.39(\mathrm{t}, J=2.0,1 \mathrm{H}, \mathrm{Pz} 4), 6.35(\mathrm{t}, J=2.0,1 \mathrm{H}, \mathrm{Pz} 4), 6.30(\mathrm{bs}$, 1H, H4), 4.28 (m buried, 1H, H5x), 4.04 (m, 1H, H2x), 3.82 (m, 1H, H2y), 3.57 (m, 1H, H6), 3.46 (d, $J=22.4,1 \mathrm{H}, \mathrm{H} 5 \mathrm{y}), 2.90$ (m, 2H, H3x and H3y), 2.60 (s, 3H, NMe), 2.01 (d, $J=8.8,1 \mathrm{H}, \mathrm{H} 7), 1.19\left(\mathrm{~d}, J=8.9,9 \mathrm{H}, \mathrm{PMe}_{3}\right) .{ }^{13} \mathrm{C} \mathrm{NMR}\left(\mathrm{CD}_{3} \mathrm{CN}, \delta\right): 177.4(\mathrm{C} 7 \mathrm{a}), 145.0$ (d, J = 2.7, Pz3), 144.2 (Pz3), $142.0(\mathrm{Pz} 3), 138.8(\mathrm{Pz} 5), 138.7$ (Pz5), 138.6 (Pz5), 134.0 (C4), 131.1 (C3a), 108.2 (Pz4), 108.1 (Pz4), 107.6 (Pz4), 59.3 (d, J= 13.8, C6), 56.5 (C2), $47.4(\mathrm{C} 7), 34.8(\mathrm{NMe}), 33.7$ (C5), $25.6(\mathrm{C} 3), 13.0\left(\mathrm{~d}, J=30.5, \mathrm{PMe}_{3}\right) .{ }^{31} \mathrm{P} \mathrm{NMR}$ $(d$-acetone, $\delta):-7.62\left(J_{\mathrm{WP}}=285\right)$. Analysis of the Mixture IR: $v_{\mathrm{BH}}=2505 \mathrm{~cm}^{-1}, v_{\mathrm{NO}}$ and $v_{\text {iminium }}=1585$ and $1608 \mathrm{~cm}^{-1} . \mathrm{CV}(\mathrm{DMA}): \mathrm{E}_{\mathrm{p}, \mathrm{a}}=1.11 \mathrm{~V} . \mathrm{HRMS}:\left[\mathrm{M}^{+}\right]=$ $\left[\mathrm{C}_{21} \mathrm{H}_{31} \mathrm{~N}_{8} \mathrm{OBPW}{ }^{+}\right]$obsd (\%), calcd (\%), ppm: $635.1936(73), 635.1938(85),-0.3$; 636.1965 (74), 636.1963 (80), 0.3; 637.1961 (100), 637.1962 (100), -0.1; 638.2002 (44), 638.2003 (43), -0.2; 639.1994 (94), 639.1994 (84), 0.0. Anal. Calc'd for $\mathrm{C}_{22} \mathrm{H}_{31} \mathrm{BF}_{3} \mathrm{~N}_{8} \mathrm{O}_{4} \mathrm{PSW}$ : C, 33.61; H, 3.97; N, 14.33. Found: C, 33.57; H, 3.80; N, 14.33.

Compound 4: Compound 1 (1.55 g, 2.67 mmol) was combined with DiPAT (0.803 g, $3.195 \mathrm{mmol})$. To this heterogeneous mixture was added a hexanes ( $24 \mathrm{~mL}$ ) solution of 1- 
methyl-1,2,3,4-tetrahydroquinoline (3.622 g, $24.606 \mathrm{mmol})$. The pale-brown and heterogeneous reaction mixture was stirred for $72 \mathrm{~h}$. The reaction mixture was filtered through a $30 \mathrm{~mL}$ medium-porosity fritted funnel, yielding a dark-yellow solid. The solid was removed from the frit and triturated with DME $(5 \mathrm{~mL})$ for $5 \mathrm{~min}$. This bright-yellow solid was collected on a $30 \mathrm{~mL}$ medium-porosity fritted funnel, washed with DME (5 $\mathrm{mL})$, and hexanes $(2 \times 30 \mathrm{~mL})$, yielding a mixture of Compounds (4A + 4B) $(1.153 \mathrm{~g}$, $1.441 \mathrm{mmol}, 54 \%)$. Major Species (Compound 4B) ${ }^{1} \mathrm{H} \mathrm{NMR}\left(\mathrm{CD}_{3} \mathrm{CN}, \delta\right): 7.99(\mathrm{~d}, J=$ 2.0, 1H, PzB3), 7.96 (d, $J=2.0,1 \mathrm{H}, \mathrm{PzC} 3), 7.91(\mathrm{~d}, J=2.0,1 \mathrm{H}, \mathrm{PzA} 5$ or PzB5), 7.90 (d, $J=2.0,1 \mathrm{H}, \mathrm{PzA} 5$ or PzB5), $7.58(\mathrm{~d}, J=2.0,1 \mathrm{H}, \mathrm{PzC} 3), 7.36(\mathrm{~d}, J=2.0,1 \mathrm{H}, \mathrm{PzA} 3), 6.42$ (t, $J=2.0,1 \mathrm{H}, \mathrm{Pz} 4 \mathrm{C}), 6.39$ (t, $J=2.0,2 \mathrm{H}, \mathrm{PzA} 4$ and PzB4), 6.35 (bs, 1H, H5), 4.33 (dd, $J=8.7,22.7,1 \mathrm{H}, \mathrm{H} 6 \mathrm{x}), 3.83(\mathrm{~m}, 1 \mathrm{H}, \mathrm{H} 2 \mathrm{x}), 3.55(\mathrm{~m}, 2 \mathrm{H}, \mathrm{H} 7$ and $\mathrm{H} 2 \mathrm{y}), 3.48(\mathrm{dd}, J=4.7$, 23.4, 1H, H6y), 2.55 (m, 2H, H4x and H4y), 2.24 (s, 3H, NMe), 2.05 (m, 1H, H3x), 2.03 $(\mathrm{d}, J=9.1,1 \mathrm{H}, \mathrm{H} 8), 1.94(\mathrm{~m}, 1 \mathrm{H}, \mathrm{H} 3 \mathrm{y}), 1.21\left(\mathrm{~d}, J=8.6,9 \mathrm{H}, \mathrm{PMe}_{3}\right) .{ }^{13} \mathrm{C} \mathrm{NMR}\left(\mathrm{CD}_{3} \mathrm{CN}\right.$,

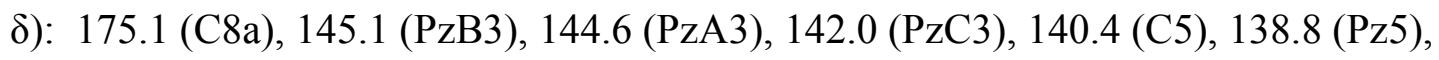
138.7 (Pz5), 138.6 (Pz5), 125.9 (C4a), 108.1 (Pz4), 108.0 (Pz4), 107.9 (Pz4), 58.1 (d, J = 13.8, C7), 54.3 (C2), 53.2 (C8), 40.2 (NMe), 33.0 (d, J = 0.7, C6), 27.2 (C4), 23.1 (C3), $13.1\left(\mathrm{~d}, J=27.3, \mathrm{PMe}_{3}\right) .{ }^{31} \mathrm{P} \mathrm{NMR}\left(\mathrm{CD}_{3} \mathrm{CN}, \delta\right):-8.4\left(J_{\mathrm{WP}}=286\right) . \quad$ Minor Species (Compound 4A) Key Features ${ }^{1} \mathrm{H}$ NMR $\left(\mathrm{CD}_{3} \mathrm{CN}, \delta\right): 6.51(\mathrm{~m}, 1 \mathrm{H}, \mathrm{H} 6), 4.72(\mathrm{dd}, J=$ 2.2, 9.3, 1H, H5), $1.27\left(\mathrm{~d}, J=9.3,9 \mathrm{H}, \mathrm{PMe}_{3}\right) .{ }^{31} \mathrm{P} \mathrm{NMR}\left(\mathrm{CD}_{3} \mathrm{CN}, \delta\right):-9.3$. Analysis of the Mixture IR: $v_{\mathrm{BH}}=2505 \mathrm{~cm}^{-1}, v_{\mathrm{NO}}$ and $v_{\text {iminium }}=1590 \mathrm{~cm}^{-1} \cdot \mathrm{CV}(\mathrm{DMA}): \mathrm{E}_{\mathrm{p}, \mathrm{a}}=0.95$ V. HRMS: $\left[\mathrm{M}^{+}\right]=\left[\mathrm{C}_{22} \mathrm{H}_{33} \mathrm{~N}_{8} \mathrm{OBPW}^{+}\right]$obsd (\%), calcd (\%), ppm: 649.2087 (70), 649.2094 (84), -1.1; 650.2109 (69), 650.2120 (80), -1.7; 651.2136 (99), 651.2118 (100), 2.7; 652.2177 (44), 652.2160 (43), 2.7; 653.2168 (100), 653.2151 (84), 2.6. Anal. Calc'd 
for $\mathrm{C}_{23} \mathrm{H}_{33} \mathrm{BF}_{3} \mathrm{~N}_{8} \mathrm{O}_{4} \mathrm{PSW} \bullet \mathrm{H}_{2} \mathrm{O}$ : C, 33.76; H, 4.31; N, 13.69. Found: C, 33.67; H, 3.97; $\mathrm{N}, 13.31$. Note: 1 equivalent of $\mathrm{H}_{2} \mathrm{O}$ confirmed via ${ }^{1} \mathrm{H}$ NMR.

Compound 5: Compound 1 (5.09 g, $8.76 \mathrm{mmol})$ was combined with DiPAT (2.84 g, $11.3 \mathrm{mmol})$. To this heterogeneous mixture was added a DME (8.3 g) solution of 1ethylindoline ( $8.83 \mathrm{~g}, 59.9 \mathrm{mmol})$. This light-brown and homogeneous solution was stirred overnight $(\sim 14 \mathrm{~h})$, yielding a precipitate. The reaction mixture was filtered through a $30 \mathrm{~mL}$ fine-porosity fritted funnel. The collected yellow solid was washed with 3 x DME (6 mL), and ether $(2 \times 30 \mathrm{~mL})$, yielding a mixture of Compounds $(\mathbf{5 A}+$ 5B) $(3.10 \mathrm{~g}, 3.88 \mathrm{mmol}, 44 \%)$. Major Species (Compound 5A) ${ }^{1} \mathrm{H}$ NMR $\left(\mathrm{CD}_{3} \mathrm{CN}, \delta\right)$ : $8.08(\mathrm{~d}, J=2.0,1 \mathrm{H}, \mathrm{PzB} 3), 7.97(\mathrm{~d}, J=2.0,1 \mathrm{H}, \mathrm{Pz} 5 \mathrm{~B}$ or Pz5C), $7.93(\mathrm{~d}, J=2.0,1 \mathrm{H}$, Pz5B or Pz5C), 7.83 (d, $J=2.0,1 \mathrm{H}, \mathrm{PzA} 5), 7.72$ (d, $J=2.0,1 \mathrm{H}, \mathrm{PzC} 3), 7.39$ (d, $J=2.0$, 1H, PzA3), 6.57 (m, 1H, H5), $6.43(\mathrm{t}, J=2.0,2 \mathrm{H}, \mathrm{PzB} 4$ and PzC4), $6.28(\mathrm{t}, J=2.0,1 \mathrm{H}$, PzA4), 4.92 (dd, $J=1.7,9.5,1 \mathrm{H}, \mathrm{H} 4), 4.15$ (m, 1H, H2x), 4.00-3.87 (m, 3H, H6 and H3a and H2y), 3.08 (dd, $J=6.9,14.2,2 \mathrm{H}, \mathrm{N}-\mathrm{Ethyl}), 2.56(\mathrm{~m}, 1 \mathrm{H}, \mathrm{H} 3 \mathrm{x}), 2.31(\mathrm{~d}, J=8.0,1 \mathrm{H}$, H7), $1.90(\mathrm{~m}, 1 \mathrm{H}, \mathrm{H} 3 \mathrm{y}), 1.24\left(\mathrm{~d}, J=9.2,9 \mathrm{H}, \mathrm{PMe}_{3}\right), 1.90\left(\mathrm{t}, J=7.2,3 \mathrm{H}, \mathrm{N}\right.$-ethyl). ${ }^{13} \mathrm{C}$

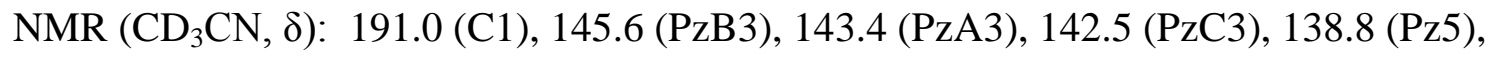
138.7 (Pz5), 138.2 (Pz5), 131.5 (d, J = 3.0, C5), 116.2 (C4), 108.4 (Pz4), 107.9 (Pz4), 107.4 (Pz4), 71.0 (d, $J=12.7, \mathrm{C} 6), 55.2$ (C2), 49.9 (C7), 45.1 (C3a), 43.6 (N-Ethyl-CH ${ }_{2}$ ), $29.3(\mathrm{C} 3), 13.5\left(\mathrm{~d}, J=30.0, \mathrm{PMe}_{3}\right), 12.1\left(\mathrm{~N}-\right.$ Ethyl- $\left.\mathrm{CH}_{3}\right) .{ }^{31} \mathrm{P}$ NMR $\left(d_{6}\right.$-acetone, $\left.\delta\right):-8.94$ $\left(J_{\mathrm{WP}}=279\right)$. Minor Species $\left(\right.$ Compound 5B) Key Features ${ }^{1} \mathrm{H}$ NMR $\left(\mathrm{CD}_{3} \mathrm{CN}, \delta\right): 1.18$ $\left(\mathrm{d}, J=8.7,9 \mathrm{H}, \mathrm{PMe}_{3}\right), 1.02(\mathrm{t}, J=7.0,3 \mathrm{H}, \mathrm{N}-\mathrm{Ethyl})$. Analysis of the Mixture: IR: $v_{\mathrm{BH}}=$ $2507 \mathrm{~cm}^{-1}, v_{\mathrm{C}=\mathrm{C}}=1699 \mathrm{~cm}^{-1}, v_{\mathrm{NO}}$ and $v_{\text {iminium }}=1581 \mathrm{~cm}^{-1} . \mathrm{CV}(\mathrm{DMA}): \mathrm{E}_{\mathrm{p}, \mathrm{a}}=1.07 \mathrm{~V}$ 
HRMS: $\left[\mathrm{M}^{+}\right]=\left[\mathrm{C}_{22} \mathrm{H}_{33} \mathrm{~N}_{8} \mathrm{OBPW}{ }^{+}\right]$obsd, calcd, ppm: 649.2084 (81), 649.2094 (84), $1.6 ; 650.2129(80), 650.2120(80), 1.4 ; 651.2126(100), 651.2118(100), 1.2 ; 652.2170$ (39), 652.2160 (43), 1.6; $653.2146(83), 653.2151(84),-0.7$.

Compound 6: Compound 1 (2.22 g, 3.82 mmol) was combined with DiPAT (1.156 g, $4.604 \mathrm{mmol})$. To this heterogeneous mixture was added 1 -isopropylindoline (3.233 g, $21.963 \mathrm{mmol})$ dissolved in DME (6 mL). This light-brown and homogeneous solution was stirred overnight $(\sim 14 \mathrm{~h})$, yielding a precipitate. The reaction mixture was filtered through a $60 \mathrm{~mL}$ medium-porosity fritted funnel. The collected light-yellow solid was washed with DME (3 x $3 \mathrm{~mL})$, and $\mathrm{Et}_{2} \mathrm{O}(2 \times 60 \mathrm{~mL})$, yielding a mixture of $(\mathbf{6 A} \mathbf{A}+\mathbf{6 B})$ (1.332 g, 1.636 mmol, 43\%). Major Species (Compound 6A) ${ }^{1} \mathrm{H}$ NMR $\left(\mathrm{CD}_{3} \mathrm{CN}, \delta\right)$ : $8.08(\mathrm{~d}, J=2.0,1 \mathrm{H}, \mathrm{PzB} 3), 7.98(\mathrm{~d}, J=2.0,1 \mathrm{H}, \mathrm{Pz} 5), 7.93(\mathrm{~d}, J=2.0,1 \mathrm{H}, \mathrm{Pz} 5), 7.83(\mathrm{~d}$, $J=2.0,1 \mathrm{H}, \mathrm{Pz} 5), 7.73(\mathrm{~d}, J=2.0,1 \mathrm{H}, \mathrm{PzC} 3), 7.46(\mathrm{~d}, J=2.0,1 \mathrm{H}, \mathrm{PzA} 3), 6.56(\mathrm{~m}, 1 \mathrm{H}$, H5), 6.43 (t, $J=2.0,2 \mathrm{H}, \mathrm{Pz} 4), 6.30$ (t, $J=2.0,1 \mathrm{H}, \mathrm{Pz} 4), 4.90$ (dd, $J=2.3,9.5,1 \mathrm{H}, \mathrm{H} 4)$, $4.08(\mathrm{~m}, 1 \mathrm{H}, \mathrm{H} 2 \mathrm{x}), 4.00(\mathrm{~m}, 1 \mathrm{H}, \mathrm{H} 6), 3.91(\mathrm{dd}, J=9.3,12.2,1 \mathrm{H}, \mathrm{H} 2 \mathrm{y}), 3.89(\mathrm{~m}, 1 \mathrm{H}$, H3a), 3.46 (m, 1H, iPr), 2.57 (m, 1H, H3x), 2.33 (d, J= 8.2, 1H, H7), 1.84 (m, 1H, H3y), $1.23\left(\mathrm{~d}, J=9.2,9 \mathrm{H}, \mathrm{PMe}_{3}\right), 1.17(\mathrm{~d}, J=6.7,3 \mathrm{H}, i \mathrm{Pr}), 1.09(\mathrm{~d}, J=6.7,1 \mathrm{H}, i \mathrm{Pr}) .{ }^{13} \mathrm{C} \mathrm{NMR}$ (CD $\left.{ }_{3} \mathrm{CN}, \delta\right): 191.0$ (C7a), 145.6 (PzB3), 143.3 (PzA3), 142.5 (PzC3), 138.8 (Pz5), 138.7 (Pz5), 138.1 (Pz5), 131.3 (d, J = 3.13, C5), 116.2 (C4), 108.4 (Pz4), 108.0 (Pz4), 107.5 (Pz4), 71.5 (d, J = 12.3, C6), 51.1 (C2), 50.1 (iPr-methine), 50.0 (C7), 45.0 (C3a), 29.3 (C3), 19.4 (iPr-methyl), 19.1 (iPr-methyl), 13.5 (d, $\left.J=31.5, \mathrm{PMe}_{3}\right) .{ }^{31} \mathrm{P} \mathrm{NMR}\left(\mathrm{CH}_{2} \mathrm{Cl}_{2}\right.$, $\delta)$ : $-10.26\left(J_{\mathrm{WP}}=281\right)$. Minor Species $($ Compound 6B $)$ Key Features ${ }^{1} \mathrm{H}$ NMR $\left(\mathrm{CD}_{3} \mathrm{CN}\right.$, $\delta): 4.30(\mathrm{~m}, 1 \mathrm{H}, \mathrm{H} 5), 2.08(\mathrm{~d}, J=8.6,1 \mathrm{H}, \mathrm{H} 7)$. Analysis of the Mixture: IR: $v_{\mathrm{BH}}=2500$ 
$\mathrm{cm}^{-1}, v_{\mathrm{NO}}$ and $v_{\text {iminium }}=1595$ and $1576 \mathrm{~cm}^{-1} . \mathrm{CV}(\mathrm{DMA}): \mathrm{E}_{\mathrm{p}, \mathrm{a}}=1.07 \mathrm{~V}$. HRMS: $\left[\mathrm{M}^{+}\right]=$ $\left[\mathrm{C}_{23} \mathrm{H}_{35} \mathrm{~N}_{8} \mathrm{OBPW}^{+}\right]$obs'd (\%), calc'd (\%), ppm: 663.2252 (75), 663.2251 (82), 0.2; 664.2277 (76), 664.2276 (81), 0.2; 665.2276 (100), 665.2275 (100), 0.2; 666.2322 (47), 666.2316 (42), 0.9; 667.2307 (93), 667.2308 (74), -0.1.

Compound 8: $\mathrm{HOTf}(1 \mathrm{~mL})$ was added to a $\mathrm{CH}_{3} \mathrm{CN}$ solution of Compound 5 ( $0.723 \mathrm{~g}$, $0.903 \mathrm{mmol}$ ), resulting in a dark-yellow, homogenous solution. To this 2-methylfuran (3 $\mathrm{mL}, 34 \mathrm{mmol}$ ) was added. The resulting dark-red homogeneous solution stirred for $1 \mathrm{~h}$. The mixture was removed from the glovebox and was treated with $100 \mathrm{~mL}$ of $\mathrm{Na}_{2} \mathrm{CO}_{3}$ (saturated, aq). The reaction mixture was extracted with DCM $(3 \times 100 \mathrm{~mL})$, and the combined organic layers were washed with deionized water $(200 \mathrm{~mL})$, dried over anhydrous $\mathrm{MgSO}_{4}$, and concentrated in vacuo. The yellow oil was redissolved in minimal DCM and then added to stirring $\mathrm{Et}_{2} \mathrm{O}(500 \mathrm{~mL})$ to induce precipitation of a light-yellow solid. The solid was collected on a $30 \mathrm{~mL}$ fine-porosity fritted funnel, washed with $\mathrm{Et}_{2} \mathrm{O}(2 \times 50 \mathrm{~mL})$, yielding 8 (0.715 g, $\left.0.809 \mathrm{mmol}, 90 \%\right) .{ }^{1} \mathrm{H} \mathrm{NMR}$ $\left(\mathrm{CD}_{3} \mathrm{CN}, \delta\right): 8.09$ (d, $\left.J=2.0,1 \mathrm{H}, \mathrm{PzB} 3\right), 7.96$ (d, $\left.J=2.0,1 \mathrm{H}, \mathrm{PzC} 5\right), 7.91$ (d, $J=2.0,1 \mathrm{H}$, PzB5), 7.85 (d, $J=2.0,1 \mathrm{H}, \mathrm{PzA} 5), 7.59$ (d, $J=2.0,1 \mathrm{H}, \mathrm{PzC} 3), 7.27$ (d, $J=2.0,1 \mathrm{H}$, PzA3), 6.43 (t, $J=2.0,1 \mathrm{H}, \mathrm{PzB} 4), 6.41$ (t, $J=2.0,1 \mathrm{H}, \mathrm{PzC} 4), 6.32(\mathrm{t}, J=2.0,1 \mathrm{H}$, PzA4), 6.18 (d, $J=3.0,1 \mathrm{H}, \mathrm{H3}$ '), 6.00 (m, 1H, H4'), 4.44 (m, 1H, H5), 4.06 (m, 1H, H2x), 3.88 (m, 1H, H2y), 3.82 (m, 1H, H6), 3.44 (m, 1H, H3a), 2.75 (m, 1H, N-Ethyl$\mathrm{CH}_{2}$ ), 2.66 (m, 1H, N-Ethyl- $\mathrm{CH}_{2}$ ), 2.49 (m, 1H, H3x), 2.31 (s, 3H, C5'Me), 2.29 (m, 1H, H4x), 2.17 (d, $J=8.9,1 \mathrm{H}, \mathrm{H} 7), 1.72$ (m, 2H, H4y and H3y), 1.06 (d, $J=9.35,9 \mathrm{H}, \mathrm{PMe}_{3}$ ), 1.03 (t, $J=7.13,3 \mathrm{H}, \mathrm{N}-$ Ethyl- $\left.\mathrm{CH}_{3}\right) .{ }^{13} \mathrm{C}$ NMR $\left(\mathrm{CD}_{3} \mathrm{CN}, \delta\right): 189.8$ (C7a), 160.4 (C2'), 
151.9 (C5'), 145.5 (PzB3), 144.9 (PzA3), 142.5 (PzC3), 138.9 (Pz5), 138.8 (Pz5), 108.5 (Pz4), 108.1 (Pz4), 107.8 (Pz4), 107.3 (C3' or C4'), 107.2 (C3' or C4'), 70.8 (d, J= 14.1, C6), $54.3(\mathrm{C} 2), 50.5(\mathrm{C} 7), 42.9(\mathrm{C} 3 \mathrm{a}$ or N-Ethyl-CH 2$), 42.8\left(\mathrm{C} 3 \mathrm{a}\right.$ or N-Ethyl- $\left.\mathrm{CH}_{2}\right), 42.3$ (C4), 28.5 (C3), 13.9 (d, $\left.J=30.1, \mathrm{PMe}_{3}\right), 13.7$ (C5'Me), 12.0 (N-Ethyl-CH$\left.{ }_{3}\right) .{ }^{31} \mathrm{P} \mathrm{NMR}$ $\left(\mathrm{CD}_{3} \mathrm{CN}, \delta\right):-8.2\left(J_{\mathrm{WP}}=283\right)$. IR: $v_{\mathrm{BH}}=2507 \mathrm{~cm}^{-1}, v_{\mathrm{NO}}$ and $v_{\text {iminium }}=1608$ and $1581 \mathrm{~cm}^{-1}$. CV (DMA): $\mathrm{E}_{\mathrm{p}, \mathrm{a}}=1.22 \mathrm{~V} . \mathrm{HRMS}:\left[\mathrm{M}^{+}\right]=\left[\mathrm{C}_{27} \mathrm{H}_{39} \mathrm{~N}_{8} \mathrm{O}_{2} \mathrm{BPW}^{+}\right]$obsd (\%), calcd (\%), ppm: 731.2521 (84), 731.2514 (82), 1.0; 732.2558 (80), 732.2539 (81), 2.6; 733.2550 (100), 733.2539 (100), 1.6; 734.2609 (45), 734.2578 (47), 4.3; 735.2580 (80), 735.2571 (83), 1.3. Anal. Calc'd for $\mathrm{C}_{28} \mathrm{H}_{39} \mathrm{BF}_{3} \mathrm{~N}_{8} \mathrm{O}_{5} \mathrm{PSW}$ : C, 38.12; H, 4.46; N, 12.70. Found: C, 38.33; H, 4.56; N, 12.62 .

Compound 9: A solution of HOTf in $\mathrm{CH}_{3} \mathrm{CN}(22 \mathrm{~mL}, 0.125 \mathrm{M})$ was added to Compound 5 (1.06 g, $1.32 \mathrm{mmol})$, resulting in a dark-yellow, homogenous solution. To this pyrazole $(501 \mathrm{mg}, 7.34 \mathrm{mmol})$ was added. The resulting light-yellow homogeneous solution stirred for $5 \mathrm{~min}$. The mixture was removed from the glovebox and was diluted with $75 \mathrm{~mL}$ DCM. This solution treated with 2 x $100 \mathrm{~mL}$ of $\mathrm{Na}_{2} \mathrm{CO}_{3}$ (saturated, aq). The reaction mixture was extracted with DCM (1x200 mL, followed by 2 x $50 \mathrm{~mL})$, and the combined organic layers were washed with deionized water $(200 \mathrm{~mL})$, dried over anhydrous $\mathrm{MgSO}_{4}$, and concentrated in vacuo. The yellow oil was redissolved in minimal DCM and then added to stirring $\mathrm{Et}_{2} \mathrm{O}(500 \mathrm{~mL})$ to induce precipitation of a white solid. The solid was collected on a $30 \mathrm{~mL}$ fine-porosity fritted funnel, washed with $\mathrm{Et}_{2} \mathrm{O}(2 \times 50 \mathrm{~mL})$, yielding 9 (1.08 g, $\left.1.24 \mathrm{mmol}, 94 \%\right) .{ }^{1} \mathrm{H} \mathrm{NMR}\left(\mathrm{CD}_{3} \mathrm{CN}, \delta\right): 8.12(\mathrm{~d}, J$ $=2.0,1 \mathrm{H}, \mathrm{PzB} 3), 7.96(\mathrm{~d}, J=2.0,1 \mathrm{H}, \mathrm{PzC} 5), 7.93(\mathrm{~d}, J=2.0,1 \mathrm{H}, \mathrm{PzB} 5), 7.89(\mathrm{dd}, J=$ 
2, 0.6, 1H, H5'), $7.86(\mathrm{~d}, J=2.0,1 \mathrm{H}, \mathrm{PzA} 5), 7.55(\mathrm{~d}, J=2.0,1 \mathrm{H}, \mathrm{PzC} 3), 7.52(\mathrm{~d}, J=2.0$, 1H, H3'), 7.30 (d, $J=2.0,1 \mathrm{H}, \mathrm{PzA} 3), 6.45$ (t, $J=2.0,1 \mathrm{H}, \mathrm{PzB} 4), 6.40(\mathrm{t}, J=2.0,1 \mathrm{H}$, PzC4), 6.38 (t, $\left.J=2.0,1 \mathrm{H}, \mathrm{H} 4{ }^{\prime}\right), 6.33$ (d, $\left.J=2.0,1 \mathrm{H}, \mathrm{PzA} 4\right), 5.82(\mathrm{~m}, 1 \mathrm{H}, \mathrm{H} 5), 4.10$ (m, 1H, H2x), 3.92 (m, 1H, H2y), 3.83 (m, 1H, H6), 3.50 (m, 1H, H3a), 2.84 (m, 1H, NEthyl- $\left.\mathrm{CH}_{2}\right), 2.75$ (m, 1H, N-Ethyl- $\left.\mathrm{CH}_{2}\right), 2.50$ (m, buried, 1H, H3x), 2.47 (m, 1H, H4x), $2.24(\mathrm{~d}, J=8.9,1 \mathrm{H}, \mathrm{H} 7), 1.88(\mathrm{~m}$, buried, 1H, H4y), $1.83(\mathrm{~m}$, buried, $1 \mathrm{H}, \mathrm{H} 3 \mathrm{y}), 1.07$ (t, $J$ $\left.=7,3 \mathrm{H}, \mathrm{N}-\mathrm{Ethyl}-\mathrm{CH}_{3}\right), 1.00\left(\mathrm{~d}, J=9.1,9 \mathrm{H}, \mathrm{PMe}_{3}\right) \cdot{ }^{13} \mathrm{C} \mathrm{NMR}\left(\mathrm{CD}_{3} \mathrm{CN}, \delta\right): 189.6(\mathrm{C} 7 \mathrm{a})$, 145.3 (d, $J=2.1, \mathrm{PzB} 3), 144.7$ (PzA3), 142.3 (PzC3), 139.4 (C3'), 138.8 (PzC5 and PzB5), 138.6 (PzA5), 128.9 (C5'), 108.5 (PzB4), 108.1 (PzC4), 107.7 (PzA4), 106.9 (C4'), 70.6 (d, $J=14.3, \mathrm{C} 6), 62.8$ (d, $J=2.6, \mathrm{C} 5), 54.4$ (C2), 49.3 (C7), 43.0 (N-Ethyl$\left.\mathrm{CH}_{2}\right), 41.8(\mathrm{C} 3 \mathrm{a}), 41.7(\mathrm{C} 4), 28.6(\mathrm{C} 3), 13.4\left(\mathrm{~d}, J=31.0, \mathrm{PMe}_{3}\right), 11.8\left(\mathrm{~N}-\mathrm{Ethyl}-\mathrm{CH}_{3}\right) .{ }^{31} \mathrm{P}$ $\operatorname{NMR}\left(\mathrm{CD}_{3} \mathrm{CN}, \delta\right):-8.8\left(J_{\mathrm{WP}}=281\right) . \mathrm{IR}: v_{\mathrm{BH}}=2511 \mathrm{~cm}^{-1}, v_{\mathrm{NO}}$ and $v_{\text {iminium }}=1612$ and $1577 \mathrm{~cm}^{-1} . \mathrm{CV}(\mathrm{DMA}): \mathrm{E}_{\mathrm{p}, \mathrm{a}}=1.34 \mathrm{~V}$. Anal. Calc'd for $\mathrm{C}_{26} \mathrm{H}_{37} \mathrm{BF}_{3} \mathrm{~N}_{10} \mathrm{O}_{4} \mathrm{PSW} \cdot 1 / 2 \mathrm{H}_{2} \mathrm{O}: \mathrm{C}$, 35.39; H, 4.37; N, 15.97. Found: C, 35.44; H, 4.28; N, 15.81. Note: $1 / 2$ equivalent of $\mathrm{H}_{2} \mathrm{O}$ confirmed via ${ }^{1} \mathrm{H}$ NMR.

Compound 10: A solution of HOTf in MeCN (13 mL, 0.125 M) was added to Compound 5 (0.704 g, $0.879 \mathrm{mmol})$, resulting in a light orange, homogenous solution. To this, 1,3-dimethyoxybenzene $(0.674 \mathrm{~g}, 4.87 \mathrm{mmol})$ was added. The light yellow solution stirred for $1 \mathrm{~h}$. The solution was removed from the glovebox and was diluted with DCM $(50 \mathrm{~mL})$. This was treated with $2 \times 50 \mathrm{~mL}$ of $\mathrm{Na}_{2} \mathrm{CO}_{3}$ (saturated, aq). The aqueous layer was back extracted with DCM ( 2 x $30 \mathrm{~mL})$, and the combined organic layers were washed with deionized water $(50 \mathrm{~mL})$ and brine $(50 \mathrm{~mL})$. This was then dried 
over anhydrous $\mathrm{MgSO}_{4}$ and concentrated in vacuo. The yellow oil was redissolved in minimal DCM and added dropwise to a stirring solution of $\mathrm{Et}_{2} \mathrm{O}(500 \mathrm{~mL})$ to induce precipitation of a light-tan solid. The powder was collected on a $30 \mathrm{~mL}$ fine-porosity fritted funnel, washed with $\mathrm{Et}_{2} \mathrm{O}(30 \mathrm{~mL})$, yielding Compound 10 (0.517 g, $0.551 \mathrm{mmol}$, 63\%). ${ }^{1} \mathrm{H}$ NMR $(d-\mathrm{MeCN}, \delta): 8.09(\mathrm{~d}, J=2.0,1 \mathrm{H}, \mathrm{PzB} 3), 7.95(\mathrm{~d}, J=2.0,1 \mathrm{H}, \mathrm{PzC} 5)$, $7.90(\mathrm{~d}, J=2.0,1 \mathrm{H}, \mathrm{PzB} 5), 7.86(\mathrm{~d}, J=2.0,1 \mathrm{H}, \mathrm{PzA} 5), 7.55(\mathrm{~d}, J=2.0,1 \mathrm{H}, \mathrm{PzC} 3), 7.53$ (d, $J=8.5,1 \mathrm{H}, \mathrm{H} 3$ ') 7.27 (d, $J=2.0,1 \mathrm{H}, \mathrm{PzA} 3), 6.65$ (dd, $\left.J=8.5,2.6,1 \mathrm{H}, \mathrm{H} 5^{\prime}\right), 6.57$ (d, $J=2.5,1 \mathrm{H}, \mathrm{H} 6$ '), 6.42 (t, $J=2.0,1 \mathrm{H}, \mathrm{PzB} 4), 6.40$ (t, $J=2.0,1 \mathrm{H}, \mathrm{PzC} 4), 6.33$ (t, $J=$ 2.0, 1H, PzA4), 4.91 (m, 1H, H5), 4.08 (m, 1H, H2x), 3.90 (m, 1H, H2y), 3.85 (s, 3H, H4' OMe), 3.82 (s, 3H, H2' OMe), 3.77 (m, 1H, H6), 3.56 (m, 1H, H3a), 2.76 (m, 1H, $N$ Ethyl $\left.\mathrm{CH}_{2}\right), 2.63$ (m, 1H, $N$-Ethyl $\left.\mathrm{CH}_{2}\right), 2.47$ (m, 1H, H3x), 2.26 (d, $\left.J=9.1,1 \mathrm{H}, \mathrm{H} 7\right)$, 2.17 (m, buried, 1H, H4x), 1.69 (m, 1H, H3y), 1.45 (m, 1H, H4y), 1.05 (t, $J=7.1,3 \mathrm{H}, N$ Ethyl $\left.\mathrm{CH}_{3}\right), 0.97$ (d, $\left.J=9.2,9 \mathrm{H}, \mathrm{PMe}_{3}\right) .{ }^{13} \mathrm{C}$ NMR $(d-\mathrm{MeCN}, \delta): 189.0(\mathrm{C} 7 \mathrm{a}), 160.4$ (OMe), 158.1 (OMe), 145.3 (d, $J=2.1, \mathrm{PzB} 3), 144.8$ (PzA3), 142.2 (PzC3), 138.7 (PzC5), 138.7 (PzA5), 138.6 (PzB5), 130.4 (C1'), 130.0 (C3’), 108.4 (PzB4), 108.0 (PzC4), 107.7 (PzA4), 106.7 (C5’), 99.0 (C6'), 74.1 (d, J = 13.7, C7), 56.1 (OMe), 56.0 (OMe), 53.9 (C2), 51.2 (C7), 45.4 (C4), 43.0 (C3a), 42.6 ( $N$-Ethyl $\mathrm{CH}_{2}$ ), 37.3 (C5), 28.2 (C3), $13.9\left(\mathrm{~d}, J=30.2, \mathrm{PMe}_{3}\right), 11.8\left(N\right.$-Ethyl $\left.\mathrm{CH}_{3}\right) .{ }^{31} \mathrm{P} \mathrm{NMR}(d-\mathrm{MeCN}, \delta):-7.66\left(J_{\mathrm{wp}}=\right.$ 292). IR: $v_{\mathrm{BH}}=2515 \mathrm{~cm}^{-1}, v_{\mathrm{NO}}$ and $v_{\text {iminium }}=1605$ and $1579 \mathrm{~cm}^{-1}$. CV (DMA): $\mathrm{E}_{\mathrm{p}, \mathrm{a}}=$ 1.15 V. HRMS: $\left[\mathrm{M}^{+}\right]=\left[\mathrm{C}_{30} \mathrm{H}_{43} \mathrm{~N}_{8} \mathrm{O}_{3} \mathrm{BPW}^{+}\right]$obs'd (\%), calc'd (\%), ppm: 787.2757 (77), 787.2776 (80), -2.5; 788.2772 (78), 788.2801 (82), -3.7; 789.2790 (100), 789.2802 (100), -1.5; 790.2809 (46), 790.2839 (49), -3.8; 791.2803 (86), 791.2834 (82), -3.9. 
Compound 11: A solution of HOTf in MeCN (1 mL, $0.125 \mathrm{M})$ was added to Compound $5(0.050 \mathrm{~g}, 0.062 \mathrm{mmol})$, resulting in a light orange, homogenous solution. To this, indole $(0.051 \mathrm{~g}, 0.437 \mathrm{mmol})$ was added. The light yellow solution stirred for $5 \mathrm{~min}$. The solution was removed from the glovebox and was diluted with DCM (15 mL). This was treated with $2 \times 20 \mathrm{~mL}$ of $\mathrm{Na}_{2} \mathrm{CO}_{3}$ (saturated, aq). The aqueous layer was back extracted with DCM (2 x $20 \mathrm{~mL})$, and the combined organic layers were washed with deionized water $(15 \mathrm{~mL})$ and brine $(15 \mathrm{~mL})$. This was then dried over anhydrous $\mathrm{MgSO}_{4}$ and concentrated in vacuo. The yellow oil was redissolved in minimal DCM and added dropwise to a stirring solution of $\mathrm{Et}_{2} \mathrm{O}(100 \mathrm{~mL})$ to induce precipitation of a light-tan solid. The powder was collected on a $15 \mathrm{~mL}$ fine-porosity fritted funnel, washed with $\mathrm{Et}_{2} \mathrm{O}(30 \mathrm{~mL})$, yielding Compound $11(0.036 \mathrm{~g}, 0.118 \mathrm{mmol}, 62 \%) .{ }^{1} \mathrm{H} \mathrm{NMR}(d-\mathrm{MeCN}$,

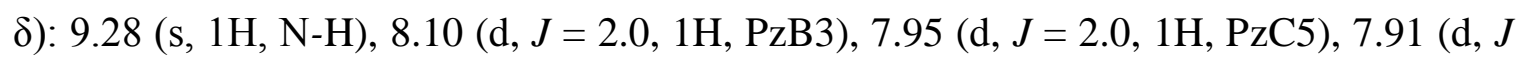
$=2.0,1 \mathrm{H}, \mathrm{PzB} 5), 7.87(\mathrm{~d}, J=2.0,1 \mathrm{H}, \mathrm{PzA} 5), 7.84\left(\mathrm{dd}, J=7.8,0.7,1 \mathrm{H}, \mathrm{H} 7{ }^{\prime}\right), 7.49(\mathrm{~d}, J$ $=2.0,1 \mathrm{H}, \mathrm{PzC} 3), 7.46\left(\mathrm{dt}, J=8.1,0.7,1 \mathrm{H}, \mathrm{H} 4^{\prime}\right), 7.34\left(\mathrm{~d}, J=2.4,1 \mathrm{H}, \mathrm{H} 2{ }^{\prime}\right), 7.31(\mathrm{~d}, J=$ 2.0, 1H, PzA3), $7.19\left(\mathrm{td}, J=7.6,1.0,1 \mathrm{H}, \mathrm{H} 5^{\prime}\right), 7.10\left(\mathrm{td}, J=7.5,1.0,1 \mathrm{H}, \mathrm{H} 6^{\prime}\right), 6.43$ (t, $J$ $=2.0,1 \mathrm{H}, \mathrm{PzB} 4), 6.38(\mathrm{t}, J=2.0,1 \mathrm{H}, \mathrm{PzC} 4), 6.34(\mathrm{t}, J=2.0,1 \mathrm{H}, \mathrm{PzA} 4), 4.79(\mathrm{~m}, 1 \mathrm{H}$, H5), 4.09 (m, 1H, H2x), 4.08 (m, 1H, H6), 3.91 (t, $J=11.0,1 \mathrm{H}, \mathrm{H} 2 \mathrm{y}), 3.60$ (m, 1H, H3a), $2.78\left(\mathrm{~m}, 1 \mathrm{H}, N\right.$-Ethyl $\left.\mathrm{CH}_{2}\right), 2.67\left(\mathrm{~m}, 1 \mathrm{H}, N\right.$-Ethyl $\left.\mathrm{CH}_{2}\right), 2.48(\mathrm{~m}, 1 \mathrm{H}, \mathrm{H} 3 \mathrm{x}), 2.33$ (d, $J=8.7,1 \mathrm{H}, \mathrm{H} 7), 2.27$ (m, 1H, H4x), 1.78 (m, 1H, H4y), 1.67 (m, 1H, H3y), 1.08 (t, $J$ $=7.4,3 \mathrm{H}, N$-Ethyl $\left.\mathrm{CH}_{3}\right), 0.97\left(\mathrm{~d}, J=9.2,9 \mathrm{H}, \mathrm{PMe}_{3}\right) .{ }^{13} \mathrm{C} \mathrm{NMR}(d-\mathrm{MeCN} \delta): 189.6$ (C7a), 145.3 (PzB3), 144.8 (PzA3), 142.2 (PzC3), 138.7 (PzB5 and PzC5), 138.1 (PzA5), 126.5 (C7a'), 123.3 (C3a' and C3'), 123.1 (C2'), 122.7 (C5'), 120.3 (C7'), 119.9 (C6'), 112.8 (C4'), 108.4 (Pz4), 108.0 (Pz4), 107.7 (Pz4), 73.0 (d, J = 13.7, C6), 54.1 (C2), 51.1 
(C7), 45.0 (C4), 43.0 (C3a), 42.7 ( $N$-Ethyl $\mathrm{CH}_{2}$ ), 37.6 (C5), 28.2 (C3), 14.1 (d, J = 31.0, $\left.\mathrm{PMe}_{3}\right), 11.9\left(N\right.$-Ethyl $\left.\mathrm{CH}_{3}\right) .{ }^{31} \mathrm{P}$ NMR $(d$-Acetone, $\delta):-7.10\left(J_{\mathrm{WP}}=287\right) . \mathrm{IR}: v_{\mathrm{BH}}=2511$ $\mathrm{cm}^{-1}, v_{\mathrm{NO}}$ and $v_{\text {iminium }}=1604$ and $1577 \mathrm{~cm}^{-1} . \mathrm{CV}(\mathrm{DMA}): \mathrm{E}_{\mathrm{p}, \mathrm{a}}=1.19 \mathrm{~V} . \mathrm{HRMS}:\left[\mathrm{M}^{+}\right]=$ $\left[\mathrm{C}_{30} \mathrm{H}_{40} \mathrm{~N}_{9} \mathrm{OBPW}{ }^{+}\right]$obs'd (\%), calc'd (\%), ppm: 766.2653 (80), 766.2674 (80), -2.7; 767.2674 (80), 767.2699 (82), -3.2; 768.2675 (100), 768.2699 (100), -3.1; 769.2717 (49), 769.2737 (49), -2.5; $770.2710(79), 770.2731(82),-2.8$.

Compound 12: A solution of HOTf in MeCN (1 mL, 0.125 M) was added to Compound $5(0.051 \mathrm{~g}, 0.064 \mathrm{mmol})$, resulting in a light orange, homogenous solution. To this, 2methylthiophene $(0.057 \mathrm{~g}, 0.581 \mathrm{mmol})$ was added. The light yellow solution stirred for 5 min. The solution was removed from the glovebox and was diluted with DCM (20 mL). This was treated with $2 \times 50 \mathrm{~mL}$ of $\mathrm{Na}_{2} \mathrm{CO}_{3}$ (saturated, aq). The aqueous layer was back extracted with DCM (2 x $20 \mathrm{~mL})$, and the combined organic layers were washed with deionized water $(40 \mathrm{~mL})$ and brine $(40 \mathrm{~mL})$. This was then dried over anhydrous $\mathrm{MgSO}_{4}$ and concentrated in vacuo. The yellow oil was redissolved in minimal DCM and added dropwise to a stirring solution of $\mathrm{Et}_{2} \mathrm{O}(50 \mathrm{~mL})$ to induce precipitation of a light-tan solid. The powder was collected on a $15 \mathrm{~mL}$ fine-porosity fritted funnel, washed with $\mathrm{Et}_{2} \mathrm{O}$ (30 $\mathrm{mL}$ ), yielding Compound 12 (0.028 g, $0.031 \mathrm{mmol}, 54 \%) .{ }^{1} \mathrm{H}$ NMR ( $d$-MeCN, $\left.\delta\right): 8.09$ $(\mathrm{d}, J=2.0,1 \mathrm{H}, \mathrm{PzB} 3), 7.96(\mathrm{~d}, J=2.0,1 \mathrm{H}, \mathrm{PzC} 5), 7.91(\mathrm{~d}, J=2.0,1 \mathrm{H}, \mathrm{PzB} 5), 7.86(\mathrm{~d}, J$ $=2.0,1 \mathrm{H}, \mathrm{PzA} 5), 7.55(\mathrm{~d}, J=2.0,1 \mathrm{H}, \mathrm{PzC} 3), 7.26(\mathrm{~d}, J=2.0,1 \mathrm{H}, \mathrm{PzA} 3), 6.89(\mathrm{~d}, J=$ 3.0, 1H, H4'), 6.68 (m, 1H, H3'), 6.43 (t, $J=2.0,1 \mathrm{H}, \mathrm{PzB} 4), 6.41$ (t, $J=2.0,1 \mathrm{H}, \mathrm{PzC} 4)$, $6.32(\mathrm{t}, J=2.0,1 \mathrm{H}, \mathrm{PzA} 4), 4.62(\mathrm{~m}, 1 \mathrm{H}, \mathrm{H} 5), 4.06(\mathrm{~m}, 1 \mathrm{H}, \mathrm{H} 2 \mathrm{x}), 3.87(\mathrm{~m}, 1 \mathrm{H}, \mathrm{H} 2 \mathrm{y})$, 3.77 (m, 1H, H6), 3.47 (m, 1H, H3a), 2.75 (m, 1H, $N$-Ethyl- $\left.\mathrm{CH}_{2}\right), 2.63$ (m, 1H, $N$-Ethyl- 
$\left.\mathrm{CH}_{2}\right), 2.47$ (m, buried, 1H, H3x), 2.46 (d, $\left.J=1,3 \mathrm{H}, 2^{\prime} \mathrm{Me}\right), 2.33$ (m, 1H, H4x), 2.21 (d, $J$ $=8.7,1 \mathrm{H}, \mathrm{H} 7), 1.71(\mathrm{~m}, 1 \mathrm{H}, \mathrm{H} 3 \mathrm{y}), 1.63(\mathrm{~m}, 1 \mathrm{H}, \mathrm{H} 4 \mathrm{y}), 1.05\left(\mathrm{~d}, J=9.2,9 \mathrm{H}, \mathrm{PMe}_{3}\right), 1.04$ $\left(\mathrm{t}, J=8.7,3 \mathrm{H}, \mathrm{N}\right.$-Ethyl- $\left.\mathrm{CH}_{3}\right) .{ }^{13} \mathrm{C}$ NMR $(d-\mathrm{MeCN}, \delta): 189.4(\mathrm{C} 7 \mathrm{a}), 152.3\left(\mathrm{C} 2\right.$ ' and $\left.\mathrm{C} 5{ }^{\prime}\right)$, 145.4 (PzB3), 144.8 (PzA3), 142.3 (PzC3), 139.1 (PzA5), 138.8 (PzB5), 138.7 (PzC5), 125.9 (C3'), 125.2 (C4'), 108.5 (PzB4), 108.1 (PzC4), 107.8 (PzA4), 73.7 (d, $J=14.3$, C6), 54.2 (C2), 50.6 (C7), 46.8 (C4), 42.69 (H3a), 42.8 (N-Ethyl-CH $\left.{ }_{2}\right), 41.88$ (C5), 28.3 (C3), $15.5\left(\mathrm{C} 2{ }^{`} \mathrm{Me}\right), 14.1$ (d, $\left.J=30, \mathrm{PMe}_{3}\right), 11.8(N$-Ethyl-CH 3$) .{ }^{31} \mathrm{P}$ NMR $(d-\mathrm{MeCN}, \delta)$ : $-8.04\left(J_{\mathrm{wp}}=284\right) . \mathrm{IR}: v_{\mathrm{BH}}=2518 \mathrm{~cm}^{-1}, v_{\mathrm{NO}}$ and $v_{\text {iminium }}=1606$ and $1577 \mathrm{~cm}^{-1} . \mathrm{CV}$ $\left(\right.$ DMA): $\mathrm{E}_{\mathrm{p}, \mathrm{a}}=1.21 \mathrm{~V} . \mathrm{HRMS}:\left[\mathrm{M}^{+}\right]=\left[\mathrm{C}_{27} \mathrm{H}_{39} \mathrm{~N}_{8} \mathrm{OBPSW}^{+}\right]$obs'd (\%), calc'd (\%), ppm: 747.2262 (77), 747.2285 (78), -3.1; 748.2296 (79), 748.2310 (79), -1.9; 749.2295 (100), 749.2308 (100), -1.7; 750.2329 (49), 750.2343 (49), -1.8; 751.2324 (85), 751.2338 (84), 1.9 .

Compound 13: A solution of HOTf in $\mathrm{MeCN}(10 \mathrm{~mL}, 0.125 \mathrm{M})$ was added to Compound 5 (0.498 g, $0.622 \mathrm{mmol})$, resulting in a light orange, homogenous solution. To this, $\mathrm{NaCNBH}_{3}(0.201 \mathrm{~g}, 3.20 \mathrm{mmol})$ was added. The light yellow solution stirred for 5 min. The solution was removed from the glovebox and was diluted with DCM $(50 \mathrm{~mL})$. This was treated with $2 \times 50 \mathrm{~mL}$ of $\mathrm{Na}_{2} \mathrm{CO}_{3}$ (saturated, aq). The aqeuous layer was back extracted with DCM (2 x $40 \mathrm{~mL})$, and the combined organic layers were washed with deionized water $(40 \mathrm{~mL})$. This was then dried over anhydrous $\mathrm{MgSO}_{4}$ and concentrated in vacuo. The yellow oil was redissolved in minimal DCM and added dropwise to a stirring solution of $\mathrm{Et}_{2} \mathrm{O}(400 \mathrm{~mL})$ to induce precipitation of a light-tan solid. The powder was collected on a $30 \mathrm{~mL}$ fine-porosity fritted funnel, washed with $\mathrm{Et}_{2} \mathrm{O}(30 \mathrm{~mL})$, 
yielding Compound $13(0.346 \mathrm{~g}, 0.431 \mathrm{mmol}, 69 \%) .{ }^{1} \mathrm{H}$ NMR $\left(d-\mathrm{CDCl}_{3}, \delta\right): 8.07(\mathrm{~d}, J=$ 2.0, 1H, PzB3), 7.95 (d, $J=2.0,1 \mathrm{H}, \mathrm{PzB} 5$ or PzC5), $7.91(\mathrm{~d}, J=2.0,1 \mathrm{H}, \mathrm{PzB} 5$ or PzC5), 7.85 (d, $J=2.0,1 \mathrm{H}, \mathrm{PzA} 5), 7.63$ (d, $J=2.0,1 \mathrm{H}, \mathrm{PzC} 3), 7.32$ (d, $J=2.0,1 \mathrm{H}, \mathrm{PzA} 3), 6.42$ (m, 2H, PzB4 and PzC4), 6.31 (t, $J=2.0,1 \mathrm{H}, \mathrm{PzA} 4), 4.06(\mathrm{~m}, 1 \mathrm{H}, \mathrm{H} 2 \mathrm{x}), 3.86(\mathrm{t}, J=$ 10.9, 1H, H2y), 3.75 (m, 1H, H6), 3.32 (m, 1H, H3a), 3.01 (m, 2H, H5), 2.80 (m, 1H, $N$ Ethyl $\mathrm{CH}_{2}$ ), 2.68 (m, 1H, $N$-Ethyl $\mathrm{CH}_{2}$ ), 2.50 (m, 1H, H3x), 2.14 (buried, 1H, H4x), 2.04 (d, $J=9.7,1 \mathrm{H}, \mathrm{H} 7), 1.73$ (m, 1H, H3y), 1.46 (m, 1H, H4y), 1.21 (d, $J=9.0,9 \mathrm{H}, \mathrm{PMe}_{3}$ ), 1.00 (t, $J=7.0,3 \mathrm{H}, N$-Ethyl $\left.\mathrm{CH}_{3}\right) .{ }^{13} \mathrm{C}$ NMR $\left(d-\mathrm{CDCl}_{3}, \delta\right): 190.7$ (C7a), 145.3 (PzB3), 144.9 (PzA3), 142.1 (PzC3), 138.7 (Pz5), 138.6 (Pz5), 138.5 (Pz5), 67.7 (d, $J=$ 14.0, C6), 53.9 (C2), 50.0 (C3a), 43.4 (C7), 42.7 (N-Ethyl $\mathrm{CH}_{2}$ ), 35.7 (C4), 28.2 (C3), 28.1 (d, $J=4.0, \mathrm{C} 5), 13.7\left(\mathrm{~d}, J=30.0, \mathrm{PMe}_{3}\right), 11.9\left(N\right.$-Ethyl $\left.\mathrm{CH}_{3}\right) .{ }^{31} \mathrm{P} \mathrm{NMR}\left(d-\mathrm{CDCl}_{3}, \delta\right):-7.99$ $\left(J_{\mathrm{wp}}=287\right)$. IR: $v_{\mathrm{BH}}=2507 \mathrm{~cm}^{-1}, v_{\mathrm{NO}}$ and $v_{\text {iminium }}=1604$ and $1577 \mathrm{~cm}^{-1} . \mathrm{CV}(\mathrm{DMA})$ : $\mathrm{E}_{\mathrm{p}, \mathrm{a}}=1.23$ V. HRMS: $\left[\mathrm{M}^{+}\right]=\left[\mathrm{C}_{22} \mathrm{H}_{35} \mathrm{~N}_{8} \mathrm{OBPW}^{+}\right]$obs'd (\%), calc'd (\%), ppm651.2240 (86), 651.2251 (84), -1.7; 652.2284 (72), 652.2276 (80), 1.2; 653.2262 (100), 653.2275 (100), -2.0; 654.2336 (41), 654.2316 (43), 3.0; 655.2328 (69), 655.2307 (84), 3.2.

Compound 14: A solution of HOTf in $\mathrm{MeCN}(3 \mathrm{~mL}, 0.125 \mathrm{M})$ was added to Compound $5(0.150 \mathrm{~g}, 0.187 \mathrm{mmol})$, resulting in a light orange, homogenous solution. To this, $\mathrm{N}$ propyl amine $(0.102 \mathrm{~g}, 1.73 \mathrm{mmol})$ was added. The light yellow solution stirred for 5 min. The solution was removed from the glovebox and was diluted with DCM $(30 \mathrm{~mL})$. This was treated with $2 \times 50 \mathrm{~mL}$ of $\mathrm{Na}_{2} \mathrm{CO}_{3}$ (saturated, aq). The aqueous layer was back extracted with DCM (2 x $30 \mathrm{~mL})$, and the combined organic layers were washed with deionized water $(30 \mathrm{~mL})$ and brine $(30 \mathrm{~mL})$. This was then dried over anhydrous $\mathrm{MgSO}_{4}$ 
and concentrated in vacuo. The yellow oil was redissolved in minimal DCM and added dropwise to a stirring solution of $\mathrm{Et}_{2} \mathrm{O}(200 \mathrm{~mL})$ to induce precipitation of a light-tan solid. The powder was collected on a $15 \mathrm{~mL}$ fine-porosity fritted funnel, washed with $\mathrm{Et}_{2} \mathrm{O}(30 \mathrm{~mL})$, yielding Compound $14(0.101 \mathrm{~g}, 0.118 \mathrm{mmol}, 63 \%) .{ }^{1} \mathrm{H} \mathrm{NMR}(d-\mathrm{MeCN}$,

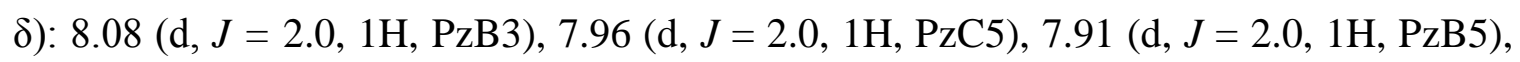
7.83 (d, $J=2.0,1 \mathrm{H}$, PzA5), 7.63 (d, $J=2.0,1 \mathrm{H}, \mathrm{PzC} 3), 7.26$ (d, $J=2.0,1 \mathrm{H}, \mathrm{PzA} 3), 6.43$ (m, 2H, PzB4 and PzC4), 6.29 (t, $J=2.0,1 \mathrm{H}, \mathrm{PzA} 4), 4.05$ (m, 1H, H2x), 4.03 (m, 1H, H5), $3.86(\mathrm{t}, J=11.4,1 \mathrm{H}, \mathrm{H} 2 \mathrm{y}), 3.47(\mathrm{~m}, 1 \mathrm{H}, \mathrm{H} 6), 3.30(\mathrm{~m}, 1 \mathrm{H}, \mathrm{H} 3 \mathrm{a}), 2.84(\mathrm{~m}, 1 \mathrm{H}, N-$ Ethyl $\mathrm{CH}_{2}$ ), 2.83 (m, 1H, H2x'), 2.73 (m, 1H, $N$-Ethyl $\mathrm{CH}_{2}$ ), 2.71 (broad, 1H, $\mathrm{NH}$ ), 2.51 (m, 1H, H2x'), 2.48 (m, 1H, H4x), 2.46 (m, 1H, H3x), 2.06 (d, $J=9.1,1 \mathrm{H}, \mathrm{H} 7), 1.78$ (m, 1H, H3y), 1.53 (m, 2H, H3'), 1.31 (buried, 1H, H4y), 1.31 (d, $J=9.4,9 H, \mathrm{PMe}_{3}$ ), 1.02 (t, $J=7.2,3 \mathrm{H}, N$-Ethyl $\left.\mathrm{CH}_{3}\right), 0.97$ (t, $J=7.4,3 \mathrm{H}, \mathrm{H} 4$ '). ${ }^{13} \mathrm{C}$ NMR $(d$-Acetone, $\delta$ ): 191.1 (C7a), 145.3 (PzB3), 144.9 (PzA3), 142.3 (PzC3), 138.7 (Pz5), 138.5 (Pz5), 138.3 (Pz5), 108.2 (Pz4), 107.9 (Pz4), 107.5 (Pz4), 75.3 (C6), 58.9 (C5), 54.2 (C2), 49.8 (C2'), 49.6 (C7), 42.7 ( $N$-Ethyl $\mathrm{CH}_{2}$ ), 42.1 (C3a), 40.1 (C4), 24.6 (C3'), 29.0 (C3), 14.2 (d, $J=29.0$, $\left.\mathrm{PMe}_{3}\right), 12.4$ ( $N$-Ethyl $\left.\mathrm{CH}_{3}\right), 11.7\left(\mathrm{C}^{\prime}\right) .{ }^{31} \mathrm{P}$ NMR $(d-\mathrm{MeCN}, \delta):-8.10\left(J_{\mathrm{wp}}=272\right) . \mathrm{IR}$ : $v_{\mathrm{BH}}=2511 \mathrm{~cm}^{-1}, v_{\mathrm{NO}}$ and $v_{\text {amidine }}=1604$ and $1574 \mathrm{~cm}^{-1}$. CV $(\mathrm{DMA}): \mathrm{E}_{\mathrm{p}, \mathrm{a}}=1.12 \mathrm{~V}$. HRMS: $\left[\mathrm{M}^{+}\right]=\left[\mathrm{C}_{25} \mathrm{H}_{42} \mathrm{~N}_{9} \mathrm{OBPW}^{+}\right]$obs'd (\%), calc'd (\%), ppm: 708.2818 (84), 708.2830 (83), -1.7; 709.2840 (85), 709.2855 (81), -2.1; 710.2833 (100), 710.2854 (100), -3.0; 711.2891 (44), 711.2894 (46), -0.4; 712.2857 (85), 712.2886 (83), -4.1.

Compound 15: A solution of HOTf in $\mathrm{MeCN}(15 \mathrm{~mL}, 0.125 \mathrm{M})$ was added to Compound 5 (0.751 g, $0.938 \mathrm{mmol})$, resulting in a light orange, homogenous solution. 
To this, a solution of imidazole $(0.318 \mathrm{~g}, 4.68 \mathrm{mmol})$ in $\mathrm{MeCN}(2 \mathrm{~mL})$ was added. The light yellow solution stirred for $45 \mathrm{~min}$. The solution was removed from the glovebox and was diluted with DCM (70 mL). This was treated with $2 \times 50 \mathrm{~mL}$ of $\mathrm{Na}_{2} \mathrm{CO}_{3}$ (saturated, aq). The aqueous layer was back extracted with DCM ( 2 x $40 \mathrm{~mL})$, and the combined organic layers were washed with deionized water $(50 \mathrm{~mL})$. This was then dried over anhydrous $\mathrm{MgSO}_{4}$ and concentrated in vacuo. The yellow oil was redissolved in minimal DCM and added dropwise to a stirring solution of $\mathrm{Et}_{2} \mathrm{O}(700 \mathrm{~mL})$ to induce precipitation of a light-tan solid. The powder was collected on a $30 \mathrm{~mL}$ fine-porosity fritted funnel, washed with $\mathrm{Et}_{2} \mathrm{O}(30 \mathrm{~mL})$, yielding Compound 15 (0.726 g, $\left.0.836 \mathrm{mmol}, 89 \%\right) .{ }^{1} \mathrm{H}$ NMR $(d-\mathrm{MeCN}, \delta): 8.11(\mathrm{~d}, J=2.0,1 \mathrm{H}, \mathrm{PzB} 3), 7.97(\mathrm{~d}, J=2.0,1 \mathrm{H}, \mathrm{PzC} 5), 7.94(\mathrm{~d}, J=$ 2.0, PzB5), 7.89 (t, $\left.J=1.0,1 \mathrm{H}, \mathrm{H} 2^{\prime}\right), 7.87$ (d, $\left.J=2.0,1 \mathrm{H}, \mathrm{PzA} 5\right), 7.61(\mathrm{~d}, J=2.0,1 \mathrm{H}$, PzC3), 7.43 (t, $\left.J=1.2,1 \mathrm{H}, \mathrm{H} 5^{\prime}\right), 7.28$ (d, $\left.J=2.0,1 \mathrm{H}, \mathrm{PzA} 3\right), 7.08$ (t, $\left.J=2.0,1 \mathrm{H}, \mathrm{H} 4{ }^{\prime}\right)$, $6.45(\mathrm{t}, J=2.0,1 \mathrm{H}, \mathrm{PzB} 4), 6.42(\mathrm{t}, J=2.0,1 \mathrm{H}, \mathrm{PzC} 4), 6.34(\mathrm{t}, J=2.0,1 \mathrm{H}, \mathrm{PzA} 4), 5.70$ (m, 1H, H5), 4.09 (m, 1H, H2x), 3.91 (m, 1H, H2y), 3.69 (m, 1H, H6), 3.51 (m, 1H, H3a), 2.81 (m, 1H, N-Ethyl $\mathrm{CH}_{2}$ ), 2.67 (m, 1H, N-Ethyl $\mathrm{CH}_{2}$ ), 2.46 (m, 1H, H4x), 2.28 $(\mathrm{d}, J=9.2,1 \mathrm{H}, \mathrm{H} 7), 1.83(\mathrm{~m}, 1 \mathrm{H}, \mathrm{H} 4 \mathrm{y}), 1.71(\mathrm{~m}, 1 \mathrm{H}, \mathrm{H} 3 \mathrm{y}), 1.06$ (t, $J=7.3,3 \mathrm{H}, N$-Ethyl $\left.\mathrm{CH}_{3}\right), 0.97\left(\mathrm{~d}, J=9.2,9 \mathrm{H}, \mathrm{PMe}_{3}\right) .{ }^{13} \mathrm{C} \mathrm{NMR}(d-\mathrm{MeCN}, \delta): 188.4(\mathrm{C} 7 \mathrm{a}), 145.3(\mathrm{PzB} 3)$, 145.2 (PzA3), 144.7 (PzC3), 139.0 (PzC5), 138.9 (PzB5), 138.8 (PzA5), 137.3 (C2’), 130.1 (C4'), 118.8 (C5’), 108.6 (PzB4), 108.2 (PzC4), 107.8 (PzA4), 69.6 (d, J = 13.8, C6), $58.1(\mathrm{~d}, J=2.5, \mathrm{C} 5), 54.4$ (C2), 49.7 (C7), 43.7 (C4), 43.0 ( $N$-Ethyl $\mathrm{CH}_{2}$ ), 41.6 (C3a), $13.7\left(\mathrm{~d}, J=31.0, \mathrm{PMe}_{3}\right), 11.8\left(N\right.$-Ethyl $\left.\mathrm{CH}_{3}\right) .{ }^{31} \mathrm{P} \mathrm{NMR}(d-\mathrm{MeCN}, \delta):-8.90\left(J_{\mathrm{wp}}=\right.$ 278). IR: $v_{\mathrm{BH}}=2515 \mathrm{~cm}^{-1}, v_{\mathrm{NO}}$ and $v_{\text {iminium }}=1608$ and $1579 \mathrm{~cm}^{-1} . \mathrm{CV}(\mathrm{DMA}): \mathrm{E}_{\mathrm{p}, \mathrm{a}}=$ 1.43 V. HRMS: $\left[\mathrm{M}^{+}\right]=\left[\mathrm{C}_{25} \mathrm{H}_{37} \mathrm{~N}_{10} \mathrm{OBPW}^{+}\right]$obs'd (\%), calc'd (\%), ppm: 717.2458 (75), 
717.2469 (82), -1.6; 718.2490 (79), 718.2494 (81), -0.6; 719.2479 (100), 719.2494 (100), $-2.0 ; 720.2520(47), 720.2533(46),-1.7 ; 721.2520(88), 721.2526(83),-0.8$.

Compound 16: A solution of HOTf in $\mathrm{MeCN}(1 \mathrm{~mL}, 0.125 \mathrm{M})$ was added to Compound $5(0.050 \mathrm{~g}, 0.062 \mathrm{mmol})$, resulting in a light orange, homogenous solution. To this, $1 H$ 1,2,3-triazole $(0.767 \mathrm{~g}, 0.581 \mathrm{mmol})$ was added. The light yellow solution stirred for 5 min. The solution was removed from the glovebox and was diluted with DCM $(20 \mathrm{~mL})$. This was treated with $2 \times 30 \mathrm{~mL}$ of $\mathrm{Na}_{2} \mathrm{CO}_{3}$ (saturated, aq). The aqueous layer was back extracted with DCM (2 x $20 \mathrm{~mL})$, and the combined organic layers were washed with deionized water $(50 \mathrm{~mL})$ and brine $(50 \mathrm{~mL})$. This was then dried over anhydrous $\mathrm{MgSO}_{4}$ and concentrated in vacuo. The yellow oil was redissolved in minimal DCM and added dropwise to a stirring solution of $\mathrm{Et}_{2} \mathrm{O}(50 \mathrm{~mL})$ to induce precipitation of a bright yellow solid. The powder was collected on a $15 \mathrm{~mL}$ fine-porosity fritted funnel, washed with $\mathrm{Et}_{2} \mathrm{O}(30 \mathrm{~mL})$, yielding Compound $16(0.043 \mathrm{~g}, 0.049 \mathrm{mmol}, 79 \%) .{ }^{1} \mathrm{H} \mathrm{NMR}(d-\mathrm{MeCN}$, $\delta): 8.15\left(\mathrm{~d}, J=0.9,1 \mathrm{H}, \mathrm{H} 5^{\prime}\right), 8.13(\mathrm{~d}, J=2.0,1 \mathrm{H}, \mathrm{PzB} 3), 7.97(\mathrm{~d}, J=2.0,1 \mathrm{H}, \mathrm{PzC} 5)$, $7.94(\mathrm{~d}, J=2.0,1 \mathrm{H}, \mathrm{PzB} 5), 7.86(\mathrm{~d}, J=2.0,1 \mathrm{H}, \mathrm{PzA} 5), 7.80\left(\mathrm{~d}, J=0.6,1 \mathrm{H}, \mathrm{H} 4^{\prime}\right), 7.57$ $(\mathrm{d}, J=2.0,1 \mathrm{H}, \mathrm{PzC} 3), 7.31(\mathrm{~d}, J=2.0,1 \mathrm{H}, \mathrm{PzA} 3), 6.46(\mathrm{t}, J=2.0,1 \mathrm{H}, \mathrm{PzB} 4), 6.40(\mathrm{t}, J$ $=2.0,1 \mathrm{H}, \mathrm{PzC} 4), 6.33(\mathrm{t}, J=2.0,1 \mathrm{H}, \mathrm{PzA} 4), 6.12(\mathrm{~m}, 1 \mathrm{H}, \mathrm{H} 5), 4.13(\mathrm{~m}, 1 \mathrm{H}, \mathrm{H} 2 \mathrm{x}), 3.94$ (m, 1H, H2y), 3.69 (m, 1H, H6), 3.54 (m, 1H, H3a), 2.87 (m, 1H, N-Ethyl $\mathrm{CH}_{2}$ ), 2.78 (m, 1H, $N$-Ethyl $\left.\mathrm{CH}_{2}\right), 2.58(\mathrm{~m}, 1 \mathrm{H}, \mathrm{H} 4 \mathrm{x}), 2.50$ (m, 1H, H3x), 2.26 (d, J = 9.0, 1H, H7), 1.96 (m, 1H, H4y), 1.84 (m, 1H, H3y), 1.07 (t, $J=7.4,3 \mathrm{H}, N$-Ethyl $\left.\mathrm{CH}_{3}\right), 1.01(\mathrm{~d}, J=9.1,9 \mathrm{H}$, $\left.\mathrm{PMe}_{3}\right) .{ }^{13} \mathrm{C}$ NMR $(d-\mathrm{MeCN}, \delta): 189.2$ (C7a), 145.2 (PzB3), 144.6 (PzA3), 142.3 (PzB3), 138.9 (Pz5), 138.8 (Pz5), 138.7 (PzA5), 135.0 (C4'), 123.7 (C5'), 108.5 (PzB4), 108.0 
(PzC4), 107.7 (PzA4), 69.6 (d, $J=14.0, \mathrm{C} 6), 61.6$ (C5), $54.4(\mathrm{C} 2), 49.1$ (C7), 43.0 (NEthyl $\left.\mathrm{CH}_{2}\right), 41.4$ (C3a), 40.7 (C4), 28.7 (C3), 13.4 (d, $\left.J=30.8, \mathrm{PMe}_{3}\right), 11.7$ (N-Ethyl $\left.\mathrm{CH}_{3}\right) .{ }^{31} \mathrm{P}$ NMR $(d-\mathrm{MeCN}, \delta):-9.00\left(J_{\mathrm{wp}}=276\right) . \mathrm{IR}: v_{\mathrm{BH}}=2515 \mathrm{~cm}^{-1}, v_{\mathrm{NO}}$ and $v_{\text {iminium }}=$ 1608 and $1581 \mathrm{~cm}^{-1}$. $\quad \mathrm{CV}$ (DMA): $\mathrm{E}_{\mathrm{p}, \mathrm{a}}=1.40$ V. HRMS: $\left[\mathrm{M}^{+}\right]=$ $\left[\mathrm{C}_{24} \mathrm{H}_{36} \mathrm{~N}_{11} \mathrm{OBPW}^{+}\right]$obs'd (\%), calc'd (\%), ppm: 718.2393 (78), 718.2422 (83), -4.0; 719.2428 (79), 719.2446 (81), -2.6; 720.2437 (100), 720.2446 (100), -1.2; 721.2459 (44), $721.2485(45),-3.5 ; 722.2453(80), 722.2478(83),-3.5$.

Compound 17: A solution of HOTf in MeCN (5 mL, $0.125 \mathrm{M}$ ) was added to Compound $5(0.250 \mathrm{~g}, 0.312 \mathrm{mmol})$, resulting in a light orange, homogenous solution. To this, piperidine $(0.133 \mathrm{~g}, 1.56 \mathrm{mmol})$ was added. The light yellow solution stirred for $5 \mathrm{~min}$. The solution was removed from the glovebox and was diluted with DCM (30 mL). This was treated with $2 \times 30 \mathrm{~mL}$ of $\mathrm{Na}_{2} \mathrm{CO}_{3}$ (saturated, aq). The aqueous layer was back extracted with DCM (2 x $20 \mathrm{~mL})$, and the combined organic layers were washed with deionized water $(15 \mathrm{~mL})$ and brine $(15 \mathrm{~mL})$. This was then dried over anhydrous $\mathrm{MgSO}_{4}$ and concentrated in vacuo. The yellow oil was redissolved in minimal DCM and added dropwise to a stirring solution of $\mathrm{Et}_{2} \mathrm{O}(200 \mathrm{~mL})$ to induce precipitation of a light-tan solid. The powder was collected on a $15 \mathrm{~mL}$ fine-porosity fritted funnel, washed with $\mathrm{Et}_{2} \mathrm{O}(50 \mathrm{~mL})$, yielding Compound 17 (0.184 g, $\left.0.208 \mathrm{mmol}, 67 \%\right) .{ }^{1} \mathrm{H}$ NMR $(d-\mathrm{MeCN}$, $\delta): 8.06(\mathrm{~d}, J=2.0,1 \mathrm{H}, \mathrm{PzB} 3), 7.96(\mathrm{~d}, J=2.0,1 \mathrm{H}, \mathrm{PzA} 5$ or PzB5), $7.90(\mathrm{~d}, J=2.0,1 \mathrm{H}$, PzA5 or PzB5), $7.84(\mathrm{~d}, J=2.0,1 \mathrm{H}, \mathrm{PzC} 5), 7.67(\mathrm{~d}, J=2.0,1 \mathrm{H}, \mathrm{PzA} 3), 7.21(\mathrm{~d}, J=2.0$, 1H, PzC3), 6.42 (m, 2H, PzA4 and PzB4), 6.30 (t, 1H, $J=2.0, \mathrm{PzC} 4), 4.30$ (dt, $J=11.0$, 3.2, 1H, H5), 4.03 (m, 1H, H2x), 3.86 (t, $J=11.0,1 \mathrm{H}, \mathrm{H} 2 \mathrm{y}), 3.54$ (m, 1H, H6), 3.33 (m, 
1H, H3a), 2.90 (broad m, 2H, H2' or H6'), 2.69 (m, 1H, N-Ethyl $\mathrm{CH}_{2}$ ), 2.53 (m, 1H, $N$ Ethyl $\mathrm{CH}_{2}$ ), 2.48 (m, 2H, H2' or H6'), 2.48 (buried, 1H, H3x), 2.10 (m, 1H, H4x), 2.03 (d, $J=8.5,1 \mathrm{H}, \mathrm{H} 7), 1.75$ (m, 1H, H3y), 1.64 (broad m, 4H, H3' and H5'), 1.51 (broad m, 2H, H4'), 1.47 (buried, 1H, H4y), 1.33 (d, $J=9.6,9 \mathrm{H}, \mathrm{PMe}_{3}$ ), 0.98 (t, $J=7.2,3 \mathrm{H}, N-$ Ethyl $\left.\mathrm{CH}_{3}\right) .{ }^{13} \mathrm{C}$ NMR (d-MeCN, $\left.\delta\right): 188.9$ (C7a), 145.4 (PzB3), 144.6 (PzC3), 142.4 (PzA3), 138.6 (Pz5), 108.2 (Pz4), 107.8 (Pz4), 107.6 (PzC4), 71.4 (d, J = 13, C6), 65.6 (d, $J=2.0, \mathrm{C} 5), 54.2$ (C2), 49.8 (C7), $42.6\left(N\right.$-Ethyl $\left.\mathrm{CH}_{2}\right), 42.1$ (C3a), 32.5 (C4), 30.8 (C2' and C6'), $28.4(\mathrm{C} 3), 27.4\left(\mathrm{C}^{\prime}{ }^{\prime}\right.$ and $\mathrm{C}^{\prime}$ '), 25.9 (C4'), $14.2\left(\mathrm{~d}, \mathrm{~J}=30, \mathrm{PMe}_{3}\right), 11.7(\mathrm{~N}-$ Ethyl $\left.\mathrm{CH}_{3}\right) .{ }^{31} \mathrm{P}$ NMR $(d-\mathrm{MeCN}, \delta):-7.96\left(J_{\mathrm{wp}}=284\right) . \mathrm{IR}: \mathrm{v}_{\mathrm{BH}}=2511 \mathrm{~cm}^{-1}, \mathrm{v}_{\mathrm{NO}}$ and $v_{\text {iminium }}=1604$ and $1577 \mathrm{~cm}^{-1} . \quad \mathrm{CV}$ (DMA): $\mathrm{E}_{\mathrm{p}, \mathrm{a}}=1.13 \mathrm{~V} . \quad$ HRMS: $\left[\mathrm{M}^{+}\right]=$ $\left[\mathrm{C}_{27} \mathrm{H}_{44} \mathrm{~N}_{9} \mathrm{OBPW}^{+}\right]$obs'd (\%), calc'd (\%), ppm: 734.2976 (100), 734.2987 (81), -1.4; 735.3003 (75), 735.3012 (81), -1.2; 736.2997 (87), 736.3011 (100), -1.9; 737.3071 (44), 737.3050 (47), 2.9; 738.3042 (87), 738.3043 (82), -0.2.

Compound 18: A solution of HOTf in $\mathrm{MeCN}(20 \mathrm{~mL}, 0.125 \mathrm{M})$ was added to Compound 5 (1.03 g, $1.28 \mathrm{mmol})$, resulting in a light orange, homogenous solution. To this, morpholine $(0.643 \mathrm{~g}, 7.38 \mathrm{mmol})$ was added. The light yellow solution stirred for 5 min. The solution was removed from the glovebox and was diluted with DCM $(50 \mathrm{~mL})$. This was treated with $2 \times 40 \mathrm{~mL}$ of $\mathrm{Na}_{2} \mathrm{CO}_{3}$ (saturated, aq). The aqueous layer was back extracted with DCM (2 x $30 \mathrm{~mL})$, and the combined organic layers were washed with deionized water $(30 \mathrm{~mL})$. This was then dried over anhydrous $\mathrm{MgSO}_{4}$ and concentrated in vacuo. The yellow oil was redissolved in minimal DCM and added dropwise to a stirring solution of $\mathrm{Et}_{2} \mathrm{O}(800 \mathrm{~mL})$ to induce precipitation of a light-tan solid. The powder 
was collected on a $30 \mathrm{~mL}$ fine-porosity fritted funnel, washed with $\mathrm{Et}_{2} \mathrm{O}(30 \mathrm{~mL})$, yielding Compound $18(0.950 \mathrm{~g}, 1.07 \mathrm{mmol}, 83 \%) .{ }^{1} \mathrm{H}$ NMR $(d-\mathrm{MeCN}, \delta): 8.06(\mathrm{~d}, J=$ 2.0, 1H, PzB3), 7.96 (d, $J=2.0,1 \mathrm{H}, \mathrm{PzC} 5), 7.90(\mathrm{~d}, J=2.0,1 \mathrm{H}, \mathrm{PzB} 5), 7.84(\mathrm{~d}, J=2.0$, 1H, PzA5), $7.68(\mathrm{~d}, J=2.0,1 \mathrm{H}, \mathrm{PzC} 3), 7.21(\mathrm{~d}, J=2.0,1 \mathrm{H}, \mathrm{PzA} 3), 6.43(\mathrm{t}, J=2.0,1 \mathrm{H}$, PzC4), $6.42(\mathrm{t}, J=2.0,1 \mathrm{H}, \mathrm{PzB} 4), 6.30(\mathrm{t}, J=2.0,1 \mathrm{H}, \mathrm{PzA} 4), 4.29$ (m, 1H, H5), 4.05 (m, 1H, H2x), 3.87 (m, 1H, H2y), 3.70 (m, 4H, H3' and H5'), 3.51 (m, 1H, H6), 3.35 (m, 1H, H3a), 2.94 (m, 2H, H2' or H6'), 2.70 (m, 1H, N-Ethyl $\left.\mathrm{CH}_{2}\right), 2.55$ (m, 1H, $N$-Ethyl $\mathrm{CH}_{2}$ ), 2.54 (m, 2H, H2' or H6'), 2.48 (m, 1H, H3x), 2.12 (dt, $J=12.3,5.1,1 \mathrm{H}, \mathrm{H} 4 \mathrm{x}), 2.03$ (d, J $=9.3,1 \mathrm{H}, \mathrm{H} 7), 1.77(\mathrm{~m}, 1 \mathrm{H}, \mathrm{H} 3 \mathrm{y}), 1.50(\mathrm{q}, J=12.3,1 \mathrm{H}, \mathrm{H} 4 \mathrm{y}), 1.33(\mathrm{~d}, J=9.4,9 \mathrm{H}$, $\left.\mathrm{PMe}_{3}\right), 0.99\left(\mathrm{t}, J=8.5,3 \mathrm{H}, N\right.$-Ethyl $\left.\mathrm{CH}_{3}\right) .{ }^{13} \mathrm{C}$ NMR $(d-\mathrm{MeCN}, \delta): 188.9(\mathrm{C} 7 \mathrm{a}), 145.3$ (PzB3), 144.6 (PzA3), 142.4 (PzC3), 138.6 (Pz5), 108.3 (Pz4), 107.9 (Pz4), 107.7 (Pz4), 70.4 (C6), 68.0 (C3' and C5'), 65.1 (C5), 54.2 (C2), 49.9 (C2' and C6'), 49.8 (C7), 42.6 (N-Ethyl $\mathrm{CH}_{2}$ ), 41.9 (C3a), 32.2 (C4), 28.4 (C3), 14.3 (d, $J=31, \mathrm{PMe}_{3}$ ), 11.7 (N-Ethyl $\left.\mathrm{CH}_{3}\right) .{ }^{31} \mathrm{P} \mathrm{NMR}(d-\mathrm{MeCN}, \delta):-7.76\left(J_{\mathrm{wp}}=286\right) . \mathrm{IR}: v_{\mathrm{BH}}=2511 \mathrm{~cm}^{-1}, v_{\mathrm{NO}}$ and $v_{\text {iminium }}=$ 1608 and $1577 \mathrm{~cm}^{-1}$. CV (DMA): $\mathrm{E}_{\mathrm{p}, \mathrm{a}}=0.76 \mathrm{~V}$. HRMS: $\left[\mathrm{M}^{+}\right]=\left[\mathrm{C}_{26} \mathrm{H}_{42} \mathrm{~N}_{9} \mathrm{O}_{2} \mathrm{BPW}^{+}\right]$ obs'd (\%), calc'd (\%), ppm: 736.2799 (82), 736.2779 (82), 2.7; 737.2819 (81), 737.2804 (81), 2.0; 738.2824 (100), 738.2804 (100), 2.7; 739.2861 (46), 739.2843 (46), 2.5; 740.2840 (83), $740.2836(83), 0.6$.

Compound 19: A solution of HOTf in MeCN (1 mL, $0.125 \mathrm{M})$ was added to Compound $5(0.050 \mathrm{~g}, 0.062 \mathrm{mmol})$, resulting in a light orange, homogenous solution. To this lithium dimethylmalonate $(0.061 \mathrm{~g}, 0.441 \mathrm{mmol})$ was added. The light yellow solution stirred for $5 \mathrm{~min}$. The solution was removed from the glovebox and was diluted with 
DCM $(15 \mathrm{~mL})$. This was treated with $2 \times 20 \mathrm{~mL}$ of $\mathrm{Na}_{2} \mathrm{CO}_{3}$ (saturated, aq). The aqueous layer was back extracted with DCM $(2 \times 20 \mathrm{~mL})$, and the combined organic layers were washed with deionized water $(20 \mathrm{~mL})$ and brine $(20 \mathrm{~mL})$. This was then dried over anhydrous $\mathrm{MgSO}_{4}$ and concentrated in vacuo. The yellow oil was redissolved in minimal DCM and added dropwise to a stirring solution of $\mathrm{Et}_{2} \mathrm{O}(100 \mathrm{~mL})$ to induce precipitation of a light-tan solid. The powder was collected on a $15 \mathrm{~mL}$ fine-porosity fritted funnel, washed with $\mathrm{Et}_{2} \mathrm{O}(30 \mathrm{~mL})$, yielding Compound 19 (0.032 g, $\left.0.034 \mathrm{mmol}, 56 \%\right) .{ }^{1} \mathrm{H}$ NMR $(d-\mathrm{MeCN}, \delta): 8.13(\mathrm{~d}, J=2.0,1 \mathrm{H}, \mathrm{PzB} 3), 7.97(\mathrm{~d}, J=2.0,1 \mathrm{H}, \mathrm{PzC} 5), 7.93(\mathrm{~d}, J=$ 2.0, 1H, PzB5), 7.81 (d, $J=2.0,1 \mathrm{H}, \mathrm{PzA} 5), 7.49$ (d, $J=2.0,1 \mathrm{H}, \mathrm{PzC} 3), 7.26(\mathrm{~d}, J=2.0$, 1H, PzA3), 6.45 (t, $J=2.0,1 \mathrm{H}, \mathrm{PzB} 4), 6.43 \mathrm{t}, J=2.0,1 \mathrm{H}, \mathrm{PzC} 4), 6.28(\mathrm{t}, J=2.0,1 \mathrm{H}$, PzA4), 4.07 (m, 1H, H2x), 3.87 (dd, $J=12.8,8.7,1 \mathrm{H}, \mathrm{H} 2 \mathrm{y}$ ), 3.77 (s, 3H, OMe), 3.75 (s, 3H, OMe), 3.46 (broad m, 2H, H5 and H2'), 3.38 (t, $J=10.2,1 \mathrm{H}, \mathrm{H} 6), 3.29$ (m, 1H, H3a), 3.08 (m, 2H, N-Ethyl $\left.\mathrm{CH}_{2}\right), 2.43(\mathrm{~m}, 1 \mathrm{H}, \mathrm{H} 3 \mathrm{x}), 2.38$ (m, 1H, H4x), 2.30 (d, J = 9.9, 1H, H7), 1.92 (m, 1H, H3y), 1.61 (dt, $J=14.9,2.5,1 \mathrm{H}, \mathrm{H} 4 \mathrm{y}), 1.26$ (d, $J=9.1,9 \mathrm{H}$, $\left.\mathrm{PMe}_{3}\right), 1.10\left(\mathrm{t}, J=7.3,3 \mathrm{H}, N\right.$-Ethyl $\left.\mathrm{CH}_{3}\right) .{ }^{13} \mathrm{C} \mathrm{NMR}(d-\mathrm{MeCN}, \delta): 191.8(\mathrm{C} 7 \mathrm{a}), 169.9$ (C1' or C3'), 169.8 (C1' or C3'), 145.4 (PzB3), 144.2 (PzA3), 142.2 (PzC3), 139.0 (Pz5), 138.7 (Pz5), 138.0 (PzA5), 108.3 (Pz4), 108.0 (Pz4), 107.5 (PzA4), 74.2 (d, J = 14.7, C6), 63.5 (C2'), 54.4 (C2), 53.3 (OMe), 53.2 (OMe), 49.4 (C7), 43.0 ( $N$-Ethyl $\left.\mathrm{CH}_{2}\right), 41.0$ (C3a), 40.3 (d, $J=2.0, \mathrm{C} 5), 30.9$ (C3), 30.4 (C4), 13.5 (d, $J=31.4, \mathrm{PMe}_{3}$ ), 11.9 (N-Ethyl $\left.\mathrm{CH}_{3}\right) .{ }^{31} \mathrm{P} \mathrm{NMR}\left(\mathrm{CH}_{2} \mathrm{Cl}_{2}, \delta\right):-7.95\left(J_{\mathrm{wp}}=280\right) . \mathrm{IR}: v_{\mathrm{BH}}=2515 \mathrm{~cm}^{-1}, v_{\text {ester }}=1732 \mathrm{~cm}^{-1}$, $v_{\text {NO }}$ and $v_{\text {iminium }}=1604$ and $1577 \mathrm{~cm}^{-1} . \mathrm{CV}(\mathrm{DMA}): \mathrm{E}_{\mathrm{p}, \mathrm{a}}=1.20 \mathrm{~V} . \mathrm{HRMS}:\left[\mathrm{M}^{+}\right]=$ $\left[\mathrm{C}_{27} \mathrm{H}_{41} \mathrm{~N}_{8} \mathrm{O}_{5} \mathrm{BPW}^{+}\right]$obs'd (\%), calc'd (\%), ppm: 781.2512 (78), 781.2518 (81), -0.7; 
782.2539 (78), 782.2543 (81), -0.5; 783.2527 (100), 783.2543 (100), -2.0; 784.2554 (44), $784.2582(47),-3.5 ; 785.2576(77), 785.2575(83), 0.2$.

Compound 20: A solution of HOTf in MeCN (1 mL, 0.125 M) was added to Compound $5(0.053 \mathrm{~g}, 0.066 \mathrm{mmol})$, resulting in a light orange, homogenous solution. To this methyl trimethylsilyl dimethylketene acetal (MTDA) (0.071 g, 0.407 mmol) was added. The light yellow solution stirred for $2 \mathrm{~min}$. The solution was removed from the glovebox and was diluted with DCM $(20 \mathrm{~mL})$. This was treated with $2 \times 20 \mathrm{~mL}$ of $\mathrm{Na}_{2} \mathrm{CO}_{3}$ (saturated, aq). The aqueous layer was back extracted with DCM (2 x $20 \mathrm{~mL})$, and the combined organic layers were washed with deionized water $(20 \mathrm{~mL})$ and brine $(20 \mathrm{~mL})$. This was then dried over anhydrous $\mathrm{MgSO}_{4}$ and concentrated in vacuo. The yellow oil was redissolved in minimal DCM and added dropwise to a stirring solution of $\mathrm{Et}_{2} \mathrm{O}$ (50 $\mathrm{mL}$ ) to induce precipitation of a yellow solid. The powder was collected on a $15 \mathrm{~mL}$ fineporosity fritted funnel, washed with $\mathrm{Et}_{2} \mathrm{O}(10 \mathrm{~mL})$, yielding Compound 20 (0.048 g, $0.053 \mathrm{mmol}, 81 \%) .{ }^{1} \mathrm{H}$ NMR $(d-\mathrm{MeCN}, \delta): 8.14(\mathrm{~d}, J=2.0,1 \mathrm{H}, \mathrm{PzB} 3), 7.98(\mathrm{~d}, J=2.0$, 1H, PzB5 or PzC5), $7.93(\mathrm{~d}, J=2.0,1 \mathrm{H}, \mathrm{PzB} 5$ or PzC5), $7.81(\mathrm{~d}, J=2.0,1 \mathrm{H}, \mathrm{PzA} 5)$, $7.65(\mathrm{~d}, J=2.0,1 \mathrm{H}, \mathrm{PzC} 3), 7.20(\mathrm{~d}, J=2.0,1 \mathrm{H}, \mathrm{PzA} 3), 6.44(\mathrm{t}, J=2.0,1 \mathrm{H}, \mathrm{Pz} 4), 6.44(\mathrm{t}$, $J=2.0,1 \mathrm{H}, \mathrm{Pz} 4), 6.27(\mathrm{t}, J=2.0,1 \mathrm{H}, \mathrm{PzA} 4), 4.03(\mathrm{td}, J=11.7,6.4,1 \mathrm{H}, \mathrm{H} 2 \mathrm{x}), 3.88(\mathrm{dd}$, $J=12.3,9.5,1 \mathrm{H}, \mathrm{H} 2 \mathrm{y}), 3.68\left(\mathrm{~s}, 3 \mathrm{H}\right.$, Acetyl $\left.\mathrm{CH}_{3}\right), 3.42(\mathrm{t}, J=11.3,1 \mathrm{H}, \mathrm{H} 6), 3.32(\mathrm{q}, J=$ 11.3, 1H, H3a), 3.22 (d, $J=8.6,1 \mathrm{H}, \mathrm{H} 5), 3.06\left(\mathrm{~m}, 2 \mathrm{H}, N\right.$-Ethyl $\left.\mathrm{CH}_{2}\right), 2.45(\mathrm{~m}, 1 \mathrm{H}, \mathrm{H} 4 \mathrm{x})$, 2.44 (m, 1H, H3x), 2.41 (d, $J=11.1,1 \mathrm{H}, \mathrm{H} 7), 1.88$ (m, 1H, H3y), 1.42 (m, 1H, H4y), $1.34\left(\mathrm{~s}, 3 \mathrm{H}, \mathrm{CH}_{3}\right), 1.21\left(\mathrm{~d}, J=9.3,9 \mathrm{H}, \mathrm{PMe}_{3}\right), 1.19\left(\mathrm{~s}, 3 \mathrm{H}, \mathrm{CH}_{3}\right), 1.07(\mathrm{t}, J=7.4,3 \mathrm{H}, N-$ Ethyl $\left.\mathrm{CH}_{3}\right) .{ }^{13} \mathrm{C}$ NMR $(d-\mathrm{MeCN}, \delta): 192.6$ (H7a), 179.1 (carbonyl) 145.1 (d, $J=2.0$, 
PzB3), 144.0 (PzA3), 142.5 (PzC3), 138.9 (Pz5), 138.7 (Pz5), 138.2 (PzA5), 108.3 (Pz4), 107.9 (Pz4), 107.4 (PzA4) , 71.5 (d, $J=13.9, \mathrm{H} 6), 54.2$ (C2), 52.7 (Acetyl $\left.\mathrm{CH}_{3}\right), 46.4$ (C5), 43.0 (N-Ethyl $\left.\mathrm{CH}_{2}\right), 40.2$ (C3a), 30.9 (C3), $28.9(\mathrm{C} 4), 25.3\left(\mathrm{CH}_{3}\right), 23.3\left(\mathrm{CH}_{3}\right), 13.1$ $\left(\mathrm{d}, J=31.0, \mathrm{PMe}_{3}\right), 12.0\left(N\right.$-Ethyl $\left.\mathrm{CH}_{3}\right) .{ }^{31} \mathrm{P}$ NMR $(d-\mathrm{MeCN}, \delta):-9.18\left(J_{\text {wp }}=285\right) . \mathrm{IR}$ : $v_{\mathrm{BH}}=2511 \mathrm{~cm}^{-1}, v_{\mathrm{NO}}$ and $v_{\text {iminium }}=1601$ and $1581 \mathrm{~cm}^{-1} . \mathrm{CV}(\mathrm{DMA}): \mathrm{E}_{\mathrm{p}, \mathrm{a}}=1.15 \mathrm{~V}$ HRMS: $\left[\mathrm{M}^{+}\right]=\left[\mathrm{C}_{27} \mathrm{H}_{43} \mathrm{~N}_{8} \mathrm{O}_{3} \mathrm{BPW}^{+}\right]$obs'd (\%), calc'd (\%), ppm: $751.2759(81), 751.2776$ (81), -2.3; 752.2776 (83), 752.2801 (81), -3.3; 753.2786 (100), 753.2801 (100), -2.0; 754.2821 (48), 754.2840 (47), -2.5; $755.2820(83), 755.2833$ (83), -1.7.

Compound 21: Compound 1 (0.300 g, $0.526 \mathrm{mmol})$ was combined with DiPAT (0.135 $\mathrm{g}, .537 \mathrm{mmol})$. To this heterogeneous mixture was added a hexanes $(5 \mathrm{~mL})$ solution of $N, N$-dimethyl-para-toluidine $(0.757 \mathrm{~g}, 4.69 \mathrm{mmol})$. The pale-brown and heterogeneous reaction mixture was stirred for $72 \mathrm{~h}$. The reaction mixture was filtered through a $15 \mathrm{~mL}$ medium-porosity fritted funnel, yielding a dark-yellow solid. The solid was removed from the frit and triturated with DME $(1 \mathrm{~mL})$ for $5 \mathrm{~min}$. This bright-yellow solid was collected on a $15 \mathrm{~mL}$ medium-porosity fritted funnel, washed with DME (1 x $1 \mathrm{~mL})$, ether ( 2 x $5 \mathrm{~mL})$ and hexanes ( 2 x $10 \mathrm{~mL})$, yielding Compound 21 (0.267 g, $0.338 \mathrm{mmol}$, 65\%). ${ }^{1} \mathrm{H}$ NMR ( $d$-MeCN, $\left.\delta\right): 8.08$ (d, $\left.J=2.0,1 \mathrm{H}, \mathrm{PzB} 3\right), 7.99(\mathrm{~d}, J=2.0,1 \mathrm{H}, \mathrm{PzC} 5)$, $7.92(\mathrm{~d}, J=2.0,1 \mathrm{H}, \mathrm{Pz} 5), 7.90(\mathrm{~d}, J=2.0,1 \mathrm{H}, \mathrm{Pz} 5), 7.67$ (d, $J=2.0,1 \mathrm{H}, \mathrm{PzC} 3), 7.07$ (d, $J=2.0,1 \mathrm{H}, \mathrm{PzA} 3), 6.47(\mathrm{t}, J=2.0,1 \mathrm{H}, \mathrm{PzC} 4), 6.41(\mathrm{t}, J=2.0,1 \mathrm{H}, \mathrm{PzB} 4), 6.36(\mathrm{t}, J=$ 2.0, 1H, PzA4), 4.70 (broad s, 1H, H5), 3.88 (m, 1H, H3), 3.52 (d, J = 23.0, 1H, H6x), 3.36 (s, 3H, N-Me A), 3.23 (d, $J=23.0,1 \mathrm{H}, \mathrm{H} 6 \mathrm{y}), 2.34$ (d, $J=8.3,1 \mathrm{H}, \mathrm{H} 2), 2.10$ (s, 2H, N-Me B), 2.90 (s, 3H, 4-Me), $1.22\left(\mathrm{~d}, J=8.9,9 \mathrm{H}, \mathrm{PMe}_{3}\right) .{ }^{13} \mathrm{C} \mathrm{NMR}(d-\mathrm{MeCN}, \delta): 181.3$ 
(C1), 145.5 (Pz3), 142.5 (Pz3), 142.3 (Pz3), 139.3 (Pz5), 139.0 (Pz5), 138.8 (Pz5), 136.5

(C4), 109.6 (C5), 108.4 (Pz4), 108.3 (Pz4), 107.6 (Pz4), 66.9 (d, J = 13.0, C3), 58.4 (C2), 42.3 (N-Me A), 40.7 (N-Me B), 31.3 (C6), 24.5 (4-Me), 14.2 (d, $\left.J=31.0, \mathrm{PMe}_{3}\right) .{ }^{31} \mathrm{P}$ $\operatorname{NMR}(d-\mathrm{MeCN}, \delta):-10.2\left(J_{\mathrm{wp}}=288\right) . \mathrm{IR}: v_{\mathrm{BH}}=2507 \mathrm{~cm}^{-1}, v_{\mathrm{NO}}$ and $v_{\text {iminium }}=1577$ and $1604 \mathrm{~cm}^{-1} . \mathrm{CV}(\mathrm{DMA}): \mathrm{E}_{\mathrm{p}, \mathrm{a}}=0.98 \mathrm{~V}$

Compound 22: Compound 1 (0.496 g, 0.85 mmol) was combined with DiPAT (0.227 g, $0.90 \mathrm{mmol})$. To this heterogeneous mixture was added a DME (1.13 g) solution of $N, N-$ dimethyl-m-toluidine $(0.820 \mathrm{~g}, 6.06 \mathrm{mmol})$. This dark-yellow and homogeneous solution was stirred overnight $(\sim 14 \mathrm{~h})$, forming a precipitate. The reaction mixture was filtered through a $15 \mathrm{~mL}$ fine-porosity fritted funnel. The collected yellow solid was washed with DME $(2 \times 2 \mathrm{~mL})$, and $\mathrm{Et}_{2} \mathrm{O}(2 \times 20 \mathrm{~mL})$, yielding a mixture of $(\mathbf{2 2 A}+\mathbf{2 2 B})(0.330$ g, 0.42 mmol, 48\%). Major Species (Compound 22B) ${ }^{1} \mathrm{H}$ NMR $\left(\mathrm{CD}_{3} \mathrm{CN}, \delta\right): 7.98(\mathrm{~d}, J=$ 2.0, 1H, PzB3), 7.94 (d, $J=2.0,1 \mathrm{H}, \mathrm{PzC} 5), 7.91(\mathrm{~d}, J=2.0,1 \mathrm{H}, \mathrm{PzB} 5), 7.88(\mathrm{~d}, J=2.0$, 1H, PzA5), 7.55 (d, $J=2.0,1 \mathrm{H}, \mathrm{PzC} 3), 7.36$ (d, $J=2.0,1 \mathrm{H}, \mathrm{PzA} 3), 6.41$ (broad, 1H, H3), 6.40 (t, $J=2.0,1 \mathrm{H}, \mathrm{PzB} 4), 6.39$ (t, $J=2.0,1 \mathrm{H}, \mathrm{PzC} 4), 6.35$ (t, $J=2.0,1 \mathrm{H}, \mathrm{PzA} 4)$, $4.32(\mathrm{dd}, J=22.3,8.7,1 \mathrm{H}, \mathrm{H} 4 \mathrm{x}), 3.58(\mathrm{~m}, 1 \mathrm{H}, \mathrm{H} 3), 3.49$ (d, $J=23.0,1 \mathrm{H}, \mathrm{H} 4 \mathrm{y}), 3.43$ (s, 3H, $N-\mathrm{CH}_{3} \mathrm{~A}$ ), 2.28 (s, 3H, $N-\mathrm{CH}_{3} \mathrm{~B}$ ), 2.14 (s, 3H, 5-Me), 1.94 (buried, 1H, H2), 1.23 (d, $\left.J=9.1,9 \mathrm{H}, \mathrm{PMe}_{3}\right) .{ }^{13} \mathrm{C} \mathrm{NMR}\left(\mathrm{CD}_{3} \mathrm{CN}, \delta\right): 178.1(\mathrm{C} 1), 144.9$ (PzB3), $143.8(\mathrm{PzA} 3)$, 141.8 (PzC3), 138.6 (Pz5), 138.5 (Pz5), 138.4 (Pz5), 121.1 (C5), 113.4 (C6), 108.1 (Pz4), 108.0 (Pz4), 107.8 (Pz4), $57.8(\mathrm{~d}, J=12.7, \mathrm{C} 3), 53.1(\mathrm{C} 3), 42.2\left(N-\mathrm{CH}_{3} \mathrm{~A}\right), 41.1\left(N-\mathrm{CH}_{3}\right.$ B), 38.2 (C4), 24.4 (C5-Me), 12.9 (d, $J=30.4, \mathrm{PMe}_{3}$ ). Minor Species (Compound 22A) ${ }^{1} \mathrm{H}$ NMR $\left(\mathrm{CD}_{3} \mathrm{CN}, \delta\right): 8.04(\mathrm{~d}, J=2.0,1 \mathrm{H}, \mathrm{PzB} 3), 7.96(\mathrm{~d}, J=2.0,1 \mathrm{H}, \mathrm{PzC} 5), 7.88(\mathrm{~m}$, 
2H, PzA5 \& PzB5), $7.69(\mathrm{~d}, J=2.0,1 \mathrm{H}, \mathrm{PzC} 3), 7.21$ (d, $J=2.0,1 \mathrm{H}, \mathrm{PzA} 3), 6.44$ (t, $J=$ 2.0, 1H, PzC4), 6.40 (t, $J=2.0,1 \mathrm{H}, \mathrm{PzB} 4), 6.39$ (t, $J=2.0,1 \mathrm{H}, \mathrm{PzA} 4), 6.27$ (broad, 1H, H4), 3.81 (m, 1H, H3), 3.46 (s, 3H, $N-\mathrm{CH}_{3} \mathrm{~A}$ ), 3.44 (buried, 1H, H6x), 3.28 (d, J= 23.0, 1H, H6y), 2.40 (dd, $J=8.0,1.9,1 \mathrm{H}, \mathrm{H} 2), 2.39$ (s, 3H, $\left.N-\mathrm{CH}_{3} \mathrm{~B}\right), 2.14$ (s, 3H, 5-Me), 1.27 $\left(\mathrm{d}, J=8.8,9 \mathrm{H}, \mathrm{PMe}_{3}\right) .{ }^{13} \mathrm{C} \mathrm{NMR}\left(\mathrm{CD}_{3} \mathrm{CN}, \delta\right): 184.6(\mathrm{C} 7 \mathrm{a}), 145.8(\mathrm{PzB} 3), 142.6(\mathrm{PzC} 3)$, 142.3 (PzA3), 138.9 (Pz5), 138.7 (Pz5), 138.6 (Pz5), 124.4 (C4), 122.9 (C5), 65.4 (d, J = 12.4, C3), 54.9 (C2), $42.7\left(N-\mathrm{CH}_{3} \mathrm{~A}\right), 41.4\left(N-\mathrm{CH}_{3} \mathrm{~B}\right), 35.8$ (C6), 20.4 (5-Me), 13.6 (d, J $\left.=31.0, \mathrm{PMe}_{3}\right)$.

Compound 23: Compound 1 (1.00 g, $1.72 \mathrm{mmol})$ was combined with DiPAT (0.519 g, $2.07 \mathrm{mmol})$. To this heterogeneous mixture was added a hexanes $(15 \mathrm{~mL})$ solution of 1,6-dimethyl-1,2,3,4-tetrahydroquinoline (2.22 $\mathrm{g}, 13.76 \mathrm{mmol})$. The pale-brown and heterogeneous reaction mixture was stirred for $72 \mathrm{~h}$. The reaction mixture was filtered through a $30 \mathrm{~mL}$ medium-porosity fritted funnel, yielding a dark-yellow solid. The solid was removed from the frit and triturated with DME $(0.8 \mathrm{~mL})$ for $5 \mathrm{~min}$. This brightyellow solid was collected on a $15 \mathrm{~mL}$ medium-porosity fritted funnel, washed with DME $(1 \times 1 \mathrm{~mL})$, ether $(2 \times 5 \mathrm{~mL})$ and hexanes $(2 \times 10 \mathrm{~mL})$, yielding a mixture of Compounds (23A + 4B) $(0.532 \mathrm{~g}, 0.653 \mathrm{mmol}, 40 \%) .{ }^{1} \mathrm{H}$ NMR $(d-\mathrm{MeCN}, \delta): 8.06(\mathrm{~d}, J$ $=2.0,1 \mathrm{H}, \mathrm{PzB} 3), 7.99(\mathrm{~d}, J=2.0,1 \mathrm{H}, \mathrm{PzC} 5), 7.93(\mathrm{~d}, J=2.0,1 \mathrm{H}, \mathrm{PzB} 5), 7.89(\mathrm{~d}, J=$ 2.0, 1H, PzA5), 7.69 (d, $J=2.0,1 \mathrm{H}, \mathrm{PzC} 3), 7.22(\mathrm{~d}, J=2.0,1 \mathrm{H}, \mathrm{PzA} 3), 6.46(\mathrm{t}, J=2.0$, $1 \mathrm{H}, \mathrm{PzC} 4), 6.42$ (t, $J=2.0,1 \mathrm{H}, \mathrm{PzB} 4), 6.36(\mathrm{t}, J=2.0,1 \mathrm{H}, \mathrm{PzA} 3), 4.55$ (bs, 1H, H5), 3.92 (m, 1H, H7), 3.69 (m, 2H, H2), 3.28 (bs, 1H, H4a), 2.27 (d, J = 8.2, 1H, H8), 2.17 (m, 1H, H3x), 2.14 (s, 3H, N-Me), 2.10 (m, 1H, H4x), 2.06 (m, 1H, H3y), 1.92 (bs, 3H, 
6-Me), $1.60(\mathrm{~m}, 1 \mathrm{H}, \mathrm{H} 4 \mathrm{y}), 1.24\left(\mathrm{~d}, J=9.1,9 \mathrm{H}, \mathrm{PMe}_{3}\right) .{ }^{13} \mathrm{C} \mathrm{NMR}(d-\mathrm{MeCN}, \delta): 185.9$ (C7a), 145.5 (PzB3), 142.3 (PzC3), 141.4 (PzA3), 139.0 (Pz5), 139.0 (Pz5), 138.8 (Pz5), 137.8 (C6), 114.4 (C5), 108.4 (Pz4), 108.2 (Pz4), 107.8 (Pz4), 69.0 (d, J = 11.8, C7), 57.5 (C8), 55.0 (C2), 40.7 (N-Me), 38.3 (C4a), 26.5 (C4), 24.5 (6-Me), 22.7 (C3), 14.3 $\left(\mathrm{d}, J=31.0, \mathrm{PMe}_{3}\right) .{ }^{31} \mathrm{P} \mathrm{NMR}(d-\mathrm{MeCN}, \delta):-10.0\left(J_{\mathrm{wp}}=286\right) . \mathrm{IR}: v_{\mathrm{BH}}=2507 \mathrm{~cm}^{-1}, v_{\mathrm{NO}}$ and $v_{\text {iminium }}=1577$ and $1601 \mathrm{~cm}^{-1} . \mathrm{CV}(\mathrm{DMA}): \mathrm{E}_{\mathrm{p}, \mathrm{a}}=1.03 \mathrm{~V}$.

Compound 24: A solution of HOTf in $\mathrm{MeCN}(0.5 \mathrm{~mL}, 0.250 \mathrm{M})$ was added to Compound 21 (0.049 g, $0.062 \mathrm{mmol})$, resulting in a light orange, homogenous solution. After $30 \mathrm{~min}$, 2-methylfuran $(0.062 \mathrm{~g}, 0.755 \mathrm{mmol})$ was added. The light yellow solution stirred for $2 \mathrm{~h}$. The solution was removed from the glovebox and was diluted with DCM (20 mL). This was treated with $2 \times 20 \mathrm{~mL}$ of $\mathrm{Na}_{2} \mathrm{CO}_{3}$ (saturated, aq). The aqueous layer was back extracted with DCM (2 x $10 \mathrm{~mL})$, and the combined organic layers were washed with deionized water $(30 \mathrm{~mL})$ and brine $(30 \mathrm{~mL})$. This was then dried over anhydrous $\mathrm{MgSO}_{4}$ and concentrated in vacuo. The yellow oil was redissolved in minimal DCM and added dropwise to a stirring solution of $\mathrm{Et}_{2} \mathrm{O}(50 \mathrm{~mL})$ to induce precipitation of a light-tan solid. The powder was collected on a $15 \mathrm{~mL}$ fine-porosity fritted funnel, washed with $\mathrm{Et}_{2} \mathrm{O}(15 \mathrm{~mL})$, yielding Compound 24 (0.038 g, $\left.0.043 \mathrm{mmol}, 69 \%\right) .{ }^{1} \mathrm{H}$ NMR ( $d$-MeCN, $\delta): 8.09(\mathrm{~d}, J=2.0,1 \mathrm{H}, \mathrm{PzB} 3), 8.00(\mathrm{~d}, J=2.0,1 \mathrm{H}, \mathrm{PzC} 5), 7.96(\mathrm{~d}, J=$ 2.0, 1H, PzB5), $7.90(\mathrm{~d}, J=2.0,1 \mathrm{H}, \mathrm{PzA} 5), 7.63(\mathrm{~d}, J=2.0,1 \mathrm{H}, \mathrm{PzC} 3), 7.18(\mathrm{t}, J=2.0$, 1H, PzA3), 6.46 (m, 2H, PzB4 \& PzC4), 6.37 (t, $J=2.0,1 \mathrm{H}, \mathrm{PzA} 4), 6.18$ (d, $J=2.9,1 \mathrm{H}$, H3'), 5.93 (broad s, 1H, H4'), 3.89 (dd, $J=13.5,9.2,1 \mathrm{H}, \mathrm{H} 3$ ), 3.27 (s, 3H, N-Me A), $2.67(\mathrm{dd}, J=18.8,5.9,1 \mathrm{H}, \mathrm{H} 6 \mathrm{x}), 2.44$ (m, 1H, H5x), 2.39 (m, 1H, H6y), 2.29 (d, $J=9.2$, 
1H, H2), 2.26 (s, 3H, 5'-Me), 1.94 (buried, 1H, H5y), 1.91 (s, 3H, N-Me B), 1.61 (s, 3H, 4-Me), $1.13\left(\mathrm{~d}, J=8.8,9 \mathrm{H}, \mathrm{PMe}_{3}\right) .{ }^{13} \mathrm{C} \mathrm{NMR}(d-\mathrm{MeCN}, \delta): 188.0(\mathrm{C} 1), 165.1$ (C2'), 151.3 (C5'), 144.6 (PzA3), 144.0 (PzB3), 142.0 (PzC3), 139.1 (Pz5), 138.9 (Pz5), 138.8 (Pz5), 108.7 (Pz4), 108.5 (Pz4), 108.0 (PzA4), 106.8 (C4'), 106.1 (C3’), 73.4 (d, J = 13.2, C3), 59.5 (C2), 42.1 (N-Me A), 41.2 (C4), 40.2 (N-Me B), 31.0 (4-Me), 30.9 (C5), $27.3(\mathrm{C} 6), 14.0\left(\mathrm{~d}, J=30.8, \mathrm{PMe}_{3}\right), 13.6\left(5^{\prime}-\mathrm{Me}\right) .{ }^{31} \mathrm{P} \mathrm{NMR}\left(d-\mathrm{CDCl}_{3}, \delta\right):-10.5\left(J_{\mathrm{wp}}=\right.$ 280).

Compound 25: A solution of HOTf in $\mathrm{MeCN}(5 \mathrm{~mL}, 0.250 \mathrm{M})$ was added to Compound $23(0.200 \mathrm{~g}, 0.245 \mathrm{mmol})$, resulting in a light orange, homogenous solution. After $1 \mathrm{~h}, 2-$ methylfuran (0.376 g, $4.57 \mathrm{mmol})$ was added. The light yellow solution stirred for $3 \mathrm{~h}$. The solution was removed from the glovebox and was diluted with DCM (20 mL). This was treated with $2 \times 20 \mathrm{~mL}$ of $\mathrm{Na}_{2} \mathrm{CO}_{3}$ (saturated, aq). The aqueous layer was back extracted with DCM (2 x $10 \mathrm{~mL})$, and the combined organic layers were washed with deionized water $(30 \mathrm{~mL})$ and brine $(30 \mathrm{~mL})$. This was then dried over anhydrous $\mathrm{MgSO}_{4}$ and concentrated in vacuo. The yellow oil was redissolved in minimal DCM and added dropwise to a stirring solution of $\mathrm{Et}_{2} \mathrm{O}(200 \mathrm{~mL})$ to induce precipitation of a light-tan solid. The powder was collected on a $15 \mathrm{~mL}$ fine-porosity fritted funnel, washed with $\mathrm{Et}_{2} \mathrm{O}(15 \mathrm{~mL})$, yielding Compound 25 (0.184 g, $\left.0.205 \mathrm{mmol}, 83 \%\right) .{ }^{1} \mathrm{H}$ NMR $(d-\mathrm{MeCN}$, $\delta): 8.09(\mathrm{~d}, J=2.0,1 \mathrm{H}, \mathrm{PzB} 3), 7.99(\mathrm{~d}, J=2.0,1 \mathrm{H}, \mathrm{Pz5}), 7.93(\mathrm{~d}, J=2.0,1 \mathrm{H}, \mathrm{Pz} 5), 7.92$ $(\mathrm{d}, J=2.0,1 \mathrm{H}, \mathrm{Pz} 5), 7.58(\mathrm{~d}, J=2.0,1 \mathrm{H}, \mathrm{PzC} 3), 7.17(\mathrm{~d}, J=2.0,1 \mathrm{H}, \mathrm{PzA} 3), 6.44(\mathrm{~m}$, 2H, PzB4 \& PzC4), 6.40 (t, $J=2.0,1 \mathrm{H}, \mathrm{PzA} 4), 6.17$ (d, J = 3.1, 1H, H3'), 5.96 (m, 1H, H4'), 3.82 (dd, $J=13.4,9.8,1 \mathrm{H}, \mathrm{H} 7), 3.62$ (m, 2H, H2), 2.85 (m, 1H, H4a), 2.30 (buried, 
1H, H8), 2.29 (s, 3H, 5'-Me), 2.09 (s, 3H, N-Me), 2.03 (buried, 1H, H5x), 1.99 (buried, 1H, H3x), 1.98 (m, 1H, H5y), 1.97 (m, 1H, H4x), 1.89 (m, 1H, H3y), 1.66 (s, 3H, 6-Me), $1.24(\mathrm{~m}, 1 \mathrm{H}, \mathrm{H} 4 \mathrm{y}), 1.08\left(\mathrm{~d}, J=8.9,9 \mathrm{H}, \mathrm{PMe}_{3}\right) .{ }^{13} \mathrm{C} \mathrm{NMR}(d-\mathrm{MeCN}, \delta): 187.6(\mathrm{C} 8 \mathrm{a})$ 165.3 (C2'), 151.8 (C5'), 144.8 (PzB3), 143.7 (PzA3), 142.1 (PzC3), 139.4 (Pz5), 139.2 (Pz5), 139.1 (Pz5), 108.8 (Pz4), 108.6 (Pz4), 108.3 (Pz4), 106.9 (C4'), 106.1 (C3'), 75.9 (d, $J=13.4, \mathrm{C} 7), 57.7$ (C8), 54.6 (C2), 47.8 (C5), 41.6 (C6), 40.5 (N-Me), 34.0 (C4a), 29.8 (6-Me), 26.8 (C4), 22.5 (C3), 14.8 (d, $\left.J=31.0, \mathrm{PMe}_{3}\right), 13.8$ (5'-Me). ${ }^{31} \mathrm{P}$ NMR $(d-$ $\left.\mathrm{CDCl}_{3}, \delta\right):-10.3\left(J_{\mathrm{wp}}=282\right)$. 


\subsection{References}

(1) Mazurais, M.; Lescot, C.; Retailleau, P.; Dauban, P. European Journal of Organic Chemistry 2014, 2014, 66.

(2) Diethelm, S.; Schindler, C. S.; Carreira, E. M. Chemistry - A European Journal 2014, 20, 6071.

(3) Hanessian, S.; Margarita, R.; Hall, A.; Johnstone, S.; Tremblay, M.; Parlanti, L. Journal of the American Chemical Society 2002, 124, 13342.

(4) Hanessian, S.; Tremblay, M.; Petersen, J. F. W. Journal of the American Chemical Society 2004, 126, 6064.

(5) Petronijevic, F. R.; Wipf, P. Journal of the American Chemical Society 2011, 133, 7704.

(6) Wipf, P.; Kim, Y.; Goldstein, D. M. Journal of the American Chemical Society 1995, 117, 11106.

(7) Wipf, P.; Rector, S. R.; Takahashi, H. Journal of the American Chemical Society 2002, 124, 14848.

(8) Wipf, P.; Spencer, S. R. Journal of the American Chemical Society 2005, $127,225$.

(9) Hoshina, Y.; Doi, T.; Takahashi, T. Tetrahedron 2007, 63, 12740.

(10) Barton, D. H. R.; Guilhem, J.; Hervé, Y.; Potier, P.; Thierry, J. Tetrahedron Letters 1987, 28, 1413.

(11) Murakami, M.; Ishida, K.; Okino, T.; Okita, Y.; Matsuda, H.; Yamaguchi, K. Tetrahedron Letters 1995, 36, 2785. 
(12) Bonjoch, J.; Catena, J.; Isábal, E.; López-Canet, M.; Valls, N.

Tetrahedron: Asymmetry 1996, 7, 1899.

(13) Sayago, F. J.; Calaza, M. I.; Jiménez, A. I.; Cativiela, C. Tetrahedron: Asymmetry 2008, 19, 2763.

(14) Jin, Z. Natural Product Reports 2011, 28, 1126.

(15) Gärtner, M.; Qu, J.; Helmchen, G. The Journal of Organic Chemistry 2012, 77, 1186 .

(16) Daly, J. W.; Nishizawa, Y.; Padgett, W. L.; Tokuyama, T.; McCloskey, P. J.; Waykole, L.; Schultz, A. G.; Aronstam, R. S. Neurochemical Research, 16, 1207.

(17) Barbe, G.; Charette, A. B. Journal of the American Chemical Society 2008, 130, 13873.

(18) Davis, R. A.; Carroll, A. R.; Quinn, R. J. Journal of Natural Products 2002, 65, 454.

(19) Chen, C. Y.; Hart, D. J. The Journal of Organic Chemistry 1990, 55, 6236.

(20) Ginn, J. D.; Padwa, A. Organic Letters 2002, 4, 1515.

(21) Zeng, Y.; Aubé, J. Journal of the American Chemical Society 2005, 127, 15712.

(22) Baeckvall, J. E.; Andersson, P. G. Journal of the American Chemical Society 1990, 112, 3683.

(23) Roche, S. P.; Porco, J. A. Angewandte Chemie International Edition 2011, $50,4068$.

(24) Welch, K. D.; Harrison, D. P.; Lis, E. C.; Liu, W.; Salomon, R. J.; Harman, W. D.; Myers, W. H. Organometallics 2007, 26, 2791. 
(25) Pienkos, J. A.; Zottig, V. E.; Iovan, D. A.; Li, M.; Harrison, D. P.; Sabat, M.; Salomon, R. J.; Strausberg, L.; Teran, V. A.; Myers, W. H.; Harman, W. D. Organometallics 2013, 32, 691.

(26) Salomon, R. J.; Todd, M. A.; Sabat, M.; Myers, W. H.; Harman, W. D. Organometallics 2010, 29, 707.

(27) Pienkos, J. A.; Knisely, A. T.; Liebov, B. K.; Teran, V.; Zottig, V. E.; Sabat, M.; Myers, W. H.; Harman, W. D. Organometallics 2013, 33, 267.

(28) Keane, J. M.; Harman, W. D. Organometallics 2005, 24, 1786.

(29) Salomon, R. J., University of Virginia, 2010.

(30) The distinct splitting pattern (qd) of the methyl on the furan moiety was used as spectroscopic handle to monitor subsequent reactivity.

(31) Keane, J. M.; Chordia, M. D.; Mocella, C. J.; Sabat, M.; Trindle, C. O.; Harman, W. D. Journal of the American Chemical Society 2004, 126, 6806.

(32) Graham, P. M.; Meiere, S. H.; Sabat, M.; Harman, W. D. Organometallics 2003, 22, 4364 .

(33) MacLeod, B. L.; Pienkos, J. A.; Myers, J. T.; Sabat, M.; Myers, W. H.; Harman, W. D. Organometallics 2014, 33, 6286. 


\section{Chapter 4:}

\section{Further Reactivity Testing of}

$N$-Ethylindolinium Complex and

Isolation of Novel Hexahydroindoles. 


\subsection{Introduction}

After an initial investigation into the reactivity of the $\mathrm{TpW}(\mathrm{NO})\left(\mathrm{PMe}_{3}\right)\left(\eta^{2}-N-\right.$ ethylindolinium) (5), it was determined that this was a viable system to possibly synthesize novel hexahydroindoles. There are multiple steps in being able to complete this goal. Initially, electrophiles other than $\mathrm{H}^{+}$, will be tested for reactivity. This will hopefully create a larger variety of molecules that could become novel organics. As with the $N, N$-dimethylaniline system (2), a problem remains with the backbonding of the metal into the iminium. This does not allow for easy metal oxidation to liberate the organics. The iminium bond of the aniline system was never reduced, which caused problems with isolation of organics. This problem is a major focus with the indoline system. Once the iminium can be reduced, the isolation of the organic molecules can be explored.

\subsection{Exploration of Electrophiles}

In the second phase of this study, we endeavored to determine the range of electrophiles that could be added to $\mathrm{C} 4$ of the indoline ring system. Of greatest concern here is the potential competing oxidation of the tungsten by the electrophile. Thus, it becomes critical that the indolinium ligand of $\mathbf{5}$ not be deprotonated, as this action would dramatically lower the $\mathrm{W}(\mathrm{I} / 0)$ reduction potential and increase the susceptibility of the metal to oxidation. 


\subsubsection{Carbon Based Electrophiles}

Types of carbon electrophiles explored included isocyanate, alkyl halide, Michael acceptor, and acetal reagents. There was no reactivity with unactivated isocyanates, such as ethyl isocyanate. In contrast, chlorosulfonyl isocyanate reacted with $\mathbf{5}$ to give one major product (26). We speculate that this product is the result of electrophilic substitution at $\mathrm{C} 4$. This new species (26) was not stable enough to isolate, however ${ }^{1} \mathrm{H}$ and ${ }^{13} \mathrm{C}$ NMR data support this assignment. In particular, proton signals from $\mathrm{H} 5, \mathrm{H} 6, \mathrm{H} 7$ and $\mathrm{H} 3 \mathrm{a}$ are observed with chemical shifts similar to compound $\mathbf{5}$, but no $\mathrm{H} 4$ signal was observed. In addition, a ${ }^{13} \mathrm{C}$ resonance for an amide carbonyl was observed at $162 \mathrm{ppm}$.

Scheme 4.1. Reactivity of indolinium complex 5 with isocyanates.
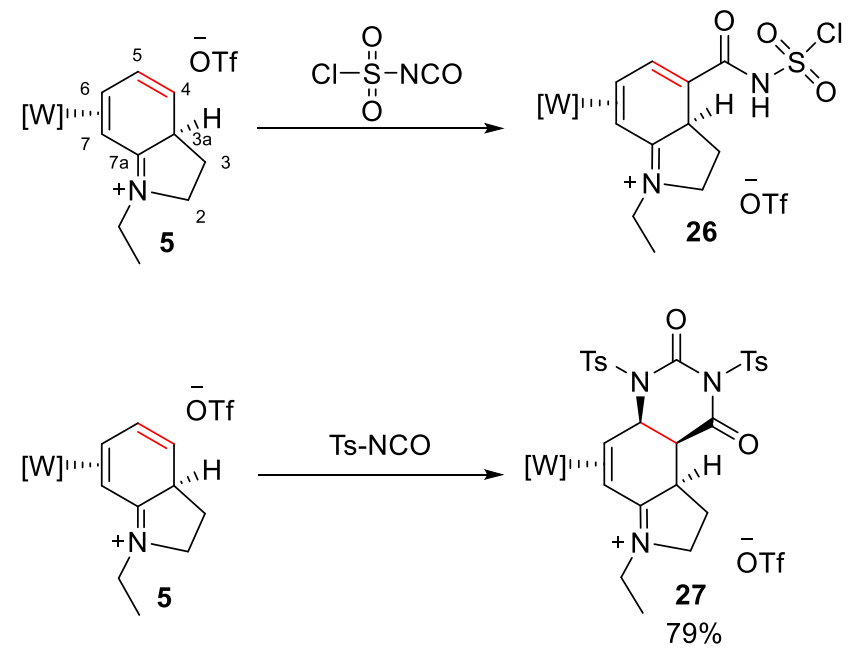

In contrast to this reaction, when $\mathbf{5}$ was combined with tosyl isocyanate, the dihydrouracil derivative $\mathbf{2 7}$ was isolated as the only major product resulting from a double addition of the isocyanate. Signals for two different tosyl groups are seen in the aromatic region of the proton spectrum and ${ }^{13} \mathrm{C}$ NMR data shows two carbonyl groups. 
Further, infrared spectroscopy indicates two carbonyl stretches at 1751 and $1728 \mathrm{~cm}^{-1}$. Methine signals for H4 and H5 were identified in both HSQC and COSY data, and the chemical shifts also show strong NOE interactions with the $\mathrm{PMe}_{3}$ signal. These observations indicate that electrophilic and nucleophilic addition reactions to form the dihydrouracil ring of $\mathbf{2 7}$ occur anti to the metal center.

Scheme 4.2. Simmons-Smith Cyclopropanation of $\mathbf{5}$.

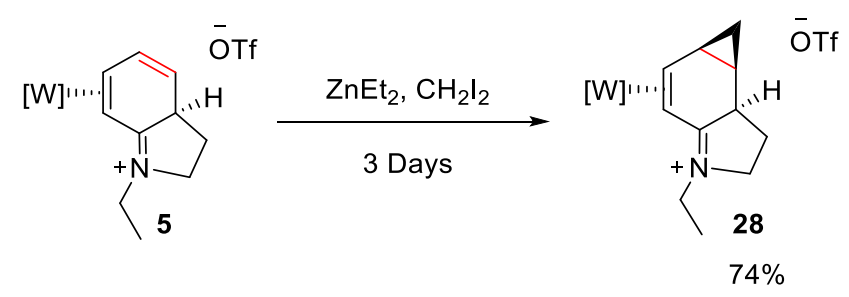

Cyclopropane rings are key features in many natural products and pharmaceutical chemicals. ${ }^{1,2}$ Previously we have shown that a Simmons-Smith cyclopronation could be performed on either a $N, N$-dimethylanilinium (2) or phenol complex of $\left\{\mathrm{TpW}(\mathrm{NO})\left(\mathrm{PMe}_{3}\right)\right\}^{3,4}$ In the case of the former system, an acid-induced ring cleavage led to both methylated and gem-dimethylated products. We hoped to incorporate such structural elements into the indoline ring system. Hence, combining 5 with $\mathrm{ZnEt}_{2}$ and $\mathrm{CH}_{2} \mathrm{I}_{2}$ in DCM resulted in the cycloproponated product 28. Key features for this product include a diastereotopic methylene group with upfield proton resonances at 0.88 and 0.04 ppm, indicative of the strained ring. As with 27, $\mathrm{H} 4$ and $\mathrm{H} 5$ both have strong NOE interactions with the $\mathrm{PMe}_{3}$ group and the methylene proton at $0.88 \mathrm{ppm}$, indicating that all three protons are syn to the metal. Cyclic voltammetric, ${ }^{13} \mathrm{C} N M R$ and IR data indicate that the iminium group in $\mathbf{2 8}$ is intact. Unfortunately, in contrast to that seen 
with the $N, N$-dimethylanilinium (2) analogue, compound 28 failed to undergo clean ringscission in the presence of triflic acid. ${ }^{3}$

\subsubsection{Fluorination of Indolinium}

Scheme 4.3. Fluorination of the indolinium complex 5 .

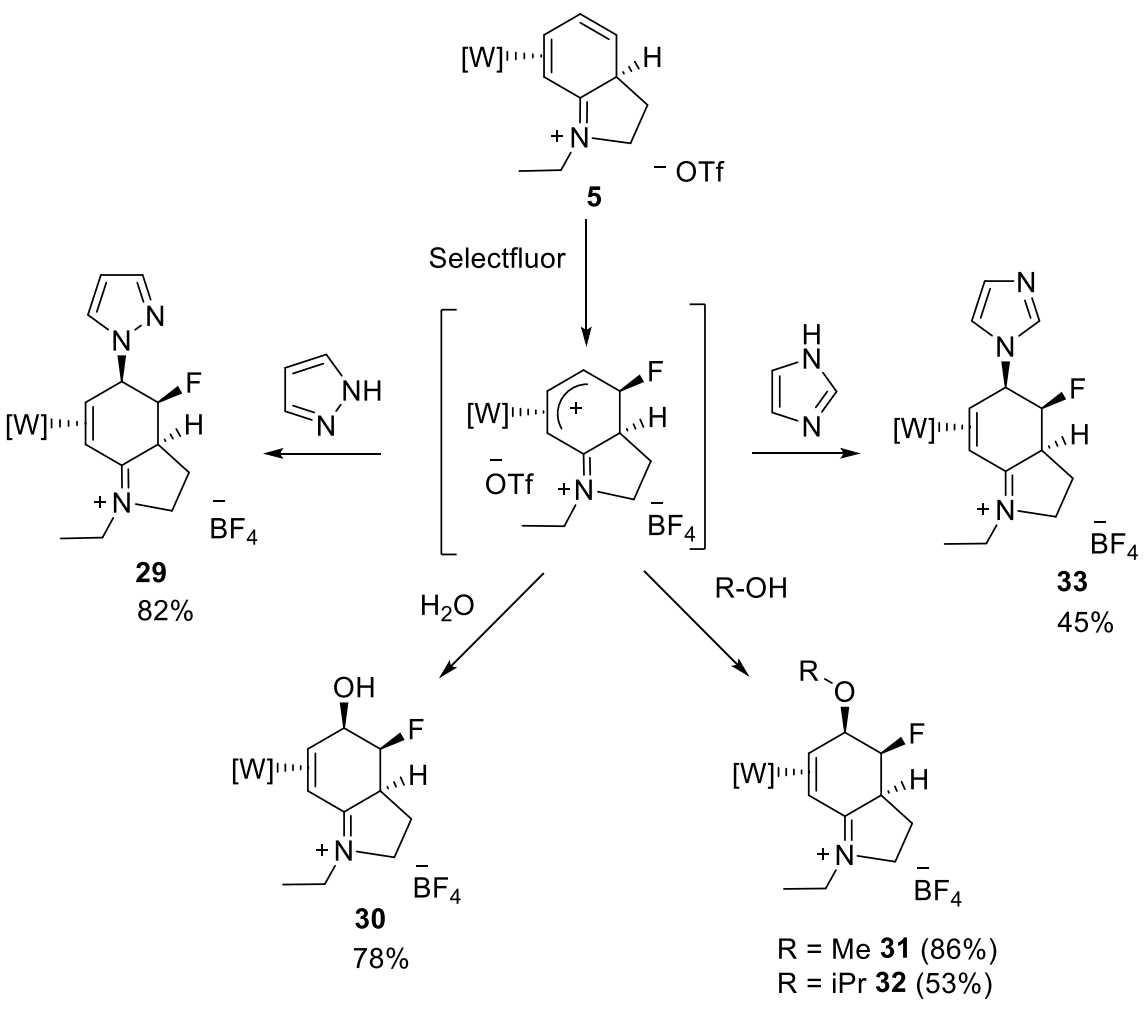

We next explored the reactivity at $\mathrm{C} 4$ of the indolinium ring of $\mathbf{5}$ with various heteroatom electrophiles. As mentioned before, of primary concern is the competing reaction of oxidation of the metal by the electrophile. In pharmaceutical design, the exchange of a proton with fluorine has become a strategy for optimizing a drug's performance. In addition to the predictable effects stemming from the large 
electronegativity of this heteroatom, fluorine substitution also increases the bioavailability and fat solubility for a compound. Some of the most popular medicines on the market, including Lipitor, Prevacid and Prozac, include at least one fluorinated functional group. ${ }^{5}$ Further, using Positron Emission Tomography (PET), fluorodeoxyglucose is an important ${ }^{18} \mathrm{~F}$ radio label used to analyze glucose uptake, blood flow and the disease states of cells in vivo. ${ }^{6}$

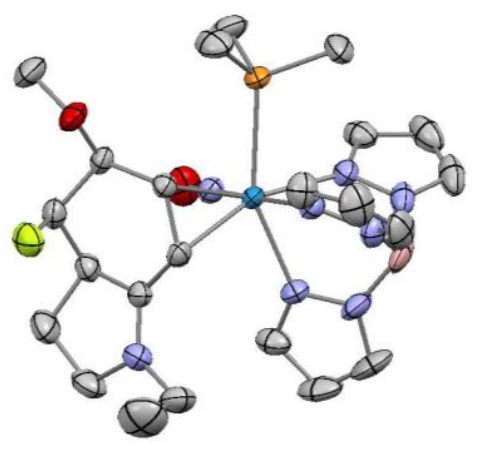

Figure 4.1. Crystal structure of complex 31 (30\% ellipsoids). Selected bond lengths

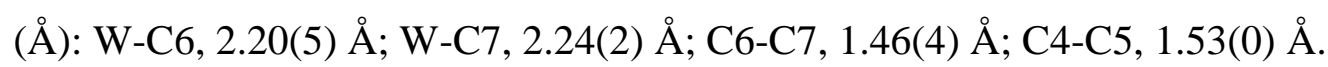

The addition of the electrophilic fluorine source Selectfluor ${ }^{\circledR}$ to the indolinium complex 5 without a nucleophile present led to its decomposition, suggesting that the purported fluorinated and dicationic allyl species is too unstable to isolate. However, we found success when various nucleophiles were added to the reaction vessel prior to the Selectfluor ${ }^{\circledR}$ addition. (Note that this is in contrast to the HOTf based addition reactions (vide supra), where the allylic intermediate was formed prior to the nucleophiles being added to the reaction mixture.) Aromatic amines (forming 29 and 33) and oxygen-based nucleophiles (forming 30-32) were successfully added to C5 following fluorine addition 
to $\mathrm{C} 4$. The addition of fluorine at $\mathrm{C} 4$ was easily identified in ${ }^{1} \mathrm{H}$ NMR spectra. Compared to the protonated analogues, the signal for $\mathrm{H} 4$ shifted 3 ppm downfield and showed a $\mathrm{H}-\mathrm{F}$ geminal coupling constant of $52 \mathrm{~Hz}$. This splitting was observed for the signals of $\mathrm{H} 3 \mathrm{a}$ and $\mathrm{H} 5$ (splitting $34 \mathrm{~Hz}$ and $30 \mathrm{~Hz}$ respectively). In the proton decoupled ${ }^{13} \mathrm{C} \mathrm{NMR}$ spectra of 29-33, C4 is shifted to approximately 90 ppm with a splitting of $182 \mathrm{~Hz}$. Fluorine was determined to add anti to metal through NOE data. H4, H5, and H3a showed strong correlations with $\mathrm{PMe}_{3}$ and to each other. These observations are consistent with the addition reactions of both nucleophile and electrophile anti to the metal and this stereochemistry was confirmed in the case of $\mathbf{3 1}$ by x-ray data (Figure 4.1). Other nucleophiles such as activated alkenes, aromatics and aliphatic amines were not successfully added in tandem with fluorine, likely owing to their incompatibility with the highly electrophilic fluorinating agent.

\subsubsection{Halogenation of Indolinium}

The additions of alternate electrophilic halide sources, such as N-chlorosuccinimide (NCS), N-bromosuccinimide (NBS), and N-iodosuccinimide (NIS), were explored. The addition of the halide source to the indolinium complex $\mathbf{5}$ without a nucleophile present was found to lead to decomposition, similar to Selectfluor ${ }^{\circledR}$. Success was found, however, when nucleophiles were already present in the reaction vessel prior to the halide addition. NCS and NBS had similar reactivity, though NBS products were generally less clean, so only NCS was continued further. The one exception, 34, can be seen in Scheme 4.4. The 
addition of chlorine instead of a proton is advantageous for possible biological uses, as chlorine is found in current pharmaceutical drugs. These include the antidepressant, Zoloft, and the blood thinner, Plavix. ${ }^{7}$

Scheme 4.4. Bromination tandem addition of 5.

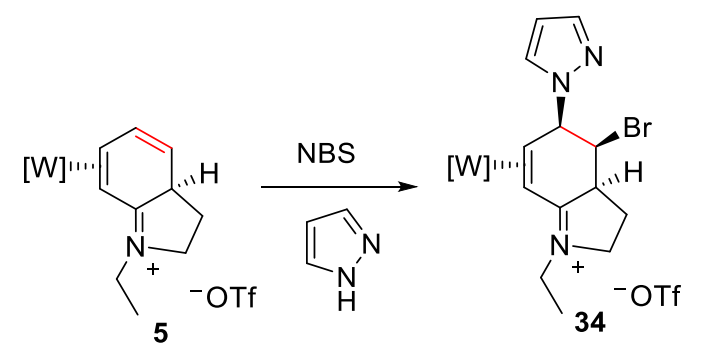

Similar to the Selectfluor ${ }^{\circledR}$ addition, aromatic amines (36 and 39) and oxygen-based (37) nucleophiles successfully added to C5 with the chlorine adding at C4. In addition, aniline (35) was successfully added. There was promising reactivity with thiophenol (38), however isolation of the product proved very difficult, so this product was not fully characterized. Other nucleophiles which were attempted included enolates, aromatics, isopropanol, amines and hydride sources. Enolates, such as MTDA and LiDMM, hydrides and aromatics, such as 2-methylfuran and 1,3-dimethoxybenzene, yielded only starting material. Propyl amine and isopropanol yielded only decomposition. This might speak to the incompatibile nature of certain nucleophiles with the electrophile source. Attempts to slow decomposition via the addition of base or low temperatures were not successful. 
Scheme 4.5. Chlorination of the indolinium complex 5.

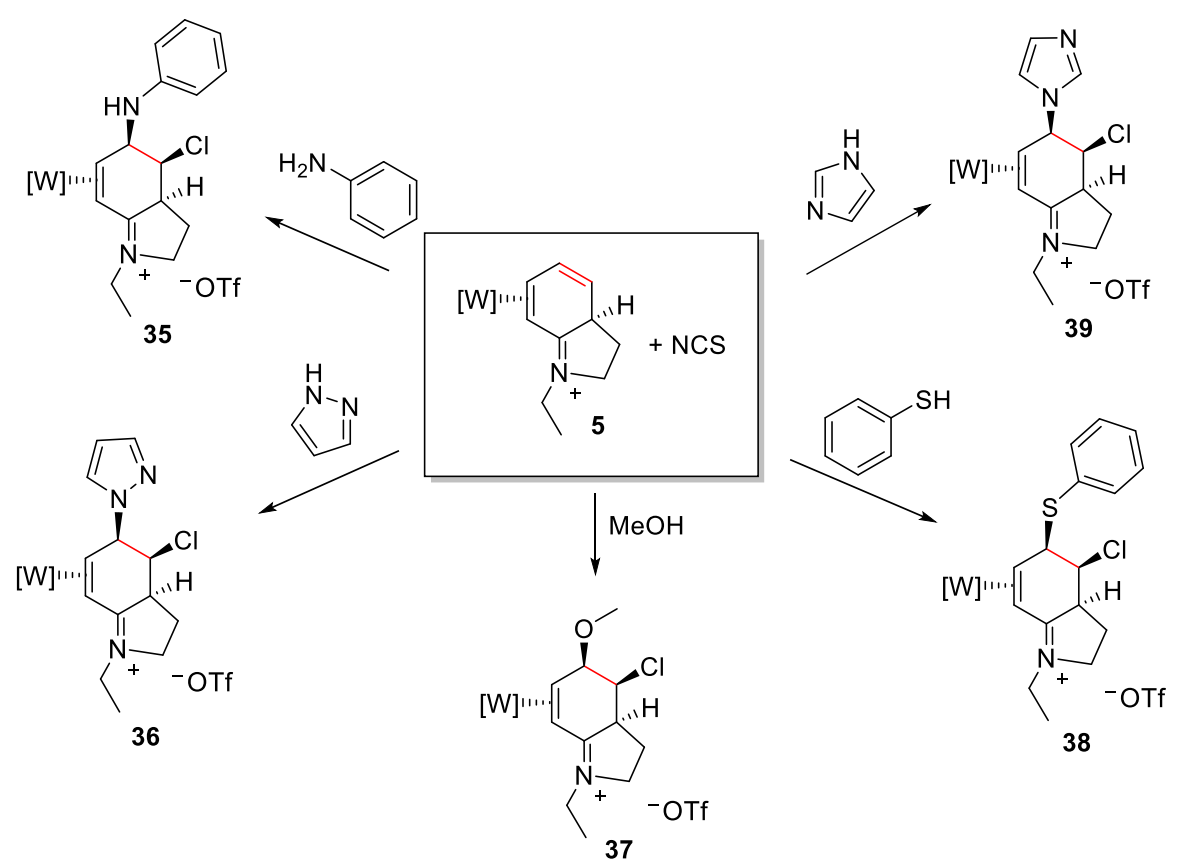

Chlorine addition to the compounds was not as simple to identify as fluorine addition, due to a lack of NMR splitting. Consistently with the fluorine system, however, the H4 proton shifted 2-4 ppm downfield in comparison to the protonated analogs. NOE data shows $\mathrm{H} 4, \mathrm{H} 5$ and $\mathrm{H} 3 \mathrm{a}$ correlating to the $\mathrm{PMe}_{3}$ ligand, indicating they are syn to the metal center, meaning additions of the chlorine and nucleophile occurred anti to the metal. The most conclusive identification technique for chlorine in these compounds comes from HRMS data showing a M+2 peaks of about a 3:1 ratio for all compounds. 
Scheme 4.6. Substitution of iodine with the indolinium complex 5 .

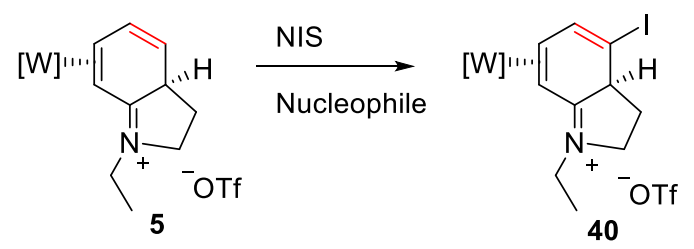

The addition of an iodo group onto the indoliinium system was attempted in order to open the possibility of subsititution reactions at the $\mathrm{C} 4$ position. If the iodine could be substituted with another nucleophile (through a $\mathrm{S}_{\mathrm{N}} 1$ or $\mathrm{S}_{\mathrm{N}} 2$ type reaction mechanism), the additions could appear as double nucleophile addition. When this was attempted however, there were unexpected results. When NIS was added with various nucleophiles to the indolinium complex $\mathbf{5}$, only one product was ever seen via ${ }^{31} \mathrm{P} \mathrm{NMR}$. Upon isolation, a substitution reaction was seen to form an iodo substituted indoline (40). In ${ }^{1} \mathrm{H}$ NMR, there is no longer a H4 peak and the H5 peak has shifted downfield 1 ppm. Further reactivity with this system was not attempted.

\subsubsection{Hydroxylation of Indolinium with mCPBA}

Epoxidation and hydroxylation of C4 and C5 on the indolinium system (5) was attempted using the peroxyacid mCPBA. When these were combined in a MeCN/DCM solution, a new species was formed (42), which was neither an epoxide nor an allylic species. It was determined that $\mathbf{4 2}$ contains a $m$-chlorobenzoate group at $\mathrm{C} 5$ and a hydroxyl group at $\mathrm{C} 4$, similar to that observed with the $N, N$-dimethylanilinium system. ${ }^{8}$ The ${ }^{13} \mathrm{C}$ NMR spectrum for 42 indicated a carbonyl signal at $166 \mathrm{ppm}$, with ${ }^{1} \mathrm{H}$ NMR 
data indicating 3 non-equivalent aromatic peaks. H4 and H5 showed an NOE interaction to $\mathrm{PMe}_{3}$, however $\mathrm{H} 4$ is shifted $2 \mathrm{ppm}$ downfield, relative to the protonated system. Infrared spectroscopy data further support this assignment showing a broad $\mathrm{OH}$ peak near $3500 \mathrm{~cm}^{-1}$ and a $\mathrm{C}=\mathrm{O}$ stretch at $1699 \mathrm{~cm}^{-1}$.

Scheme 4.7. Hydroxylation of the indolinium complex 5.

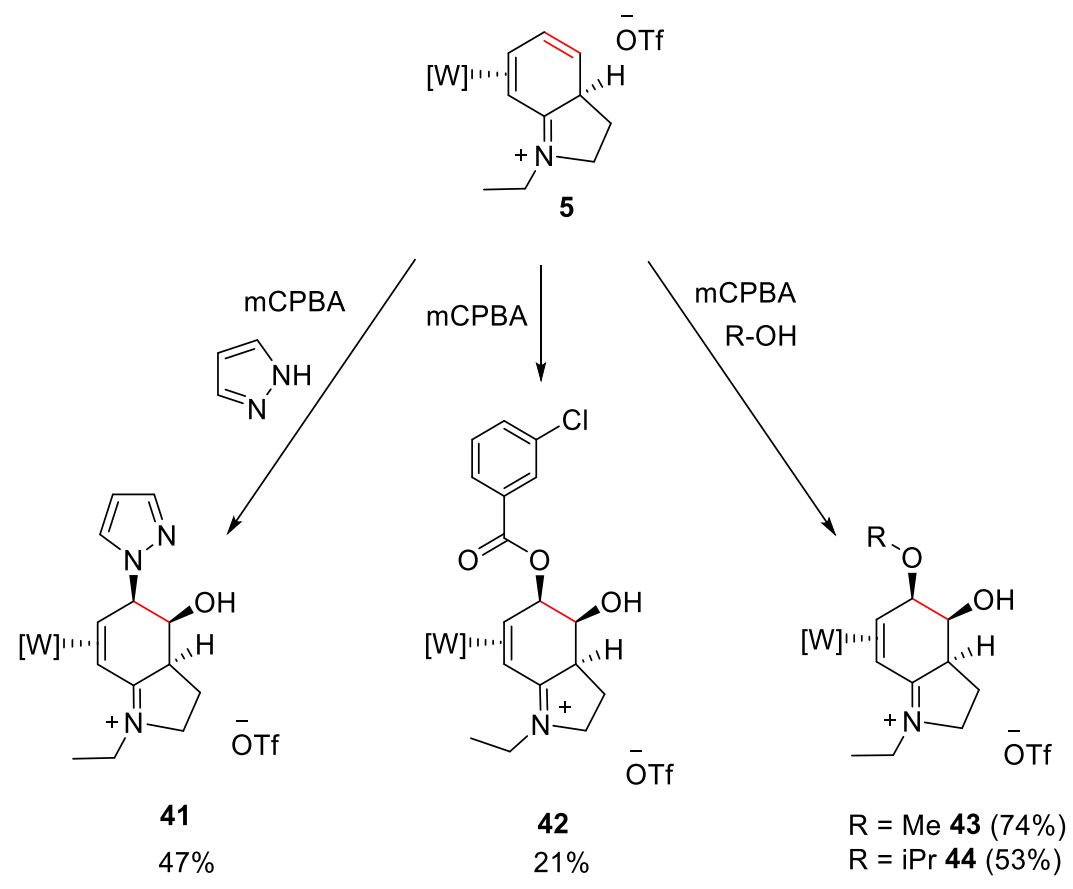

Upon addition of other nucleophiles to a mixture of $\mathbf{5}$ and mCPBA, such as pyrazole (41), methanol (43) and isopropanol (44), the resulting products no longer display spectroscopic features from the $m$-chlorobenzoate group. This indicates that the nucleophiles added instead. Stereochemistry at H5 is maintained however, as indicated by NOE data. This observation indicates that the carboxylate anion must leave before the new nucleophile adds (i.e., a $\mathrm{S}_{\mathrm{N}} 1$-type reaction mechanism) or the nucleophiles add preferentially due to excess. Compounds 41-44 were isolated in the pure form most easily 
when the nucleophile was added to the reaction mixture prior to the addition of mCPBA. Like the Selectfluor ${ }^{\circledR}$ case, other nucleophiles were not successfully added most likely due to the incompatibility with mCPBA.

Scheme 4.8. The formation of cyclic imidates from the hydroxylation product 43.

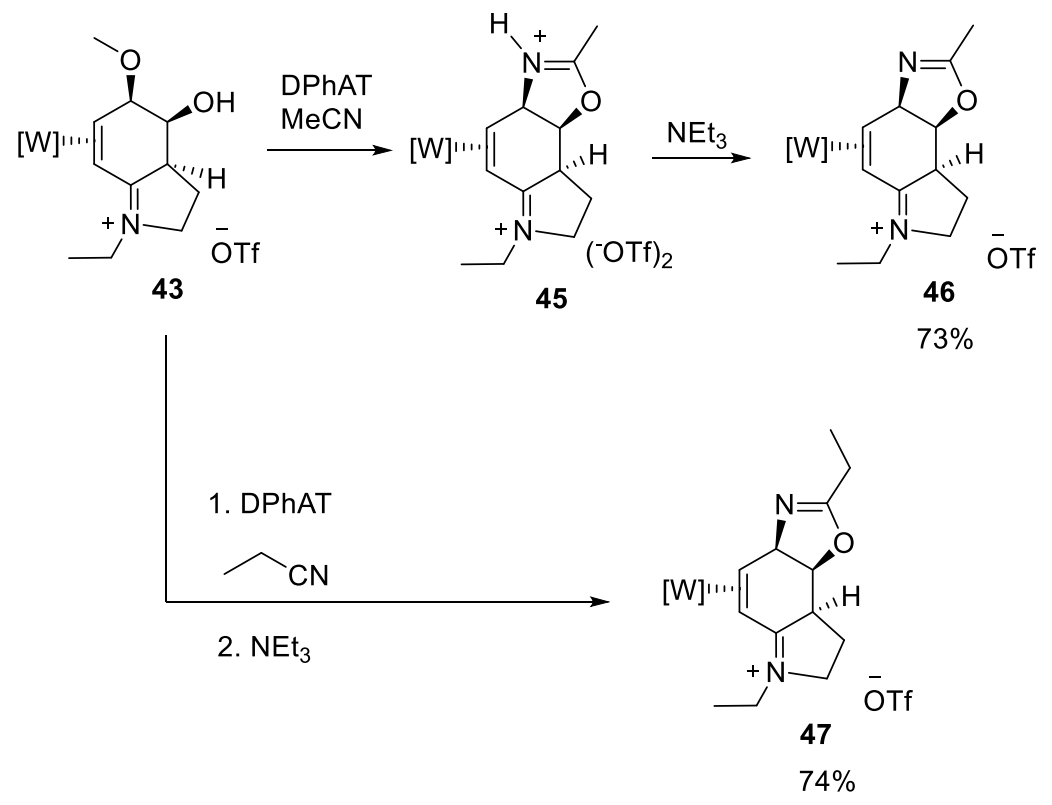

In order to combat the limited reactivity at $\mathrm{C} 5$, a methoxy group at $\mathrm{C} 5$ on the $\mathrm{N}, \mathrm{N}$ dimethylanilinium analogue could be protonated (diphenylammonium triflate (DPhAT) in $\mathrm{MeCN}$ ) to act as a leaving group, allowing a new nucleophile, such as 1,3,5trimethyoxybenzene to be added at $\mathrm{C} 5 .{ }^{9}$ Upon the addition of DPhAT to a solution of $\mathbf{4 3}$ and 1,3,5-trimethyoxybenzene, the resulting complex (45) did not incorporate the arene. The proton spectrum shows a new methyl signal at $2.50 \mathrm{ppm}$ along with a peak at 11.7 ppm, the latter of which does not correlate to any carbon in an HSQC experiment. Further, an additional ${ }^{13} \mathrm{C}$ peak at $177 \mathrm{ppm}$ appears that was not present in that of $\mathbf{4 3}$. We hypothesize that the methoxy group of $\mathbf{4 3}$ was protonated then replaced by acetonitrile 
and the resulting nitrilium ion then reacted with the adjacent $\mathrm{OH}$ group to form the cyclic imidate 45. Upon addition of triethylamine, complex46 results, which shows proton and carbon NMR data similar to $\mathbf{4 5}$, but with several peaks shifted upfield and the loss of the 11.7 signal in the proton spectrum. Together, proton and carbon NMR spectra along with COSY and NOESY data support the assignment of $\mathbf{4 5}$ and 46 as the cis-fused cyclic imidates shown in Scheme 8. Analogous reactions were attempted with propionitrile and benzonitrile, and by ${ }^{31} \mathrm{P}$ data, only propionitrile gave a clean product analogous to $\mathbf{4 6}$, while benzonitrile gave multiple products. The propionitrile analogue was isolated and characterized (47). Due to the similarities between 46 and 47, only compound 46 was brought forward (vide infra).

\subsection{Reduction of Iminium}

Scheme 4.9. Overall synthetic scheme of hexahydroindoles

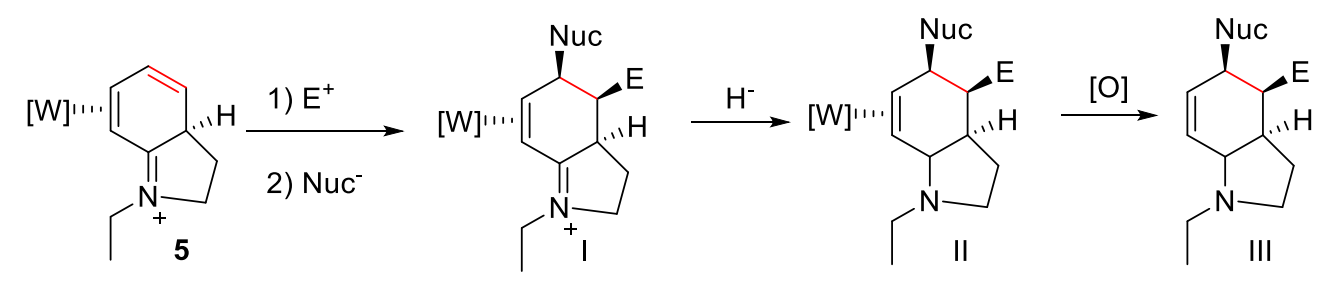

Similar to the $N, N$-dimethylanilinium complex (2), the reduction potentials of these iminium complexes are approximately $1.40 \mathrm{~V} \cdot{ }^{9,10}$ This potential is too high to easily oxidize the metal to remove the organic compounds without using harsh oxidants, such as ceric ammonium nitrate $(\mathrm{CAN})$. In order to make the metal system easier to oxidize, the 
iminium bond needs to be reduced to minimize the back-bonding from the metal. This should reduce the reduction potential to a range which allows for the organic ligand to be liberated. This type of reaction can be seen in an overall Scheme 4.9. Beginning with an addition product (I), the iminium isreduced to yield an amine (II). This could then be oxidized to yield a hexahydroindole that is completely novel (III). Iminiums in typical organic reactions are reduced using $\mathrm{NaCNBH}_{3}$ and $\mathrm{NaBH}_{4}$ in $\mathrm{MeOH}$. Using these conditions when in coordination to the metal complex, no reactivity was observed. It was believed that using a stronger hydride source, such as lithium aluminum hydride, the iminium could be reduced while not affecting the ligand or the metal center.

Preliminarily, lithium aluminum hydride in diethyl ether was used to reduce the iminium bond of tetrahydroindolium complexes cleanly without degradation of the metal complex. Upon further exploration, it was determined that using dimethyoxyethane (DME) as a solvent improved solubility and produced cleaner products. ${ }^{11}$ There are theories that DME has the ability to chelate the lithium cations, solvating them better than ether, making the ion less available to further react with the metal systems. Thus, the corresponding tetrahydroindolium complexes were reduced to form the hexahydroindole complexes 48-57. Many of these reductions were found to contain small amounts (5$15 \%$ ) of an unknown side product. These side products were different for every reaction, but in every case a doublet for the impurity (resembling a Tp signal) appeared downfield 
Scheme 4.10. Reduction of the iminium bond in the tetrahydroindolium complexes.
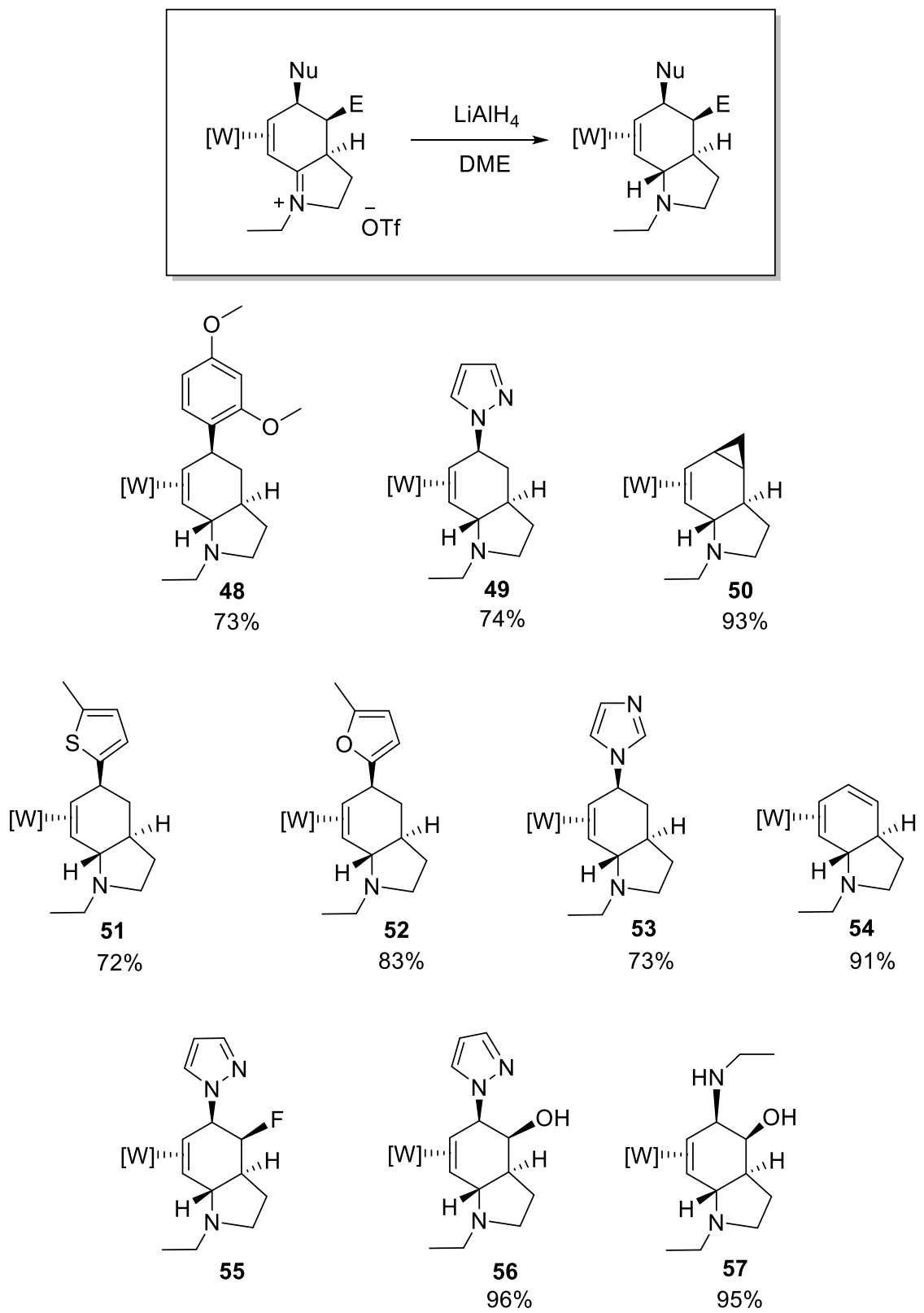

of $9.5 \mathrm{ppm}$. These impurities do not impact the subsequent step and were not removed, but could possibly indicate that certain nucleophiles are not compatible with these 
strongly hydridic conditions. There might also be the possibility of that the $\left\{\mathrm{TpW}(\mathrm{NO})\left(\mathrm{PMe}_{3}\right)\right\}$ system is not completely inert to these conditions as well.

The reduction of the iminium group of the tetrahydroindolium complexes has several distinguishing features. Significantly, for every product the chemical shift of the Tp PzA3 peak shifts dramatically downfield to $\sim 9.5 \mathrm{ppm}$. The characteristic doublet of H7 now becomes more complex due to the coupling with H7a. Further, H7a couples strongly to $\mathrm{H} 3 \mathrm{a}(\sim 12 \mathrm{~Hz})$, but these do not show a NOE correlation, indicating a Karplus angle near 180 degrees. These observations are consistent with an assignment of $\mathrm{H} 7 \mathrm{a}$ anti to the metal, with $\mathrm{H} 4, \mathrm{H} 5$ and $\mathrm{H} 3 \mathrm{a}$ all syn to the metal. A crystal structure is reported for 52, which confirms both the predicted trans- ring juncture and the addition of the 2methylfuran anti to the metal. In total, three new stereocenters are selectively created relative to the initial stereochemistry of the tungsten stereocenter. (Figure 4.2). Of note, this trans ring junction is present in a number of perhydroindole natural products. ${ }^{12-16}$ Consistent with the decreased $\pi$ acidity of the ligand, these compounds much easier to oxidize than their iminium precursors $\left(\mathrm{E}_{\mathrm{p}, \mathrm{a}} \approx 0.5 \mathrm{~V}, \mathrm{cf} . \sim 1.2 \mathrm{~V} @ 100 \mathrm{mV} / \mathrm{s}\right)$.

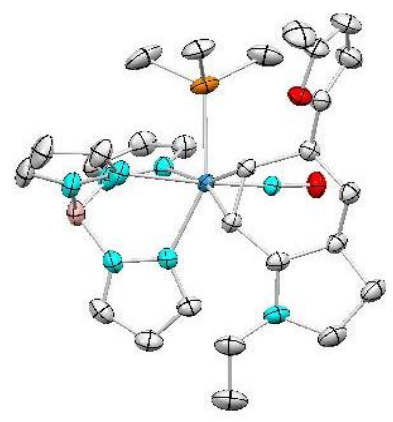

Figure 4.2. Crystal structure of compound $\mathbf{5 2}$ (30\% ellipsoids). Selected bond lengths

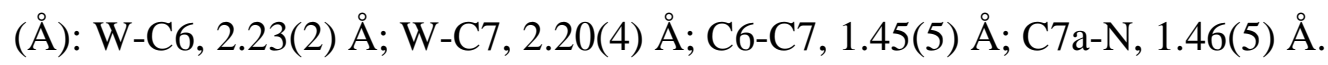


Unfortunately tetrahydroindolium complexes with aliphatic amine groups such as those derived from propyl amine (14), piperidine (17) and morpholine (18), decomposed under the reducing conditions. The decomposition contained some consistent NMR features, including alternate peaks for the PzA3 in the $9.5 \mathrm{ppm}$ region. The reduction of 16 leads to the identification of one possible hydride addition product. Upon addition of LAH to the triazole addition product, $\mathbf{5 8}$ is formed. This is identified by the characteristic downfield PzA3, but also by the lack of triazole peaks. A new set of geminal protons, as indicated by HSQC, have an NOE interaction with the $\mathrm{PMe}_{3}$ and a COSY interaction to the H4 geminal set. This is indicative that the nucleophile has been displaced by a hydride. The bridgehead position ( $\mathrm{H} 7 \mathrm{a})$ proton was also identified as a hydride addition anti to the metal.

Scheme 4.11. Displacement of triazole with hydride of $\mathbf{1 6 .}$

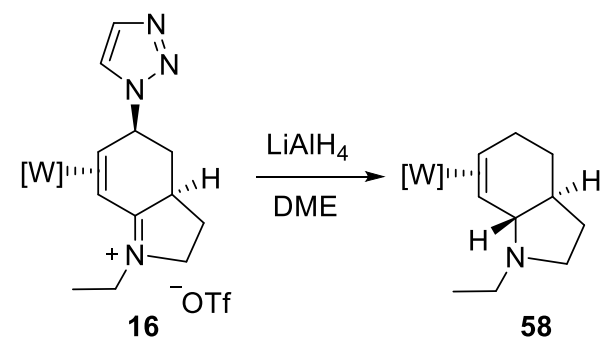

The iminium reduction was limited to reactions that did not contain carbonyls or other reducible functional groups. For example, when compound $\mathbf{4 6}$ was subjected to $\mathrm{LAH}$, the imidate ring resulted in the ethylamino derivative (57). Due to the clean reduction, compound $\mathbf{5 7}$ was continued onto oxidation. The fluorinated compound 29 was also not reduced cleanly to $\mathbf{5 5}$, but the impure complex was carried forward into the 
final decomplexation step (vide infra). All chlorinated compounds were not able to be reduced, but yielded only decomposition products.

\subsection{Oxidation and Isolation of Hexahydroindole Organics}

The decrease in the $\pi$-acidity of the ligand following the reduction of the conjugated iminium group of the tetrahydroindolinium complexes results in a substantial decrease in the $d^{5} / d^{6}$ reduction potential of the complex. While formal reduction potentials are not available, the anodic peak potentials $\left(\mathrm{E}_{\mathrm{p}, \mathrm{a}}=\right.$ for the reduced products are $\left.\sim 0.4\right)$ lowered about a volt compared to their tetrahydroindolium precursors. As a result, mild oxidants can be used to effect the oxidative cleavage.

In the exploration of various oxidants ranged in reductive potential from $0.5 \mathrm{~V}$ to 2.0 V. ${ }^{17}$ These included ferrecinium $\mathrm{PF}_{6}$, silver salts, CAN, DDQ and $\mathrm{NOPF}_{6}$. In the preliminary investigation, 2,3-dichloro-5,6-dicyano-1,4-benzoquinone (DDQ) in acetonitrile was used as the oxidant. This was possible for both 2-methylfuran and pyrazole. Upon further investigation, it was determined that nitrosonium hexafluorophosphate $\left(\mathrm{NOPF}_{6}\right)$ in acetone produced cleaner oxidations with higher yields overall. For example, experiments using furan 52 showed a NMR-based yield of 16\% with DDQ but a 57\% NMR yield with $\mathrm{NOPF}_{6}$. The only compound which this did not improve yields, but rather caused no organic products to be isolated, was with the pyrazole addition. Other oxidants were not moved forward with due to lack of oxidation or poor NMR yield results. 
Scheme 4.12. Decomplexation of the final hexahydroindole products.

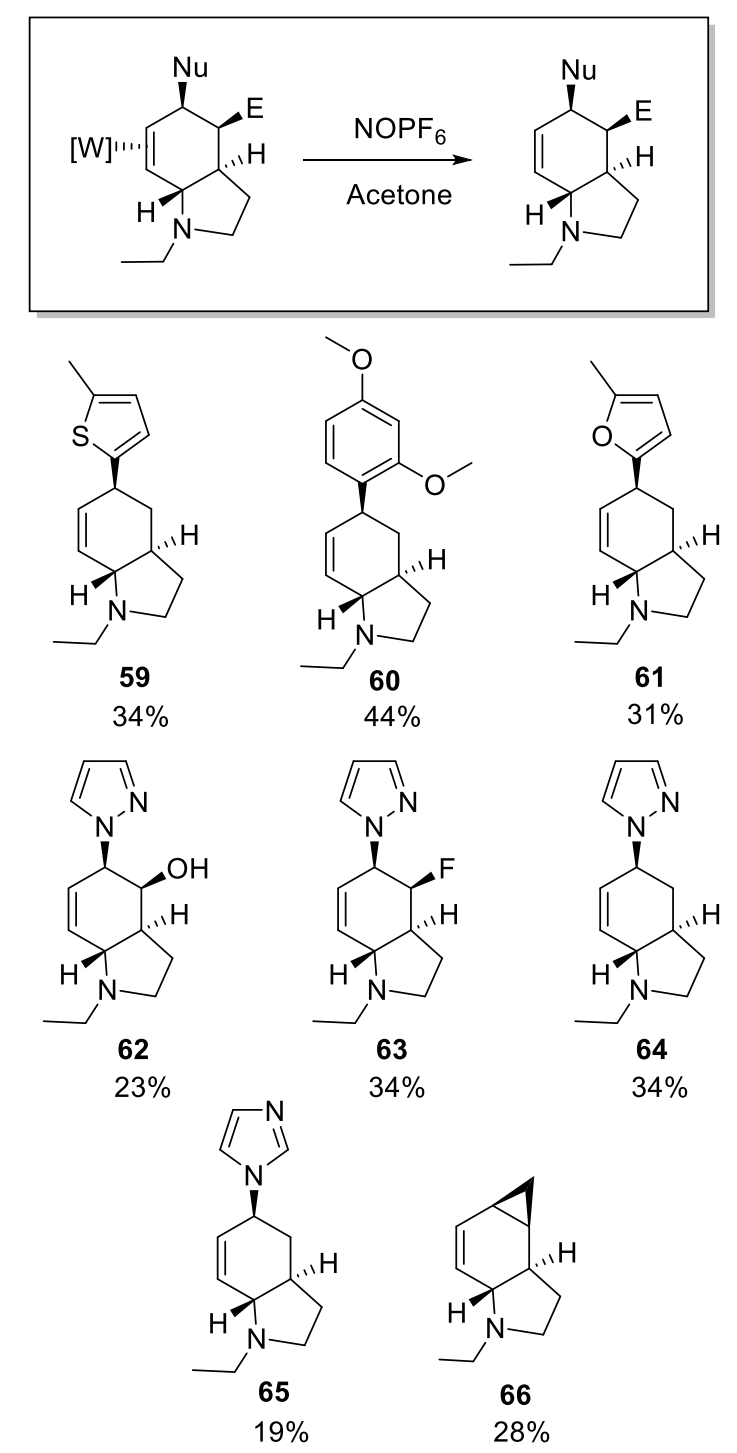

The ease of isolation of the resulting organics varied greatly depending on the functional groups involved. The majority of these species were isolated on alumina preparatory TLC plates. Compound 65, however, required isolation with Florisil as the solid phase because alumina was too polar to allow for isolation. Even with Florisil as the stationary phase, $\mathrm{MeCN} / \mathrm{MeOH}$ mixtures were required for elution, with resulting low 
yields. This loss of yield appears to be related to the chromatography process, since NMR yields were typically $>50 \%$ yield. The novel hexahydroindoles 59-66 were identified using proton, carbon, NOESY and COSY data to confirm that the stereocenters were unaltered following decomplexation from the metal center.

To our knowledge, these hexahydroindoles are entirely unique. The most similar compounds that could be found in the Scifinder database are the natural products in the Stemona, Amaryllidaceae, Aeruginosin and Sceletium alkaloid groups. The ability to fully saturate this system would be beneficial in expanding the possible biological activity. Examples of this reactivity can be seen in Scheme 4.13, with dihydroxylation through $\mathrm{OsO}_{4}$ and hydrogenation over carbon. Due to the small yields $(<20 \mathrm{mg})$ of the organic compounds, these were only preliminarily tested, though there are literature procedures suggesting this is possible. ${ }^{18}$ The addition of $\mathrm{OsO}_{4}$ to compound $\mathbf{6 1}$ yielded only decomposition of the furan ring, showing a limitation of further reactivity stemming from the nucleophile.

Scheme 4.13. Examples of further elaboration to perhydroindoles

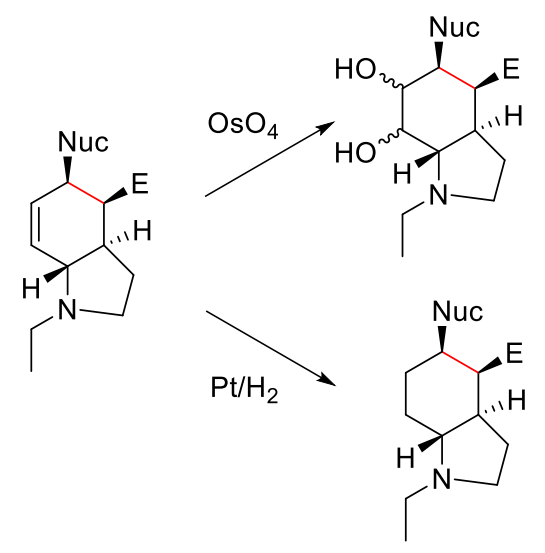




\subsection{Conclusion}

Despite the obvious biological significance, there are few examples of hexahydroindoles or perhydroindoles with multiple stereocenters or functional groups that have been synthesized, other than those directly inspired from natural sources. Described herein is a general strategy to prepare such compounds that could provide an unusually high level of chemical diversity (Figure 4.3). Importantly, these new alkaloidlike compounds each contain an isolated alkene available for further elaboration. Most significantly, they are all derived from a simple indoline core, which itself could be modified prior to its coordination and elaboration.

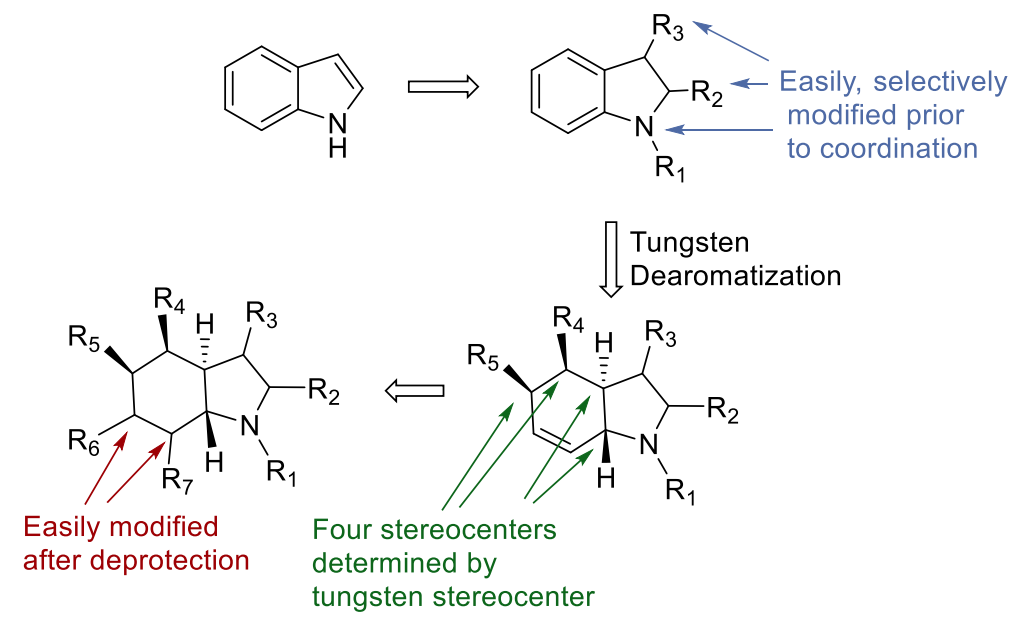

Figure 4.3. Dearomatization as part of creating a diverse chemical library. 


\subsection{Experimental Section}

General Methods: NMR spectra were obtained on a 300, 500, 600, or $800 \mathrm{MHz}$ spectrometer. All chemical shifts are reported in ppm and proton and carbon shifts are referenced to tetramethylsilane (TMS) utilizing residual ${ }^{1} \mathrm{H}$ or ${ }^{13} \mathrm{C}$ signals of the deuterated solvents as an internal standard. Phosphorus NMR signals are referenced to $85 \% \mathrm{H}_{3} \mathrm{PO}_{4}(\delta=0.00)$ using a triphenylphosphate external standard $(\delta=-16.58)$. Coupling constants $(J)$ are reported in hertz $(\mathrm{Hz})$. Infrared spectra (IR) were recorded as a glaze on a spectrometer fitted with a Horizontal Attenuated Total Reflectance (HATR) accessory, or on a FT-IR spectrometer equipped with a diamond anvil ATR assembly. Electrochemical experiments were performed under a dinitrogen atmosphere using a potentiostat. Cyclic voltammetry data was taken at ambient temperature $\left(\sim 25^{\circ} \mathrm{C}\right)$ at 100 $\mathrm{mV} / \mathrm{s}$ in a standard three-electrode cell with a glassy carbon working electrode, $\mathrm{N}, \mathrm{N}$ dimethylacetamide (DMA) or acetonitrile (MeCN) solvent (unless otherwise specified), and tetrabutylammonium hexafluorophosphate (TBAH) electrolyte (approx. 0.5 M). All potentials are reported versus NHE (Normal Hydrogen Electrode) using cobaltocenium hexafluorophosphate $\left(\mathrm{E}_{1 / 2}=-0.78 \mathrm{~V}\right)$, ferrocene $\left(\mathrm{E}_{1 / 2}=+0.55 \mathrm{~V}\right)$, or decamethylferrocene $\left(\mathrm{E}_{1 / 2}=+0.04 \mathrm{~V}\right)$ as an internal standard. The peak-to-peak separation was less than 100 $\mathrm{mV}$ for all reversible couples. High-resolution mass spectra were acquired in ESI mode, from samples dissolved in a 3:1 acetonitrile/water solution containing sodium trifluoroacetate (NaTFA). Mass spectra are reported as $\mathrm{M}^{+}$for monocationic complexes, or as $\left[\mathrm{M}+\mathrm{H}^{+}\right]$or $\left[\mathrm{M}+\mathrm{Na}^{+}\right]$for neutral complexes, using $\left[\mathrm{Na}(\mathrm{NaTFA})_{\mathrm{x}}\right]^{+}$clusters as an internal standard. In all cases, observed isotopic envelopes were consistent with the 
molecular composition reported. For organic products, the monoisotopic ion is reported; for complexes, the major peaks in the isotopic envelope are reported. Unless otherwise noted, all synthetic reactions were performed in a glovebox under a dry nitrogen atmosphere. $\mathrm{CH}_{2} \mathrm{Cl}_{2}$ and benzene were purified by passage through a column packed with activated alumina. Other solvents and liquid reagents were thoroughly purged with dry nitrogen prior to use. Triflate salts of amines were synthesized by addition of an $\mathrm{Et}_{2} \mathrm{O}$ solution of triflic acid to the appropriate conjugate base dissolved in $\mathrm{Et}_{2} \mathrm{O}$. Deuterated solvents were used as received from Cambridge Isotopes. Pyrazole (Pz) protons of the (trispyrazolyl) borate (Tp) ligand were uniquely assigned (eg., "PzB3") using a combination of 2-dimensional NMR data and phosphorus-proton NOE interactions. BH peaks (around 4-5 ppm) are not identified due to their quadrupole broadening; IR data is used to confirm the presence of a $\mathrm{BH}$ (around $2500 \mathrm{~cm}^{-1}$ ). $\mathrm{OH}$ and $\mathrm{NH}$ peaks are not always identified due to exchange with water in solvent. Where appropriate, $\mathrm{OH}$ peaks have been confirmed with IR data.

DFT Calculations. Initial structures were built in GAUSSVIEW (5.0.8) and optimized with the PM6 semiempirical method in GAUSSIAN 09. These structures were refined stepwise in Gaussian using B3LYP and a series of basis functions incorporating LANL2 pseudopotentials and associated basis functions provided in the GAUSSIAN package. The most demanding calculations reported here put the LANL2DZ pseudopotential and its basis only on the W atom and used the 6-31G(d) basis for all other atoms. 


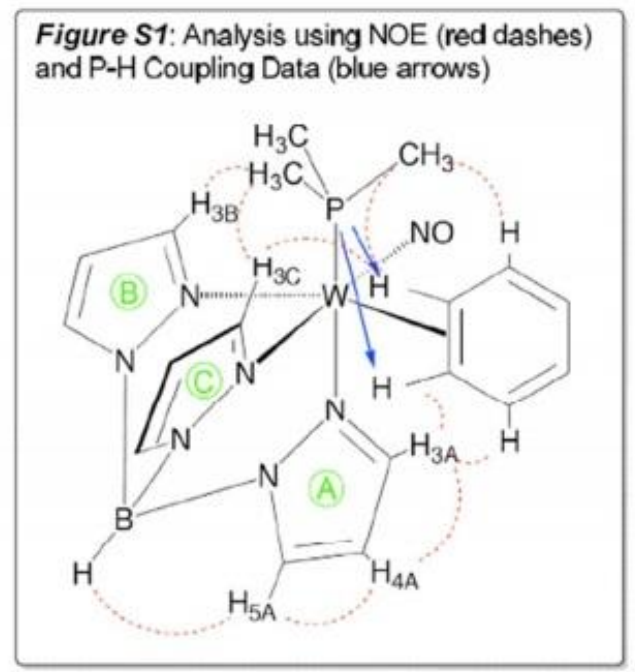

Compound 27: To a solution of Compound 5 (0.050 g, 0.062 mmols) in MeCN (1 mL) was combined with Ts-ICN $(0.153 \mathrm{mg}, 1.08 \mathrm{mmols})$ in a 4-dram vial charged with a stirbar. This homogeneous solution was stirred for $2 \mathrm{~d}$. The solution was then removed from inert atmosphere and added dropwise to stirring $\mathrm{Et}_{2} \mathrm{O}(75 \mathrm{~mL})$. The yellow powder was collected on a $15 \mathrm{~mL}$ medium-porosity frit and washed with $\mathrm{Et}_{2} \mathrm{O}(30 \mathrm{~mL})$ yielding Compound 27 (0.059 g, 0.049 mmol, 79\%). ${ }^{1} \mathrm{H}$ NMR ( $d$-MeCN, $\left.\delta\right): 8.15$ (d, $J=2.0,1 \mathrm{H}$, PzB3), 8.02 (d, $J=2.0,1 \mathrm{H}$, PzC5), 7.97 (d, $J=8.1,2 \mathrm{H}, \mathrm{H} 12$ '), 7.97 (buried, 1H, PzB5), $7.96\left(\mathrm{~d}, J=8.1,2 \mathrm{H}, \mathrm{H} 7{ }^{\prime}\right), 7.87(\mathrm{~d}, J=2.0,1 \mathrm{H}, \mathrm{PzA} 5), 7.59(\mathrm{~d}, J=2.0,1 \mathrm{H}, \mathrm{PzC} 3), 7.43$ (d, $\left.J=8.1,1 \mathrm{H}, \mathrm{H} 8^{\prime}\right), 7.34(\mathrm{~d}, J=8.1,1 \mathrm{H}, \mathrm{H} 13$ ') 7.37 (d, $J=2.0,1 \mathrm{H}, \mathrm{PzA} 3), 6.55$ (t, $J=$ 2.0, 1H, PzC4), $6.47(\mathrm{t}, J=2.0,1 \mathrm{H}, \mathrm{PzB} 4), 6.31(\mathrm{t}, J=2.0,1 \mathrm{H}, \mathrm{PzA} 4), 6.11(\mathrm{~m}, 1 \mathrm{H}, \mathrm{H} 5)$, 4.06 (m, 2H, H2), 3.81 (t, $J=8.7,1 \mathrm{H}, \mathrm{H} 3 \mathrm{a}), 3.63$ (m, 1H, H6), 3.25 (t, $J=3.9,1 \mathrm{H}, \mathrm{H} 4)$, 2.44 (s, 3H, 9’Me), 2.49 (m, 2H, H3), 2.49 (buried, 1H, N-Ethyl $\mathrm{CH}_{2}$ ), 2.35 (s, 3H, 14'Me), 2.06 (m, 1H, $N$-Ethyl $\left.\mathrm{CH}_{2}\right), 1.85$ (d, $\left.J=8.6,1 \mathrm{H}, \mathrm{H} 7\right), 1.28(\mathrm{~d}, J=9.6,9 \mathrm{H}$, $\left.\mathrm{PMe}_{3}\right), 0.77\left(\mathrm{t}, J=6.9,3 \mathrm{H}, N\right.$-Ethyl $\left.\mathrm{CH}_{3}\right) \cdot{ }^{13} \mathrm{C}$ NMR $(d-\mathrm{MeCN}, \delta): 183.8(\mathrm{C} 7 \mathrm{a}), 169.3$ 
(C2' and $\mathrm{C}^{\prime}$ '), 147.7 (C9' or C14'), 147.6 (C9' or C14'), 145.1 (PzB3), 144.7 (PzA3), 141.6 (PzC3), 139.2 (Pz5), 139.1 (Pz5), 139.0 (Pz5), 136.2 (C6’ or C11'), 134.7 (C6’or C11'), 130.7 (C8'), 130.6 (C13'), 130.5 (C12' or C7'), 129.9 (C12' or C7'), 108.7 (PzB4 or PzC4), 108.6 (PzB4 or PzC4), 107.9 (PzA4), 67.8 (d, $J=14.0, \mathrm{C} 5), 57.8$ (C6), 55.4 (C2), 53.1 (C4), 50.5 (C7), 45.4 (C3a), 42.8 (N-Ethyl $\mathrm{CH}_{2}$ ), 22.8 (C3), 21.7 (C9’Me), $21.6\left(\mathrm{C}_{14}{ }^{\mathrm{M}} \mathrm{Me}\right), 14.1\left(\mathrm{~d}, J=29, \mathrm{PMe}_{3}\right), 10.8\left(N\right.$-Ethyl $\left.\mathrm{CH}_{3}\right) .{ }^{31} \mathrm{P}$ NMR $(d-\mathrm{MeCN}, \delta)$ : $9.44\left(J_{\mathrm{wp}}=279\right)$. IR: $v_{\mathrm{BH}}=2495 \mathrm{~cm}^{-1}, v_{\mathrm{NO}}=1728 \mathrm{~cm}^{-1}, v_{\mathrm{NO}}$ and $v_{\text {iminium }}=1624 \mathrm{~cm}^{-1}$ and $1589 \mathrm{~cm}^{-1} . \mathrm{CV}$ (DMA): $\mathrm{E}_{\mathrm{p}, \mathrm{a}}=1.40 \mathrm{~V}$. HRMS: $\left[\mathrm{M}^{+}\right]=\left[\mathrm{C}_{25} \mathrm{H}_{44} \mathrm{BN}_{9} \mathrm{OPW}^{+}\right]=$obsd (\%), calcd (\%), ppm: 1043.2392 (65), 1043.2390 (69), 0.2; 1044.2429 (78), 1044.2413 (78), 1.5; 1045.2372 (100), 1045.2412 (100), -3.8; 1046.2392 (52), 1046.2440 (59), -4.6; 1047.2424 (90), 1047.2441 (84), -1.6; 1048.2456 (33), 1048.2463 (36), -0.7.

Compound 28: In a flame dried $100 \mathrm{~mL}$ round bottom flask charged with a stirbar, a solution of $\mathrm{CH}_{2} \mathrm{I}_{2}(1.54 \mathrm{~g}, 5.75 \mathrm{mmol})$ was added in DCM $(10 \mathrm{~mL})$ and stirred. A solution of $\mathrm{ZnEt}_{2}$ (254 mg, $\left.2.06 \mathrm{mmol}\right)$ in $\mathrm{DCM}(15 \mathrm{~mL})$ was added dropswise to the reaction solution, creating a milky white solution. A solution of Compound 5 (201 mg, 0.251 mmol) in DCM (5 mL) was added to the reaction solution and stirred $3 \mathrm{~d}$. The reaction was removed from the glovebox and diluted with DCM $(50 \mathrm{~mL})$. This was treated $2 \times 50$ $\mathrm{mL}$ of $\mathrm{NH}_{4} \mathrm{Cl}$ (saturated, aq). The aqueous layer was back extracted with DCM (2 x 50 $\mathrm{mL})$, and the combined organic layers were washed with deionized water $(100 \mathrm{~mL})$. This was then dried over anhydrous $\mathrm{MgSO}_{4}$ and concentrated in vacuo. The yellow oil was redissolved in minimal DCM and added dropwise to a stirring solution of $\mathrm{Et}_{2} \mathrm{O}(200 \mathrm{~mL})$ to induce precipitation of a tan solid. The powder was collected on a $15 \mathrm{~mL}$ fine-porosity 
fritted funnel, washed with $\mathrm{Et}_{2} \mathrm{O}$ (30 mL), yielding Compound 28 (152 mg, 0.187 mmol, 74\%). ${ }^{1} \mathrm{H}$ NMR $(d-\mathrm{MeCN}, \delta): 8.12(\mathrm{~d}, J=2.0,1 \mathrm{H}, \mathrm{PzB} 3), 7.95(\mathrm{~d}, J=2.0,1 \mathrm{H}, \mathrm{PzC} 5)$, $7.92(\mathrm{~d}, J=2.0,1 \mathrm{H}, \mathrm{PzB} 5), 7.82(\mathrm{~d}, J=2.0,1 \mathrm{H}, \mathrm{PzA} 5), 7.67(\mathrm{~d}, J=2.0,1 \mathrm{H}, \mathrm{PzC} 3), 7.23$ $(\mathrm{d}, J=2.0,1 \mathrm{H}, \mathrm{PzA} 3), 6.44(\mathrm{t}, J=2.0,1 \mathrm{H}, \mathrm{PzB} 4), 6.41(\mathrm{t}, J=2.0,1 \mathrm{H}, \mathrm{PzC} 4), 6.29(\mathrm{t}, J=$ 2.0, 1H, PzA4), 4.12 (m, 1H, H6), 4.07 (m, 1H, H2), 3.84 (dd, J = 11.8, 9.0, 1H, H2), 3.77 (m, 1H, H3a), 2.87 (m, 2H, N-Ethyl $\mathrm{CH}_{2}$ ), 2.50 (m, 1H, H3y), 2.04 (d, $J=9.3,1 \mathrm{H}$, H7), 1.92 (m, 1H, H3x), 1.86 (m, 1H, H5), 1.54 (m, 1H, H4), 1.25 (d, J = 9.0, 9H, $\mathrm{PMe}_{3}$ ), $1.00\left(\mathrm{t}, J=7.5,3 \mathrm{H}, N\right.$-Ethyl $\left.\mathrm{CH}_{3}\right), 0.88(\mathrm{td}, J=8.0,4.6,1 \mathrm{H}, \mathrm{H} 8 \mathrm{x}), 0.04(\mathrm{q}, J=5.4,1 \mathrm{H}$, H8y). ${ }^{13} \mathrm{C}$ NMR $(d-\mathrm{MeCN}, \delta): 190.4$ (C7a), 145.3 (d, $\left.J=2.1, \mathrm{PzB} 3\right), 144.5$ (PzA3), 142.3 (PzC3), 138.7 (2C, PzC5 and PzB5), 138.2 (PzA5), 108.4 (PzB4), 108.0 (PzC4), 107.5 (PzA4), 74.4 (d, $J=14.5, \mathrm{C} 6), 54.3$ (C2), 49.8 (C7), 43.2 (C3a), 42.7 (N-Ethyl $\mathrm{CH}_{2}$ ), 29.4 (C3), 19.1 (d, $\left.J=3.3, \mathrm{C} 5\right), 17.9$ (C4), 14.7 (C8), 13.0 (d, $J=31.0, \mathrm{PMe}_{3}$ ), $11.9(N$-Ethyl-CH 3$) .{ }^{31} \mathrm{P}$ NMR $(d-\mathrm{MeCN}, \delta):-7.64\left(J_{\mathrm{wp}}=283\right) . \mathrm{IR}: \mathrm{v}_{\mathrm{BH}}=2506 \mathrm{~cm}^{-1}, \mathrm{v}_{\mathrm{NO}}$ and $v_{\text {iminium }}=1598$ and $1575 \mathrm{~cm}^{-1} . \mathrm{CV}(\mathrm{DMA}): \mathrm{E}_{\mathrm{p}, \mathrm{a}}=1.18 \mathrm{~V}$. HRMS: $\left[\mathrm{M}^{+}\right]=$ $\left[\mathrm{C}_{23} \mathrm{H}_{35} \mathrm{~N}_{8} \mathrm{OBPW}{ }^{+}\right]$obs'd (\%), calc'd (\%), ppm: 663.2242 (78), 663.2251 (84), -1.4; 664.2265 (78), $664.2276(81),-1.7 ; 665.2272$ (100), 665.2275 (100), -0.5; 666.2317 (46), $666.2316(44), 0.2 ; 667.2299(92), 667.2308(83),-1.3$.

Compound 29: To a 4-dram vial, Selectfluor ${ }^{\circledR}(0.100,0.308 \mathrm{mmol})$ was added and stirred with MeCN (4 mL) for 10 min. A solution of Compound 5 (0.101 g, 0.126 mmol) and pyrazole $(0.108 \mathrm{~g}, 1.58 \mathrm{mmol})$ was dissolved in DCM $(4 \mathrm{~mL})$. this solution was added to the Selectfluor ${ }^{\circledR}$ and the yellow solution was stirred for $1 \mathrm{~h}$. The solution was removed from the glovebox and was diluted with DCM $(20 \mathrm{~mL})$. This was treated with 2 
x $20 \mathrm{~mL}$ of $\mathrm{Na}_{2} \mathrm{CO}_{3}$ (saturated, aq). The aqueous layer was back extracted with DCM (2 x $20 \mathrm{~mL})$, and the combined organic layers were washed with deionized water $(20 \mathrm{~mL})$. This was then dried over anhydrous $\mathrm{MgSO}_{4}$ and concentrated in vacuo. The yellow oil was redissolved in minimal DCM and added dropwise to a stirring solution of $\mathrm{Et}_{2} \mathrm{O}$ (100 $\mathrm{mL})$ to induce precipitation of a light-tan solid. The powder was collected on a $15 \mathrm{~mL}$ fine-porosity fritted funnel, yielding Compound 29 (0.085 g, $0.103 \mathrm{mmol}, 82 \%) .{ }^{1} \mathrm{H}$ NMR $(d-\mathrm{MeCN}, \delta): 8.11(\mathrm{~d}, J=2.0,1 \mathrm{H}, \mathrm{PzB} 3), 8.04\left(\mathrm{t}, J=2.5,1 \mathrm{H}, \mathrm{H} 5{ }^{\prime}\right), 7.98$ (d, $J=$ 2.0, 1H, PzC5), $7.93(\mathrm{~d}, J=2.0,1 \mathrm{H}, \mathrm{PzB} 5), 7.88(\mathrm{~d}, J=2.0,1 \mathrm{H}, \mathrm{PzA} 5), 7.62(\mathrm{~d}, J=2.0$, 1H, PzC3), 7.55 (dd, $J=1.8,0.4,1 \mathrm{H}, \mathrm{H} 3$ ') 7.35 (d, $J=2.0,1 \mathrm{H}, \mathrm{PzA} 3), 6.47$ (t, $J=2.0$, $1 \mathrm{H}, \mathrm{H} 4$ ') $6.45(\mathrm{t}, J=2.0,1 \mathrm{H}, \mathrm{PzB} 4), 6.43(\mathrm{t}, J=2.0,1 \mathrm{H}, \mathrm{PzC} 4), 6.34(\mathrm{t}, J=2.0,1 \mathrm{H}$, PzA4), 6.04 (dt, $J=34.2,3.1,1 \mathrm{H}, \mathrm{H} 5), 4.93(\mathrm{dt}, J=51.9,2.2,1 \mathrm{H}, \mathrm{H} 4), 4.18$ (m, 1H, H2), $4.01(\mathrm{~m}, 1 \mathrm{H}, \mathrm{H} 2), 3.81(\mathrm{dt}, J=34.0,9.3,1 \mathrm{H}, \mathrm{H} 3 \mathrm{a}), 3.67(\mathrm{~m}, 1 \mathrm{H}, \mathrm{H} 6), 2.85(\mathrm{~m}, 1 \mathrm{H}, N-$ Ethyl $\mathrm{CH}_{2}$ ), 2.73 (m, 1H, N-Ethyl $\left.\mathrm{CH}_{2}\right), 2.52$ (m, 1H, H3), 2.35 (d, J=8.5, 1H, H7), 2.24 $(\mathrm{m}, 1 \mathrm{H}, \mathrm{H} 3), 1.06\left(\mathrm{t}, J=7.3,3 \mathrm{H}, N\right.$-Ethyl $\left.\mathrm{CH}_{3}\right), 0.90\left(\mathrm{~d}, J=9.2,9 \mathrm{H}, \mathrm{PMe}_{3}\right) .{ }^{13} \mathrm{C} \mathrm{NMR}$

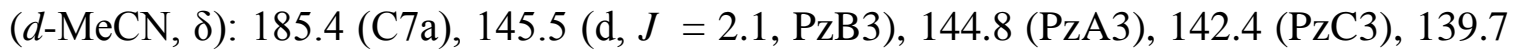
(C3’), 139.0 (PzC5), 139.9 (PzB5), 138.8 (PzA5), 130.4 (C5'), 108.6 (PzB4), 108.2 (PzC4), 107.9 (C4'), 107.8 (PzA4), 95.6 (d, $J=184.2, \mathrm{C} 4), 65.6$ (dd, $J=16.7,2.7, \mathrm{C} 5)$, $64.1(\mathrm{~d}, J=14.1, \mathrm{C} 6), 54.7$ (C2), 49.3 (C7), $43.6\left(N\right.$-Ethyl $\left.\mathrm{CH}_{2}\right), 22.3(\mathrm{C} 3), 13.4(\mathrm{~d}, J=$ 31.0, $\left.\mathrm{PMe}_{3}\right), 11.9\left(N\right.$-Ethyl $\left.\mathrm{CH}_{3}\right) .{ }^{31} \mathrm{P} \mathrm{NMR}(d-\mathrm{MeCN}, \delta):-8.81\left(J_{\mathrm{wp}}=276\right) . \mathrm{IR}: v_{\mathrm{BH}}=$ $2360 \mathrm{~cm}^{-1}, v_{\mathrm{NO}}$ and $v_{\text {iminium }}=1615$ and $1574 \mathrm{~cm}^{-1} . \mathrm{CV}(\mathrm{DMA}): \mathrm{E}_{\mathrm{p}, \mathrm{a}}=1.45 \mathrm{~V}$. HRMS: $\left[\mathrm{M}^{+}\right]=\left[\mathrm{C}_{25} \mathrm{H}_{36} \mathrm{~N}_{10} \mathrm{OBFPW}{ }^{+}\right]$obs'd (\%), calc'd (\%), ppm: 735.2353 (77), $735.2375(82)$, 
$-3.0 ; 736.2381(79), 736.2400(81),-2.6 ; 737.2373(100), 737.2399(100),-3.6 ; 738.2410$ (46), 738.2438 (46), -3.8; $739.2411(90), 739.2432(83),-2.8$.

Compound 30: To a 4-dram vial, Selectfluor ${ }^{\circledR}(0.100,0.308 \mathrm{mmol})$ was added and stirred with $\mathrm{MeCN}$ (4 mL) for $10 \mathrm{~min}$. A solution of Compound 5 (0.101 g, $0.126 \mathrm{mmol})$ and water $(1 \mathrm{~mL})$ in $\mathrm{MeCN}(4 \mathrm{~mL})$ was added to the Selectfluor ${ }^{\circledR}$. The yellow solution was stirred overnight. The solution was removed from the glovebox and was diluted with DCM $(20 \mathrm{~mL})$. This was treated with $2 \times 20 \mathrm{~mL}$ of $\mathrm{Na}_{2} \mathrm{CO}_{3}$ (saturated, aq). The aqueous layer was back extracted with DCM $(2 \times 20 \mathrm{~mL})$, and the combined organic layers were washed with deionized water $(20 \mathrm{~mL})$. This was then dried over anhydrous $\mathrm{MgSO}_{4}$ and concentrated in vacuo. The yellow oil was redissolved in minimal DCM and added dropwise to a stirring solution of $\mathrm{Et}_{2} \mathrm{O}(100 \mathrm{~mL})$ to induce precipitation of a light-tan solid. The powder was collected on a $15 \mathrm{~mL}$ fine-porosity fritted funnel, yielding Compound 30 (0.077 g, 0.099 mmol, 78\%). ${ }^{1} \mathrm{H}$ NMR ( $d$-MeCN, $\left.\delta\right): 8.09$ (d, $J=2.0,1 \mathrm{H}$, PzB3 or PzC3), 7.97 (d, $J=2.0,1 \mathrm{H}, \mathrm{PzB} 5$ or PzC5), 7.92 (d, $J=2.0,1 \mathrm{H}, \mathrm{PzB} 5$ or PzC5), $7.85(\mathrm{~d}, J=2.0,1 \mathrm{H}, \mathrm{PzA} 5), 7.64(\mathrm{~d}, J=2.0,1 \mathrm{H}, \mathrm{PzB} 3$ or $\mathrm{PzC} 3), 7.30(\mathrm{~d}, J=2.0,1 \mathrm{H}$, PzA3), $6.44(\mathrm{t}, J=2.0,2 \mathrm{H}, \mathrm{Pz} 4), 6.31(\mathrm{t}, J=2.0,1 \mathrm{H}, \mathrm{PzA} 4), 5.02(\mathrm{dd}, J=30.4,8.2,1 \mathrm{H}$, H5), 4.85 (dt, $J=52.4,1.5,1 \mathrm{H}, \mathrm{H} 4), 4.12(\mathrm{~m}, 1 \mathrm{H}, \mathrm{H} 2 \mathrm{x}), 3.96$ (d, $J=10.2,1 \mathrm{H}, \mathrm{H} 2 \mathrm{y}), 3.64$ (t, $J=9.7,1 \mathrm{H}, \mathrm{H} 3 \mathrm{a}, 3.34(\mathrm{~m}, 1 \mathrm{H}, \mathrm{H} 6), 2.83\left(\mathrm{~m}, 1 \mathrm{H}, N\right.$-Ethyl $\left.\mathrm{CH}_{2}\right), 2.71(\mathrm{~m}, 1 \mathrm{H}, N$-Ethyl $\left.\mathrm{CH}_{2}\right), 2.48(\mathrm{~m}, 1 \mathrm{H}, \mathrm{H} 3 \mathrm{x}), 2.24(\mathrm{~m}, 1 \mathrm{H}, \mathrm{H} 3 \mathrm{y}), 2.19$ (broad s, 1H, OH), $2.17(\mathrm{~d}, J=9.1$, 1H, H7), 1.30 (d, $\left.J=9.4,9 \mathrm{H}, \mathrm{PMe}_{3}\right), 1.01\left(\mathrm{t}, J=7.2,3 \mathrm{H}, N\right.$-Ethyl $\left.\mathrm{CH}_{3}\right) .{ }^{13} \mathrm{C} \mathrm{NMR}(d-$ MeCN, 8): 186.5 (C7a), 145.6 (Pz3), 144.6 (PzA3), 142.4 (Pz3), 138.8 (3C, Pz5), 108.4 (Pz4), 108.2 (Pz4), 108.0 (PzA4), 97.5 (d, $J=179.0, \mathrm{C} 4), 73.0$ (dd, $J=18.4,2.7, \mathrm{C} 5)$, 
$68.9(\mathrm{~d}, J=13.9, \mathrm{C} 6), 54.7(\mathrm{C} 2), 48.9(\mathrm{C} 7), 47.2(\mathrm{~d}, J=20.1, \mathrm{C} 3 \mathrm{a}), 43.6\left(N-\mathrm{Ethyl}_{\mathrm{CH}}\right)$, $22.4(\mathrm{~d}, J=3.8, \mathrm{C} 3), 13.9\left(\mathrm{~d}, J=29.5, \mathrm{PMe}_{3}\right), 12.0\left(N\right.$-Ethyl $\left.\mathrm{CH}_{2}\right) .{ }^{31} \mathrm{P} \mathrm{NMR}(d-\mathrm{MeCN}$, $\delta):-8.63\left(J_{\mathrm{wp}}=298\right)$. IR: $v_{\mathrm{OH}}=3145 \mathrm{~cm}^{-1} v_{\mathrm{BH}}=2511 \mathrm{~cm}^{-1}, v_{\mathrm{NO}}$ and $v_{\text {iminium }}=1613$ and $1581 \mathrm{~cm}^{-1}$. CV (DMA): $\mathrm{E}_{\mathrm{p}, \mathrm{a}}=1.37 \mathrm{~V}$. HRMS: $\left[\mathrm{M}^{+}\right]=\left[\mathrm{C}_{22} \mathrm{H}_{34} \mathrm{~N}_{8} \mathrm{O}_{2} \mathrm{BFPW}^{+}\right]$obs'd (\%), calc'd (\%), ppm: 685.2088 (75), 685.2106 (84), -2.6; 686.2124 (76), 686.2131 (80), -1.1; 687.2119 (100), 687.2130 (100), -1.6; 688.2157 (44), 688.2171 (43), -2.1; 689.2159 (88), $689.2162(84),-0.5$.

Compound 31: To a 4-dram vial, Selectfluor ${ }^{\circledR}(0.100,0.308 \mathrm{mmol})$ was added and stirred with $\mathrm{MeCN}$ (4 mL) for $10 \mathrm{~min}$. A solution of Compound $5(0.100 \mathrm{~g}, 0.125 \mathrm{mmol})$ in $\mathrm{MeOH}(4 \mathrm{~mL})$ was added to the Selectfluor ${ }^{\circledR}$. The yellow solution was stirred overnight. The solution was removed from the glovebox and was diluted with DCM (20 mL). This was treated with $2 \times 20 \mathrm{~mL}$ of $\mathrm{Na}_{2} \mathrm{CO}_{3}$ (saturated, aq). The aqueous layer was back extracted with DCM (2 x $20 \mathrm{~mL})$, and the combined organic layers were washed with deionized water $(20 \mathrm{~mL})$. This was then dried over anhydrous $\mathrm{MgSO}_{4}$ and concentrated in vacuo. The yellow oil was redissolved in minimal DCM and added dropwise to a stirring solution of $\mathrm{Et}_{2} \mathrm{O}(100 \mathrm{~mL})$ to induce precipitation of a light-tan solid. The powder was collected on a $15 \mathrm{~mL}$ fine-porosity fritted funnel, yielding Compound 31 (0.087 g, $0.108 \mathrm{mmol}, 86 \%) .{ }^{1} \mathrm{H}$ NMR $(d-\mathrm{MeCN}, \delta): 8.09$ (d, $\left.J=2.0,1 \mathrm{H}, \mathrm{PzB} 3\right), 7.97(\mathrm{~d}, J=2.0$, 1H, PzC5), $7.93(\mathrm{~d}, J=2.0,1 \mathrm{H}, \mathrm{PzB} 5), 7.85(\mathrm{~d}, J=2.0,1 \mathrm{H}, \mathrm{PzA} 5), 7.66(\mathrm{~d}, J=2.0,1 \mathrm{H}$, PzC3), $7.30(\mathrm{~d}, J=2.0,1 \mathrm{H}, \mathrm{PzA} 3), 6.44(\mathrm{t}, J=2.0,1 \mathrm{H}, \mathrm{PzB} 4), 6.42(\mathrm{t}, J=2.0,1 \mathrm{H}$, PzC4), $6.31(\mathrm{~d}, J=2.0,1 \mathrm{H}, \mathrm{PzA} 4), 5.24(\mathrm{~d}, J=52.6,1 \mathrm{H}, \mathrm{H} 4), 4.63(\mathrm{dtd}, J=30.0,2.8$, 0.9, 1H, H5), 4.14 (m, 1H, H2x), 3.98 (t, $J=10.9,1 \mathrm{H}, \mathrm{H} 2 \mathrm{y}), 3.59$ (dt, $J=34.0,10.0,1 \mathrm{H}$, 
H3a), 3.47 (s, 3H, OMe), 3.34 (m, 1H, H6), 2.82 (m, 1H, $N$-Ethyl $\left.\mathrm{CH}_{2}\right), 2.70(\mathrm{~m}, 1 \mathrm{H}, N$ Ethyl $\left.\mathrm{CH}_{2}\right), 2.50(\mathrm{~m}, 1 \mathrm{H}, \mathrm{H} 3 \mathrm{x}), 2.24(\mathrm{~m}, 1 \mathrm{H}, \mathrm{H} 3 \mathrm{y}), 2.17$ (d, $\left.J=8.8,1 \mathrm{H}, \mathrm{H} 7\right), 1.26$ (d, $J=$ 9.3, 9H, $\left.\mathrm{PMe}_{3}\right), 1.01$ (t, $J=7.2,3 \mathrm{H}, N$-Ethyl $\left.\mathrm{CH}_{3}\right) .{ }^{13} \mathrm{C} \mathrm{NMR}(d-\mathrm{MeCN}, \delta): 186.1(\mathrm{C} 7 \mathrm{a})$, 145.5 (PzB3), 144.6 (PzA3), 142.4 (PzC3), 138.8 (Pz5), 138.7 (Pz5), 138.6 (Pz5), 108.4 (Pz4), 108.0 (Pz4), 107.6 (PzA4), 91.5 (d, $J=182.0, \mathrm{C} 4), 82.1$ (dd, $J=18.2,3.0, \mathrm{C} 5)$, 66.9 (d, $J=14.0, \mathrm{C} 6), 56.4(\mathrm{OMe}), 54.6(\mathrm{C} 2), 48.6(\mathrm{C} 7), 46.6(\mathrm{~d}, J=19.0, \mathrm{C} 3 \mathrm{a}), 43.5(N-$ Ethyl $\left.\mathrm{CH}_{2}\right), 22.3(\mathrm{~d}, J=3.6, \mathrm{H} 3), 13.7\left(\mathrm{~d}, J=31.0, \mathrm{PMe}_{3}\right), 11.8\left(N\right.$-Ethyl $\left.\mathrm{CH}_{3}\right) .{ }^{31} \mathrm{P} \mathrm{NMR}$ $(d-\mathrm{MeCN}, \delta):-8.58\left(J_{\mathrm{wp}}=280\right) . \mathrm{IR}: v_{\mathrm{BH}}=2511 \mathrm{~cm}^{-1}, v_{\mathrm{NO}}$ and $v_{\text {iminium }}=1616$ and 1574 $\mathrm{cm}^{-1} . \mathrm{CV}(\mathrm{DMA}): \mathrm{E}_{\mathrm{p}, \mathrm{a}}=1.30$ V. HRMS: $\left[\mathrm{M}^{+}\right]=\left[\mathrm{C}_{23} \mathrm{H}_{36} \mathrm{~N}_{8} \mathrm{O}_{2} \mathrm{BFPW}^{+}\right]$obs'd $(\%)$, calc'd (\%), ppm: 699.2230 (76), 699.2262 (84), -4.6; 700.2264 (78), 700.2288 (81), -3.4; 701.2261 (100), 701.2287 (100), -3.7; 702.2305 (45), 702.2327 (44), -3.2; 703.2289 (87), $703.2319(83),-4.3$.

Compound 32: To a 4-dram vial, Selectfluor ${ }^{\circledR}(0.103,0.317 \mathrm{mmol})$ was added and stirred with $\mathrm{MeCN}(4 \mathrm{~mL})$ for $10 \mathrm{~min}$. A solution of Compound 5 (0.101 g, $0.126 \mathrm{mmol})$ and isopropanol $(0.5 \mathrm{~mL})$ in $\mathrm{MeCN}(4 \mathrm{~mL})$ was added to the Selectfluor ${ }^{\circledR}$. The yellow solution turned red while it stirred overnight. The solution was removed from the glovebox and was diluted with DCM $(20 \mathrm{~mL})$. This was treated with $2 \times 20 \mathrm{~mL}$ of $\mathrm{Na}_{2} \mathrm{CO}_{3}$ (saturated, aq). The aqueous layer was back extracted with DCM $(2 \times 20 \mathrm{~mL})$, and the combined organic layers were washed with deionized water $(20 \mathrm{~mL})$. This was then dried over anhydrous $\mathrm{MgSO}_{4}$ and concentrated in vacuo. The yellow oil was redissolved in minimal DCM and added dropwise to a stirring solution of $\mathrm{Et}_{2} \mathrm{O}(100 \mathrm{~mL})$ to induce precipitation of a tan solid. The powder was collected on a $15 \mathrm{~mL}$ fine-porosity 
fritted funnel, yielding Compound 32 (0.055 g, $0.067 \mathrm{mmol}, 53 \%) .{ }^{1} \mathrm{H}$ NMR ( $d$-MeCN, $\delta): 8.09$ (d, $J=2.0,1 \mathrm{H}, \mathrm{PzB} 3), 7.97$ (d, $J=2.0,1 \mathrm{H}, \mathrm{PzC} 5), 7.93$ (d, $J=2.0,1 \mathrm{H}, \mathrm{PzB} 5)$, $7.85(\mathrm{~d}, J=2.0,1 \mathrm{H}, \mathrm{PzA} 5), 7.65(\mathrm{~d}, J=2.0,1 \mathrm{H}, \mathrm{PzC} 3), 7.28(\mathrm{~d}, J=2.0,1 \mathrm{H}, \mathrm{PzA} 3)$, $6.44(\mathrm{t}, J=2.0,1 \mathrm{H}, \mathrm{PzB} 4), 6.42(\mathrm{t}, J=2.0,1 \mathrm{H}, \mathrm{PzC} 4), 6.31(\mathrm{t}, J=2.0,1 \mathrm{H}, \mathrm{PzA} 4), 5.20$ $(\mathrm{dt}, J=51.9,1.9,1 \mathrm{H}, \mathrm{H} 4), 4.90(\mathrm{dt}, J=28.8,2.6,1 \mathrm{H}, \mathrm{H} 5), 4.13(\mathrm{~m}, 1 \mathrm{H}, \mathrm{H} 2), 3.98(\mathrm{~m}$, 1H, H2), 3.95 (buried, 1H, iPr-CH), 3.61 (dt, $J=33.1,9.2,1 \mathrm{H}, \mathrm{H} 3 \mathrm{a}$ ), 3.34 (m, 1H, H6), $2.81\left(\mathrm{~m}, 1 \mathrm{H}, N\right.$-Ethyl $\left.\mathrm{CH}_{2}\right), 2.66\left(\mathrm{~m}, 1 \mathrm{H}, N\right.$-Ethyl $\left.\mathrm{CH}_{2}\right), 2.48(\mathrm{~m}, 1 \mathrm{H}, \mathrm{H} 3), 2.24(\mathrm{~m}, 1 \mathrm{H}$, H3), 2.20 (d, $J=8.9,1 \mathrm{H}, \mathrm{H} 7), 1.28\left(\mathrm{~d}, J=9.2,9 \mathrm{H}, \mathrm{PMe}_{3}\right), 1.26\left(\mathrm{~d}, J=6.1,6 \mathrm{H}, i \operatorname{Pr}-\mathrm{CH}_{3}\right)$, $1.00\left(\mathrm{t}, J=7.3,3 \mathrm{H}, N\right.$-Ethyl $\left.\mathrm{CH}_{3}\right) .{ }^{13} \mathrm{C}$ NMR $(d-\mathrm{MeCN}, \delta): 186.1$ (C7a), $145.4(\mathrm{PzB} 3)$, 144.6 (PzA3), 142.3 (PzC3), 138.8 (3C, Pz5), 108.5 (PzC4), 108.1 (PzB4), 107.7 (PzA4), $92.9(\mathrm{~d}, J=183.1, \mathrm{C} 4), 77.7$ (dd, $J=17.7,2.7, \mathrm{C} 5), 69.7(i \mathrm{PrCH}), 68.1(\mathrm{~d}, J=14.1, \mathrm{C} 6)$, 54.7 (C2), 49.1 (C7), 46.7 (C3a), $43.5\left(N\right.$-Ethyl $\left.\mathrm{CH}_{2}\right), 24.1\left(i \mathrm{PrCH}_{3}\right), 22.5(\mathrm{C} 3), 22.3$ $\left(i \mathrm{PrCH}_{3}\right), 14.1\left(\mathrm{~d}, J=30.7, \mathrm{PMe}_{3}\right), 11.9\left(N\right.$-Ethyl $\left.\mathrm{CH}_{3}\right) .{ }^{31} \mathrm{P}$ NMR $(d-\mathrm{MeCN}, \delta)$ : 8.35( $\left.J_{\mathrm{wp}}=277\right)$. IR: $v_{\mathrm{BH}}=2516 \mathrm{~cm}^{-1}, v_{\mathrm{NO}}$ and $v_{\text {iminium }}=1614$ and $1578 \mathrm{~cm}^{-1} . \mathrm{CV}(\mathrm{DMA})$ : $\mathrm{E}_{\mathrm{p}, \mathrm{a}}=1.36$ V. HRMS: $\left[\mathrm{M}^{+}\right]=\left[\mathrm{C}_{25} \mathrm{H}_{40} \mathrm{~N}_{8} \mathrm{O}_{2} \mathrm{BFPW}^{+}\right]$obs'd (\%), calc'd (\%), ppm: 727.2572 (77), 727.2576 (83), -0.5; 728.2603 (76), 728.2601 (81), 0.3; 729.2608 (100), 729.2600 (100), 1.1; 730.2646 (47), 730.2640 (45), 0.8; 731.2640 (87), 731.2632 (83), 1.0.

Compound 33: To a 4-dram vial, Selectfluor ${ }^{\circledR}(0.100 \mathrm{~g}, 0.308 \mathrm{mmol})$ was added and stirred with MeCN (4 mL) for $10 \mathrm{~min}$. A solution of Compound 5 (0.100 g, $0.125 \mathrm{mmol})$ and imidazole $(110 \mathrm{~g}, 1.62 \mathrm{mmol})$ was dissolved in DCM $(4 \mathrm{~mL})$. The solution was added to the Selectfluor ${ }^{\circledR}$ and the yellow solution was stirred for $30 \mathrm{~min}$. The solution 
was removed from the glovebox and was diluted with DCM $(20 \mathrm{~mL})$. This was treated with 2 x $20 \mathrm{~mL}$ of $\mathrm{Na}_{2} \mathrm{CO}_{3}$ (saturated, aq). The aqueous layer was back extracted with DCM (2 x $20 \mathrm{~mL})$, and the combined organic layers were washed with deionized water $(20 \mathrm{~mL})$. This was then dried over anhydrous $\mathrm{MgSO}_{4}$ and concentrated in vacuo. The yellow oil was redissolved in minimal DCM and added dropwise to a stirring solution of $\mathrm{Et}_{2} \mathrm{O}(100 \mathrm{~mL})$ to induce precipitation of a light-tan solid. The powder was collected on a 15 mL fine-porosity fritted funnel, yielding Compound 33 (0.047 g, 0.057 mmol, 45\%). ${ }^{1} \mathrm{H}$ NMR $(d-\mathrm{MeCN}, \delta): 8.11(\mathrm{~d}, J=2.0,1 \mathrm{H}, \mathrm{PzB} 3), 7.95(\mathrm{~d}, J=2.0,1 \mathrm{H}, \mathrm{PzC} 5), 7.94(\mathrm{~d}, J$ $=2.0,1 \mathrm{H}$, PzB5), $7.94(\mathrm{~m}, 1 \mathrm{H}, \mathrm{H} 2$ ') $7.88(\mathrm{~d}, J=2.0,1 \mathrm{H}, \mathrm{PzA} 5), 7.64(\mathrm{~d}, J=2.0,1 \mathrm{H}$, PzC3), 7.47 (dt, $\left.J=1.9,1.3,1 \mathrm{H}, \mathrm{H} 4^{\prime}\right), 7.34(\mathrm{~d}, J=2.0,1 \mathrm{H}, \mathrm{PzA} 3), 7.05(\mathrm{t}, J=1.0,1 \mathrm{H}$, H5'), $6.45(\mathrm{t}, J=2.0,1 \mathrm{H}, \mathrm{PzB} 4), 6.43(\mathrm{t}, J=2.0,1 \mathrm{H}, \mathrm{PzC} 4), 6.34(\mathrm{t}, J=2.0,1 \mathrm{H}, \mathrm{PzA} 4)$, $5.79(\mathrm{dt}, J=33.4,2.9,1 \mathrm{H}, \mathrm{H} 5), 4.90(\mathrm{dt}, J=52.0,2.0,1 \mathrm{H}, \mathrm{H} 4), 4.20(\mathrm{~m}, 1 \mathrm{H}, \mathrm{H} 2 \mathrm{x}), 4.02$ (t, $J=10.5,1 \mathrm{H}, \mathrm{H} 2 \mathrm{y}), 3.80(\mathrm{dt}, J=33.0,9.6,1 \mathrm{H}, \mathrm{H} 3 \mathrm{a}), 3.56(\mathrm{~m}, 1 \mathrm{H}, \mathrm{H} 6), 2.84(\mathrm{~m}, 1 \mathrm{H}$, $N$-Ethyl $\left.\mathrm{CH}_{2}\right), 2.68\left(\mathrm{~m}, 1 \mathrm{H}, N\right.$-Ethyl $\left.\mathrm{CH}_{2}\right), 2.53$ (m, 1H, H3x), 2.36 (d, $\left.J=9.2,1 \mathrm{H}, \mathrm{H} 7\right)$, $2.24(\mathrm{~m}, 1 \mathrm{H}, \mathrm{H} 3 \mathrm{y}), 1.05$ (t, $J=7.3,3 \mathrm{H}, N$-Ethyl $\left.\mathrm{CH}_{3}\right), 0.9\left(\mathrm{~d}, J=9.1,9 \mathrm{H}, \mathrm{PMe}_{3}\right) .{ }^{13} \mathrm{C}$ NMR ( $d$-MeCN, $)$ ) 184.8 (d, $J=3.5, \mathrm{C} 7 \mathrm{a}), 145.5$ (PzB3), 144.8 (PzC5), 142.5 (PzC3), 139.2 (Pz5), 139.1 (Pz5), 139.0 (Pz5), 138.3 (C2'), 130.2 (C5'), 108.8 (PzB4), 108.3 (PzC4), 108.0 (PzA4), 96.3 (d, $J=184.0, \mathrm{C} 4), 64.0$ (d, $J=14, \mathrm{C} 6), 60.7$ (dd, $J=18.0$, 2.0, C5), 54.9 (C2), 49.7 (C7), 47.1 (d, $J=19.0, \mathrm{C} 3 \mathrm{a}), 43.7\left(N\right.$-Ethyl $\left.\mathrm{CH}_{2}\right), 22.4(\mathrm{~d}, J=3$, C3), $13.7\left(\mathrm{~d}, J=31.0, \mathrm{PMe}_{3}\right), 12.0\left(N\right.$-Ethyl $\left.\mathrm{CH}_{3}\right) .{ }^{31} \mathrm{P}$ NMR $(d-\mathrm{MeCN}, \delta):-9.04\left(J_{\mathrm{wp}}=\right.$ 276). IR: $v_{\mathrm{BH}}=2511 \mathrm{~cm}^{-1}, v_{\mathrm{NO}}$ and $v_{\text {iminium }}=1616$ and $1581 \mathrm{~cm}^{-1} . \mathrm{CV}(\mathrm{DMA}): \mathrm{E}_{\mathrm{p}, \mathrm{a}}=$ 1.51 V. HRMS: $\left[\mathrm{M}^{+}\right]=\left[\mathrm{C}_{25} \mathrm{H}_{36} \mathrm{~N}_{10} \mathrm{OBFPW}^{+}\right]$obs'd (\%), calc'd (\%), ppm: $735.2376(76)$, 
735.2375 (82), 0.1; 736.2405 (79), 736.2400 (81), 0.7; 737.2398 (100), 737.2399 (100), 0.2; 738.2436 (43), 738.2438 (46), -0.3; 739.2411 (83), 739.2432 (83), -2.8.

Compound 34: In an oven dried round bottom flask, a mixture of Compound 5 (503 mg, $0.628 \mathrm{mmol})$ and pyrazole $(213 \mathrm{mg}, 3.12 \mathrm{mmol})$ was dissolved in DCM (10 mL). A solution of NBS (224 mg, $1.26 \mathrm{mmol})$ in $\mathrm{MeCN}(10 \mathrm{~mL})$ was added to the reaction mixture and stirred 10 minutes. The mixture was removed from the glovebox and concentrated in vacuo. The yellow oil was redissolved in minimal DCM and added dropwise to a stirring solution of $\mathrm{Et}_{2} \mathrm{O}(500 \mathrm{~mL})$ to induce precipitation of a tan solid. The powder was collected on a $30 \mathrm{~mL}$ fine porosity fritted funnel, yielding Compound 34 (0.468 g, $0.494 \mathrm{mmol}, 79 \%) .{ }^{1} \mathrm{H}$ NMR ( $d$-MeCN, $\left.\delta\right): 8.25$ (d, $\left.J=2.0,1 \mathrm{H}, \mathrm{PzA} 3\right), 8.10$ (d, $J=2.0,1 \mathrm{H}, \mathrm{PzB} 3), 7.98$ (d, $J=2.0, \mathrm{H}, \mathrm{PzC} 5), 7.93$ (d, $J=2.0,1 \mathrm{H}, \mathrm{PzA} / \mathrm{B} 5), 7.87$ (d, $J=2.0,1 \mathrm{H}, \mathrm{PzA} 5 / \mathrm{B} 5), 7.69$ (d, $J=2.0,1 \mathrm{H}, \mathrm{PzC} 3), 7.56$ (d, $J=2.0,1 \mathrm{H}, \mathrm{C} 3$ '), 7.37 (d, $J=2.0,1 \mathrm{H}, \mathrm{C} 5$ '), 6.44 (m, 3H, Pz4), $6.33(\mathrm{t}, J=2.0,1 \mathrm{H}, \mathrm{H} 4$ ') $, 6.17(\mathrm{~m}, 1 \mathrm{H}, \mathrm{H} 5), 4.81(\mathrm{t}$, $J=3.0,1 \mathrm{H}, \mathrm{H} 4), 4.20(\mathrm{~m}, 1 \mathrm{H}, \mathrm{H} 2 \mathrm{x}), 4.06$ (m, 1H, H2y), 3.95 (t, $J=8.7,1 \mathrm{H}, \mathrm{H} 3 \mathrm{a}), 3.80$ (m, 1H, H6), 2.87 (m, 1H, N-Ethyl $\left.\mathrm{CH}_{2}\right), 2.69$ (m, 1H, N-Ethyl $\left.\mathrm{CH}_{2}\right), 2.57$ (m, 1H, H3x), 2.37 (d, $J=8.8,1 \mathrm{H}, \mathrm{H} 7), 2.29$ (m, 1H, H3y), 1.08 (t, $J=7.2,3 \mathrm{H}, \mathrm{N}$-Ethyl $\mathrm{CH}_{3}$ ), 0.88 (d, $\left.J=9.2,9 \mathrm{H}, \mathrm{PMe}_{3}\right) .{ }^{13} \mathrm{C} \mathrm{NMR}(d-\mathrm{MeCN}, \delta): 185.7$ (C7a), 145.5 (PzB3), 144.7 (C5'), 142.6 (PzC3), 139.8 (C3’), 139.0 (Pz5), 138.9 (Pz5), 138.8 (Pz5), 130.4 (PzA3), 108.5 (Pz4), 108.1 (Pz4), 107.7 (Pz4), 107.0 (C4'), 67.2 (2C, C4 and C5), 64.3 (d, $J=14.1$, C6), 55.3 (C2), 48.9 (C7), 48.5 (C3a), 43.7 (N-Ethyl $\mathrm{CH}_{2}$ ), 24.8 (C3), 13.5 (d, $J=30.5$, $\left.\mathrm{PMe}_{3}\right), 11.9\left(\mathrm{~N}-\mathrm{Ethyl} \mathrm{CH}_{3}\right) .{ }^{31} \mathrm{P} \mathrm{NMR}\left(\mathrm{CH}_{2} \mathrm{Cl}_{2}, \delta\right):-9.23\left(J_{\mathrm{wp}}=272\right) . \mathrm{IR}: v_{\mathrm{BH}}=2503 \mathrm{~cm}^{-}$ ${ }^{1}, v_{\mathrm{NO}}$ and $v_{\text {iminium }}=1620$ and $1577 \mathrm{~cm}^{-1} . \mathrm{CV}(\mathrm{DMA}): \mathrm{E}_{\mathrm{p}, \mathrm{a}}=1.46 \mathrm{~V}$. 
Compound 35: A solution of N-chlorosuccidimide (0.040 g, $0.299 \mathrm{mmol})$ in $\mathrm{MeCN}$ (2 $\mathrm{mL})$ was added to a mixture of Compound $5(0.101 \mathrm{~g}, 0.126 \mathrm{mmol})$ and aniline $(0.130 \mathrm{~g}$, $1.40 \mathrm{mmol})$ in $\mathrm{MeCN}(2 \mathrm{~mL})$, resulting in a dark yellow, homogenous solution. The solution stirred for 4 hour. The mixture was concentrated in vacuo. The yellow oil was redissolved in minimal DCM and added dropwise to a stirring solution of $\mathrm{Et}_{2} \mathrm{O}(100 \mathrm{~mL})$ to induce precipitation of a light-tan solid. The powder was collected on a $15 \mathrm{~mL}$ fine porosity fritted funnel, washed with $\mathrm{Et}_{2} \mathrm{O}(10 \mathrm{~mL})$, yielding Compound 35 (0.098 g, 0.106 mmol, $84 \%) .{ }^{1} \mathrm{H}$ NMR $(d-\mathrm{MeCN}, \delta): 8.11(\mathrm{~d}, J=2.0,1 \mathrm{H}, \mathrm{PzB} 3), 7.99(\mathrm{~d}, J=2.0$, 1H, PzC5), $7.93(\mathrm{~d}, J=2.0,1 \mathrm{H}, \mathrm{PzB} 5), 7.86(\mathrm{~d}, J=2.0,1 \mathrm{H}, \mathrm{PzA} 5), 7.62(\mathrm{~d}, J=2.0,1 \mathrm{H}$, PzC3), 7.36 (d, $J=2.0,1 \mathrm{H}, \mathrm{PzA} 3), 7.23$ (t, $J=7.7,2 \mathrm{H}, \mathrm{H} 2$ ' \& H6'), 6.79 (d, $J=8.2,2 \mathrm{H}$, H3' \& H5'), 6.77 (tt, $\left.J=7.3,0.8,1 \mathrm{H}, \mathrm{H} 4^{\prime}\right), 6.47$ (t, $\left.J=2.0,1 \mathrm{H}, \mathrm{PzC} 4\right), 6.44$ (t, $J=2.0$, 1H, PzB4), 6.32 (t, $J=2.0,1 \mathrm{H}, \mathrm{PzA} 4), 5.31(\mathrm{dt}, J=11.1,3.2,1 \mathrm{H}, \mathrm{H} 5), 4.88($ broad s, $1 \mathrm{H}$, NH), 4.87 (m, 1H, H4), $4.13(\mathrm{~m}, 1 \mathrm{H}, \mathrm{H} 2 \mathrm{x}), 4.00$ (t, $J=11.1,1 \mathrm{H}, \mathrm{H} 2 \mathrm{y}), 3.90(\mathrm{t}, J=8.2$, 1H, H3a), 3.33 (m, 1H, H6), 2.81 (m, 1H, N-Ethyl $\left.\mathrm{CH}_{2}\right), 2.63$ (m, 1H, N-Ethyl $\mathrm{CH}_{2}$ ), $2.51(\mathrm{~m}, 1 \mathrm{H}, \mathrm{H} 3 \mathrm{x}), 2.23$ (m, 1H, H3y), 2.19 (d, $J=8.7,2 \mathrm{H}, \mathrm{H} 7), 1.23$ (d, $J=9.6,9 \mathrm{H}$, $\left.\mathrm{PMe}_{3}\right), 1.03\left(\mathrm{t}, J=7.3,3 \mathrm{H}, \mathrm{N}-\mathrm{Ethyl} \mathrm{CH}_{3}\right) .{ }^{13} \mathrm{C} \mathrm{NMR}(d-\mathrm{MeCN}, \delta): 184.8(\mathrm{C} 7 \mathrm{a}), 147.2$ (C1'), 145.8 (PzB3), 144.7 (PzA3), 142.2 (PzC3), 138.9 (Pz5), 138.8 (Pz5), 138.6 (Pz5), 130.6 (C2’ \& C6’), 119.3 (C4'), 114.8 (C3’ \& C5’) 108.4 (Pz4), 108.2 (Pz4), 107.6 (PzA4), 70.0 (C4), 66.0 (d, $J=14.8, \mathrm{C} 6), 57.5$ (d, $J=2.8, \mathrm{C} 5), 55.1(\mathrm{C} 2), 49.6$ (C7), 48.5 (C3a), 43.5 (N-Ethyl $\mathrm{CH}_{2}$ ), 23.6 (C3), 14.1 (d, $\left.J=30.9,1 \mathrm{H}, \mathrm{PMe}_{3}\right), 11.9$ (N-Ethyl $\left.\mathrm{CH}_{3}\right)$. ${ }^{31} \mathrm{P}$ NMR $(d-\mathrm{MeCN}, \delta):-8.62\left(J_{\mathrm{wp}}=280\right) . \mathrm{IR}: v_{\mathrm{BH}}=2401 \mathrm{~cm}^{-1}, v_{\mathrm{NO}}$ and $v_{\text {iminium }}=1577$ and $1620 \mathrm{~cm}^{-1}$. CV (DMA): $\mathrm{E}_{\mathrm{p}, \mathrm{a}}=1.20 \mathrm{~V}$. 
Compound 36: A solution of N-chlorosuccidimide (0.068 g, $0.509 \mathrm{mmol})$ in $\mathrm{MeCN}$ (4 mL)was added to a mixture of Compound $5(0.200 \mathrm{~g}, 0.249 \mathrm{mmol})$ and pyrazole $(0.086$ $\mathrm{g}, 1.26 \mathrm{mmol})$ in DCM (4 mL), resulting in a dark yellow, homogenous solution. The solution stirred for 1 hour. The mixture was removed from the glovebox and was diluted with DCM (40 mL). This was treated with $2 \times 40 \mathrm{~mL}$ of $\mathrm{Na}_{2} \mathrm{CO}_{3}$ (saturated, aq). The aqueous layer was back extracted with DCM (2 x $20 \mathrm{~mL})$. This was then dried over anhydrous $\mathrm{MgSO}_{4}$ and concentrated in vacuo. The yellow oil was redissolved in minimal DCM and added dropwise to a stirring solution of $\mathrm{Et}_{2} \mathrm{O}(200 \mathrm{~mL})$ to induce precipitation of a light-tan solid. The powder was collected on a $30 \mathrm{~mL}$ fine porosity fritted funnel, washed with $\mathrm{Et}_{2} \mathrm{O}(10 \mathrm{~mL})$, yielding Compound $36(0.103 \mathrm{~g}, 0.114 \mathrm{mmol}, 46 \%) .{ }^{1} \mathrm{H}$ NMR ( $d$-Acetone, $\delta): 8.40(\mathrm{~d}, J=2.0,1 \mathrm{H}, \mathrm{PzA} 3), 8.28(\mathrm{~d}, J=2.0,1 \mathrm{H}, \mathrm{PzB} 3), 8.15(\mathrm{~d}, J$ $=2.0,1 \mathrm{H}$, PzC5), $8.09(\mathrm{~d}, J=2.0,1 \mathrm{H}, \mathrm{PzB} 5), 8.02(\mathrm{~d}, J=2.0,1 \mathrm{H}, \mathrm{PzA} 5), 7.98(\mathrm{~d}, J=$ 2.0, 1H, PzC3), 7.61 (d, $\left.J=2.0,1 \mathrm{H}, \mathrm{H} 5^{\prime}\right), 7.56\left(\mathrm{~d}, J=2.0,1 \mathrm{H}, \mathrm{H} 3^{\prime}\right), 6.53(\mathrm{t}, J=2.0,1 \mathrm{H}$, PzB4), 6.52 (t, $J=2.0,1 \mathrm{H}, \mathrm{PzC} 4), 6.44(\mathrm{t}, J=2.0,1 \mathrm{H}, \mathrm{PzA} 4), 6.41\left(\mathrm{t}, J=2.0,1 \mathrm{H}, \mathrm{H} 4{ }^{\prime}\right)$, $6.35(\mathrm{~m}, 1 \mathrm{H}, \mathrm{H} 5), 4.81(\mathrm{t}, J=2.9,1 \mathrm{H}, \mathrm{H} 4), 4.50(\mathrm{~m}, 1 \mathrm{H}, \mathrm{H} 2 \mathrm{x}), 4.26(\mathrm{~m}, 1 \mathrm{H}, \mathrm{H} 2 \mathrm{y}), 4.10$ (t, $J=8.4,1 \mathrm{H}, \mathrm{H} 3 \mathrm{a}), 4.02(\mathrm{~m}, 1 \mathrm{H}, \mathrm{H} 6), 3.10\left(\mathrm{~m}, 1 \mathrm{H}, \mathrm{N}-\mathrm{Ethyl} \mathrm{CH}_{2}\right), 2.90$ (m, 1H, N-Ethyl $\left.\mathrm{CH}_{2}\right), 2.73(\mathrm{~m}, 1 \mathrm{H}, \mathrm{H} 3 \mathrm{x}), 2.54(\mathrm{~d}, J=8.7,1 \mathrm{H}, \mathrm{H} 7), 2.39(\mathrm{~m}, 1 \mathrm{H}, \mathrm{H} 3 \mathrm{y}), 1.18(\mathrm{t}, J=7.2$, 3H, N-Ethyl $\left.\mathrm{CH}_{3}\right), 1.00\left(\mathrm{~d}, J=9.2,9 \mathrm{H}, \mathrm{PMe}_{3}\right) .{ }^{13} \mathrm{C}$ NMR $(d$-Acetone, $\delta): 185.6(\mathrm{C} 7 \mathrm{a})$, 145.8 (PzB3), 145.2 (C5'), 142.8 (PzC3), 139.7 (C3’), 139.1 (Pz5), 139.0 (Pz5), 138.9 (Pz5), 130.6 (PzA3), 108.6 (Pz4), 108.2 (Pz4), 107.8 (Pz4), 107.1 (C4'), 71.5 (C4), 67.4 (d, $J=2.9$, C5), 63.8 (d, $J=14.8$, C6), 55.3 (C2), 49.4 (C7), 48.9 (C3a), 43.8 (N-Ethyl $\left.\mathrm{CH}_{2}\right), 23.7(\mathrm{C} 3), 13.6\left(\mathrm{~d}, J=30.8, \mathrm{PMe}_{3}\right), 12.1\left(\mathrm{~N}-\mathrm{Ethyl} \mathrm{CH}_{3}\right) .{ }^{31} \mathrm{P} \mathrm{NMR}\left(\mathrm{CH}_{2} \mathrm{Cl}_{2}, \delta\right)$ : - 
$8.83\left(J_{\mathrm{wp}}=275\right)$. IR: $v_{\mathrm{BH}}=2519 \mathrm{~cm}^{-1}, v_{\mathrm{NO}}$ and $v_{\text {iminium }}=1620$ and $1577 \mathrm{~cm}^{-1} . \mathrm{CV}(\mathrm{DMA})$ : $\mathrm{E}_{\mathrm{p}, \mathrm{a}}=1.43 \mathrm{~V} .\left[\mathrm{M}^{+}=\mathrm{C}_{25} \mathrm{H}_{36} \mathrm{~N}_{10} \mathrm{OBPClW}^{+}\right]=$obs'd (\%), calc'd (\%), ppm: 751.2061 (68), 751.2080 (65), -2.5; 752.2076 (76), 752.2103 (69), -3.5; 753.2080 (100), 753.2093 (100), -1.7; 754.2109 (59), 754.2118 (57), -1.2; 755.2108 (100), 755.2119 (91), -1.4; 756.2148 (33), 756.2142 (31), 0.8; 757.2087 (29), $757.2116(24),-3.9$.

Compound 37: A solution of N-chlorosuccidimide $(0.067 \mathrm{~g}, 0.501 \mathrm{mmol})$ in $\mathrm{MeOH}(2$ $\mathrm{mL}$ )was added to a mixture of Compound $5(0.201 \mathrm{~g}, 0.251 \mathrm{mmol})$ in $\mathrm{MeOH}(2 \mathrm{~mL})$, resulting in a dark yellow, homogenous solution. The solution stirred for $30 \mathrm{~min}$. The mixture was removed from the glovebox and was diluted with DCM (50 mL). This was treated with $2 \times 40 \mathrm{~mL}$ of $\mathrm{Na}_{2} \mathrm{CO}_{3}$ (saturated, aq). The aqueous layer was back extracted with DCM $(2 \times 20 \mathrm{~mL})$. This was then dried over anhydrous $\mathrm{MgSO}_{4}$ and concentrated in vacuo. The yellow oil was redissolved in minimal DCM and added dropwise to a stirring solution of $\mathrm{Et}_{2} \mathrm{O}(200 \mathrm{~mL})$ to induce precipitation of a light-tan solid. The powder was collected on a $30 \mathrm{~mL}$ fine porosity fritted funnel, washed with $\mathrm{Et}_{2} \mathrm{O}(10 \mathrm{~mL})$, yielding Compound 37 (0.176 g, 0.203 mmol, $81 \%) .{ }^{1} \mathrm{H}$ NMR $(d-\mathrm{MeCN}, \delta): 8.09(\mathrm{~d}, J=2.0,1 \mathrm{H}$, PzB3), $7.97(\mathrm{~d}, J=2.0,1 \mathrm{H}, \mathrm{PzC} 5), 7.93(\mathrm{~d}, J=2.0,1 \mathrm{H}, \mathrm{PzB} 5), 7.85(\mathrm{~d}, J=2.0,1 \mathrm{H}$, PzA5), $7.64(\mathrm{~d}, J=2.0,1 \mathrm{H}, \mathrm{PzC} 3), 7.32(\mathrm{~d}, J=2.0,1 \mathrm{H}, \mathrm{PzA} 3), 6.44(\mathrm{t}, J=2.0,1 \mathrm{H}$, PzB4), $6.42(\mathrm{t}, J=2.0,1 \mathrm{H}, \mathrm{PzC} 4), 6.30(\mathrm{t}, J=2.0,1 \mathrm{H}, \mathrm{PzA} 4), 4.93(\mathrm{td}, J=3.3,1.0,1 \mathrm{H}$, H4), $4.81(\mathrm{t}, J=2.76,1 \mathrm{H}, \mathrm{H} 5), 4.14(\mathrm{~m}, 1 \mathrm{H}, \mathrm{H} 2 \mathrm{x}), 4.03(\mathrm{td}, J=11.4,2.5,1 \mathrm{H}, \mathrm{H} 2 \mathrm{y}), 3.77$ (t, $J=9.1,1 \mathrm{H}, \mathrm{H} 31), 3.42(\mathrm{~s}, 3 \mathrm{H}, \mathrm{OMe}), 3.33(\mathrm{qd}, J=9.4,3.0,1 \mathrm{H}, \mathrm{H} 6), 2.85(\mathrm{~m}, 1 \mathrm{H}, \mathrm{N}-$ Ethyl $\mathrm{CH}_{2}$ ), 2.66 (m, 1H, N-Ethyl $\left.\mathrm{CH}_{2}\right), 2.51$ (m, 1H, H3x), 2.31 (m, 1H, H3y), 2.19 (d, J $=7.7,1 \mathrm{H}, \mathrm{H} 7), 1.26\left(\mathrm{~d}, J=9.5,9 \mathrm{H}, \mathrm{PMe}_{3}\right), 1.03\left(\mathrm{t}, J=7.6,3 \mathrm{H}, \mathrm{N}-\mathrm{Ethyl} \mathrm{CH}_{3}\right) .{ }^{13} \mathrm{C} \mathrm{NMR}$ 


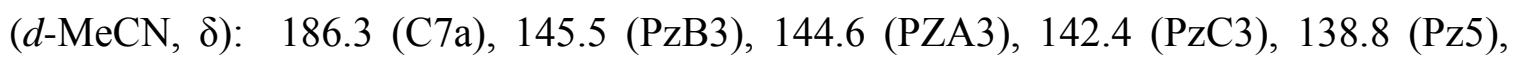
138.7 (Pz5), 138.6 (Pz5), 108.4 (Pz4), 107.9 (Pz4), 107.6 (PzA4), 83.0 (d, J = 2.7, C5), 67.6 (C4), 67.4 (d, J = 14.4, C6), 56.2 (OMe), 55.1 (C2), 48.4 (C7), 47.4 (C3a), 43.5 (NEthyl $\left.\mathrm{CH}_{2}\right), 23.1(\mathrm{C} 3), 13.7$ (d, $\left.J=31.0, \mathrm{PMe}_{3}\right), 11.9\left(\mathrm{~N}-\right.$ Ethyl $\left.\mathrm{CH}_{3}\right) .{ }^{31} \mathrm{P} \mathrm{NMR}(d-\mathrm{MeCN}$, $\delta):-8.80\left(J_{\mathrm{wp}}=283\right) . \mathrm{IR}: \mathrm{v}_{\mathrm{BH}}=2359 \mathrm{~cm}^{-1}, v_{\mathrm{NO}}$ and $v_{\text {iminium }}=1576$ and $1617 \mathrm{~cm}^{-1} . \mathrm{CV}$ $(\mathrm{DMA}): \mathrm{E}_{\mathrm{p}, \mathrm{a}}=1.39 \mathrm{~V}$.

Compound 39: A solution of N-chlorosuccidimide (0.033 g, $0.247 \mathrm{mmol})$ in $\mathrm{MeCN}$ (2 $\mathrm{mL})$ was added to a mixture of Compound $5(0.100 \mathrm{~g}, 0.125 \mathrm{mmol})$ and imidazole $(0.084$ $\mathrm{g}, 1.23 \mathrm{mmol})$ in $\mathrm{MeCN}(2 \mathrm{~mL})$, resulting in a dark yellow, homogenous solution. The solution stirred for $30 \mathrm{~min}$. The mixture was removed from the glovebox and concentrated in vacuo. The yellow oil was redissolved in minimal DCM and added dropwise to a stirring solution of $\mathrm{Et}_{2} \mathrm{O}(100 \mathrm{~mL})$ to induce precipitation of a light-tan solid. The powder was collected on a $30 \mathrm{~mL}$ fine porosity fritted funnel, washed with $\mathrm{Et}_{2} \mathrm{O}(10 \mathrm{~mL})$, yielding Compound $39(0.100 \mathrm{~g}, 0.111 \mathrm{mmol}, 89 \%) .{ }^{1} \mathrm{H}$ NMR $(d-\mathrm{MeCN}$, $\delta): 8.11(\mathrm{~d}, J=2.0,1 \mathrm{H}, \mathrm{PzB} 3), 7.99(\mathrm{~d}, J=2.0,1 \mathrm{H}, \mathrm{Pz} 5), 7.93(\mathrm{~d}, J=2.0,1 \mathrm{H}, \mathrm{Pz} 5), 7.88$ (d, $J=2.0,1 \mathrm{H}, \mathrm{Pz} 5), 7.70(\mathrm{~d}, J=2.0,1 \mathrm{H}, \mathrm{PzC} 3), 7.57$ (broad s, 1H, H2'), 7.37 (d, $J=$ 2.0, 1H, PzA3), 7.24 (broad s, 1H, H4'/H5'), 7.09 (broad s, 1H, H4'/H5'), 6.44 (m, 2H, PzB4 \& PzC4), 6.34 (t, $J=2.0,1 \mathrm{H}, \mathrm{PzA} 4), 6.04$ (t, $J=3.9,1 \mathrm{H}, \mathrm{H} 5), 4.67$ (t, $J=2.6,1 \mathrm{H}$, H4), 4.18 (m, 1H, H2x), 4.06 (m, 1H, H2y), 4.02 (t, $J=8.6,1 \mathrm{H}, \mathrm{H} 3 \mathrm{a}), 3.61$ (m, 1H, H6), $2.86\left(\mathrm{~m}, 1 \mathrm{H}, \mathrm{N}-\right.$ Ethyl $\left.\mathrm{CH}_{2}\right), 2.66\left(\mathrm{~m}, 1 \mathrm{H}, \mathrm{N}\right.$-Ethyl $\left.\mathrm{CH}_{2}\right), 2.56(\mathrm{~m}, 1 \mathrm{H}, \mathrm{H} 3 \mathrm{x}), 2.35$ (d, $J=$ 8.8, 1H, H7), 2.29 (m, 1H, H3y), 1.07 (t, $J=7.2,3 \mathrm{H}, \mathrm{N}-$ Ethyl $\left.\mathrm{CH}_{3}\right), 0.87$ (d, $J=9.1,9 \mathrm{H}$, $\left.\mathrm{PMe}_{3}\right) .{ }^{13} \mathrm{C}$ NMR $(d-\mathrm{MeCN}, \delta): 184.8$ (C7a), 145.4 (PZB3), 144.7 (PzA3), 142.6 (PzC3), 
139.0 (Pz5), 138.9 (Pz5), 138.8 (Pz5), 129.5 (C4'/C5'), 122.9 (C2'), 120.9 (C4'/C5'), 108.6 (Pz4), 108.2 (Pz4), 107.8 (PzA4), 72.1 (C4), 63.7 (d, $J=14.1, \mathrm{C} 6), 62.5$ (C5), 55.4 (C2), 49.4 (C7), 48.4 (C3a), $43.7\left(\mathrm{~N}-\mathrm{Ethyl} \mathrm{CH}_{2}\right), 23.3(\mathrm{C} 3), 13.6$ (d, $\left.J=31.0, \mathrm{PMe}_{3}\right)$, $11.9\left(\mathrm{~N}-\right.$ Ethyl $\left.\mathrm{CH}_{3}\right) .{ }^{31} \mathrm{P}$ NMR $(d-\mathrm{MeCN}, \delta):-9.02\left(J_{\mathrm{wp}}=280\right) . \mathrm{IR}: v_{\mathrm{BH}}=2507 \mathrm{~cm}^{-1}, v_{\mathrm{NO}}$ and $v_{\text {iminium }}=1573$ and $1617 \mathrm{~cm}^{-1} . \mathrm{CV}(\mathrm{DMA}): \mathrm{E}_{\mathrm{p}, \mathrm{a}}=1.11 \mathrm{~V}$.

Compound 40: A solution of $\mathrm{N}$-iodosuccidimide (0.028 g, $0.125 \mathrm{mmol})$ in $\mathrm{MeCN}(0.5$ $\mathrm{mL})$ was added to a mixture of Compound $5(0.050 \mathrm{~g}, 0.062 \mathrm{mmol})$ and pyrazole $(0.026$ $\mathrm{g}, 0.381 \mathrm{mmol})$ in DCM $(0.5 \mathrm{~mL})$, resulting in a dark yellow, homogenous solution. The solution stirred for 1 hour. The mixture was removed from the glovebox and was diluted with DCM $(20 \mathrm{~mL})$. This was treated with $2 \times 20 \mathrm{~mL}$ of $\mathrm{Na}_{2} \mathrm{CO}_{3}$ (saturated, aq). The aqueous layer was back extracted with DCM $(2 \times 10 \mathrm{~mL})$. This was then dried over anhydrous $\mathrm{MgSO}_{4}$ and concentrated in vacuo. The yellow oil was redissolved in minimal DCM and added dropwise to a stirring solution of $\mathrm{Et}_{2} \mathrm{O}(50 \mathrm{~mL})$ to induce precipitation of a brown solid. The powder was collected on a $15 \mathrm{~mL}$ fine porosity fritted funnel, washed with $\mathrm{Et}_{2} \mathrm{O}(\mathrm{x} 0 \mathrm{~mL})$, yielding Compound 40 (0.038 g, $\left.0.041 \mathrm{mmol}, 66 \%\right) .{ }^{1} \mathrm{H}$ NMR $(d-\mathrm{MeCN}, \delta): 8.21(\mathrm{~d}, J=2.0,1 \mathrm{H}, \mathrm{PzB} 3), 7.97(\mathrm{~d}, J=2.0,1 \mathrm{H}, \mathrm{PzC} 5), 7.93(\mathrm{~d}, J=$ 2.0, 1H, PzB5), 7.83 (d, $J=2.0,1 \mathrm{H}, \mathrm{PzA} 5), 7.67$ (d, $J=2.0,1 \mathrm{H}, \mathrm{PzC} 3), 7.40$ (d, $J=2.0$, 1H, PzA3), 7.17 (dd, $J=2.9,2.6,1 \mathrm{H}, \mathrm{H} 5), 6.44(\mathrm{t}, J=2.0,1 \mathrm{H}, \mathrm{PzB} 4), 6.43(\mathrm{t}, J=2.0$, 1H, PzC4), 6.30 (t, J = 2.0, 1H, PzA4), 4.18 (m, 1H, H3a), 4.15 (m, 1H, H2x), 3.94 (dd, $J$ $=12.9,9.1,1 \mathrm{H}, \mathrm{H} 2 \mathrm{y}), 3.88(\mathrm{~m}, 1 \mathrm{H}, \mathrm{H} 6), 3.07$ (m, 2H, N-Ethyl $\left.\mathrm{CH}_{2}\right), 2.60(\mathrm{~m}, 1 \mathrm{H}, \mathrm{H} 3 \mathrm{x})$, $2.46(\mathrm{~d}, J=7.9,1 \mathrm{H}, \mathrm{H} 7), 2.02(\mathrm{~m}, 1 \mathrm{H}, \mathrm{H} 3 \mathrm{y}), 1.24\left(\mathrm{~d}, J=9.2,9 \mathrm{H}, \mathrm{PMe}_{3}\right), 1.08(\mathrm{t}, J=7.2$ 3H, N-Ethyl $\left.\mathrm{CH}_{3}\right) .{ }^{13} \mathrm{C}$ NMR $(d-\mathrm{MeCN}, \delta): 188.4$ (C7a), 145.6 (PzB3), 143.3 (C5), 
143.1 (PzA3), 142.5 (PzC3), 138.8 (Pz5), 138.7 (Pz5), 138.2 (PzA5), 108.4 (Pz4), 107.9 (Pz4), 107.4 (PzA4), 81.5 (C4), 72.7 (d, J = 13.1, C6), 53.6 (C2), 52.2 (C3a), 49.2 (C7), 44.2 (N-Ethyl $\left.\mathrm{CH}_{2}\right), 32.9$ (C3), 13.4 (d, $\left.J=30.5, \mathrm{PMe}_{3}\right), 11.8\left(\mathrm{~N}-\mathrm{Ethyl} \mathrm{CH}_{3}\right) .{ }^{31} \mathrm{P} \mathrm{NMR}$ $\left(\mathrm{CH}_{2} \mathrm{Cl}_{2}, \delta\right):-9.00\left(J_{\mathrm{wp}}=283\right) . \mathrm{IR}: v_{\mathrm{BH}}=2480 \mathrm{~cm}^{-1}, v_{\mathrm{NO}}$ and $v_{\text {iminium }}=1601$ and $1581 \mathrm{~cm}^{-}$ 1. $\mathrm{CV}(\mathrm{DMA}): \mathrm{E}_{\mathrm{p}, \mathrm{a}}=1.11 \mathrm{~V} \cdot\left[\mathrm{M}^{+}=\mathrm{C}_{22} \mathrm{H}_{32} \mathrm{~N}_{8} \mathrm{OBPIW}^{+}\right]=$obs'd (\%), calc'd (\%), ppm: 775.1062 (85), 775.1061 (84), 0.1; 776.1093 (79), 776.1086 (80), 0.9; 777.1097 (100), 777.1085 (100), 1.6; 778.1141 (44), 778.1126 (43), 1.9; 779.1125 (86), 779.1117 (84), 1.0 .

Compound 41: In a 4-dram vial, mCPBA $(0.032 \mathrm{~g}, 0.185 \mathrm{mmol})$ was dissolved in $\mathrm{MeCN}$ ( $1 \mathrm{~mL})$. To this, a solution of Compound 5 (0.050 g, $0.062 \mathrm{mmol})$ in $\mathrm{MeCN}$ (1 mL) was added. This solution was stirred for $10 \mathrm{~min}$. The yellow solution was added dropwise to a stirring solution of $\mathrm{Et}_{2} \mathrm{O}(50 \mathrm{~mL})$ to induce precipitation of a tan solid. The powder was collected on a $15 \mathrm{~mL}$ fine-porosity fritted funnel, yielding Compound 41 (0.013 g, 0.013 mmol, 21\%). ${ }^{1} \mathrm{H}$ NMR (d-Acetone, $\left.\delta\right): 8.24(\mathrm{~d}, J=2.0,1 \mathrm{H}, \mathrm{PzB} 3), 8.11(\mathrm{~d}, J=2.0,1 \mathrm{H}$, PzC5), $8.08(\mathrm{~d}, J=2.0,1 \mathrm{H}, \mathrm{PzB} 5), 7.99$ (m, 2H, PzA5 and H7'), 7.97 (t, $J=1.2,1 \mathrm{H}$, H5'), 7.93 (d, $J=2.0,1 \mathrm{H}, \mathrm{PzC} 3), 7.67$ (dq, $\left.J=8.0,2.1,1 \mathrm{H}, \mathrm{H} 4^{\prime}\right), 7.55$ (m, 1H, H3'), $7.45(\mathrm{~d}, J=2.0,1 \mathrm{H}, \mathrm{PzA} 3), 6.52(\mathrm{t}, J=2.0,1 \mathrm{H}, \mathrm{PzB} 4), 6.50(\mathrm{t}, J=2.0,1 \mathrm{H}, \mathrm{PzC} 4), 6.39$ $(\mathrm{t}, J=2.0,1 \mathrm{H}, \mathrm{PzA} 4), 5.21(\mathrm{~d}, J=5.4,1 \mathrm{H}, \mathrm{H} 5), 4.58(\mathrm{~m}, 1 \mathrm{H}, \mathrm{H} 4), 4.40(\mathrm{~m}, 1 \mathrm{H}, \mathrm{H} 2 \mathrm{x})$, $4.17(\mathrm{t}, J=10.8,1 \mathrm{H}, \mathrm{H} 2 \mathrm{y}), 3.92(\mathrm{td}, J=9.6,2.8,1 \mathrm{H}, \mathrm{H} 3 \mathrm{a}), 3.58(\mathrm{dd}, J=12.8,6.7,1 \mathrm{H}$, H6), 3.10 (m, 1H, N-Ethyl $\left.\mathrm{CH}_{2}\right), 2.99$ (m, 1H, N-Ethyl $\mathrm{CH}_{2}$ ), 2.99 (broad s, 1H, OH), $2.51(\mathrm{~m}, 1 \mathrm{H}, \mathrm{H} 3 \mathrm{x}), 2.42(\mathrm{~m}, 1 \mathrm{H}, \mathrm{H} 3 \mathrm{y}), 2.40(\mathrm{~d}, J=8.9,1 \mathrm{H}, \mathrm{H} 7), 1.36(\mathrm{~d}, J=9.1,9 \mathrm{H}$, $\mathrm{PMe}_{3}$ ), 1.11 (t, $J=7.0,3 \mathrm{H}, N$-Ethyl $\left.\mathrm{CH}_{3}\right) .{ }^{13} \mathrm{C}$ NMR (d-Acetone, $\left.\delta\right): 186.5$ (C7a), 166.3 
(C1'), 145.1 (d, $J=2.0$, PzB3), 144.6 (PzA3), 142.1 (PzC3), 138.8 (Pz5), 138.7 (Pz5), 138.5 (PzA5), 134.8 (C2'), 133.6 (C4' and C6'), 130.1 (C7'), 128.9 (C5'), 108.4 (Pz4), 108.2 (Pz4), 107.5 (PzA4), 78.8 (d, $J=3.8, \mathrm{C} 5), 78.2(\mathrm{H} 4), 72.3$ (d, $J=13.0, \mathrm{C} 6), 54.6$ (C2), 48.7 (C7), 44.0 (C3a), $43.0\left(N\right.$-Ethyl $\left.\mathrm{CH}_{2}\right), 23.5$ (C3), 12.8 (d, $\left.J=30.5, \mathrm{PMe}_{3}\right), 12.0$ $\left(N\right.$-Ethyl $\left.\mathrm{CH}_{2}\right) .{ }^{31} \mathrm{P}$ NMR $(d$-Acetone, $\delta):-8.95\left(J_{\mathrm{wp}}=278\right) . \mathrm{IR}: v_{\mathrm{OH}}=3402 \mathrm{~cm}^{-1}, v_{\mathrm{BH}}=$ $2511 \mathrm{~cm}^{-1}, v_{\mathrm{NO}}, v_{\text {ester }}$ and $v_{\text {iminium }}=1699,1613$ and $1575 \mathrm{~cm}^{-1} . \mathrm{CV}(\mathrm{DMA}): \mathrm{E}_{\mathrm{p}, \mathrm{a}}=1.27 \mathrm{~V}$ HRMS: $\left[\mathrm{M}^{+}\right]=\left[\mathrm{C}_{29} \mathrm{H}_{38} \mathrm{~N}_{8} \mathrm{O}_{4} \mathrm{BPClW}^{+}\right]$obs'd (\%), calc'd (\%), ppm: 821.2013 (51), 821.2023 (64), -1.2; 822.2046 (52), 822.2046 (69), 0.0; 823.2007 (100), 823.2037 (100), -3.6; 824.2034 (47), 824.2062 (59), -3.4; 825.2054 (88), 825.2063 (91), -1.0.

Compound 42: In a 4-dram vial, mCPBA (0.034 g, $0.192 \mathrm{mmol})$ was dissolved in $\mathrm{MeCN}$ (2 mL). To this, a solution of Compound $5(0.050 \mathrm{~g}, 0.062 \mathrm{mmol})$ and pyrazole $(0.025 \mathrm{~g}$, $0.367 \mathrm{mmol})$ in MeCN (2 mL) was added. This solution was stirred overnight, turning orange. The solution was removed from the glovebox and was diluted with DCM (30 $\mathrm{mL}$ ). This was treated with $2 \times 20 \mathrm{~mL}$ of $\mathrm{Na}_{2} \mathrm{CO}_{3}$ (saturated, aq). The aqueous layer was back extracted with DCM (2 x $20 \mathrm{~mL})$, and the combined organic layers were washed with deionized water $(20 \mathrm{~mL})$. This was then dried over anhydrous $\mathrm{MgSO}_{4}$ and concentrated in vасио. The yellow oil was redissolved in minimal DCM and added dropwise to a stirring solution of $\mathrm{Et}_{2} \mathrm{O}(50 \mathrm{~mL})$ to induce precipitation of a tan solid. The powder was collected on a $15 \mathrm{~mL}$ fine-porosity fritted funnel, yielding Compound 42 (0.026 g, $0.029 \mathrm{mmol}, 47 \%) .{ }^{1} \mathrm{H}$ NMR $(d-\mathrm{MeCN}, \delta): 8.09$ (d, $\left.J=2.0,1 \mathrm{H}, \mathrm{PzB} 3\right), 8.05$ (d, $\left.J=2.0,1 \mathrm{H}, \mathrm{H} 5^{\prime}\right), 7.97(\mathrm{~d}, J=2.0,1 \mathrm{H}, \mathrm{PzC} 5), 7.92(\mathrm{~d}, J=2.0,1 \mathrm{H}, \mathrm{PzB} 5), 7.86(\mathrm{~d}, J=$ 2.0, 1H, PzA5), 7.59 (d, $J=2.0,1 \mathrm{H}, \mathrm{PzC} 3), 7.57$ (d, $\left.J=2.0,1 \mathrm{H}, \mathrm{HC} 3^{\prime}\right), 7.33$ (d, $J=2.0$, 
1H, PzA3), 6.43 (m, 1H, PzB4 and H4'), 6.41 (t, $J=2.0,1 \mathrm{H}, \mathrm{PzC} 4), 6.33(\mathrm{t}, J=2.0,1 \mathrm{H}$, PzA4), 5.97 (m, 1H, H5), 4.11 (m, 1H, H2x), 4.06 (m, 1H, H4), 3.98 (t, $J=10.7,1 \mathrm{H}$, H2y), 3.69 (m, 1H, H6), 3.68 (m, 1H, N-Ethyl $\left.\mathrm{CH}_{2}\right), 3.66$ (m, 1H, H3a), 2.79 (m, 1H, $N$ Ethyl $\mathrm{CH}_{2}$ ), 2.68 (m, 1H, $N$-Ethyl $\mathrm{CH}_{2}$ ), 2.41 (broad, 1H, $\left.\mathrm{OH}\right), 2.39$ (m, 1H, H3x), 2.27 (m, 1H, H3y), 2.26 (d, $J=8.6,1 \mathrm{H}, \mathrm{H} 7), 1.04$ (t, $J=7.4,3 \mathrm{H}, N$-Ethyl $\mathrm{CH}_{3}$ ), 0.88 (d, $J=$ 9.3, 9H, $\left.\mathrm{PMe}_{3}\right) .{ }^{13} \mathrm{C}$ NMR ( $d$-MeCN, $\left.\delta\right): 188.1$ (C7a), 145.4 (d, $J=2.1$, PzB3), 144.7 (PzA3), 142.3 (PzC3), 139.8 (C3’), 138.9 (Pz5), 138.8 (Pz5), 138.7 (Pz5), 130.8 (C5'), 108.5 (C4'), 108.1 (PzC4), 107.7 (PzA4), 107.2 (PzB4), 76.1 (C4), 67.1 (d, J = 2.6, C5), 65.2 (d, $J=14.0, \mathrm{C} 6), 55.0$ (C2), 49.7 (C7), 48.6 (C3a), 43.3 ( $N$-Ethyl $\mathrm{CH}_{2}$ ), 22.0 (C3), $13.5\left(\mathrm{~d}, J=31.0, \mathrm{PMe}_{3}\right), 12.0\left(N\right.$-Ethyl $\left.\mathrm{CH}_{3}\right) .{ }^{31} \mathrm{P} \mathrm{NMR}(d-\mathrm{MeCN}, \delta):-8.95\left(J_{\mathrm{wp}}=277\right)$. IR: $v_{\mathrm{BH}}=2500 \mathrm{~cm}^{-1}, v_{\mathrm{NO}}$ and $v_{\text {iminium }}=1614$ and $1578 \mathrm{~cm}^{-1} . \mathrm{CV}(\mathrm{DMA}): \mathrm{E}_{\mathrm{p}, \mathrm{a}}=1.30 \mathrm{~V}$. HRMS: $\left[\mathrm{M}^{+}\right]=\left[\mathrm{C}_{25} \mathrm{H}_{37} \mathrm{~N}_{10} \mathrm{O}_{2} \mathrm{BPW}^{+}\right]$obs'd (\%), calc'd (\%), ppm: 733.2408 (74), 733.2418 (82), -1.4; 734.2440 (79), 734.2443 (81), -0.5; 735.2443 (100), 735.2443 (100), 0.0; 736.2475 (47), 736.2482 (46), -0.9; 737.2469 (88), 737.2475 (83), -0.8.

Compound 43: In a 4-dram vial, mCPBA (0.128 g, $0.743 \mathrm{mmol})$ was dissolved in $\mathrm{MeCN}$ (4 mL). To this, a solution of Compound $5(0.201 \mathrm{~g}, 0.251 \mathrm{mmol})$ in $\mathrm{MeOH}(4 \mathrm{~mL})$ was added. This solution was stirred overnight, turning orange. The solution was removed from the glovebox and was diluted with DCM $(30 \mathrm{~mL})$. This was treated with 2 x $20 \mathrm{~mL}$ of $\mathrm{Na}_{2} \mathrm{CO}_{3}$ (saturated, aq). The aqueous layer was back extracted with DCM $(2 \times 20 \mathrm{~mL})$, and the combined organic layers were washed with deionized water $(20 \mathrm{~mL})$. This was then dried over anhydrous $\mathrm{MgSO}_{4}$ and concentrated in vacuo. The yellow oil was redissolved in minimal DCM and added dropwise to a stirring solution of $\mathrm{Et}_{2} \mathrm{O}(200 \mathrm{~mL})$ 
to induce precipitation of a tan solid. The powder was collected on a $30 \mathrm{~mL}$ fine-porosity fritted funnel, yielding Compound 43 (0.156 g, 0.184 mmol, 74\%). ${ }^{1} \mathrm{H}$ NMR $(d-\mathrm{MeCN}$, $\delta): 8.08(\mathrm{~d}, J=2.0,1 \mathrm{H}, \mathrm{PzB} 3), 7.96(\mathrm{~d}, J=2.0,1 \mathrm{H}, \mathrm{PzC} 5), 7.92(\mathrm{~d}, J=2.0,1 \mathrm{H}, \mathrm{PzB} 5)$, $7.84(\mathrm{~d}, J=2.0,1 \mathrm{H}, \mathrm{PzA} 5), 7.62(\mathrm{~d}, J=2.0,1 \mathrm{H}, \mathrm{PzC} 3), 7.28(\mathrm{~d}, J=2.0,1 \mathrm{H}, \mathrm{PzA} 3), 6.43$ $(\mathrm{t}, J=2.0,1 \mathrm{H}, \mathrm{PzB} 4), 6.41(\mathrm{t}, J=2.0,1 \mathrm{H}, \mathrm{PzC} 4), 6.30(\mathrm{t}, J=2.0,1 \mathrm{H}, \mathrm{PzA} 4), 4.58(\mathrm{~m}$, 1H, H5), 4.32 (t, $J=2.19,1 \mathrm{H}, \mathrm{H} 4), 4.10(\mathrm{~m}, 1 \mathrm{H}, \mathrm{H} 2 \mathrm{x}), 3.96(\mathrm{~m}, 1 \mathrm{H}, \mathrm{H} 2 \mathrm{y}), 3.48$ (t, $J=$ 9.0, 1H, H3a), 3.44 (s, 3H, OMe), 3.24 (m, 1H, H6), 2.79 (m, 1H, N-Ethyl $\mathrm{CH}_{2}$ ), 2.68 (m, 1H, N-Ethyl $\mathrm{CH}_{2}$ ), 2.35 (m, 2H, H3), 2.28 (broad,1H, OH), 2.08 (d, $\left.J=8.8,1 \mathrm{H}, \mathrm{H} 7\right)$, $1.27\left(\mathrm{~d}, J=9.1,9 \mathrm{Me}, \mathrm{PMe}_{3}\right), 1.01\left(\mathrm{t}, J=7.2,3 \mathrm{H}, N\right.$-Ethyl $\left.\mathrm{CH}_{3}\right) .{ }^{13} \mathrm{C} \mathrm{NMR}(d-\mathrm{MeCN}, \delta)$ : 188.6 (C7a), 145.4 (d, $J=2.1$, PzB3), 144.6 (PzA3), 142.3 (PzC3), 138.8 (Pz5), 138.7 (Pz5), 138.5 (Pz5), 108.4 (Pz4), 108.0 (Pz4), 107.6 (PzA4), 83.9 (d, J = 3.0, C5), 70.9 (C4), 67.5 (d, $J=13.9, \mathrm{C} 6), 54.9$ (C2), 56.5 (OMe), 48.9 (C7), 47.4 (C3a), 43.3 (N-Ethyl $\left.\mathrm{CH}_{2}\right), 22.1(\mathrm{C} 3), 13.8\left(\mathrm{~d}, J=30.5, \mathrm{PMe}_{3}\right), 12.0\left(N\right.$-Ethyl $\left.\mathrm{CH}_{3}\right) .{ }^{31} \mathrm{P} \mathrm{NMR}(d-\mathrm{MeCN}, \delta)$ : $8.66\left(J_{\mathrm{wp}}=298\right) . \mathrm{IR}: v_{\mathrm{OH}}=3467 \mathrm{~cm}^{-1}, v_{\mathrm{BH}}=2511 \mathrm{~cm}^{-1}, v_{\mathrm{NO}}$ and $v_{\text {iminium }}=1618$ and 1577 $\mathrm{cm}^{-1}$. CV (DMA): $\mathrm{E}_{\mathrm{p}, \mathrm{a}}=1.32$ V. HRMS: $\left[\mathrm{M}^{+}\right]=\left[\mathrm{C}_{23} \mathrm{H}_{37} \mathrm{~N}_{8} \mathrm{O}_{3} \mathrm{BPW}^{+}\right]$obs'd (\%), calc'd (\%), ppm: 697.2278 (76), 697.2306 (83), -4.0; 698.2304 (76), 698.2331 (80), -3.9; 699.2302 (100), 699.2330 (100), -4.0; 700.2354 (45), 700.2371 (44), -2.4; 701.2345 (90), $701.2362(84),-2.5$.

Compound 44: In a 4-dram vial, mCPBA $(0.033 \mathrm{~g}, 0.191 \mathrm{mmol})$ was dissolved in $\mathrm{MeCN}$ ( $1 \mathrm{~mL})$. To this, a solution of Compound $5(0.051 \mathrm{~g}, 0.064 \mathrm{mmol})$ and isoproponal $(0.5$ $\mathrm{mL})$ in $\mathrm{MeCN}(1 \mathrm{~mL})$ was added. This solution was stirred overnight, turning orange. The solution was removed from the glovebox and was diluted with DCM (20 mL). This 
was treated with $2 \times 20 \mathrm{~mL}$ of $\mathrm{Na}_{2} \mathrm{CO}_{3}$ (saturated, aq). The aqueous layer was back extracted with DCM (2 x $20 \mathrm{~mL}$ ), and the combined organic layers were washed with deionized water $(20 \mathrm{~mL})$. This was then dried over anhydrous $\mathrm{MgSO}_{4}$ and concentrated in vacuo. The yellow oil was redissolved in minimal DCM and added dropwise to a stirring solution of $\mathrm{Et}_{2} \mathrm{O}(50 \mathrm{~mL})$ to induce precipitation of a tan solid. The powder was collected on a $15 \mathrm{~mL}$ fine-porosity fritted funnel, yielding Compound 44 (0.055 g, 0.067 mmol, 53\%). ${ }^{1} \mathrm{H}$ NMR $(d-\mathrm{MeCN}, \delta): 8.08(\mathrm{~d}, J=2.0,1 \mathrm{H}, \mathrm{PzB} 3), 7.96(\mathrm{~d}, J=2.0,1 \mathrm{H}$, PzC5), $7.92(\mathrm{~d}, J=2.0,1 \mathrm{H}, \mathrm{PzB} 5), 7.84(\mathrm{~d}, J=2.0,1 \mathrm{H}, \mathrm{PzA} 5), 7.60(\mathrm{~d}, J=2.0,1 \mathrm{H}$, PzC3), $7.26(\mathrm{~d}, J=2.0,1 \mathrm{H}, \mathrm{PzA} 3), 6.44(\mathrm{t}, J=2.0,1 \mathrm{H}, \mathrm{PzB} 4), 6.42(\mathrm{t}, J=2.0,1 \mathrm{H}$, PzC4), 6.30 (t, $J=2.0,1 \mathrm{H}, \mathrm{PzA} 4), 4.88(\mathrm{~m}, 1 \mathrm{H}, \mathrm{H} 5), 4.27$ (t, $J=2.57,1 \mathrm{H}, \mathrm{H} 4), 4.08$ (m, 1H, H2x), 3.94 (m, 1H, H2y), 3.93 (m, 1H, iPr-CH), 3.50 (t, $J=9.4,1 \mathrm{H}, \mathrm{H} 3 \mathrm{a}), 3.24$ (m, 1H, H6), 2.77 (m, 1H, N-Ethyl $\left.\mathrm{CH}_{2}\right), 2.63\left(\mathrm{~m}, 1 \mathrm{H}, N\right.$-Ethyl $\left.\mathrm{CH}_{2}\right), 2.35(\mathrm{~m}, 1 \mathrm{H}, \mathrm{H} 3 \mathrm{x})$, 2.33 (broad, OH), $2.31(\mathrm{~m}, 1 \mathrm{H}, \mathrm{H} 3 \mathrm{y}), 2.10$ (d, $J=9.0,1 \mathrm{H}, \mathrm{H} 7), 1.28$ (buried, 6H, $i$ Pr$\left.\mathrm{CH}_{3}\right), 1.28\left(\mathrm{~d}, J=9.6,9 \mathrm{H}, \mathrm{PMe}_{3}\right), 1.00\left(\mathrm{t}, J=7.3,3 \mathrm{H}, N\right.$-Ethyl $\left.\mathrm{CH}_{3}\right) .{ }^{13} \mathrm{C} \mathrm{NMR}(d-$ MeCN, 8): 188.4 (C7a), 145.2 (PzB3), 144.6 (PzA3), 142.2 (PzC3), 138.8 (Pz5), 138.7 (Pz5), 138.6 (Pz5), 108.4 (Pz4), 108.1 (Pz4), 107.6 (PzA4), 78.8 (d, J = 2.6, C5), 72.1 (C4), 69.6 (iPr-CH), $68.2(\mathrm{~d}, J=13.8, \mathrm{C} 6), 54.9(\mathrm{C} 2), 49.5(\mathrm{C} 7), 47.4(\mathrm{C} 3 \mathrm{a}), 43.1(N-$ Ethyl $\left.\mathrm{CH}_{2}\right), 24.2\left(i \mathrm{Pr}-\mathrm{CH}_{3}\right), 22.2(\mathrm{C} 3), 14.0\left(\mathrm{~d}, J=30.5, \mathrm{PMe}_{3}\right), 11.9\left(N-\right.$ Ethyl $\left.\mathrm{CH}_{3}\right) .{ }^{31} \mathrm{P}$ $\operatorname{NMR}(d-\mathrm{MeCN}, \delta):-8.62\left(J_{\mathrm{wp}}=276\right) . \mathrm{IR}: v_{\mathrm{OH}}=3116 \mathrm{~cm}^{-1}, v_{\mathrm{BH}}=2508 \mathrm{~cm}^{-1}, v_{\mathrm{NO}}$ and $v_{\text {iminium }}=1614$ and $1574 \mathrm{~cm}^{-1} . \quad \mathrm{CV}(\mathrm{DMA}): \mathrm{E}_{\mathrm{p}, \mathrm{a}}=1.29 \mathrm{~V} . \quad$ HRMS: $\left[\mathrm{M}^{+}\right]=$ $\left[\mathrm{C}_{25} \mathrm{H}_{41} \mathrm{~N}_{8} \mathrm{O}_{3} \mathrm{BPW}^{+}\right]$obs'd (\%), calc'd (\%), ppm: 725.2603 (76), 725.2619 (82), -2.2; 
726.2618 (80), 726.2644 (81), -3.6; 727.2630 (100), 727.2644 (100), -1.9; 728.2669 (49), 728.2684 (46), -2.0; $729.2673(91), 729.2676(83),-0.4$.

Compound 46: In a 4-dram vial, Compound 43 (0.150 g, $0.177 \mathrm{mmol})$ and diphenyl ammonium triflate $(0.085 \mathrm{~g}, 0.266 \mathrm{mmol})$ were dissolved in $\mathrm{MeCN}(8 \mathrm{~mL})$ and stirred. After 2 h, 5 drops of triethylamine was added to the reaction. The solution was added dropwise to stirring $\mathrm{Et}_{2} \mathrm{O}(175 \mathrm{~mL})$ to induce precipitation of a yellow-tan solid. The powder was collected on a $30 \mathrm{~mL}$ fine-porosity fritted funnel, yielding Compound 46 (0.110 g, $0.128 \mathrm{mmol}, 73 \%) .{ }^{1} \mathrm{H}$ NMR $(d-\mathrm{MeCN}, \delta): 8.09$ (d, $\left.J=2.0,1 \mathrm{H}, \mathrm{PzB} 3\right), 7.96(\mathrm{~d}$, $J=2.0,1 \mathrm{H}, \mathrm{PzC} 5), 7.94(\mathrm{~d}, J=2.0,1 \mathrm{H}, \mathrm{PzB} 5), 7.86(\mathrm{~d}, J=2.0,1 \mathrm{H}, \mathrm{PzA} 5), 7.65(\mathrm{~d}, J=$ 2.0, 1H, PzC3), $7.23(\mathrm{~d}, J=2.0,1 \mathrm{H}, \mathrm{PzA} 3), 6.45(\mathrm{t}, J=2.0,1 \mathrm{H}, \mathrm{PzB} 4), 6.42(\mathrm{t}, J=2.0$, $1 \mathrm{H}, \mathrm{PzC} 4), 6.32(\mathrm{t}, J=2.0,1 \mathrm{H}, \mathrm{PzA} 4), 5.02(\mathrm{dd}, J=8.6,0.7,1 \mathrm{H}, \mathrm{H} 5), 4.84(\mathrm{dd}, J=8.6$, 5.4, 1H, H4), 4.11 (m, 1H, H2x), 3.95 (t, $J=10.1,1 \mathrm{H}, \mathrm{H} 2 \mathrm{y}), 3.84$ (m, 1H, H3a), 3.40 (m, 1H, H6), 2.83 (m, 2H, N-Ethyl $\mathrm{CH}_{2}$ ), 2.46 (m, 1H, H3x), 2.26 (m, 1H, H3y), 2.20 (d, J= 9.3, 1H, H7), 1.96 (d, $J=1.0,3 \mathrm{H}$, imidate $\left.\mathrm{CH}_{3}\right), 1.26\left(\mathrm{~d}, J=9.3,9 \mathrm{H}, \mathrm{PMe}_{3}\right), 1.02(\mathrm{t}, J=$ 7.4, 3H, $N$-Ethyl $\left.\mathrm{CH}_{3}\right) .{ }^{13} \mathrm{C} \mathrm{NMR}(d-\mathrm{MeCN}, \delta): 185.3$ (C7a), 163.6 (imidate C), 145.1 (PzB3), 144.4 (PzA3), 142.1 (PzC3), 108.9 (Pz5), 108.8 (Pz5), 108.7 (PzA5), 108.5 (Pz4), 108.2 (Pz4), 107.6 (PzA4), 81.0 (C4), 71.2 (d, $J=13.5, \mathrm{C} 6), 68.6$ (d, J = 3.9, C5), 54.5 (C2), $48.4(\mathrm{C} 7), 43.3(\mathrm{C} 3 \mathrm{a}), 42.9$ (N-Ethyl $\left.\mathrm{CH}_{2}\right), 23.7(\mathrm{C} 3), 14.0$ (imidate $\mathrm{CH}_{3}$ ), 12.9 $\left(\mathrm{d}, J=12.9, \mathrm{PMe}_{3}\right), 11.9\left(N\right.$-Ethyl $\left.\mathrm{CH}_{3}\right) .{ }^{31} \mathrm{P} \mathrm{NMR}(d-\mathrm{MeCN}, \delta):-7.39\left(J_{\mathrm{wp}}=280\right) . \mathrm{IR}$ : $v_{\mathrm{BH}}=2511 \mathrm{~cm}^{-1}, v_{\mathrm{NO}}, v_{\text {iminium }}$ and $v_{\text {imidate }}=1608,1670$, and $1574 \mathrm{~cm}^{-1} . \mathrm{CV}(\mathrm{DMA}): \mathrm{E}_{\mathrm{p}, \mathrm{a}}=$ 1.18 V. HRMS: $\left[\mathrm{M}^{+}\right]=\left[\mathrm{C}_{24} \mathrm{H}_{36} \mathrm{~N}_{9} \mathrm{O}_{2} \mathrm{BPW}^{+}\right]$obs'd (\%), calc'd (\%), ppm: 706.2292 (78), 
706.2309 (83), -2.5; 707.2330 (78), 707.2334 (81), -0.6; 708.2321 (100), 708.2334 (100), $-1.8 ; 709.2373$ (45), 709.2373 (45), -0.1; 710.2363 (81), 710.2366 (83), -0.4.

Compound 47: In a 4-dram vial, Compound 43 (0.100 g, $0.117 \mathrm{mmol})$ and diphenyl ammonium triflate $(0.057 \mathrm{~g}, 0.178 \mathrm{mmol})$ were dissolved in propionitrile $(5 \mathrm{~mL})$ and stirred. After 15 h, 4 drops of triethylamine was added to the reaction. The solution removed from the glovebox and evaporated to dryness. The oil was redissolved in minimal DCM and added dropwise to stirring $\mathrm{Et}_{2} \mathrm{O}(105 \mathrm{~mL})$ to induce precipitation of a yellow-tan solid. The powder was collected on a $15 \mathrm{~mL}$ fine-porosity fritted funnel, yielding Compound 47 (0.76 g, $0.087 \mathrm{mmol}, 74 \%) .{ }^{1} \mathrm{H}$ NMR $(d-\mathrm{MeCN}, \delta): 8.10(\mathrm{~d}, J=$ 2.0, 1H, PzB3), 7.96 (d, $J=2.0,1 \mathrm{H}, \mathrm{PzC} 5), 7.94(\mathrm{~d}, J=2.0,1 \mathrm{H}, \mathrm{PzB} 5), 7.86(\mathrm{~d}, J=2.0$, 1H, PzA5), $7.65(\mathrm{~d}, J=2.0,1 \mathrm{H}, \mathrm{PzC} 3), 7.23(\mathrm{~d}, J=2.0,1 \mathrm{H}, \mathrm{PzA} 3), 6.45(\mathrm{t}, J=2.0,1 \mathrm{H}$, PzB4), $6.43(\mathrm{t}, J=2.0,1 \mathrm{H}, \mathrm{PzC} 4), 6.32(\mathrm{t}, J=2.0,1 \mathrm{H}, \mathrm{PzA} 4), 5.01(\mathrm{~d}, J=8.5,1 \mathrm{H}, \mathrm{H} 5)$, $4.86(\mathrm{dd}, J=8.9,5.2,1 \mathrm{H}, \mathrm{H} 4), 4.11(\mathrm{~m}, 1 \mathrm{H}, \mathrm{H} 2 \mathrm{x}), 3.95(\mathrm{t}, J=10.9,1 \mathrm{H}, \mathrm{H} 2 \mathrm{y}), 3.88(\mathrm{~m}$, 1H, H3a), 3.41 (m, 1H, H6), 3.13 (m, 2H, $N$-Ethyl $\left.\mathrm{CH}_{2}\right), 2.85$ (m, 1H, imidate $\mathrm{CH}_{2}$ ), 2.81 (m, 1H, imidate $\left.\mathrm{CH}_{2}\right), 2.47$ (m, 1H, H3x), 2.29 (m, 1H, H3y), 2.18 (d, J = 9.3, 1H, H7), $1.26\left(\mathrm{~d}, J=9.2,9 \mathrm{H}, \mathrm{PMe}_{3}\right), 1.25\left(\mathrm{t}, J=7.3,3 \mathrm{H}, N\right.$-Ethyl $\left.\mathrm{CH}_{3}\right), 0.98(\mathrm{t}, J=7.2,3 \mathrm{H}$, imidate $\left.\mathrm{CH}_{3}\right) .{ }^{13} \mathrm{C} \mathrm{NMR}(d-\mathrm{MeCN}, \delta): 185.2(\mathrm{C} 7 \mathrm{a}), 166.7$ (imidate C), 145.1 (PzB3), 144.4 (PzA3), 142.1 (PzC3), 138.9 (Pz5), 138.7 (Pz5), 138.6 (PzA5), 108.4 (Pz4), 108.1 (Pz4), 107.6 (Pz4), 80.9 (C4), 71.3 (d, $J=12.9, \mathrm{C} 6), 68.4$ (C5), 54.6 (C2), 48.6 (C7), 47.9 ( $N$-Ethyl $\mathrm{CH}_{2}$ ), 43.3 (C3a), 42.8 (imidate $\mathrm{CH}_{2}$ ), 23.7 (C3), 12.9 (d, $J=30.8, \mathrm{PMe}_{3}$ ), 11.9 (imidate $\left.\mathrm{CH}_{3}\right), 9.1\left(N\right.$-Ethyl $\left.\mathrm{CH}_{3}\right) .{ }^{31} \mathrm{P}$ NMR $(d$-MeCN, $\delta):-7.42\left(J_{\mathrm{wp}}=277\right)$. IR: $v_{\mathrm{BH}}=2511 \mathrm{~cm}^{-1}, v_{\mathrm{NO}}, v_{\text {iminium }}$ and $v_{\text {imidate }}=1699,1612$, and $1574 \mathrm{~cm}^{-1} . \mathrm{CV}(\mathrm{DMA}): \mathrm{E}_{\mathrm{p}, \mathrm{a}}=$ 
1.36 V. HRMS: $\left[\mathrm{M}^{+}\right]=\left[\mathrm{C}_{25} \mathrm{H}_{38} \mathrm{~N}_{9} \mathrm{O}_{2} \mathrm{BPW}^{+}\right]$obs'd (\%), calc'd (\%), ppm: 720.2438 (77), 720.2466 (82), -3.9; 721.2481 (79), 721.2491 (81), -1.4; 722.2472 (100), 722.2490 (100), -2.5; 723.2504 (48), $723.2530(46),-3.6 ; 724.2520(90), 724.2523(83),-0.4$.

Compound 48: To an oven dried $100 \mathrm{~mL}$ round bottom flask, DME (10 mL) was added and stirred. A solution of Compound 10 (0.103 g, $0.109 \mathrm{mmol})$ in DCM (2 mL) was added to the DME. To this stirring solution, lithium aluminum hydride was added $(0.022$ $\mathrm{g}, 0.581 \mathrm{mmol})$ and stirred for $1 \mathrm{~h}$. The reaction was quenched with $\mathrm{H}_{2} \mathrm{O}(8 \mathrm{~mL})$ and the solution was removed from the glovebox and was diluted with DCM (50 mL). This was treated with $2 \times 20 \mathrm{~mL}$ of $\mathrm{Na}_{2} \mathrm{CO}_{3}$ (saturated, aq). The aqueous layer was back extracted with DCM (1 x $20 \mathrm{~mL})$, and the combined organic layers were washed with deionized water $(20 \mathrm{~mL})$. This was then dried over anhydrous $\mathrm{MgSO}_{4}$ and concentrated in vacuo to a yellow oil, yielding Compound $48(0.069 \mathrm{~g}, 0.079 \mathrm{mmol}, 73 \%) .{ }^{1} \mathrm{H} \mathrm{NMR}\left(d-\mathrm{CDCl}_{3}\right.$, $\delta): 9.62(\mathrm{~d}, J=2.0,1 \mathrm{H}, \mathrm{PzA} 3), 8.18(\mathrm{~d}, J=2.0,1 \mathrm{H}, \mathrm{PzB} 3), 7.68(\mathrm{~d}, J=2.0,1 \mathrm{H}, \mathrm{PzC} 5)$, 7.67 (d, $J=2.0,1 \mathrm{H}, \mathrm{PzB} 5), 7.66$ (buried, 1H, H3'), 7.48 (d, $J=2.0,1 \mathrm{H}$, PzA5), 7.32 (d, $J$ $=2.0,1 \mathrm{H}, \mathrm{PzC} 3), 6.61\left(\mathrm{dd}, J=8.3,2.3,1 \mathrm{H}, \mathrm{H} 5^{\prime}\right), 6.43\left(\mathrm{~d}, J=2.3,1 \mathrm{H}, \mathrm{H} 6{ }^{\prime}\right), 6.27$ (t, $J=$ 2.0, 1H, PzB4), $6.15(\mathrm{t}, J=2.0,1 \mathrm{H}, \mathrm{PzC} 4), 6.05(\mathrm{t}, J=2.0,1 \mathrm{H}, \mathrm{PzA} 4), 4.86(\mathrm{t}, J=8.7$, 1H, H5), 4.09 (dd, $J=10.4,3.3,1 \mathrm{H}, 3.86$ (s, 3H, 4’OMe), 3.82 (d, $J=0.9,3 \mathrm{H}, 2$ 'OMe), 3.29 (m, 1H, H2x), 2.85 (m, 1H, H6), 2.67 (m, 1H, N-Ethyl $\left.\mathrm{CH}_{2}\right), 2.58$ (m, 1H, H3a), $2.29(\mathrm{~m}, 1 \mathrm{H}, \mathrm{H} 4 \mathrm{x}), 2.02(\mathrm{~m}, 2 \mathrm{H}, \mathrm{H} 3 \mathrm{x}$ and H2y), 1.99 (buried, 1H, H7), $1.73(\mathrm{~m}, 1 \mathrm{H}, N-$ Ethyl $\left.\mathrm{CH}_{2}\right), 1.21(\mathrm{~m}, 1 \mathrm{H}, \mathrm{H} 3 \mathrm{y}), 1.11(\mathrm{~m}, 1 \mathrm{H}, \mathrm{H} 4 \mathrm{y}), 0.99$ (t, $J=7.4,3 \mathrm{H}, N$-Ethyl $\left.\mathrm{CH}_{3}\right)$, $0.87\left(\mathrm{~d}, J=8.6,9 \mathrm{H}, \mathrm{PMe}_{3}\right) .{ }^{13} \mathrm{C} \mathrm{NMR}\left(d-\mathrm{CDCl}_{3}, \delta\right): 160.9\left(\mathrm{C} 2{ }^{\prime}\right), 158.1(\mathrm{C} 4 '), 156.7$ (C1'), 151.1 (PzA3), 142.6 (PzB3), 139.8 (PzC3), 136.3 (PzC5), 135.4 (PzB5), 134.7 
(PzA5), 129.9 (C3'), 106.4 (PzB4), 106.2 (PzC4), 105.2 (C5'), 104.5 (PzA4), 78.6 (C7a), $58.8(\mathrm{~d}, J=10.8, \mathrm{C} 6), 57.5(\mathrm{C} 7), 55.4$ (C4’OMe), 55.3 (C2’OMe), $51.6(\mathrm{C} 2), 49.4(N-$ Ethyl $\mathrm{CH}_{2}$ ), 43.8 (C4), 38.4 (C5), 38.3 (C3a), 28.6 (C3), 13.4 (d, $J=27.0, \mathrm{PMe}_{3}$ ), 13.1 $\left(N\right.$-Ethyl $\left.\mathrm{CH}_{3}\right) .{ }^{31} \mathrm{P} \mathrm{NMR}\left(d-\mathrm{CDCl}_{3}, \delta\right):-8.93\left(J_{\mathrm{wp}}=280\right) . \mathrm{IR}: v_{\mathrm{BH}}=2484 \mathrm{~cm}^{-1}, v_{\mathrm{NO}}=$ $1543 \mathrm{~cm}^{-1} \cdot \mathrm{CV}(\mathrm{DMA}): \mathrm{E}_{\mathrm{p}, \mathrm{a}}=0.32 \mathrm{~V} . \mathrm{HRMS}:\left[\mathrm{M}+\mathrm{H}^{+}\right]=\left[\mathrm{C}_{30} \mathrm{H}_{44} \mathrm{~N}_{8} \mathrm{O}_{3} \mathrm{BPW}+\mathrm{H}^{+}\right]$obs'd (\%), calc'd (\%), ppm: 789.2922 (79), 789.2933 (80), -1.4; 790.2959 (80), 790.2958 (82), 0.1; 791.2952 (100), 791.2958 (100), -0.8; 792.2991 (48), 792.2996 (49), -0.6; 793.2985 (79), $793.2990(82),-0.6$.

Compound 49: Outside of the glovebox, in a flame dried round bottom flask, $\mathrm{LiAlH}_{4}$ $(0.220 \mathrm{~g}, 0.580 \mathrm{mmol})$ was added to a stirring mixture of Compound 9 (1.01 g, 1.16 $\mathrm{mmol}$ ) in $\mathrm{Et}_{2} \mathrm{O}(350 \mathrm{~mL})$. After $1 \mathrm{hr}$, the grey, heterogeneous solution was filtered through a $60 \mathrm{~mL}$ M frit packed with $3 \mathrm{~cm}$ of celite. The frit was washed with $10 \mathrm{~mL} \mathrm{Et}_{2} \mathrm{O}$ and the filtrate was concentrated in vacuo. The resulting clear oil was redissolved in DCM (100 mL) and washed twice with $30 \mathrm{~mL}$ of $\mathrm{Na}_{2} \mathrm{CO}_{3}$ (saturated, aq). The combined aqueous layers were back extracted with DCM $(2 \times 50 \mathrm{~mL})$. The resulting organic fractions were washed 1 x $40 \mathrm{~mL}$ water and dried over anhydrous $\mathrm{MgSO}_{4}$. Concentration of the solution in vacuo produced a yellow power of Compound 49 (0.742 g, 0.854 mmol, 74\%). ${ }^{1} \mathrm{H}$ NMR $\left(\mathrm{CDCl}_{3}, \delta\right): 9.48(\mathrm{~d}, J=2.0,1 \mathrm{H}, \mathrm{PzA} 3), 8.15(\mathrm{~d}, J=2.0,1 \mathrm{H}$, PzB3), 7.75 (dd, $J=2.0,0.5,1 \mathrm{H}, \mathrm{H} 5$ ') 7.69 (m, 2H, PzC5 and PzB5), 7.54 (d, $J=2.0$, $\left.1 \mathrm{H}, \mathrm{H} 3{ }^{\prime}\right), 7.48(\mathrm{~d}, J=2.0,1 \mathrm{H}, \mathrm{PzA} 5), 7.24(\mathrm{~d}, J=2.0,1 \mathrm{H}, \mathrm{PzC} 3), 6.31(\mathrm{t}, J=2.0,1 \mathrm{H}$, H4'), 6.29 (t, $J=2.0,1 \mathrm{H}, \mathrm{PzB} 4), 6.17$ (t, $J=2.0,1 \mathrm{H}, \mathrm{PzC} 4), 6.06(\mathrm{t}, J=2.0,1 \mathrm{H}, \mathrm{PzA} 4)$, $5.84(\mathrm{t}, J=8,1 \mathrm{H}, \mathrm{H} 5), 4.17(\mathrm{dd}, J=10.3,3.7,1 \mathrm{H}, \mathrm{H} 7 \mathrm{a}), 3.25(\mathrm{~m}, 1 \mathrm{H}, \mathrm{H} 2 \mathrm{x}), 3.03(\mathrm{~m}, 1 \mathrm{H}$, 
H6), 2.55 (m, 1H, N-Ethyl- $\mathrm{CH}_{2}$ ), 2.46 (m, buried, 1H, H3a), 2.40 (m, 1H, H4y), 2.04 (m, buried, 1H, H2x), $2.02(\mathrm{~m}, 1 \mathrm{H}, \mathrm{H} 3 \mathrm{x}), 1.82(\mathrm{dd}, J=11.9,3.7, \mathrm{H} 7), 1.71$ (m, 1H, N-Ethyl$\left.\mathrm{CH}_{2}\right), 1.50(\mathrm{~m}, 1 \mathrm{H}, \mathrm{H} 4 \mathrm{x}) 1.26(\mathrm{~m}, 1 \mathrm{H}, \mathrm{H} 3 \mathrm{y}), 0.92\left(\mathrm{t}, J=6.2,3 \mathrm{H}, \mathrm{N}-\mathrm{Ethyl}-\mathrm{CH}_{3}\right), 0.82$ (d, $\left.J=9,9 \mathrm{H}, \mathrm{PMe}_{3}\right) .{ }^{13} \mathrm{C} \mathrm{NMR}\left(\mathrm{CDCl}_{3}, \delta\right): 151.0(\mathrm{PzA} 3), 142.8(\mathrm{PzB} 3), 139.9(\mathrm{PzC} 3)$, 138.3 (C3’), 136.6 (PzC5), 135.7 (PzB5), 134.9 (PzA5), 127.5 (C5'), 106.7 (PzB4), 105.9 (PzC4), 105.5 (C4'), 104.7 (PzA4), 77.1 (C7a), 64.2 (d, J = 3.7, C5), 55.2 (C7), 53.8 (d, J = 11.3, H6), $51.4(\mathrm{C} 2), 49.1\left(\mathrm{~N}-\mathrm{Ethyl}-\mathrm{CH}_{2}\right), 42.2(\mathrm{C} 4), 37.4(\mathrm{C} 3 \mathrm{a}), 28.3(\mathrm{C} 3), 13.0(\mathrm{~N}-$ Ethyl- $\left.\mathrm{CH}_{3}\right), 12.9\left(\mathrm{~d}, J=27.4, \mathrm{PMe}_{3}\right) .{ }^{31} \mathrm{P} \mathrm{NMR}\left(\mathrm{CDCl}_{3}, \delta\right):-11.3\left(J_{\mathrm{WP}}=268\right) . \quad \mathrm{IR}: v_{\mathrm{BH}}=$ $2492 \mathrm{~cm}^{-1}, \quad v_{\mathrm{NO}}=1544 \mathrm{~cm}^{-1} \cdot \mathrm{CV}(\mathrm{DMA}): \mathrm{E}_{\mathrm{p}, \mathrm{a}}=0.48 \mathrm{~V}$. HRMS: $\quad[\mathrm{M}+]=$ $\left[\mathrm{C}_{25} \mathrm{H}_{38} \mathrm{~N}_{10} \mathrm{OBPW}+\mathrm{H}^{+}\right]$obsd (\%), calcd (\%), ppm: 719.2634 (90), 719.2626 (82), 1.1; 720.2651 (95), 720.2651 (81), 0.0; 721.2674 (100), 721.2650 (100), 3.3; 722.2686 (67), 722.2689 (46), -0.4; 723.2705 (81), 723.2682 (83), 3.1.

Compound 50: To an oven dried $50 \mathrm{~mL}$ round bottom flask, DME (22 mL) was added and stirred. A solution of Compound 28 (0.115 g, $0.144 \mathrm{mmol})$ in DCM (2 mL) was added to the DME. To this stirring solution, $\mathrm{LiAlH}_{4}$ was added $(0.028 \mathrm{~g}, 0.737 \mathrm{mmol})$ and stirred for $10 \mathrm{~min}$. The reaction was quenched with $\mathrm{H}_{2} \mathrm{O}(5 \mathrm{~mL})$ and the solution was removed from the glovebox and was diluted with DCM $(50 \mathrm{~mL})$. This was treated with $20 \mathrm{~mL} \mathrm{Na} \mathrm{CO}_{3}$ (saturated, aq). The aqueous layer was back extracted with DCM (1 x 20 $\mathrm{mL})$, and the combined organic layers were washed with deionized water $(20 \mathrm{~mL})$. This was then dried over anhydrous $\mathrm{MgSO}_{4}$ and concentrated in vacuo to a yellow oil, yielding Compound $50(0.087 \mathrm{~g}, 0.130 \mathrm{mmol}, 93 \%) .{ }^{1} \mathrm{H} \mathrm{NMR}\left(d-\mathrm{CDCl}_{3}, \delta\right): 9.56(\mathrm{~d}, J=$ 2.0, 1H, PzA3), 8.19 (d, $J=2.0,1 \mathrm{H}, \mathrm{PzB} 3), 7.71(\mathrm{~d}, J=2.0,1 \mathrm{H}, \mathrm{PzB} 5), 7.70(\mathrm{~d}, J=2.0$, 
1H, PzC5), $7.45(\mathrm{~d}, J=2.0,1 \mathrm{H}, \mathrm{PzA} 5), 7.31(\mathrm{~d}, J=2.0,1 \mathrm{H}, \mathrm{PzC} 3), 6.30(\mathrm{t}, J=2.0,1 \mathrm{H}$, PzB4), 6.17 (t, $J=2.0,1 \mathrm{H}, \mathrm{PzC} 4), 6.03$ (t, $J=2.0,1 \mathrm{H}, \mathrm{PzA} 4), 3.73$ (dt, $J=10.7,1.0,1 \mathrm{H}$, H7a), 3.28 (m, 1H, H2x), 2.96 (m, 1H, H6), 2.72 (m, 1H, H3a), 2.57 (m, 1H, N-Ethyl $\left.\mathrm{CH}_{2}\right), 1.99$ (m, 2H, H3x and $\left.\mathrm{H} 2 \mathrm{y}\right), 1.64$ (m, 1H, N-Ethyl $\left.\mathrm{CH}_{2}\right), 1.55$ (m, 2H, H5 and H7), $1.48(\mathrm{~m}, 1 \mathrm{H}, \mathrm{H} 3 \mathrm{y}), 1.34(\mathrm{~m}, 1 \mathrm{H}, \mathrm{H} 4), 1.12\left(\mathrm{~d}, J=8.5,9 \mathrm{H}, \mathrm{PMe}_{3}\right), 0.93(\mathrm{t}, J=7.4,3 \mathrm{H}, N-$ Ethyl $\left.\mathrm{CH}_{3}\right), 0.59$ (dd, $\left.J=9.1,4.5,1 \mathrm{H}, \mathrm{H} 8 \mathrm{x}\right), 0.44(\mathrm{td}, J=8.1,4.2,1 \mathrm{H}, \mathrm{H} 8 \mathrm{y}) .{ }^{13} \mathrm{C} \mathrm{NMR}$ (d-CDCl $\left.{ }_{3}, \delta\right): 151.3$ (PzA3), 142.7 (PzB3), 139.8 (PzC3), 136.4 (Pz5), 135.5 (Pz5), 134.4 (PzA5), 106.4 (PzB4), 105.6 (PzC4), 104.6 (PzA4), 70.2 (C7a), 55.2 (C7), 54.3 (d, J = 21.0, C6), 51.7 (C2), 48.9 ( $N$-Ethyl $\mathrm{CH}_{2}$ ), 38.1 (C3a), 27.4 (C3), 18.9 (d, $J=4.0, \mathrm{C} 5$ ), $16.7(\mathrm{C} 4), 12.6\left(\mathrm{~d}, J=27.0, \mathrm{PMe}_{3}\right), 12.6\left(N-\right.$ Ethyl $\left.\mathrm{CH}_{3}\right), 10.2(\mathrm{C} 8), .{ }^{31} \mathrm{P} \mathrm{NMR}\left(d-\mathrm{CDCl}_{3}\right.$, $\delta):-9.42\left(J_{\mathrm{wp}}=274\right) . \mathrm{IR}: v_{\mathrm{BH}}=2488 \mathrm{~cm}^{-1}, v_{\mathrm{NO}}=1543 \mathrm{~cm}^{-1} . \mathrm{CV}(\mathrm{DMA}): \mathrm{E}_{\mathrm{p}, \mathrm{a}}=0.43 \mathrm{~V}$. HRMS: $\left[\mathrm{M}+\mathrm{H}^{+}\right]=\left[\mathrm{C}_{22} \mathrm{H}_{34} \mathrm{~N}_{8} \mathrm{OBPW}+\mathrm{H}^{+}\right]$obs'd (\%), calc'd (\%), ppm: 651.2246 (82), 651.2251 (84), -0.8; $652.2251(77), 652.2276(80),-3.9 ; 653.2284(100), 653.2275$ (100), $1.4 ; 654.2312$ (45), $654.2316(43),-0.6 ; 655.2289$ (86), 655.2307 (84), -2.8.

Compound 51: Outside of the glovebox, in a flame dried round bottom flask, $\mathrm{LiAlH}_{4}$ (0.040 g, $1.05 \mathrm{mmol})$ was added to a stirring solution of Compound $12(0.180 \mathrm{~g}, 0.203$ $\mathrm{mmol})$ in $\mathrm{Et}_{2} \mathrm{O}(60 \mathrm{~mL})$. After $30 \mathrm{~min}$, the grey, heterogeneous mixture was filtered through a $30 \mathrm{~mL}$ medium-porosity fritted funnel packed with $3 \mathrm{~cm}$ of celite. The frit was washed with $\mathrm{Et}_{2} \mathrm{O}(10 \mathrm{~mL})$ and the filtrate was concentrated in vacuo. The resulting clear oil was redissolved in DCM $(50 \mathrm{~mL})$ and washed with $\mathrm{Na}_{2} \mathrm{CO}_{3}(2 \times 100 \mathrm{~mL}$, saturated, aq). The combined aqueous layers were back extracted with DCM (2 x $20 \mathrm{~mL})$. The resulting organic fractions were washed with water $(1 \times 30 \mathrm{~mL})$ and dried over anhydrous 
$\mathrm{MgSO}_{4}$. Concentration of the solution in vacuo produced a yellow power of Compound 51 (0.110 g, $0.146 \mathrm{mmol}, 72 \%) .{ }^{1} \mathrm{H}$ NMR $\left(d-\mathrm{CDCl}_{3}, \delta\right): 9.53$ (d, $\left.J=2.0,1 \mathrm{H}, \mathrm{PzA} 3\right), 8.15$ $(\mathrm{d}, J=2.0,1 \mathrm{H}, \mathrm{PzB} 3), 7.70(\mathrm{~d}, J=2.0,1 \mathrm{H}, \mathrm{PzC} 5), 7.68(\mathrm{~d}, J=2.0,1 \mathrm{H}, \mathrm{PzB} 5), 7.47(\mathrm{~d}, J$ $=2.0,1 \mathrm{H}$, PzA5), $7.26(\mathrm{~d}, J=2.0,1 \mathrm{H}, \mathrm{PzC} 3), 6.70\left(\mathrm{~d}, J=3.2,1 \mathrm{H}, \mathrm{H} 5^{\prime}\right), 6.56(\mathrm{dq}, J=$ 3.3, 1.1, 1H, H3'), 6.28 (t, $J=2.0,1 \mathrm{H}, \mathrm{PzB} 4), 6.19$ (t, $J=2.0,1 \mathrm{H}, \mathrm{PzC} 4), 6.05$ (t, $J=2.0$, 1H, PzA4), 4.54 (m, 1H, H5), 4.05 (dd, $J=10.4,3.7,1 \mathrm{H}, \mathrm{H} 7 \mathrm{a}), 3.23$ (m, 1H, H2x), 2.90 (m, 1H, H6), 2.53 (m, 1H, N-Ethyl $\mathrm{CH}_{2}$ ), 2.47 (s, 3H, 2'- $\left.\mathrm{CH}_{3}\right), 2.43$ (m, 1H, H3a), 2.24 (m, 1H, H4x), 2.02 (m, 1H, H3x), 1.99 (m, 1H, H2y), 1.80 (dd, J = 11.7, 3.7, 1H, H7), $1.67\left(\mathrm{~m}, 1 \mathrm{H}, N\right.$-Ethyl $\left.\mathrm{CH}_{2}\right), 1.37$ (q, $\left.J=12.6,1 \mathrm{H}, \mathrm{H} 4 \mathrm{y}\right), 1.23(\mathrm{~m}, 1 \mathrm{H}, \mathrm{H} 3 \mathrm{y}), 0.92$ (t, $J=$ 7.1, 3H, $N$-Ethyl $\left.\left.\mathrm{CH}_{3}\right), 0.88\left(\mathrm{~d}, J=8.4,9 \mathrm{H}, \mathrm{PMe}_{3}\right) .{ }^{13} \mathrm{C} \mathrm{NMR}_{\left(d-\mathrm{CDCl}_{3}, \delta\right): 157.3(\mathrm{C} 2}{ }^{\prime}\right)$, 151.1 (PzA3), 142.8 (PzB3), 140.0 (PzC3), 136.7 (C5'), 136.5 (Pz5), 135.6 (Pz5), 134.8 (PzA5), 124.3 (C3’), 123.3 (C5'), 106.6 (PzB4), 105.8 (PzC4), 104.6 (PzA4), 78.1 (C7a), 57.6 (d, $J=20.0, \mathrm{C} 6), 56.9$ (C7), $51.5(\mathrm{C} 2), 49.3\left(N\right.$-Ethyl $\left.\mathrm{CH}_{2}\right), 46.1(\mathrm{C} 4), 42.1$ (d, $J=$ 6.1, C5), 38.6 (C3a), 28.3 (C3), $15.7\left(2^{\prime}-\mathrm{CH}_{3}\right), 13.7$ (d, $\left.J=28.0, \mathrm{PMe}_{3}\right), 13.1$ (N-Ethyl $\left.\mathrm{CH}_{3}\right) .{ }^{31} \mathrm{P} \mathrm{NMR}\left(d-\mathrm{CDCl}_{3}, \delta\right):-9.24\left(J_{\mathrm{wp}}=281\right) . \mathrm{IR}: \mathrm{v}_{\mathrm{BH}}=2480 \mathrm{~cm}^{-1}, \mathrm{v}_{\mathrm{NO}}=1550 \mathrm{~cm}^{-1}$. CV (DMA): $\mathrm{E}_{\mathrm{p}, \mathrm{a}}=0.40$ V. HRMS: $\left[\mathrm{M}^{+}\right]=\left[\mathrm{C}_{27} \mathrm{H}_{40} \mathrm{~N}_{8} \mathrm{OBPSW}+\mathrm{H}^{+}\right]$obs'd (\%), calc'd (\%), ppm: 749.2417 (77), 749.2442 (78), -3.3; 750.2439 (78), 750.2466 (79), -3.7; 751.2444 (100), 751.2464 (100), -2.7; 752.2475 (49), 752.2499 (49), -3.2; 753.2487 (90), $753.2495(84),-1.0$.

Compound 52: Outside of the glovebox, $\mathrm{LiAlH}_{4}(0.156 \mathrm{~g}, 4.110 \mathrm{mmol})$ was added to a stirring mixture of Compound 8 (0.715 g, $0.809 \mathrm{mmol})$ in $\mathrm{Et}_{2} \mathrm{O}(100 \mathrm{~mL})$. After $30 \mathrm{~min}$, the grey, heterogeneous solution was filtered through a $60 \mathrm{~mL} \mathrm{M}$ frit packed with $3 \mathrm{~cm}$ of 
celite. The frit was washed with an additional $50 \mathrm{~mL}$ of $\mathrm{Et}_{2} \mathrm{O}$ and the filtrate was concentrated in vacuo. The resulting clear oil was redissolved in DCM (50 mL) and washed with $50 \mathrm{~mL}$ of $\mathrm{Na}_{2} \mathrm{CO}_{3}$ (saturated, aq). The aqueous layer was back extracted with DCM $(2 \times 50 \mathrm{~mL})$. The resulting organic fractions were combined, washed with deionized water $(100 \mathrm{~mL})$, and dried over anhydrous $\mathrm{MgSO}_{4}$. Concentrating the solution in vacuo produced a yellow powder of Compound $52(0.525 \mathrm{~g}, 0.715 \mathrm{mmol}, 83 \%) .{ }^{1} \mathrm{H}$ NMR ( $d$-acetone, $\delta): 9.48(\mathrm{~d}, J=2.0,1 \mathrm{H}, \mathrm{PzA} 3), 8.16(\mathrm{~d}, J=2.0,1 \mathrm{H}, \mathrm{PzB} 3), 7.93(\mathrm{~m}$, 2H, PzB5 and PzC5), $7.68(\mathrm{~d}, J=2.0,1 \mathrm{H}, \mathrm{PzA} 5), 7.42(\mathrm{~d}, J=2.0,1 \mathrm{H}, \mathrm{PzC} 3), 6.40(\mathrm{t}, J$ $=2,1 \mathrm{H}, \mathrm{PzB} 4), 6.32(\mathrm{t}, J=2.0,1 \mathrm{H}, \mathrm{PzC} 4), 6.13(\mathrm{t}, J=2.0,1 \mathrm{H}, \mathrm{PzA} 4), 6.11(\mathrm{~d}, J=3.0$, 1H, H3'), 5.96 (m, 1H, H4'), 4.42 (m, 1H, H5), 4.02 (dd, J = 3.7, 10.4, 1H, H7a), 3.26 (m, 1H, H2x), 3.03 (m, 1H, H6), 2.56 (m, 1H, N-Ethyl-CH $\left.\mathrm{CH}_{2}\right), 2.50$ (m, 1H, H3a), 2.27 (s, 3H, C2’Me), 2.08 (m, 1H, H4x), $2.00(\mathrm{~m}, 1 \mathrm{H}, \mathrm{H} 2 \mathrm{y}), 1.93$ (m, 1H, H3x), 1.70 (dd, $J=$ 3.7, 11.6, 1H, H7), 1.93 (m, 1H, H4y), 1.66 (m, 1H, N-Ethyl- $\left.\mathrm{CH}_{2}\right), 1.28$ (m, 1H, H3y), $0.94\left(\mathrm{~d}, J=8.44,9 \mathrm{H}, \mathrm{PMe}_{3}\right) .{ }^{13} \mathrm{C}$ NMR $(d$-acetone, $\delta): 164.6\left(\mathrm{C} 2^{\prime}\right), 151.3(\mathrm{PzA} 3), 149.9$ (C5’), 143.7 (PzB3), 141.3 (PzC3), 137.7 (Pz5), 136.8 (Pz5), 136.0 (Pz5), 107.4 (Pz4), 107.0 (C3' or C4'), 106.9 (Pz4), 105.2 (C3' or C4'), 105.3 (Pz4), 78.80 (C7a), 56.3 (C7), $54.0(\mathrm{~d}, J=11.5, \mathrm{C} 6), 52.1(\mathrm{C} 2), 49.9\left(\mathrm{~N}-\mathrm{Ethyl}^{\left.-\mathrm{CH}_{2}\right),} 42.0(\mathrm{C} 4), 40.6\right.$ (C5), 39.0 (C3a),

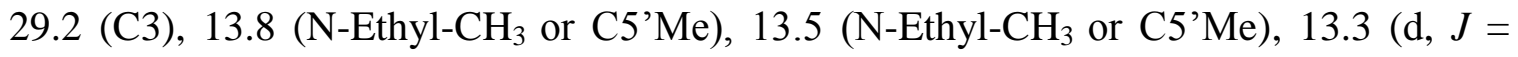
$\left.27.4, \mathrm{PMe}_{3}\right) .{ }^{31} \mathrm{P} \mathrm{NMR}\left(\mathrm{CDCl}_{3}, \delta\right):-10.6\left(J_{\mathrm{WP}}=270\right) . \mathrm{IR}: \mathrm{v}_{\mathrm{BH}}=2484 \mathrm{~cm}^{-1}, v_{\mathrm{NO}}=1539$ $\mathrm{cm}^{-1} \cdot \mathrm{CV}$ (DMA): $\mathrm{E}_{\mathrm{p}, \mathrm{a}}=0.38 \mathrm{~V} . \quad$ HRMS: $[\mathrm{M}+\mathrm{H}]^{+}=\left[\mathrm{C}_{27} \mathrm{H}_{40} \mathrm{BN}_{8} \mathrm{O}_{2} \mathrm{PW}+\mathrm{H}^{+}\right]$obsd (\%), calcd (\%), ppm: 733.2657 (85), $733.2670(82),-1.8 ; 734.2684(81), 734.2696(81)$, -1.6; 735.2685 (100), 735.2695 (100), -1.4; 736.2732 (47), 736.2734 (47), -0.3; 737.2726 
(80), 737.2727 (83), -0.2. Anal. Calc'd for $\mathrm{C}_{27} \mathrm{H}_{40} \mathrm{BN}_{8} \mathrm{O}_{2} \mathrm{PW}$ : C, 44.16; H, 5.49; N, 15.26. Found: C, 44.01; H, 5.38; N, 15.10.

Compound 53: To an oven dried $500 \mathrm{~mL}$ round bottom flask, DME (200 mL) was added and stirred. A solution of Compound 15 (0.760 g, $0.875 \mathrm{mmol})$ in DCM (5 mL) was added to the DME. To this stirring solution, $\mathrm{LiAlH}_{4}$ was added $(0.166 \mathrm{~g}, 0.4 .37 \mathrm{mmol})$ and stirred for $1 \mathrm{hr}$. The reaction was quenched with $\mathrm{H}_{2} \mathrm{O}(50 \mathrm{~mL})$ and the solution was removed from the glovebox and was diluted with DCM $(200 \mathrm{~mL})$. This was treated with $2 \times 100 \mathrm{~mL}$ of $\mathrm{Na}_{2} \mathrm{CO}_{3}$ (saturated, aq). The aqueous layer was back extracted with DCM (1 x $300 \mathrm{~mL}$ ), and the combined organic layers were washed with deionized water (50 $\mathrm{mL}$ ). This was then dried over anhydrous $\mathrm{MgSO}_{4}$ and concentrated in vacuo to a yellow oil, yielding Compound 53 (0.465 g, 0.636 mmol, 73\%). ${ }^{1} \mathrm{H} \mathrm{NMR}\left(d-\mathrm{CDCl}_{3}, \delta\right): 9.43$ (d, $J=2.0,1 \mathrm{H}, \mathrm{PzA} 3), 8.14(\mathrm{~d}, J=2.0,1 \mathrm{H}, \mathrm{PzB} 3), 7.79\left(\mathrm{t}, J=1.0,1 \mathrm{H}, \mathrm{H} 2{ }^{\prime}\right), 7.72(\mathrm{~d}, J=$ 2.0, 1H, PzC5), 7.70 (d, $J=2.0,1 \mathrm{H}, \mathrm{PzB} 5), 7.49$ (d, $J=2.0,1 \mathrm{H}$, PzA5), 7.30 (t, $J=1.1$, $\left.1 \mathrm{H}, \mathrm{H} 5^{\prime}\right), 7.16(\mathrm{~d}, J=2.0,1 \mathrm{H}, \mathrm{PzC} 3), 7.12\left(\mathrm{t}, J=1.0,1 \mathrm{H}, \mathrm{H} 4^{\prime}\right), 6.31(\mathrm{t}, J=2.0,1 \mathrm{H}$, PzB4), $6.21(\mathrm{t}, J=2.0,1 \mathrm{H}, \mathrm{PzC} 4), 6.06(\mathrm{t}, J=2.0,1 \mathrm{H}, \mathrm{PzA} 4), 5.61(\mathrm{t}, J=8.8,1 \mathrm{H}, \mathrm{H} 5)$, 4.09 (dd, $J=10.3,3.9,1 \mathrm{H}, \mathrm{H} 7 \mathrm{a}), 3.24$ (m, 1H, H2x), 2.77 (m, 1H, H6), 2.51 (dd, $J=$ 11.1, 7.5, 1H, $N$-Ethyl $\left.\mathrm{CH}_{2}\right), 2.42$ (m, 1H, H3a), 2.36 (m, 1H, H4x), 2.02 (m, 1H, H2y), $2.00(\mathrm{~m}, 1 \mathrm{H}, \mathrm{H} 3 \mathrm{x}), 1.77(\mathrm{dd}, J=11.8,3.6,1 \mathrm{H}, \mathrm{H} 7), 1.69\left(\mathrm{~m}, 1 \mathrm{H}, N\right.$-Ethyl $\left.\mathrm{CH}_{2}\right), 1.42$ (m, 1H, H4y), 1.22 (m, 1H, H3y), 0.91 (t, $J=7.4,3 \mathrm{H}, N$-Ethyl $\left.\mathrm{CH}_{3}\right), 0.79$ (d, $J=8.2,9 \mathrm{H}$, $\left.\mathrm{PMe}_{3}\right) . \quad{ }^{13} \mathrm{C}$ NMR $\left(d-\mathrm{CDCl}_{3}, \delta\right): 151.0$ (PzA3), 142.7 (PzB3), 139.7 (PzC3), 136.3 (PzC5), 135.8 (PzB5), 135.1 (PzA5), 106.8 (PzB4), 106.1 (PzC4), 104.7 (PzA4), 77.3 (C7a), 59.4 (d, $J=3.3, \mathrm{C} 5), 55.6(\mathrm{C} 7), 53.6$ (d, $J=24.2, \mathrm{C} 6), 51.4$ (C2), 49.1 ( $N$-Ethyl 
$\left.\mathrm{CH}_{2}\right), 43.7$ (C4), 37.3 (C3a), $24.0(\mathrm{C} 3), 13.1\left(N\right.$-Ethyl $\left.\mathrm{CH}_{3}\right), 13.0\left(\mathrm{~d}, J=27.0, \mathrm{PMe}_{3}\right) .{ }^{31} \mathrm{P}$ $\operatorname{NMR}\left(d-\mathrm{CDCl}_{3}, \delta\right):-11.75\left(J_{\mathrm{wp}}=275\right) . \mathrm{IR}: v_{\mathrm{BH}}=2492 \mathrm{~cm}^{-1}, v_{\mathrm{NO}}=1535 \mathrm{~cm}^{-1} \cdot \mathrm{CV}$ (DMA): $\mathrm{E}_{\mathrm{p}, \mathrm{a}}=0.55 \mathrm{~V}$. HRMS: $\left[\left(\mathrm{M}-\mathrm{H}^{-}\right)^{+}\right]=\left[\left(\mathrm{C}_{25} \mathrm{H}_{38} \mathrm{~N}_{10} \mathrm{OBPW}-\mathrm{H}^{-}\right)^{+}\right]$obs'd (\%), calc'd (\%), ppm: 717.2439 (66), 717.2469 (82), -4.2; 718.2499 (72), 718.2494 (81), 0.7; 719.2499 (100), 719.2494 (100), 0.8; 720.2551 (60), 720.2533 (46), 2.6; 721.2523 (85), $721.2526(83),-0.4$.

Compound 54: To an oven dried $250 \mathrm{~mL}$ round bottom flask, DME (100 mL) was added and stirred. A solution of Compound $5(0.508 \mathrm{~g}, 0.634 \mathrm{mmol})$ in DCM $(5 \mathrm{~mL})$ was added to the DME. To this stirring solution, $\mathrm{LiAlH}_{4}$ was added $(0.119 \mathrm{~g}, 3.13 \mathrm{mmol})$ and stirred for 10 min. The reaction was quenched with $\mathrm{H}_{2} \mathrm{O}(8 \mathrm{~mL})$ and the solution was removed from the glovebox and was diluted with DCM $(80 \mathrm{~mL})$. This was treated with 2 x $20 \mathrm{~mL}$ of $\mathrm{Na}_{2} \mathrm{CO}_{3}$ (saturated, aq). The aqueous layer was back extracted with DCM (1 x $20 \mathrm{~mL})$, and the combined organic layers were washed with deionized water $(20 \mathrm{~mL})$. This was then dried over anhydrous $\mathrm{MgSO}_{4}$ and concentrated in vacuo to a yellow oil, yielding Compound 54 (0.378 g, $0.580 \mathrm{mmol}, 91 \%) .{ }^{1} \mathrm{H} \mathrm{NMR}\left(d-\mathrm{CDCl}_{3}, \delta\right)$ : $9.58(\mathrm{~d}, J=$ 2.0, 1H, PzA3), $8.16(\mathrm{~d}, J=2.0,1 \mathrm{H}, \mathrm{PzB} 3), 7.70(\mathrm{~m}, 2 \mathrm{H}, \mathrm{PzC} 5$ and PzB5), $7.47(\mathrm{~d}, J=$ 2.0, 1H, PzA5), 7.34 (d, $J=2.0,1 \mathrm{H}, \mathrm{PzC} 3), 6.38(\mathrm{~m}, 1 \mathrm{H}, \mathrm{H} 5), 6.28(\mathrm{t}, J=2.0,1 \mathrm{H}$, PzB4), 6.19 (t, $J=2.0,1 \mathrm{H}$, PzC4), 6.08 (t, $J=2.0,1 \mathrm{H}$, PzA4), 5.30 (dd, $J=9.3,1.7,1 \mathrm{H}$, H4), $3.94(\mathrm{dt}, J=11.7,2.2,1 \mathrm{H}, \mathrm{H} 7 \mathrm{a}), 3.39$ (m, 1H, H2x), 3.00 (dd, $J=11.5,10.7,1 \mathrm{H}$, H3a), $2.90(\mathrm{~m}, 1 \mathrm{H}, \mathrm{H} 6), 2.76\left(\mathrm{~m}, 1 \mathrm{H}, \mathrm{N}\right.$-Ethyl $\left.\mathrm{CH}_{2}\right), 2.16(\mathrm{~m}, 1 \mathrm{H}, \mathrm{H} 2 \mathrm{y}), 2.01(\mathrm{~m}, 1 \mathrm{H}$, H3x), $1.78\left(\mathrm{~m}, 1 \mathrm{H}, N\right.$-Ethyl $\left.\mathrm{CH}_{2}\right), 1.72$ (dt, $\left.J=10.5,2.0,1 \mathrm{H}, \mathrm{H} 7\right), 1.43$ (m, 1H, H3y), $1.18\left(\mathrm{~d}, J=8.8,9 \mathrm{H}, \mathrm{PMe}_{3}\right), 1.00\left(\mathrm{t}, J=7.3,3 \mathrm{H}, N\right.$-Ethyl $\left.\mathrm{CH}_{3}\right) .{ }^{13} \mathrm{C} \mathrm{NMR}\left(d-\mathrm{CDCl}_{3}, \delta\right)$ : 
150.9 (PzA3), 143.1 (PzB3), 136.6 (PzB5 or PzC5), 135.6 (PzB5 or PzC5), 134.4 (PzA5), 131.5 (d, $J=3.0, \mathrm{C} 5), 140.0$ (PzC3), 123.9 (C4), 106.3 (PzB4), 105.7 (PzC4), 104.7 (PzA4), 78.7 (C7a), 54.8 (d, $J=11.0, \mathrm{C} 6), 54.2$ (C7), 52.8 (C2), 49.0 ( $N$-Ethyl $\left.\mathrm{CH}_{2}\right), 40.7$ (C3a), $26.1(\mathrm{C} 3), 13.4\left(\mathrm{~d}, J=27.4, \mathrm{PMe}_{3}\right), 13.2\left(N\right.$-Ethyl $\left.\mathrm{CH}_{2}\right) .{ }^{31} \mathrm{P}$ NMR $(d-$ $\left.\mathrm{CDCl}_{3}, \delta\right):-11.38\left(J_{\mathrm{wp}}=273\right)$. IR: $v_{\mathrm{BH}}=2484 \mathrm{~cm}^{-1}, v_{\mathrm{NO}}=1554 \mathrm{~cm}^{-1} . \mathrm{CV}(\mathrm{DMA}): \mathrm{E}_{\mathrm{p}, \mathrm{a}}=$ 0.54 V. HRMS: $\left[\mathrm{M}+\mathrm{H}^{+}\right]=\left[\mathrm{C}_{22} \mathrm{H}_{34} \mathrm{~N}_{8} \mathrm{OBPW}+\mathrm{H}^{+}\right]$obsd (\%), calcd (\%), ppm: 651.2246 (82), 651.2251 (84), -0.8; 652.2251 (77), 652.2276 (80), -3.9; 653.2284 (100), 653.2275 (100), 1.4; 654.2312 (45), 654.2316 (43), -0.6; 655.2289 (86), 655.2307 (84), -2.8.

Compound 56: To an oven dried $50 \mathrm{~mL}$ round bottom flask, DME (25 mL) was added and stirred. A solution of Compound $41(0.200 \mathrm{~g}, 0.226 \mathrm{mmol})$ in DCM (5 mL) was added to the DME. To this stirring solution, $\mathrm{LiAlH}_{4}$ was added $(0.043 \mathrm{~g}, 1.13 \mathrm{mmol})$ and stirred for $5 \mathrm{~min}$. The now clear solution was quenched with $\mathrm{H}_{2} \mathrm{O}(10 \mathrm{~mL})$ and the solution was removed from the glovebox and was diluted with DCM $(50 \mathrm{~mL})$. This was treated with $20 \mathrm{~mL}$ of $\mathrm{Na}_{2} \mathrm{CO}_{3}$ (saturated, aq). The aqueous layer was back extracted with DCM (1 x $20 \mathrm{~mL})$, and the combined organic layers were washed with deionized water $(20 \mathrm{~mL})$. This was then dried over anhydrous $\mathrm{MgSO}_{4}$ and concentrated in vacuo to a yellow oil, yielding Compound $56(0.161 \mathrm{~g}, 0.218 \mathrm{mmol}, 96 \%) .{ }^{1} \mathrm{H}$ NMR $\left(d-\mathrm{CDCl}_{3}, \delta\right)$ : 9.44 (d, $J=2.0,1 \mathrm{H}, \mathrm{PzA} 3), 8.11$ (d, $J=2.0,1 \mathrm{H}, \mathrm{PzB} 3), 7.77$ (d, $J=2.0,1 \mathrm{H}, \mathrm{H} 5$ '), 7.70 (d, $J=2.0,1 \mathrm{H}, \mathrm{PzC} 5), 7.68$ (d, $J=2.0,1 \mathrm{H}, \mathrm{PzB} 5), 7.64(\mathrm{~d}, J=2.0,1 \mathrm{H}, \mathrm{H} 3$ ') 7.49 (d, $J=$ 2.0, 1H, PzA5), 7.26 (d, $J=2.0,1 \mathrm{H}, \mathrm{PzC} 3), 6.36$ (t, $J=2.0,1 \mathrm{H}, \mathrm{H} 4$ '), 6.28 (t, $J=2.0$, 1H, PzB4), 6.19 (t, $J=2.0,1 \mathrm{H}, \mathrm{PzC} 4), 6.07$ (t, $J=2.0,1 \mathrm{H}, \mathrm{PzA} 4), 5.85(\mathrm{t}, J=3.7,1 \mathrm{H}$, H5), 4.61 (dd, $J=11.2,4.6,1 \mathrm{H}, \mathrm{H7a}$ ), 3.98 (d, $J=3.7,1 \mathrm{H}, \mathrm{H} 4), 3.26$ (td, $J=9.3,4.4$, 
1H, H2x), 3.11 (m, 1H, H6), 2.49 (m, 1H, N-Ethyl $\mathrm{CH}_{2}$ ), 2.46 (buried, 1H, H3a), 2.02 (m, 1H, H2y), 1.91 (m, 1H, H3x), 1.82 (m, 1H, H3y), 1.71 (buried, 1H, H7), 1.70 (m, 1H, NEthyl $\left.\mathrm{CH}_{2}\right), 0.91\left(\mathrm{t}, J=7.1,3 \mathrm{H}, N\right.$-Ethyl $\left.\mathrm{CH}_{3}\right), 0.69\left(\mathrm{~d}, J=8.2,9 \mathrm{H}, \mathrm{PMe}_{3}\right) .{ }^{13} \mathrm{C} \mathrm{NMR}(d-$ $\left.\mathrm{CDCl}_{3}, \delta\right): 151.1$ (PzA3), 142.9 (PzB3), 140.1 (PzC3), 139.5 (C3’), 136.6 (Pz5), 135.7 (Pz5), 135.0 (PzA5), 129.9 (C5'), 106.7 (PzB4), 105.9 (PzC4 and C4'), 104.8 (PzA4), 74.1 (C4), 68.7 (C7a), 67.6 (d, $J=3.0, \mathrm{C} 5), 54.0$ (C7), 51.6 (C2), 50.1 (d, $J=11.5, \mathrm{C} 6)$, 49.6 (N-Ethyl $\left.\mathrm{CH}_{2}\right), 42.3$ (C3a), 22.7 (C3), $13.1\left(N\right.$-Ethyl $\left.\mathrm{CH}_{3}\right), 13.0\left(\mathrm{~d}, J=27.0, \mathrm{PMe}_{3}\right)$. ${ }^{31} \mathrm{P}$ NMR $\left(d-\mathrm{CDCl}_{3}, \delta\right):-12.42\left(J_{\mathrm{wp}}=264\right) . \mathrm{IR}: v_{\mathrm{OH}}=3402 \mathrm{~cm}^{-1}, v_{\mathrm{BH}}=2360 \mathrm{~cm}^{-1}, v_{\mathrm{NO}}=$ $1535 \mathrm{~cm}^{-1}$. CV (DMA): $\mathrm{E}_{\mathrm{p}, \mathrm{a}}=0.53 \mathrm{~V} . \mathrm{HRMS}:\left[\mathrm{M}+\mathrm{H}^{+}\right]=\left[\mathrm{C}_{25} \mathrm{H}_{38} \mathrm{~N}_{10} \mathrm{O}_{2} \mathrm{~B} \mathrm{~W}+\mathrm{H}^{+}\right]$obs'd (\%), calc'd (\%), ppm: 735.2590 (89), 735.2575 (82), 2.0; 736.2611 (95), 736.2600 (81), 1.5; 737.2592 (100), 737.2599 (100), -1.0; 738.2628 (59), 738.2638 (46), -1.4; 739.2615 (78), $739.2632(83),-2.2$.

Compound 57: To an oven dried 4-dram vial, DME (10 mL) was added and stirred. A solution of Compound $47(0.200 \mathrm{~g}, 0.232 \mathrm{mmol})$ in DCM $(3 \mathrm{~mL})$ was added to the DME. To this stirring solution, $\mathrm{LiAlH}_{4}$ was added $(0.045 \mathrm{~g}, 1.18 \mathrm{mmol})$ and stirred for 30 min until the solution became clear. The reaction was quenched with $\mathrm{H}_{2} \mathrm{O}(10 \mathrm{~mL})$ and the solution was removed from the glovebox and was diluted with DCM (50 mL). This was treated with $2 \times 20 \mathrm{~mL}$ of $\mathrm{Na}_{2} \mathrm{CO}_{3}$ (saturated, aq). The aqueous layer was back extracted with DCM (1 x $20 \mathrm{~mL})$, and the combined organic layers were washed with deionized water $(40 \mathrm{~mL})$. This was then dried over anhydrous $\mathrm{MgSO}_{4}$ and concentrated in vacuo to a yellow oil, yielding Compound 57 (0.158 g, $0.221 \mathrm{mmol}, 95 \%) .{ }^{1} \mathrm{H}$ NMR $\left(d-\mathrm{CDCl}_{3}, \delta\right): 9.49(\mathrm{~d}, J=2.0,1 \mathrm{H}, \mathrm{PzA} 3), 8.13(\mathrm{~d}, J=2.0,1 \mathrm{H}, \mathrm{PzB} 3), 7.70(\mathrm{~d}, J=2.0$ 
1H, PzC5), 7.68 (d, $J=2.0,1 \mathrm{H}, \mathrm{PzB} 5), 7.45(\mathrm{~d}, J=2.0,1 \mathrm{H}, \mathrm{PzA} 5), 7.29$ (d, $J=2.0,1 \mathrm{H}$, PzC3), $6.26(\mathrm{t}, J=2.0,1 \mathrm{H}, \mathrm{PzB} 4), 6.19(\mathrm{t}, J=2.0,1 \mathrm{H}, \mathrm{PzC} 4), 6.00(\mathrm{t}, J=2.0,1 \mathrm{H}$, PzA4), 4.19 (dd, $J=11.6,3.4,1 \mathrm{H}, \mathrm{H7a}), 4.00$ (m, 1H, H4), 3.83 (dd, $J=5.0,1.9,1 \mathrm{H}$, H5), 3.25 (m, 1H, H3x), 2.83 (m, 1H, H2’x), 2.65 (m, 1H, H2’y), 2.51 (m, 1H, N-Ethyl $\mathrm{CH}_{2}$ ), 2.45 (m, 1H, H3a), 2.30 (m, 1H, H6), 1.99 (m, 1H, H3y), 1.90 (m, 1H, H2x), 1.81 (m, 1H, H2y), 1.67 (m, 1H, N-Ethyl $\mathrm{CH}_{2}$ ), 1.60 (dd, $\left.J=11.7,3.5,1 \mathrm{H}, \mathrm{H} 7\right), 1.16$ (t, $J=$ 7.1, 3H, H3'), 1.13 (d, $\left.J=8.3,9 \mathrm{H}, \mathrm{PMe}_{3}\right), 0.90\left(\mathrm{t}, J=7.1,3 \mathrm{H}, N\right.$-Ethyl $\left.\mathrm{CH}_{3}\right) .{ }^{13} \mathrm{C}$ NMR $\left(d-\mathrm{CDCl}_{3}, \delta\right): 151.0(\mathrm{PzA} 3), 142.9$ (PzB3), 139.9 (PzC3), 136.5 (Pz5), 135.6 (Pz5), 134.8 (PzA5), 106.4 (PzB4), 105.7 (PzC4), 104.6 (PzA4), 69.4 (C7a), 67.1 (C4), 62.8 (d, $J=3.2, \mathrm{C} 5), 55.6(\mathrm{~d}, J=11.2, \mathrm{C} 6), 54.3(\mathrm{C} 7), 51.8(\mathrm{C} 3), 49.4\left(N\right.$-Ethyl $\left.\mathrm{CH}_{2}\right), 42.5$ (C3a), 41.9 (C2'), 23.0 (C2), 16.0 (C3'), 13.4 (d, $\left.J=27.6, \mathrm{PMe}_{3}\right), 13.0\left(N\right.$-Ethyl $\left.\mathrm{CH}_{3}\right) .{ }^{31} \mathrm{P}$ $\operatorname{NMR}\left(d-\mathrm{CDCl}_{3}, \delta\right):-11.2\left(J_{\mathrm{wp}}=271\right) . \mathrm{IR}: v_{\mathrm{BH}}=2484 \mathrm{~cm}^{-1}, v_{\mathrm{NO}}=1535 \mathrm{~cm}^{-1} . \mathrm{CV}$ (DMA): $\mathrm{E}_{\mathrm{p}, \mathrm{a}}=0.36 \mathrm{~V}$. HRMS: $\left[\mathrm{M}+\mathrm{H}^{+}\right]=\left[\mathrm{C}_{24} \mathrm{H}_{41} \mathrm{~N}_{9} \mathrm{O}_{2} \mathrm{BPW}+\mathrm{H}^{+}\right]$obs'd (\%), calc'd (\%), ppm: 712.2784 (81), 712.2779 (83), 0.7; 713.2803 (74), 713.2804 (81), -0.1; 714.2820 (100), 714.2803 (100), 2.4; 715.2822 (44), 715.2843 (45), -2.9; 716.2868 (78), 716.2835 (83), 4.5.

Compound 58: To an oven dried $100 \mathrm{~mL}$ round bottom flask, DME (20 mL) was added and stirred. A solution of Compound 16 (0.205 g, $0.235 \mathrm{mmol})$ in DCM (1 mL) was added to the DME. To this stirring solution, lithium aluminum hydride was added $(0.049$ g, $1.29 \mathrm{mmol})$ and stirred for $15 \mathrm{hr}$. The reaction was quenched with $\mathrm{H}_{2} \mathrm{O}(10 \mathrm{~mL})$ and the mixture was removed from the glovebox and was diluted with DCM (50 mL). This was treated with $2 \times 30 \mathrm{~mL}$ of $\mathrm{Na}_{2} \mathrm{CO}_{3}$ (saturated, aq). The aqueous layer was back 
extracted with DCM (1 x $20 \mathrm{~mL})$, and the combined organic layers were washed deionized water $(50 \mathrm{~mL})$. This was then dried over anhydrous $\mathrm{MgSO}_{4}$ and concentrated in vacuo to a yellow oil, yielding Compound 58 (0.138 g, $0.210 \mathrm{mmol}, 89 \%) .{ }^{1} \mathrm{H}$ NMR $\left(d-\mathrm{CDCl}_{3}, \delta\right): 9.50(\mathrm{~d}, J=2.0,1 \mathrm{H}, \mathrm{PzA} 3), 8.13(\mathrm{~d}, J=2.0,1 \mathrm{H}, \mathrm{PzB} 3), 7.68(\mathrm{~d}, J=2.0$, 1H, PzC5), 7.67 (d, $J=2.0,1 \mathrm{H}, \mathrm{PzB} 5), 7.47(\mathrm{~d}, J=2.0,1 \mathrm{H}, \mathrm{PzA} 5), 7.29(\mathrm{~d}, J=2.0,1 \mathrm{H}$, PzC3), $6.27(\mathrm{t}, J=2.0,1 \mathrm{H}, \mathrm{PzC} 4), 6.18(\mathrm{t}, J=2.0,1 \mathrm{H}, \mathrm{PzB} 4), 6.06(\mathrm{t}, J=2.0,1 \mathrm{H}$, PzA4), 3.87 (dd, $J=10.5,3.5,1 \mathrm{H}, \mathrm{H} 7 \mathrm{a}), 3.24$ (m, 1H, H2x), 2.90 (m, 2H, H5), 2.75 (m, 1H, H6), 2.60 (m, 1H, N-Ethyl $\mathrm{CH}_{2}$ ), 2.22 (m, 1H, H3a), 1.98 (m, 1H, H2y), 1.97 (m, 1H, H3x ), 1.86 (m, 1H, H4x), 1.67 (m, 1H, H7), 1.65 (m, 1H, N-Ethyl $\left.\mathrm{CH}_{2}\right), 1.26(\mathrm{~m}, 1 \mathrm{H}$, H3y), 1.24 (m, 1H, H4y), 1.08 (d, $\left.J=8.1,9 \mathrm{H}, \mathrm{PMe}_{3}\right), 0.95\left(\mathrm{t}, J=7.1,3 \mathrm{H}, \mathrm{N}-\mathrm{Ethyl}_{\mathrm{CH}}\right)$. ${ }^{13} \mathrm{C}$ NMR $\left(d-\mathrm{CDCl}_{3}, \delta\right): 151.0$ (PzA3), 142.8 (PzB3), $139.7(\mathrm{PzC} 3), 136.3(\mathrm{Pz} 5), 135.4$ (Pz5), 134.6 (PzA5), 106.4 (PzC4), 105.7 (PzB4), 104.7 (PzA4), 78.7 (C7a), 56.3 (C7), 51.5 (C2), 51.3 (d, $J=12.0, \mathrm{C} 6), 49.6\left(\mathrm{~N}-\mathrm{Ethyl} \mathrm{CH}_{2}\right), 38.9(\mathrm{C} 3 \mathrm{a}), 32.3(\mathrm{C} 4), 29.3$ (d, $J=$ 4.2, C5), $28.5(\mathrm{C} 3), 13.4\left(\mathrm{~d}, J=26.8, \mathrm{PMe}_{3}\right), 13.1\left(\mathrm{~N}-\right.$ Ethyl $\left.\mathrm{CH}_{3}\right) .{ }^{31} \mathrm{P} \mathrm{NMR}\left(d-\mathrm{CDCl}_{3}\right.$, $\delta):-9.17\left(J_{\mathrm{wp}}=274\right)$.

Compound 59: Outside of the box, $\mathrm{NOPF}_{6}(0.036 \mathrm{~g}, 0.205 \mathrm{mmol})$ was added to a vigorously stirring solution of Compound 51 (0.100 g, $0.133 \mathrm{mmol})$ in $\mathrm{MeCN}$ (5 mL). After 2 days, the solution was diluted with $50 \mathrm{~mL}$ DCM and treated with $2 \times 20 \mathrm{~mL}$ of $\mathrm{Na}_{2} \mathrm{CO}_{3}$ (saturated, aq). The aqueous layer was back extracted with DCM (2 x $\left.20 \mathrm{~mL}\right)$, and the combined organic layers were washed with deionized water $(30 \mathrm{~mL})$. The organic layer was then dried over anhydrous $\mathrm{MgSO}_{4}$ and concentrated in vacuo. The brown oil was redissolved in minimal DCM and added dropwise to a stirring solution of $\mathrm{Et}_{2} \mathrm{O}$ (100 
$\mathrm{mL}$ ) to induce precipitation of a brown solid. The precipitate was collected on a $30 \mathrm{~mL}$ fine-porosity fritted funnel with $2 \mathrm{~cm}$ celite. To the filtrate was added triethylamine (10 $\mathrm{mL}$ ) and the solution was added to a $4 \mathrm{~cm}$ silica column set with $10 \%$ TEA in $\mathrm{Et}_{2} \mathrm{O}$. The column was washed with $10 \%$ TEA in $\mathrm{Et}_{2} \mathrm{O}(50 \mathrm{~mL})$. The filtrate was concentrated in vacuo to a 4-dram vial. The residue was loaded onto a $20 \mathrm{~cm}$ x $20 \mathrm{~cm}$ x $1000 \mu \mathrm{m} \mathrm{Al}_{2} \mathrm{O}_{3}$ preparatory TLC plate with $2 \times 0.3 \mathrm{~mL}$ DCM. The plate was developed using a $10 \%$ $\mathrm{Et}_{2} \mathrm{O} / \mathrm{HLPC}$ hexanes solution. A band, which stained positive with $\mathrm{KMnO}_{4}$, was scraped and placed in a round bottom flask with $70 \mathrm{~mL}$ HPLC EtOAc and sonicated for $15 \mathrm{~min}$. The slurry was filtered on a $30 \mathrm{~mL}$ fine-porosity fritted funnel and washed with $50 \mathrm{~mL}$ HPLC EtOAc. The filtrate was concentrated in vacuo. The oil was collected, yielding Compound $59(0.011 \mathrm{~g}, 0.045 \mathrm{mmol}, 34 \%) .{ }^{1} \mathrm{H} \mathrm{NMR}\left(d-\mathrm{CDCl}_{3}, \delta\right): 6.59(\mathrm{dd}, J=3.3$, 0.4, 1H, H4'), 6.55 (m, 1H, H3'), 6.01 (dt, $J=9.9,1.9,1 \mathrm{H}, \mathrm{H6}), 5.72$ (dtd, $J=10.0,2.6$, 0.9, 1H, H7), 3.83 (m, 1H, H5), 3.36 (m, 1H, H2x), 3.02 (m, 1H, $N$-Ethyl $\mathrm{CH}_{2}$ ), 2.53 (m, 1H, H7a), 2.43 (d, $\left.J=1.0,3 \mathrm{H}, 2^{\prime} \mathrm{Me}\right), 2.36$ (m, 1H, H4x), 2.30 (m, 1H, H2y), 2.14 (m, 1H, N-Ethyl $\mathrm{CH}_{2}$ ), 2.03 (m, 1H, H3a), 1.92 (m, 1H, H3x), 1.61 (m, 1H, H4y), 1.47 (m,

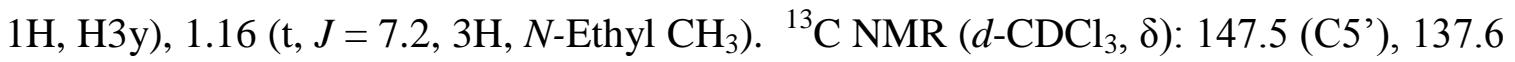
(C2’), 131.7 (C7), 127.5 (C6), 124.7 (C3’), 122.8 (C4'), 67.5 (C7a), 52.7 (C2), 48.9 (NEthyl $\mathrm{CH}_{2}$ ), 43.1 (C3a), 40.3 (C5), 37.4 (C4), 27.4 (C3), 15.4 (C2’Me), 13.8 (N-Ethyl $\left.\mathrm{CH}_{3}\right) . \mathrm{HRMS}:\left[\mathrm{M}+\mathrm{H}^{+}\right]=\left[\mathrm{C}_{15} \mathrm{H}_{21} \mathrm{NS}+\mathrm{H}^{+}\right]$obs'd, calc'd, ppm: 248.1476, 248.1467, 3.4.

Compound 60: Outside of the box, $\mathrm{NOPF}_{6}(0.046 \mathrm{~g}, 0.263 \mathrm{mmol})$ was added to a vigorously stirring solution of Compound 48 (0.125 g, $0.158 \mathrm{mmol})$ in acetone $(5 \mathrm{~mL})$. After $2 \mathrm{~h}$, the solution was diluted with $50 \mathrm{~mL}$ DCM and treated with 2 x $20 \mathrm{~mL}$ of 
$\mathrm{Na}_{2} \mathrm{CO}_{3}$ (saturated, aq). The aqueous layer was back extracted with DCM $(2 \times 20 \mathrm{~mL})$, and the combined organic layers were washed with deionized water $(30 \mathrm{~mL})$. The organic layer was then dried over anhydrous $\mathrm{MgSO}_{4}$ and concentrated in vacuo. The brown oil was redissolved in minimal DCM and added dropwise to a stirring solution of $\mathrm{Et}_{2} \mathrm{O}$ (200 $\mathrm{mL}$ ) to induce precipitation of a brown solid. The precipitate was collected on a $30 \mathrm{~mL}$ fine-porosity fritted funnel with $2 \mathrm{~cm}$ celite. To the filtrate was added triethylamine (12 $\mathrm{mL}$ ) and the solution was added to a $4 \mathrm{~cm}$ silica column set with $10 \%$ TEA in $\mathrm{Et}_{2} \mathrm{O}$. The column was washed with $10 \%$ TEA in $\mathrm{Et}_{2} \mathrm{O}(50 \mathrm{~mL})$. The filtrate was concentrated in vacuo to a 4-dram vial. The residue was loaded onto a $20 \mathrm{~cm}$ x $20 \mathrm{~cm}$ x $1000 \mu \mathrm{m} \mathrm{Al}_{2} \mathrm{O}_{3}$ preparatory TLC place with $2 \times 0.3 \mathrm{~mL}$ DCM. The plate was developed using $\mathrm{Et}_{2} \mathrm{O}$. A band which stained positive with $\mathrm{KMnO}_{4}$ and was UV active was scraped and placed in a round bottom flask with $70 \mathrm{~mL}$ HPLC EtOAc and sonicated for $15 \mathrm{~min}$. The slurry was filtered on a $30 \mathrm{~mL}$ fine-porosity fritted funnel and washed with $50 \mathrm{~mL}$ HPLC EtOAc. The filtrate was concentrated in vacuo. The oil was collected, yielding Compound 60 (0.020 g, $0.070 \mathrm{mmol}, 44 \%) .{ }^{1} \mathrm{H} \mathrm{NMR}\left(d-\mathrm{CDCl}_{3}, \delta\right): 7.03$ (dd, $J=6.9,1.9,1 \mathrm{H}, \mathrm{H} 6$ '), 6.44 (s, 1H, H3'), 6.43 (dd, $\left.J=7.4,2.4,1 \mathrm{H}, \mathrm{H} 5^{\prime}\right), 6.06$ (d, $\left.J=9.7,1 \mathrm{H}, \mathrm{H} 7\right), 5.59$ (dt, $J=$ 10.3, 2.5, 1H, H6), 3.91 (m, 1H, H5), 3.80 (s, 3H, OMe), 3.79 (s, 3H, OMe). 3.35 (m, 1H, H2x), 3.05 (m, 1H, N-Ethyl $\mathrm{CH}_{2}$ ), 2.53 (d, $\left.J=8.5,1 \mathrm{H}, \mathrm{H} 7 \mathrm{a}\right), 2.30$ (m, 1H, H4x), 2.27 (buried, 1H, H2y), 2.13 (m, 1H, $N$-Ethyl $\mathrm{CH}_{2}$ ), 2.06 (m, 1H, H3a), 1.38 (m, 2H, H3), 1.29 $(\mathrm{m}, 1 \mathrm{H}, \mathrm{H} 4 \mathrm{y}), 1.16\left(\mathrm{t}, J=7.2,3 \mathrm{H}, N\right.$-Ethyl $\left.\mathrm{CH}_{3}\right) \cdot{ }^{13} \mathrm{C} \mathrm{NMR}\left(d-\mathrm{CDCl}_{3}, \delta\right): 159.0\left(\mathrm{C} 2{ }^{\prime}\right.$ or C4'), 157.7 (C2' or C4'), 132.4 (C7), 128.2 (C1'), 127.5 (C6’), 126.5 (C6), 104.1 (C5'), 98.4 (C3'), 67.7 (C7a), 55.3 (OMe), 55.2 (OMe), 52.5 (C2), 48.7 ( $N$-Ethyl $\left.\mathrm{CH}_{2}\right), 43.1$ 
(C3a), 37.5 (C5), 35.4 (C4), 27.3 (C3), 13.5 (N-Ethyl $\left.\mathrm{CH}_{3}\right) . \quad$ HRMS: [M+H $\left.{ }^{+}\right]=$ $\left[\mathrm{C}_{18} \mathrm{H}_{25} \mathrm{NO}_{2}+\mathrm{H}^{+}\right]$obs'd , calc'd, ppm: 288.1953, 288.1958.

Compound 61: In a glovebox, DDQ $(0.124 \mathrm{~g}, 0.546 \mathrm{mmol})$ was added to a vigorously stirring solution of Compound $52(0.204 \mathrm{~g}, 0.278 \mathrm{mmol})$ in acetonitrile (10 mL). After 1 $\mathrm{hr}$, the solution was removed from the box and diluted with $100 \mathrm{~mL}$ DCM and treated with 2 x $50 \mathrm{~mL}$ of $\mathrm{Na}_{2} \mathrm{CO}_{3}$ (saturated, aq). The aqueous layer was back extracted with DCM (2 x $50 \mathrm{~mL})$, and the combined organic layers were washed deionized water (50 $\mathrm{mL}$ ). The organic was then dried over anhydrous $\mathrm{MgSO}_{4}$ and concentrated in vacuo. The brown oil was redissolved in minimal DCM and added dropwise to a stirring solution of $\mathrm{Et}_{2} \mathrm{O}(200 \mathrm{~mL})$ to induce precipitation of a brown solid. The precipitate was collected on a $15 \mathrm{~mL}$ fine porosity fritted funnel with $2 \mathrm{~cm}$ celite. To the filtrate was added $20 \mathrm{~mL}$ of triethylamine (TEA) and the solution was added to a $4 \mathrm{~cm}$ silica column set with $10 \%$ TEA in $\mathrm{Et}_{2} \mathrm{O}$. The column was washed with $50 \mathrm{~mL}$ of $10 \%$ TEA in $\mathrm{Et}_{2} \mathrm{O}$. The filtrate was concentrated in vacuo to a vial. The residue was loaded onto a $20 \mathrm{~cm}$ x $20 \mathrm{~cm}$ x $1000 \mu \mathrm{m}$ $\mathrm{Al}_{2} \mathrm{O}_{3}$ preparatory TLC place with $2 \times 0.3 \mathrm{~mL}$ DCM. The plate was developed using a $33 \% \mathrm{Et}_{2} \mathrm{O} / \mathrm{HLPC}$ Hexanes solution. A band which stained positive with $\mathrm{KMnO}_{4}\left(R_{f}=\right.$ $0.45-0.60$ ) was collected and placed in a round bottom flask with $50 \mathrm{~mL}$ HPLC EtOAc and sonicated for 15 min to break up alumina. The slurry was filtered on a $30 \mathrm{~mL}$ fine porosity fritted funnel and washed with $50 \mathrm{~mL}$ HPLC EtOAc. The filtrate was then stripped to dryness. The oil was collected yielding $61(0.008 \mathrm{~g}, 0.034 \mathrm{mmol}, 13 \%) .{ }^{1} \mathrm{H}$ $\operatorname{NMR}\left(\mathrm{CDCl}_{3}, \delta\right): 6.07(\mathrm{dt}, J=10,2,1 \mathrm{H}, \mathrm{H} 7), 5.86(\mathrm{~d}, J=3,1 \mathrm{H}, \mathrm{H} 3$ '), $5.84(\mathrm{dd}, J=3,1$, 1H, H4'), 5.74 (m, 1H, H6), 3.63 (m, 1H, H5), 3.35 (q, J = 10, 1H, H2x), 3.00 (m, 1H, N- 
Ethyl- $\mathrm{CH}_{2}$ ), 2.51 (d, $\left.J=10,1 \mathrm{H}, \mathrm{H} 7 \mathrm{a}\right), 2.30$ (m, 1H, H2y), 2.25 (s, 3H, C2’Me), 2.29 (m, buried, 1H, H4x), 2.12 (m, 1H, N-Ethyl- $\left.\mathrm{CH}_{2}\right), 2.00$ (m, 1H, H3a), 1.90 (m, 1H, H3x), $1.61(\mathrm{~m}, 1 \mathrm{H}, \mathrm{H} 4 \mathrm{y}), 1.47(\mathrm{~m}, 1 \mathrm{H}, \mathrm{H} 3 \mathrm{y}), 1.13\left(\mathrm{t}, J=7,3 \mathrm{H}, \mathrm{N}-\mathrm{Ethyl}-\mathrm{CH}_{3}\right) .{ }^{13} \mathrm{C}$ NMR $\left(\mathrm{CDCl}_{3}, \delta\right): 156.5\left(\mathrm{C} 2^{\prime}\right), 150.7$ (C5'), 129.6 (C6), 127.8 (C7), 105.9 (C4'), 104.6 (C3'), 67.5 (C7a), 52.7 (C2), 48.9 (N-Ethyl-CH2), 42.7 (C3a), 38.5 (C5), 33.06 (C4), 27.5 (C3), 13.7 (N-Ethyl-CH 3$), 13.6\left(\mathrm{C} 2^{\prime} \mathrm{Me}\right)$. HRMS: $\left[\mathrm{M}^{+} \mathrm{H}^{+}\right]=\left[\mathrm{C}_{15} \mathrm{H}_{21} \mathrm{NO}+\mathrm{H}^{+}\right]$obsd $(\%)$, calcd (\%), ppm: 232.1685 (100), $232.1696(100),-4.7$.

Compound 62: Outside of the box, $\mathrm{NOPF}_{6}(0.089 \mathrm{~g}, 0.508 \mathrm{mmol})$ was added to a vigorously stirring solution of Compound $56(0.237 \mathrm{~g}, 0.321 \mathrm{mmol})$ in acetone $(20 \mathrm{~mL})$. After $1.5 \mathrm{~h}$, the solution was diluted with $50 \mathrm{~mL}$ DCM and treated with $2 \times 20 \mathrm{~mL}$ of $\mathrm{Na}_{2} \mathrm{CO}_{3}$ (saturated, aq). The aqueous layer was back extracted with DCM (2 x $\left.20 \mathrm{~mL}\right)$, and the combined organic layers were washed with deionized water $(30 \mathrm{~mL})$. The organic layer was then dried over anhydrous $\mathrm{MgSO}_{4}$ and concentrated in vacuo. The brown oil was redissolved in minimal DCM and added dropwise to a stirring solution of $\mathrm{Et}_{2} \mathrm{O}$ (200 $\mathrm{mL}$ ) to induce precipitation of a brown solid. The precipitate was collected on a $30 \mathrm{~mL}$ fine-porosity fritted funnel with $2 \mathrm{~cm}$ celite. The filtrate was concentrated in a 4-dram vial in vacuo. The residue was loaded onto a $20 \mathrm{~cm}$ × $20 \mathrm{~cm}$ x $1000 \mu \mathrm{m} \mathrm{Al}_{2} \mathrm{O}_{3}$ preparatory TLC place with $2 \times 0.3 \mathrm{~mL}$ DCM. The plate was developed using a $10 \%$ HPLC EtOAc/Et $2 \mathrm{O}$ solution. A band which stained positive with $\mathrm{KMnO}_{4}\left(\mathrm{R}_{\mathrm{f}} \approx 0.9-.19\right)$, was collected and placed in a round bottom flask with $70 \mathrm{~mL}$ HPLC EtOAc and sonicated for $15 \mathrm{~min}$. The slurry was filtered on a $30 \mathrm{~mL}$ fine-porosity fritted funnel and washed with $50 \mathrm{~mL}$ HPLC EtOAc. The filtrate was concentrated in vacuo. The oil was 
collected yielding Compound $62(0.017 \mathrm{~g}, 0.072 \mathrm{mmol}, 23 \%) .{ }^{1} \mathrm{H}$ NMR $\left(d-\mathrm{CDCl}_{3}, \delta\right)$ : $7.56\left(\mathrm{~d}, J=1.7,1 \mathrm{H}, \mathrm{H} 3^{\prime}\right), 7.51$ (d, $J=2.3,1 \mathrm{H}, \mathrm{H} 5$ '), 6.30 (t, $J=2.0,1 \mathrm{H}, \mathrm{H} 4$ ') 6.26 (dt, $J$ $=10.2,2.0,1 \mathrm{H}, \mathrm{H6}), 5.64(\mathrm{~m}, 1 \mathrm{H}, \mathrm{H} 7), 5.06(\mathrm{~m}, 1 \mathrm{H}, \mathrm{H} 5), 4.43(\mathrm{~d}, J=3.7,1 \mathrm{H}, \mathrm{H} 4), 3.30$ (m, 1H, H2x), 3.09 (dt, $J=10.1,3.9,1 \mathrm{H}, \mathrm{H7a}), 3.03\left(\mathrm{~m}, 1 \mathrm{H}, N\right.$-Ethyl $\left.\mathrm{CH}_{2}\right), 2.27(\mathrm{~m}, 1 \mathrm{H}$, H2y), $2.17\left(\mathrm{~m}, 1 \mathrm{H}, N\right.$-Ethyl $\left.\mathrm{CH}_{2}\right), 2.14(\mathrm{~m}, 1 \mathrm{H}, \mathrm{H} 3 \mathrm{a}), 1.91(\mathrm{~m}, 1 \mathrm{H}, \mathrm{H} 3 \mathrm{x}), 1.82(\mathrm{~m}, 1 \mathrm{H}$, H3y), 1.14 (t, $J=7.18,3 \mathrm{H}, N$-Ethyl $\left.\mathrm{CH}_{3}\right) .{ }^{13} \mathrm{C}$ NMR $\left(d-\mathrm{CDCl}_{3}, \delta\right): 140.3\left(\mathrm{C}^{\prime}\right), 131.2$ (C6), 129.5 (C5'), 125.2 (C7), 105.6 (C4'), 69.0 (C4), 63.4 (C5), 60.4 (C7a), 52.1 (C2), 48.9 (N-Ethyl $\left.\mathrm{CH}_{2}\right), 46.9(\mathrm{C} 3 \mathrm{a}), 22.9(\mathrm{C} 3), 13.7\left(N\right.$-Ethyl $\left.\mathrm{CH}_{3}\right)$. HRMS: $\left[\mathrm{M}+\mathrm{H}^{+}\right]=$ $\left[\mathrm{C}_{13} \mathrm{H}_{19} \mathrm{~N}_{3} \mathrm{O}+\mathrm{H}^{+}\right]$obs'd (\%), calc'd (\%), ppm: 234.1597, 234.1601, -1.7.

Compound 63: Outside of the box, $\mathrm{NOPF}_{6}(0.082 \mathrm{~g}, 0.468 \mathrm{mmol})$ was added to a vigorously stirring solution of Compound $55(0.225 \mathrm{~g}, 0.304 \mathrm{mmol})$ in acetone $(5 \mathrm{~mL})$. After $1 \mathrm{~h}$, the solution was diluted with $50 \mathrm{~mL}$ DCM and treated with $2 \times 20 \mathrm{~mL}$ of $\mathrm{Na}_{2} \mathrm{CO}_{3}$ (saturated, aq). The aqueous layer was back extracted with DCM $(2 \times 20 \mathrm{~mL})$, and the combined organic layers were washed with deionized water $(30 \mathrm{~mL})$. The organic layer was then dried over anhydrous $\mathrm{MgSO}_{4}$ and concentrated in vacuo. The brown oil was redissolved in minimal DCM and added dropwise to a stirring solution of $\mathrm{Et}_{2} \mathrm{O}$ (200 $\mathrm{mL}$ ) to induce precipitation of a brown solid. The precipitate was collected on a $30 \mathrm{~mL}$ fine-porosity fritted funnel with $2 \mathrm{~cm}$ celite. The filtrate was concentrated in vacuo. The residue was loaded onto a $20 \mathrm{~cm} \times 20 \mathrm{~cm} \times 1000 \mu \mathrm{m} \mathrm{Al}_{2} \mathrm{O}_{3}$ preparatory TLC place with 2 x $0.3 \mathrm{~mL}$ DCM. The plate was developed using $\mathrm{Et}_{2} \mathrm{O}$. A band, which stained positive with $\mathrm{KMnO}_{4}\left(\mathrm{R}_{\mathrm{f}} \approx 0.03-.25\right)$, was collected and placed in a round bottom flask with 70 $\mathrm{mL}$ HPLC EtOAc and sonicated for $15 \mathrm{~min}$. The slurry was filtered on a $30 \mathrm{~mL}$ fine- 
porosity fritted funnel and washed with $50 \mathrm{~mL}$ HPLC EtOAc. The filtrate was concentrated in vacuo. The oil was collected yielding Compound 63 (0.024 g, 0.105 mmol, 34\%). ${ }^{1} \mathrm{H}$ NMR ( $\left.d-\mathrm{CDCl}_{3}, \delta\right): 7.54$ (d, $\left.J=1.6,1 \mathrm{H}, \mathrm{H} 3{ }^{\prime}\right), 7.44$ (t, $\left.J=1.8,1 \mathrm{H}, \mathrm{H} 5^{\prime}\right)$, $6.33(\mathrm{dt}, J=10.1,1.9,1 \mathrm{H}, \mathrm{H} 7), 6.27$ (t, $J=2.1,1 \mathrm{H}, \mathrm{H} 4$ ') $, 5.72(\mathrm{~m}, 1 \mathrm{H}, \mathrm{H} 6), 5.26$ (m, 1H, H5), $5.14(\mathrm{dd}, J=53.3,3.8,1 \mathrm{H}, \mathrm{H} 4), 3.34(\mathrm{~m}, 1 \mathrm{H}, \mathrm{H} 2 \mathrm{x}), 3.06\left(\mathrm{~m}, 1 \mathrm{H}, N\right.$-Ethyl $\left.\mathrm{CH}_{2}\right)$, $2.99(\mathrm{~d}, J=11.2,1 \mathrm{H}, \mathrm{H} 7 \mathrm{a}), 2.28(\mathrm{~m}, 1 \mathrm{H}, \mathrm{H} 2 \mathrm{y}), 2.22$ (buried, 1H, H3a), $2.18(\mathrm{~m}, 1 \mathrm{H}, N-$ Ethyl $\left.\mathrm{CH}_{2}\right), 1.88(\mathrm{~m}, 2 \mathrm{H}, \mathrm{H} 3), 1.15\left(\mathrm{t}, J=7.0,3 \mathrm{H}, N\right.$-Ethyl $\left.\mathrm{CH}_{3}\right) .{ }^{13} \mathrm{C} \mathrm{NMR}\left(d-\mathrm{CDCl}_{3}, \delta\right)$ : 139.5 (C3’), 131.4 (C7), 129.0 (C5'), 124.3 (C6), 105.7 (C4'), 88.8 (d, J = 185.1, C4), $62.5(\mathrm{~d}, J=18.4, \mathrm{C} 5), 60.3(\mathrm{~d}, J=6.0, \mathrm{C} 7 \mathrm{a}), 51.9(\mathrm{C} 2), 48.8\left(N\right.$-Ethyl $\left.\mathrm{CH}_{2}\right), 46.0(\mathrm{~d}, J=$ 18.7, C3a), 22.5 (C3), $13.6\left(N\right.$-Ethyl $\left.\mathrm{CH}_{3}\right)$. HRMS: $\left[\mathrm{M}^{+} \mathrm{H}^{+}\right]=\left[\mathrm{C}_{13} \mathrm{H}_{18} \mathrm{~N}_{3} \mathrm{~F}+\mathrm{H}^{+}\right]$obs'd, calc'd, ppm: 236.1559, 236.1558, 0.6.

Compound 64: In a glovebox, DDQ $(0.057 \mathrm{~g}, 0.251 \mathrm{mmol})$ was added to a vigorously stirring solution of Compound $49(0.101 \mathrm{~g}, 0.143 \mathrm{mmol})$ in acetonitrile (7 mL). After 1 $\mathrm{hr}$, the solution was removed from the box and diluted with $50 \mathrm{~mL}$ DCM and treated with $2 \times 20 \mathrm{~mL}$ of $\mathrm{Na}_{2} \mathrm{CO}_{3}$ (saturated, aq). The aqueous layer was back extracted with DCM (2 x $20 \mathrm{~mL}$ ), and the combined organic layers were washed deionized water $(30 \mathrm{~mL})$. The organic was then dried over anhydrous $\mathrm{MgSO}_{4}$ and concentrated in vacuo to a vial. The residue was loaded onto a $20 \mathrm{~cm}$ x $20 \mathrm{~cm}$ x $1000 \mu \mathrm{m} \mathrm{Al}_{2} \mathrm{O}_{3}$ preparatory TLC place with 2 x $0.3 \mathrm{~mL}$ DCM. The plate was developed using $\mathrm{Et}_{2} \mathrm{O}$. A band which stained positive with $\mathrm{KMnO}_{4}\left(R_{f}=0.34-0.51\right)$ was collected and placed in a round bottom flask with $50 \mathrm{~mL}$ HPLC EtOAc and sonicated for 15 min to break up alumina. The slurry was filtered on a $30 \mathrm{~mL}$ fine porosity fritted funnel and washed with $50 \mathrm{~mL}$ HPLC EtOAc. The filtrate was 
then stripped to dryness. The oil was collected yielding 64 (0.010 g, 0.049 mmol, 34\%). ${ }^{1} \mathrm{H}$ NMR $\left(\mathrm{CDCl}_{3}, \delta\right): 7.52\left(\mathrm{dd}, J=1.8,0.53,1 \mathrm{H}, \mathrm{H} 3{ }^{\prime}\right), 7.45\left(\mathrm{dd}, J=2.3,0.5,1 \mathrm{H}, \mathrm{H} 5^{\prime}\right)$, $6.25\left(\mathrm{t}, J=2.37,1 \mathrm{H}, \mathrm{H} 4^{\prime}\right), 6.23(\mathrm{t}, J=1.9,1 \mathrm{H}, \mathrm{H} 6), 5.72(\mathrm{t}, J=1.9,1 \mathrm{H}, \mathrm{H} 7), 5.13(\mathrm{~m}$, 1H, H5), 3.33 (m, 1H, H2x), 3.00 (m, 1H, N-Ethyl- $\left.\mathrm{CH}_{2}\right), 2.66(\mathrm{dt}, J=9.67,3.11,1 \mathrm{H}$, H7a), 2.50 (ddd, $J=7.02,6.29$ 1.09, 1H, H4x), 2.30 (m, 1H, H2y), 2.15 (m, 1H, N-Ethyl$\mathrm{CH}_{2}$ ), 2.05 (m, 1H, H3a), 1.94 (m, 1H, H3x), 1.84 (m, 1H, H4y), 1.53 (m, 1H, H3y), 1.14 $\left(\mathrm{t}, J=7.31,3 \mathrm{H}, \mathrm{N}-\mathrm{Ethyl}-\mathrm{CH}_{3}\right) .{ }^{13} \mathrm{C} \mathrm{NMR}\left(\mathrm{CDCl}_{3}, \delta\right): 139.9(\mathrm{C} 3$ '), 131.5 (C6), 127.9 (C7), 127.6 (C5'), 105.6 (C4'), 67.5 (C7a), 60.8 (C5), 52.4 (C2), 49.1 (N-Ethyl-CH (N), $^{\prime}$ 42.5 (C3a), 35.9 (C4), 27.3 (C3), 14.0 (N-Ethyl- $\left.\mathrm{CH}_{3}\right)$. HRMS: $\left[\mathrm{M}^{+} \mathrm{H}^{+}\right]=\left[\mathrm{C}_{13} \mathrm{H}_{19} \mathrm{~N}_{3}+\mathrm{H}^{+}\right]$ obsd (\%), calcd (\%), ppm: 218.1658 (100), $218.1652(100), 2.9$.

Compound 65: Outside of the box, $\mathrm{NOPF}_{6}(0.096 \mathrm{~g}, 0.548 \mathrm{mmol})$ was added to a vigorously stirring solution of Compound 53 (0.199 g, $0.272 \mathrm{mmol})$ in acetone (10 mL). After overnight, the solution was diluted with $50 \mathrm{~mL}$ DCM and treated with $2 \times 20 \mathrm{~mL}$ of $\mathrm{Na}_{2} \mathrm{CO}_{3}$ (saturated, aq). The aqueous layer was back extracted with DCM $(2 \times 20 \mathrm{~mL})$, and the combined organic layers were washed with deionized water $(30 \mathrm{~mL})$. The organic layer was then dried over anhydrous $\mathrm{MgSO}_{4}$ and concentrated in vacuo. The brown oil was redissolved in minimal DCM and added dropwise to a stirring solution of hexanes $(150 \mathrm{~mL})$ to induce precipitation of a brown solid. The precipitate was collected on a 30 $\mathrm{mL}$ fine-porosity fritted funnel with $2 \mathrm{~cm}$ celite. This filtrate was concentrated in vacuo and redissolved in minimal DCM. The solution was added to a $4 \mathrm{~cm}$ florisil column set with DCM. The column was washed with DCM (10 mL), followed by MeCN (25 mL). The column was washed with $5 \% \mathrm{MeOH} / \mathrm{MeCN}(10 \mathrm{~mL})$ and $10 \% \mathrm{MeOH} / \mathrm{MeCN}(10$ 
$\mathrm{mL}$ ), which were collected and concentrated. The majority of product was retrieved from a wash with 50\% $\mathrm{MeOH} / \mathrm{MeCN}(10 \mathrm{~mL})$ and $\mathrm{MeOH}(5 \mathrm{~mL})$. These were concentrated in vacuo. The product was identified using NMR. The oil collected yielded Compound 65 (0.009 g, 0.041mmol, 19\%). ${ }^{1} \mathrm{H}$ NMR $\left(d-\mathrm{CDCl}_{3}, \delta\right): 7.54(\mathrm{~s}, 1 \mathrm{H}, \mathrm{H} 2$ '), $7.06(\mathrm{t}, J=1.0$, 1H, H5'), 6.94 (t, $\left.J=1.0,1 \mathrm{H}, \mathrm{H} 4^{\prime}\right), 6.23(\mathrm{dt}, J=9.9,1.7,1 \mathrm{H}, \mathrm{H} 7), 5.66$ (m, 1H, H6), 4.95 (m, 1H, H5), 3.31 (m, 1H, H2x), 3.00 (m, 1H, $N$-Ethyl $\left.\mathrm{CH}_{2}\right), 2.58(\mathrm{dt}, J=9.8,3.5$, 1H, H7a), 2.49 (m, 1H, H4x), 2.28 (td, $J=10.8,3.3,1 \mathrm{H}, \mathrm{H} 2 \mathrm{y}), 2.15$ (m, 1H, $N$-Ethyl $\mathrm{CH}_{2}$ ), 2.03 (m, 1H, H3a), 1.93 (m, 1H, H3x), 1.69 (m, 1H, H4y), 1.49 (m, 1H, H3y), 1.13 $\left(\mathrm{t}, J=7.1,3 \mathrm{H}, \mathrm{N}\right.$-Ethyl $\left.\mathrm{CH}_{3}\right) .{ }^{13} \mathrm{C} \mathrm{NMR}\left(d-\mathrm{CDCl}_{3}, \delta\right): 136.2(\mathrm{C} 2$ ') $, 132.0(\mathrm{C} 7), 129.8$ (C5'), 127.7 (C6), 117.6 (C4'), 67.4 (C7a), 56.2 (C5), 52.2 (C2), $49.2\left(N\right.$-Ethyl $\left.\mathrm{CH}_{2}\right)$, $42.5(\mathrm{C} 3 \mathrm{a}), 37.2(\mathrm{C} 4), 27.2(\mathrm{C} 3), 14.0\left(\mathrm{~N}\right.$-Ethyl $\left.\mathrm{CH}_{3}\right)$. HRMS: $\left[\mathrm{M}+\mathrm{H}^{+}\right]=\left[\mathrm{C}_{13} \mathrm{H}_{19} \mathrm{~N}_{3}+\mathrm{H}^{+}\right]$ obs'd, calc'd, ppm: 218.1643, 218.1652, -4.0.

Compound 66: Outside of the box, $\mathrm{NOPF}_{6}(0.093 \mathrm{~g}, 0.531 \mathrm{mmol})$ was added to a vigorously stirring solution of Compound 50 (0.235 g, $0.352 \mathrm{mmol})$ in acetone $(10 \mathrm{~mL})$. After $18 \mathrm{~h}$, the solution was diluted with $40 \mathrm{~mL}$ DCM and treated with $2 \times 20 \mathrm{~mL}$ of $\mathrm{Na}_{2} \mathrm{CO}_{3}$ (saturated, aq). The aqueous layer was back extracted with DCM (2 x $\left.20 \mathrm{~mL}\right)$, and the combined organic layers were washed with deionized water $(30 \mathrm{~mL})$. The organic layer was then dried over anhydrous $\mathrm{MgSO}_{4}$ and concentrated in vacuo. The brown oil was redissolved in minimal DCM and added dropwise to a stirring solution of $\mathrm{Et}_{2} \mathrm{O}$ (200 $\mathrm{mL}$ ) to induce precipitation of a brown solid. The precipitate was collected on a $30 \mathrm{~mL}$ fine-porosity fritted funnel with $2 \mathrm{~cm}$ celite. The filtrate was concentrated in vacuo to a 4dram vial. The residue was loaded onto a $20 \mathrm{~cm}$ x $20 \mathrm{~cm}$ x $1000 \mu \mathrm{m} \mathrm{Al}_{2} \mathrm{O}_{3}$ preparatory 
TLC place with DCM $(2 \times 0.3 \mathrm{~mL})$. The plate was developed using $\mathrm{Et}_{2} \mathrm{O}$. A band, which stained positive with $\mathrm{KMnO}_{4}\left(\mathrm{R}_{\mathrm{f}} \approx 0.03-.25\right)$, was collected and placed in a round bottom flask with $70 \mathrm{~mL}$ HPLC EtOAc and sonicated for $15 \mathrm{~min}$. The slurry was filtered on a 30 $\mathrm{mL}$ fine-porosity fritted funnel and washed with $50 \mathrm{~mL}$ HPLC EtOAc. The filtrate was evaporated in vacuo. The oil was collected yielding Compound 66 (0.016 g, 0.099 mmol, 28\%). ${ }^{1} \mathrm{H}$ NMR $\left(d-\mathrm{CDCl}_{3}, \delta\right): 5.97$ (m, 1H, H6), $5.74(\mathrm{dd}, J=9.8,1,1 \mathrm{H}, \mathrm{H} 7)$, $3.39(\mathrm{~m}, 1 \mathrm{H}, \mathrm{H} 2 \mathrm{x}), 2.91\left(\mathrm{~m}, 1 \mathrm{H}, N\right.$-Ethyl $\left.\mathrm{CH}_{2}\right), 2.33(\mathrm{td}, J=10.6,3.2,1 \mathrm{H}, \mathrm{H} 2 \mathrm{y}), 2.21(\mathrm{dt}$, $J=11.1,2.0,1 \mathrm{H}, \mathrm{H} 7 \mathrm{a}), 2.05\left(\mathrm{~m}, 1 \mathrm{H}, N\right.$-Ethyl $\left.\mathrm{CH}_{2}\right), 2.01(\mathrm{~m}, 1 \mathrm{H}, \mathrm{H} 3 \mathrm{x}), 1.95(\mathrm{~m}, 1 \mathrm{H}$, H3a), 1.59 (m, 1H, H3y), 1.34 (m, 2H, H4 and H5), 1.10 (t, $J=7.3,3 \mathrm{H}, N$-Ethyl $\mathrm{CH}_{3}$ ), $0.91(\mathrm{q}, J=5.4,1 \mathrm{H}, \mathrm{H} 8 \mathrm{x}), 0.711(\mathrm{td}, J=8.39,5.0,1 \mathrm{H}, \mathrm{H} 8 \mathrm{y}) .{ }^{13} \mathrm{C} \mathrm{NMR}\left(d-\mathrm{CDCl}_{3}, \delta\right)$ : 129.0 (C6), 125.7 (C7), 63.6 (C7a), 53.8 (C2), 49.4 (N-Ethyl $\mathrm{CH}_{2}$ ), 40.4 (C3a), 27.2 (C3), $14.9\left(\mathrm{C} 4\right.$ or C5), $13.7(\mathrm{C} 8), 12.7\left(\mathrm{C} 4\right.$ or C5), $8.11\left(N\right.$-Ethyl $\left.\mathrm{CH}_{3}\right) . \mathrm{HRMS}:\left[\mathrm{M}^{+} \mathrm{H}^{+}\right]=$ $\left[\mathrm{C}_{11} \mathrm{H}_{17} \mathrm{~N}+\mathrm{H}^{+}\right]$obs'd, calc'd, ppm: 164.1428, 164.1434, -3.5. 


\subsection{References}

(1) Thibodeaux, C. J.; Chang, W.-c.; Liu, H.-w. Chemical Reviews 2012, 112, 1681.

(2) Wessjohann, L. A.; Brandt, W.; Thiemann, T. Chemical Reviews 2003, $103,1625$.

(3) Pienkos, J. A.; Knisely, A. T.; Liebov, B. K.; Teran, V.; Zottig, V. E.; Sabat, M.; Myers, W. H.; Harman, W. D. Organometallics 2014, 33, 267.

(4) Zottig, V. E.; Todd, M. A.; Nichols-Nielander, A. C.; Harrison, D. P.; Sabat, M.; Myers, W. H.; Harman, W. D. Organometallics 2010, 29, 4793.

(5) Wang, J.; Sánchez-Roselló, M.; Aceña, J. L.; del Pozo, C.; Sorochinsky, A. E.; Fustero, S.; Soloshonok, V. A.; Liu, H. Chemical Reviews 2014, 114, 2432.

(6) Rigo, P.; Paulus, P.; Kaschten, B. J.; Hustinx, R.; Bury, T.; Jerusalem, G.; Benoit, T.; Foidart-Willems, J. Eur J Nucl Med 1996, 23, 1641.

(7) Whitfield, R. ICIS Chemical Business 2009.

(8) Salomon, R. J.; Todd, M. A.; Sabat, M.; Myers, W. H.; Harman, W. D. Organometallics 2010, 29, 707.

(9) Pienkos, J. A.; Zottig, V. E.; Iovan, D. A.; Li, M.; Harrison, D. P.; Sabat, M.; Salomon, R. J.; Strausberg, L.; Teran, V. A.; Myers, W. H.; Harman, W. D. Organometallics 2013, 32, 691.

(10) MacLeod, B. L.; Pienkos, J. A.; Myers, J. T.; Sabat, M.; Myers, W. H.; Harman, W. D. Organometallics 2014, 33, 6286. 
(11) Seyden-Penne, J. Reductions by the Alumino- and Borohydrides in Organic Synthesis; 2 ed.; Wiley-VCH: New York, NY, 1997.

(12) Aloise Pilli, R.; da Conceicao Ferreira de Oliveira, M. Natural Product Reports 2000, 17, 117.

(13) Goldstein, D. M.; Wipf, P. Tetrahedron Letters 1996, 37, 739.

(14) Morimoto, Y.; Iwahashi, M.; Nishida, K.; Hayashi, Y.; Shirahama, H. Angewandte Chemie International Edition in English 1996, 35, 904.

(15) Wipf, P.; Kim, Y.; Goldstein, D. M. Journal of the American Chemical Society 1995, 117, 11106.

(16) Wipf, P.; Rector, S. R.; Takahashi, H. Journal of the American Chemical Society 2002, 124, 14848.

(17) Connelly, N. G.; Geiger, W. E. Chemical Reviews 1996, 96, 877.

(18) Zhang, J.; Wang, Y.-Q.; Wang, X.-W.; Li, W.-D. Z. The Journal of Organic Chemistry 2013, 78, 6154. 


\section{Chapter 5:}

New Branches of Reactivity with $N$ Ethylindolinium and $N, N$ -

\section{Dimethylanilinium Systems}




\subsection{Introduction}

There has been an large amount of work completed with the $N, N$ dimethylanilinium complex, but because of the inability to reduce the iminium, most of it did not come to fruition. This issue was solved in the $N$-ethylindolinium system. A range of novel hexahydroindoles with various functional groups have been synthesized using the $N$-ethylindolinium complex. This breadth has been limited by the necessity of an iminium reduction and nucleophiles' tolerance to those conditions. The following chapter will explore the reactivity surrounding the reduced complex and other reactivity that was discovered through this iminium reduction. This will be explored in both the indoline and aniline systems.

\subsection{NCS/NBS Reactivity Expansion}

As previously shown in chapter 4 , halides, as electrophiles, were relatively unstable towards LAH reduction conditions. Only one example of both a hydroxylated and a fluorinated organic were isolated. The reason behind this instability was not initially understood, but this has been explored further through the use of NCS and NBS as the electrophile source.

When chlorinated or brominated additions products were subjected to LAH reduction conditions, multiple products were observed. This result was more prominent in the brominated products, but both systems were susceptible. Upon reduction of compound 36, similar features to the protonated analog were identified via ${ }^{1} \mathrm{H}$ NMR. The PzA3 peak shifted downfield to $9.4 \mathrm{ppm}$, while the pyrazole peaks remained intact.

Through ${ }^{1} \mathrm{H}$ NMR, the major product was identified as 68. Unfortunately, over time in 
solution, this complex appears to decompose via ${ }^{1} \mathrm{H}$ and ${ }^{31} \mathrm{P}$ NMR to multiple products which did not allow for 2D NMR analysis. Upon the reduction of compound 34, there were two equal products. Both of these new systems include a shifted PzA3 peak, but the pyrazole peaks (H3'-H5') could not easily be identified. This was not entirely discouraging, due to this similarity to the fluorinated counterpart, which still yields a novel organic molecule.

Scheme 5.1. Oxidation of 67 and 68

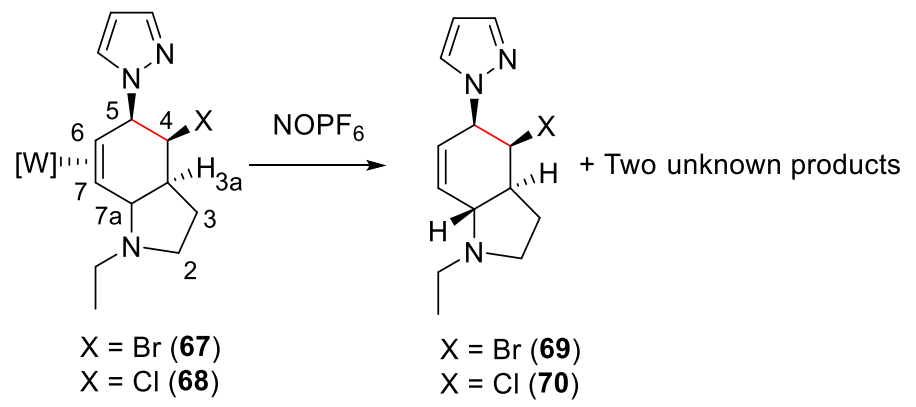

Oxidation of these reduced species was attempted with $\mathrm{NOPF}_{6}$ in acetone, similar to previous experiments (Scheme 5.1). Through a basic workup procedure and a basic alumina preparatory TLC plate, organic products were isolated after identification with KMnO4 staining. The stained band for compound $\mathbf{7 0}$ contained three species and corresponding band for compound 69 contained two equal species, as determined via ${ }^{1} \mathrm{H}$ NMR. Close identification of this shows that the two species of $\mathbf{6 9}$ are the same as two in 70. Upon further investigation, it appears that the pyrazole is intact in all of three species, along with the alkene at $\mathrm{C} 6$ and $\mathrm{C} 7$. This implies that something occurred at the $\mathrm{C} 4$ position. In 69, the bromide is being possibly displaced easier than the chloride in $\mathbf{7 0 .}$

The general trend of reactivity is consistent with a $S_{N} 1$ or $S_{N} 2$ type reaction mechanism. Bromine is a better leaving group than chlorine, meaning that it would be 
more susceptible to being replaced by another nucleophile. ${ }^{1}$ The chlorine species might be able to retain the chlorine, which accounts for the third desired product where bromie would not. ${ }^{1} \mathrm{H}$ NMR data agrees with this theory with a proton at $5.30 \mathrm{ppm}$, which could be the $\mathrm{H} 4$ proton of 70. The fluorinated analog has the corresponding proton at $5.14 \mathrm{ppm}$. The full identification of the two unknown products was not possible due to overlapping peaks. This was not the first instance that $S_{N} 1$ type reactivity has been assumed for these types of dearomatized systems. Previous work with $\mathrm{TpW}(\mathrm{NO})\left(\mathrm{PMe}_{3}\right)\left(\eta^{2}\right.$-anthracene) showed reactivity which supports this conclusion. ${ }^{2}$ The addition of NBS with various nucleophiles, including LiDMM, imidazole and aniline, produced a double nucleophilic addition product rather than a tandem electrophile-nucleophile addition. A variety of reaction conditions were attempted with this system, including adding triethylamine (TEA) to scavenge acidic byproducts. This can be seen in Scheme 5.2 with aniline as a nucleophile.

Scheme 5.2. Double nucleophilic addition of aniline with anthrecene

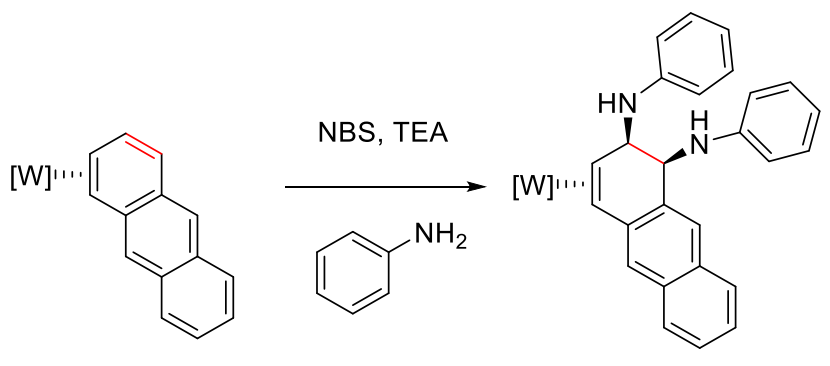

\subsubsection{Elimination of Halide}

As seen in chapter 4, NCS was able to add in a tandem addition fashion with various nucleophiles (34-39). Unlike the anthracene example, there was never a double 
nucleophilic addition product seen, even when left overnight. Upon addition of LAH however, these addition products began to decompose. This gives a starting point for the reaction conditions which could displace the halide. Rather than beginning with the ethylindoline complex (5), the addition products (34-39) will be used for further elaboration with strong nucleophiles. Initially, propyl amine and aniline were tested for reactivity in displacing the halide of compound 34. Aniline yielded decomposition, while propyl amine yielded a new clean product. When monitored using ${ }^{31} \mathrm{P}$ NMR, a new peak is seen after 5 minutes, with a $J_{\mathrm{W}-\mathrm{P}}$ of $268 \mathrm{~Hz}$ and a slight downfield shift. Using ${ }^{1} \mathrm{H}$ NMR and 2D NMR, it was determined that the new product did not integrate propyl amine and this complex was identified as compound 71 (Scheme 5.3). Aromatic peaks between 6 and 7 ppm indicate that the new complex retains the pyrazole peaks. A broad multiplet at 6.27 ppm has a COSY interaction with the H5 peak, but nothing else. There is now a partial positive charge at $\mathrm{C} 4$, corresponding to a shift downfield.H4 has an NOE interaction with $\mathrm{H} 3$ due to the loss of the $\mathrm{H} 3 \mathrm{a}$ bridgehead. IR data and cyclic voltammetry confirms that the iminium is intact.

Scheme 5.3. Elimination of $\mathbf{3 4}$ with propyl amine

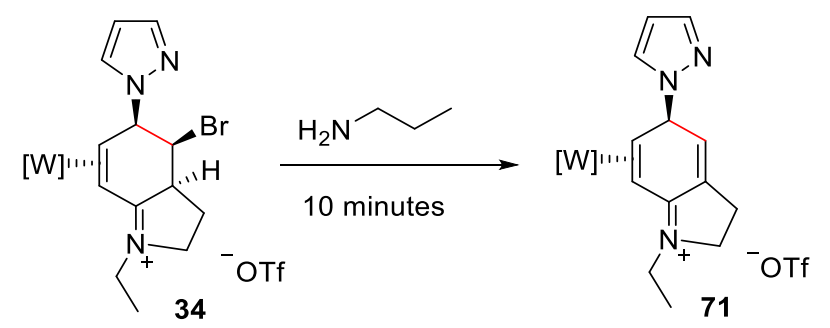

This same elimination reaction was attempted with the other tandem addition products with NCS. These can be seen in Scheme 5.4. Compound 72 was stable when isolated from solution, however compounds $\mathbf{7 3}$ and $\mathbf{7 4}$ were difficult to isolate and 
degraded in solution, therefore could not be fully identified using $2 \mathrm{D}$ NMR. Using ${ }^{1} \mathrm{H}$ NMR, however, these species were spectroscopically similar with their more stable analogs. There are large downfield shifts attributed to the $\mathrm{H} 4$ protons for the new alkene position. This elimination could not be completed with triethylamine or diisopropyl amine, most likely due to the steric hindrance at the bridgehead position being syn to the metal. This same type of reactivity was seen in the $N, N$-dimethylaniline system only with a NCS and methoxy addition, which could be eliminated using $\mathrm{NaBH}_{4}$ or diethylamine. ${ }^{3}$

Scheme 5.4. Elimination of $\mathbf{3 5}, \mathbf{3 7}$ and 39 with propyl amine (isolated as triflate salts)

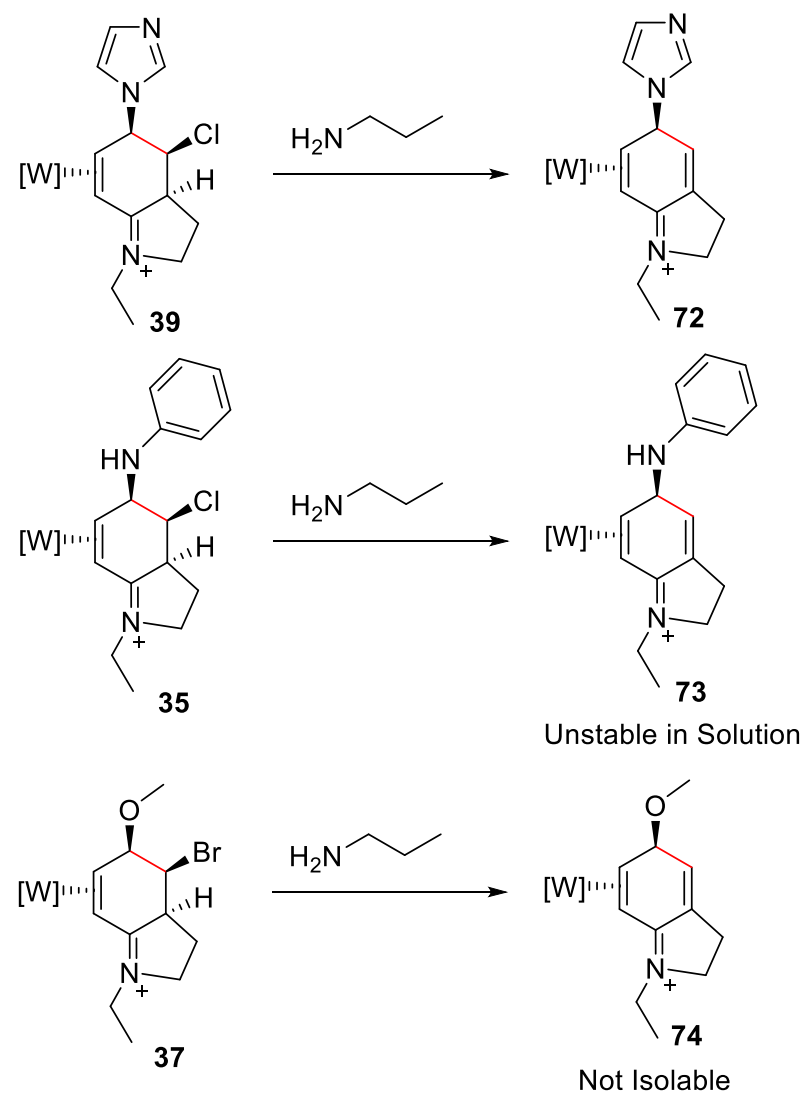




\subsubsection{Reactivity of Elimination Product}

The new bond orientation of this system is reminiscent of the original paraprotonated indoline and quinoline complexes discussed in chapter 3 . These are expected to have a position for nucleophilic attack at the $\mathrm{C} 4$ position. This reactivity of $\mathbf{4 B}$ was only investigated in a cursory manner, though no of the results were promising. Compounds 71-73 introduce a new take on this isomer. It is already known that the system is stable without the $\mathrm{C} 3 \mathrm{a}-\mathrm{C} 4$ alkene, so new addition products should be possible. It was determined that a strong nucleophile would be necessary to add to this system, but also a source for protonation at the bridgehead position would be crucial to complete the reaction.

Scheme 5.5. Scheme of reactivity for elimination products

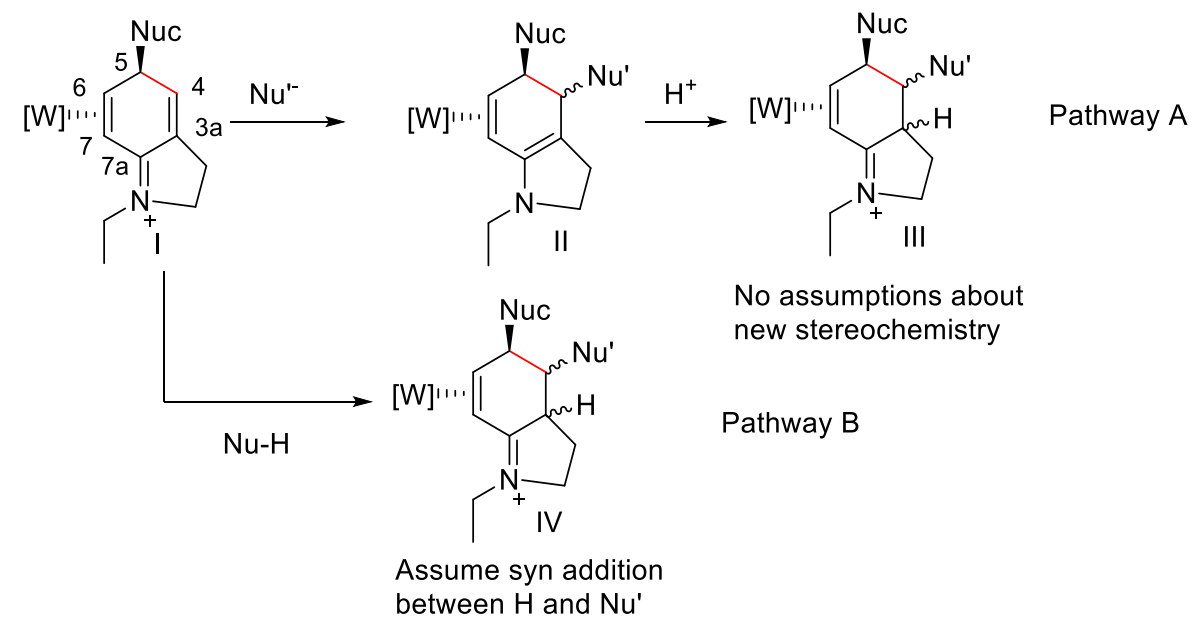

This can be through two different reactions mechanisms. Pathway A (Scheme 5.5) involves the addition of a nucleophile at $\mathrm{C} 4$ in compound $\mathbf{I}$, removing the iminium and forming an enamine (II). This system is very reactive and would pick up a proton to reform the stabilizing iminium (III). This system would have the typical stereochemistry 
assumed of reagents adding most-likely anti to the metal center. There would be no necessity for these additions to be syn to one another as they are occurring in separate steps. With pathway B, this would be a concerted reaction. The nucleophile can deliver the nucleophile and a proton for the bridgehead (C3a), never creating the enamine, but rather going to the addition product directly (IV). This system should have syn stereochemistry between the two new additions as they should be delivered on the same side of the ligand. As usual it would be assumed that additions should occur anti to the metal center.

The system which was used for these tests was the pyrazole elimination product. The ability to add nucleophiles at the $\mathrm{C} 4$ position proved to be more difficult than expected. Initial tests were attempted by adding nucleophiles to the elimination species. Nucleophiles tested included imidazole, thiophenol, aniline, and propyl amine. It was believed that these nucleophiles would be able to donate a proton and a nucleophile at the same time. These reactions were followed in deuterated solvent with ${ }^{1} \mathrm{H}$ NMR or ${ }^{31} \mathrm{P}$ NMR with times ranging from 20 minutes to over a week. Only thiophenol showed any reactivity, though the product was not clean upon isolation.

Table 5.1. Nucleophiles and their corresponding pKas

\begin{tabular}{|l|l|}
\hline Nucleophile & pKa \\
\hline $\mathrm{MeOH}_{2}{ }^{+}$ & -2.2 \\
\hline Anilinium triflate & 4.6 \\
\hline Thiophenol & 7 \\
\hline Piperidine or Morpholine $\mathrm{H}^{+}$ & 8.3 \\
\hline Phenol & 9.9 \\
\hline Propyl ammonium triflate & 10.6 \\
\hline DiPAT & 11.0 \\
\hline
\end{tabular}


Thiophenol is the only acidic nucleophile which was tried however, which led to the concept of adding acid to the nucleophile addition reaction conditions. In order to test the range of acidity that this system can withstand, the nucleophiles in table 1 were investigated. Unfortunately, protonated methanol and phenol both led to decomposition of the starting material with no clean product ever visualized via ${ }^{31} \mathrm{P}$ NMR or ${ }^{1} \mathrm{H}$ NMR. The addition of DiPAT and propyl ammonium triflate alone did not lead to decomposition, but no addition products were seen. Attempts to combine amine nucleophiles with DiPAT in order to increase the proton source were unsuccessful and remained as the elimination starting material. The combination of thiophenol and DiPAT with 71 did produce a new product; however, this proved to be compound $\mathbf{7 5}$ in scheme 5.6. The nitrogen of pyrazole can be protonated and allow for thiophenol to displace the pyrazole. Using ${ }^{1} \mathrm{H}$ and 2D NMR techniques this product was fully identified. The characteristic doublet at approximately $6.3 \mathrm{ppm}$ for the $\mathrm{H} 4$ position remains intact and the H5 proton shifts upfield to $4.82 \mathrm{ppm}$ due to the lack of anisotropy from the pyrazole ring. The H5 proton has a NOE interaction with the $\mathrm{PMe}_{3}$ ligand, showing that the pyrazole leaves prior to the thiophenol addition. The pyrazole peaks are also gone in the aromatic region, but phenol peaks are now present. Other attempts to broaden the scope of nucleophiles for the tandem addition through exchange generally caused decomposition, as seen in chapter 4 , so this was a new type of reaction mechanism not seen previously with the indoline complex. 
Scheme 5.6. Exchange of pyrazole for thiophenol

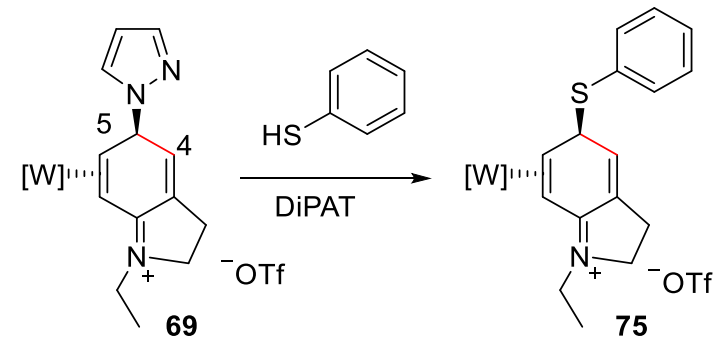

In a final attempt for nucleophilic additions, the elimination reaction was left overnight starting from compound 34. After 5 minutes, the elimination product was seen in solution via ${ }^{1} \mathrm{H}$ NMR, but the reaction continued for 15 hours. The reaction was monitored in situ with ${ }^{1} \mathrm{H}$ NMR, and showed the disappearance of the $\mathrm{H} 4$ and $\mathrm{H} 5$ peaks of the elimination product and the appearance of multiple $\mathrm{CH}_{2}$ groups in the aliphatic region between 2-3 ppm and a new doublet of doublet at $5.74 \mathrm{ppm}$. Using 2D NMR, this product was found to be compound 76. A strong COSY interaction between $\mathrm{H} 4$ and $\mathrm{H} 5$ was found, though no NOE interactions were shown between them. H5 maintains an NOE interaction to the $\mathrm{PMe}_{3}$ and with $\mathrm{H} 3 \mathrm{a}$, indicating the bridgehead proton was again syn to the metal. These protons also had a NOE interaction to the $1 * \mathrm{CH}_{2}$ protons (Scheme 5.7). Contrary to all other additions to this system, it appears that the propyl amine has added syn to the metal complex. 
Scheme 5.7. Double nucleophilic addition of propyl amine onto 34 (isolated as triflate salts)

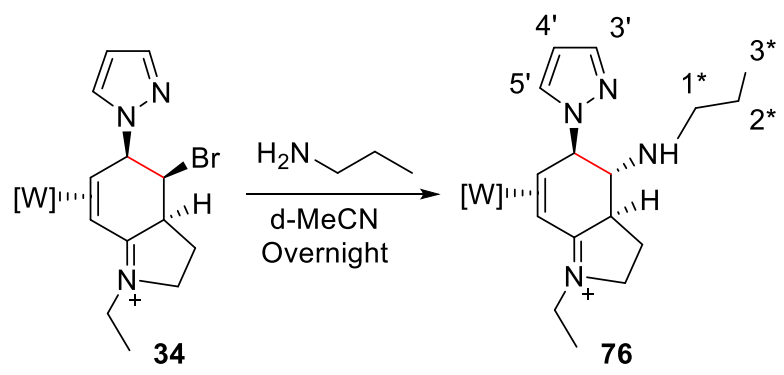

This same type of reaction was attempted with the aniline (35) and imidazole (39) addition products (Scheme 5.8). Both reactions were followed in situ with ${ }^{1} \mathrm{H}$ NMR. In the imidazole case, after 20 minutes the elimination product was observed, but after 48 hours no change was observed. With the aniline case, at 20 minutes the elimination product observed, with characteristic peaks at 6.38 and $5.05 \mathrm{ppm}$, but at 2 hours a product was seen in a ratio of 1.5:1 to the elimination. In an attempt to push the reaction forward, 0.5 equivalents of DiPAT were added. At 4 hours, the product was seen in a 3.5:1 ratio. A new peak at 4.26 is in the correct region for either $\mathrm{H} 4$ or $\mathrm{H} 5$. Unfortunately, ${ }^{31} \mathrm{P}$ NMR showed a large amount of decomposition of the metal occurring faster than the reaction. This product could not to be isolated cleanly. 
Scheme 5.8. Long term reactivity of 35 and 39 with propyl amine
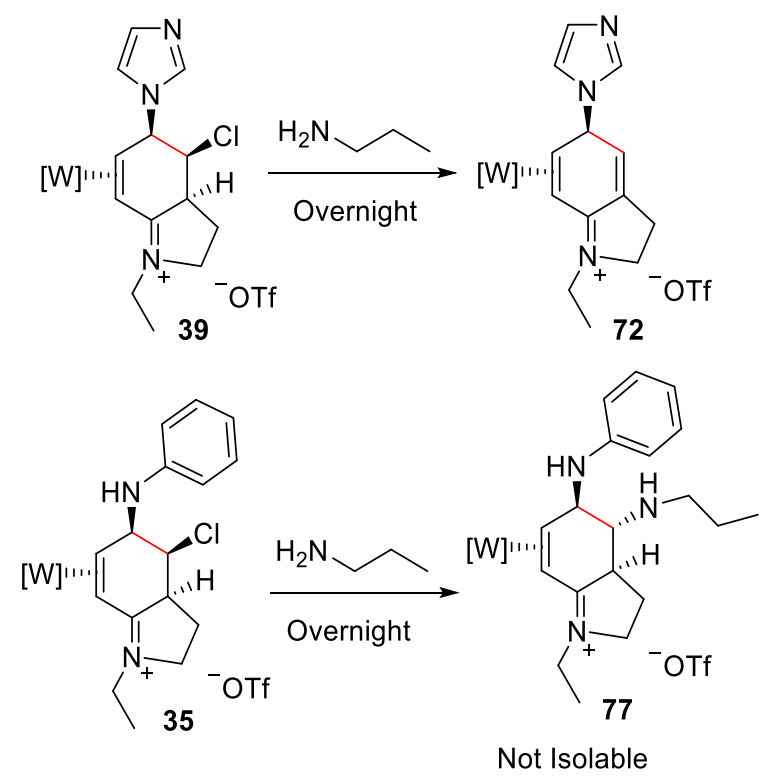

Scheme 5.9. Proposed scheme of intramolecular double nucleophilic addition with ethylenediamine

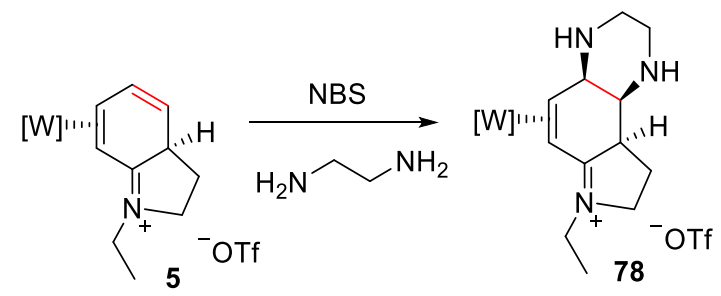

An attempt for an intramolecular double nucleophilic additions was attempted with ethylenediamine with NBS or NCS. It was believed that having the nucleophile be able to close on itself, allowed for a higher possibility of a double addition product. With NCS, this reaction only yielded decomposition. With NBS, when followed with ${ }^{1} \mathrm{H}$ NMR, a product was seen after 1 night and was the major species after 4 days. There was 
significant decomposition as well, which made identification difficult. Nothing conclusive was determined by either ${ }^{31} \mathrm{P}$ or ${ }^{1} \mathrm{H}$ NMR.

\subsection{Additions to the Reduced Indoline Complex}

In a recent paper out of the Harman lab, the addition of MTDA can create an opportunity to complete iodolactonizations (Scheme 5.10). ${ }^{5}$ This new functional group is common in natural products with perhydroindole cores, such as Stenine. ${ }^{6}$ One of the largest downfalls of the indoline and aniline systems is that the reduction of the iminium can limit the number of functional groups available to the final organic molecules. Any system with a carbonyl is reduced and many aliphatic amine addition products resulted in decomposition. In order to branch into this type of reactivity, the addition reactions must occur after the iminium is reduced.

Scheme 5.10. Iodolactonization of MTDA addition on naphthalene

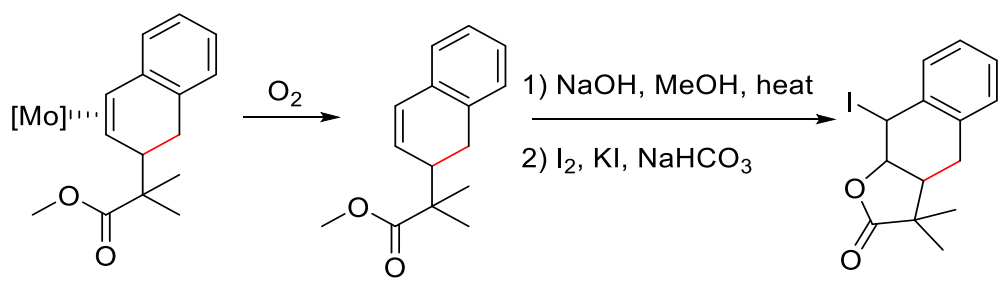

Using the reaction conditions similar from additions in chapter 3, HOTf leveled in $\mathrm{MeCN}$ was added to the reduced starting material, $\mathbf{5 4}$, followed by the addition of excess MTDA to synthesize a tandem addition product. Unfortunately this only led to decomposition of the metal complex when monitored via ${ }^{1} \mathrm{H}$ or ${ }^{31} \mathrm{P}$ NMR. Presumably 
these reaction conditions allow for the triflic acid to oxidize the metal rather than adding as an electrophile, or both could occur. Preliminary attempts to complete this reaction at low temperature $\left(-30^{\circ} \mathrm{C}\right)$ yielded multiple products, but the metal did not decompose, according to ${ }^{31} \mathrm{P}$ NMR. A difficulty may stem from the protonation of the nitrogen as well as creating the allyl.

Scheme 5.11. Addition of MTDA to 54

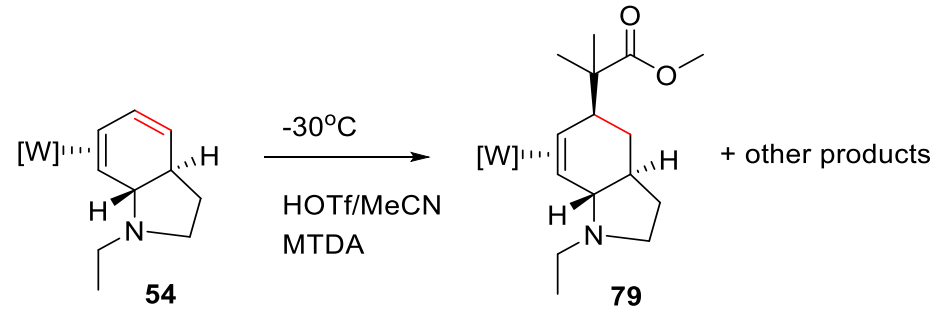

A weaker acid, DPhAT, was attempted for the same addition reaction. Under these conditions a new product was cleanly formed, when monitored by ${ }^{31} \mathrm{P}$ NMR spectroscopy. Whereas the starting material has a $J_{\mathrm{W}-\mathrm{P}}$ of $273 \mathrm{~Hz}$, the new product had a coupling constant of $278 \mathrm{~Hz}$. This is not consistent with an allylic, cationic species. Using cyclic voltammetry and ${ }^{13} \mathrm{C}$ NMR, it was determined that an iminium formed, with a new reduction potential at $0.62 \mathrm{~V}$ and a carbon NMR peak at $199 \mathrm{ppm}$. Based on previously unpublished work with aniline, ${ }^{3}$ this species was identified as the ring turn product, complex 80 (Scheme 5.12). 
Scheme 5.12. Ring turn of $\mathbf{5 4}$ to yield 80

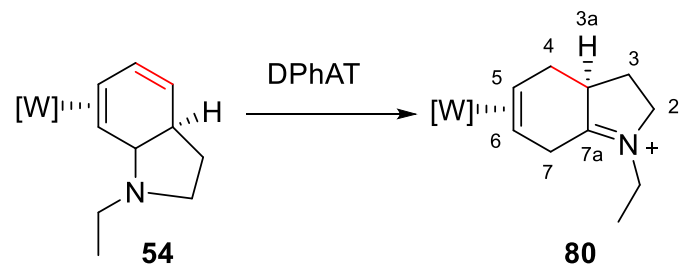

\subsection{Ring Turn Reactivity}

With the anilinium system, a reaction scheme was devised to possibly make additions to the ring turn system, creating functionality at a new position on the ring (Scheme 5.13). Using DPhAT as an acid source with the reduced aniline complex (I), the ring turn product is formed (II). Using a sterically hindered base, the enamine intermediate (III) is formed in situ. This could not be isolated away from its silyated byproducts. ${ }^{3}$ Two electrophiles were attempted: allyl bromide and bromopropane. While bromopropane added through the nitrogen, allyl bromide created a product with the desired form of IV. It was determined that soft nucleophiles add at C2 and hard nucleophiles add at the nitrogen position.

Scheme 5.13. Ring turn scheme of $N, N$-dimethylaniline complex<smiles>CC(C)C1=CC=C(N(C)C)CC1</smiles> 
Using the same reaction scheme, the compound $\mathbf{8 0}$ was subjected to the elimination conditions with KHMDS in toluene. A new carbon resonance occurs at 145 ppm for the quaternary at $\mathrm{C} 7 \mathrm{a}$ and the iminium peak for compound $\mathbf{8 0}$ is gone. There is also no longer an iminium in IR, only a signal for the NO ligand. In ${ }^{1} \mathrm{H}$ NMR, a new peak at 5.09 ppm for $\mathrm{H} 7$ occurs with COSY interactions to C6 and NOE interactions with the PzA3 proton. Contrary to the aniline system, which could not be purified, this enamine (81) can be isolated separate from the silylated counterparts.

Scheme 5.14. Ring turn elimination and addition of allyl bromide

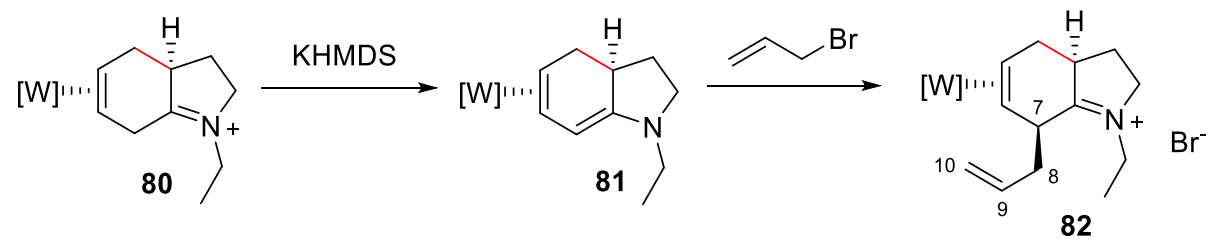

The addition of electrophiles to the elimination product has been limited. The most successful addition has been of allyl bromide to yield $\mathbf{8 2}$. The iminium can again be seen with IR and ${ }^{13} \mathrm{C}$ NMR. When following the reaction with ${ }^{1} \mathrm{H}$ NMR there are 3 new peaks between 5 and 6 ppm for $\mathrm{H} 9$ and $\mathrm{H} 10$ of the electrophile. There is a strong COSY interaction between $\mathrm{H} 7$ and the new geminal set at H8. Stereochemistry is confirmed as anti to the metal via NOE interactions between $\mathrm{H} 7, \mathrm{H} 3 \mathrm{a}$ and the Tp set. HMBC shows a correlation between the iminium carbon at $\mathrm{C} 7 \mathrm{a}$ and $\mathrm{H} 7$ also. When this reaction was attempted without isolating the elimination intermediate, the product was unclean, so the intermediate isolation allows for cleaner products overall, possibly from reagent compatibility issues with the impurities. 
Other electrophiles which were attempted included 2,2-dimethyoxypropane, methyl vinyl ketone (MVK), thiophenol and acetyl chloride. The acetal yielded multiple products, but they could not be identified. The addition of MVK, when watched with ${ }^{1} \mathrm{H}$ NMR in situ, showed the creation of a new product, though it could notbe identified or isolated. The addition of thiophenol showed two products, most likely stemming from nitrogen and carbon addition. When left over a week to look for equilibration towards one product there was no change seen. The addition of acetyl chloride showed a new proton appearing at $5.1 \mathrm{ppm}$, which is promising for a new $\mathrm{H} 7$ proton, but there were multiple products.

Scheme 5.15. Reduction of 80 iminium with $\mathrm{NaCNBH}_{3}$

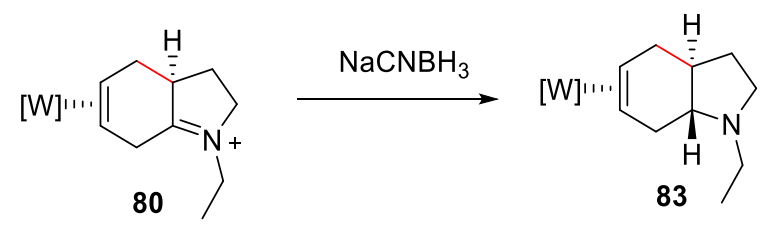

Previously, the ability to reduce the iminium was difficult due to the metal back bonding into the system. The ring turn product $\mathbf{8 0}$ does not have that conjugated $\pi$-system , so the iminium should be easier to reduce. It was believed that the addition of strong nucleophiles could possibly add at the C7a position to functionalize that bridgehead. When attempts were made with Grignard reagents, either multiple products or starting material were isolated. The addition of MTDA and LiDMM yielded only starting material. The addition of NaI to activate the MTDA was successful, but yielded 3 equal products. The cleanest addition to the iminium was with $\mathrm{NaCNBH}_{3}$, the common reducing agent used for reducing organic iminium bonds. Using ${ }^{1} \mathrm{H}$ and $2 \mathrm{D} \mathrm{NMR}$, this 
new product was fully identified. There is no longer a carbon resonance above $190 \mathrm{ppm}$, indicating the iminium is gone. $\mathrm{H} 3 \mathrm{a}$ maintains an NOE interaction with the $\mathrm{PMe}_{3}$ ligand, but the new $\mathrm{H} 7 \mathrm{a}$ does not have an NOE to $\mathrm{H} 3 \mathrm{a}$, indicating a trans juncture.

When these same $\mathrm{NaCNBH}_{3}$ conditions were attempted on compound 82, no reaction was seen. When the stronger reducing agent, LAH, was used, a new product was seen via ${ }^{31} \mathrm{P}$ NMR after 10 minutes. Using ${ }^{1} \mathrm{H}$ and $2 \mathrm{D}$ NMR, the new compound was identified as compound 84 (Scheme 5.16). The stereochemistry at the H7a bridgehead is unique to this reaction, due to the cis ring juncture. There is an NOE interaction between the PMe and one geminal proton on $\mathrm{H} 4$. This same proton has an NOE interaction with the $\mathrm{H} 3 \mathrm{a}$ bridgehead. This indicates the $\mathrm{H} 3 \mathrm{a}$ bridgehead is syn with the metal center. The $\mathrm{H} 3$ a proton has an $\mathrm{NOE}$ interaction with the new $\mathrm{H} 7$ a proton, indicating the new bridgehead is syn to the metal. It is believed that the sterics from the new functionality at C7 has the ability to force the hydride addition syn to the metal center, and the distance of H7a from the metal allows this to be possible.

Scheme 5.16. Reduction of iminium in $\mathbf{8 2}$ to yield cis ring juncture

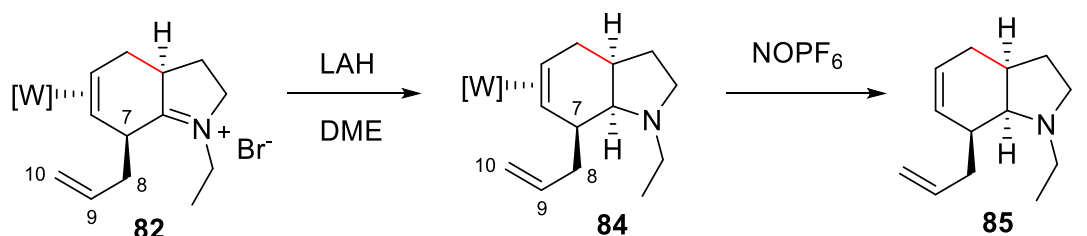

Oxidation of $\mathbf{8 4}$ with $\mathrm{NOPF}_{6}$ led to promising results, leading to an NMR yield of $88 \% .1 \mathrm{H}$ NMR of a crude mixture showed the characteristic $\mathrm{H} 9$ proton as a multiplet at 
$5.78 \mathrm{ppm}$ and a geminal set at 4.75 and 4.92 for $\mathrm{H} 10$. The $\mathrm{CH}_{2}$ groups of 85 on the pyrole ring were also still intact.

\subsection{Further Aniline Exploration}

One of the greatest problems with the $N, N$-dimethylanilinium complex was the fact that the organic molecules lost their aniline core and became $\alpha, \beta$-unsaturated enones during oxidation. These are biologically damaging and are rejected by biological libraries. This whole problem stemmed from the fact that the iminium of the addition products could not be reduced. By applying the same reaction conditions for the indoline reductions to the aniline system, it would be possible to synthesize the desired aniline derivatives. This scheme can be seen in scheme 5.17. By starting out with an addition product (I), LAH in DME or $\mathrm{Et}_{2} \mathrm{O}$ can be used to reduce the iminium bond stereoselectively to yield II. Using a weak oxidant, the novel organic (III) could then be isolated.

Scheme 5.17. Scheme to yield novel cyclohexenamine organics from $N, N$ dimethylanilinium complex

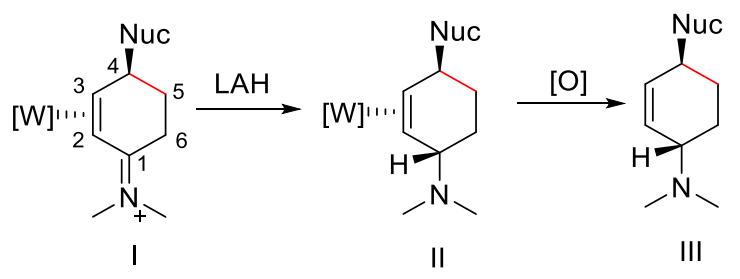


Scheme 5.18. Reduction of the iminium bond in aniline addition products (86-92)
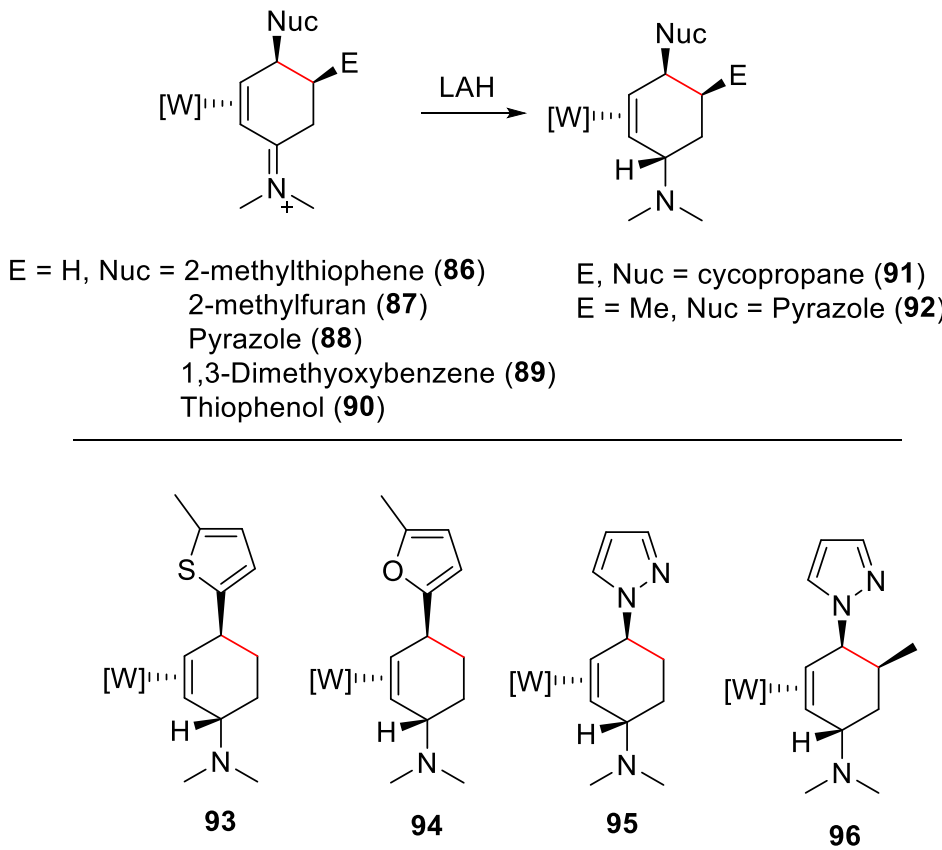<smiles>COc1ccc(C2CC[C@H](N(C)C)[C@H](C)C2)c(OC)c1</smiles>

97

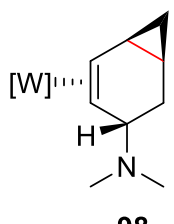

98

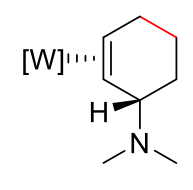

99

This was applied to various addition products (86-92), which have previously been published. ${ }^{7-9}$ The products of these reductions (93-99) can be seen in Scheme 5.18. These have all been identified via ${ }^{1} \mathrm{H},{ }^{13} \mathrm{C}$ and $2 \mathrm{D}$ NMR. Through all of the systems there is a new proton resonance between 3.8 and $4.1 \mathrm{ppm}$ which has a COSY interaction with the $\mathrm{H} 2$ proton and the $\mathrm{H} 6$ geminal set. There is no NOE interaction between $\mathrm{H} 1$ and $\mathrm{H} 4$, nor between $\mathrm{H} 1$ and either the Tp set or $\mathrm{PMe}_{3}$. This indicates that the hydride addition occurs anti to the metal as expected. Cyclic voltammetry and IR both show that the 
iminium has been reduced, with a lower reduction potential and a loss of the iminium peak. As with the indoline reduction, simple amine or enolate nucleophiles were not stable to the reduction conditions. The thiophenol addition $(\mathbf{9 0})$ was subjected to these reduction conditions and produced a new product which lacked any phenol NMR resonance. Using HSQC, a new geminal set was observed, leading to the conclusion that the thiophenol was displaced by a hydride generating compound $\mathbf{9 9 .}$

\subsubsection{Isolation of Novel Aniline Organics}

Isolation of these novel aniline based organics proved to be challenging. Upon oxidation of 94 and 95 (with $\mathrm{NOPF}_{6}$ and DDQ respectively), the new novel organics 100 and 101 were isolated cleanly after a basic alumina preparatory TLC. The yields for these isolations were discouraging at $14 \%$ for compound $\mathbf{1 0 0}$ and $12 \%$ for compound $\mathbf{1 0 1}$. It is believed that these novel organics might be volatile, meaning that each step of the isolation reduces the yield dramatically. Systems such as $N, N$-dimethylcyclohexamine are frequently isolated as a $\mathrm{HCl}$ salt in order to increase stability and increase the boiling point of the desired compound. As a free amine, $N, N$-dimethylamine has a boiling point of $162^{\circ} \mathrm{C}$, which is close to many solvents. As the $\mathrm{HCl}$ salt, the melting point increases to $224^{\circ} \mathrm{C}$, now a solid at room temperature 
Scheme 5.19. Oxidation to yield novel aminocyclohexenes

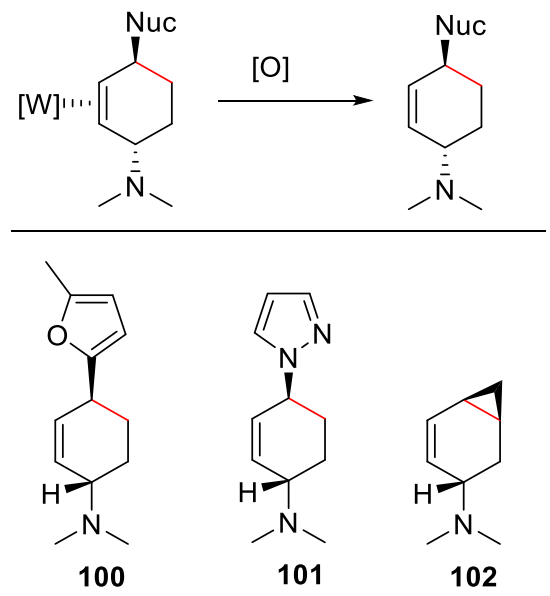

Using this knowledge, the isolation of these systems as the protonated salts was attempted. Two different pathways were tried. The first pathway stemmed from the knowledge that HOTf leveled in MeCN can oxidize these reduced complexes, so an in situ oxidation and protonation was attempted with compound 95, as seen in Scheme 5.20 as pathway A. Unfortunately the organic was never seen when followed by ${ }^{1} \mathrm{H}$ NMR. The alternate pathway was the oxidation of these systems with $\mathrm{NOPF}_{6}$ followed by a minimal workup, then protonating the amine with either $\mathrm{HCl}$ or HOTf, as seen in Pathway B.

Scheme 5.20. Novel organic isolation with protonation of amine

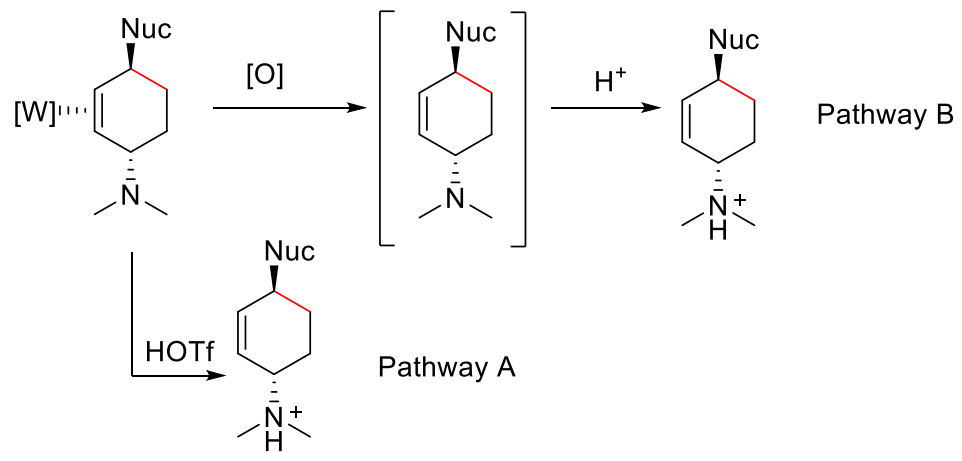


Pathway B was most successful with compound $\mathbf{9 8}$, the cycloproponated product. This functional group was assumed to be unstable towards excess acid due to possible ring opening, so the strength of acid necessary to protonate the amine would be limited. Following oxidation with $\mathrm{NOPF}_{6}$ in acetone, 1.1 equivalents of $4 \mathrm{M} \mathrm{HCl}$ in dioxane were added in hopes of a chlorine salt being formed. This reaction was not conclusive, though a protonated system was observed. This protonated species was more prevalent using HOTf in DCM. In ${ }^{1} \mathrm{H}$ NMR, the dimethylamino group becomes two defined doublets at $2.84 \mathrm{ppm}$, integrating for 3 protons each after protonation. The cyclopropane ring remains intact, unless a large excess of acid is used, as seen by a geminal set at 0.41 and $0.95 \mathrm{ppm}$. A NH peak also appears at $8.6 \mathrm{ppm}$. Unfortunately this protonation was not perfectly clean, so an alumina column was necessary. Upon isolation of the final product, it was determined that the organic had deprotonated. The NH peak was gone and the dimethylamino group was now a singlet integrating for 6 . The yield for the isolation of 102 was $37 \%$, a large increase from the previous isolations. This showed the ability to isolate, in now moderate yields, cyclohexamine derivatives.

Cyclohexamines, are prevalent in many biological systems and have many medicinal uses. Ketamine, a common anesthetic for surgeries, has a cyclohexamine core. This drug is so common that is considered a "core" medicine in the World Health Organization's Essential Drugs List. ${ }^{10}$ Tetrasubstituted aminocyclohexenes, such as conduramines also have biological acitivy, including $\beta$-glucosidase inhibitors. ${ }^{11,12}$ Even Streptomycin, an antibiotic, has a cyclohexamine core. 


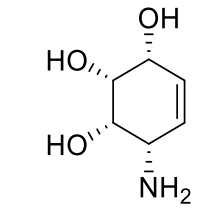

(-)-Conduramine-D1

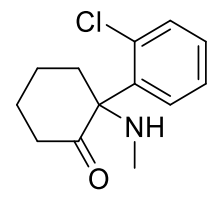

Ketamine

Figure 5.1. Structures of (-)-Conduramine-D1 and Ketamine

\subsubsection{New Allyl Generation from Aniline Complex}

In order to possibly branch out from the limited cyclohexamine derivative world, a novel reaction pathway was envisioned (Scheme 5.21). Using a weak acid, the amine could be protonated, while not oxidizing the metal. The amine could act as a leaving group and create a new allylic position (I). From here two pathways were possible. First, a new nucleophile could be added (II) to eventually to create cyclohexene derivative (III). Alternatively, the base could be added to recreate a diene type compound (IV). Here a typical tandem addition could be possible (V) to eventually create a more functionalized derivative (VI). Important concepts to keep in mind were the ability to protonate the amine while not decomposing the metal and also the ability for the amine to leave, creating the allyl (I). 
Scheme 5.21. Reaction scheme for new cyclohexene organic products

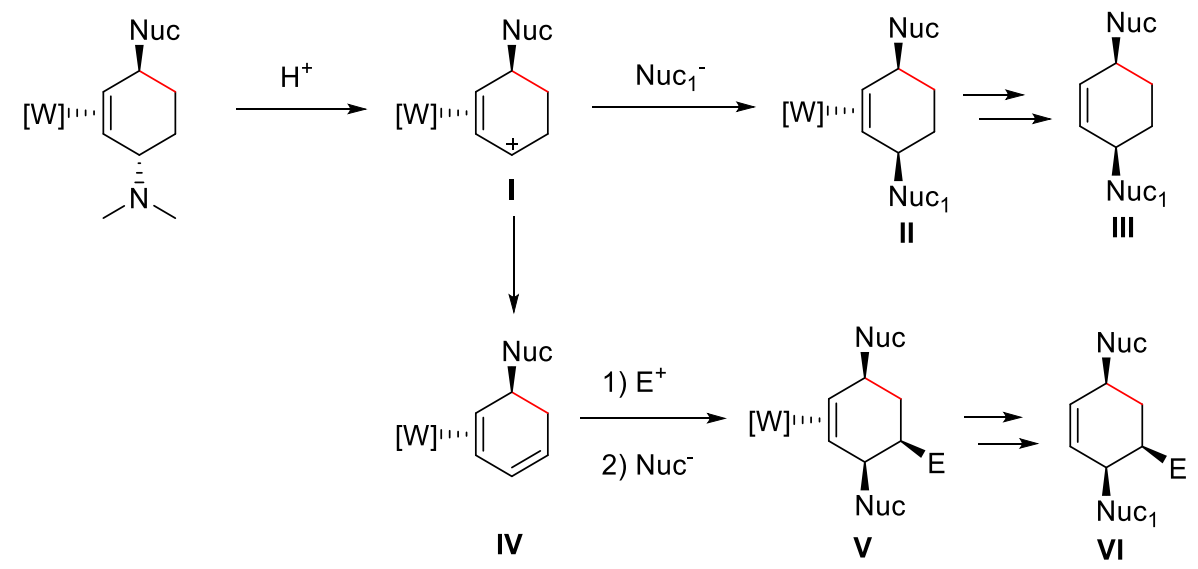

The majority of this initial work was completed with compound $\mathbf{9 8}$. This was due to the easy NMR handle of the cyclopropane group, but also the sensitivity of this functional group to acid. It was known the HOTf in $\mathrm{MeCN}(\mathrm{pKa} \approx-5)$ was strong enough to oxidize the metal, so weaker acids were attempted. These included DiPAT (11.0), TEAOTf (pka = 10.7), DPhAT (0.78) and HOTf in MeOH (-2.2). Upon addition of TEAOTf and DiPAT, a new species is created within 10 minutes. This new product appears to be the protonated intermediate (103) seen in Scheme 5.22. In ${ }^{1} \mathrm{H}$ NMR, a new $\mathrm{NH}$ peak can be seen along with a broadening of the peaks for the dimethylamino substituent. When these reactions were left for multiple days, a new product was seen growing in, though never reaching completion. With HOTf in $\mathrm{MeOH}$, the same new product grew in, though followed closely by decomposition, as seen in ${ }^{31} \mathrm{P}$ NMR. 
Scheme 5.22. Allyl 104 Creation from 98 with DPhAT

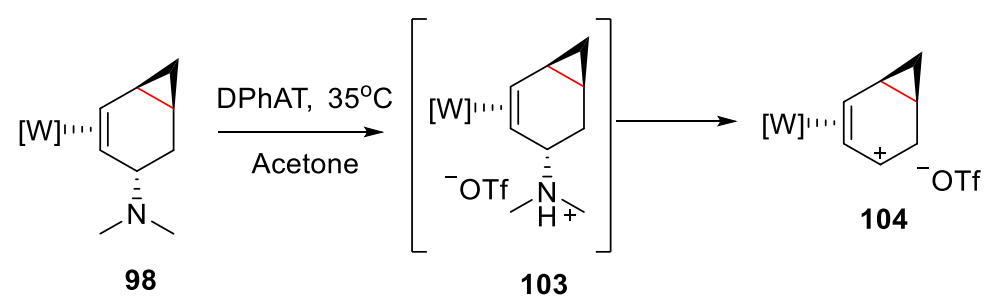

When compound $\mathbf{9 8}$ was combined with DPhAT and left for 5 days and followed with ${ }^{31}$ P NMR, a new product slowly grew in. This new species has a new chemical shift at $-6.40 \mathrm{ppm}$ and a $J_{\mathrm{W}-\mathrm{P}}$ of $270 \mathrm{~Hz}$. This shift and coupling constant are consistent with previous work done to determine the features of allylic species. ${ }^{13}$ In order to increase the rate of the amine loss, this reaction was repeated overnight at $35^{\circ} \mathrm{C}$ in acetone. After 18 hours, the allylic cation was isolated after precipitation into hexanes. This was fully characterized using ${ }^{1} \mathrm{H}$ and 2D NMR spectroscopic techniques. Previous work has shown the preference for the positive charge to be localized at the 1 position (Figure 5.2). This is shown in ${ }^{1} \mathrm{H}$ NMR shifts in compound $\mathbf{1 0 5}$ with $\mathrm{H} 1$ at 6.59 ppm, $\mathrm{H} 2$ at 5.13 ppm and $\mathrm{H} 3$ at 4.38 ppm. ${ }^{13}$ Only $\mathrm{H} 3$ has an NOE interaction with the $\mathrm{PMe}_{3}$ ligand. $\mathrm{H} 1$ and $\mathrm{H} 2$ have NOE interactions with the PzA3 proton. These same conclusions are shown in compound 104. $\mathrm{H} 1$ appears at $6.68 \mathrm{ppm}$ with an NOE to PzA3 and a COSY interaction to a geminal set at $\mathrm{H} 6 . \mathrm{H} 2$ is consistent at 5.10 and $\mathrm{H} 3$ appears at $4.81 \mathrm{ppm}$. $\mathrm{H} 3$ has a strong NOE interaction with the $\mathrm{PMe}_{3}$ and a COSY interaction with the $\mathrm{H} 4$ proton of the cyclopropane ring. This could be isolated in a $76 \%$ yield. Nucleophilic additions were attempted with pyrazole, MTDA and $\mathrm{MeOH}$, though none showed a clean product. This is being investigated further by another graduate student. 


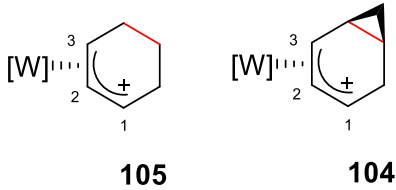

Figure 5.2. Comparison of known allyl 105 and 104

\subsection{Conclusions:}

Since the ability to reduce the conjugated iminium bond in the indoline system was discovered, the possibilities of both the indoline and aniline systems have grown substantially. The synthesis of novel, tetrasubstituted cyclohexeneamine has been completed. There has also been new functionalization of the indoline system at the H4 and $\mathrm{H} 7$ positions. Aniline has been expanded further into the field of saturated cyclohexenes. The future of this work could be expansive and this work continues with future graduate students. 


\subsection{Experimental Section:}

General Methods: NMR spectra were obtained on a 300, 500, 600, or $800 \mathrm{MHz}$ spectrometer. All chemical shifts are reported in ppm and proton and carbon shifts are referenced to tetramethylsilane (TMS) utilizing residual ${ }^{1} \mathrm{H}$ or ${ }^{13} \mathrm{C}$ signals of the deuterated solvents as an internal standard. Phosphorus NMR signals are referenced to $85 \% \mathrm{H}_{3} \mathrm{PO}_{4}(\delta=0.00)$ using a triphenylphosphate external standard $(\delta=-16.58)$. Coupling constants $(J)$ are reported in hertz $(\mathrm{Hz})$. Infrared spectra (IR) were recorded as a glaze on a spectrometer fitted with a Horizontal Attenuated Total Reflectance (HATR) accessory, or on a FT-IR spectrometer equipped with a diamond anvil ATR assembly. Electrochemical experiments were performed under a dinitrogen atmosphere using a potentiostat. Cyclic voltammetry data was taken at ambient temperature $\left(\sim 25^{\circ} \mathrm{C}\right)$ at 100 $\mathrm{mV} / \mathrm{s}$ in a standard three-electrode cell with a glassy carbon working electrode, $N, N$ dimethylacetamide (DMA) or acetonitrile $(\mathrm{MeCN})$ solvent (unless otherwise specified), and tetrabutylammonium hexafluorophosphate (TBAH) electrolyte (approx. 0.5 M). All potentials are reported versus NHE (Normal Hydrogen Electrode) using cobaltocenium hexafluorophosphate $\left(E_{1 / 2}=-0.78 \mathrm{~V}\right)$, ferrocene $\left(\mathrm{E}_{1 / 2}=+0.55 \mathrm{~V}\right)$, or decamethylferrocene $\left(\mathrm{E}_{1 / 2}=+0.04 \mathrm{~V}\right)$ as an internal standard. The peak-to-peak separation was less than 100 $\mathrm{mV}$ for all reversible couples. High-resolution mass spectra were acquired in ESI mode, from samples dissolved in a 3:1 acetonitrile/water solution containing sodium trifluoroacetate (NaTFA). Mass spectra are reported as $\mathrm{M}^{+}$for monocationic complexes, or as $\left[\mathrm{M}+\mathrm{H}^{+}\right]$or $\left[\mathrm{M}+\mathrm{Na}^{+}\right]$for neutral complexes, using $\left[\mathrm{Na}(\mathrm{NaTFA})_{\mathrm{x}}\right]^{+}$clusters as an internal standard. In all cases, observed isotopic envelopes were consistent with the 
molecular composition reported. For organic products, the monoisotopic ion is reported; for complexes, the major peaks in the isotopic envelope are reported. Unless otherwise noted, all synthetic reactions were performed in a glovebox under a dry nitrogen atmosphere. $\mathrm{CH}_{2} \mathrm{Cl}_{2}$ and benzene were purified by passage through a column packed with activated alumina. Other solvents and liquid reagents were thoroughly purged with dry nitrogen prior to use. Triflate salts of amines were synthesized by addition of an $\mathrm{Et}_{2} \mathrm{O}$ solution of triflic acid to the appropriate conjugate base dissolved in $\mathrm{Et}_{2} \mathrm{O}$. Deuterated solvents were used as received from Cambridge Isotopes. Pyrazole $(\mathrm{Pz})$ protons of the (trispyrazolyl) borate (Tp) ligand were uniquely assigned (eg., "PzB3") using a combination of 2-dimensional NMR data and phosphorus-proton NOE interactions. Compounds 86-92 and 105 were reported with full characterization previously. BH peaks (around 4-5 ppm) are not identified due to their quadrupole broadening; IR data is used to confirm the presence of a $\mathrm{BH}$ (around $2500 \mathrm{~cm}^{-1}$ ). $\mathrm{OH}$ and $\mathrm{NH}$ peaks are not always identified due to exchange with water in solvent. Where appropriate, $\mathrm{OH}$ peaks have been confirmed with IR data. 


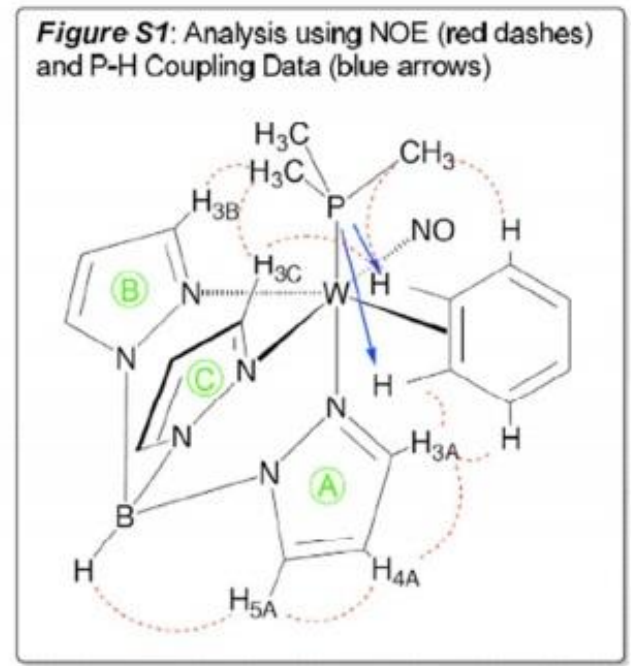

Compound 71: In a vial with a stirbar, Compound 34 (203 mg, $0.225 \mathrm{mmol})$ was dissolved in acetone $(5 \mathrm{~mL})$. Propyl amine $(152 \mathrm{mg}, 2.57 \mathrm{mmol})$ was added to the reaction mixture and stirred 45 minutes. The mixture was added dropwise to a stirring solution of $\mathrm{Et}_{2} \mathrm{O}(200 \mathrm{~mL})$ to induce precipitation of a yellow solid. The powder was collected on a $15 \mathrm{~mL}$ fine porosity fritted funnel, yielding Compound $71(0.143 \mathrm{~g}, 0.165$ mmol, 73\%). ${ }^{1} \mathrm{H}$ NMR $(d-\mathrm{MeCN}, \delta): 8.06(\mathrm{~d}, J=2.0,1 \mathrm{H}, \mathrm{PzB} 3), 7.97(\mathrm{~d}, J=2.0,1 \mathrm{H}$, PzC5), 7.93 (d, $J=2.0,1 \mathrm{H}, \mathrm{Pz} 5), 7.91(\mathrm{~d}, J=2.0,1 \mathrm{H}, \mathrm{Pz} 5), 7.66(\mathrm{~d}, J=2.0,1 \mathrm{H}, \mathrm{H} 5$ '), $7.60(\mathrm{~d}, J=2.0,1 \mathrm{H}, \mathrm{PzC} 3), 7.54\left(\mathrm{~d}, J=2.0,1 \mathrm{H}, \mathrm{H} 3{ }^{\prime}\right), 7.31(\mathrm{~d}, J=2.0,1 \mathrm{H}, \mathrm{PzA} 3), 6.42$ (t, $J=2.0,1 \mathrm{H}, \mathrm{PzB} 4), 6.39(\mathrm{t}, J=2.0,1 \mathrm{H}, \mathrm{PzC} 4), 6.37\left(\mathrm{t}, J=2.0,1 \mathrm{H}, \mathrm{H} 4{ }^{\prime}\right), 6.36(\mathrm{t}, J=$ 2.0, 1H, PzA4), 6.27 (m, 1H, H4), 5.84 (d, J = 4.2, 1H, H5), 4.08 (m, 1H, H2x), 4.03 (m, 1H, H2y), 3.62 (dd, $J=12.4,8.3,1 \mathrm{H}, \mathrm{H} 6), 3.00$ (m, 2H, H3), 2.89 (m, 1H, N-Ethyl $\mathrm{CH}_{2}$ ), $2.84\left(\mathrm{~m}, 1 \mathrm{H}, \mathrm{N}-\mathrm{Ethyl} \mathrm{CH}_{2}\right), 2.26(\mathrm{~d}, J=8.2,1 \mathrm{H}, \mathrm{H} 7), 1.25\left(\mathrm{~d}, J=9.1,9 \mathrm{H}, \mathrm{PMe}_{3}\right), 1.06(\mathrm{t}$, $\left.J=7.3,3 \mathrm{H}, \mathrm{N}-\mathrm{Ethyl} \mathrm{CH}_{3}\right) .{ }^{13} \mathrm{C} \mathrm{NMR}(d-\mathrm{MeCN}, \delta): 174.0(\mathrm{C} 7 \mathrm{a}), 145.0(\mathrm{PzB} 3), 144.0$ (PzA3) 142.3 (PzC3), 139.7 (C3'), 139.1 (Pz5), 138.9 (Pz5), 138.7 (Pz5), 137.8 (C3a), 
128.0 (C5'), 127.3 (C4), 108.4 (Pz4), 108.1 (Pz4), 107.6 (Pz4), 106.9 (C4'), 66.3 (d, J = 13.1, C6), 62.3 (d, $J=3.6, \mathrm{C} 5), 53.4(\mathrm{C} 2), 45.7$ (C7), $43.3\left(\mathrm{~N}-\mathrm{Ethyl} \mathrm{CH}_{2}\right), 25.6(\mathrm{C} 3), 12.9$ $\left(\mathrm{d}, J=30.5, \mathrm{PMe}_{3}\right), 12.0\left(\mathrm{~N}-\right.$ Ethyl $\left.\mathrm{CH}_{3}\right) .{ }^{31} \mathrm{P} \mathrm{NMR}\left(\mathrm{CH}_{2} \mathrm{Cl}_{2}, \delta\right):-8.29\left(J_{\mathrm{wp}}=268\right) . \mathrm{IR}$ : $v_{\mathrm{BH}}=2511 \mathrm{~cm}^{-1}, v_{\mathrm{NO}}$ and $v_{\text {iminium }}=1608$ and $1585 \mathrm{~cm}^{-1} . \mathrm{CV}(\mathrm{DMA}): \mathrm{E}_{\mathrm{p}, \mathrm{a}}=1.33 \mathrm{~V} .\left[\mathrm{M}^{+}\right.$ $\left.=\mathrm{C}_{25} \mathrm{H}_{35} \mathrm{~N}_{10} \mathrm{OBPW}^{+}\right]=$obsd (\%), calcd (\%), ppm: 715.2336 (82), 715.2313 (82), 3.2; 716.2342 (80), 716.2338 (81), 0.6; 717.2348 (100), 717.2337 (100), 1.5; 718.2374 (48), $718.2376(46),-0.3 ; 719.2356(91), 719.2369(83),-1.9$.

Compound 72: In a vial with a stirbar, Compound 39 (45 mg, $0.050 \mathrm{mmol})$ was dissolved in acetone $(1 \mathrm{~mL})$. Propyl amine $(42 \mathrm{mg}, 0.710 \mathrm{mmol})$ was added to the reaction mixture and stirred 30 minutes. The mixture was added dropwise to a stirring solution of $\mathrm{Et}_{2} \mathrm{O}(50 \mathrm{~mL})$ to induce precipitation of a yellow solid. The powder was collected on a $15 \mathrm{~mL}$ fine porosity fritted funnel, yielding Compound $72(0.033 \mathrm{~g}$, 0.038mmol, 88\%). ${ }^{1} \mathrm{H}$ NMR $\left(d-\mathrm{CD}_{3} \mathrm{CN}, \delta\right): 8.05$ (d, $\left.J=2.0,1 \mathrm{H}, \mathrm{PzB} 3\right), 7.98(\mathrm{~d}, J=2.0$, 1H, PzC5), 7.93 (d, $J=2.0,1 \mathrm{H}$, PzA5), $7.91(\mathrm{~d}, J=2.0,1 \mathrm{H}, \mathrm{PzB} 5), 7.79$ (s, 1H, H2'), $7.68(\mathrm{~d}, J=2.0,1 \mathrm{H}, \mathrm{PzC} 3), 7.31(\mathrm{~d}, J=2.0,1 \mathrm{H}, \mathrm{PzA} 3), 7.21$ (broad s, 1H, H4' or H5'), 7.02 (broad s, 1H, H4' or H5'), 6.42 (t, $J=2.0,1 \mathrm{H}, \mathrm{PzB} 4), 6.40$ (t, $J=2.0,1 \mathrm{H}, \mathrm{PzC} 4)$, $6.36(\mathrm{t}, J=2.0,1 \mathrm{H}, \mathrm{PzA} 4), 6.23(\mathrm{~d}, J=4.2,1 \mathrm{H}, \mathrm{H} 4), 5.63(\mathrm{~d}, J=4.6,1 \mathrm{H}, \mathrm{H} 5), 4.06(\mathrm{~m}$, 1H, H2x), 4.01 (m, 1H, H2y), 3.60 (dd, J = 12.2, 8.4, 1H, H6), 2.94 (m, 2H, N-Ethyl $\left.\mathrm{CH}_{2}\right), 2.83(\mathrm{~m}, 2 \mathrm{H}, \mathrm{H} 3), 2.36(\mathrm{~d}, J=8.2,1 \mathrm{H}, \mathrm{H} 7), 1.20\left(\mathrm{~d}, J=9.0,9 \mathrm{H}, \mathrm{PMe}_{3}\right), 1.09$ (t, $J$ = 7.2, 3H, N-Ethyl $\left.\mathrm{CH}_{3}\right) .{ }^{13} \mathrm{C} \mathrm{NMR}\left(d-\mathrm{CD}_{3} \mathrm{CN}, \delta\right): 173.7$ (C7a), $145.0(\mathrm{PzB} 3), 144.0$ (PzA3), 142.4 (PzC3), 139.1 (Pz5), 139.0 (Pz5), 138.7 (Pz5), 136.7 (C2’), 129.7 (C4’or C5'), 128.5 (C4), 118.1 (C4' or C5'), 108.4 (Pz4), 108.1 (Pz4), 107.7 (PzA4), 65.5 (d, J= 
13.9, 1H, C6), 57.5 (d, $J=4.1, \mathrm{C} 5), 53.4(\mathrm{C} 2), 46.1$ (C3 and C7), $25.5\left(\mathrm{~N}-\mathrm{Ethyl} \mathrm{CH}_{2}\right)$, $13.0\left(\mathrm{~d}, J=30.7,9 \mathrm{H}, \mathrm{PMe}_{3}\right), 12.0\left(\mathrm{~N}-\mathrm{Ethyl} \mathrm{CH}_{3}\right) .{ }^{31} \mathrm{P} \mathrm{NMR}\left(\mathrm{CD}_{3} \mathrm{CN}, \delta\right):-9.01\left(J_{\mathrm{wp}}=\right.$ 272).

Compound 75: In a vial with a stirbar, Compound 71 (50 mg, $0.057 \mathrm{mmol})$ was dissolved in MeCN (3 mL). A mixture of thiophenol (52 mg, $0.472 \mathrm{mmol}$ ) and diisopropyl ammonium triflate $(16 \mathrm{mg}, 0.064 \mathrm{mmmol})$ in $\mathrm{MeCN}(2 \mathrm{~mL})$ was added to the reaction mixture and stirred overnight. The mixture was removed from the glovebox and diluted with $20 \mathrm{~mL}$ DCM. This was treated with $30 \mathrm{~mL} \mathrm{Na}_{2} \mathrm{CO}_{3}$ (saturated, aq). The aqueous layer was back extracted with DCM (1 x $20 \mathrm{~mL})$, and the combined organic layers were washed with deionized water $(20 \mathrm{~mL})$. ). This was then dried over anhydrous $\mathrm{MgSO}_{4}$ and concentrated in vacuo. The oil was redissolved in minimal DCM and added dropwise to a stirring solution of $\mathrm{Et}_{2} \mathrm{O}(50 \mathrm{~mL})$ to induce precipitation of a yellow solid. The powder was collected on a $15 \mathrm{~mL}$ fine porosity fritted funnel, yielding Compound 75 (0.019 g, $0.038 \mathrm{mmol}, 13 \%) .{ }^{1} \mathrm{H} \mathrm{NMR}\left(d-\mathrm{CD}_{3} \mathrm{CN}, \delta\right): 8.14(\mathrm{~d}, J=2.0,1 \mathrm{H}, \mathrm{PzB} 3)$, $8.11(\mathrm{~d}, J=2.0,1 \mathrm{H}, \mathrm{PzC} 5), 8.04(\mathrm{~d}, J=2.0,1 \mathrm{H}, \mathrm{PzC} 3), 8.03(\mathrm{~d}, J=2.0,1 \mathrm{H}, \mathrm{PzB} 5), 7.96$ (d, $J=2.0,1 \mathrm{H}, \mathrm{PzA} 5), 7.70\left(\mathrm{~d}, J=7.01,2 \mathrm{H}, \mathrm{H} 2^{\prime}\right.$ and $\left.\mathrm{H}^{\prime}{ }^{\prime}\right), 7.39$ (m, 3H, H3', H4', and H5'), $7.34(\mathrm{~d}, J=2.0,1 \mathrm{H}, \mathrm{PzA} 3), 6.54(\mathrm{t}, J=2.0,1 \mathrm{H}, \mathrm{PzC} 4), 6.46(\mathrm{t}, J=2.0,1 \mathrm{H}$, PzC4), $6.38(\mathrm{~m}, 1 \mathrm{H}, \mathrm{H} 4), 6.31(\mathrm{t}, J=2.0,1 \mathrm{H}, \mathrm{PzA} 4), 4.82(\mathrm{~d}, J=3.3,1 \mathrm{H}, \mathrm{H} 5), 4.03$ (m, 1H, H6), 4.02 (m, 1H, H2x), 3.75 (m, 1H, H2y), 3.03 (m, 1H, H3x), 2.95 (m, 1H, H3y), 2.82 (m, 1H, N-Ethyl $\left.\mathrm{CH}_{2}\right), 2.56$ (m, 1H, N-Ethyl $\left.\mathrm{CH}_{2}\right), 1.82(\mathrm{~d}, J=8.5,1 \mathrm{H}, \mathrm{H} 7), 1.37$ $\left(\mathrm{d}, J=9.0,9 \mathrm{H}, \mathrm{PMe}_{3}\right), 0.80\left(\mathrm{t}, J=7.3,3 \mathrm{H}, \mathrm{N}-\mathrm{Ethyl} \mathrm{CH}_{3}\right) .{ }^{13} \mathrm{C} \mathrm{NMR}\left(d-\mathrm{CD}_{3} \mathrm{CN}, \delta\right)$ : 175.1 (C7a), 145.2 (PzB3), 144.3 (PzC3), 142.3 (PzA3), 139.5 (C3' and C5'), 138.9 (C2' 
and C6'), 138.9 (Pz5), 138.8 (Pz5), 138.7 (Pz5), 133.4 (C3a), 131.5 (C4), 131.2 (C1'), 130.3 (C4'), 108.3 (PzB4 and PzC4), 107.5 (PzA4), 66.4 (d, $J=12.8, \mathrm{C} 6), 53.0$ (C5), $52.8(\mathrm{C} 2), 47.1(\mathrm{C} 7), 42.9\left(\mathrm{~N}-\right.$ Ethyl $\left.\mathrm{CH}_{2}\right), 25.5(\mathrm{C} 3), 13.2\left(\mathrm{~d}, J=30.1, \mathrm{PMe}_{3}\right), 12.4(\mathrm{~N}-$ Ethyl $\left.\mathrm{CH}_{3}\right) .{ }^{31} \mathrm{P} \mathrm{NMR}\left(\mathrm{CD}_{3} \mathrm{CN}, \delta\right):-8.44\left(J_{\mathrm{wp}}=268\right)$.

Compound 76: In a vial with a stirbar, Compound 34 (30 mg, $0.032 \mathrm{mmol})$ was dissolved in MeCN (1.5 mL). Propyl amine (21 $\mathrm{mg}, 0.355 \mathrm{mmol}$ ) was added to the reaction mixture and stirred overnight. The mixture was removed from the glovebox and diluted with $10 \mathrm{~mL}$ DCM. This was treated with $10 \mathrm{~mL} \mathrm{Na}_{2} \mathrm{CO}_{3}$ (saturated, aq). The aqueous layer was back extracted with DCM $(1 \times 20 \mathrm{~mL})$, and the combined organic layers were washed with deionized water $(20 \mathrm{~mL})$. This was then dried over anhydrous $\mathrm{MgSO}_{4}$ and concentrated in vacuo. The oil was redissolved in minimal DCM and added dropwise to a stirring solution of $\mathrm{Et}_{2} \mathrm{O}(25 \mathrm{~mL})$ to induce precipitation of a yellow solid. The powder was collected on a $15 \mathrm{~mL}$ fine porosity fritted funnel, yielding Compound 76 (0.005 g, $0.005 \mathrm{mmol}, 16 \%) .{ }^{1} \mathrm{H}$ NMR $(d-\mathrm{MeCN}, \delta): 8.09$ (d, $\left.J=2.0,1 \mathrm{H}, \mathrm{PzB} 3\right), 7.96$ (d, $J=2.0,1 \mathrm{H}, \mathrm{PzC} 5), 7.92(\mathrm{~d}, J=2.0,1 \mathrm{H}, \mathrm{PzB} 5$ or H5'), 7.91 (d, $J=2.0,1 \mathrm{H}, \mathrm{PzB} 5$ or H5'), 7.87 (d, $J=2.0,1 \mathrm{H}, \mathrm{PzA} 5), 7.56$ (d, $J=2.0,1 \mathrm{H}, \mathrm{H} 3$ '), 7.50 (d, $J=2.0,1 \mathrm{H}, \mathrm{PzC} 3)$, $7.31(\mathrm{~d}, J=2.0,1 \mathrm{H}, \mathrm{PzA} 3), 6.44(\mathrm{t}, J=2.0,1 \mathrm{H}, \mathrm{PzB} 4), 6.42(\mathrm{t}, J=2.0,1 \mathrm{H}, \mathrm{H} 4$ ') $6.40(\mathrm{t}$, $J=2.0,1 \mathrm{H}, \mathrm{PzC} 4), 6.34(\mathrm{t}, J=2.0,1 \mathrm{H}, \mathrm{PzA} 4), 5.74(\mathrm{dd}, J=9.8,3.0,1 \mathrm{H}, \mathrm{H} 5), 4.10(\mathrm{~m}$, 1H, H2x), 3.95 (t, $J=11.2,1 \mathrm{H}, \mathrm{H} 2 \mathrm{y}), 3.83$ (m, 1H, H6), 3.53 (m, 1H, H3a), 2.86 (dd, $J=$ 12.2, 9.9, 1H, H4), $2.77\left(\mathrm{~m}, 1 \mathrm{H}, \mathrm{N}-\mathrm{E}\right.$ thyl $\left.\mathrm{CH}_{2}\right), 2.63\left(\mathrm{~m}, 1 \mathrm{H}, \mathrm{N}-\mathrm{Ethyl} \mathrm{CH}_{2}\right), 2.52(\mathrm{~m}, 1 \mathrm{H}$, H3x), $2.42\left(\mathrm{~m}, 1 \mathrm{H}, \mathrm{H} 1 \mathrm{x}^{*}\right), 2.19$ (d, $\left.J=8.7,1 \mathrm{H}, \mathrm{H} 7\right), 2.02$ (m, 1H, H1y*), 1.96 (m, 1H, H3y), 1.26 (m, 2H, H2*), 1.05 (t, $J=7.2,3 \mathrm{H}, \mathrm{N}-\mathrm{Ethyl}_{\mathrm{CH}}$ ), 0.92 (d, $J=9.2,9 \mathrm{H}, \mathrm{PMe}_{3}$ ), 
$0.78(\mathrm{t}, J=7.4,3 \mathrm{H}, \mathrm{H} 3 *) .{ }^{13} \mathrm{C} \mathrm{NMR}(d-\mathrm{MeCN}, \delta): 188.5(\mathrm{C} 7 \mathrm{a}), 145.3$ (PzB3), 144.7

(PzA3), 142.1 (PzC3), 139.6 (C3'), 138.8 (Pz5), 138.7 (2C, Pz5), 130.1 (C5'), 108.4

(Pz4), 108.1 (Pz4), 107.9 (Pz4), 107.1 (C4'), 70.1 (C4), 68.0 (d, J = 2.5, C5), 67.7 (d, $J=$ 13.0, C6), 54.7 (C2), 49.5 (C7), 47.7 (C1*), 47.0 (C3a), 43.0 (N-Ethyl $\mathrm{CH}_{2}$ ), 26.3 (C3), $24.5\left(\mathrm{C} 2^{*}\right), 13.4\left(\mathrm{~d}, J=31.0, \mathrm{PMe}_{3}\right), 11.9\left(\mathrm{C} 3^{*}\right), 11.7\left(\mathrm{~N}-\right.$ Ethyl $\left.\mathrm{CH}_{3}\right) .{ }^{31} \mathrm{P} \mathrm{NMR}$ $\left(\mathrm{CH}_{2} \mathrm{Cl}_{2}, \delta\right):-8.78\left(J_{\mathrm{wp}}=279\right)$. IR: $v_{\mathrm{BH}} 2499 \mathrm{~cm}^{-1}, v_{\mathrm{NO}}$ and $v_{\text {iminium }}=1608$ and $1577 \mathrm{~cm}^{-1}$. $\mathrm{CV}$ (DMA): $\mathrm{E}_{\mathrm{p}, \mathrm{a}}=1.37 \mathrm{~V} \cdot\left[\mathrm{M}^{+}=\mathrm{C}_{28} \mathrm{H}_{44} \mathrm{~N}_{11} \mathrm{OBPW}^{+}\right]=\operatorname{obsd}(\%)$, calcd (\%), ppm: 774.3029 (80), 774.3048 (81), -2.5; 775.3061 (82), 775.3073 (82), -1.5; 776.3053 (100), 776.3073 (100), -2.6; 777.3089 (48), 777.3110 (48), -2.7; 778.3075 (81), 778.3105 (82), 3.9.

Compound 80: In a vial, Compound $54(0.501 \mathrm{~g}, 0.768 \mathrm{mmol})$ was dissolved in DCM $(10 \mathrm{~mL})$. To this solution, diphenyl ammonium triflate $(270 \mathrm{mg}, 0.846 \mathrm{mmol})$ was added and the solution stirred for $2 \mathrm{~min}$. The reaction mixture was added dropwise to a stirring solution of $\mathrm{Et}_{2} \mathrm{O}(500 \mathrm{~mL})$ to induce precipitation of a light-tan solid. The powder was collected on a $30 \mathrm{~mL}$ fine porosity fritted funnel, washed with $\mathrm{Et}_{2} \mathrm{O}(20 \mathrm{~mL})$, yielding Compound $80(0.543 \mathrm{~g}, 0.677 \mathrm{mmol}, 88 \%) .{ }^{1} \mathrm{H}$ NMR $\left(d-\mathrm{CD}_{3} \mathrm{CN}, \delta\right): 8.13(\mathrm{~d}, J=2.0$, 1H, PzA3), 8.06 (d, $J=2.0,1 \mathrm{H}, \mathrm{PzB} 3), 7.90$ (d, $J=2.0,1 \mathrm{H}, \mathrm{PzB} 5), 7.84$ (d, $J=2.0,1 \mathrm{H}$, PzC5), 7.80 (d, $J=2.0,1 \mathrm{H}, \mathrm{PzA} 5), 7.41$ (d, $J=2.0,1 \mathrm{H}, \mathrm{PzC} 3), 6.41$ (t, $J=2.0,1 \mathrm{H}$, PzB4), 6.33 (t, $J=2.0,1 \mathrm{H}$, PzA4), $6.26(\mathrm{t}, J=2.0,1 \mathrm{H}, \mathrm{PzC} 4), 4.05(\mathrm{~m}, 2 \mathrm{H}, \mathrm{H} 2), 3.94(\mathrm{~m}$, 1H, H7x), 3.78 (m, 1H, H7y), 3.76 (m, 2H, N-Ethyl $\mathrm{CH}_{2}$ ), 3.16 (m, 2H, H4), 2.69 (m, 1H, H5), 2.49 (m, 1H, H3x), 2.09 (m, 1H, H3y), 1.29 (t, $J=7.3,3 \mathrm{H}, \mathrm{N}$-Ethyl $\left.\mathrm{CH}_{3}\right), 1.19$ (buried, $1 \mathrm{H}, \mathrm{H} 6), 1.19$ (d, $\left.J=8.5,9 \mathrm{H}, \mathrm{PMe}_{3}\right) .{ }^{13} \mathrm{C} \mathrm{NMR}\left(d-\mathrm{CD}_{3} \mathrm{CN}, \delta\right): 199.0$ (C7a), 
144.9 (PzB3), 143.5 (PzA3), 142.0, (PzC3), 138.2 (PzC5), 137.6 (PzB5), 137.2 (PzA5), 107.7 (PzB4), 107.1 (PzA4), 107.0 (PzC4), 58.0, (C2), 52.0 (d, J = 13.0, C5), 49.6 (C3a), 48.1 (C6), 45.7 (N-Ethyl $\mathrm{CH}_{2}$ ), 35.2 (C4), 32.4 (C7), 28.0 (C3), 13.4 (d, J = 31.0, $\left.\mathrm{PMe}_{3}\right), 12.4\left(\mathrm{~N}-\right.$ Ethyl $\left.\mathrm{CH}_{3}\right) .{ }^{31} \mathrm{P} \mathrm{NMR}\left(d-\mathrm{CD}_{3} \mathrm{CN}, \delta\right):-10.6\left(J_{\mathrm{wp}}=278\right) . \mathrm{IR}: v_{\mathrm{BH}}=2484$ $\mathrm{cm}^{-1}, v_{\mathrm{NO}}$ and $v_{\text {imminium }}=1547$ and $1535 \mathrm{~cm}^{-1}(\mathrm{DMA}): \mathrm{E}_{\mathrm{p}, \mathrm{a}}=0.62 \mathrm{~V}$. HRMS: $\left[\mathrm{C}_{22} \mathrm{H}_{35} \mathrm{~N}_{8} \mathrm{OBPW}^{+}\right]=$obs'd (\%), calc'd (\%), ppm: 651.2249 (87), 651.2251 (84), -0.3; 652.2282 (79), 652.2276 (80), 0.9; 653.2261 (100), 653.2275 (100), -2.1; 654.2329 (41), 654.2316 (43), 2.0; 655.2327 (71), 655.2307 (84), 3.0.

Compound 81: In a vial, Compound 80 (0.100 g, $0.124 \mathrm{mmol})$ was dissolved in $\mathrm{MeCN}$ (1 $\mathrm{mL})$. To this solution, KHMDS $(0.7 \mathrm{M}$ in toluene, $0.250 \mathrm{~mL})$ was added and the solution stirred for $10 \mathrm{~min}$. The reaction mixture was evaporated to dryness and redissolved in minimal DCM. This solution was added dropwise to a stirring solution of hexanes $(50 \mathrm{~mL})$ to induce precipitation of a light-tan solid. The powder was collected on a $15 \mathrm{~mL}$ fine porosity fritted funnel, washed with hexanes $(5 \mathrm{~mL})$, yielding Compound $81(0.059 \mathrm{~g}, 0.090 \mathrm{mmol}, 73 \%) .{ }^{1} \mathrm{H}$ NMR $\left(d-\mathrm{CDCl}_{3}, \delta\right): 8.39(\mathrm{~d}, J=2.0,1 \mathrm{H}, \mathrm{PzA} 3), 7.98$ (d, $J=2.0,1 \mathrm{H}, \mathrm{PzB} 3), 7.83(\mathrm{~d}, J=2.0,1 \mathrm{H}, \mathrm{PzB} 5), 7.80$ (d, $J=2.0,1 \mathrm{H}, \mathrm{PzC} 5), 7.74(\mathrm{~d}, J$ $=2.0,1 \mathrm{H}, \mathrm{PzA} 5), 7.42(\mathrm{~d}, J=2.0,1 \mathrm{H}, \mathrm{PzC} 3), 6.34(\mathrm{t}, J=2.0,1 \mathrm{H}, \mathrm{PzC} 4), 6.26(\mathrm{t}, J=2.0$, 1H, PzA4), $6.22(\mathrm{t}, J=2.0,1 \mathrm{H}, \mathrm{PzB} 4), 5.09(\mathrm{dd}, J=6.1,2.3,1 \mathrm{H}, \mathrm{H} 7), 3.11(\mathrm{~m}, 1 \mathrm{H}$, H4x), 3.08 (m, 1H, N-Ethyl $\mathrm{CH}_{2}$ ), 3.06 (m, 1H, H2x), 3.00 (m, 1H, N-Ethyl $\left.\mathrm{CH}_{2}\right), 2.97$ (m, 1H, H2y), 2.93 (m, 1H, H4y), 2.82 (m, 1H, H3a), 2.58 (m, 1H, H5), 2.04 (m, 1H, H3x), 1.56 (m, 1H, H6), 1.39 (m, 1H, H3y), 1.23 (d, J = 8.16, 9H, PMe $\left.)_{3}\right), 1.09$ (t, $J=6.8$, 3H, N-Ethyl $\left.\mathrm{CH}_{3}\right) .{ }^{13} \mathrm{C} \mathrm{NMR}\left(d-\mathrm{CDCl}_{3}, \delta\right): 145.3$ (C7a), 144.0 (PzB3), 143.8 (PzA3), 
141.8 (PzC3), 137.6 (Pz5), 136.7 (Pz5), 136.3 (PzA5), 107.1 (PzC4), 106.6 (PzC4), 106.3 (PzA4), 92.6 (C7), 54.4 (C6), 52.9 (C5), 50.1 (C2), 42.5 (N-Ethyl $\mathrm{CH}_{2}$ ), 39.1 (C4), 37.5 (C3a), $31.2(\mathrm{C} 3), 13.3\left(\mathrm{~d}, J=28.0, \mathrm{PMe}_{3}\right), 12.0\left(\mathrm{~N}-\mathrm{Ethyl} \mathrm{CH}_{3}\right) .{ }^{31} \mathrm{P} \mathrm{NMR}\left(d-\mathrm{CDCl}_{3}, \delta\right)$ : $10.5\left(J_{\mathrm{wp}}=282\right)$. IR: $v_{\mathrm{BH}}=2509 \mathrm{~cm}^{-1}, v_{\mathrm{NO}}=1533 \mathrm{~cm}^{-1}(\mathrm{DMA}): \mathrm{E}_{\mathrm{p}, \mathrm{a}}=0.60 \mathrm{~V}$.

Compound 82: In a vial, Compound 81 (0.050 g, $0.076 \mathrm{mmol})$ was dissolved in $\mathrm{MeCN}$ ( $1 \mathrm{~mL})$. To this solution, allyl bromide $(0.050 \mathrm{~g}, 0.413 \mathrm{mmol})$ was added and the solution stirred for $30 \mathrm{~min}$. The reaction mixture was evaporated to dryness and redissolved in minimal DCM. This solution was added dropwise to a stirring solution of $\mathrm{Et}_{2} \mathrm{O}(50 \mathrm{~mL})$ to induce precipitation of a light-tan solid. The powder was collected on a $15 \mathrm{~mL}$ fine porosity fritted funnel, washed with $\mathrm{Et}_{2} \mathrm{O}(5 \mathrm{~mL})$, yielding Compound 82 (0.036 g, 0.043 mmol, $56 \%) .{ }^{1} \mathrm{H}$ NMR $\left(d-\mathrm{CD}_{3} \mathrm{CN}, \delta\right): 8.04(\mathrm{~d}, J=2.0,1 \mathrm{H}, \mathrm{PzA} 3), 8.03(\mathrm{~d}, J=2.0,1 \mathrm{H}$, PzC3), $7.87(\mathrm{~d}, J=2.0,2 \mathrm{H}, \mathrm{PzA} 5$ and PzB5), $7.80(\mathrm{~d}, J=2.0,1 \mathrm{H}, \mathrm{PzC} 5), 7.57(\mathrm{~d}, J=$ 2.0, 1H, PzB3), 6.38 (t, $J=2.0,1 \mathrm{H}, \mathrm{PzB} 4), 6.33(\mathrm{t}, J=2.0,1 \mathrm{H}, \mathrm{PzC} 4), 6.31(\mathrm{t}, J=2.0$, 1H, PzA4),5.91 (m, 1H, H9), 5.11 (dd, $J=17.3,1.1,1 \mathrm{H}, \mathrm{H} 10 \mathrm{x}$ ), 5.06 (dd, $J=10.7,0.79$, 1H, H10y), 4.26 (t, J = 5.3, 1H, H7), 4.18 (m, 1H, N-Ethyl $\left.\mathrm{CH}_{2}\right), 3.94$ (m, 2H, H2), 3.84 (m, 1H, N-Ethyl $\mathrm{CH}_{2}$ ), 3.64 (t, $\left.J=13.0,1 \mathrm{H}, \mathrm{H} 4 \mathrm{x}\right), 3.50$ (m, 1H, H3a), 2.90 (dd, $J=14.0$, 6.6, 1H, H4y), 2.72 (tt, $J=10.5,2.8,1 \mathrm{H}, \mathrm{H} 5), 2.59$ (m, 2H, H8), 2.47 (m, 1H, H3x), 1.91 (m, 1H, H3y), 1.42 (m, 1H, H6), 1.36 (t, $J=7.26,3 \mathrm{H}, \mathrm{N}-$ Ethyl $\left.\mathrm{CH}_{3}\right), 1.17$ (d, $J=8.4,9 \mathrm{H}$, $\mathrm{PMe}_{3}$ ). ${ }^{13} \mathrm{C}$ NMR (d- $\left.\mathrm{CD}_{3} \mathrm{CN}, \delta\right): 200.1$ (C7a), 144.7 (PzA3), 143.3 (PzC3), 142.6 (PzB3), 138.5 (Pz5), 137.5 (Pz5), 137.4 (Pz5),135.5 (C9), 119.2 (C10), 107.6 (PzB4), 107.2 (Pz4), 107.1 (Pz4), $57.4(\mathrm{C} 2), 52.2$ (d, $J=12.8, \mathrm{C} 5), 48.8$ (C6), 48.3 (C3a), 46.6 (N-Ethyl $\mathrm{CH}_{2}$ ), 45.5 (C8), 42.4 (C7), 30.7 (d, $J=3.8$, C4), 29.3 (C3), 13.4 (d, $J=28.5$, 
$\left.\mathrm{PMe}_{3}\right), 13.0\left(\mathrm{~N}-\mathrm{Ethyl} \mathrm{CH}_{3}\right) .{ }^{31} \mathrm{P}$ NMR $(\mathrm{DME}, \delta):-10.7\left(J_{\mathrm{wp}}=264\right) . \mathrm{IR}: v_{\mathrm{BH}}=2494 \mathrm{~cm}^{-1}$, $v_{\mathrm{NO}}$ and $v_{\text {iminium }}=1650 \mathrm{~cm}^{-1}$ and $1532 \mathrm{~cm}^{-1}(\mathrm{DMA}): \mathrm{E}_{\mathrm{p}, \mathrm{a}}=0.67 \mathrm{~V}$. Compound 83: In a vial, Compound 80 (0.031 g, $0.039 \mathrm{mmol})$ was dissolved in $\mathrm{MeOH}(1 \mathrm{~mL})$. To this solution, $\mathrm{NaCNBH}_{3}(7 \mathrm{mg}, 0.111 \mathrm{mmol})$ was added and the solution stirred for $10 \mathrm{~min}$. The reaction was quenched with $2 \mathrm{~mL}$ distilled $\mathrm{H}_{2} \mathrm{O}$ and extracted once with DCM (5 mL). The organic layer was dried with $\mathrm{Na}_{2} \mathrm{SO}_{4}$, filtered and concentrated to dryness to a yellow oil, yielding Compound 83 (0.017 g, 0.026 mmol, $67 \%) .{ }^{1} \mathrm{H}$ NMR $\left(d-\mathrm{CDCl}_{3}, \delta\right)$ : $8.08(\mathrm{~d}, J=2.0,1 \mathrm{H}, \mathrm{PzA} 3), 8.04(\mathrm{~d}, J=2.0,1 \mathrm{H}, \mathrm{PzB} 3), 7.89(\mathrm{~d}, J=2.0,1 \mathrm{H}, \mathrm{PzB} 5), 7.81$ $(\mathrm{d}, J=2.0,1 \mathrm{H}, \mathrm{PzC} 5), 7.77(\mathrm{~d}, J=2.0,1 \mathrm{H}, \mathrm{PzA} 5), 7.39$ (d, $J=2.0,1 \mathrm{H}, \mathrm{PzC} 3), 6.39(\mathrm{t}, J$ $=2.0,1 \mathrm{H}, \mathrm{PzB} 4), 6.31(\mathrm{t}, J=2.0,1 \mathrm{H}, \mathrm{PzA} 4), 6.25(\mathrm{t}, J=2.0,1 \mathrm{H}, \mathrm{PzC} 4), 3.67(\mathrm{~m}, 1 \mathrm{H}$, H2x), 3.68 (buried, 1H, H7a), 3.42 (m, 1H, N-Ethyl $\mathrm{CH}_{2}$ ), 2.93 (m, 1H, N-Ethyl $\mathrm{CH}_{2}$ ), 2.88 (m, 1H, H2y), 2.80 (m, 1H, H3a), 2.70 (m, 2H, H7), 2.60 (m, 1H, H5), 2.33 (m, 1H, H6), 2.28 (m, 1H, H4x), 2.18 (m, 1H, H4y), 1.27 (t, $\left.J=7.2,3 \mathrm{H}, \mathrm{N}-\mathrm{Ethyl} \mathrm{CH}_{3}\right), 1.22$ (d, $J$ $\left.=8.5,9 \mathrm{H}, \mathrm{PMe}_{3}\right), 1.01(\mathrm{~m}, 2 \mathrm{H}, \mathrm{H} 3) .{ }^{13} \mathrm{C} \mathrm{NMR}\left(d-\mathrm{CDCl}_{3}, \delta\right): 145.1(\mathrm{PzB} 3), 144.2(\mathrm{PzA} 3)$, 142.3( PzC3), 137.9 (PzC5), 137.3 (PzB5), 136.6 (PzA5), 107.5 (PzB4), 106.9 (PzA4), 106.8 (PzC4), 72.3 (C7a), 53.7 (C2), 50.2 (d, $J=13.0, \mathrm{C} 5), 49.4\left(\mathrm{~N}-\mathrm{Ethyl}_{\mathrm{CH}}\right.$ ), 44.9 (C3), 39.3 (C3a), 30.6 (C6), 28.6 (C4), 27.9 (C7), 13.7 (d, $J=28.1, \mathrm{PMe}_{3}$ ), 10.7 (N-Ethyl $\left.\mathrm{CH}_{3}\right) .{ }^{31} \mathrm{P}$ NMR $\left(d-\mathrm{CDCl}_{3}, \delta\right):-11.5\left(J_{\mathrm{wp}}=279\right) . \mathrm{IR}: \mathrm{v}_{\mathrm{BH}}=2486 \mathrm{~cm}^{-1}, v_{\mathrm{NO}}=1543 \mathrm{~cm}^{-1}$ $(D M A): E_{p, a}=0.43 \mathrm{~V}$.

Compound 84: To an oven dried vial, DME $(15 \mathrm{~mL})$ was added and stirred. A solution of Compound $82(0.255 \mathrm{~g}, 0.329 \mathrm{mmol})$ in DCM (2 mL) was added to the DME. To this stirring solution, lithium aluminum hydride was added $(0.040 \mathrm{~g}, 1.05 \mathrm{mmol})$ and stirred 
for 15 min. The reaction was quenched with $\mathrm{H}_{2} \mathrm{O}(5 \mathrm{~mL})$ and the mixture was removed from the glovebox and was diluted with DCM $(20 \mathrm{~mL})$. This was treated with 2 x $30 \mathrm{~mL}$ of $\mathrm{Na}_{2} \mathrm{CO}_{3}$ (saturated, aq). The aqueous layer was back extracted with DCM (1 x 15 $\mathrm{mL})$, and the combined organic layers were washed deionized water $(10 \mathrm{~mL})$. This was then dried over anhydrous $\mathrm{MgSO}_{4}$ and concentrated in vacuo to a yellow oil, yielding Compound 85 (0.163 g, $0.234 \mathrm{mmol}, 72 \%) .{ }^{1} \mathrm{H}$ NMR $\left(d-\mathrm{CD}_{3} \mathrm{CN}, \delta\right): 8.19$ (d, $J=2.0$, 1H, PzA3), 8.00 (d, $J=2.0,1 \mathrm{H}, \mathrm{PzB} 3), 7.83$ (d, $J=2.0,1 \mathrm{H}, \mathrm{Pz} 5), 7.82$ (d, $J=2.0,1 \mathrm{H}$, Pz5), 7.79 (d, $J=2.0,1 \mathrm{H}, \mathrm{Pz} 5), 7.38$ (d, $J=2.0,1 \mathrm{H}, \mathrm{PzC} 3), 6.34$ (t, $J=2.0,1 \mathrm{H}, \mathrm{PzB} 4)$, 6.24 (m, 2H, PzA4 \& PzC4), 5.92 (m, 1H, H9), 4.86 (dt, $J=10.7,1.8,1 \mathrm{H}, \mathrm{H} 10 \mathrm{x}), 4.81$ (dq, $J=7.4,1.6,1 \mathrm{H}, \mathrm{H} 10 \mathrm{y}), 3.31$ (m, 1H, H4x), 3.19 (m, 1H, H7a), 3.06 (m, 1H, H2x), 2.98 (m, 1H, H4y), 2.95 (m, 1H, N-Ethyl $\mathrm{CH}_{2}$ ), 2.82 (m, 1H, H5), 2.47 (m, 1H, H8x), 2.46 (m, 1H, H2y), 2.36 (m, 1H, N-Ethyl $\mathrm{CH}_{2}$ ), 2.14 (m, 1H, H3a), 2.08 (dd, $J=13.0$, 4.9, 1H, H8y), 2.04 (m, 1H, H7), 1.76 (m, 1H, H3x), 1.31 (m, 1H, H3y), 1.13 (d, $J=8.3$, 9H, $\left.\mathrm{PMe}_{3}\right), 1.04\left(\mathrm{t}, J=6.9,3 \mathrm{H}, \mathrm{N}-\mathrm{Ethyl} \mathrm{CH}_{3}\right), 0.82(\mathrm{dt}, J=11.2,2.7,1 \mathrm{H}, \mathrm{H} 6) .{ }^{13} \mathrm{C} \mathrm{NMR}$ (d- $\mathrm{CD}_{3} \mathrm{CN}, \delta$ ): 143.9 (PzB3), 142.9 (PzA3), 141.5 (PzC3), 138.2 (C9), 139.9 (Pz5), 137.6 (Pz5), 137.3 (Pz5), 116.0 (C10), 107.3 (PzB4), 106.9 (Pz4), 106.3 (Pz4), 73.9 (C7), 57.3 (C6), 56.1 (d, $J=11.2, \mathrm{C} 5), 53.7$ (C2), 51.8 (N-Ethyl $\mathrm{CH}_{2}$ ), 47.8 (C7a), 42.6 (C8), 41.4 (C3a), 39.1 (d, $J=3.8, \mathrm{C} 4$ ), 30.7 (C3), 14.4 (N-Ethyl $\mathrm{CH}_{3}$ ), 13.3 (d, $J=28.0, \mathrm{PMe}_{3}$ ). ${ }^{31} \mathrm{P}$ NMR $\left(d-\mathrm{CD}_{3} \mathrm{CN}, \delta\right):-8.03\left(J_{\mathrm{wp}}=287\right) . \mathrm{IR}: v_{\mathrm{BH}}=2483 \mathrm{~cm}^{-1}, v_{\mathrm{NO}}=1530 \mathrm{~cm}^{-1}$ (DMA): $\mathrm{E}_{\mathrm{p}, \mathrm{a}}=0.31 \mathrm{~V}$.

Compound 93: To an oven dried vial, DME ( $5 \mathrm{~mL})$ was added and stirred. A solution of Compound $86(0.072 \mathrm{~g}, 0.082 \mathrm{mmol})$ in DCM $(1 \mathrm{~mL})$ was added to the DME. To this 
stirring solution, lithium aluminum hydride was added $(0.017 \mathrm{~g}, 0.448 \mathrm{mmol})$ and stirred for $5 \mathrm{~min}$. The reaction was quenched with $\mathrm{H}_{2} \mathrm{O}(2 \mathrm{~mL})$ and the mixture was removed from the glovebox and was diluted with DCM $(10 \mathrm{~mL})$. This was treated with $2 \times 20 \mathrm{~mL}$ of $\mathrm{Na} 2 \mathrm{CO} 3$ (saturated, aq). The aqueous layer was back extracted with DCM (1 x 10 $\mathrm{mL}$ ), and the combined organic layers were washed deionized water (20 $\mathrm{mL})$. This was then dried over anhydrous $\mathrm{MgSO}_{4}$ and concentrated in vacuo to a yellow oil, yielding Compound $93(0.048 \mathrm{~g}, 0.066 \mathrm{mmol}, 81 \%) .{ }^{1} \mathrm{H} \mathrm{NMR}\left(d-\mathrm{CDCl}_{3}, \delta\right): 9.19(\mathrm{~d}, J=2.0,1 \mathrm{H}$, PzA3), 8.10 (d, $J=2.0,1 \mathrm{H}, \mathrm{PzB} 3), 7.71(\mathrm{~d}, J=2.0,1 \mathrm{H}, \mathrm{PzC} 5), 7.65(\mathrm{~d}, J=2.0,1 \mathrm{H}$, PzB5), $7.50(\mathrm{~d}, J=2.0,1 \mathrm{H}, \mathrm{PzA} 5), 7.22(\mathrm{~d}, J=2.0,1 \mathrm{H}, \mathrm{PzC} 3), 6.75(\mathrm{~d}, J=3.0,1 \mathrm{H}$, H4'), 6.58 (m, 1H, H3'), 6.25 (t, $J=2.0,1 \mathrm{H}, \mathrm{PzB} 4), 6.20$ (t, $J=2.0,1 \mathrm{H}, \mathrm{PzC} 4), 6.01(\mathrm{t}, J$ $=2.0,1 \mathrm{H}, \mathrm{PzA} 4), 4.30(\mathrm{t}, J=7.6,1 \mathrm{H}, \mathrm{H} 4), 4.10(\mathrm{dt}, J=11.5,3.7,1 \mathrm{H}, \mathrm{H} 1), 3.00$ (ddd, $J=$ 16.4, 12.0, 2.5, 1H, H3), 2.48 (s, 3H, 2’Me), 2.15 (m, 1H, H5x), 1.94 (m, 1H, H6x), 1.88 (s, 6H, N-(CH3) 2$), 1.77$ (m, 1H, H6y), $1.76(\mathrm{~m}, 1 \mathrm{H}, \mathrm{H} 2), 1.61$ (m, 1H, H5y), 0.93 (d, J= 8.2, 9H, $\left.\mathrm{PMe}_{3}\right) .{ }^{13} \mathrm{C} \mathrm{NMR}\left(d-\mathrm{CDCl}_{3}, \delta\right): 150.1$ (PzA3), 142.7 (PzB3), 140.0 (PzC3), 136.4 (PzC5), 135.5 (PzB5), 135.3 (PzA5), 124.3 (C3’), 122.5 (C4'), 106.3 (PzB4), 105.8 (PzC4), 104.4 (PzA4), 71.9 (C2' or C5'), $71.4(\mathrm{C} 1), 59.2$ (C2' or $\mathrm{C}^{\prime}$ '), 58.4 (d, $J=11.6$, C3), $56.3(\mathrm{C} 2), 44.1\left(\mathrm{~N}-\left(\mathrm{CH}_{3}\right)_{2}\right), 40.3(\mathrm{~d}, J=4.0, \mathrm{C} 4), 36.9$ (C5), $26.6(\mathrm{C} 6), 15.7$ (2’Me), $13.8\left(\mathrm{~d}, J=27.0, \mathrm{PMe}_{3}\right) .{ }^{31} \mathrm{P} \mathrm{NMR}\left(d-\mathrm{CDCl}_{3}, \delta\right):-10.6\left(J_{\mathrm{wp}}=270\right) . \mathrm{IR}: \mathrm{v}_{\mathrm{BH}}=2360 \mathrm{~cm}^{-1}$, $v_{\mathrm{NO}}=1559 \mathrm{~cm}^{-1}$

Compound 94: To an oven dried vial, DME $(10 \mathrm{~mL})$ was added and stirred. A solution of Compound $87(0.081 \mathrm{~g}, 0.095 \mathrm{mmol})$ in DCM $(1 \mathrm{~mL})$ was added to the DME. To this stirring solution, lithium aluminum hydride was added $(0.020 \mathrm{~g}, 0.527 \mathrm{mmol})$ and stirred 
for $5 \mathrm{~min}$. The reaction was quenched with $\mathrm{H}_{2} \mathrm{O}(5 \mathrm{~mL})$ and the mixture was removed from the glovebox and was diluted with DCM $(10 \mathrm{~mL})$. This was treated with $2 \times 20 \mathrm{~mL}$ of $\mathrm{Na} 2 \mathrm{CO} 3$ (saturated, aq). The aqueous layer was back extracted with DCM (1 x 10 $\mathrm{mL})$, and the combined organic layers were washed deionized water $(20 \mathrm{~mL})$. This was then dried over anhydrous $\mathrm{MgSO}_{4}$ and concentrated in vacuo to a yellow oil, yielding Compound 94 (0.066 g, $0.090 \mathrm{mmol}, 96 \%) .{ }^{1} \mathrm{H} \mathrm{NMR}\left(d-\mathrm{CDCl}_{3}, \delta\right): 9.20(\mathrm{~d}, J=2.0,1 \mathrm{H}$, PzA3), 8.10 (d, $J=2.0,1 \mathrm{H}, \mathrm{PzB} 3), 7.70(\mathrm{~d}, J=2.0,1 \mathrm{H}, \mathrm{PzC} 5), 7.65(\mathrm{~d}, J=2.0,1 \mathrm{H}$, PzB5), 7.49 (d, $J=2.0,1 \mathrm{H}, \mathrm{PzA} 5), 7.28(\mathrm{~d}, J=2.0,1 \mathrm{H}, \mathrm{PzC} 3), 6.25(\mathrm{t}, J=2.0,1 \mathrm{H}$, PzB4), 6.19 (t, $J=2.0,1 \mathrm{H}, \mathrm{PzC} 4), 6.03(\mathrm{~d}, J=2.89,1 \mathrm{H}, \mathrm{H} 3$ '), $6.01(\mathrm{t}, J=2.0,1 \mathrm{H}$, PzA4), 5.90 (dd, $J=2.9,1.0,1 \mathrm{H}, \mathrm{H} 4$ '), 4.10 (m, 2H, H1 \& H4), 3.06 (ddd, $J=16.9,12.5$, 2.8, 1H, H3), 2.33 (s, 3H, 5'-Me), 2.05 (m, 1H, H5x), 1.96 (m, 1H, H6x), 1.88 (broad, 6H, N-( $\left.\left(\mathrm{CH}_{3}\right)_{2}\right), 1.73$ (buried, 1H, H2), 1.71 (m, 1H, H6y), 1.68 (m, 1H, H5y), 0.95 (d, J= 8.2, 9H, $\left.\mathrm{PMe}_{3}\right) .{ }^{13} \mathrm{C} \mathrm{NMR}_{(}\left(d-\mathrm{CDCl}_{3}, \delta\right): 163.7$ (PzA3), 150.1 (C2' or C5'), 149.5 (C2' or C5'), 142.8 (PzB3), 140.1 (PzC3), 136.4 (Pz5), 135.5 (Pz5), 135.3 (Pz5), 106.3 (Pz4), 105.9 (C3' or C4'), 105.7 (Pz4), 104.7 (C3' or C4'), 104.4 (Pz4), 70.8 (C1), 55.6 (C2), $54.7(\mathrm{~d}, J=12.0, \mathrm{C} 3), 44.1\left(\mathrm{~N}-\left(\mathrm{CH}_{3}\right)_{2}\right), 38.1(\mathrm{~d}, J=3.9, \mathrm{C} 4), 31.8(\mathrm{C} 5), 26.4$ (C6), 13.9 (5'-Me), $13.4\left(\mathrm{~d}, J=27.4, \mathrm{PMe}_{3}\right) \cdot{ }^{31} \mathrm{P} \mathrm{NMR}\left(d-\mathrm{CDCl}_{3}, \delta\right):-10.1\left(J_{\mathrm{wp}}=271\right) .(\mathrm{DMA}): \mathrm{E}_{\mathrm{p}, \mathrm{a}}$ $=0.29 \mathrm{~V}$.

Compound 95: To an oven dried vial, DME (4 mL) was added and stirred. A solution of Compound $88(0.051 \mathrm{~g}, 0.061 \mathrm{mmol})$ in DCM $(0.5 \mathrm{~mL})$ was added to the DME. To this stirring solution, lithium aluminum hydride was added $(0.013 \mathrm{~g}, 0.342 \mathrm{mmol})$ and stirred for 5 min. The reaction was quenched with $\mathrm{H}_{2} \mathrm{O}(1 \mathrm{~mL})$ and the mixture was removed 
from the glovebox and was diluted with DCM $(10 \mathrm{~mL})$. This was treated with 2 x $20 \mathrm{~mL}$ of $\mathrm{Na} 2 \mathrm{CO} 3$ (saturated, aq). The aqueous layer was back extracted with DCM (1 x 10 $\mathrm{mL})$, and the combined organic layers were washed deionized water $(20 \mathrm{~mL})$. This was then dried over anhydrous $\mathrm{MgSO}_{4}$ and concentrated in vacuo to a yellow oil, yielding Compound 95 (0.038 g, 0.055 mmol, 86\%). ${ }^{1} \mathrm{H}$ NMR $\left(d-\mathrm{CDCl}_{3}, \delta\right): 9.20$ (d, $J=2.0,1 \mathrm{H}$, PzA3), $8.12(\mathrm{~d}, J=2.0,1 \mathrm{H}, \mathrm{PzB} 3), 7.82\left(\mathrm{~d}, J=2.0,1 \mathrm{H}, \mathrm{H} 5^{\prime}\right), 7.71(\mathrm{~d}, J=2.0,1 \mathrm{H}$, PzC5), $7.67(\mathrm{~d}, J=2.0,1 \mathrm{H}, \mathrm{PzB} 5), 7.57\left(\mathrm{~d}, J=2.0,1 \mathrm{H}, \mathrm{H} 3^{\prime}\right), 7.51(\mathrm{~d}, J=2.0,1 \mathrm{H}$, PzA5), 7.28 (d, $J=2.0,1 \mathrm{H}, \mathrm{PzC} 3), 6.30$ (t, $J=2.0,1 \mathrm{H}, \mathrm{H} 4$ '), 6.27 (t, $J=2.0,1 \mathrm{H}, \mathrm{PzB} 4)$, $6.19(\mathrm{t}, J=2.0,1 \mathrm{H}, \mathrm{PzC} 4), 6.03(\mathrm{t}, J=2.0,1 \mathrm{H}, \mathrm{PzA} 4), 5.56(\mathrm{t}, J=6.9,1 \mathrm{H}, \mathrm{H} 4), 4.16(\mathrm{dt}$, $J=11.4,3.7,1 \mathrm{H}, \mathrm{H} 1), 3.11(\mathrm{~m}, 1 \mathrm{H}, \mathrm{H} 3), 2.30$ (m, 1H, H5x), 1.99 (m, 1H, H6x), 1.91 (s, 6H, N-(CH$\left.)_{2}\right), 1.86$ (m, 1H, H5y), 1.80 (m, 1H, H2), 1.79 (m, 1H, H6y), 0.92 (d, J = 8.19, 9H, $\left.\mathrm{PMe}_{3}\right) .{ }^{13} \mathrm{C} \mathrm{NMR}\left(d-\mathrm{CDCl}_{3}, \delta\right): 150.1$ (PzA3), 142.7 (PzB3), 140.0 (PzC3), 138.3 (C3'), 136.5 (PzC5), 135.7 (PzB5), 135.4 (PzA5), 127.6 (C5'), 106.5 (PzB4), 105.9 (PzC4), 104.9 (C4’), 104.5 (PzA4), 70.0 (C1), 62.9 (d, $J=4.0, \mathrm{C} 4), 54.6$ (C2), 54.4 (d, $J$ $=12.3, \mathrm{C} 3), 44.1\left(\mathrm{~N}-\left(\mathrm{CH}_{3}\right)_{2}\right), 32.3(\mathrm{C} 5), 25.3(\mathrm{C} 6), 13.1\left(\mathrm{~d}, J=27.6, \mathrm{PMe}_{3}\right) .{ }^{31} \mathrm{P} \mathrm{NMR}(d-$ $\left.\mathrm{CDCl}_{3}, \delta\right):-11.8\left(J_{\mathrm{wp}}=266\right)$.

Compound 96: Outside of the glovebox, in a flame dried round bottom flask, $\mathrm{LiAlH}_{4}$ (0.050g, $1.32 \mathrm{mmol})$ was added to a stirring mixture of Compound $92(0.218 \mathrm{~g}, 0.255$ $\mathrm{mmol})$ in $\mathrm{Et}_{2} \mathrm{O}(120 \mathrm{~mL})$. After $10 \mathrm{~min}$, the grey, heterogeneous solution was quenched with $10 \mathrm{~mL} \mathrm{H}_{2}$. The solution was diluted with DCM $(100 \mathrm{~mL})$ and washed twice with $30 \mathrm{~mL}$ of $\mathrm{Na}_{2} \mathrm{CO}_{3}$ (saturated, aq). The combined aqueous layers were back extracted with DCM $(2 \times 50 \mathrm{~mL})$. The resulting organic fractions were washed $1 \times 40 \mathrm{~mL}$ water and 
dried over anhydrous $\mathrm{MgSO}_{4}$. Concentration of the solution in vacuo produced a yellow power of Compound 96 (0.168 g, $0.237 \mathrm{mmol}, 93 \%) .{ }^{1} \mathrm{H} \mathrm{NMR}\left(d-\mathrm{CDCl}_{3}, \delta\right): 9.38(\mathrm{~d}, J$ $=2.0,1 \mathrm{H}, \mathrm{PzA} 3), 8.12(\mathrm{~d}, J=2.0,1 \mathrm{H}, \mathrm{PzB} 3), 7.83\left(\mathrm{~d}, J=2.0,1 \mathrm{H}, \mathrm{H} 3{ }^{\prime}\right), 7.71(\mathrm{~d}, J=2.0$, 1H, Pz5), 7.68 (d, $J=2.0,1 \mathrm{H}, \mathrm{Pz} 5), 7.53(\mathrm{~d}, J=2.0,1 \mathrm{H}, \mathrm{H} 5$ '), $7.51(\mathrm{~d}, J=2.0,1 \mathrm{H}, \mathrm{Pz} 5)$, $7.31(\mathrm{~d}, J=2.0,1 \mathrm{H}, \mathrm{PzC} 3), 6.33\left(\mathrm{t}, J=2.0,1 \mathrm{H}, \mathrm{H} 4{ }^{\prime}\right), 6.27(\mathrm{t}, J=2.0,1 \mathrm{H}, \mathrm{PzB} 4), 6.17$ (t, $J=2.0,1 \mathrm{H}, \mathrm{PzC} 4), 6.04(\mathrm{t}, J=2.0,1 \mathrm{H}, \mathrm{PzA} 4), 5.53(\mathrm{~m}, 1 \mathrm{H}, \mathrm{H} 4), 4.44(\mathrm{~m}, 1 \mathrm{H}, \mathrm{H} 1), 3.00$ (m, 1H, H3), 2.57 (m, 1H, H5), 2.05 (buried, 1H, H6x), 1.99 (broad s, $\left.\left(\mathrm{N}_{-} \mathrm{CH}_{3}\right)_{2}\right), 1.81$ (dd, $J=11.6,3.8,1 \mathrm{H}, \mathrm{H6y}), 1.57$ (m, 1H, H2), 0.98 (d, $\left.J=8.1,9 \mathrm{H}, \mathrm{PMe}_{3}\right), 0.79$ (d, $J=$ 7.1, 3H, 4-Me). ${ }^{13} \mathrm{C}$ NMR ( $\left.d-\mathrm{CDCl}_{3}, \delta\right): 150.1$ (PzA3), 142.8 (PzB3), 140.2 (PzC3), 137.3 (C3'), 136.6 (Pz5), 135.6 (Pz5), 135.3 (Pz5), 128.6 (C5'), 105.0 (C4’) 106.4 (Pz4), 105.8 (Pz4), 104.6 (Pz4), 67.5 (d, $J=3.9, \mathrm{C} 4), 55.2(\mathrm{~d}, J=11.5, \mathrm{C} 3), 52.4(\mathrm{C} 2), 44.1$ $\left(\left(\mathrm{N}-\mathrm{CH}_{3}\right)_{2}\right), 32.6(\mathrm{C} 1), 31.0(\mathrm{C} 6), 18.9(\mathrm{C} 5), 15.3(4-\mathrm{Me}), 13.0\left(\mathrm{~d}, J=27.6, \mathrm{PMe}_{3}\right) .{ }^{31} \mathrm{P}$ $\operatorname{NMR}\left(d-\mathrm{CDCl}_{3}, \delta\right):-8.91\left(J_{\mathrm{wp}}=274\right) . \mathrm{IR}: v_{\mathrm{BH}}=2362 \mathrm{~cm}^{-1}, v_{\mathrm{NO}}=1556 \mathrm{~cm}^{-1}$.

Compound 97: To an oven dried $100 \mathrm{~mL}$ round bottom flask, DME (5 mL) was added and stirred. A solution of Compound $89(0.070 \mathrm{~g}, 0.076 \mathrm{mmol})$ in DCM (1 mL) was added to the DME. To this stirring solution, lithium aluminum hydride was added $(0.016$ $\mathrm{g}, 0.421 \mathrm{mmol})$ and stirred for $5 \mathrm{~min}$. The reaction was quenched with $\mathrm{H}_{2} \mathrm{O}(5 \mathrm{~mL})$ and the mixture was removed from the glovebox and was diluted with DCM (10 mL). This was treated with $2 \times 20 \mathrm{~mL}$ of $\mathrm{Na} 2 \mathrm{CO} 3$ (saturated, aq). The aqueous layer was back extracted with DCM (1 x $20 \mathrm{~mL})$, and the combined organic layers were washed deionized water $(20 \mathrm{~mL})$. This was then dried over anhydrous $\mathrm{MgSO}_{4}$ and concentrated in vacuo to a yellow oil, yielding Compound 97 (0.046 g, $0.060 \mathrm{mmol}, 78 \%) .{ }^{1} \mathrm{H}$ NMR 
$\left(d-\mathrm{CDCl}_{3}, \delta\right): 9.26(\mathrm{~d}, J=2.0,1 \mathrm{H}, \mathrm{PzA} 3), 8.13(\mathrm{~d}, J=2.0,1 \mathrm{H}, \mathrm{PzB} 3), 7.70(\mathrm{~d}, J=2.0$, 1H, PzB5), 7.67 (d, $J=8.6,1 \mathrm{H}, \mathrm{H} 5$ '), $7.64(\mathrm{~d}, J=2.0,1 \mathrm{H}, \mathrm{PzC} 5), 7.51(\mathrm{~d}, J=2.0,1 \mathrm{H}$, PzA5), 7.20 (d, $J=2.0,1 \mathrm{H}, \mathrm{PzC} 3), 6.66$ (dd, $J=8.4,2.4,1 \mathrm{~h}, \mathrm{H6}$ '), 6.44 (d, $J=2.3,1 \mathrm{H}$, H3'), 6.24 (t, $J=2.0,1 \mathrm{H}, \mathrm{PzB} 4), 6.17$ (t, $J=2.0,1 \mathrm{H}, \mathrm{PzC} 4), 6.02$ (t, $J=2.0,1 \mathrm{H}, \mathrm{PzA} 4)$, $4.62(\mathrm{t}, J=8.4,1 \mathrm{H}, \mathrm{H} 4), 4.11(\mathrm{~m}, 1 \mathrm{H}, \mathrm{H} 1), 3.80$ (s, 3H, OMe), 3.79 (s, 3H, OMe), 2.94 (m, 1H, H3), $\left.2.10(\mathrm{~m}, 1 \mathrm{H}, \mathrm{H} 6 \mathrm{x}), 1.92\left(\operatorname{broad}, 6 \mathrm{H},\left(\mathrm{N}-\mathrm{CH}_{3}\right)_{2}\right)\right), 1.90(\mathrm{~m}, 1 \mathrm{H}, \mathrm{H} 2), 1.89$ (m, 2H, H5), 1.32 (m, 1H, H6y), $0.86\left(\mathrm{~d}, J=8.3,9 \mathrm{H}, \mathrm{PMe}_{3}\right) .{ }^{13} \mathrm{C} \mathrm{NMR}\left(d-\mathrm{CDCl}_{3}, \delta\right): 160.9$ (C2' or C4'), 158.2 (C2’ or C4'), 157.2 (C1'), 150.2 (PzA3), 142.6 (PzB3), 139.9 (PzC3), 136.2 (PzB5), 135.5 (Pz5), 135.4 (Pz5), 129.5 (C5'), 106.2 (PzB4), 105.7 (PzC4), 104.4 (PzA4), 97.9 (C3’), 72.5 (C1), 58.7 (d, $J=11.0, \mathrm{C} 3), 57.5$ (C2), 55.4 (OMe), 55.3 (OMe), $44.2\left(\left(\mathrm{~N}_{-} \mathrm{CH}_{3}\right)_{2} \& \mathrm{C} 5\right), 36.0$ (C6), 35.1 (d, $\left.J=4.5, \mathrm{C} 4\right), 13.7\left(\mathrm{~d}, J=27.1, \mathrm{PMe}_{3}\right)$. ${ }^{31} \mathrm{P} \mathrm{NMR}\left(d-\mathrm{CDCl}_{3}, \delta\right):-7.97\left(J_{\mathrm{wp}}=276\right)$.

Compound 98: To an oven dried $100 \mathrm{~mL}$ round bottom flask, DME (40 mL) was added and stirred. A solution of Compound 91 (0.301 g, $0.382 \mathrm{mmol})$ in DCM (3 mL) was added to the DME. To this stirring solution, lithium aluminum hydride was added $(0.074$ $\mathrm{g}, 1.95 \mathrm{mmol})$ and stirred for $5 \mathrm{~min}$. The reaction was quenched with $\mathrm{H}_{2} \mathrm{O}(10 \mathrm{~mL})$ and the mixture was removed from the glovebox and was diluted with DCM (50 mL). This was treated with $2 \times 40 \mathrm{~mL}$ of $\mathrm{Na}_{2} \mathrm{CO}_{3}$ (saturated, aq). The aqueous layer was back extracted with DCM (1 x $50 \mathrm{~mL})$, and the combined organic layers were washed deionized water $(50 \mathrm{~mL})$. This was then dried over anhydrous $\mathrm{MgSO}_{4}$ and concentrated in vacuo to a yellow oil, yielding Compound 98 (0.229 g, $0.357 \mathrm{mmol}, 94 \%) .{ }^{1} \mathrm{H}$ NMR $\left(d-\mathrm{CDCl}_{3}, \delta\right): 9.26(\mathrm{~d}, J=2.0,1 \mathrm{H}, \mathrm{PzA} 3), 8.12(\mathrm{~d}, J=2.0,1 \mathrm{H}, \mathrm{PzB} 3), 7.69(\mathrm{~d}, J=2.0$ 
1H, PzC5), $7.66(\mathrm{~d}, J=2.0,1 \mathrm{H}, \mathrm{PzB} 5), 7.48(\mathrm{~d}, J=2.0,1 \mathrm{H}, \mathrm{PzA} 5), 7.29(\mathrm{~d}, J=2.0,1 \mathrm{H}$, PzC3), $6.26(\mathrm{t}, J=2.0,1 \mathrm{H}, \mathrm{PzB} 4), 6.19(\mathrm{t}, J=2.0,1 \mathrm{H}, \mathrm{PzC} 4), 6.00(\mathrm{t}, J=2.0,1 \mathrm{H}$, PzA4), 3.82 (d, $J=10.4,1 \mathrm{H}, \mathrm{H} 1), 3.08$ (t, $J=13.0,1 \mathrm{H}, \mathrm{H} 3), 2.20$ (d, $J=11.8,1 \mathrm{H}, \mathrm{H} 6 \mathrm{x}$ ), $\left.2.04(\mathrm{t}, J=11.8,1 \mathrm{H}, \mathrm{H6y}), 1.91\left(\text { broad s, 6H, N-( } \mathrm{CH}_{3}\right)_{2}\right), 1.57(\mathrm{~d}, J=12.0,1 \mathrm{H}, \mathrm{H} 2), 1.47$ (m, 1H, H4), $1.31(\mathrm{~m}, 1 \mathrm{H}, \mathrm{H} 5), 1.11$ (d, $\left.J=8.0,9 \mathrm{H}, \mathrm{PMe}_{3}\right), 0.45$ (m, 1H, H7x), 0.44 (m, 1H, H7y). ${ }^{13} \mathrm{C}$ NMR (d-CDCl $\left.3, \delta\right): 150.1$ (PzA3), 142.7 (PzB3), 139.9 (PzC3), 136.3 (Pz5), 135.4 (Pz5), 135.0 (PzA5), 106.3 (PzB4), 105.8 (PzC4), 104.3 (PzA4), 65.9 (C1), $55.0(\mathrm{C} 2), 54.6(\mathrm{~d}, J=11.0, \mathrm{C} 3), 44.3\left(\mathrm{~N}-\left(\mathrm{CH}_{3}\right)_{2}\right), 26.7(\mathrm{C} 6), 17.2(\mathrm{~d}, J=4.8, \mathrm{C} 4), 13.2$ (C5), 12.7 (d, $\left.J=27.0, \mathrm{PMe}_{3}\right), 11.7(\mathrm{C} 7) .{ }^{31} \mathrm{P} \mathrm{NMR}\left(d-\mathrm{CDCl}_{3}, \delta\right):-10.60\left(J_{\mathrm{wp}}=270\right)$. IR: $v_{\mathrm{BH}}=2360 \mathrm{~cm}^{-1}, v_{\mathrm{NO}}=1559 \mathrm{~cm}^{-1}$.

Compound 99: To an oven dried $100 \mathrm{~mL}$ round bottom flask, DME (6 mL) was added and stirred. A solution of Compound 90 (0.084 g, $0.094 \mathrm{mmol})$ in DCM (1 mL) was added to the DME. To this stirring solution, lithium aluminum hydride was added $(0.020$ $\mathrm{g}, 0.527 \mathrm{mmol})$ and stirred for $15 \mathrm{~min}$. The reaction was quenched with $\mathrm{H}_{2} \mathrm{O}(5 \mathrm{~mL})$ and the mixture was removed from the glovebox and was diluted with DCM (10 mL). This was treated with $2 \times 20 \mathrm{~mL}$ of $\mathrm{Na}_{2} \mathrm{CO}_{3}$ (saturated, aq). The aqueous layer was back extracted with DCM (1 x $20 \mathrm{~mL})$, and the combined organic layers were washed deionized water $(20 \mathrm{~mL})$. This was then dried over anhydrous $\mathrm{MgSO}_{4}$ and concentrated in vacuo to a yellow oil, yielding Compound 99 (0.048 g, $0.076 \mathrm{mmol}, 81 \%) .{ }^{1} \mathrm{H}$ NMR $\left(d-\mathrm{CDCl}_{3}, \delta\right): 9.37(\mathrm{~d}, J=2.0,1 \mathrm{H}, \mathrm{PzA} 3), 8.11(\mathrm{~d}, J=2.0,1 \mathrm{H}, \mathrm{PzB} 3), 7.68(\mathrm{~d}, J=2.0$ 1H, PzA5), $7.66(\mathrm{~d}, J=2.0,1 \mathrm{H}, \mathrm{PzC} 5), 7.48(\mathrm{~d}, J=2.0,1 \mathrm{H}, \mathrm{PzB} 5), 7.29(\mathrm{~d}, J=2.0,1 \mathrm{H}$, PzC3), $6.25(\mathrm{t}, J=2.0,1 \mathrm{H}, \mathrm{PzB} 4), 6.18(\mathrm{t}, J=2.0,1 \mathrm{H}, \mathrm{PzC} 4), 6.03(\mathrm{t}, J=2.0,1 \mathrm{H}$, 
PzA4), 3.97 (dt, $J=11.3,3.7,1 \mathrm{H}, \mathrm{H} 1), 2.92$ (m, 1H, H4x), 2.83 (m, 1H, H3), 2.65 (m, 1H, H4y), $1.99\left(\mathrm{~N}-\left(\mathrm{CH}_{3}\right)_{2}\right), 1.98$ (buried, 1H, H5x), 1.90 (m, 1H, H6x), 1.77 (m, 1H, H2), 1.69 (m, 1H, H5y), 1.60 (m, 1H, H6y), 1.09 (d, $\left.J=8.2,9 \mathrm{H}, \mathrm{PMe}_{3}\right) .{ }^{13} \mathrm{C} \mathrm{NMR}\left(d-\mathrm{CDCl}_{3}\right.$, ઈ): 150.3 (PzA3), 142.8 (PzB3), 139.9 (PzC3), 136.4 (Pz5), 135.4 (Pz5), 135.1 (Pz5), 106.2 (Pz4), 105.7 (Pz4), 104.6 (Pz4), 71.9 (C1), 55.4 (C5), 53.1 (d, J = 11.9, C3), 44.3 $\left(\mathrm{N}-\left(\mathrm{CH}_{3}\right)_{2}\right), 27.8(\mathrm{~d}, J=4.1, \mathrm{C} 4), 26.6(\mathrm{C} 6), 24.9(\mathrm{C} 2), 13.4\left(\mathrm{~d}, J=27.0, \mathrm{PMe}_{3}\right) .{ }^{31} \mathrm{P}$ $\operatorname{NMR}\left(d-\mathrm{CDCl}_{3}, \delta\right):-8.92\left(J_{\mathrm{wp}}=281\right)$.

Compound 100: Outside of the box, $\mathrm{NOPF}_{6}(0.117 \mathrm{~g}, 0.668 \mathrm{mmol})$ was added to a vigorously stirring solution of Compound $94(0.295 \mathrm{~g}, 0.416 \mathrm{mmol})$ in acetone $(10 \mathrm{~mL})$. After $1 \mathrm{hr}$, the solution was diluted with $40 \mathrm{~mL}$ DCM and treated with $2 \times 20 \mathrm{~mL}$ of $\mathrm{Na}_{2} \mathrm{CO}_{3}$ (saturated, aq). The aqueous layer was back extracted with DCM $(2 \times 20 \mathrm{~mL})$, and the combined organic layers were washed deionized water $(30 \mathrm{~mL})$. The organic was then dried over anhydrous $\mathrm{MgSO}_{4}$ and concentrated in vacuo. The brown oil was redissolved in minimal DCM and added dropwise to a stirring solution of $\mathrm{Et}_{2} \mathrm{O}(300 \mathrm{~mL})$ to induce precipitation of a brown solid. The precipitate was collected on a $60 \mathrm{~mL}$ fine porosity fritted funnel with $2 \mathrm{~cm}$ celite. The filtrate was concentrated in vacuo to a vial. The residue was loaded onto a $20 \mathrm{~cm}$ × $20 \mathrm{~cm}$ x $1000 \mu \mathrm{m} \mathrm{Al} \mathrm{O}_{3}$ preparatory TLC place with 2 x $0.3 \mathrm{~mL}$ DCM. The plate was developed using $30 \% \mathrm{Et}_{2} \mathrm{O}$ in hexanes. A band which stained positive with $\mathrm{KMnO}_{4}$ (r.f. $\left.\approx 0.10-.43\right)$ and placed in a round bottom flask with $70 \mathrm{~mL}$ HPLC EtOAc and sonicated for 15 min to break up alumina. The slurry was filtered on a $30 \mathrm{~mL}$ fine porosity fritted funnel and washed with $50 \mathrm{~mL}$ HPLC EtOAc. The filtrate was then stripped to dryness. The oil was collected yielding Compound 100 
(0.012 g, $0.058 \mathrm{mmol}, 14 \%) .{ }^{1} \mathrm{H} \mathrm{NMR}\left(d-\mathrm{CDCl}_{3}, \delta\right): 5.88(\mathrm{~m}, 1 \mathrm{H}, \mathrm{H} 3), 5.85(\mathrm{~m}, 1 \mathrm{H}, \mathrm{H} 3$ ' or H4'), 5.84 (m, 1H, H3' or H4'), 5.78 (m, 1H, H2), 3.40 (m, 1H, H4), 3.23 (m, 1H, H1), 2.31 (s, 6H, N-(CH$\left.)_{2}\right), 2.25$ (s, 3H, 2’Me), 2.15 (m, 1H, H5x), 1.88 (m, 1H, H6x), 1.65 (m, 1H, H5y), 1.60 (m, 1H, H6y). ${ }^{13} \mathrm{C}$ NMR ( $\left.d-\mathrm{CDCl}_{3}, \delta\right): 157.9$ (C5'), 150.6 (C2'), 130.7 (C3), 130.2 (C2), 105.8 (C3' or C4'), 104.5 (C3' or C4'), 60.3 (C1), 40.9 (N$\left.\left(\mathrm{CH}_{3}\right)_{2}\right), 36.1$ (C4), 28.0 (C5), 22.3 (C6), 13.5 (2’Me).

Compound 101: Outside of the box, DDQ (0.200 g, $0.881 \mathrm{mmol})$ was added to a vigorously stirring solution of Compound 95 (0.304 g, $0.438 \mathrm{mmol})$ in $\mathrm{MeCN}(10 \mathrm{~mL})$. After $20 \mathrm{~min}$, the solution was diluted with $40 \mathrm{~mL}$ DCM and treated with $2 \times 20 \mathrm{~mL}$ of $\mathrm{Na}_{2} \mathrm{CO}_{3}$ (saturated, aq). The aqueous layer was back extracted with DCM $(2 \times 20 \mathrm{~mL})$, and the combined organic layers were washed deionized water $(30 \mathrm{~mL})$. The organic was then dried over anhydrous $\mathrm{MgSO}_{4}$ and concentrated in vacuo. The brown oil was redissolved in minimal DCM and added dropwise to a stirring solution of $\mathrm{Et}_{2} \mathrm{O}(300 \mathrm{~mL})$ to induce precipitation of a brown solid. The precipitate was collected on a $60 \mathrm{~mL}$ fine porosity fritted funnel with $2 \mathrm{~cm}$ celite. The filtrate was concentrated in vacuo to a vial. The residue was loaded onto a $20 \mathrm{~cm}$ x $20 \mathrm{~cm}$ x $1000 \mu \mathrm{m} \mathrm{Al} \mathrm{O}_{3}$ preparatory TLC place with $2 \times 0.3 \mathrm{~mL}$ DCM. The plate was developed using $\mathrm{Et}_{2} \mathrm{O}$. A band which stained positive with $\mathrm{KMnO}_{4}$ (r.f. $\approx 0.20-.43$ ) and placed in a round bottom flask with $70 \mathrm{~mL}$ HPLC EtOAc and sonicated for 15 min to break up alumina. The slurry was filtered on a $30 \mathrm{~mL}$ fine porosity fritted funnel and washed with $50 \mathrm{~mL}$ HPLC EtOAc. The filtrate was then stripped to dryness. The oil was collected yielding Compound 101 (0.010 g, 0.052 mmol, 12\%). ${ }^{1} \mathrm{H}$ NMR $\left(d-\mathrm{CDCl}_{3}, \delta\right): 7.51\left(\mathrm{~d}, J=1.6,1 \mathrm{H}, \mathrm{H} 3{ }^{\prime}\right), 7.40(\mathrm{~d}, J=2.3,1 \mathrm{H}$, 
H5'), 6.24 (t, $J=2.0,1 \mathrm{H}, \mathrm{H} 4$ '), 5.98 (m, 1H, H2), 5.89 (m, 1H, H3), 4.94 (m, 1H, H4), $3.33(\mathrm{~m}, 1 \mathrm{H}, \mathrm{H} 1), 2.32$ (m, 1H, H5x), $2.31\left(\mathrm{~s}, 6 \mathrm{H}, \mathrm{N}-\left(\mathrm{CH}_{3}\right)_{2}\right), 1.92$ (m, 1H, H6x), 1.88 (m, 1H, H5y), 1.68 (m, 1H, H6y). ${ }^{13} \mathrm{C} \mathrm{NMR}\left(d-\mathrm{CDCl}_{3}, \delta\right): 139.2(\mathrm{C} 3$ ') 133.4 (C2), 129.3 (C3), 127.1 (C5'), 105.4 (C4'), 60.0 (C1), 58.7 (C4), 40.9 (N-( $\left.\left.\mathrm{CH}_{3}\right)_{2}\right), 30.7$ (C5), 21.8 (C6).

Compound 102: Outside of the box, $\mathrm{NOPF}_{6}(0.104 \mathrm{~g}, 0.594 \mathrm{mmol})$ was added to a vigorously stirring solution of Compound 98 (0.251 g, $0.392 \mathrm{mmol})$ in acetone $(15 \mathrm{~mL})$. After $30 \mathrm{~min}$, the solution was diluted with $50 \mathrm{~mL}$ DCM and treated with $2 \times 30 \mathrm{~mL}$ of $\mathrm{Na}_{2} \mathrm{CO}_{3}$ (saturated, aq). The aqueous layer was back extracted with DCM (2 x $\left.20 \mathrm{~mL}\right)$, and the combined organic layers were washed deionized water $(30 \mathrm{~mL})$. The organic was then dried over anhydrous $\mathrm{MgSO}_{4}$ and concentrated in vacuo. The brown oil was redissolved in minimal DCM and added dropwise to a stirring solution of $\mathrm{Et}_{2} \mathrm{O}(250 \mathrm{~mL})$ to induce precipitation of a brown solid. The precipitate was collected on a $30 \mathrm{~mL}$ fine porosity fritted funnel with $2 \mathrm{~cm}$ celite. The filtrate was concentrated in vacuo. The oil was redissolved in $20 \mathrm{~mL}$ DCM and a solution of HOTf $(0.076 \mathrm{~g}, 0.506 \mathrm{mmol})$ in $1 \mathrm{~mL}$ DCM was added to the stirring reaction. The solution was concentrated in vacu. The residue was loaded onto a $20 \mathrm{~cm}$ x $20 \mathrm{~cm}$ x $1000 \mu \mathrm{m}$ silica preparatory TLC place with 2 x $0.3 \mathrm{~mL}$ DCM. The plate was developed using 15\% MeOH/DCM. A band which stained positive with $\mathrm{KMnO}_{4}$ (r.f. $\approx 0.10-.43$ ) and placed in a round bottom flask with $70 \mathrm{~mL}$ HPLC EtOAc and sonicated for 15 min to break up alumina. The slurry was filtered on a $30 \mathrm{~mL}$ fine porosity fritted funnel and washed with $50 \mathrm{~mL} \mathrm{HPLC}$ EtOAc. The filtrate was then stripped to dryness. The oil was collected yielding Compound 102 (0.020 g, 0.144 
mmol, 37\%). ${ }^{1} \mathrm{H} \mathrm{NMR}\left(d-\mathrm{CDCl}_{3}, \delta\right): 6.50(\mathrm{~m}, 1 \mathrm{H}, \mathrm{H} 3), 5.41(\mathrm{~m}, 1 \mathrm{H}, \mathrm{H} 2), 3.64(\mathrm{~m}, 1 \mathrm{H}$, H1), 2.81 (s, 6H, N-(CH$\left.)_{2}\right), 2.43$ (m, 1H, H6x), 1.83 (m, 1H, H6y), $1.43(\mathrm{~m}, 1 \mathrm{H}, \mathrm{H} 4)$, 1.39 (m, 1H, H5), 0.98 (m, 1H, H7x), 0.41 (m, 1H, H7y). $\left.{ }^{13} \mathrm{C} \mathrm{NMR} \mathrm{(d-CDCl} l_{3}, \delta\right): 138.0$ (C3), $115.3(\mathrm{C} 2), 58.4(\mathrm{C} 1), 39.8\left(\mathrm{~N}-\left(\mathrm{CH}_{3}\right)_{2}\right), 19.5$ (C6), 13.7 (C7), 9.3 (C4 or $\left.\mathrm{C} 5\right), 9.6$ (C4 or $\mathrm{C} 5)$.

Compound 104: A solution of DPhAT $(0.036 \mathrm{mg}, 0.051 \mathrm{mmol})$ in acetone $(0.5 \mathrm{~mL})$ was added to a stirring mixture of Compound $98(0.036 \mathrm{~g}, 0.112 \mathrm{mmol})$ in acetone $(1 \mathrm{~mL})$. This mixture was place in an oil bath at $35^{\circ} \mathrm{C}$ overnight. The mixture was added to $50 \mathrm{~mL}$ stirring hexanes and the tan precipitate was collected on a $15 \mathrm{~mL} \mathrm{~F}$ frit to collect Compound $104(0.043 \mathrm{~g}, 0.057 \mathrm{mmol}, 78 \%) .{ }^{1} \mathrm{H}$ NMR ( $d$-Acetone, $\left.\delta\right) 146.4(\mathrm{~d}, J=2.0$, 1H, PzA3), 8.32 (d, $J=2.0,1 \mathrm{H}, \mathrm{Pz} 5), 8.27$ (d, $J=2.0,1 \mathrm{H}, \mathrm{PzC} 3), 8.19$ (d, $J=2.0,1 \mathrm{H}$, Pz5), 8.15 (d, $J=2.0,1 \mathrm{H}, \mathrm{Pz} 5), 6.93(\mathrm{~d}, J=2.0,1 \mathrm{H}, \mathrm{PzB} 3), 6.68$ (t, $J=7.0,1 \mathrm{H}, \mathrm{H} 1)$, 6.62 (m, 2H, PzA4 \& PzC4), 6.38 (t, $J=2.0,1 \mathrm{H}, \mathrm{PzB} 4), 5.09$ (dd, $J=15.7,7.7,1 \mathrm{H}, \mathrm{H} 2)$, 4.80 (m, 1H, H3), 3.87 (m, 1H, H6x), 3.60 (m, 1H, H6y), 2.63 (m, 1H, H5), 1.65 (m, 1H, $\mathrm{H} 4), 1.39$ (d, $\left.J=9.8,9 \mathrm{H}, \mathrm{PMe}_{3}\right), 1.14$ (m, 1H, H7x), 0.42 (m, 1H, H7y). ${ }^{13} \mathrm{C} \mathrm{NMR}(d-$ Acetone, 8): 149.3 (PzC3), 146.4 (PzA3), 143.5 (PzB3), 140.4 (C1), 139.6 (Pz5), 139.5 (Pz5), 139.4 (Pz5), 109.5 (Pz4), 109.1 (Pz4), 108.0 (Pz4), 100.9 (C2),71.0 (C3), 45.5 (C7), 36.0 (C5), 21.7 (C6), 12.9 (d, $\left.J=31.0, \mathrm{PMe}_{3}\right) .{ }^{31} \mathrm{P}$ NMR ( $d$-Acetone, $\left.\delta\right):-7.27\left(J_{\mathrm{wp}}\right.$ $=270)$. 


\subsection{References:}

(1) Carey, F. A.; Sundberg, R. J. Advanced Organic Chemistry Part A:

Structure and Mechanisms; Springer: New York, 2007.

(2) Strausberg, L., University of Virginia, 2013.

(3) Salomon, R. J., University of Virginia, 2010.

(4) Ripin, D. H., Evans, D.A. 2002.

(5) Myers, J. T.; Shivokevich, P. J.; Pienkos, J. A.; Sabat, M.; Myers, W. H.;

Harman, W. D. Organometallics 2015, 34, 3648.

(6) Pilli, R. A.; Rosso, G. B.; de Oliveira, M. d. C. F. Natural Product Reports 2010, 27, 1908.

(7) Pienkos, J. A.; Knisely, A. T.; Liebov, B. K.; Teran, V.; Zottig, V. E.; Sabat, M.; Myers, W. H.; Harman, W. D. Organometallics 2014, 33, 267.

(8) Pienkos, J. A.; Zottig, V. E.; Iovan, D. A.; Li, M.; Harrison, D. P.; Sabat, M.; Salomon, R. J.; Strausberg, L.; Teran, V. A.; Myers, W. H.; Harman, W. D. Organometallics 2013, 32, 691.

(9) Salomon, R. J.; Todd, M. A.; Sabat, M.; Myers, W. H.; Harman, W. D. Organometallics 2010, 29, 707.

(10) Ketamine: Use and Abuse; CRC Press: Boca Raton, FL, 2015.

(11) Łysek, R.; Schütz, C.; Vogel, P. Bioorganic \& Medicinal Chemistry Letters 2005, 15, 3071.

(12) Rajender, A.; Rao, B. V. Tetrahedron Letters 2013, 54, 2329. 
(13) Harrison, D. P.; Nichols-Nielander, A. C.; Zottig, V. E.; Strausberg, L.; Salomon, R. J.; Trindle, C. O.; Sabat, M.; Gunnoe, T. B.; Iovan, D. A.; Myers, W. H.; Harman, W. D. Organometallics 2011, 30, 2587. 
Appendix 


\section{Compound 3:}
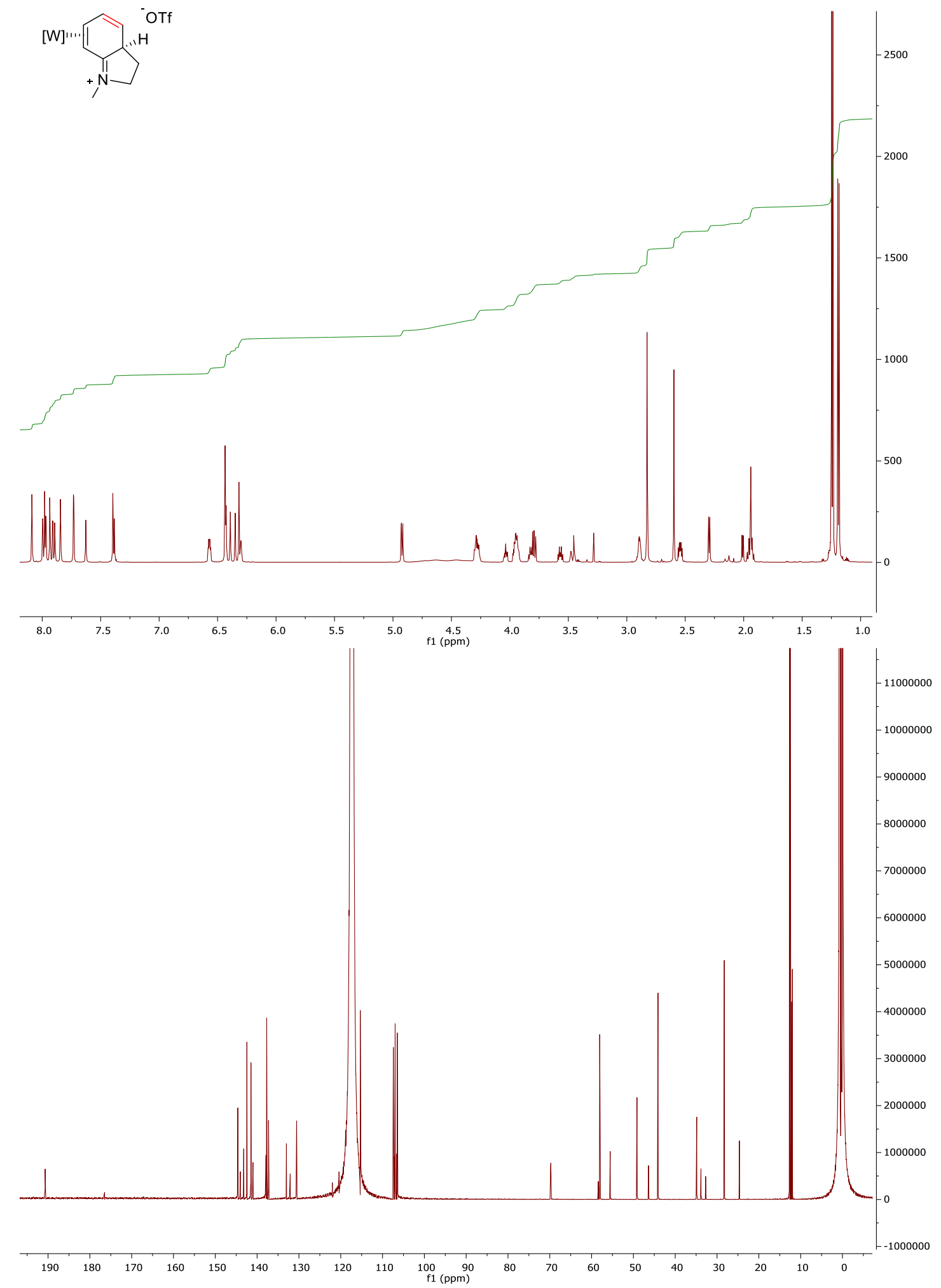
Compound 4:

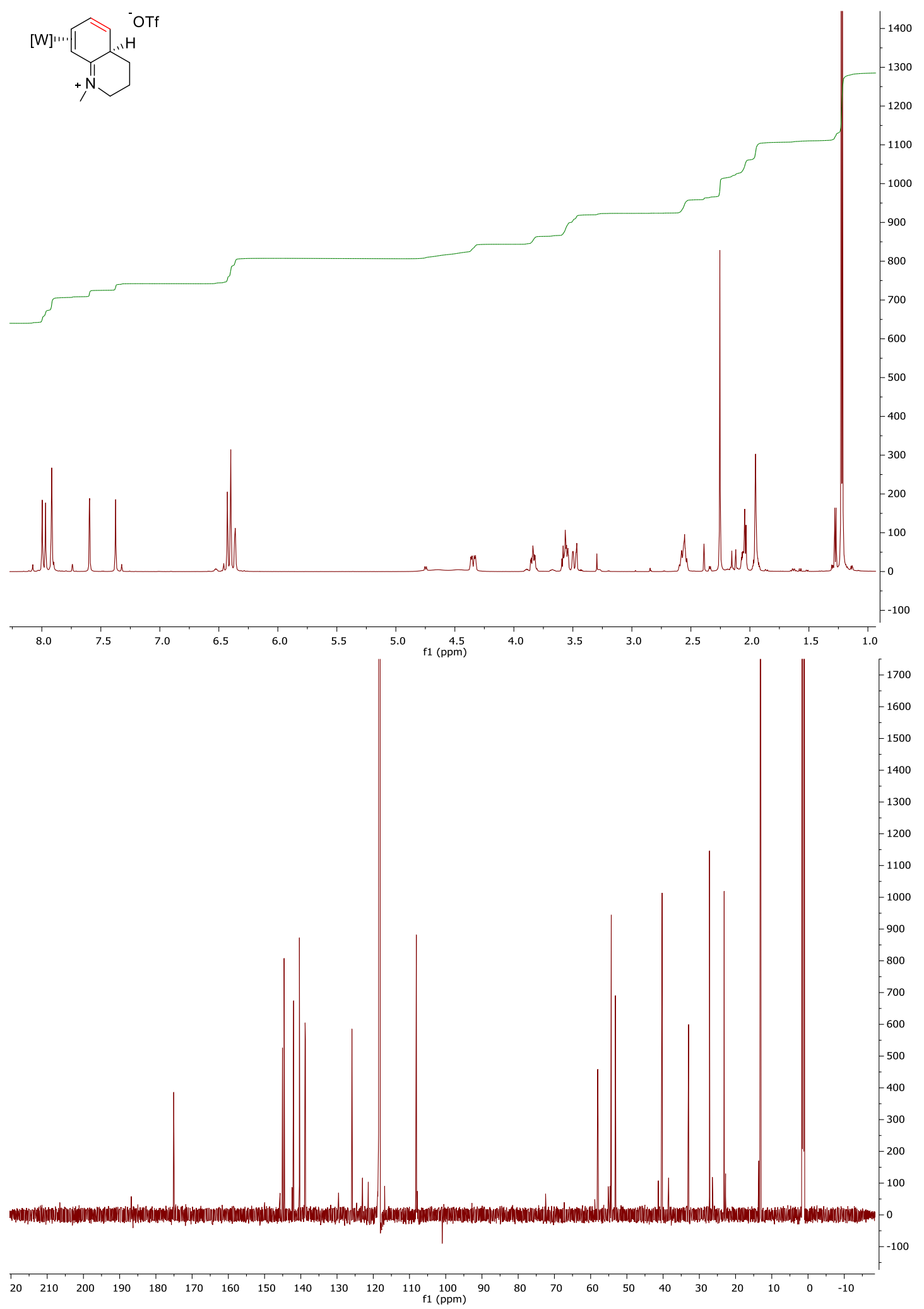


Compound 4 X-Ray Crystal Structure:

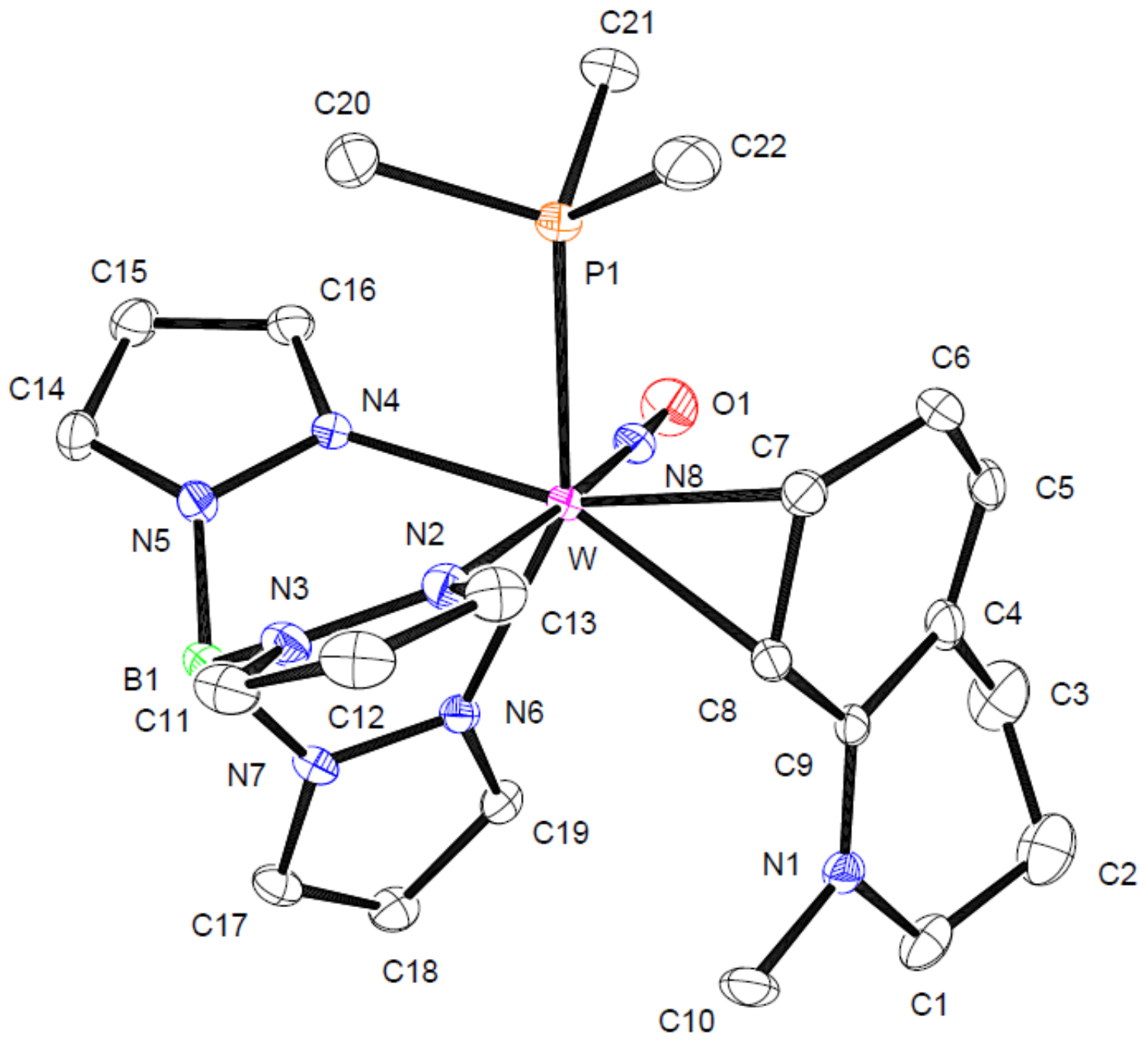


Table 1. Crystal data and structure refinement for C23H33BF3N8O4PSW.

Empirical formula

Formula weight

Temperature

Wavelength

Crystal system

Space group

Unit cell dimensions

$104.2540(5)^{\circ}$

Volume

$\mathrm{Z}$

Density (calculated)

Absorption coefficient

$\mathrm{F}(000)$

Crystal size

Theta range for data collection

Index ranges

Reflections collected

Independent reflections

Completeness to theta $=25.242^{\circ}$

Absorption correction

Refinement method

Data / restraints / parameters

Goodness-of-fit on $\mathrm{F}^{2}$

Final $R$ indices [I>2sigma(I)]

$\mathrm{R}$ indices (all data)

Largest diff. peak and hole

\section{C23 H33 B F3 N8 O4 P S W}

800.26

153(2) K

$0.71073 \AA$

Monoclinic

P 21/c

$\mathrm{a}=15.2910(5) \AA$

$\mathrm{b}=12.7130(4) \AA$

$\mathrm{c}=16.0828(5) \AA$

$3030.16(17) \AA^{3}$

4

$1.754 \mathrm{Mg} / \mathrm{m}^{3}$

$3.996 \mathrm{~mm}^{-1}$

1584

$0.320 \times 0.280 \times 0.240 \mathrm{~mm}^{3}$

3.461 to $26.370^{\circ}$.

$-19<=\mathrm{h}<=19,-15<=\mathrm{k}<=15,-20<=1<=20$

41902

$6174[\mathrm{R}($ int $)=0.0204]$

$99.7 \%$

Empirical

Full-matrix least-squares on $\mathrm{F}^{2}$

6174 / 135 / 395

1.009

$\mathrm{R} 1=0.0169, \mathrm{wR} 2=0.0435$

$\mathrm{R} 1=0.0196, \mathrm{wR} 2=0.0451$

0.915 and -0.354 e. $\AA^{-3}$ 


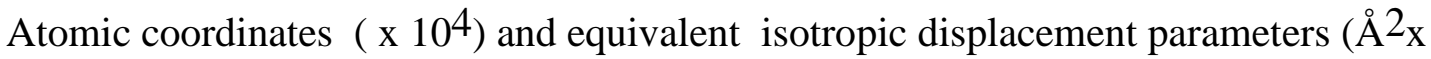
$10^{3}$ ) for $\mathrm{C} 23 \mathrm{H} 33 \mathrm{BF} 3 \mathrm{~N} 8 \mathrm{O} 4 \mathrm{PSW}$. U(eq) is defined as one third of the trace of the orthogonalized $\mathrm{U}^{\mathrm{ij}}$ tensor.

\begin{tabular}{|c|c|c|c|c|}
\hline & $\mathrm{x}$ & $\mathrm{y}$ & $\mathrm{z}$ & $\mathrm{U}(\mathrm{eq})$ \\
\hline $\mathrm{W}$ & $2595(1)$ & $2622(1)$ & $4408(1)$ & $22(1)$ \\
\hline $\mathrm{S}(1)$ & $7651(1)$ & $2636(1)$ & $2967(1)$ & $36(1)$ \\
\hline $\mathrm{P}(1)$ & $2180(1)$ & $879(1)$ & $3667(1)$ & $33(1)$ \\
\hline $\mathrm{F}(1)$ & 8101(1) & 1339(1) & $4269(1)$ & $53(1)$ \\
\hline $\mathrm{F}(2)$ & $9215(1)$ & $2060(2)$ & $3922(1)$ & $73(1)$ \\
\hline $\mathrm{F}(3)$ & $8477(1)$ & $823(1)$ & $3139(1)$ & $64(1)$ \\
\hline $\mathrm{O}(1)$ & 1016(1) & $2742(2)$ & $5191(2)$ & $50(1)$ \\
\hline $\mathrm{O}(2)$ & $7595(2)$ & $3440(2)$ & $3567(1)$ & $76(1)$ \\
\hline $\mathrm{O}(3)$ & $6839(1)$ & $2034(2)$ & $2644(1)$ & $60(1)$ \\
\hline $\mathrm{O}(4)$ & $8110(2)$ & $2937(2)$ & $2329(1)$ & $51(1)$ \\
\hline $\mathrm{N}(1)$ & $2222(1)$ & $5776(1)$ & $4116(1)$ & $28(1)$ \\
\hline $\mathrm{N}(2)$ & $3872(1)$ & $2473(1)$ & $3972(1)$ & $29(1)$ \\
\hline $\mathrm{N}(3)$ & $4677(1)$ & $2323(1)$ & $4557(1)$ & $29(1)$ \\
\hline $\mathrm{N}(4)$ & $3230(1)$ & $1554(1)$ & $5460(1)$ & $26(1)$ \\
\hline $\mathrm{N}(5)$ & $4142(1)$ & $1508(2)$ & $5770(1)$ & $28(1)$ \\
\hline $\mathrm{N}(6)$ & $3428(1)$ & $3656(1)$ & $5385(1)$ & $24(1)$ \\
\hline $\mathrm{N}(7)$ & $4321(1)$ & $3437(2)$ & $5724(1)$ & $26(1)$ \\
\hline $\mathrm{N}(8)$ & $1636(1)$ & $2703(1)$ & $4845(1)$ & $30(1)$ \\
\hline $\mathrm{C}(1)$ & $1745(2)$ & $6702(2)$ & $4351(2)$ & $44(1)$ \\
\hline $\mathrm{C}(2)$ & $763(2)$ & $6643(3)$ & $4023(2)$ & $66(1)$ \\
\hline $\mathrm{C}(3)$ & $430(2)$ & $5592(3)$ & $4157(3)$ & $67(1)$ \\
\hline $\mathrm{C}(4)$ & $869(2)$ & $4741(2)$ & $3767(2)$ & $37(1)$ \\
\hline$C(5)$ & $439(2)$ & $3950(2)$ & $3273(2)$ & $48(1)$ \\
\hline $\mathrm{C}(6)$ & $860(2)$ & $3168(2)$ & $2842(2)$ & $51(1)$ \\
\hline$C(7)$ & 1881(2) & $3133(2)$ & $3110(2)$ & $34(1)$ \\
\hline $\mathrm{C}(8)$ & $2343(2)$ & $4048(2)$ & $3562(1)$ & $27(1)$ \\
\hline $\mathrm{C}(9)$ & 1843(1) & $4881(2)$ & $3824(1)$ & $25(1)$ \\
\hline $\mathrm{C}(10)$ & $3183(2)$ & $5977(2)$ & $4211(2)$ & $42(1)$ \\
\hline
\end{tabular}




$\begin{array}{lrrrr}\mathrm{C}(11) & 5339(2) & 2222(2) & 4147(2) & 38(1) \\ \mathrm{C}(12) & 4975(2) & 2304(2) & 3284(2) & 42(1) \\ \mathrm{C}(13) & 4062(2) & 2462(2) & 3196(2) & 37(1) \\ \mathrm{C}(14) & 4342(2) & 796(2) & 6408(2) & 37(1) \\ \mathrm{C}(15) & 3551(2) & 365(2) & 6520(2) & 40(1) \\ \mathrm{C}(16) & 2875(2) & 874(2) & 5922(2) & 33(1) \\ \mathrm{C}(17) & 4666(2) & 4150(2) & 6340(1) & 33(1) \\ \mathrm{C}(18) & 3995(2) & 4840(2) & 6407(2) & 35(1) \\ \mathrm{C}(19) & 3232(2) & 4496(2) & 5808(1) & 28(1) \\ \mathrm{C}(20) & 3054(2) & -112(2) & 4012(2) & 51(1) \\ \mathrm{C}(21) & 1181(2) & 254(2) & 3848(2) & 49(1) \\ \mathrm{C}(22) & 1994(2) & 795(2) & 2510(2) & 53(1) \\ \mathrm{C}(23) & 8409(2) & 1671(2) & 3600(2) & 37(1) \\ \mathrm{B}(1) & 4735(2) & 2381(2) & 5526(2) & 30(1)\end{array}$


Compound 5:

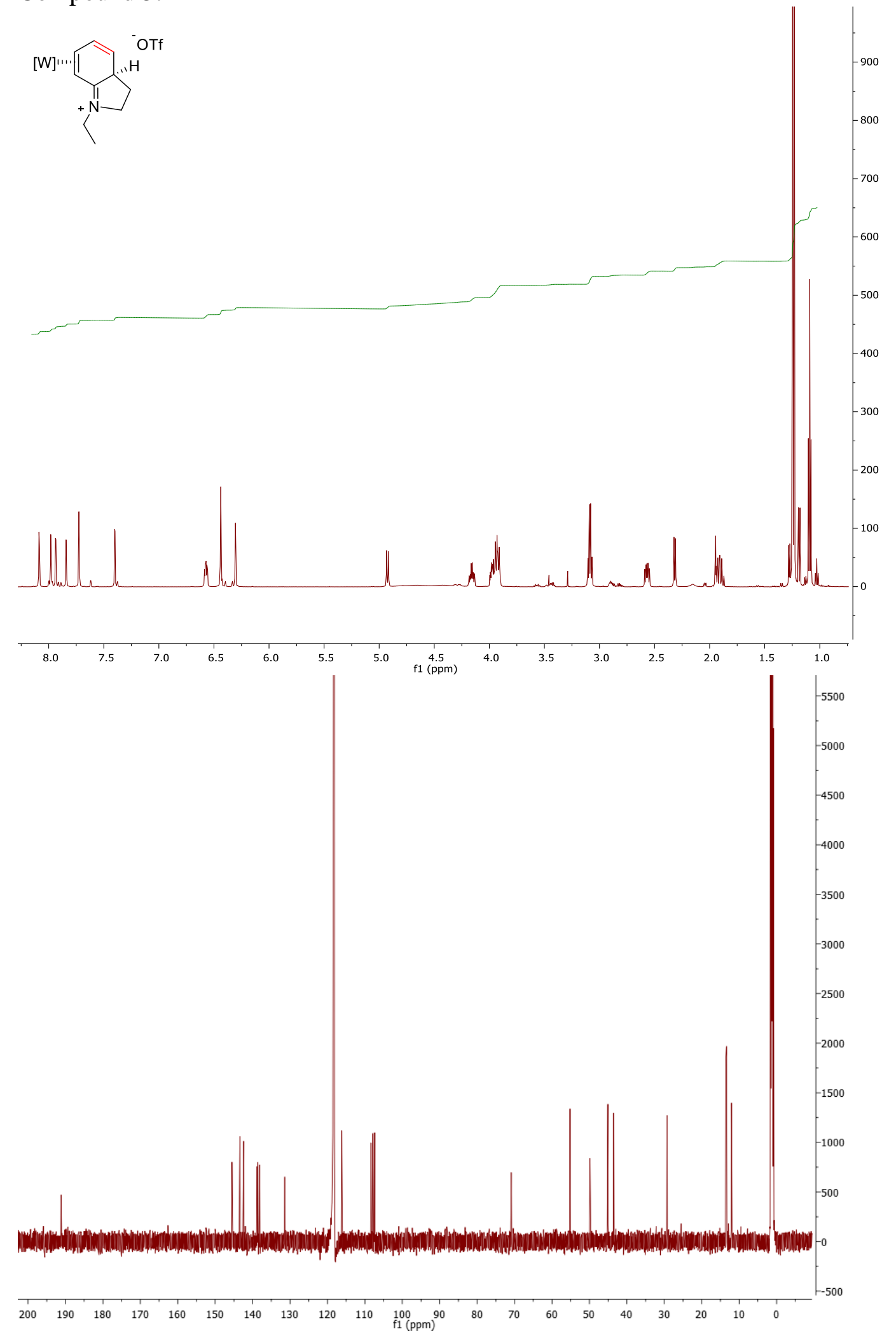


Compound 6:

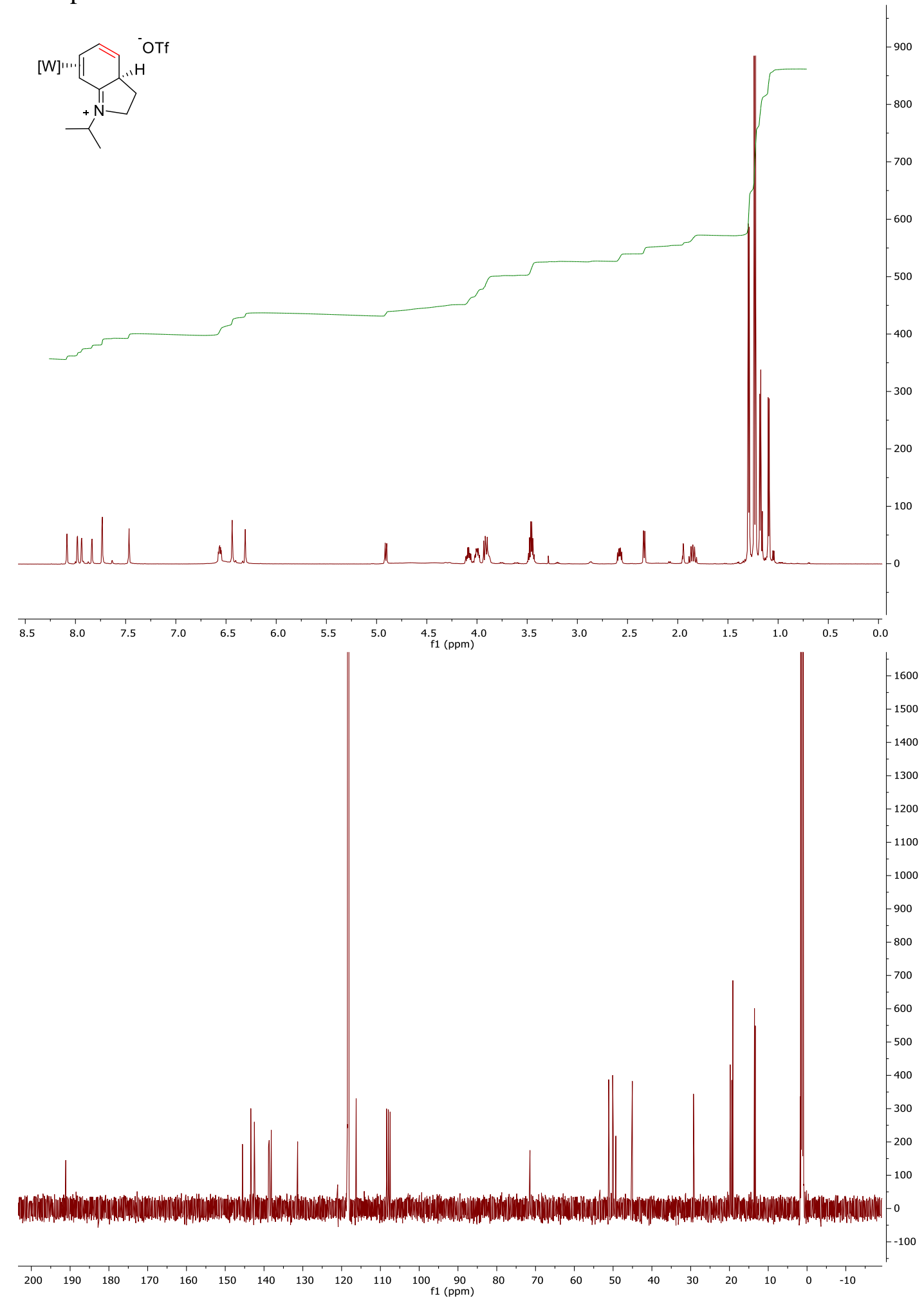


Compound 8:

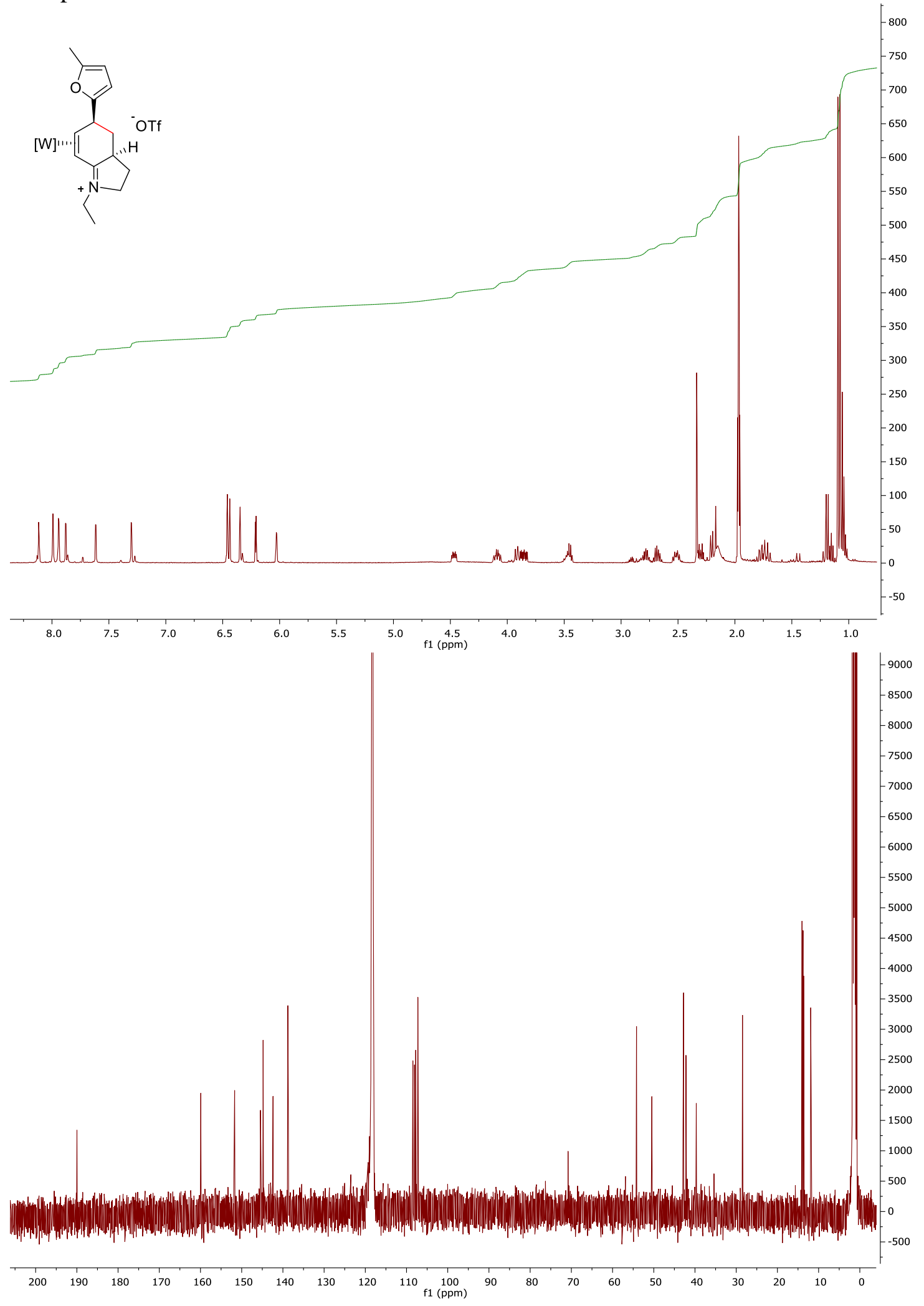




\section{Compound 9:}

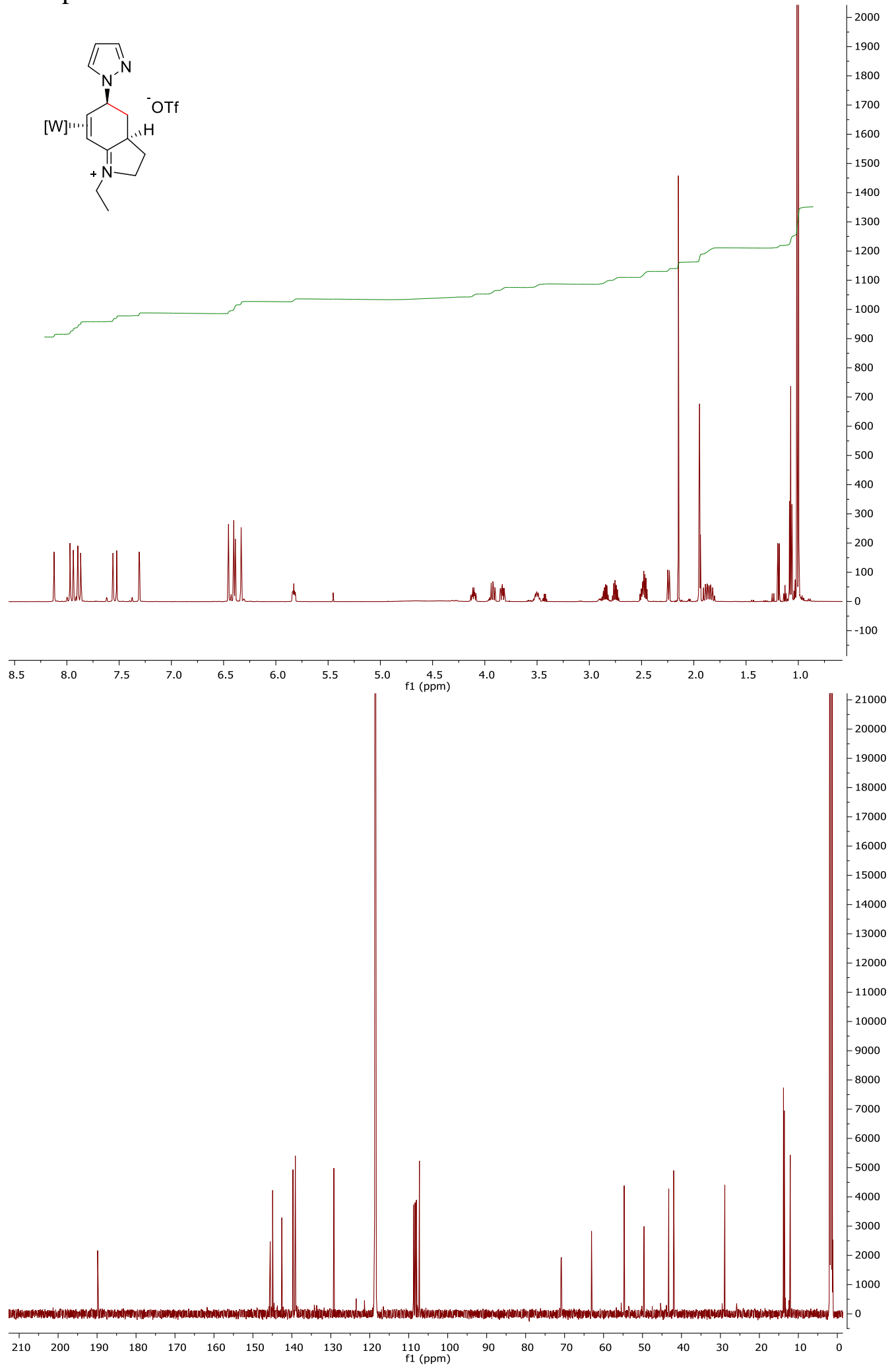


Compound 9-iPr X-ray Crystal Structure:

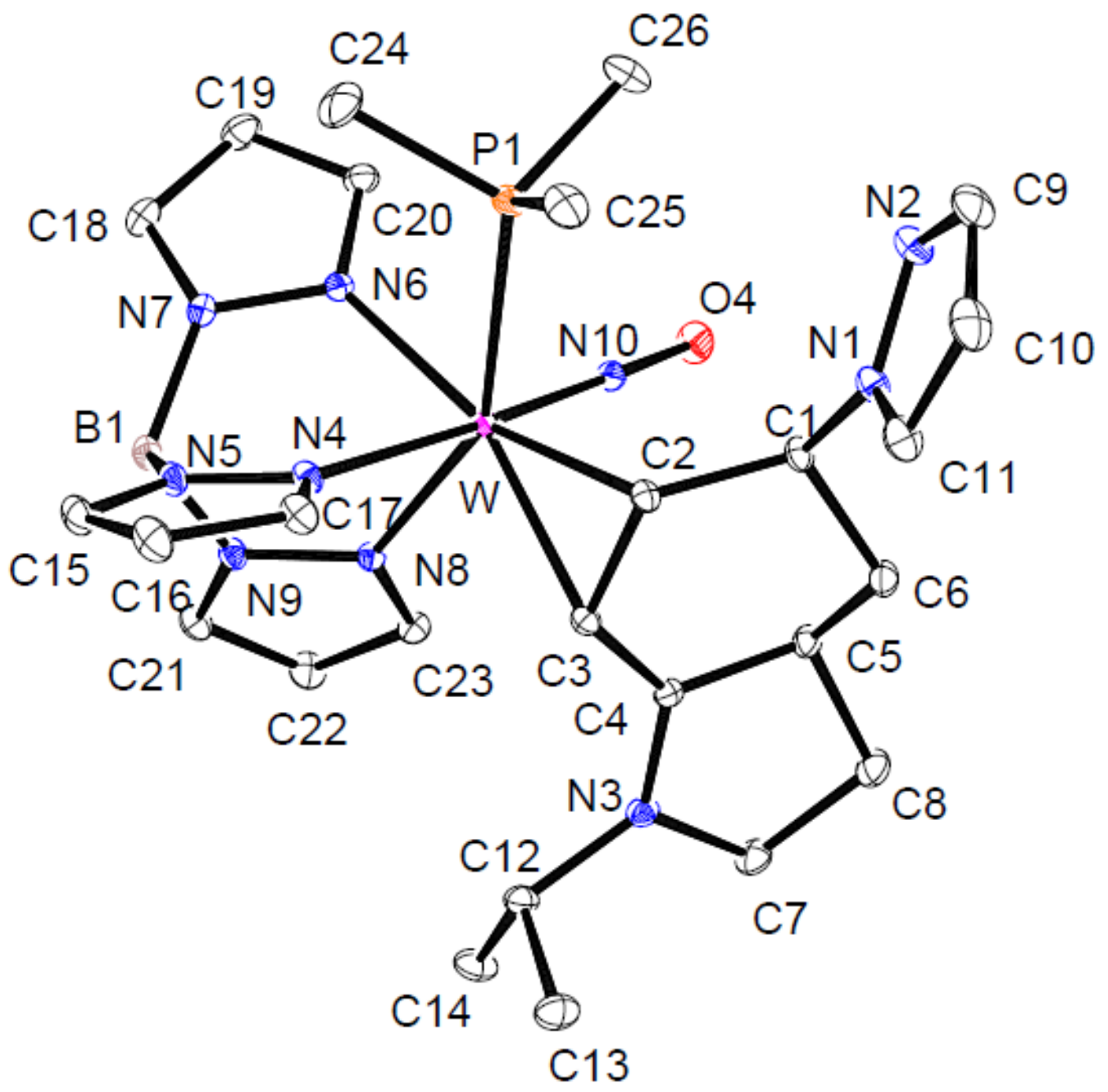


Table 1. Crystal data and structure refinement for C27H39BF3N10O4PSW.

Empirical formula

Formula weight

Temperature

Wavelength

Crystal system

Space group

Unit cell dimensions

$98.1216(5)^{\circ}$

Volume

Z

Density (calculated)

Absorption coefficient

$\mathrm{F}(000)$

Crystal size

Theta range for data collection

Index ranges

Reflections collected

Independent reflections

Completeness to theta $=25.242^{\circ}$

Absorption correction

Refinement method

Data / restraints / parameters

Goodness-of-fit on $\mathrm{F}^{2}$

Final $\mathrm{R}$ indices [I>2sigma(I)]

$\mathrm{R}$ indices (all data)

Largest diff. peak and hole

\section{C27 H39 B F3 N10 O4 P S W}

882.37

153(2) K

$0.71073 \AA$

Monoclinic

P 21/n

$a=9.7292(3) \AA$

$\mathrm{b}=23.4747(7) \AA$

$\mathrm{c}=15.2996(5) \AA$

3459.23(19) $\AA^{3}$

4

$1.694 \mathrm{Mg} / \mathrm{m}^{3}$

$3.511 \mathrm{~mm}^{-1}$

1760

$0.410 \times 0.370 \times 0.340 \mathrm{~mm}^{3}$

3.354 to $37.024^{\circ}$.

$-15<=\mathrm{h}<=15,-39<=\mathrm{k}<=38,-24<=1<=25$

84192

$16495[\mathrm{R}(\mathrm{int})=0.0153]$

$99.6 \%$

Empirical

Full-matrix least-squares on $\mathrm{F}^{2}$

16495 / 0 / 454

1.178

$\mathrm{R} 1=0.0242, \mathrm{wR} 2=0.0532$

$\mathrm{R} 1=0.0262, \mathrm{wR} 2=0.0538$

2.854 and -1.615 e. $\AA^{-3}$ 
Table 2. Atomic coordinates ( $x 10^{4}$ ) and equivalent isotropic displacement parameters $\left(\AA^{2} \times 10^{3}\right)$ for $\mathrm{C} 27 \mathrm{H} 39 \mathrm{BF} 3 \mathrm{~N} 10 \mathrm{O} 4 \mathrm{PSW}$. U(eq) is defined as one third of the trace of the orthogonalized $\mathrm{U}^{\mathrm{ij}}$ tensor.

\begin{tabular}{|c|c|c|c|c|}
\hline & $\mathrm{x}$ & $\mathrm{y}$ & $\mathrm{z}$ & $\mathrm{U}(\mathrm{eq})$ \\
\hline W & $9283(1)$ & 4139(1) & $7872(1)$ & $13(1)$ \\
\hline$S(1)$ & $-445(1)$ & $1446(1)$ & $8189(1)$ & $29(1)$ \\
\hline $\mathrm{P}(1)$ & $7172(1)$ & 3901(1) & $6762(1)$ & $21(1)$ \\
\hline $\mathrm{F}(1)$ & $2206(2)$ & $1217(1)$ & $8584(1)$ & $79(1)$ \\
\hline $\mathrm{F}(2)$ & $1682(2)$ & $1635(1)$ & $7365(1)$ & $59(1)$ \\
\hline $\mathrm{F}(3)$ & 1691(3) & $2100(1)$ & $8566(2)$ & $94(1)$ \\
\hline $\mathrm{O}(1)$ & $-594(2)$ & $886(1)$ & $7793(2)$ & $48(1)$ \\
\hline $\mathrm{O}(2)$ & $-618(2)$ & $1484(1)$ & 9111(1) & $49(1)$ \\
\hline $\mathrm{O}(3)$ & $-1107(2)$ & 1898(1) & $7635(1)$ & $47(1)$ \\
\hline $\mathrm{O}(4)$ & $7686(2)$ & $4833(1)$ & $9025(1)$ & $29(1)$ \\
\hline $\mathrm{N}(1)$ & $6342(2)$ & $2978(1)$ & $8738(1)$ & $22(1)$ \\
\hline $\mathrm{N}(2)$ & $5066(2)$ & $3187(1)$ & $8424(1)$ & $29(1)$ \\
\hline $\mathrm{N}(3)$ & $11542(2)$ & $3655(1)$ & $10267(1)$ & $20(1)$ \\
\hline $\mathrm{N}(4)$ & $10468(1)$ & $3669(1)$ & $6952(1)$ & $18(1)$ \\
\hline $\mathrm{N}(5)$ & $11360(2)$ & $3954(1)$ & 6494(1) & $19(1)$ \\
\hline $\mathrm{N}(6)$ & $9253(1)$ & $4855(1)$ & $6949(1)$ & $18(1)$ \\
\hline $\mathrm{N}(7)$ & $10269(2)$ & $4935(1)$ & $6432(1)$ & $20(1)$ \\
\hline $\mathrm{N}(8)$ & $11299(1)$ & $4554(1)$ & $8254(1)$ & $16(1)$ \\
\hline $\mathrm{N}(9)$ & $12129(1)$ & $4702(1)$ & $7639(1)$ & $19(1)$ \\
\hline $\mathrm{N}(10)$ & $8347(1)$ & $4550(1)$ & $8562(1)$ & $17(1)$ \\
\hline $\mathrm{C}(1)$ & $7465(2)$ & $3378(1)$ & $9027(1)$ & $19(1)$ \\
\hline $\mathrm{C}(2)$ & $8639(2)$ & $3338(1)$ & $8445(1)$ & $17(1)$ \\
\hline $\mathrm{C}(3)$ & $10075(2)$ & $3462(1)$ & $8856(1)$ & $16(1)$ \\
\hline $\mathrm{C}(4)$ & $10335(2)$ & $3621(1)$ & 9761(1) & $16(1)$ \\
\hline $\mathrm{C}(5)$ & $9170(2)$ & $3726(1)$ & $10292(1)$ & $20(1)$ \\
\hline$C(6)$ & $8009(2)$ & $3299(1)$ & $10009(1)$ & $22(1)$ \\
\hline $\mathrm{C}(7)$ & $11415(2)$ & 3793(1) & 11199(1) & $26(1)$ \\
\hline $\mathrm{C}(8)$ & $9885(2)$ & $3674(1)$ & $11251(1)$ & $29(1)$ \\
\hline
\end{tabular}




$\begin{array}{lrrrr}\mathrm{C}(9) & 4313(2) & 2723(1) & 8173(2) & 35(1) \\ \mathrm{C}(10) & 5088(2) & 2222(1) & 8324(2) & 34(1) \\ \mathrm{C}(11) & 6399(2) & 2403(1) & 8688(1) & 27(1) \\ \mathrm{C}(12) & 12908(2) & 3525(1) & 9992(1) & 21(1) \\ \mathrm{C}(13) & 13288(2) & 2910(1) & 10232(2) & 31(1) \\ \mathrm{C}(14) & 14025(2) & 3932(1) & 10425(2) & 29(1) \\ \mathrm{C}(15) & 11946(2) & 3586(1) & 5982(1) & 25(1) \\ \mathrm{C}(16) & 11429(2) & 3047(1) & 6102(1) & 29(1) \\ \mathrm{C}(17) & 10509(2) & 3118(1) & 6715(1) & 24(1) \\ \mathrm{C}(18) & 10021(2) & 5424(1) & 5973(1) & 27(1) \\ \mathrm{C}(19) & 8817(2) & 5666(1) & 6186(1) & 29(1) \\ \mathrm{C}(20) & 8378(2) & 5297(1) & 6805(1) & 23(1) \\ \mathrm{C}(21) & 13169(2) & 5038(1) & 8021(1) & 23(1) \\ \mathrm{C}(22) & 13025(2) & 5109(1) & 8900(1) & 25(1) \\ \mathrm{C}(23) & 11840(2) & 4798(1) & 9012(1) & 19(1) \\ \mathrm{C}(24) & 7387(3) & 4102(1) & 5635(1) & 40(1) \\ \mathrm{C}(25) & 6630(2) & 3164(1) & 6571(1) & 30(1) \\ \mathrm{C}(26) & 5595(2) & 4261(1) & 6959(2) & 35(1) \\ \mathrm{C}(27) & 1371(2) & 1608(1) & 8178(2) & 37(1) \\ \mathrm{B}(1) & 11641(2) & 4597(1) & 6646(1) & 21(1)\end{array}$




\section{Compound 10:}
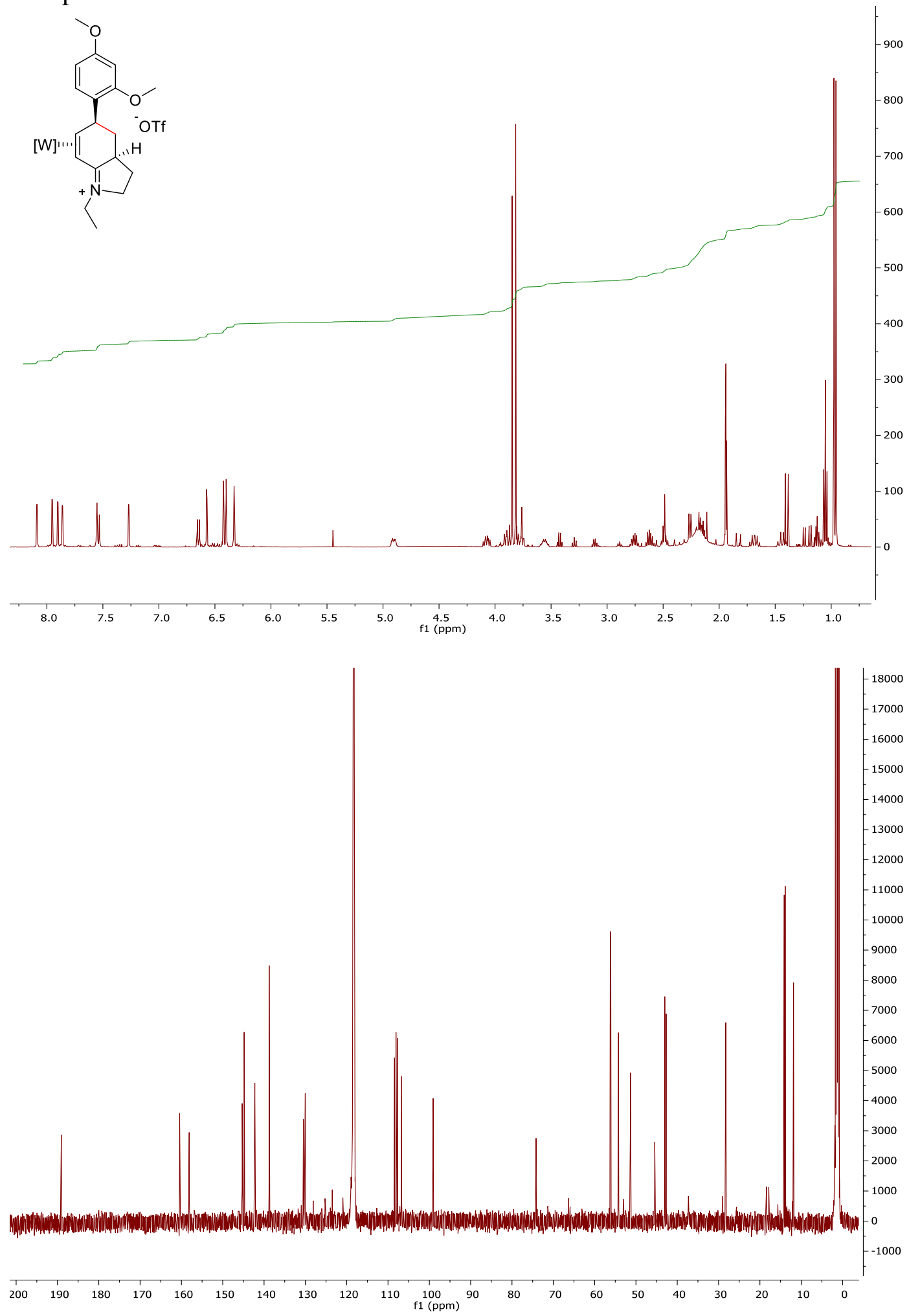
Compound 11:
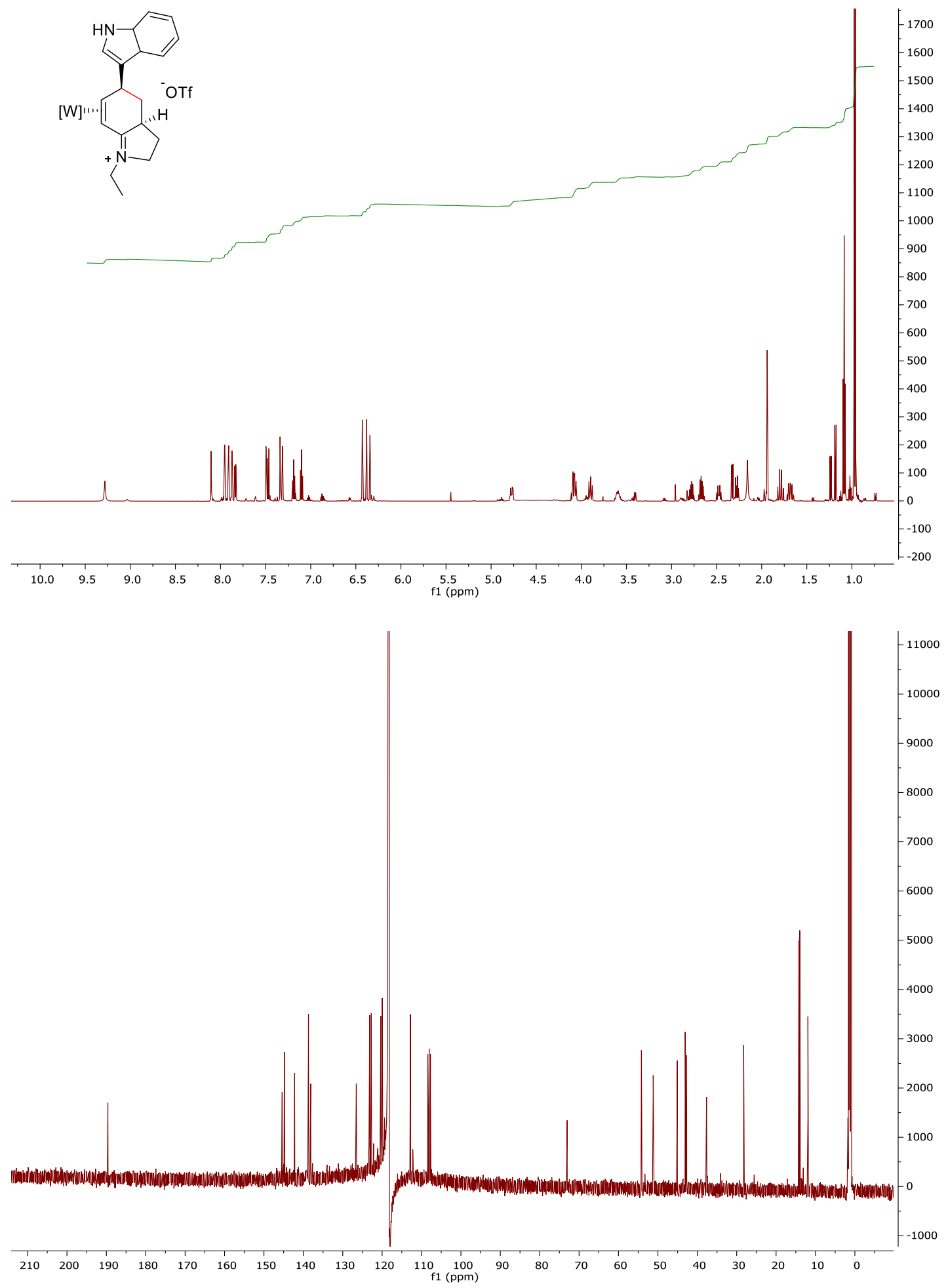
Compound 12:

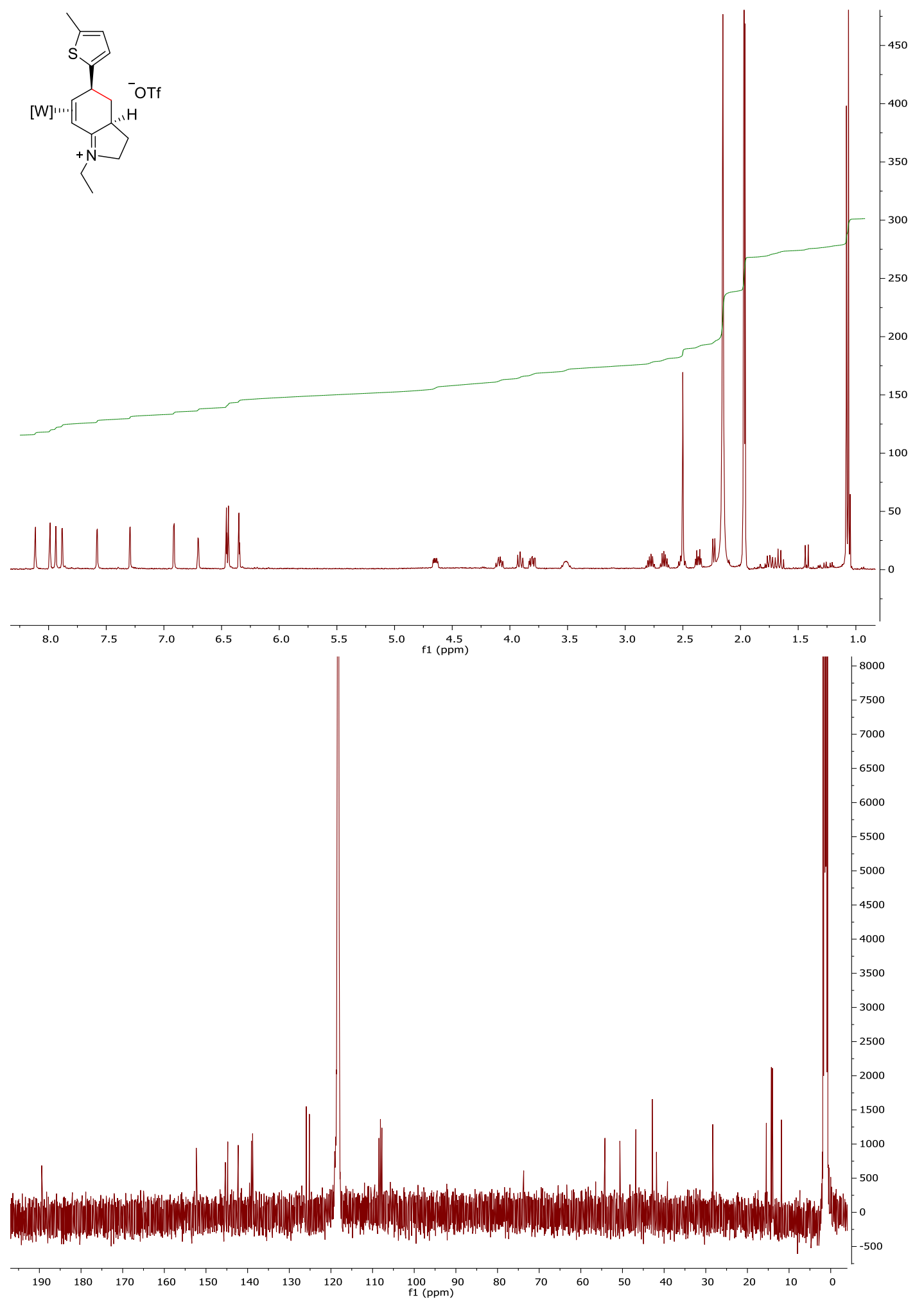


Compound 13:
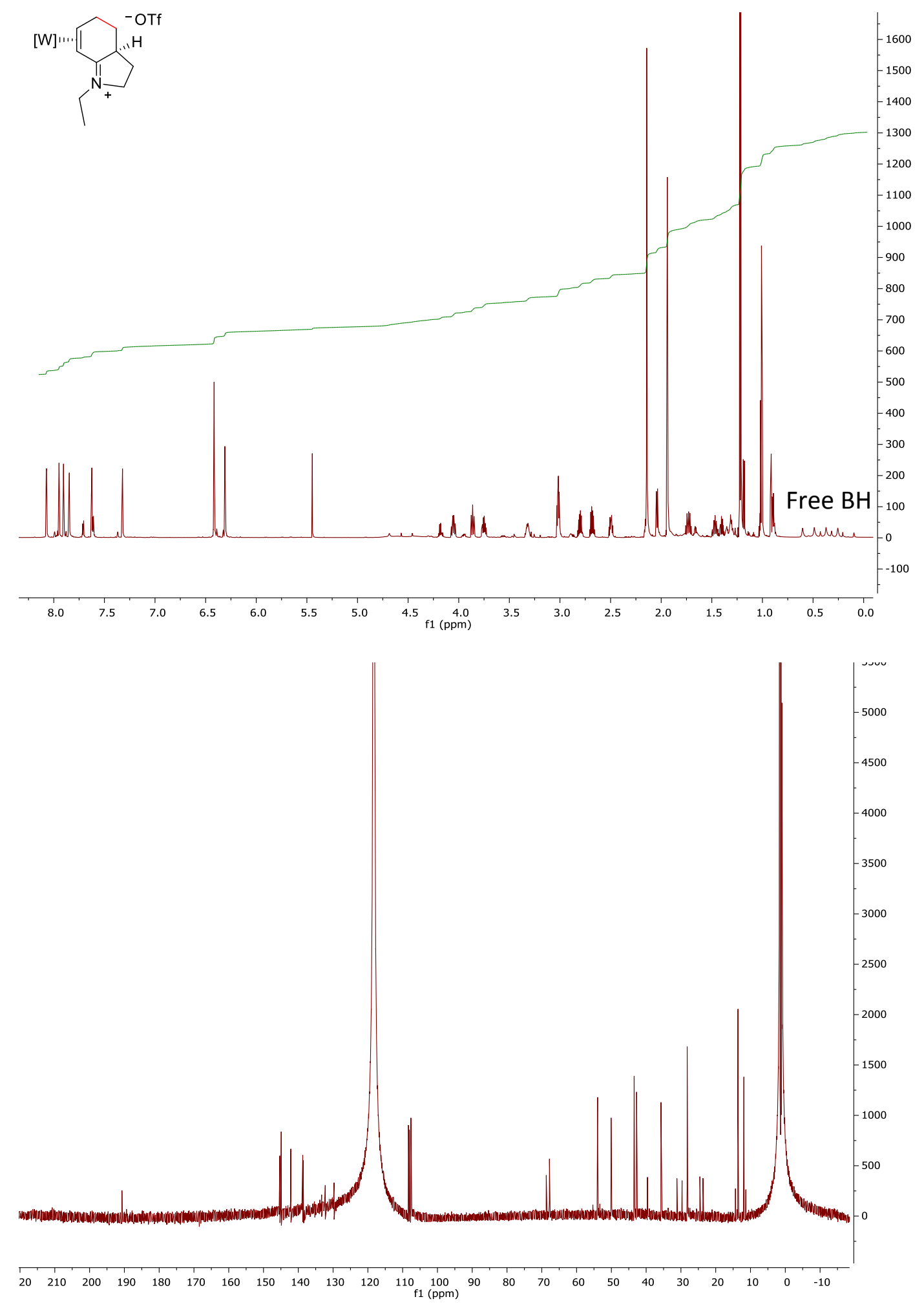


\section{Compound 14:}

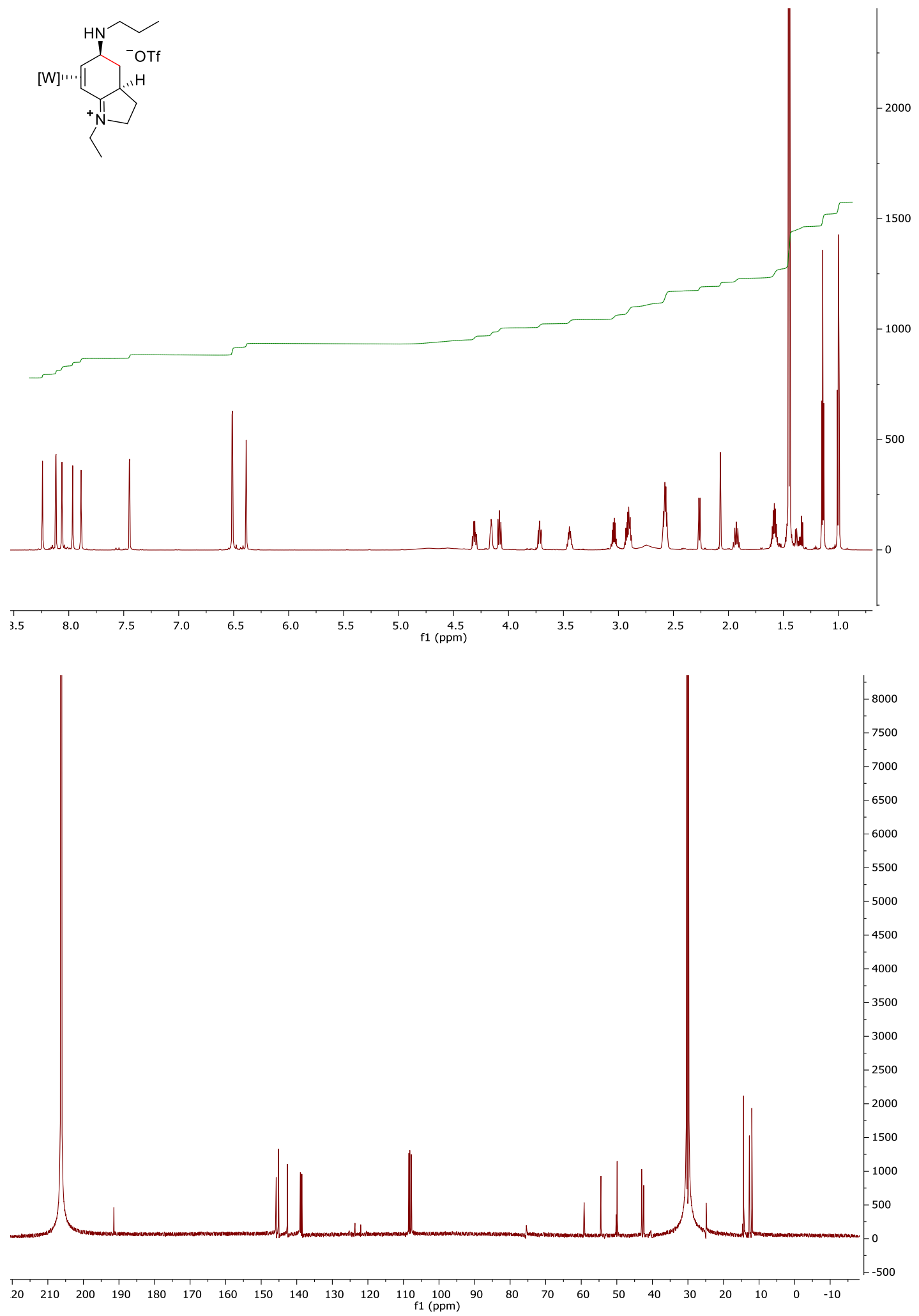


Compound 15:
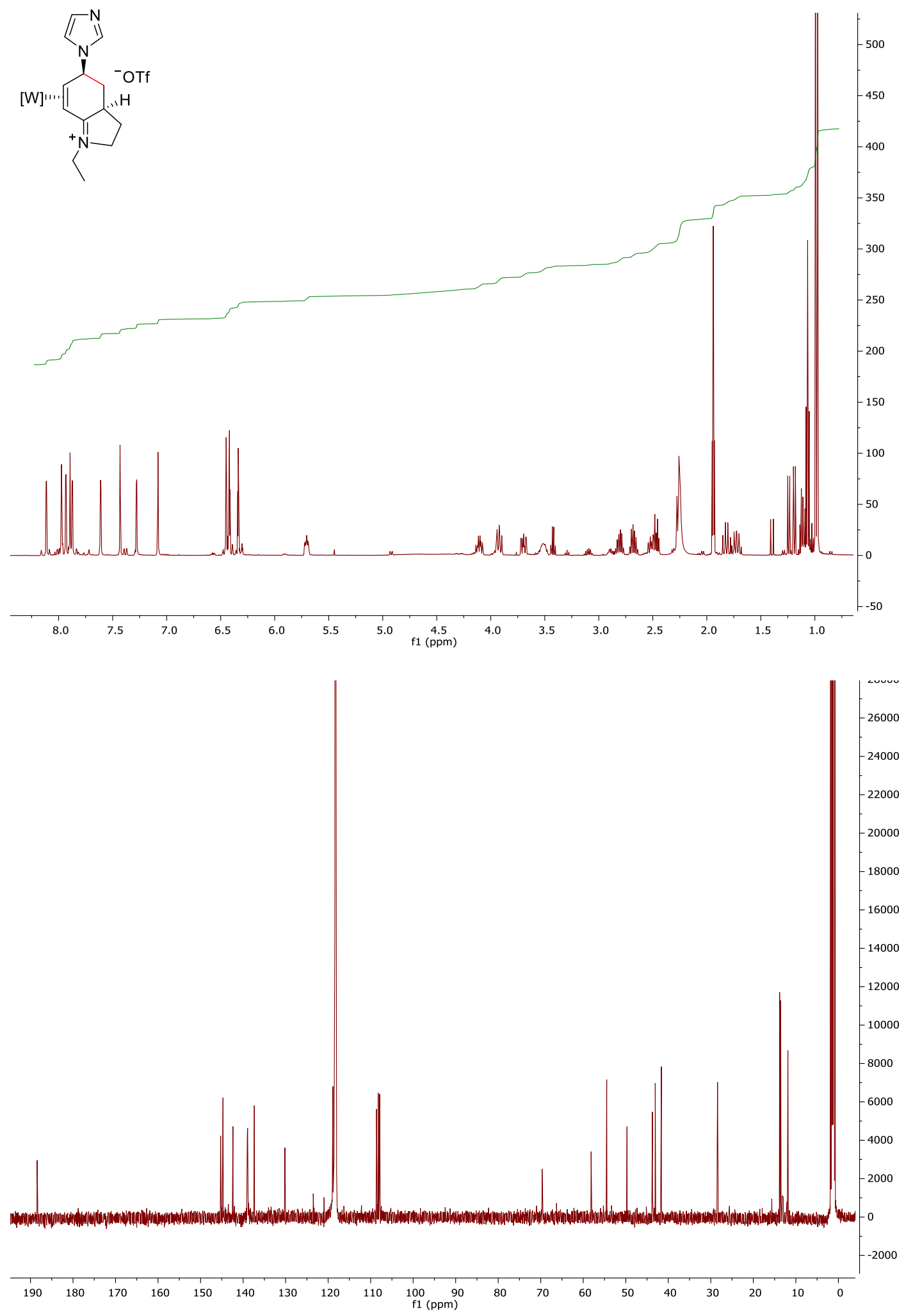
Compound 16:

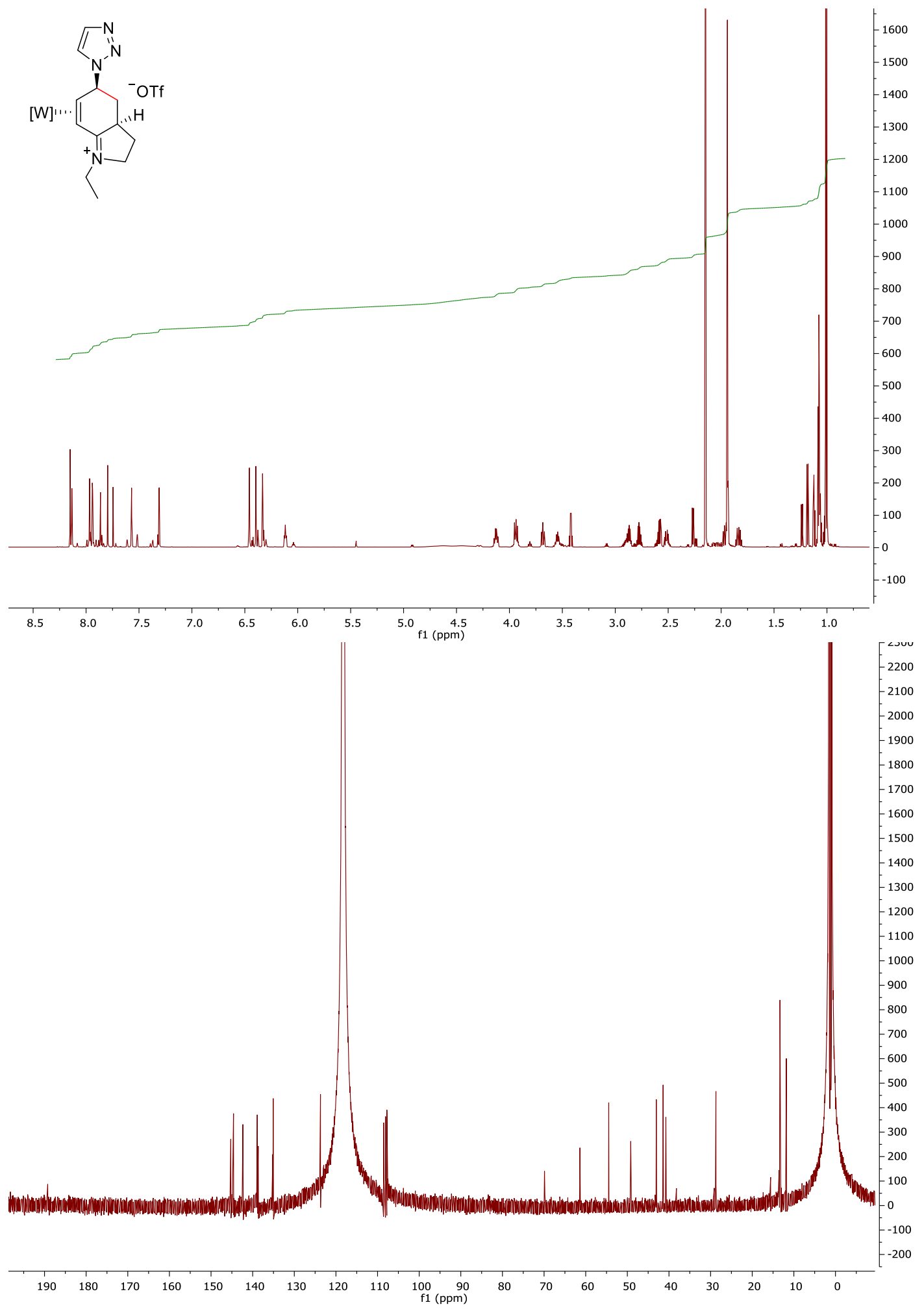




\section{Compound 17:}

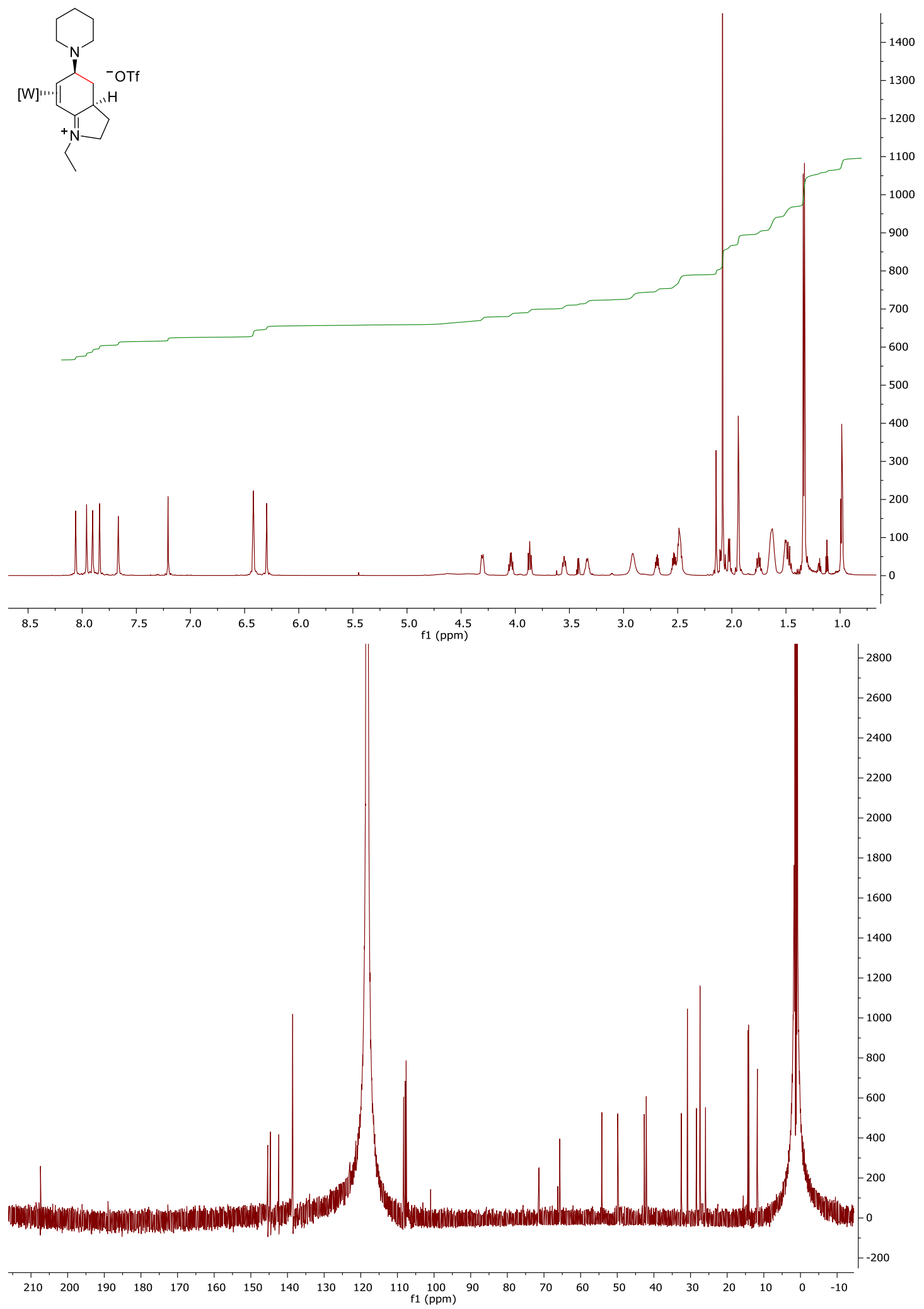


Compound 18:
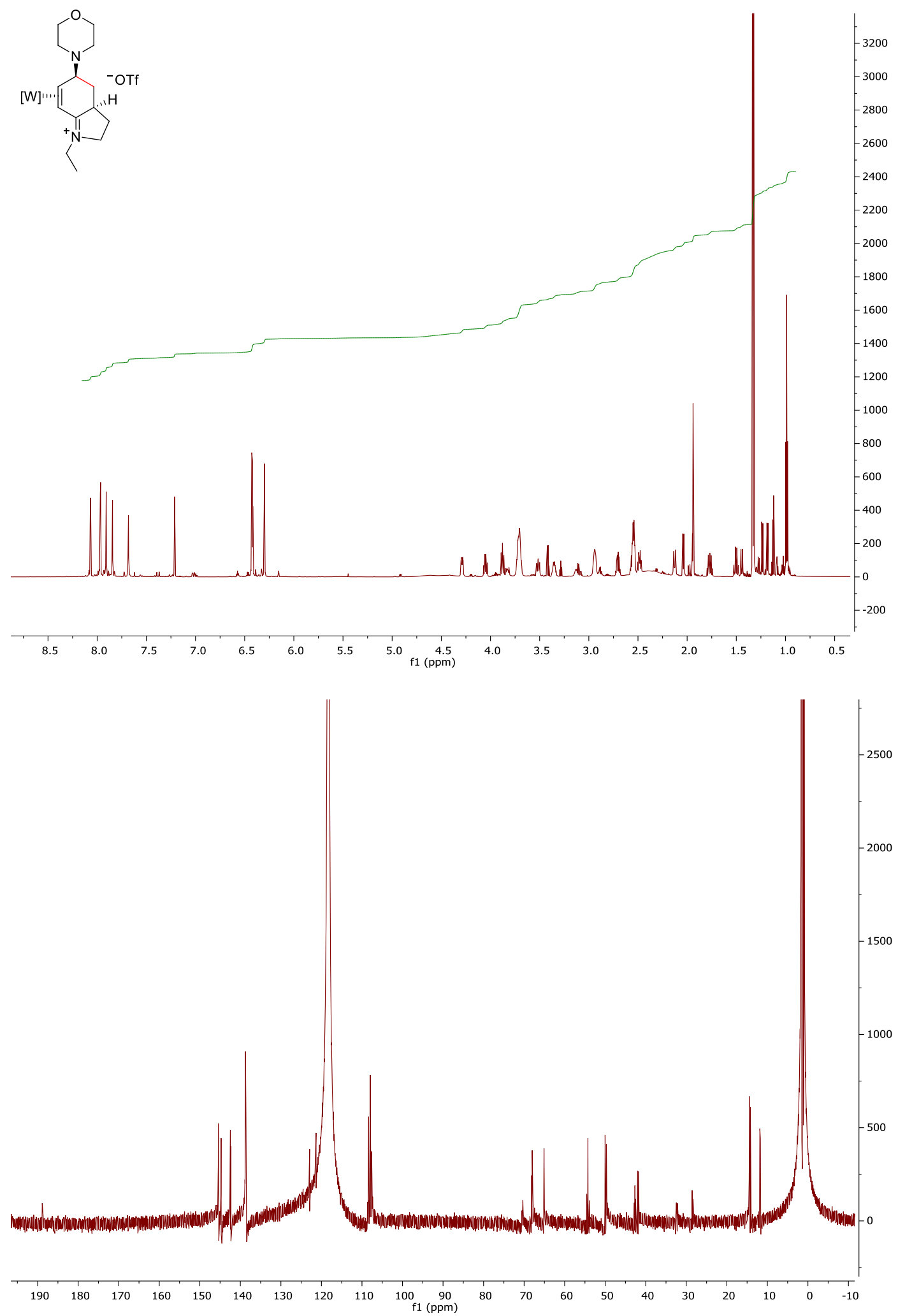


\section{Compound 19:}

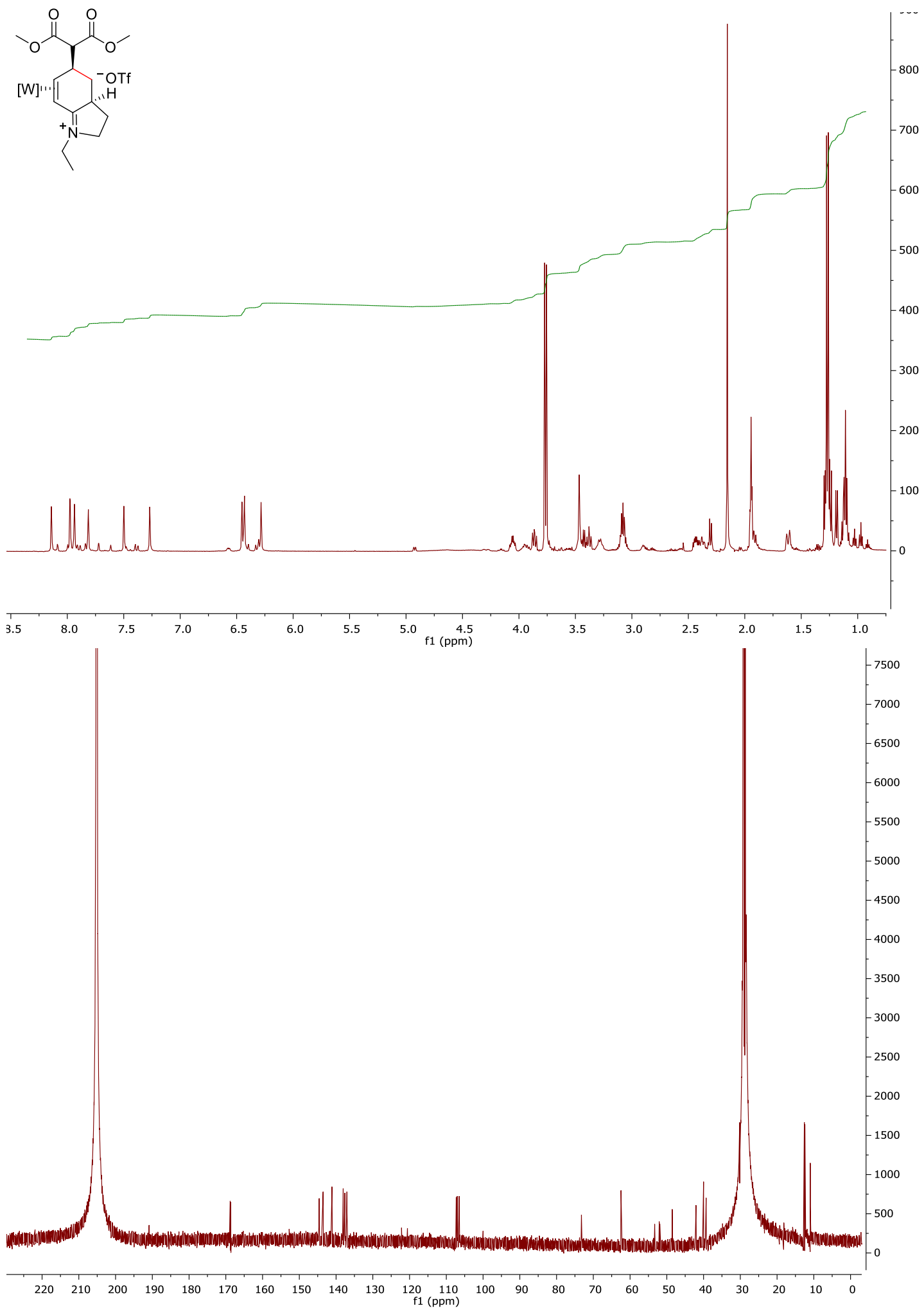


Compound 20:

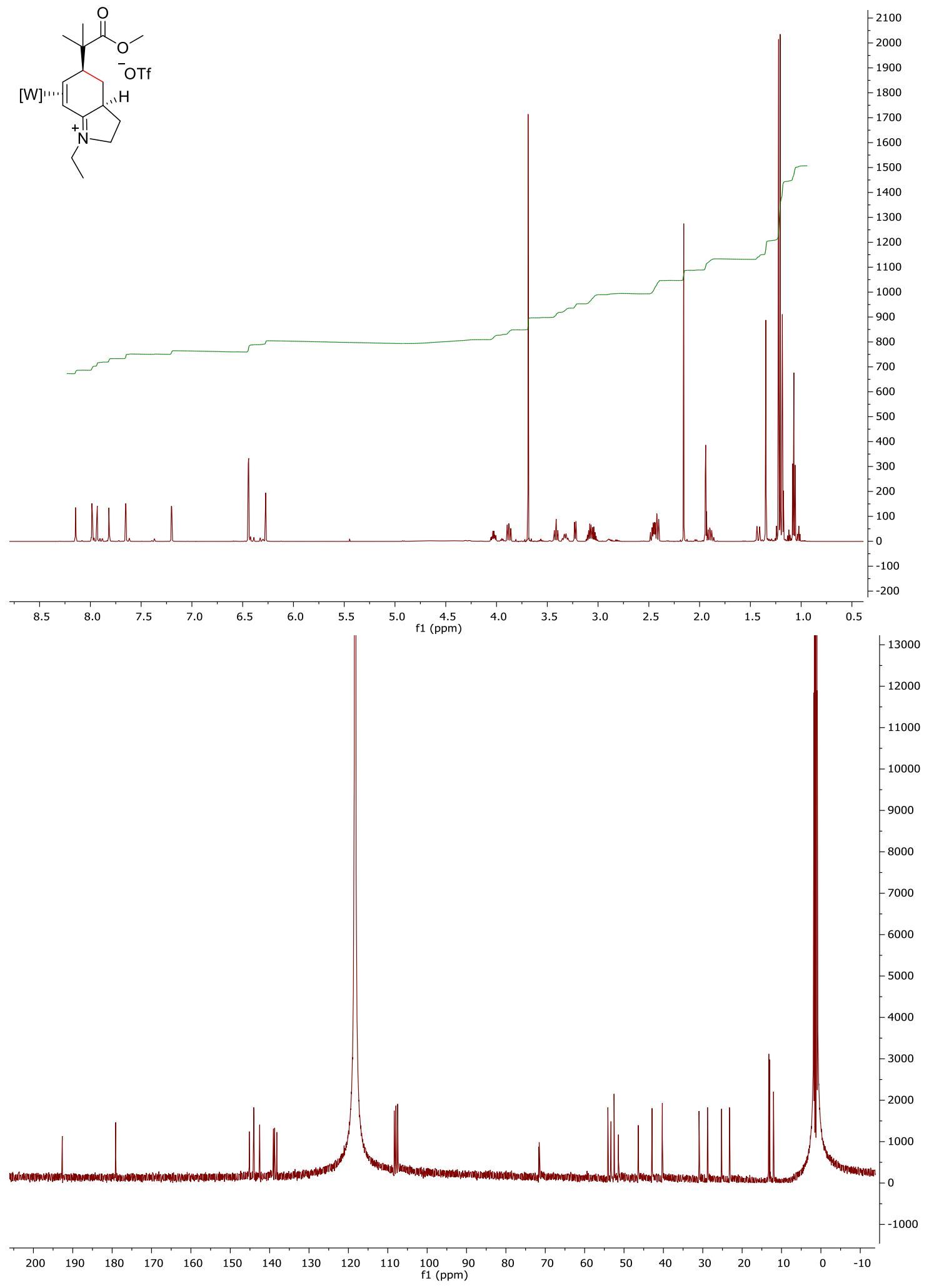


Compound 21:

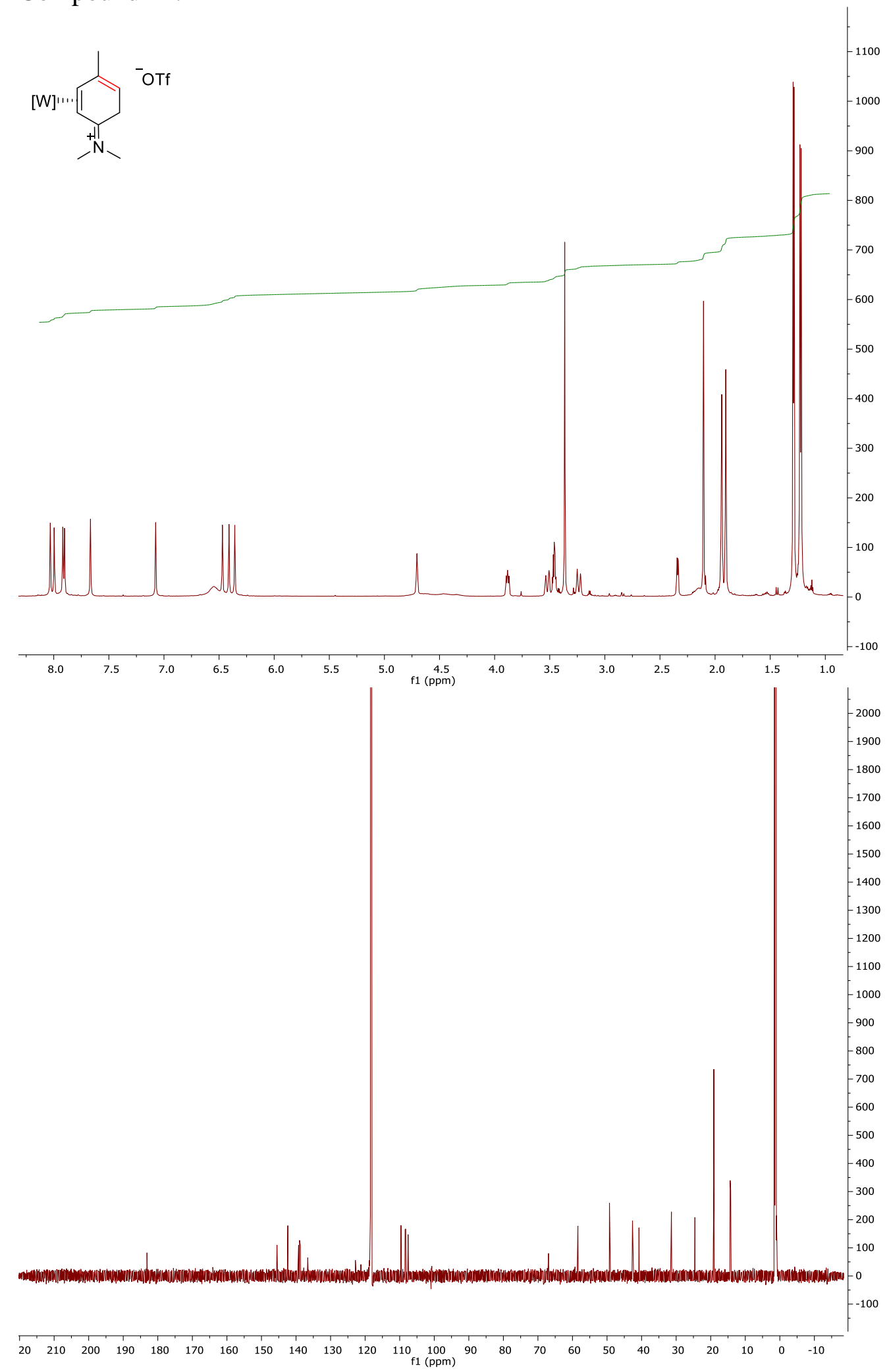


Compound 22:

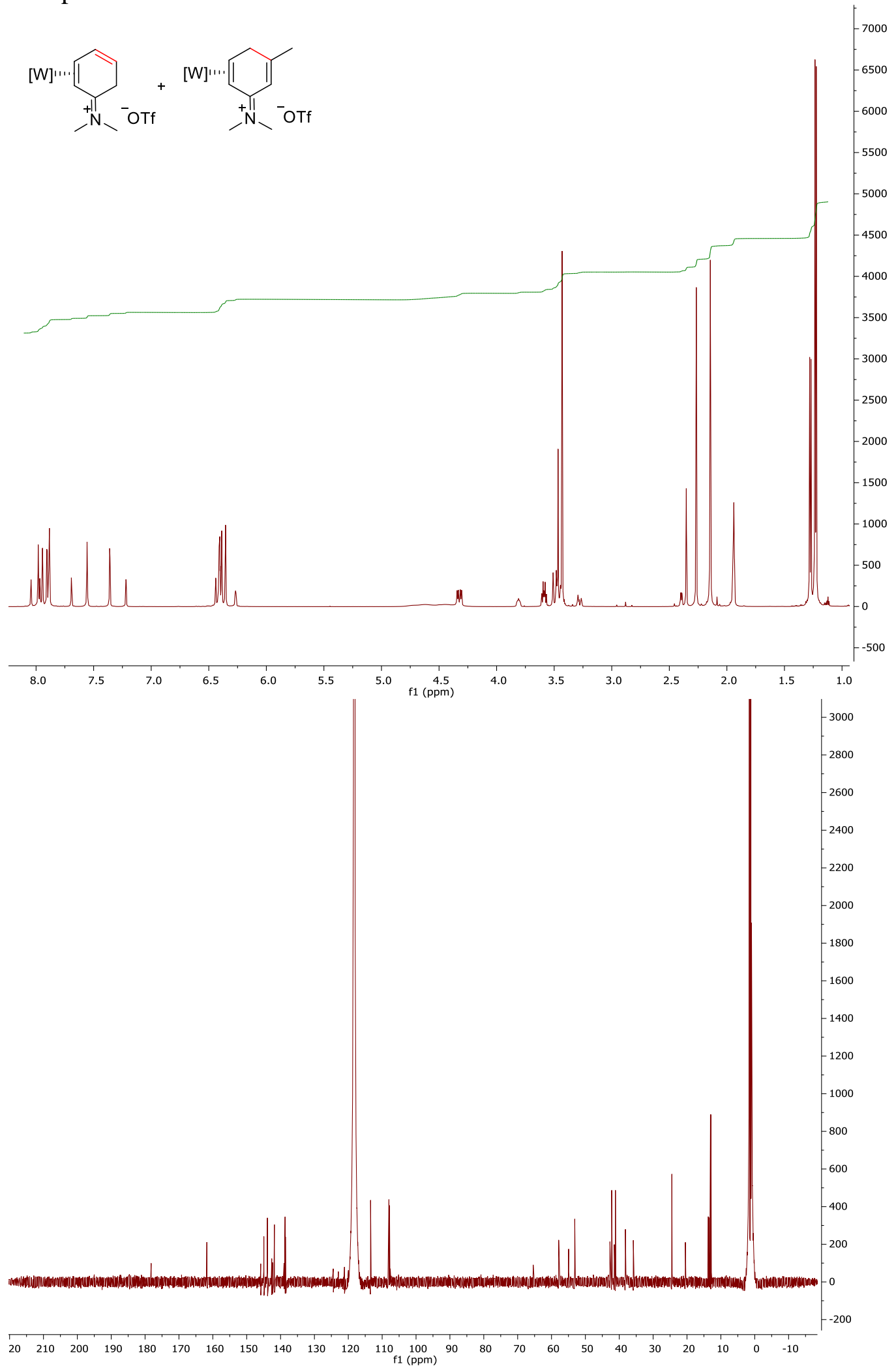


Compound 23:

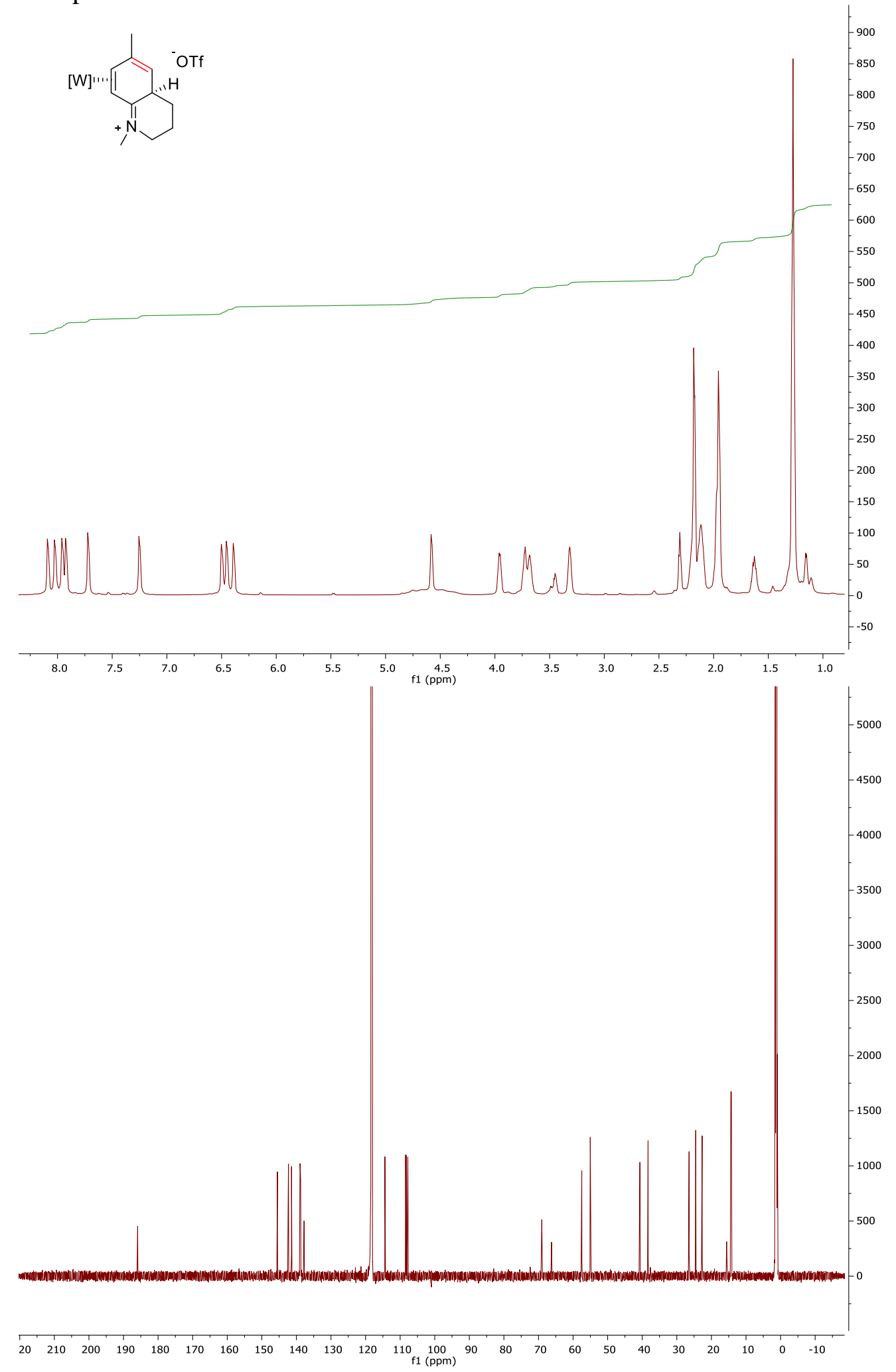


Compound 24:
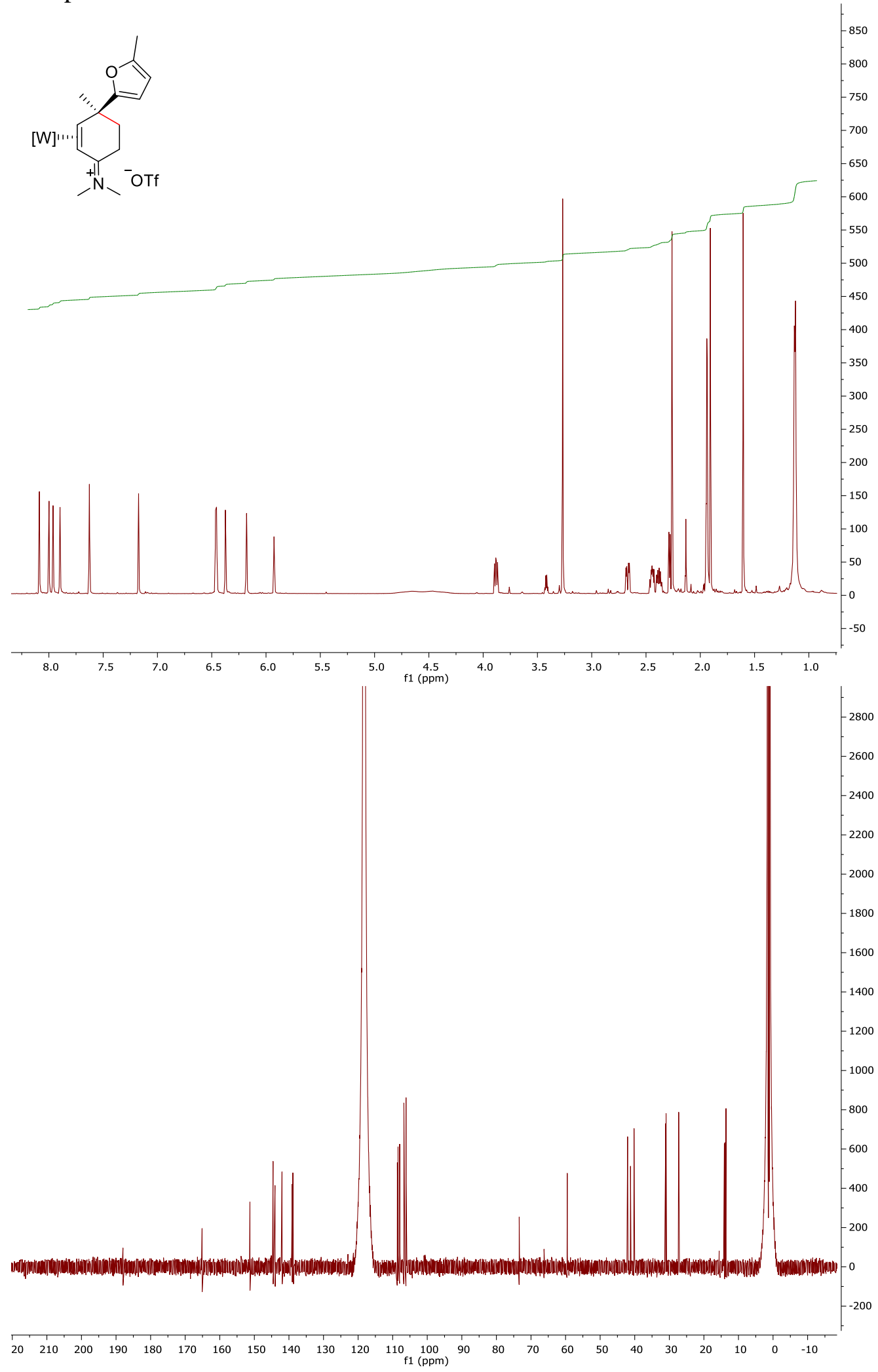
Compound 25:

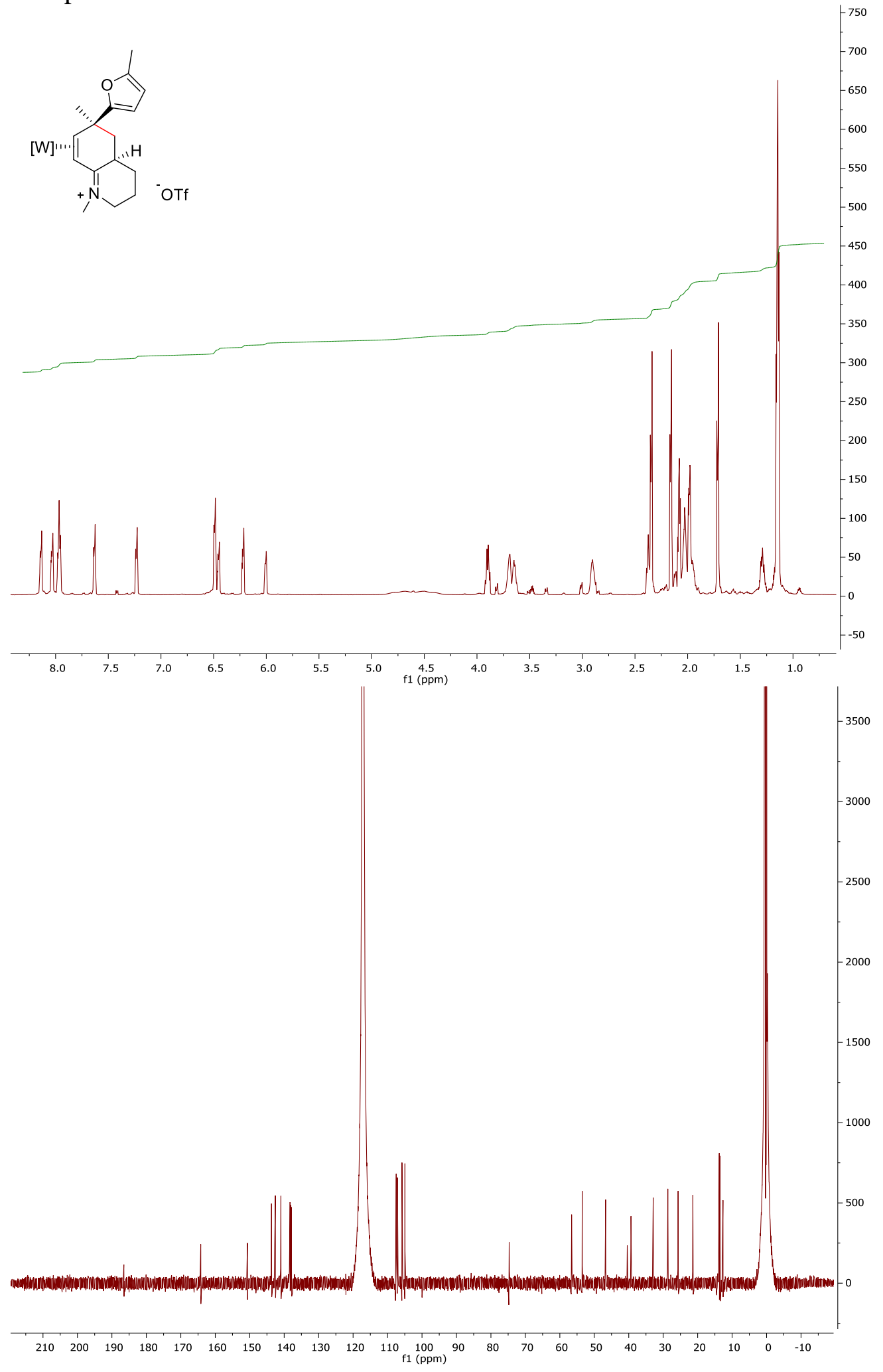


Compound 27:

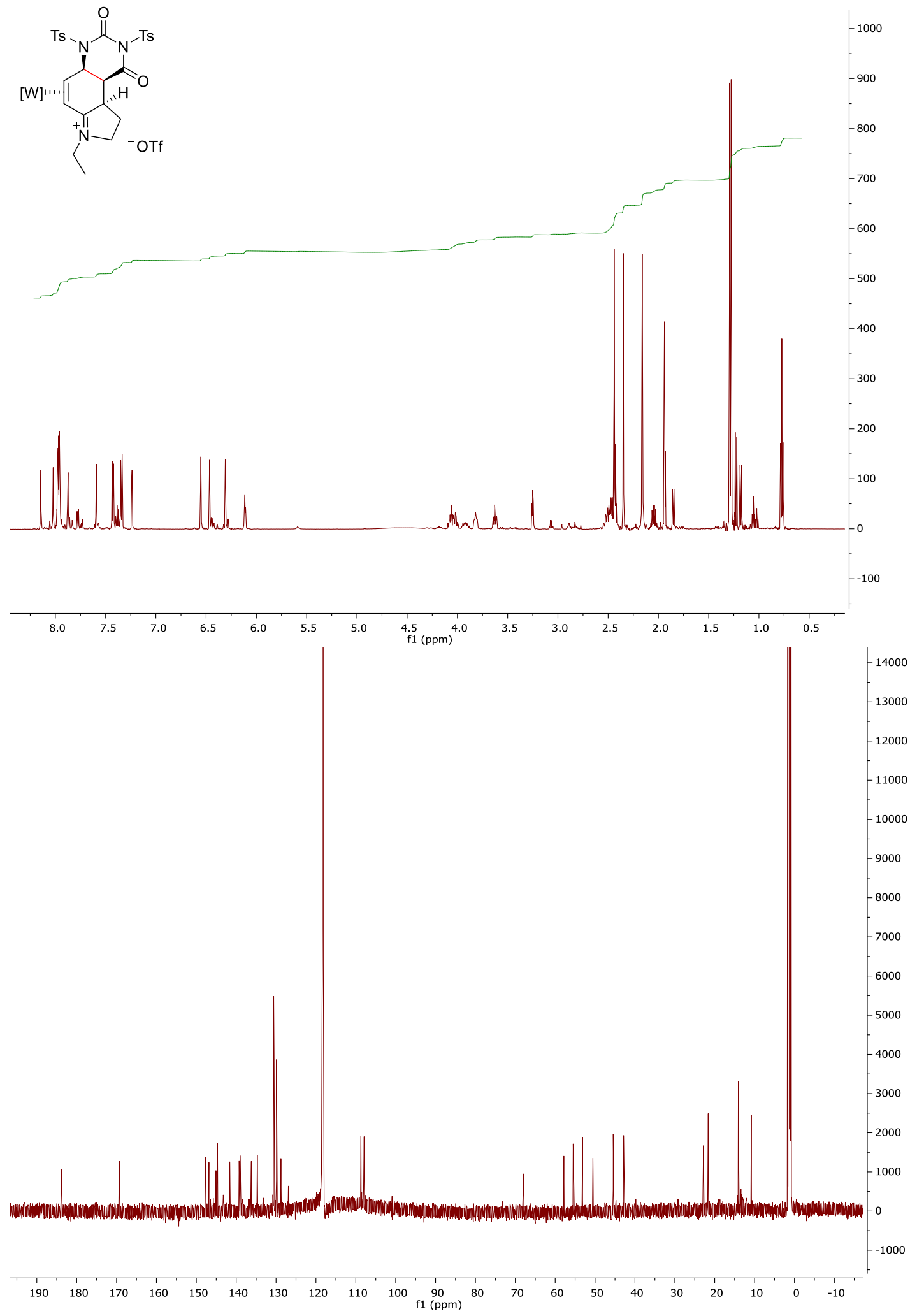


Compound 28:

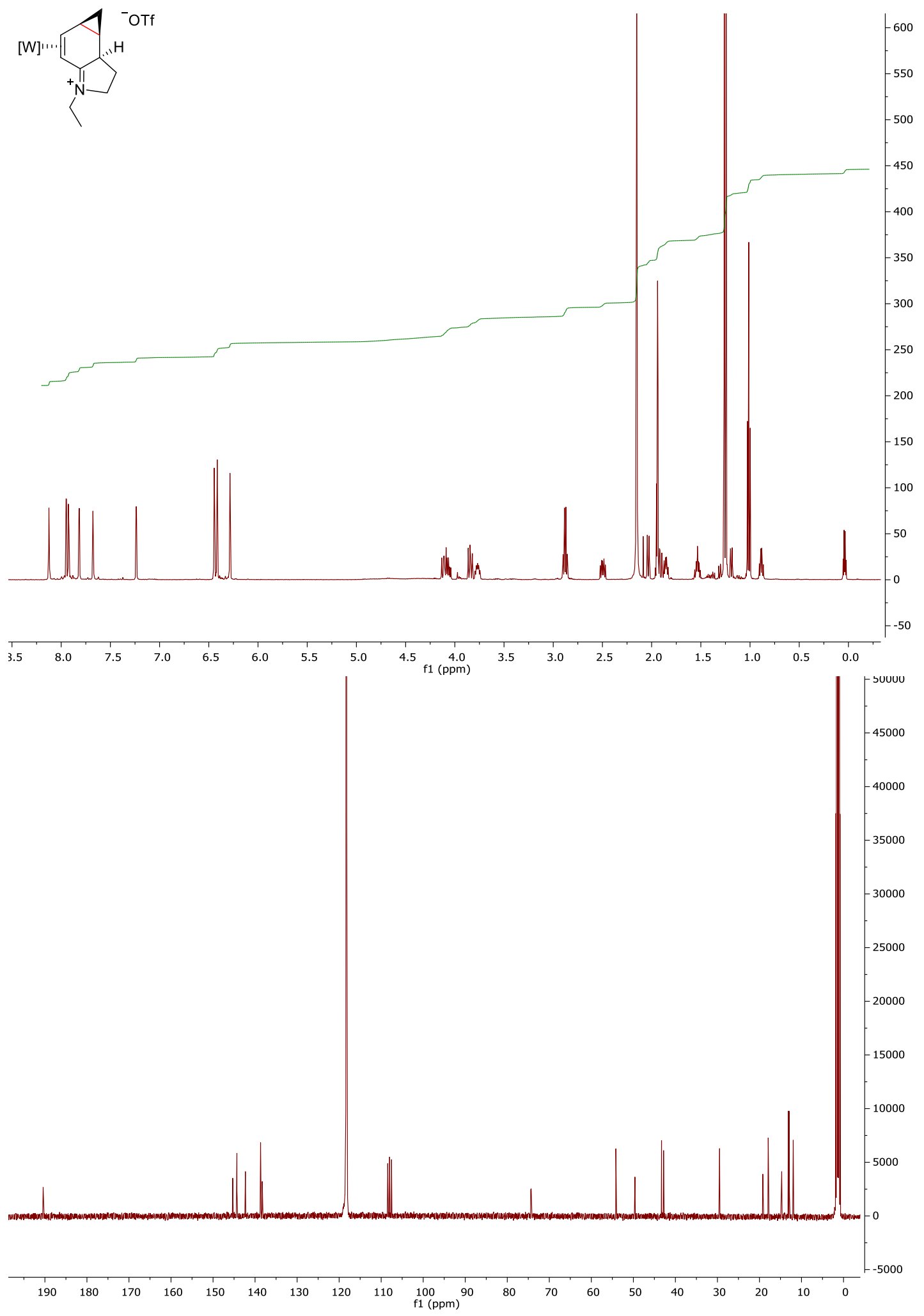


Compound 29:

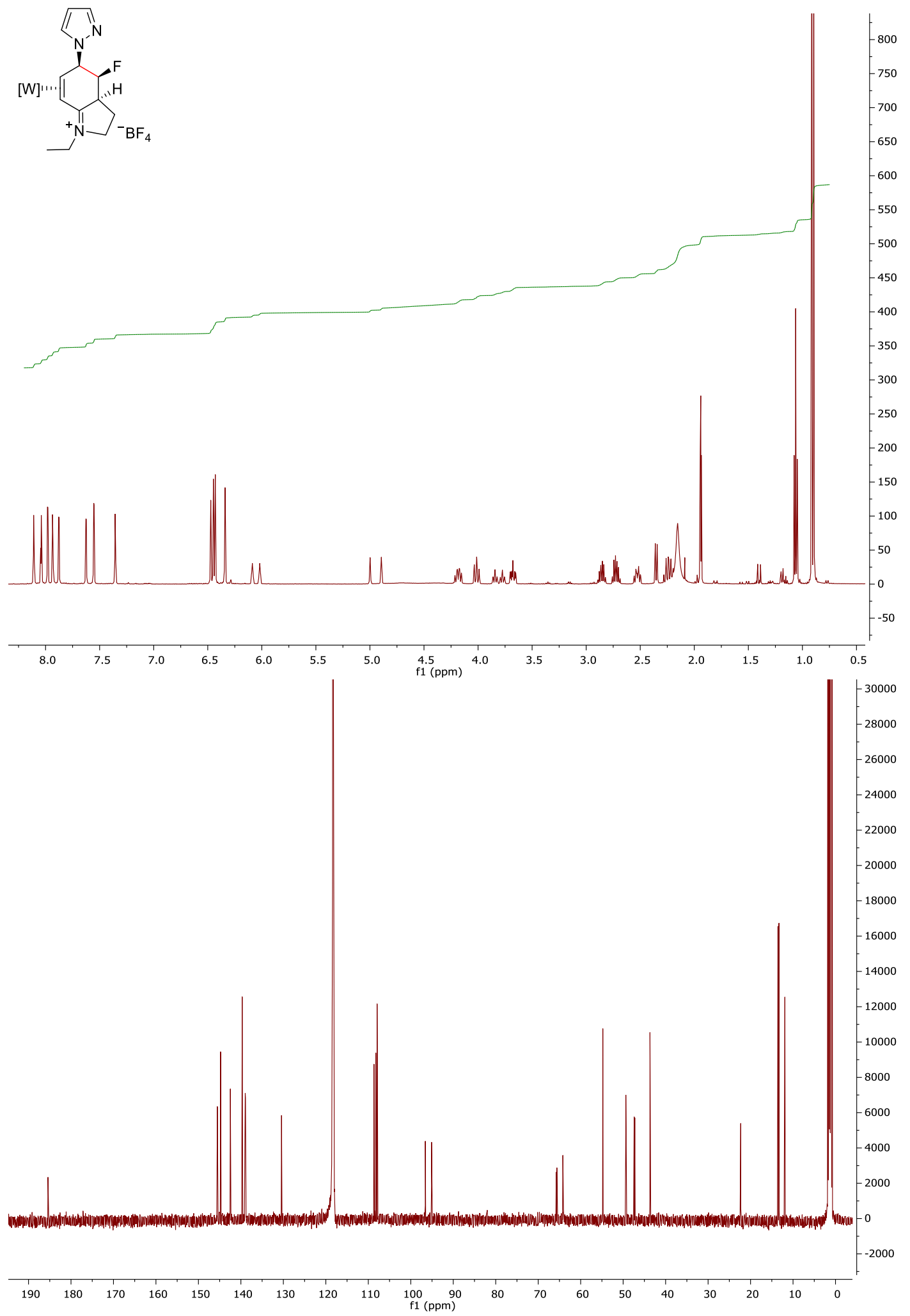


Compound 30:

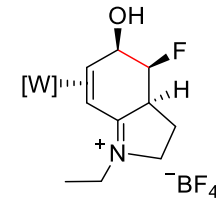

$\mathrm{BF}_{4}$
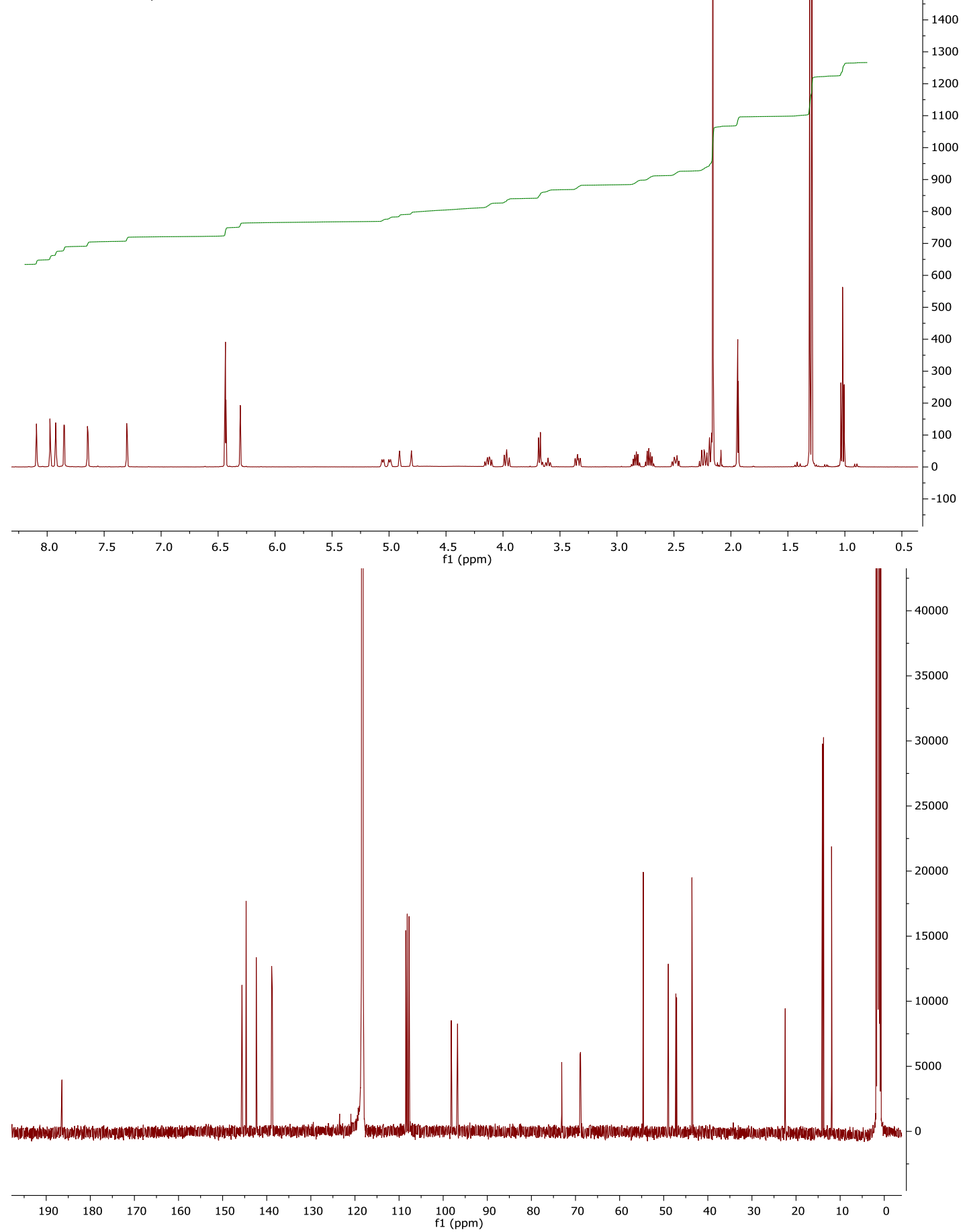
Compound 31:
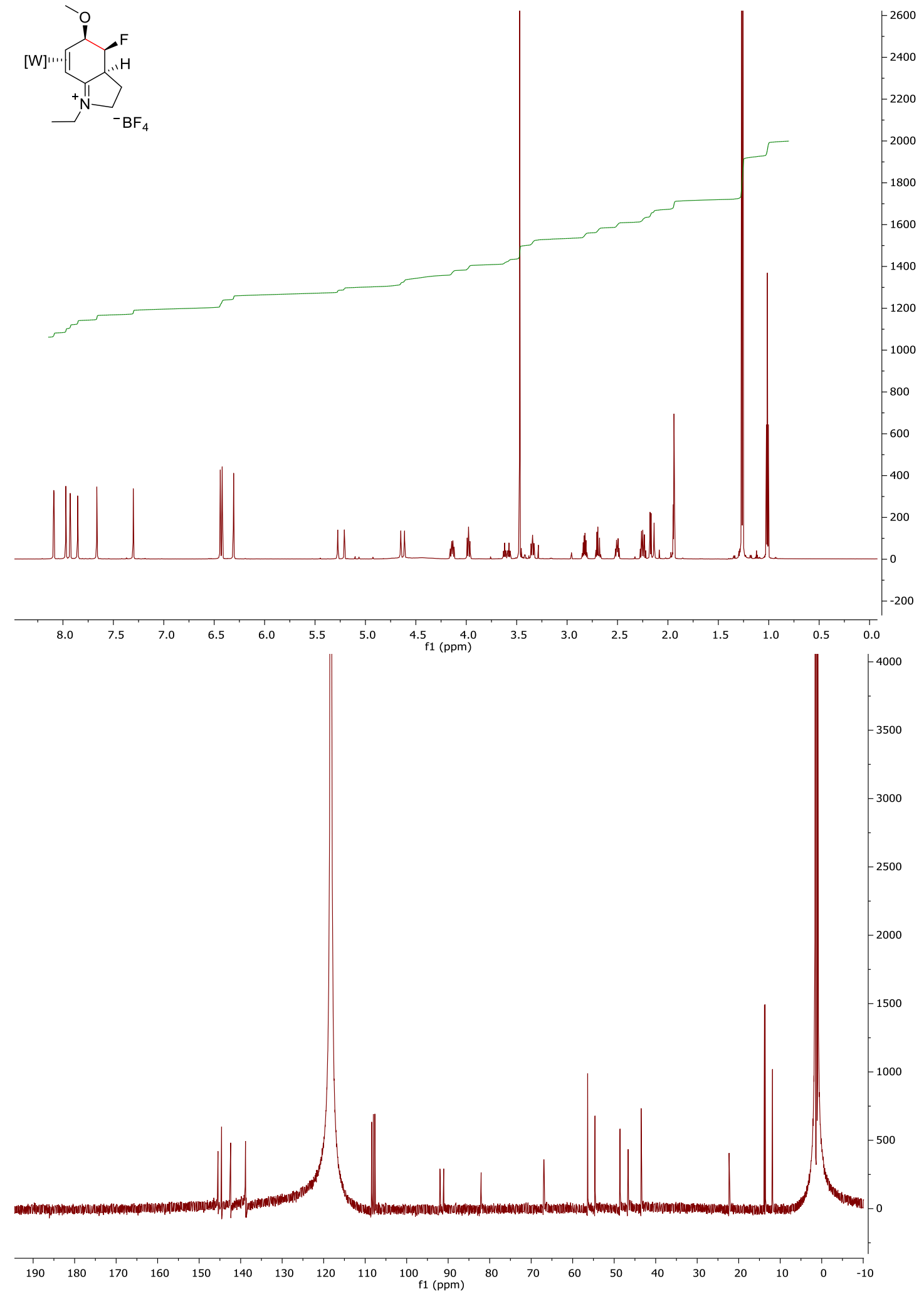
Compound 31 X-Ray Crystal Sturcutre:

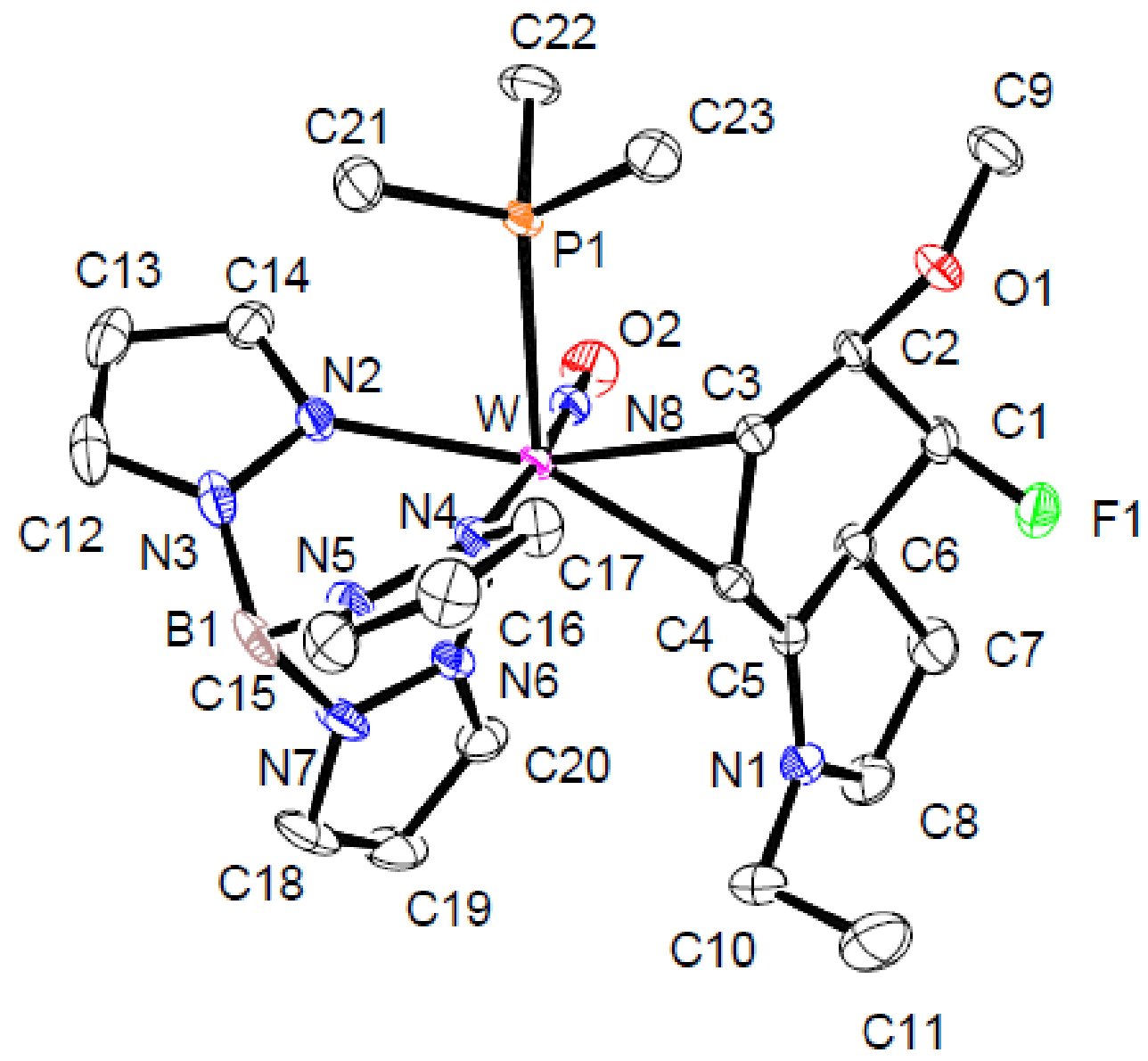


Table 1. Crystal data and structure refinement for C23H36B2F5N8O2PW.

Empirical formula

Formula weight

Temperature

Wavelength

Crystal system

Space group

Unit cell dimensions

$100.752(1)^{\circ}$

Volume

$\mathrm{Z}$

Density (calculated)

Absorption coefficient

$\mathrm{F}(000)$

Crystal size

Theta range for data collection

Index ranges

Reflections collected

Independent reflections

Completeness to theta $=25.242^{\circ}$

Absorption correction

Refinement method

Data / restraints / parameters

Goodness-of-fit on $\mathrm{F}^{2}$

Final $\mathrm{R}$ indices [I $>2 \operatorname{sigma}(\mathrm{I})]$

$\mathrm{R}$ indices (all data)

Largest diff. peak and hole

\section{C23 H36 B2 F5 N8 O2 P W}

788.04

233(2) K

$0.71073 \AA$

Monoclinic

$\mathrm{C} 2 / \mathrm{c}$

$a=15.6659(9) \AA$

$\mathrm{b}=16.0480(9) \AA$

$\mathrm{c}=25.489(2) \AA$

6295.5(6) $\AA^{3}$

8

$1.663 \mathrm{Mg} / \mathrm{m}^{3}$

$3.785 \mathrm{~mm}^{-1}$

3120

$0.470 \times 0.380 \times 0.340 \mathrm{~mm}^{3}$

3.514 to $26.372^{\circ}$.

$-19<=\mathrm{h}<=19,-20<=\mathrm{k}<=20,-31<=\mathrm{l}<=31$

41847

$6398[\mathrm{R}(\mathrm{int})=0.0208]$

$99.6 \%$

Empirical

Full-matrix least-squares on $\mathrm{F}^{2}$

6398 / 0 / 396

1.232

$\mathrm{R} 1=0.0353, \mathrm{wR} 2=0.0821$

$\mathrm{R} 1=0.0361, \mathrm{wR} 2=0.0826$

1.491 and -2.391 e. $\AA^{-3}$ 
Table 2. Atomic coordinates ( $\times 10^{4}$ ) and equivalent isotropic displacement parameters $\left(\AA^{2} \times 10^{3}\right)$ for $\mathrm{C} 23 \mathrm{H} 36 \mathrm{~B} 2 \mathrm{~F} 5 \mathrm{~N} 8 \mathrm{O} 2 \mathrm{PW}$. U(eq) is defined as one third of the trace of the orthogonalized $\mathrm{Uij}^{\mathrm{ij}}$ tensor.

\begin{tabular}{|c|c|c|c|c|}
\hline & $\mathrm{x}$ & $\mathrm{y}$ & $\mathrm{z}$ & $\mathrm{U}(\mathrm{eq})$ \\
\hline W & 1987(1) & $1076(1)$ & $3560(1)$ & $26(1)$ \\
\hline $\mathrm{P}(1)$ & $3592(1)$ & $1408(1)$ & $3651(1)$ & $34(1)$ \\
\hline $\mathrm{F}(1)$ & $1073(2)$ & $3515(2)$ & $4472(1)$ & $53(1)$ \\
\hline $\mathrm{F}(2)$ & $3032(9)$ & $8285(5)$ & $6785(3)$ & $207(5)$ \\
\hline $\mathrm{F}(3)$ & $3502(5)$ & $9404(4)$ & $6355(3)$ & $134(2)$ \\
\hline $\mathrm{F}(4)$ & $2752(8)$ & $8321(8)$ & $5966(5)$ & $257(7)$ \\
\hline $\mathrm{F}(5)$ & $3968(6)$ & $8184(5)$ & $6309(7)$ & $271(8)$ \\
\hline $\mathrm{O}(1)$ & $2772(3)$ & $3403(2)$ & $4373(2)$ & $42(1)$ \\
\hline $\mathrm{O}(2)$ & 1399(3) & $2222(3)$ & $2639(2)$ & $57(1)$ \\
\hline $\mathrm{N}(1)$ & $-278(3)$ & $1866(3)$ & $3982(2)$ & $40(1)$ \\
\hline $\mathrm{N}(2)$ & $2375(3)$ & 137(3) & $3023(2)$ & $37(1)$ \\
\hline $\mathrm{N}(3)$ & $2308(3)$ & $-692(3)$ & $3125(2)$ & $44(1)$ \\
\hline $\mathrm{N}(4)$ & $2426(3)$ & $140(3)$ & $4201(2)$ & $34(1)$ \\
\hline $\mathrm{N}(5)$ & $2290(3)$ & $-686(3)$ & $4097(2)$ & $45(1)$ \\
\hline $\mathrm{N}(6)$ & $833(3)$ & 276(3) & $3367(2)$ & $36(1)$ \\
\hline $\mathrm{N}(7)$ & $914(3)$ & $-563(3)$ & $3429(2)$ & $50(1)$ \\
\hline $\mathrm{N}(8)$ & $1646(3)$ & $1765(3)$ & $3017(2)$ & $33(1)$ \\
\hline $\mathrm{C}(1)$ & $1240(4)$ & $3411(3)$ & $3953(2)$ & $37(1)$ \\
\hline $\mathrm{C}(2)$ & $2125(3)$ & 2987(3) & $3995(2)$ & $32(1)$ \\
\hline$C(3)$ & $2124(3)$ & 2071(3) & $4166(2)$ & $29(1)$ \\
\hline $\mathrm{C}(4)$ & $1292(3)$ & $1653(3)$ & $4162(2)$ & $31(1)$ \\
\hline$C(5)$ & $517(3)$ & 2063(3) & $3940(2)$ & $32(1)$ \\
\hline$C(6)$ & $516(3)$ & 2881(3) & $3649(2)$ & $36(1)$ \\
\hline$C(7)$ & $-414(4)$ & $3213(4)$ & $3628(3)$ & $56(2)$ \\
\hline$C(8)$ & $-941(4)$ & $2452(4)$ & $3709(3)$ & $54(2)$ \\
\hline$C(9)$ & $3082(4)$ & $4147(4)$ & $4173(3)$ & $57(2)$ \\
\hline$C(10)$ & $-527(4)$ & $1142(4)$ & $4273(3)$ & $54(2)$ \\
\hline $\mathrm{C}(11)$ & $-591(7)$ & $1372(7)$ & $4836(3)$ & $89(3)$ \\
\hline
\end{tabular}




$\begin{array}{lrrrr}\mathrm{C}(12) & 2597(4) & -1133(4) & 2742(3) & 60(2) \\ \mathrm{C}(13) & 2853(4) & -588(5) & 2394(3) & 60(2) \\ \mathrm{C}(14) & 2692(4) & 196(4) & 2575(2) & 46(1) \\ \mathrm{C}(15) & 2633(5) & -1130(4) & 4535(3) & 58(2) \\ \mathrm{C}(16) & 2988(5) & -592(5) & 4924(3) & 62(2) \\ \mathrm{C}(17) & 2851(4) & 194(4) & 4703(2) & 49(1) \\ \mathrm{C}(18) & 128(5) & -912(5) & 3260(3) & 68(2) \\ \mathrm{C}(19) & -460(4) & -308(5) & 3092(3) & 66(2) \\ \mathrm{C}(20) & 7(4) & 424(4) & 3158(2) & 46(1) \\ \mathrm{C}(21) & 4264(4) & 490(4) & 3634(3) & 60(2) \\ \mathrm{C}(22) & 3835(4) & 2076(4) & 3126(3) & 58(2) \\ \mathrm{C}(23) & 4177(4) & 1916(5) & 4247(3) & 58(2) \\ \mathrm{B}(1) & 1808(5) & -980(4) & 3552(3) & 53(2) \\ \mathrm{B}(2) & 3328(6) & 8575(6) & 6384(4) & 63(2)\end{array}$


Compound 32:
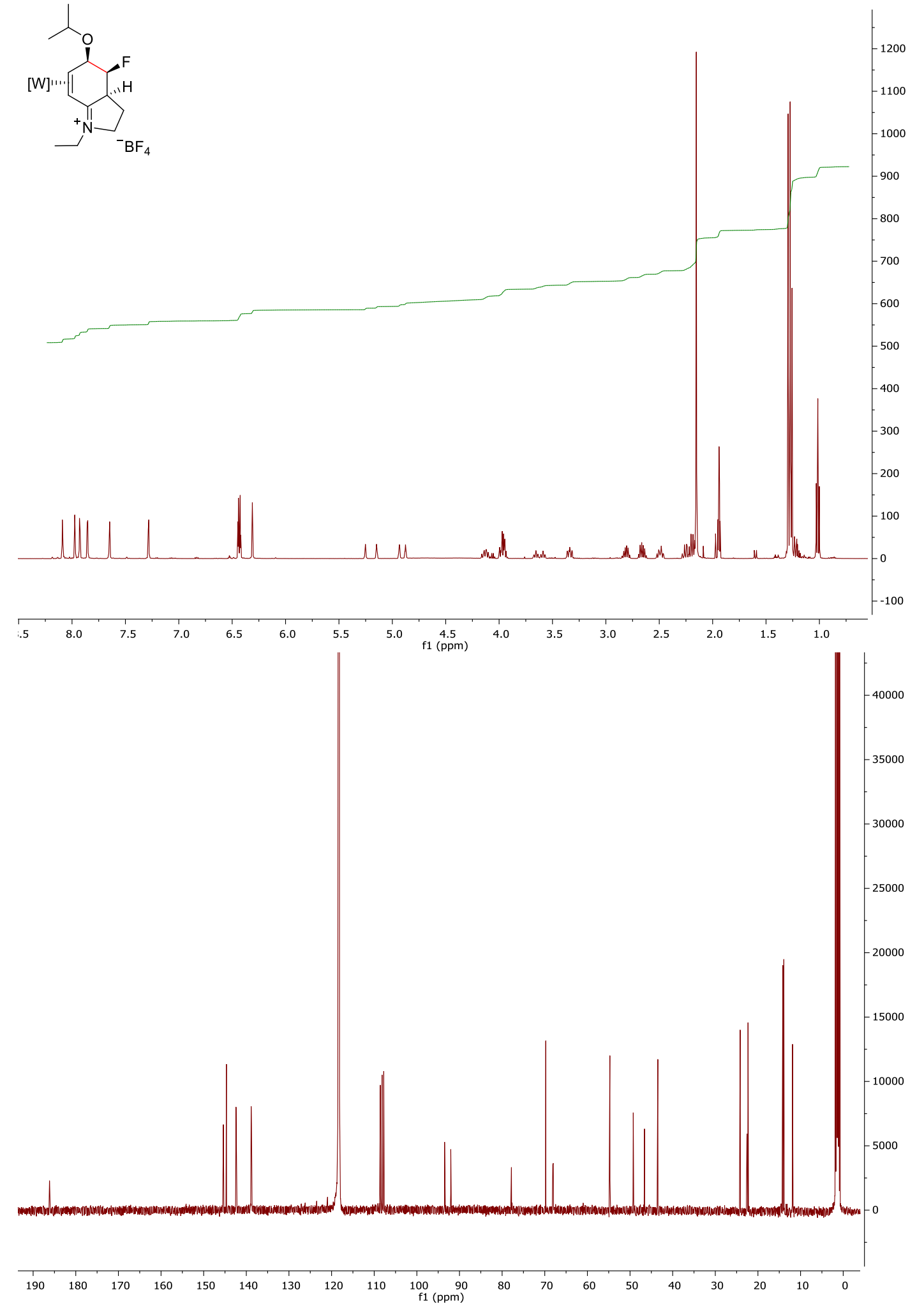
Compound 33:
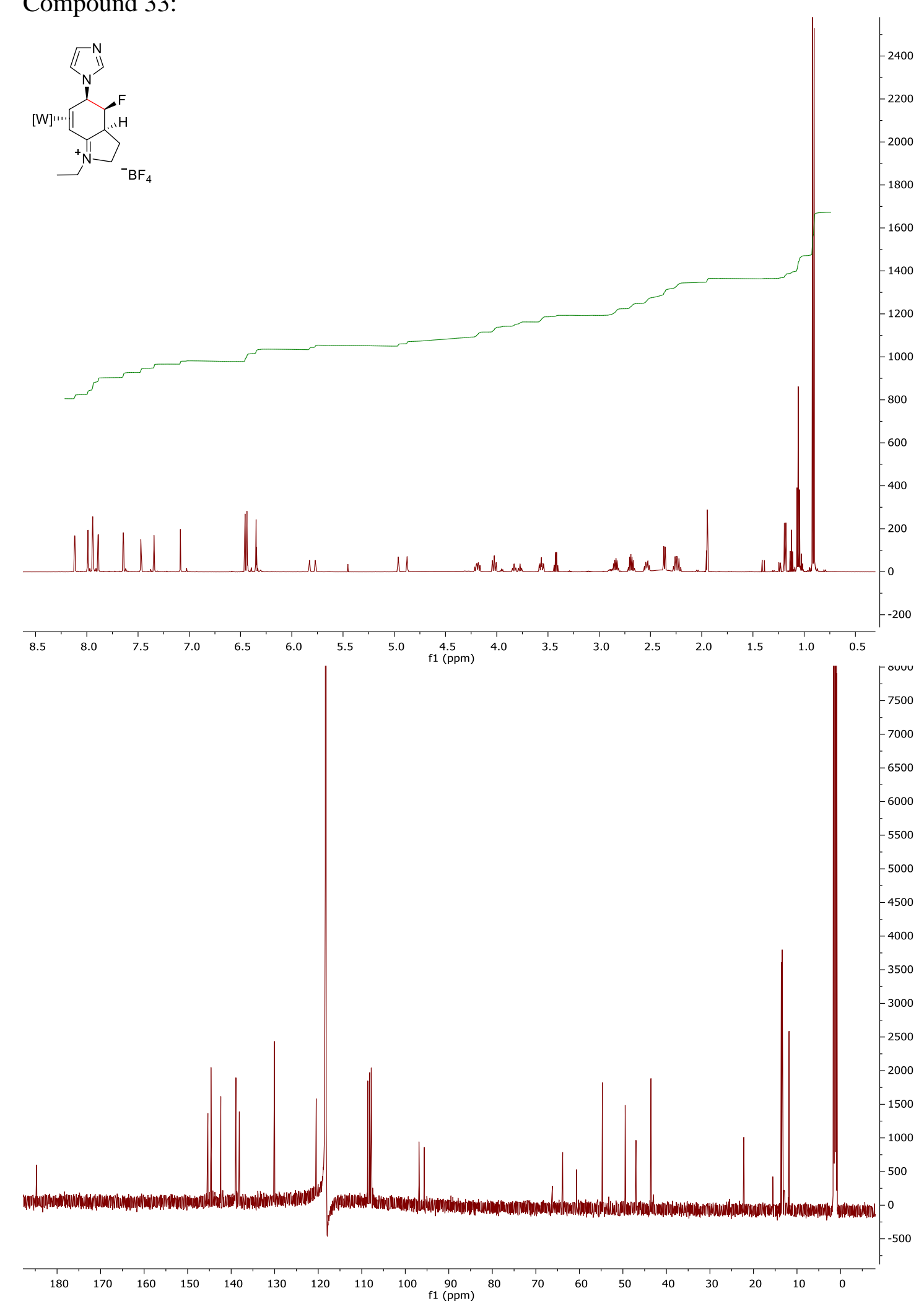
Compound 34:

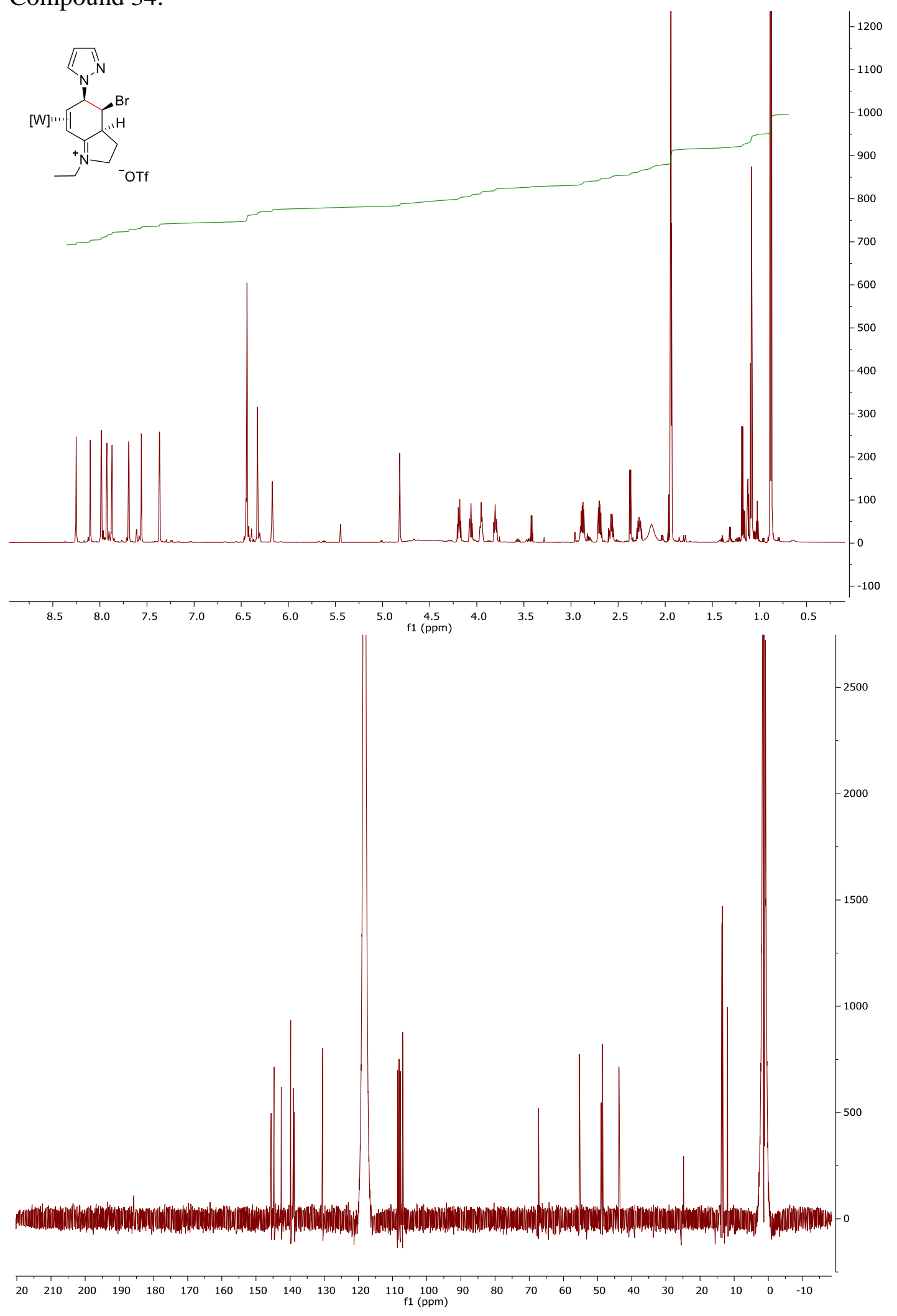


Compound 35:

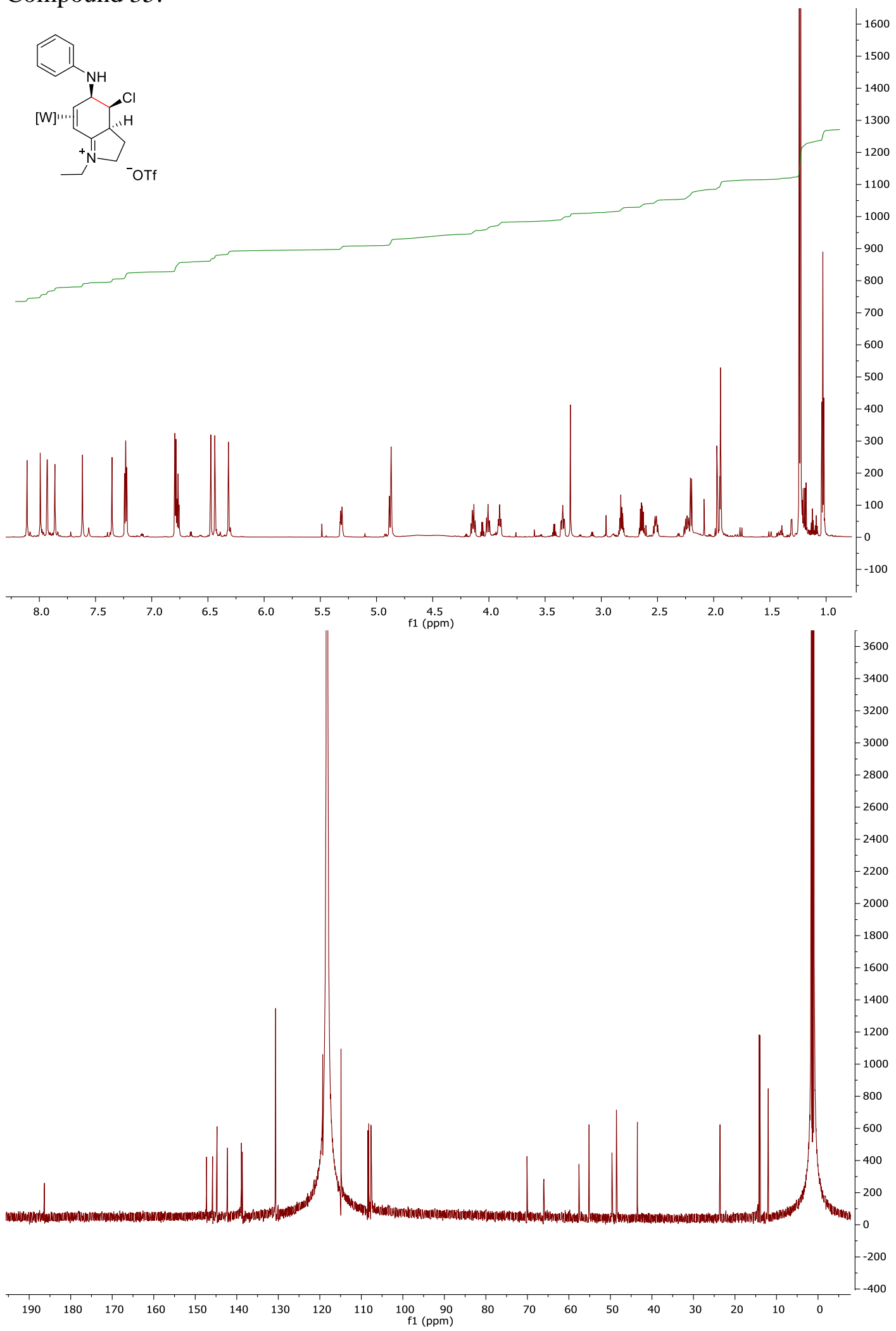


Compound 36:

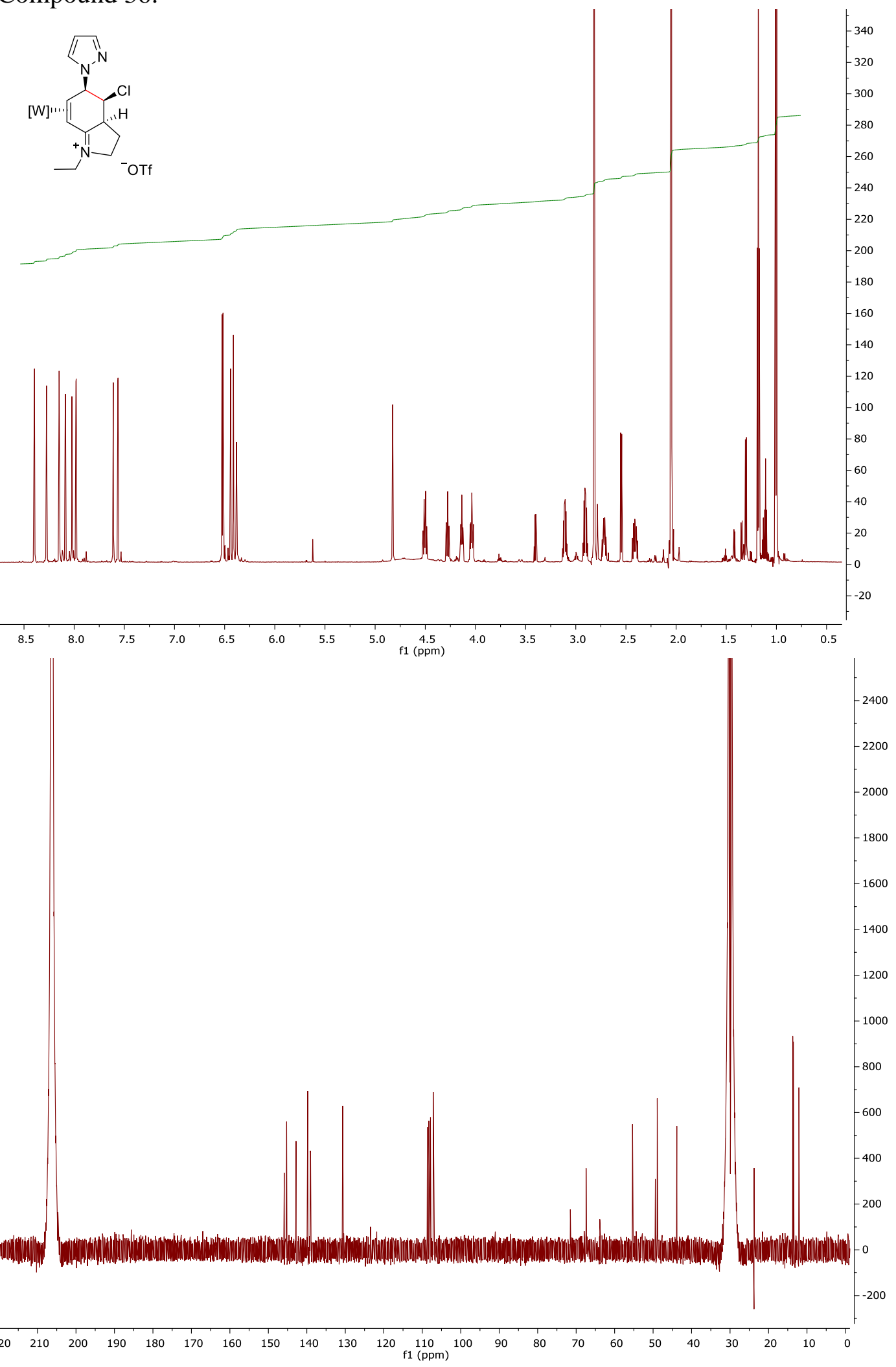


Compound 37:
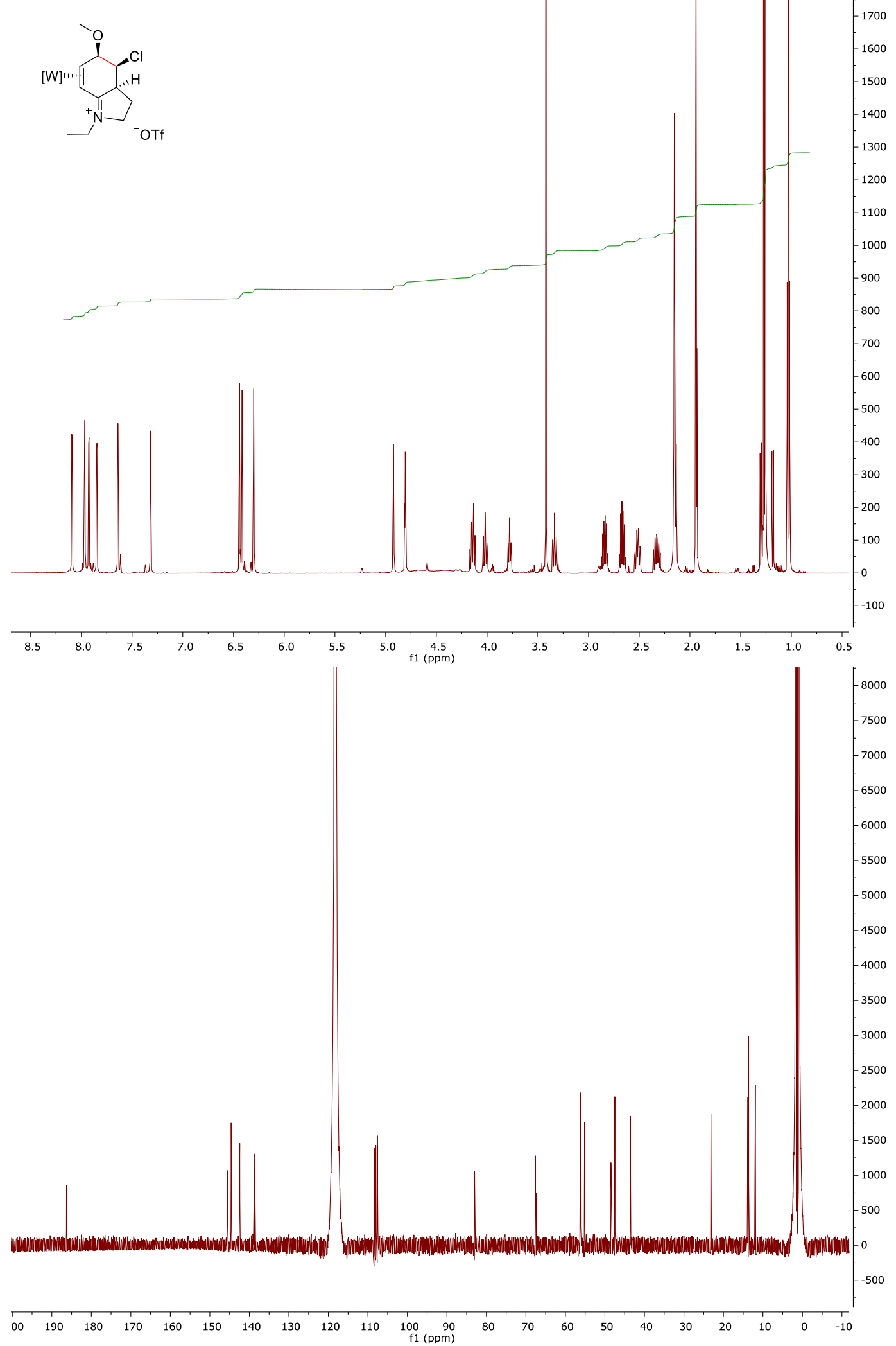
Compound 39:
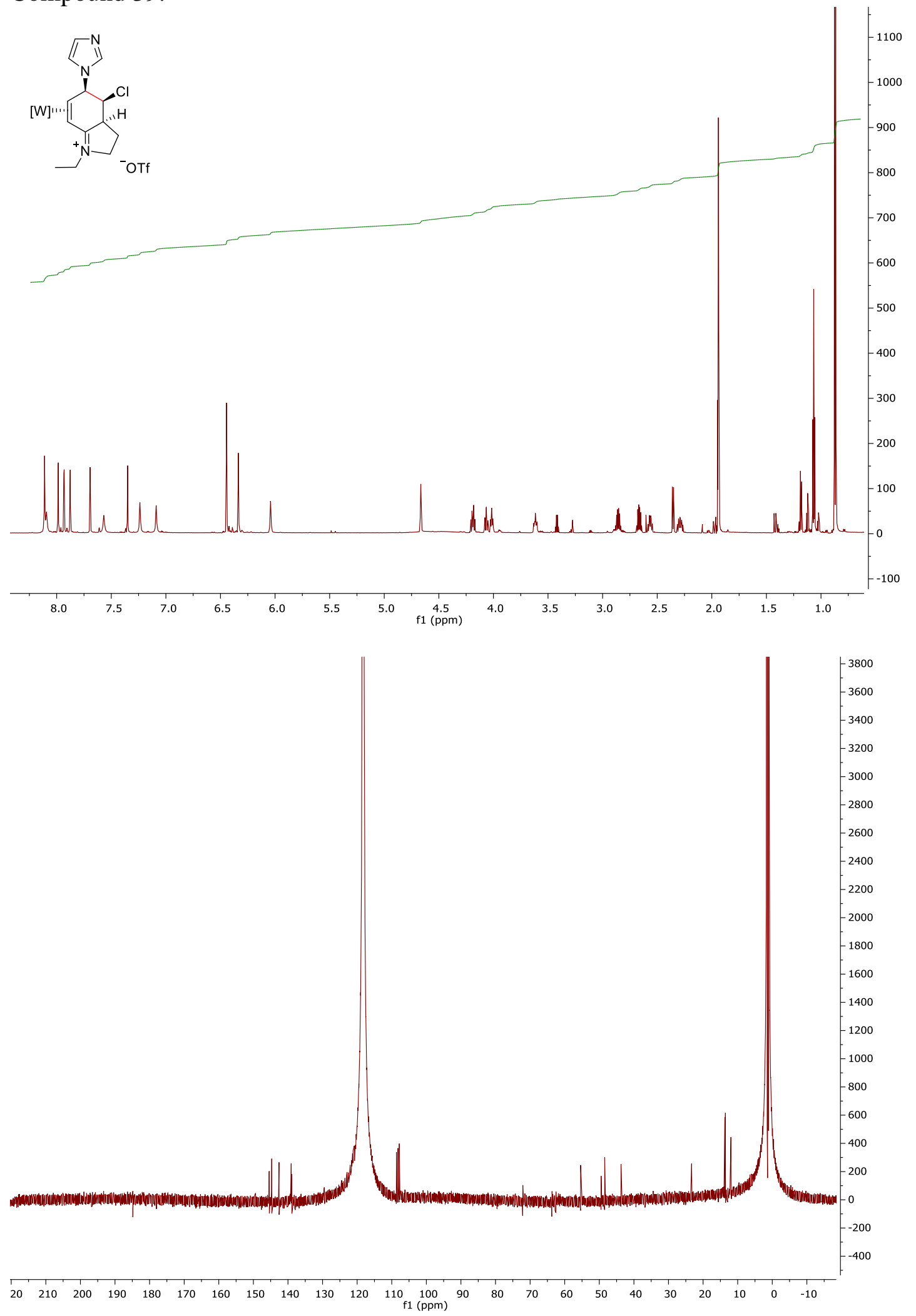
Compound 40:
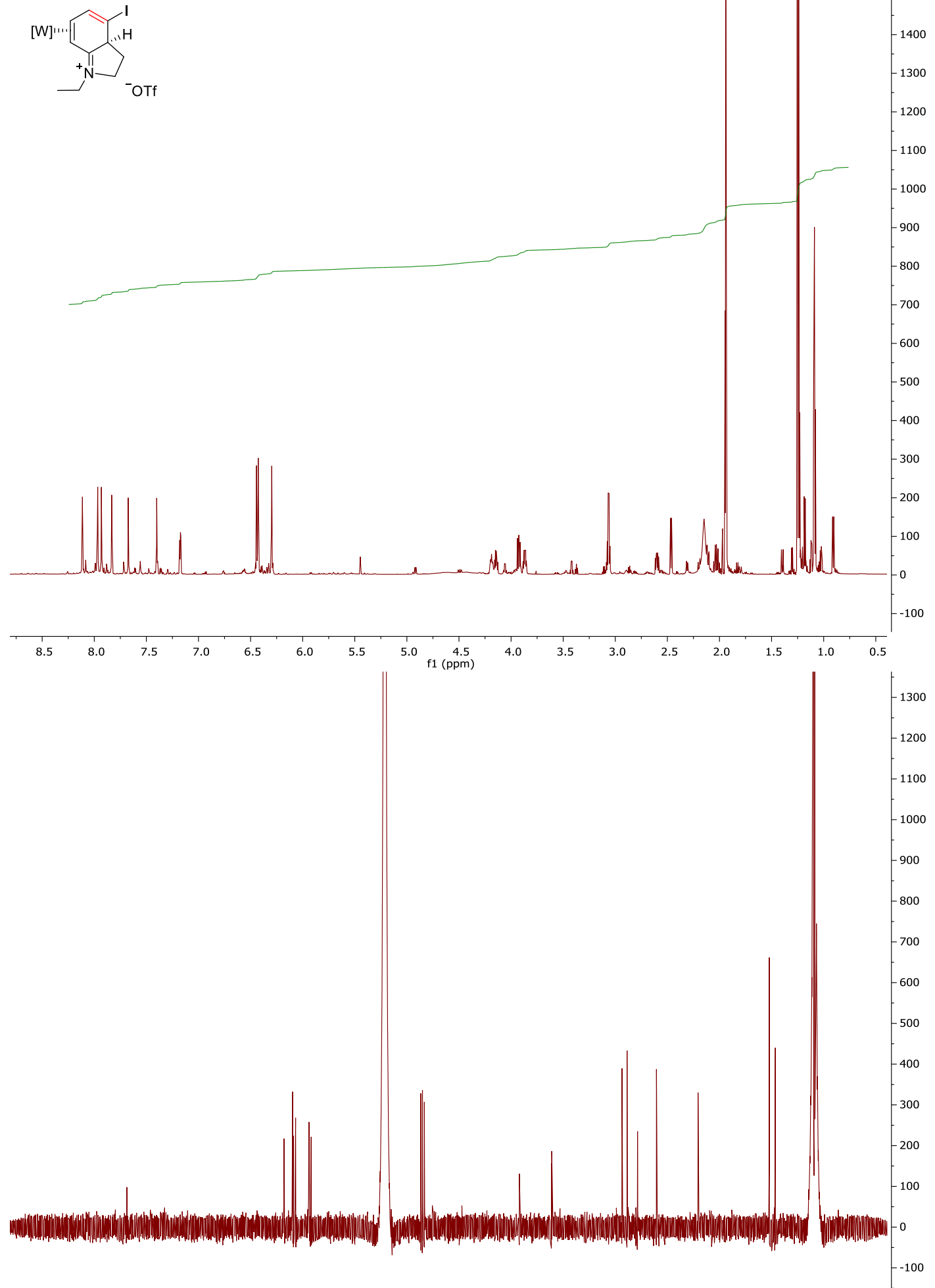

\begin{tabular}{lllllllllllllllllllllllll}
\hline 20 & 210 & 200 & 190 & 180 & 170 & 160 & 150 & 140 & 130 & 120 & 110 & 100 & 90 & 80 & 70 & 60 & 50 & 40 & 30 & 20 & 10 & 0 & -10
\end{tabular} 
Compound 41:
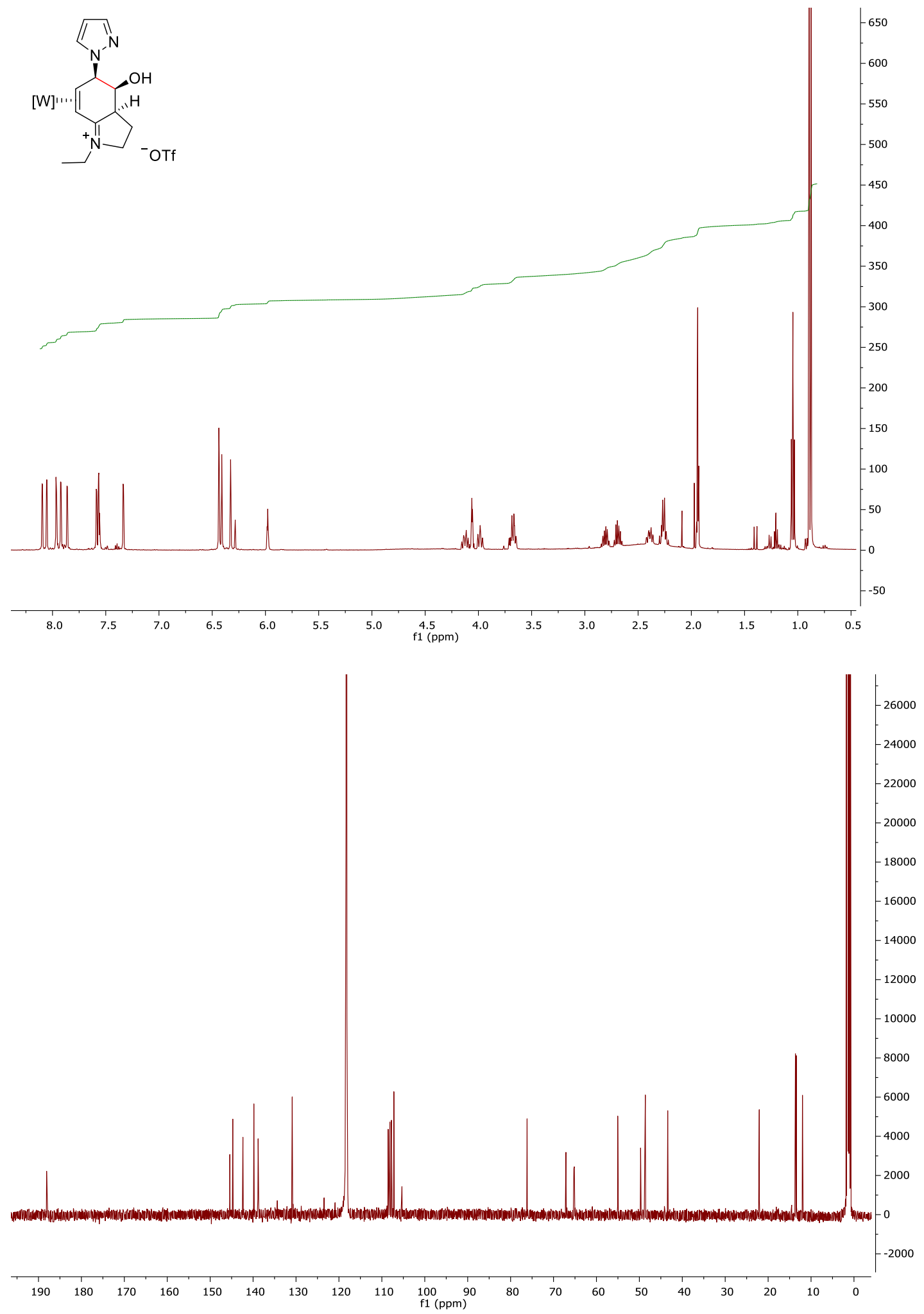
Compound 42:
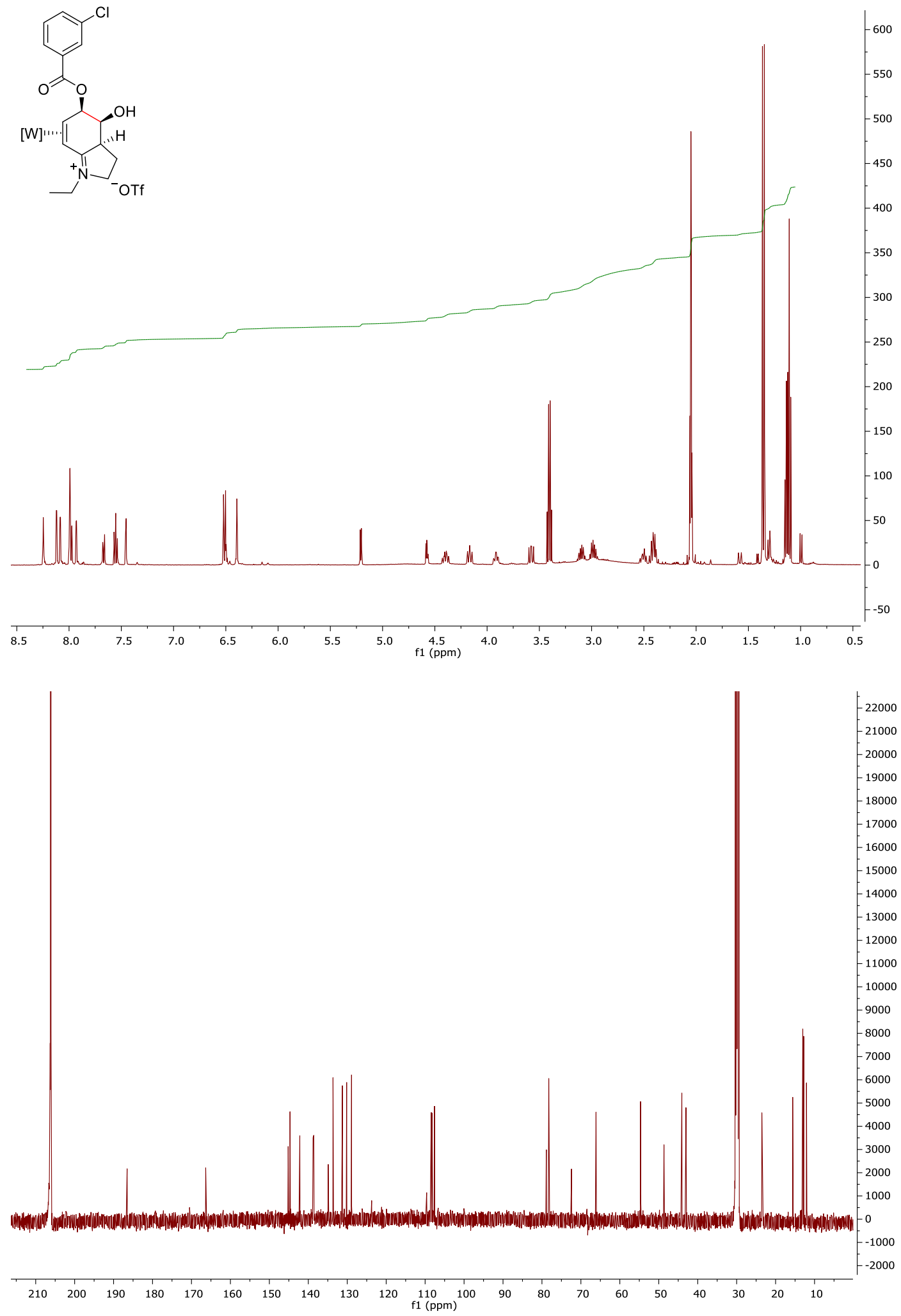
Compound 43:
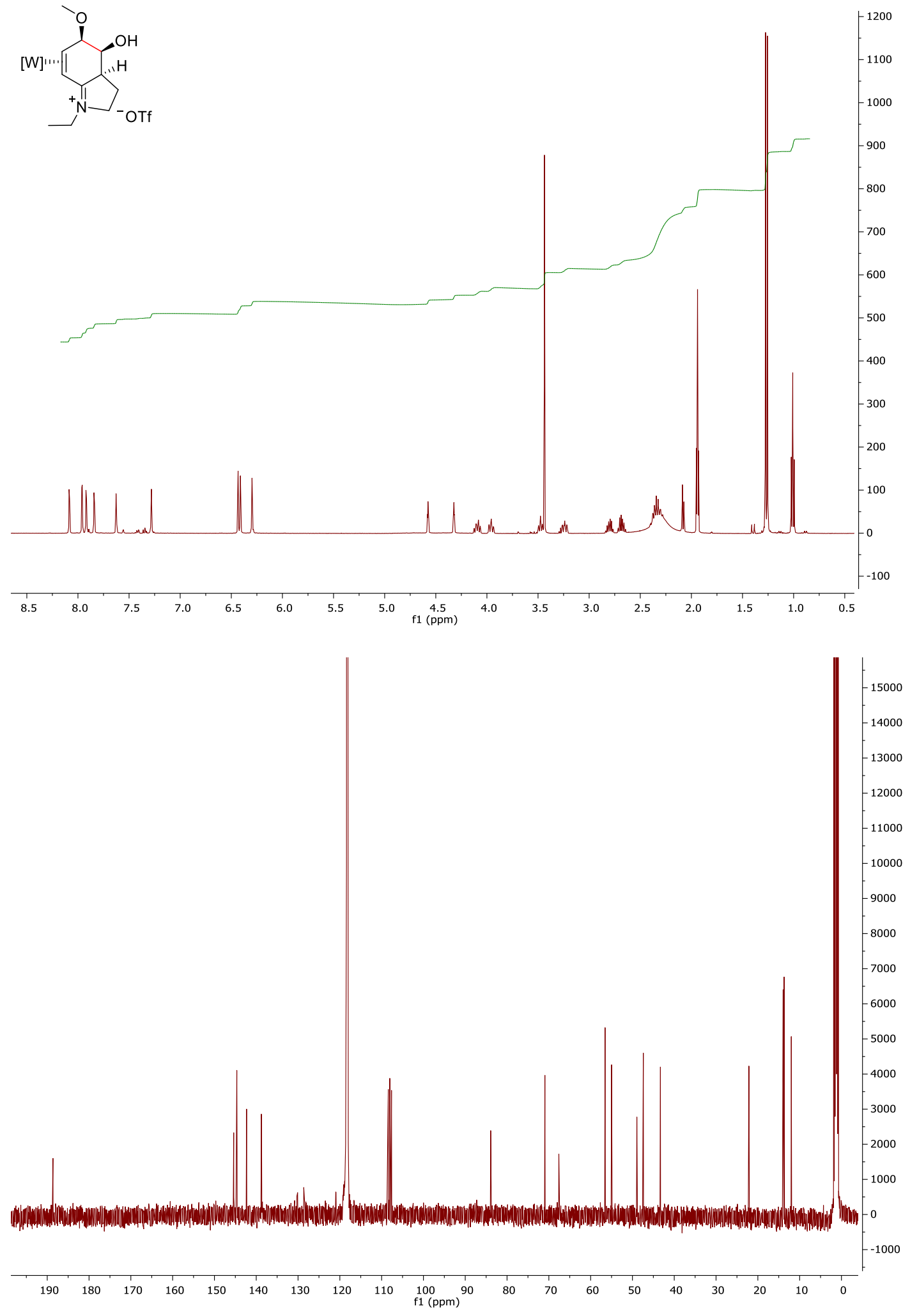
Compound 44:
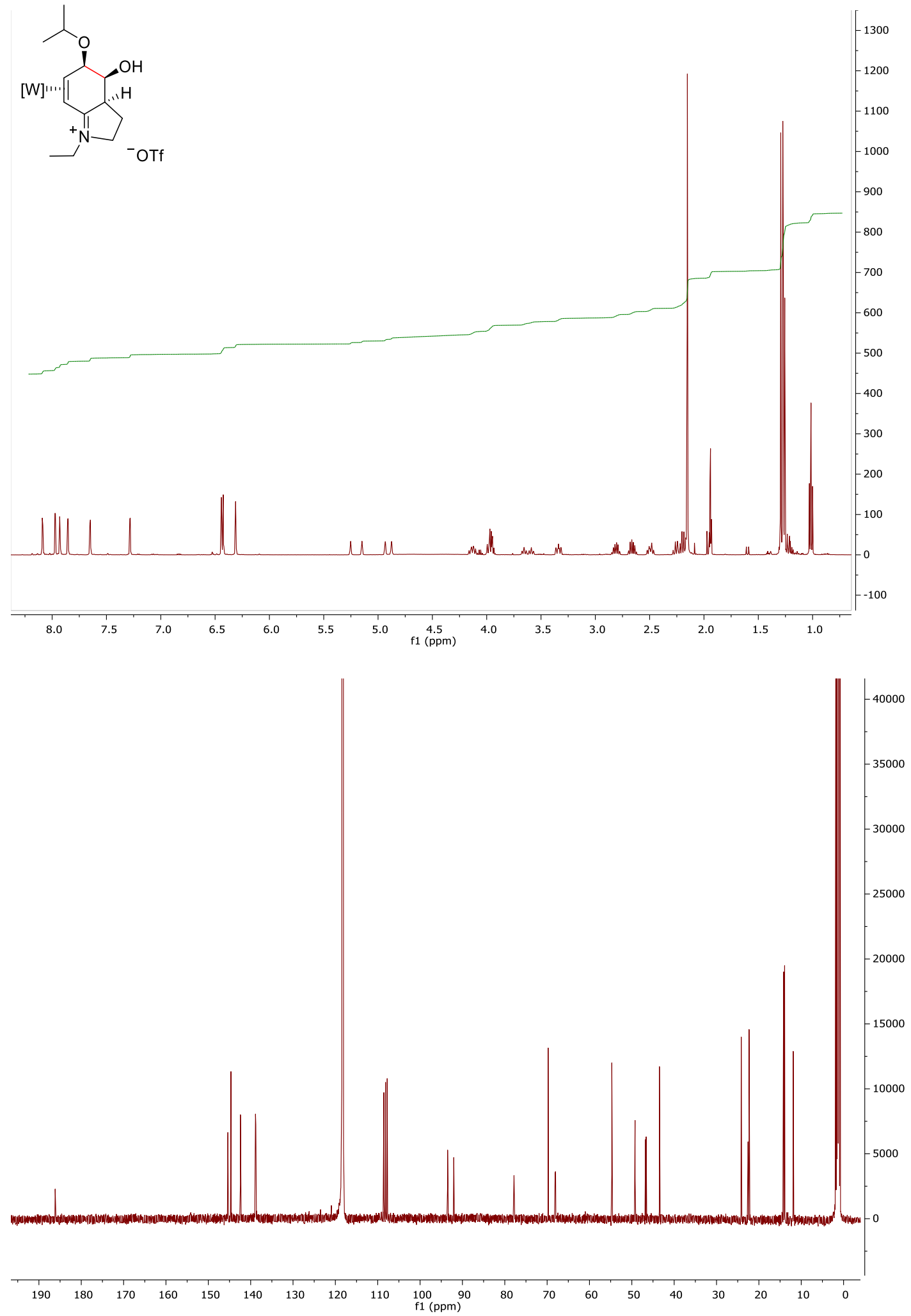
Compound 46:
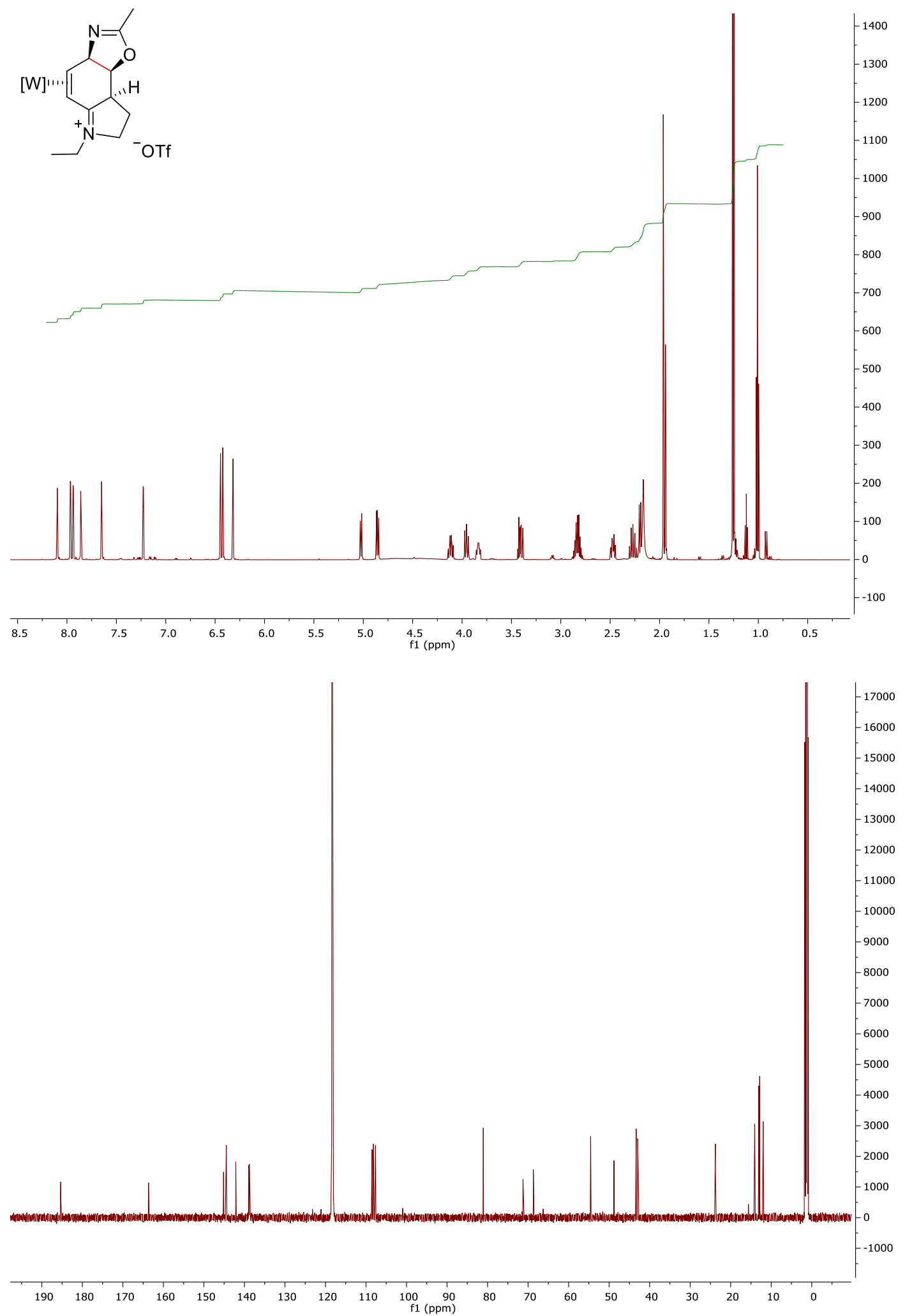
Compound 47:
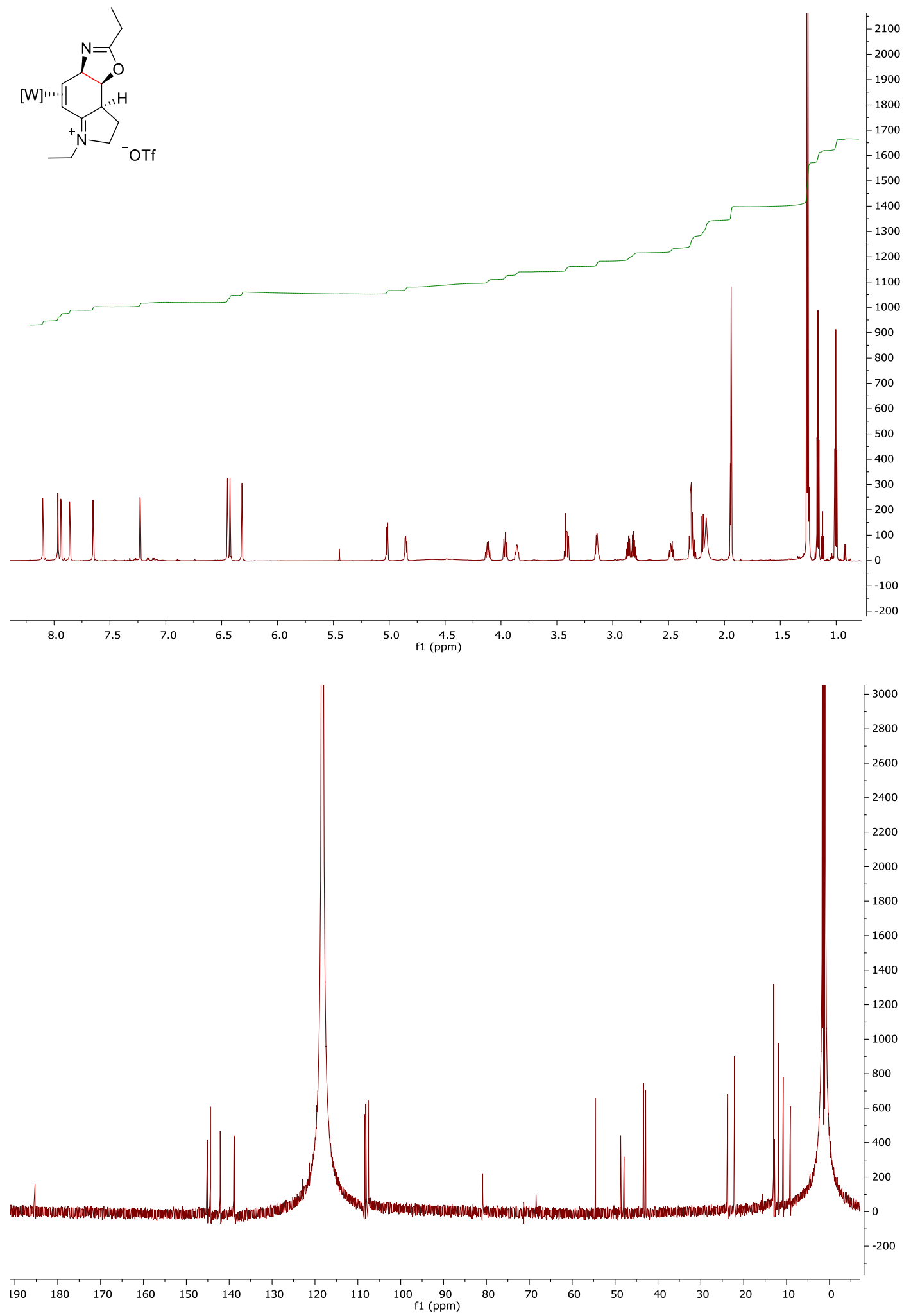
Compound 48:
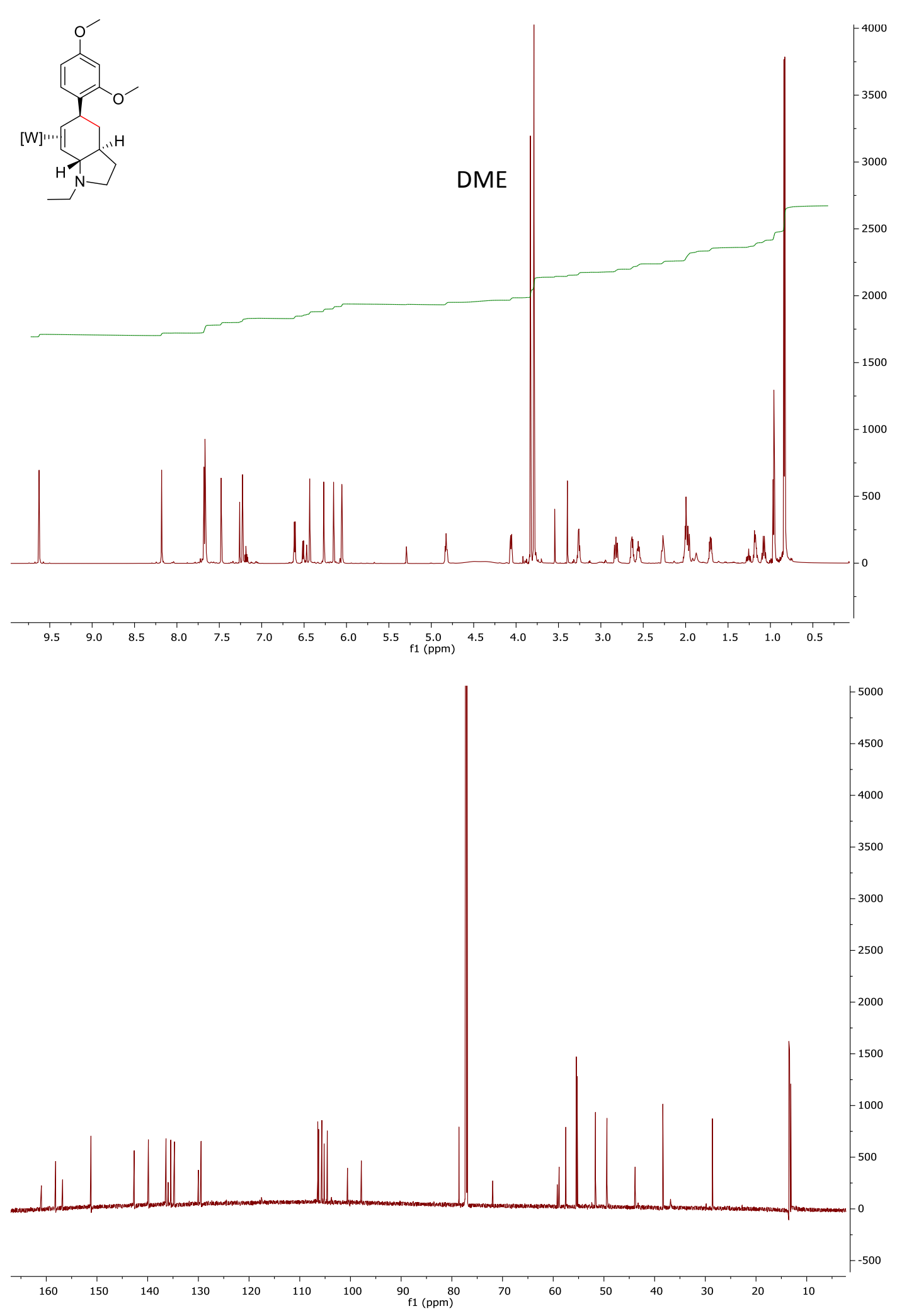


\section{Compound 49:}
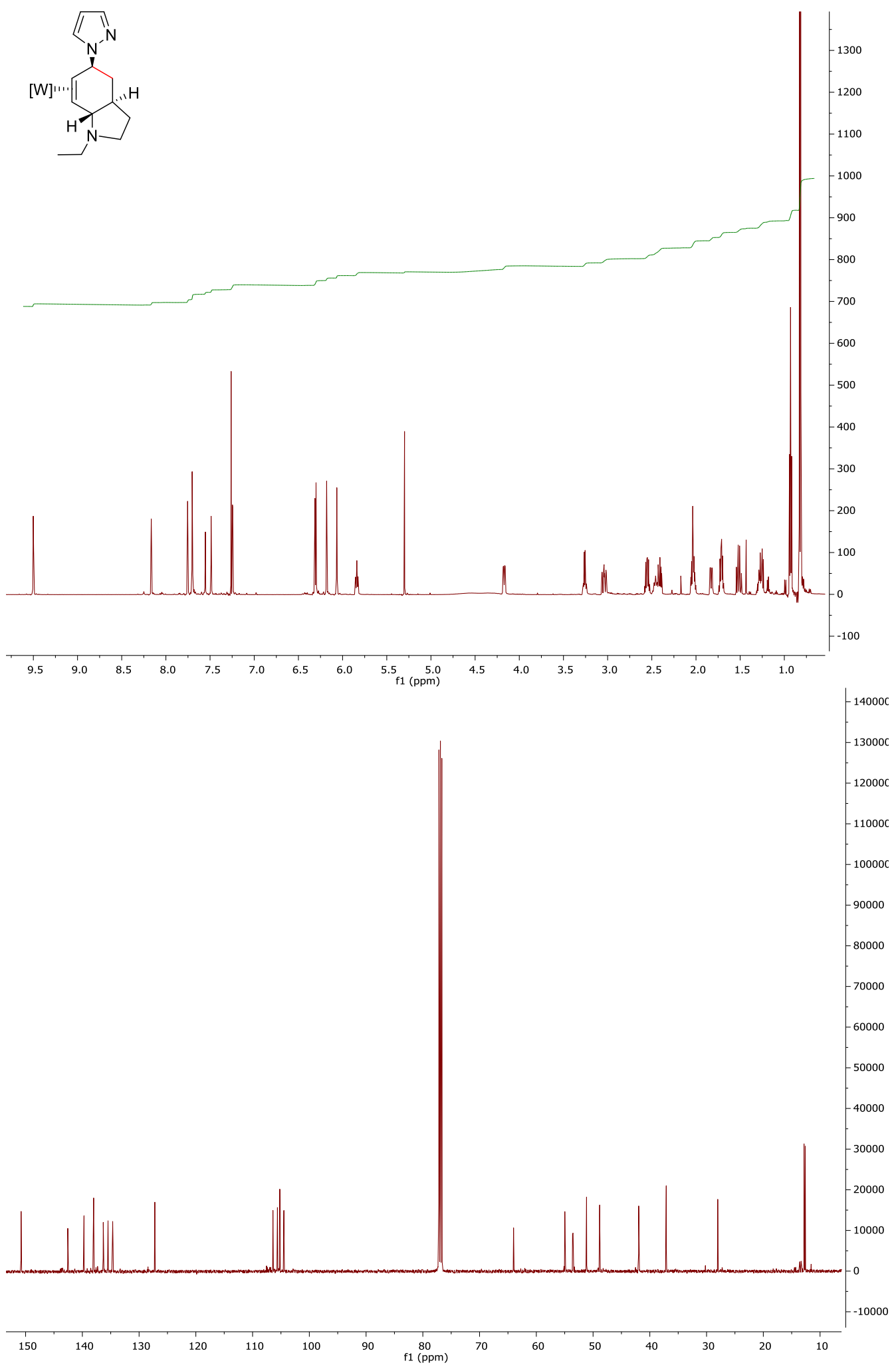
Compound 50:
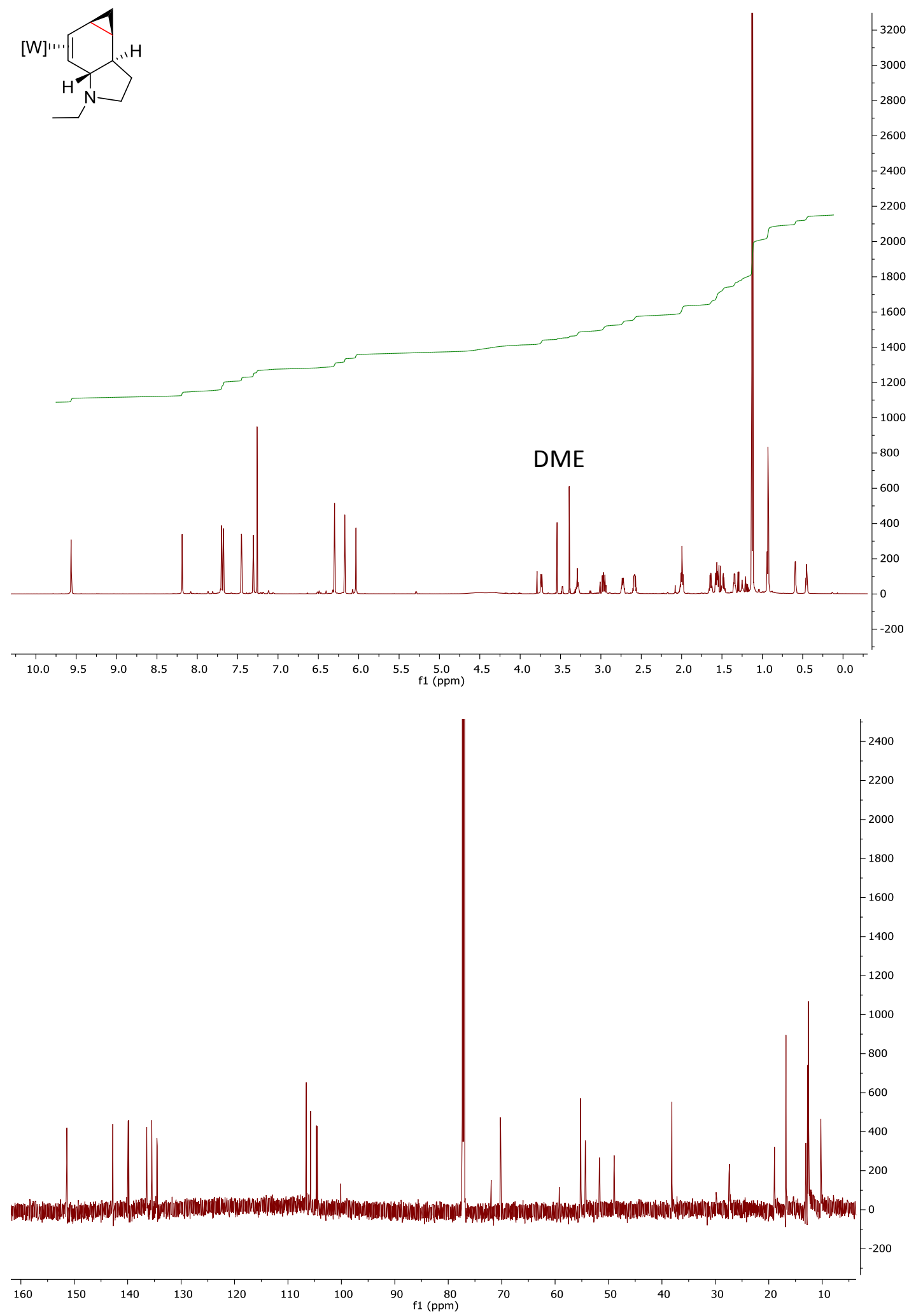
Compound 51:
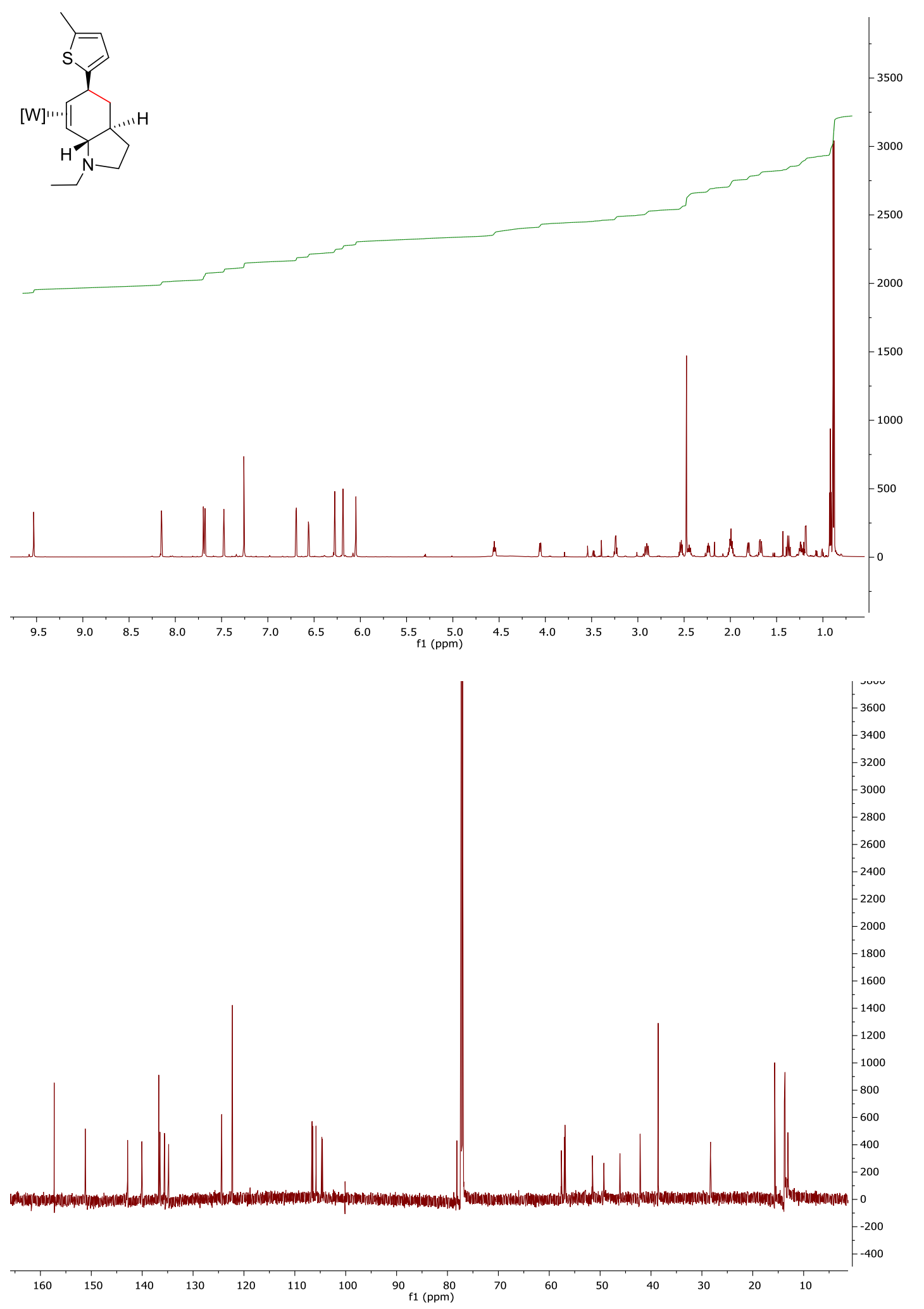


\section{Compound 52:}

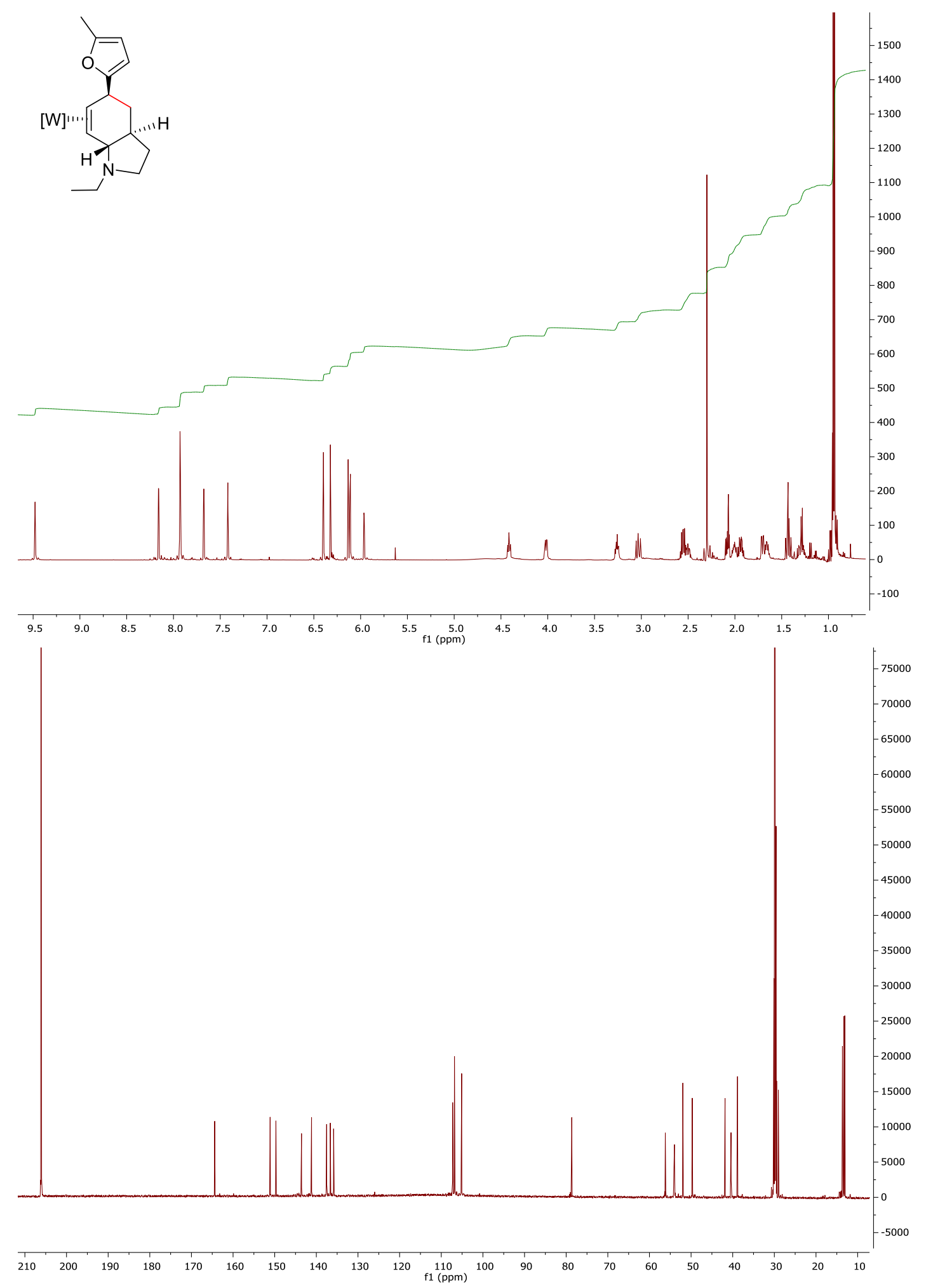


Compound 53:
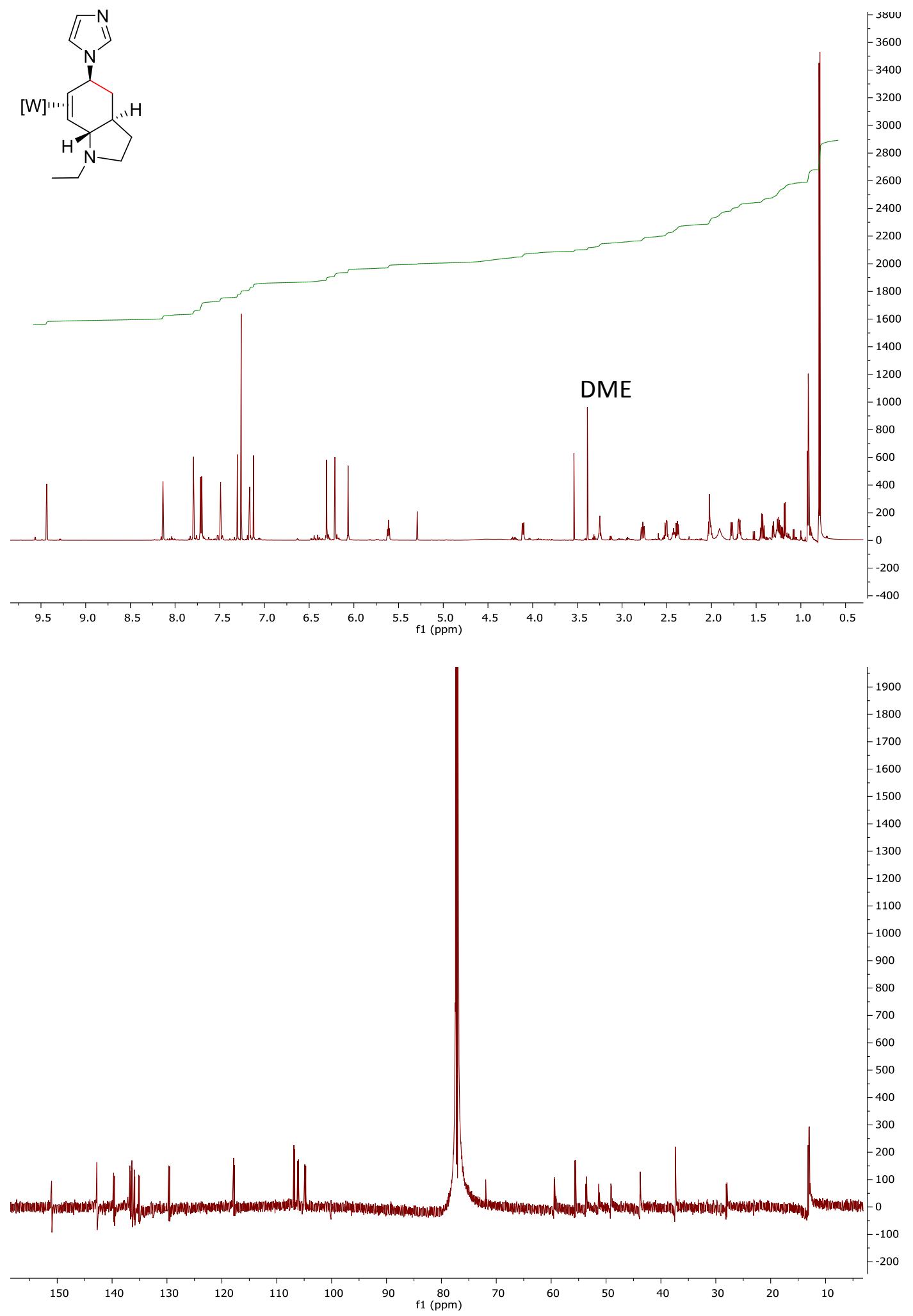


\section{Compound 54:}
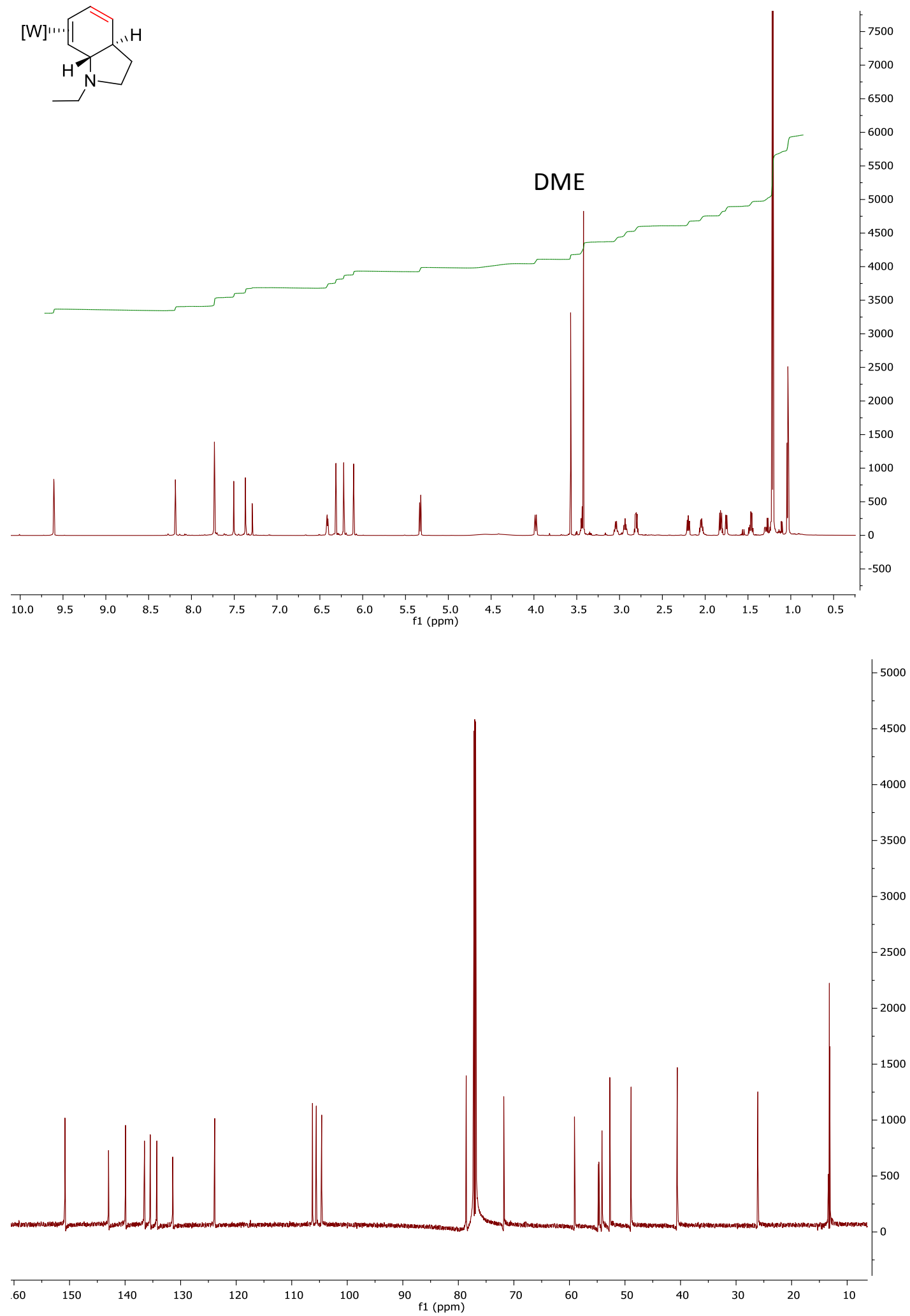
Compound 56:
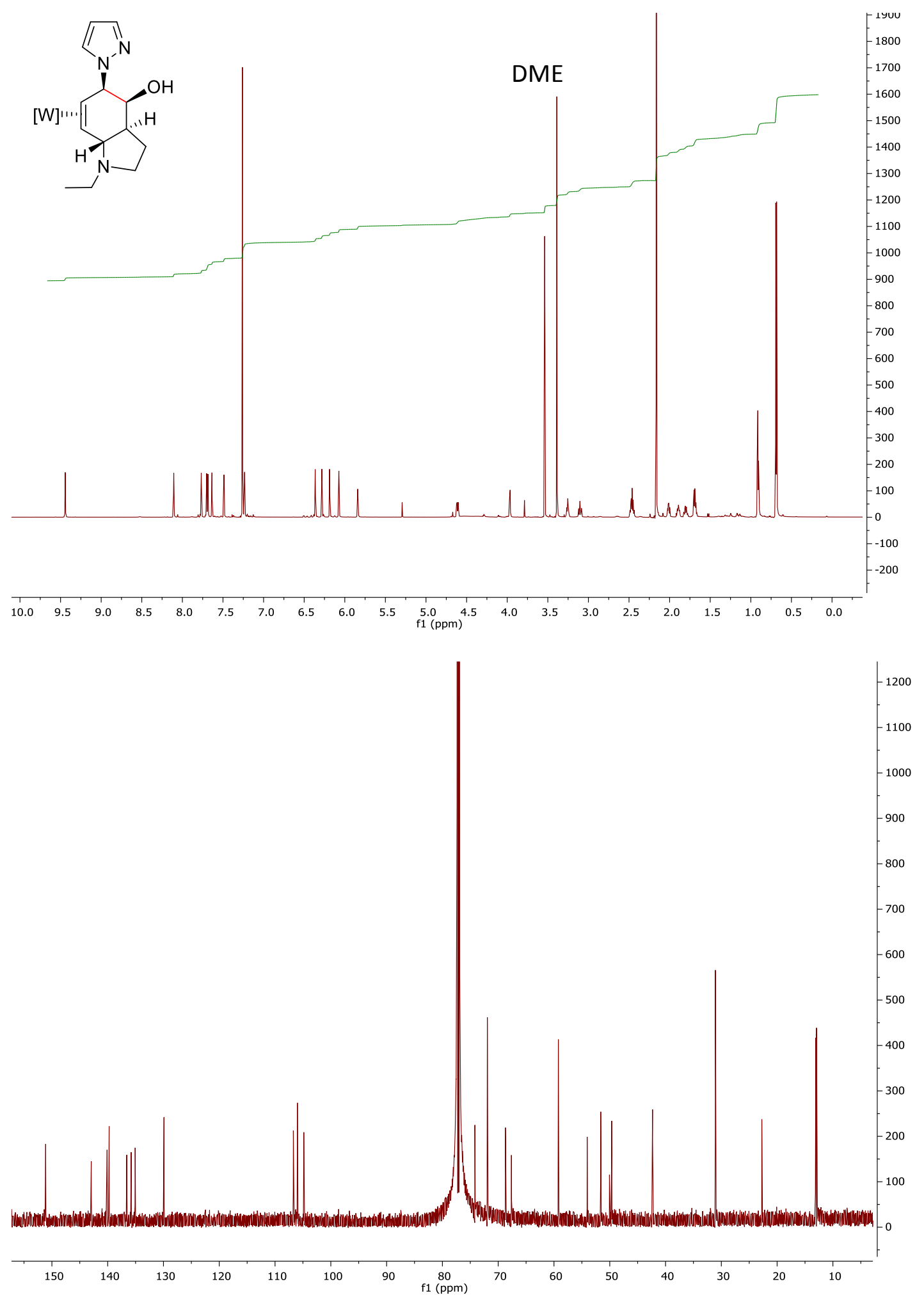
Compound 57:
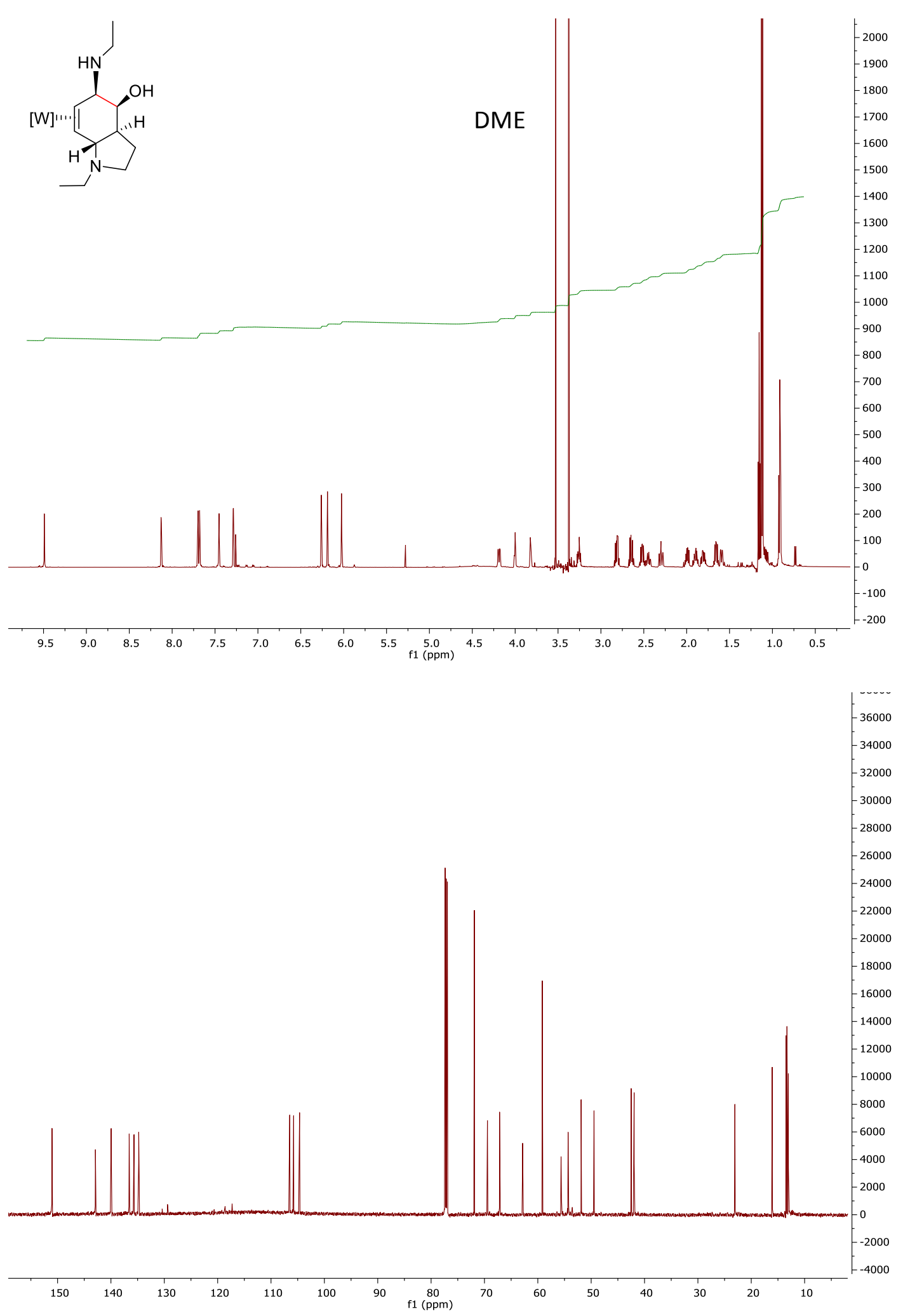


\section{Compound 58:}

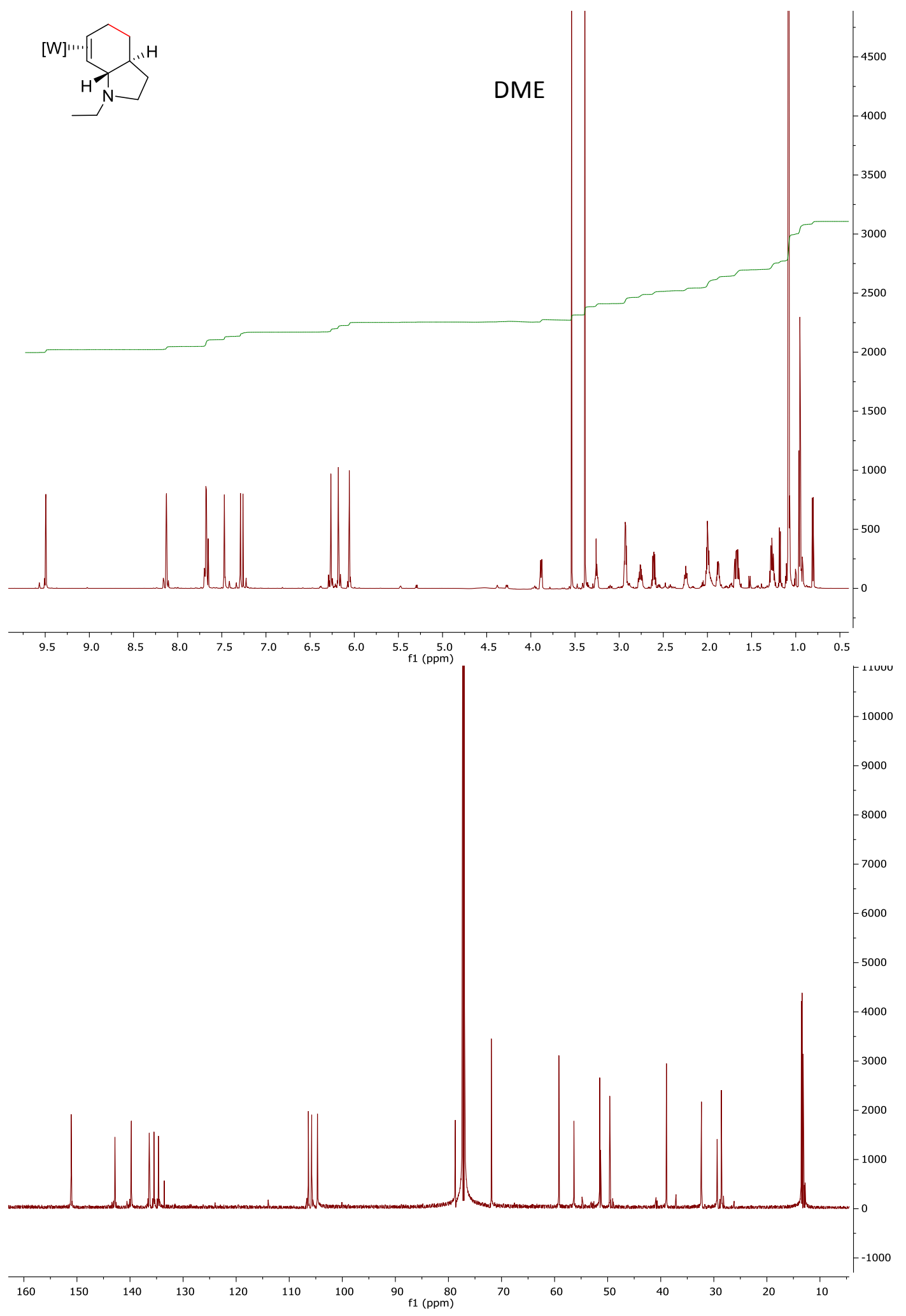


Compound 59:

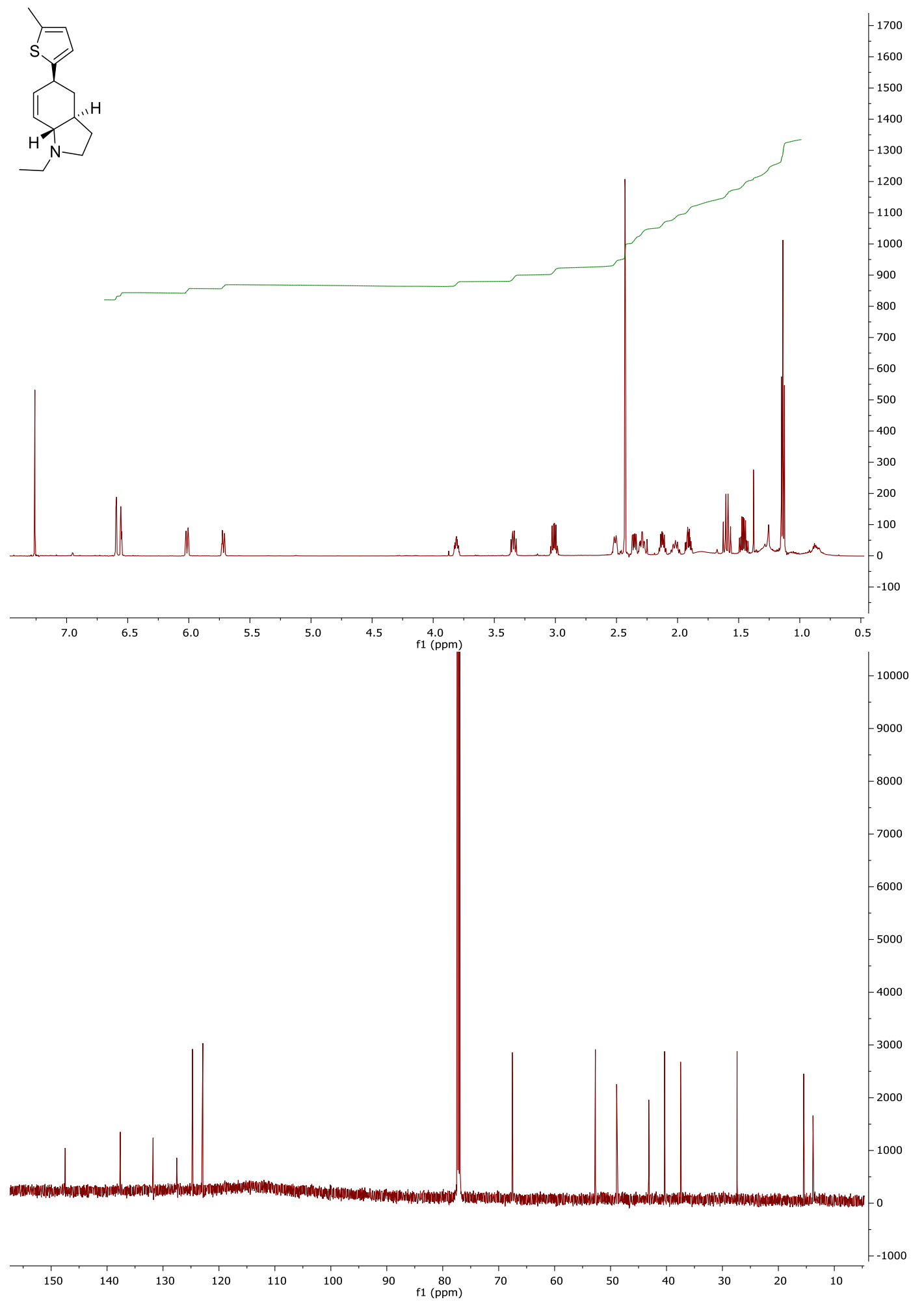


Compound 60:

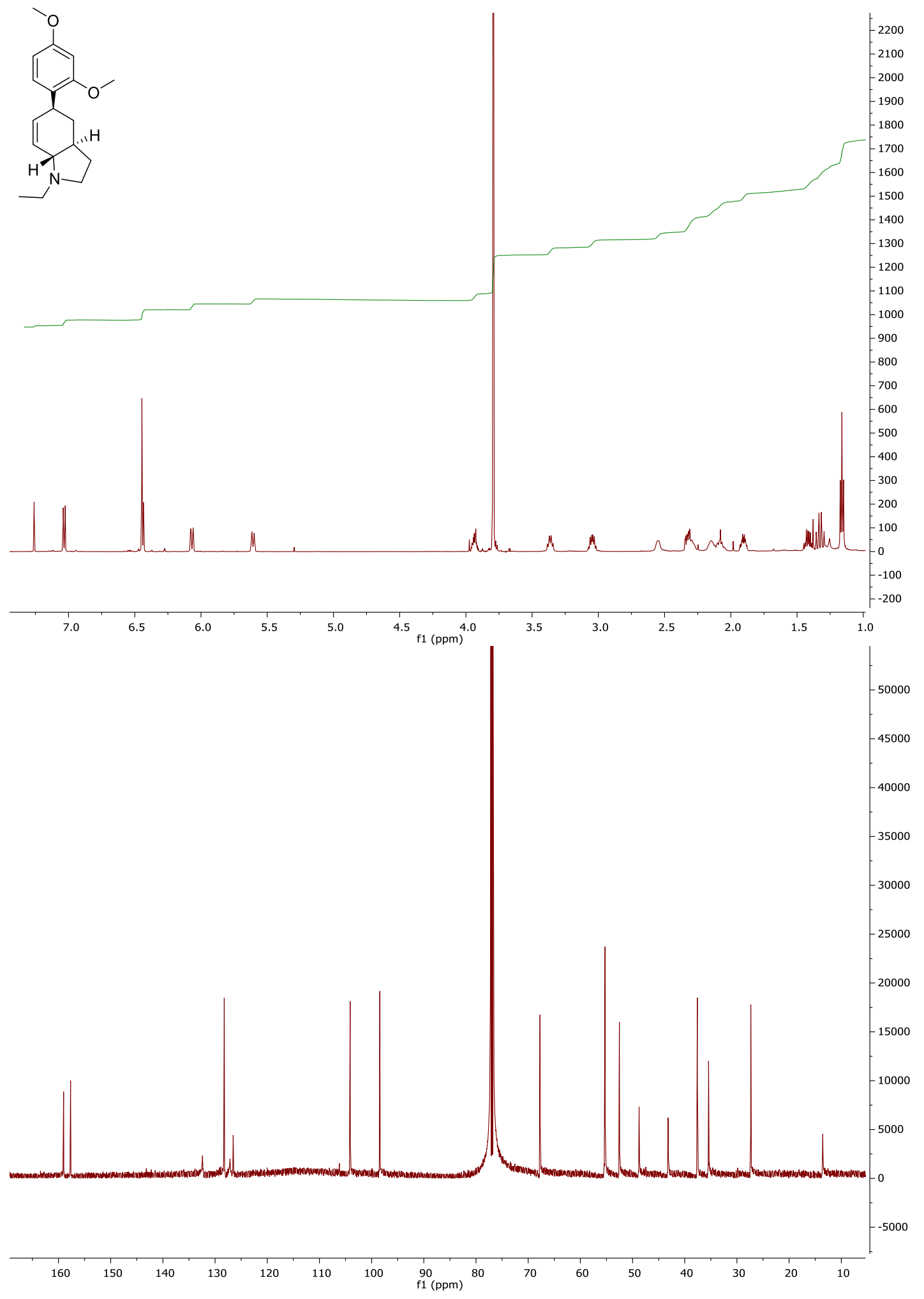


Compound 61:

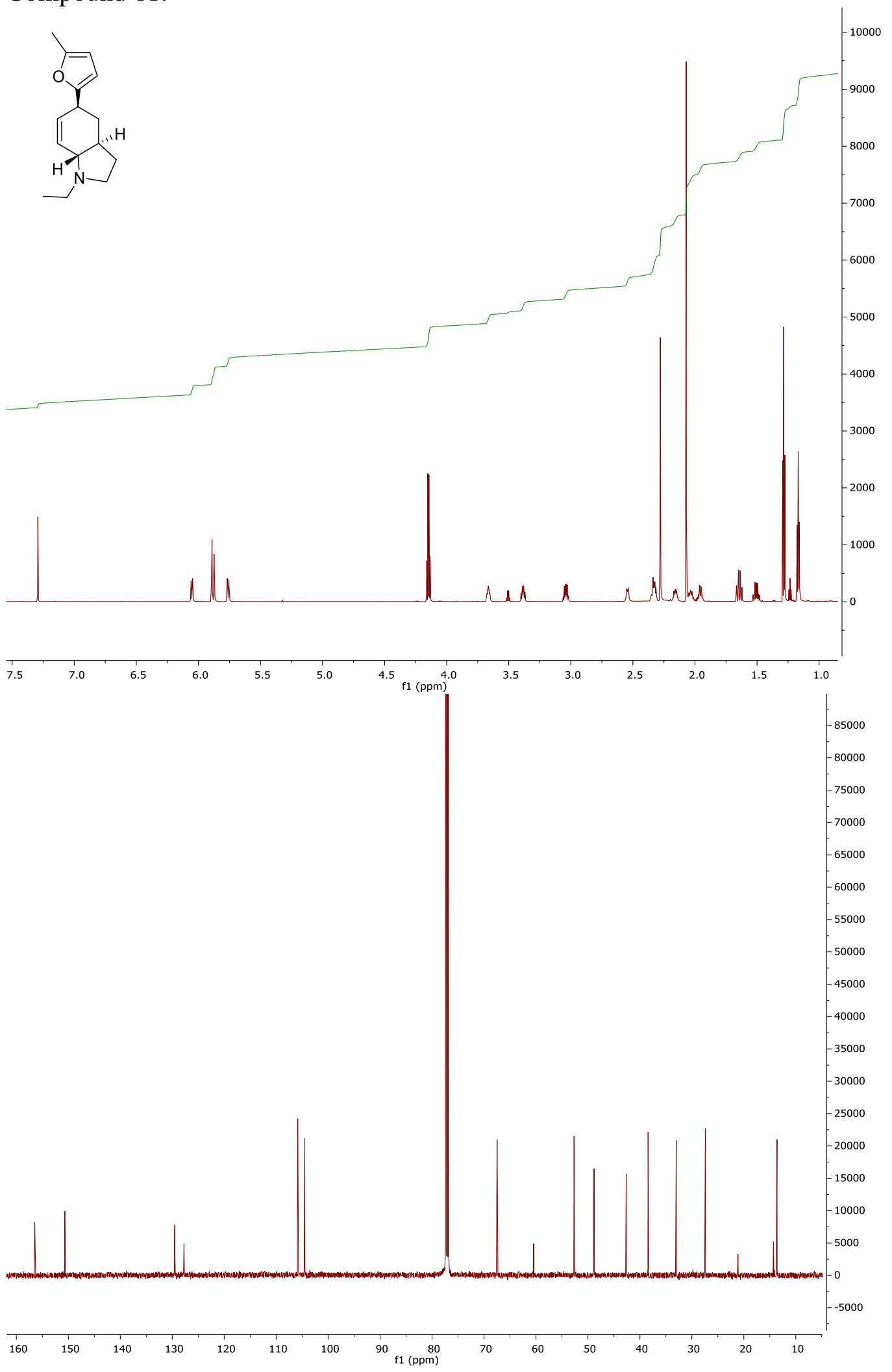




\section{Compound 62:}
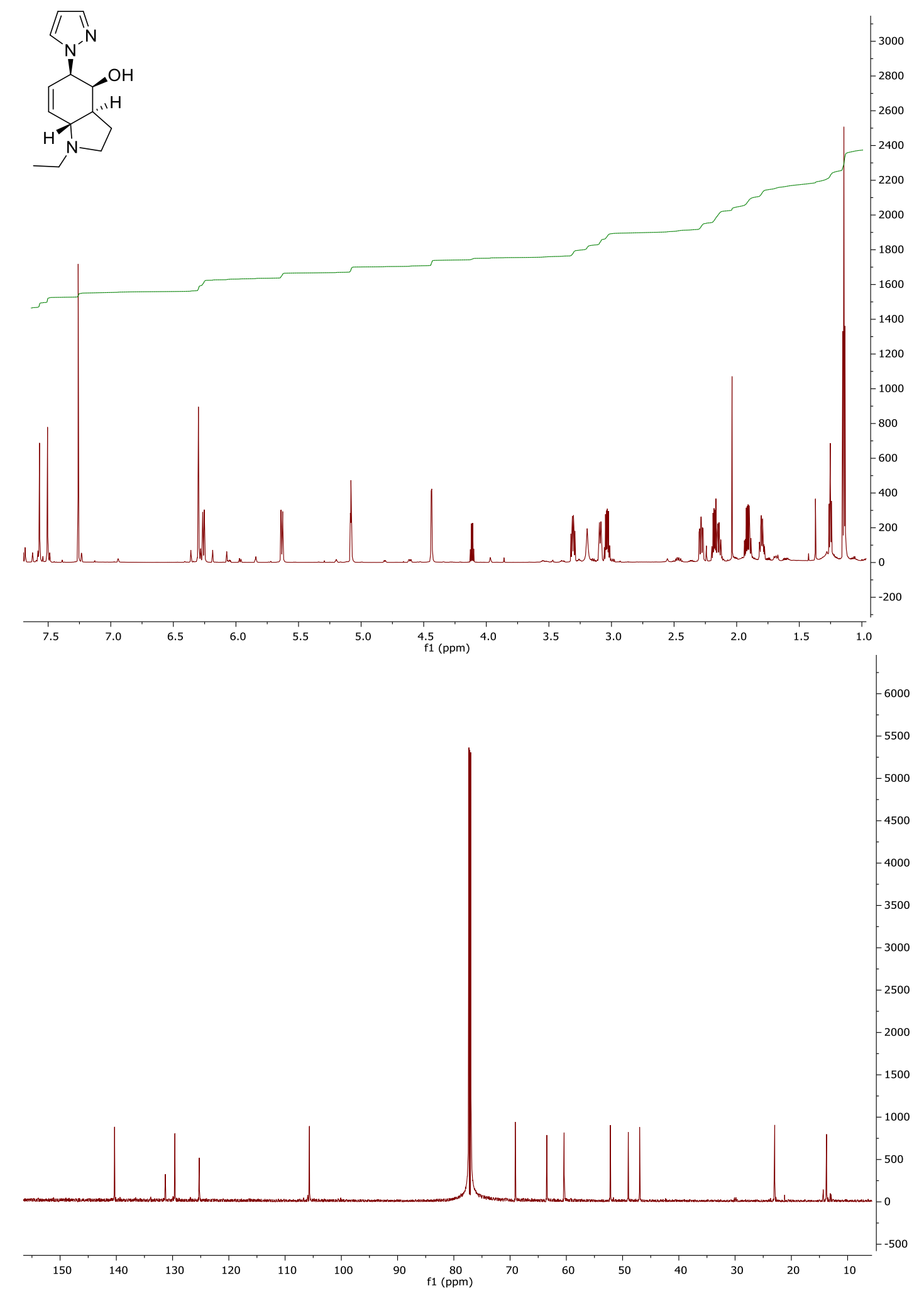


\section{Compound 63:}
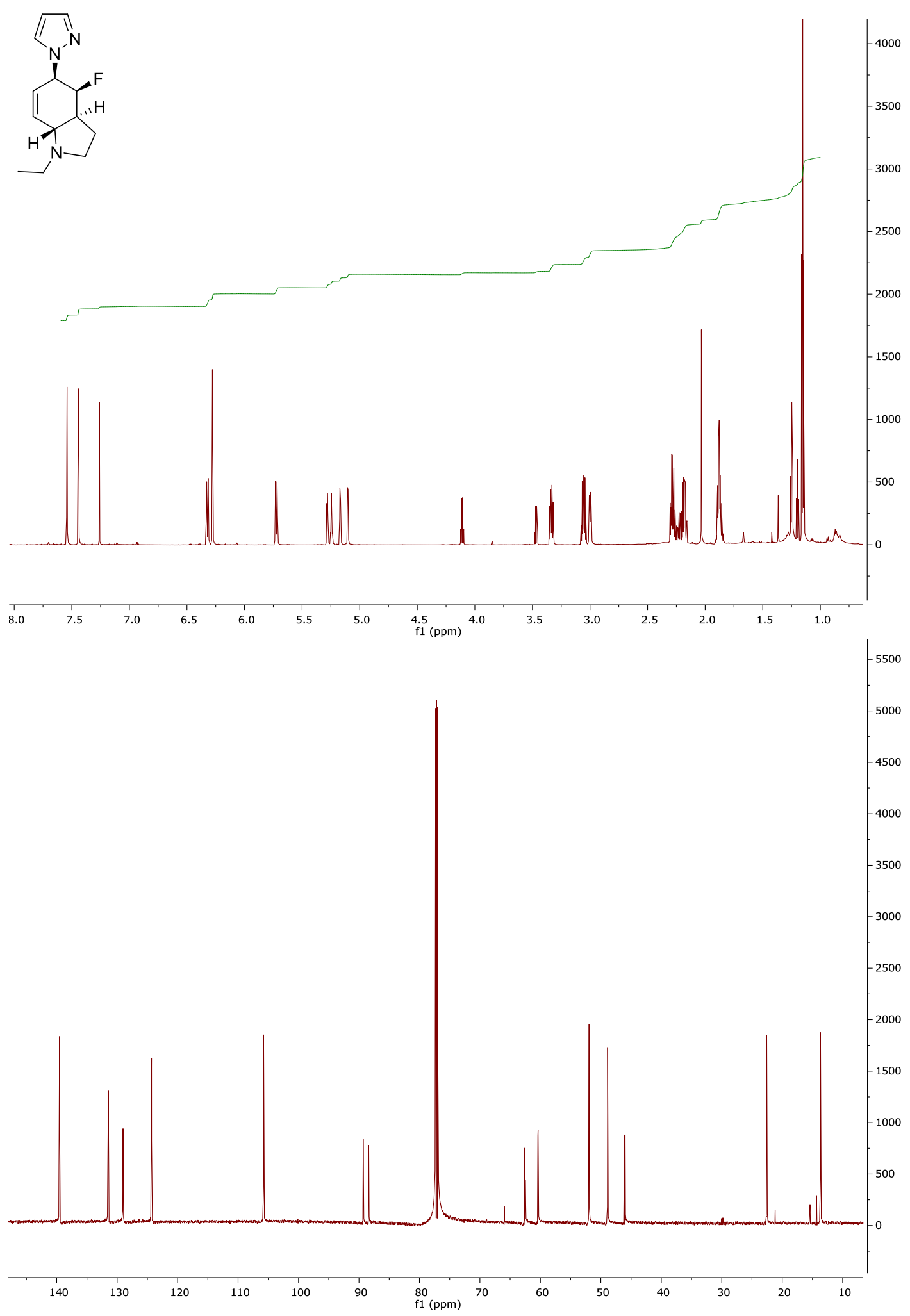
Compound 64:

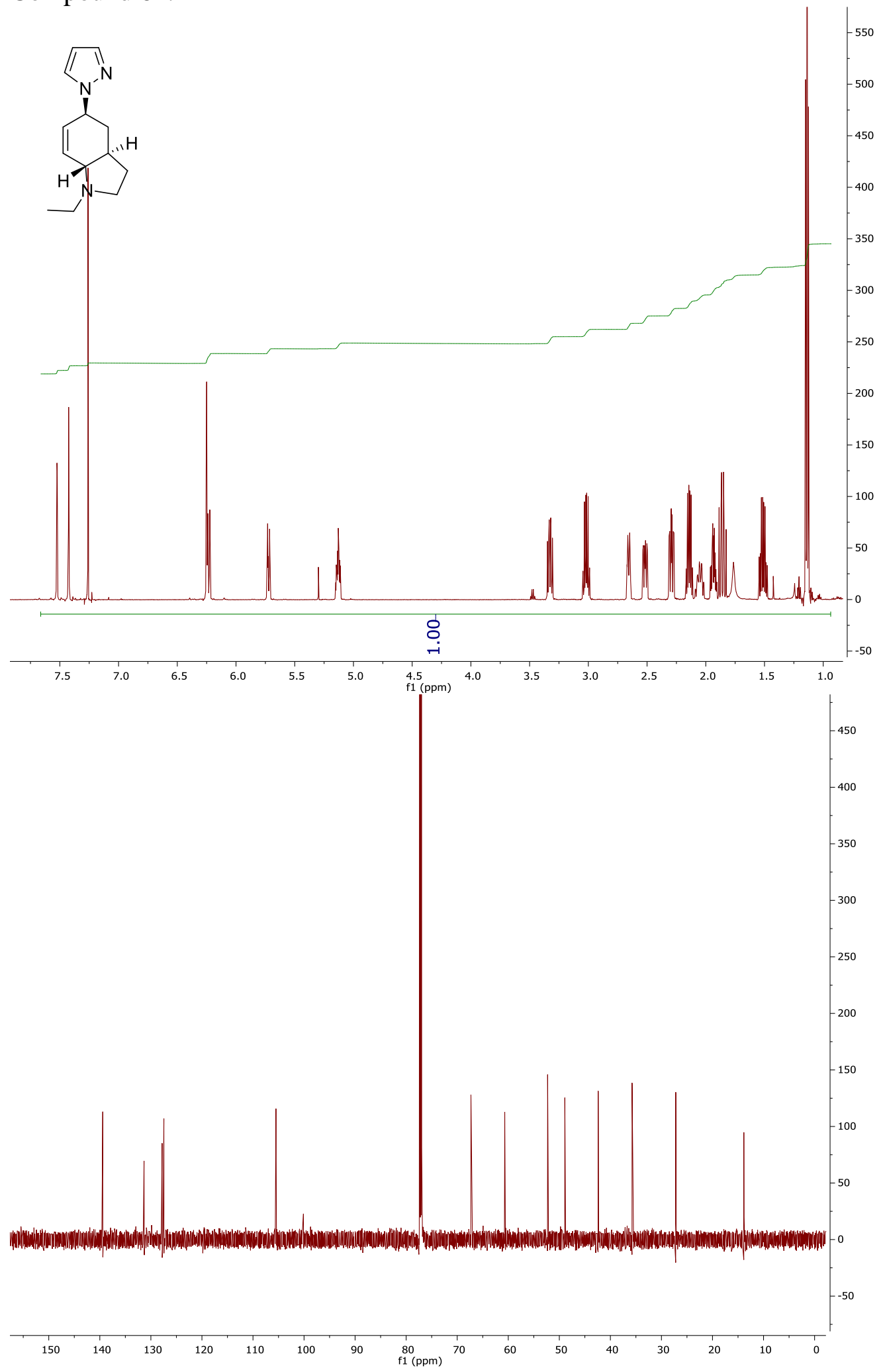


Compound 65:
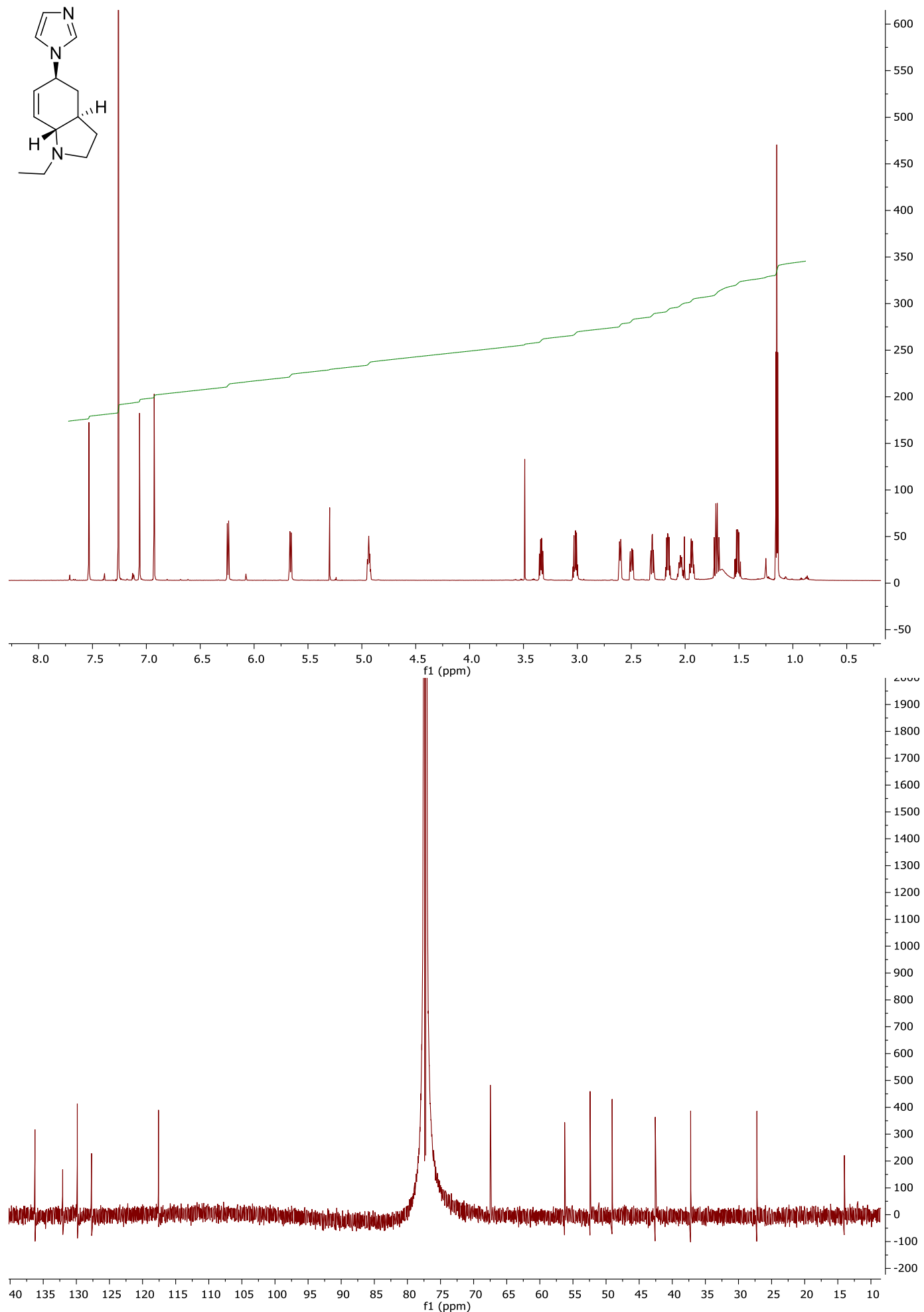
Compound 66:

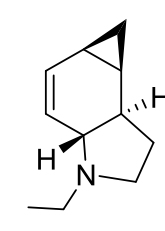




\section{Compound 71:}
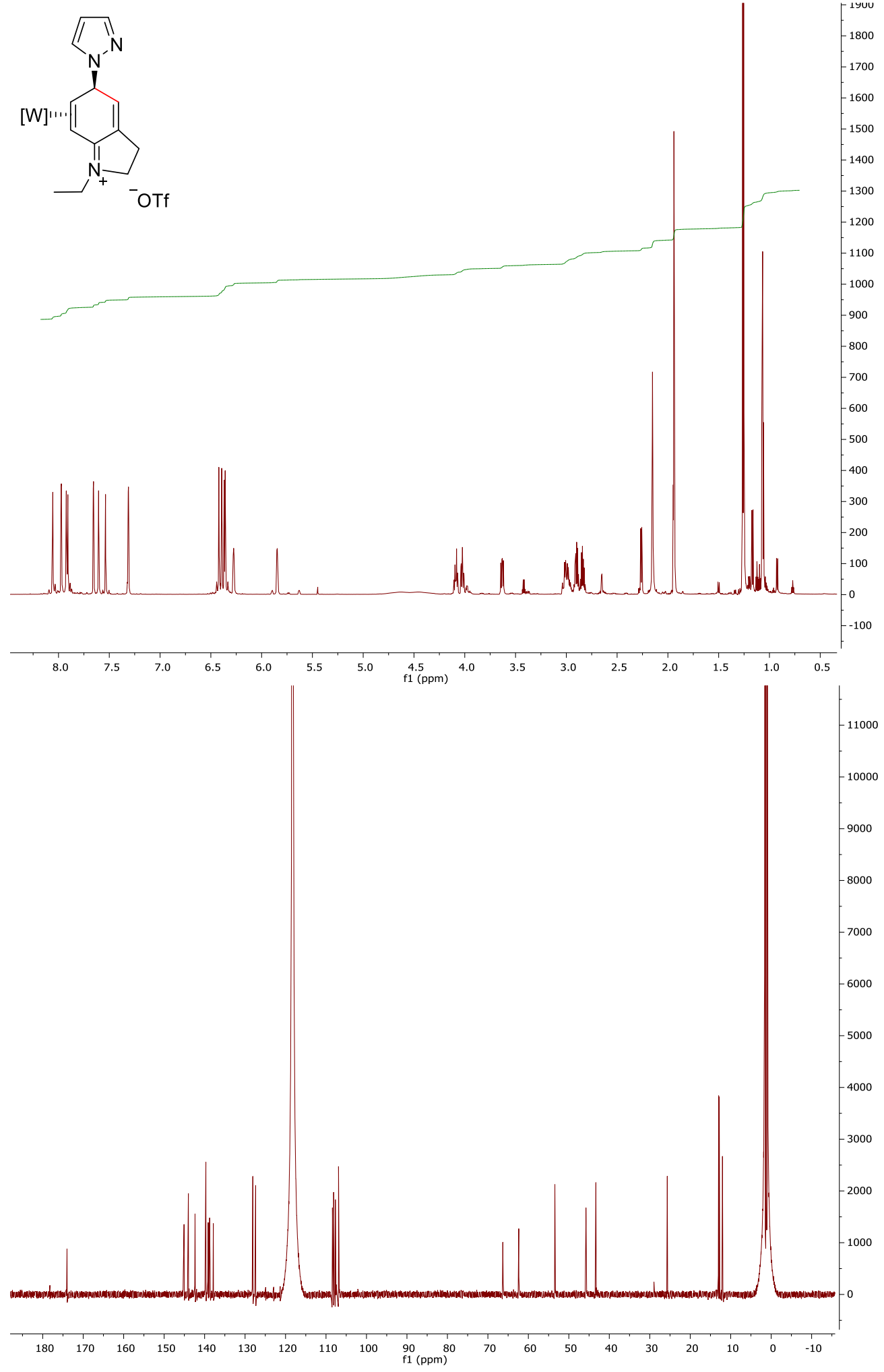
Compound 72:

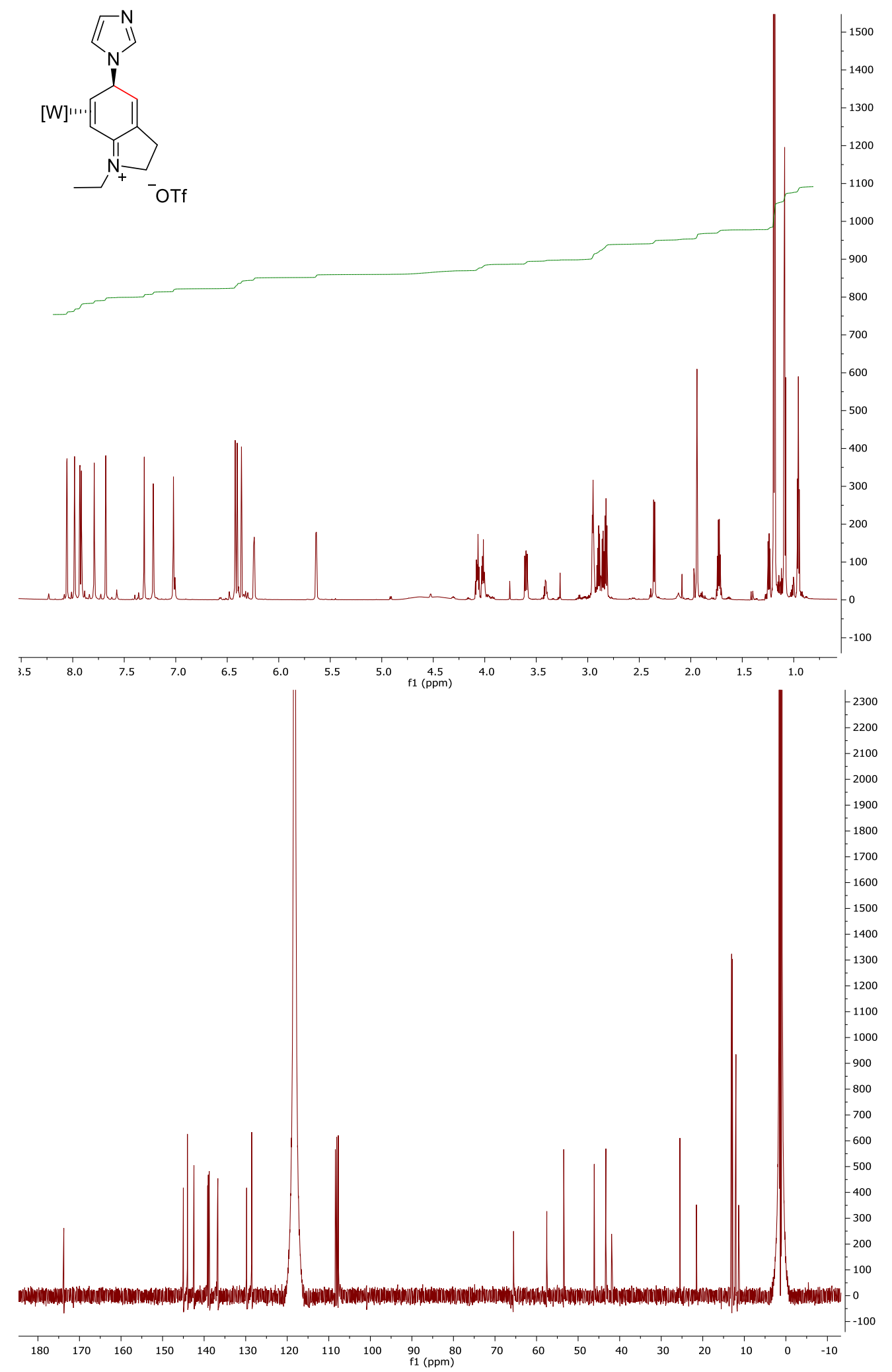


Compound 75:
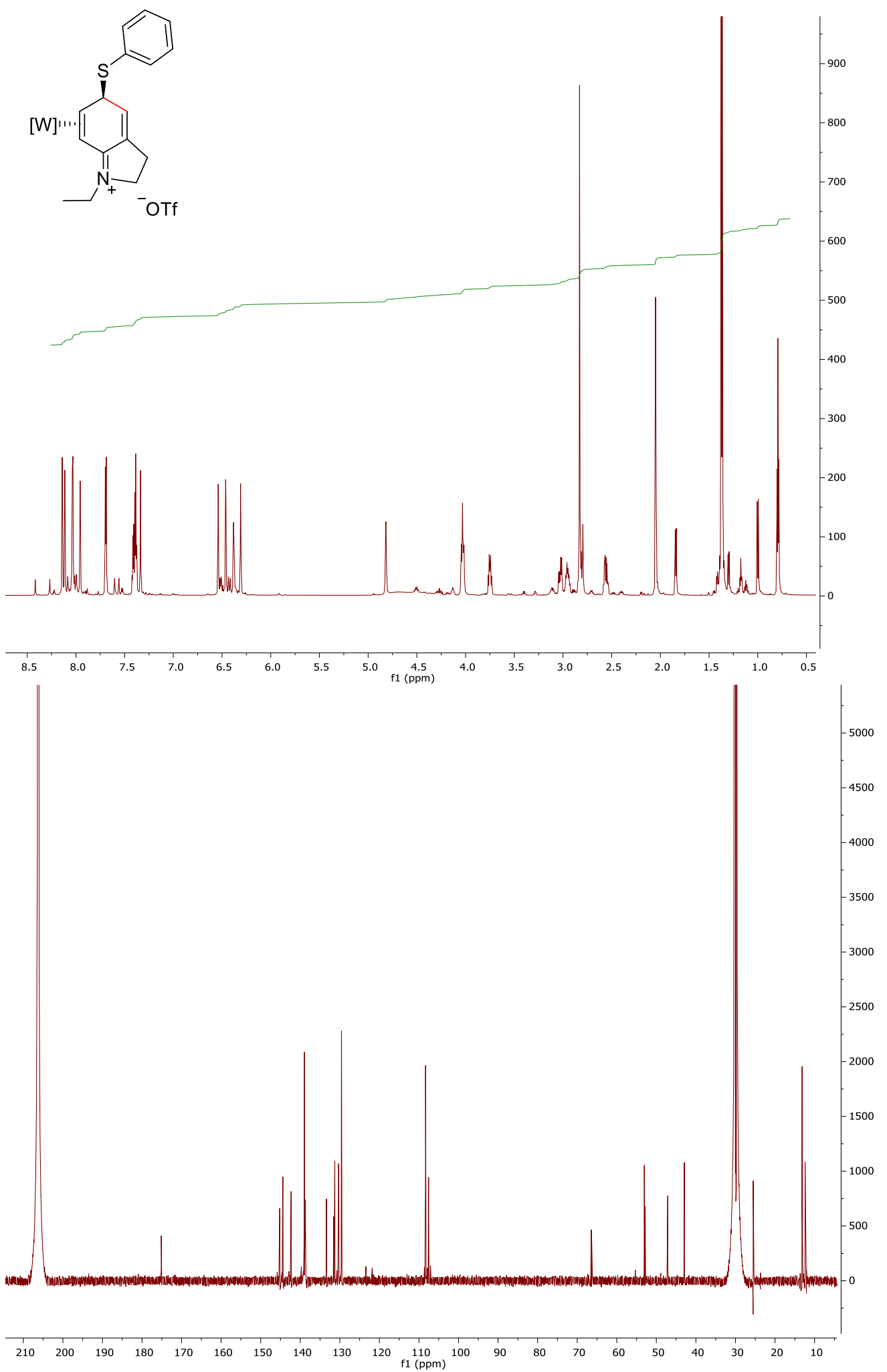
Compound 76:<smiles>CCCN[C@H]1C2CC[N+](CC)=C2C=C(C)C[C@H]1n1cccn1</smiles>
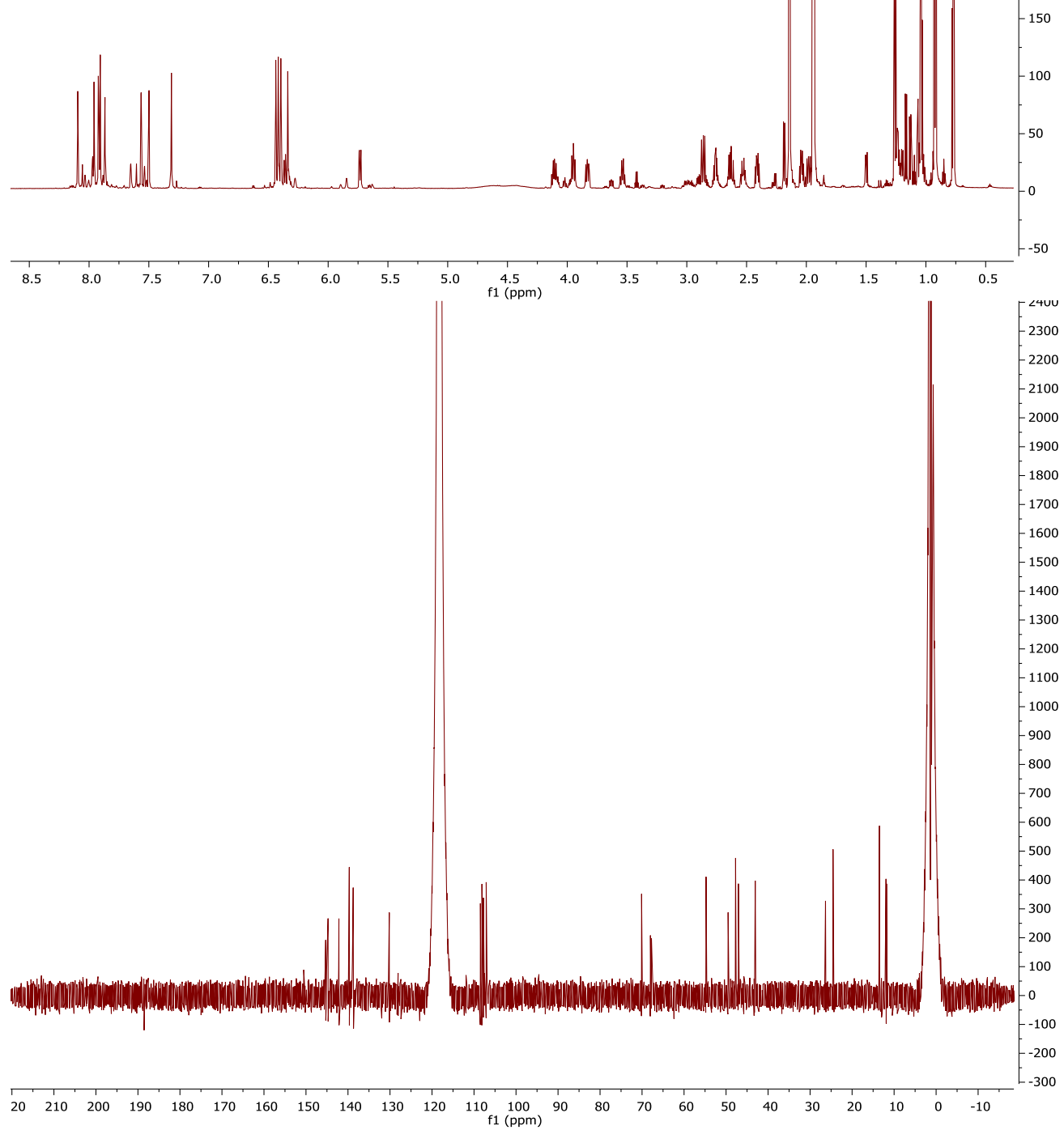
Compound 80:
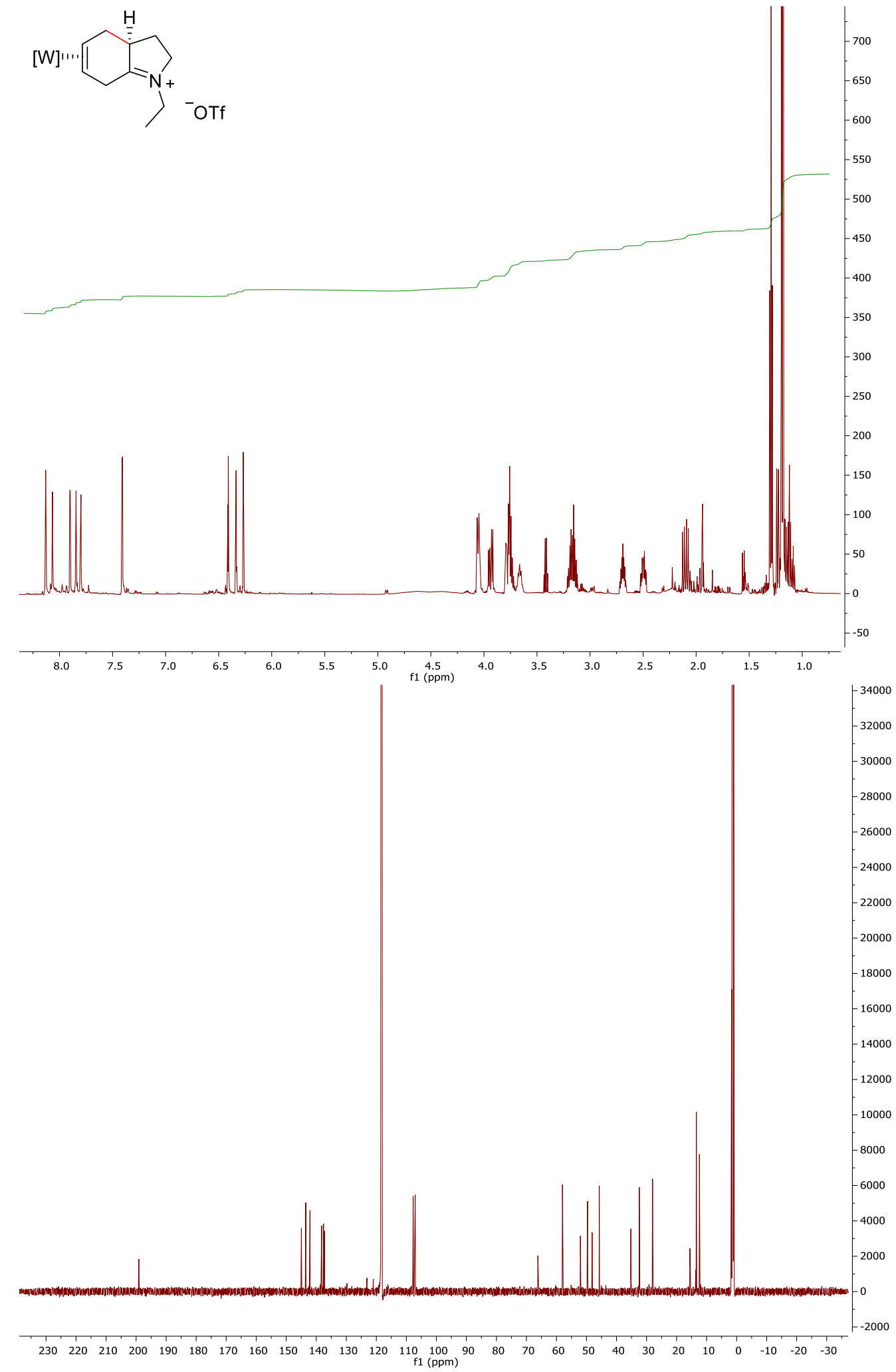
Compound 81:

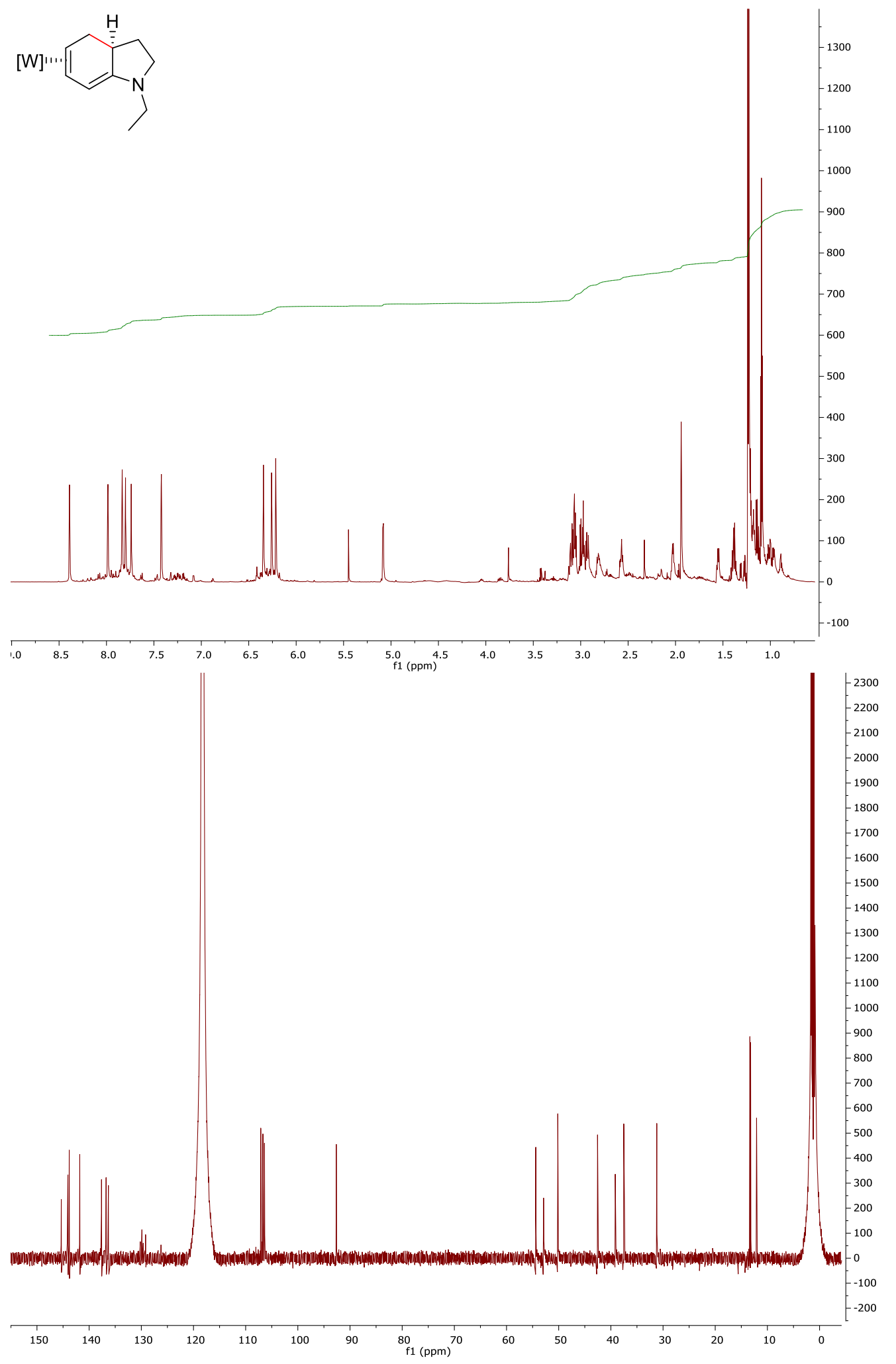




\section{Compound 82:}

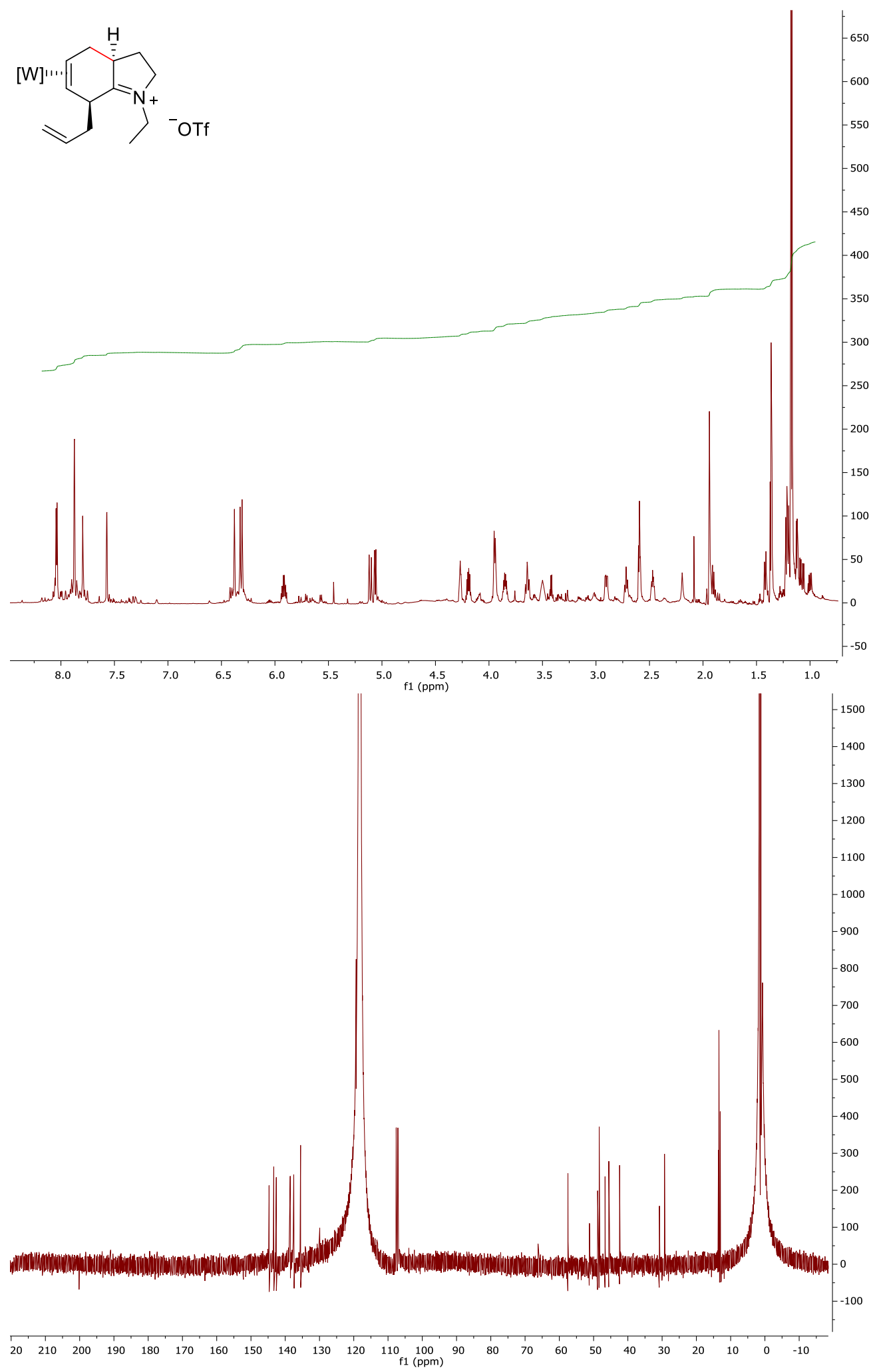


Compound 83:

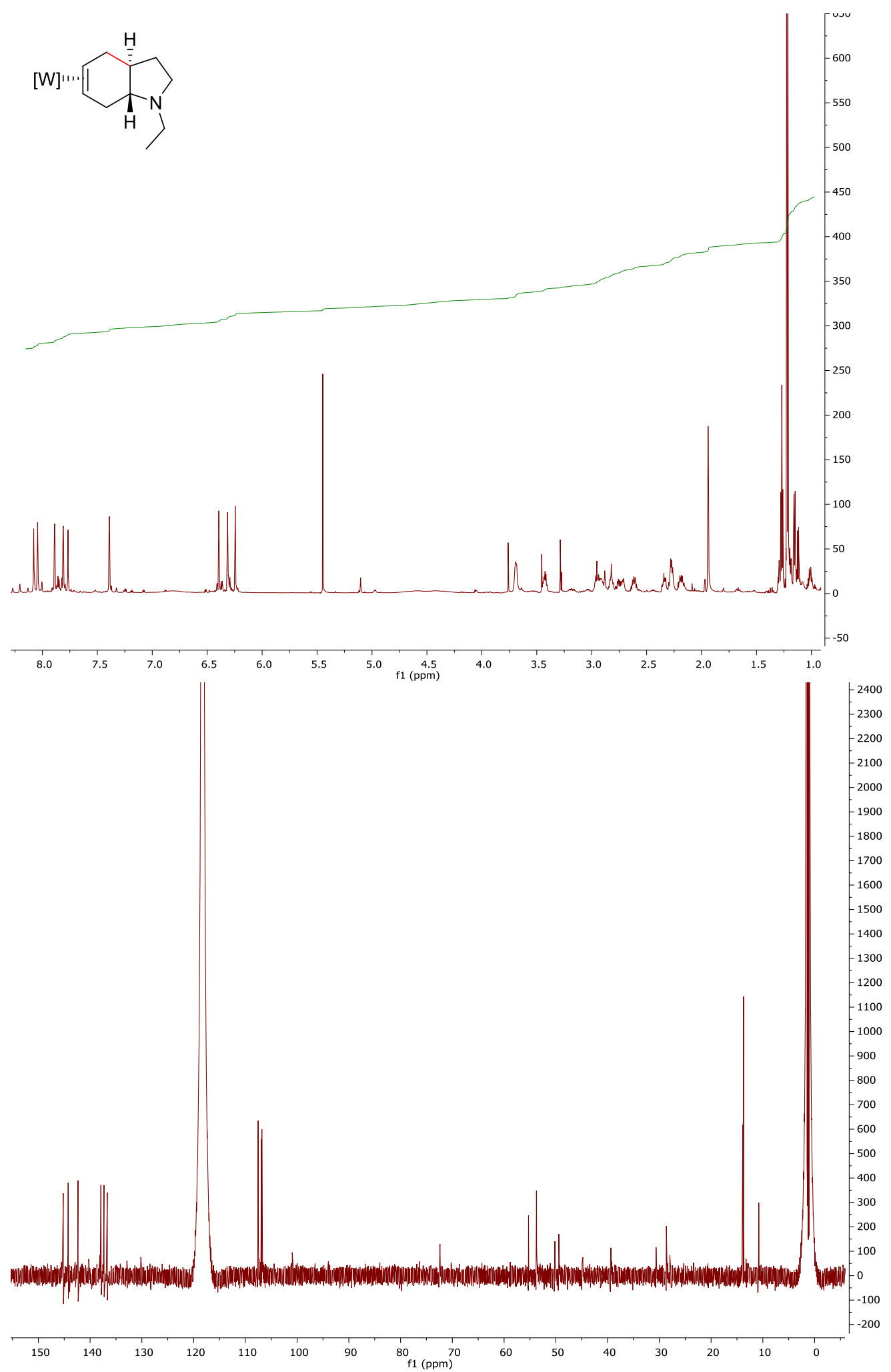


Compound 84:

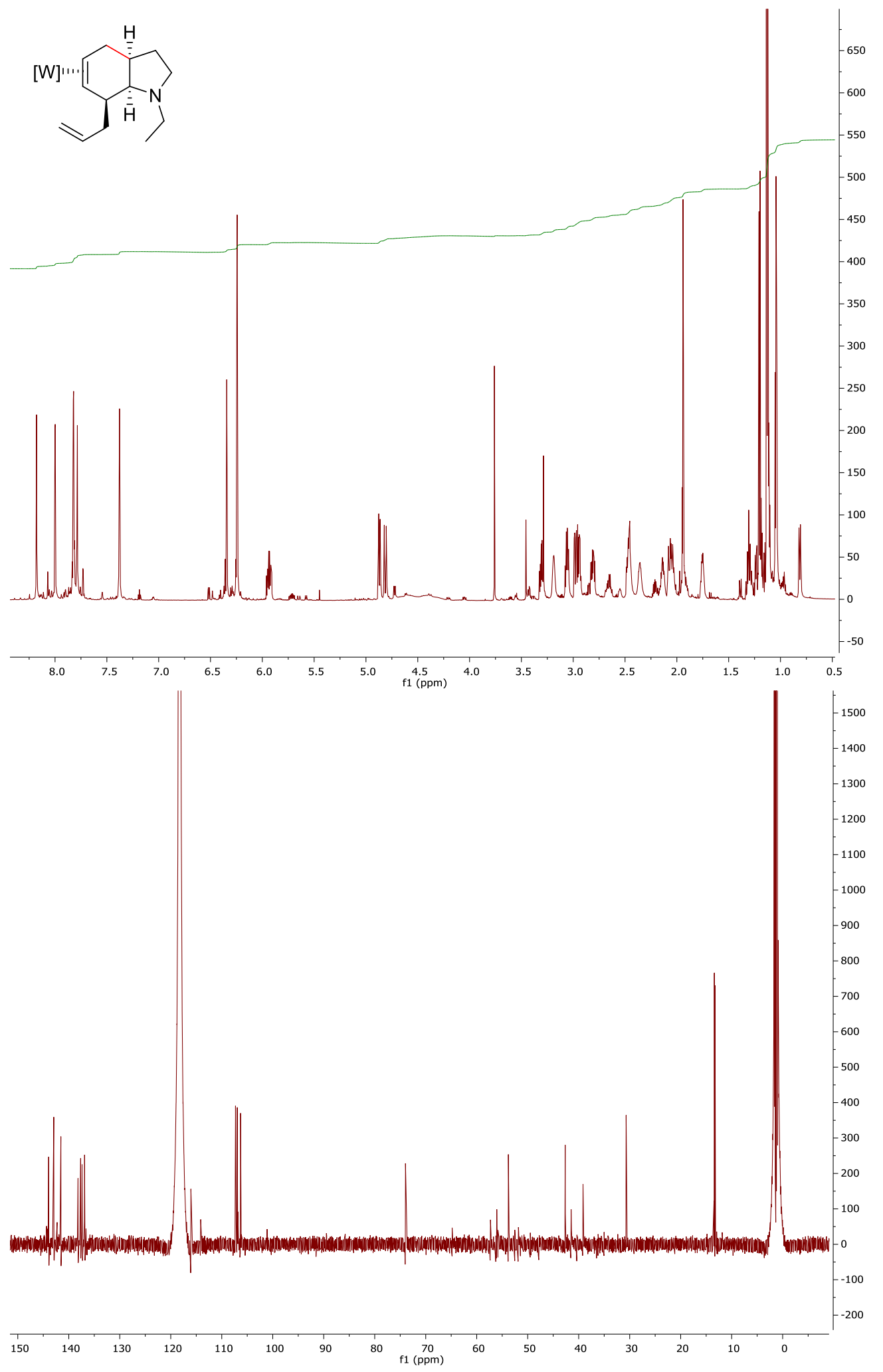


Compound 93:

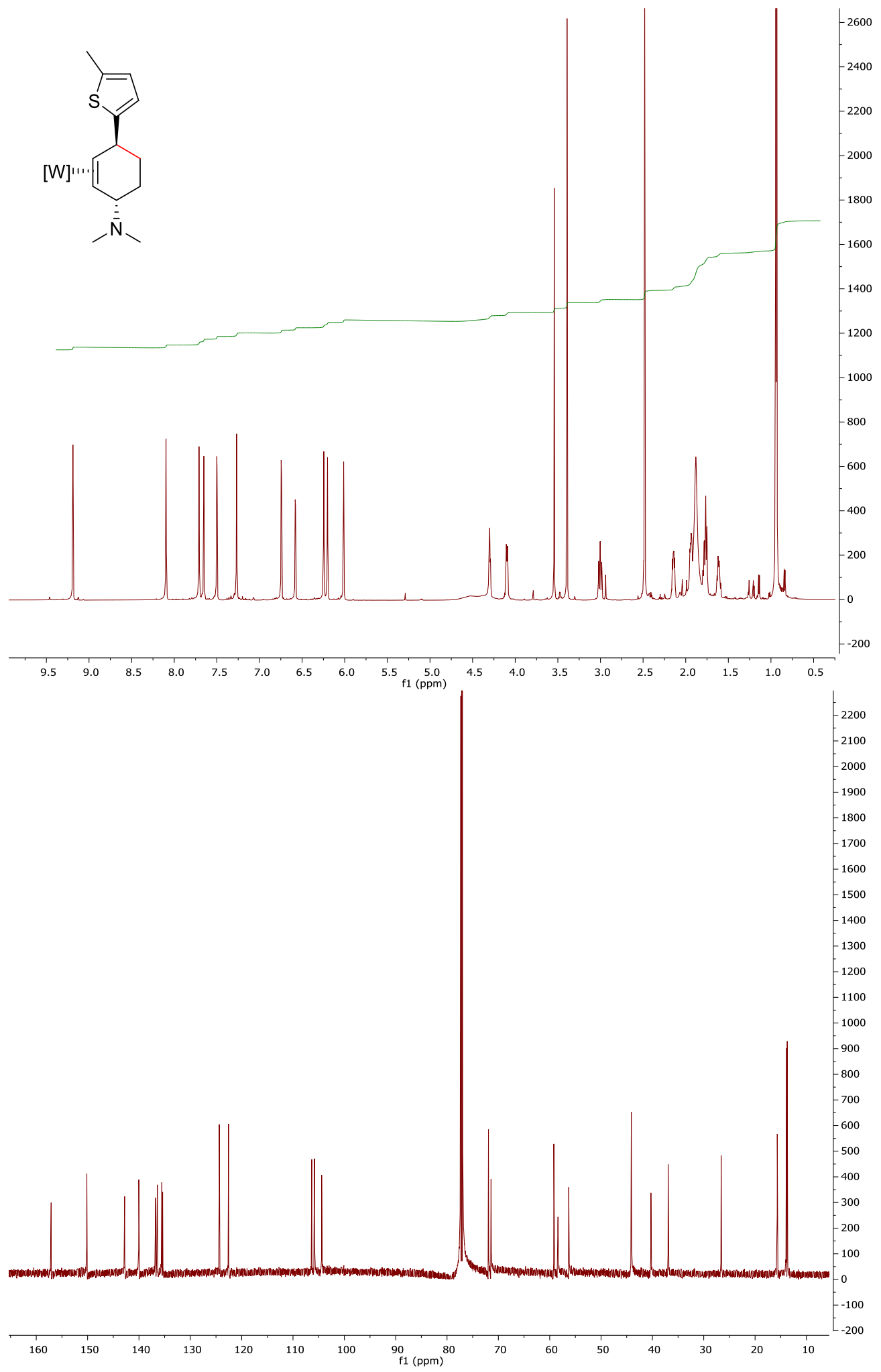




\section{Compound 94:}

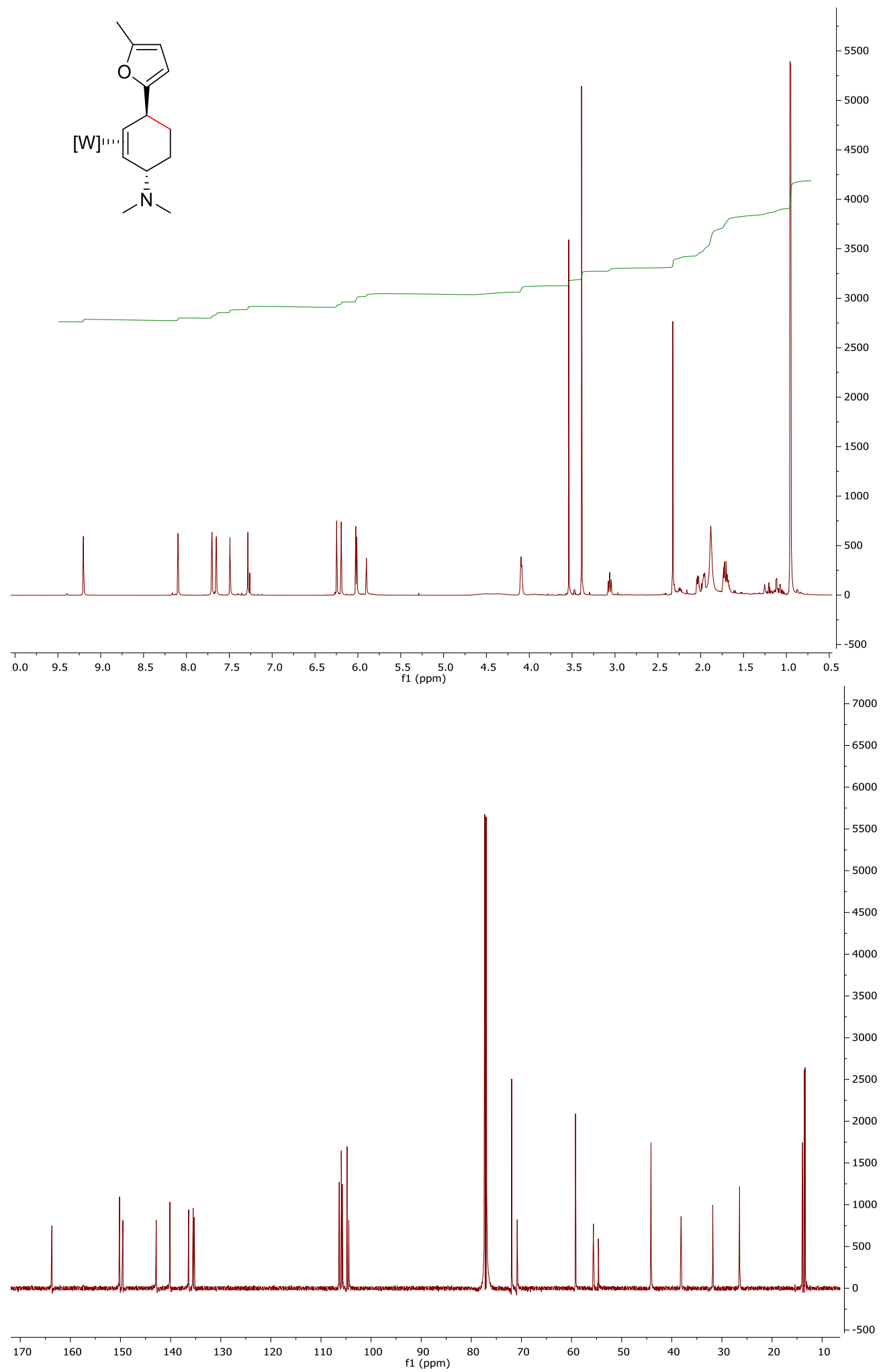


Compound 95:

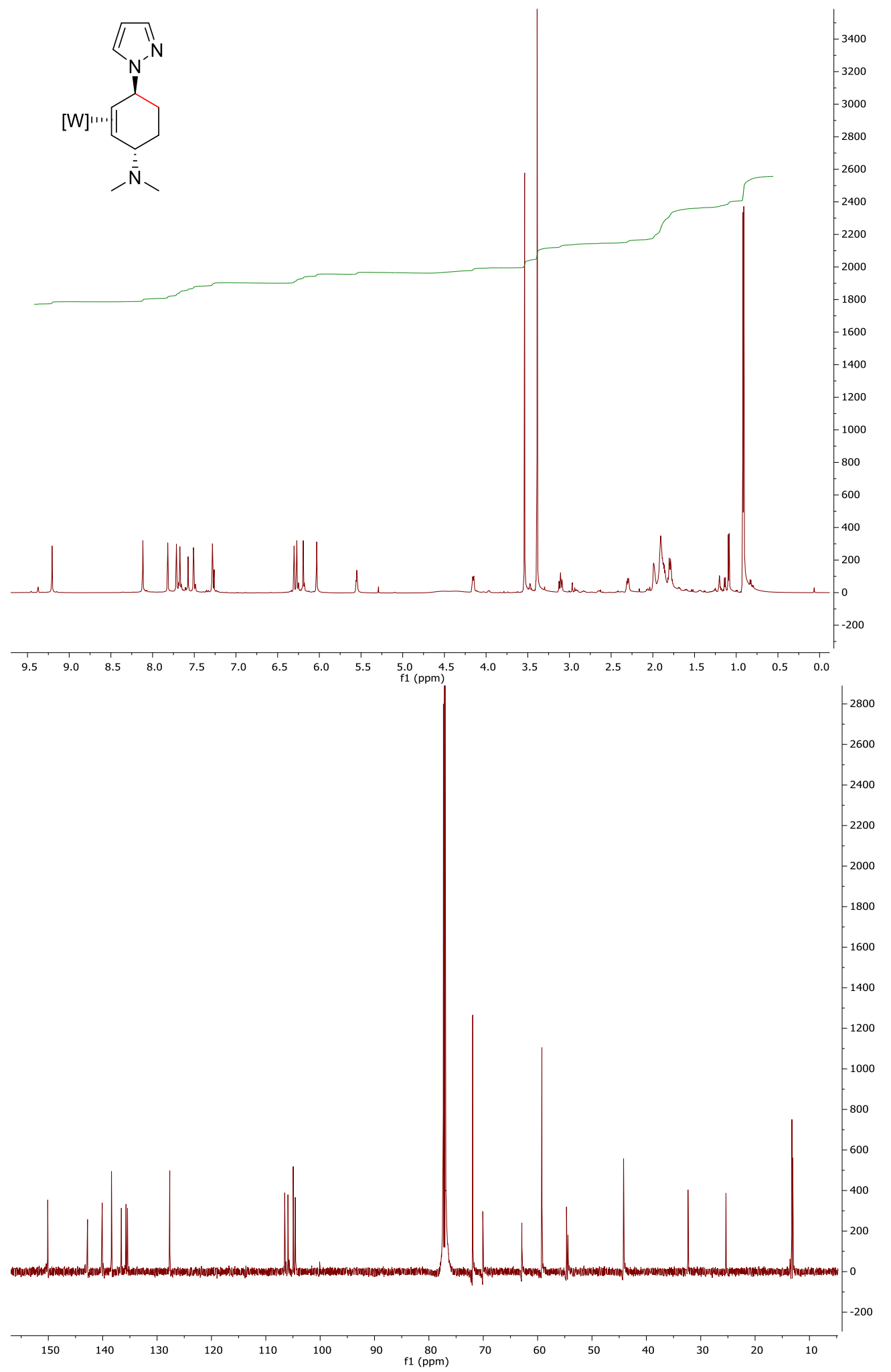


Compound 96:

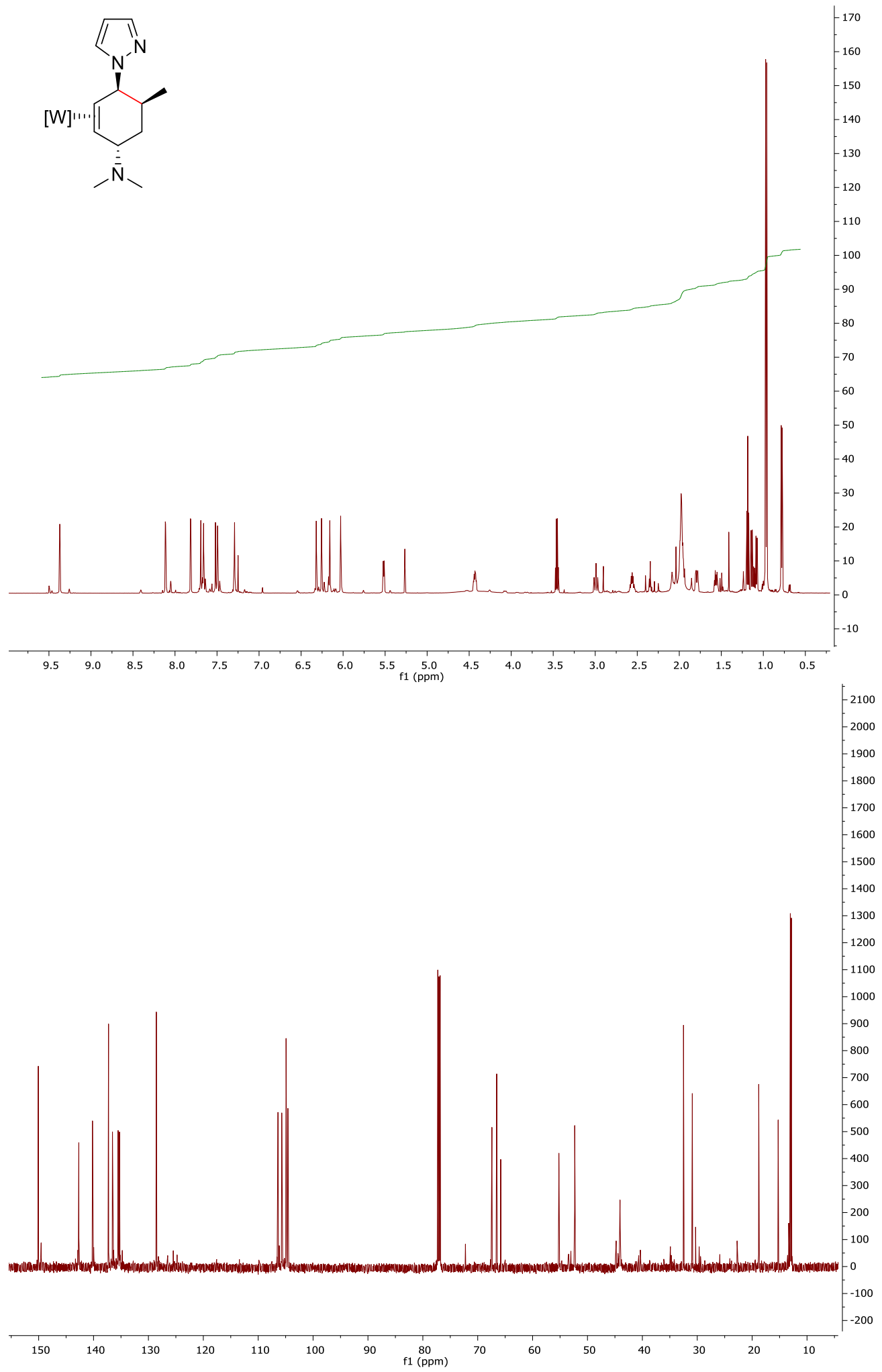




\section{Compound 97:}

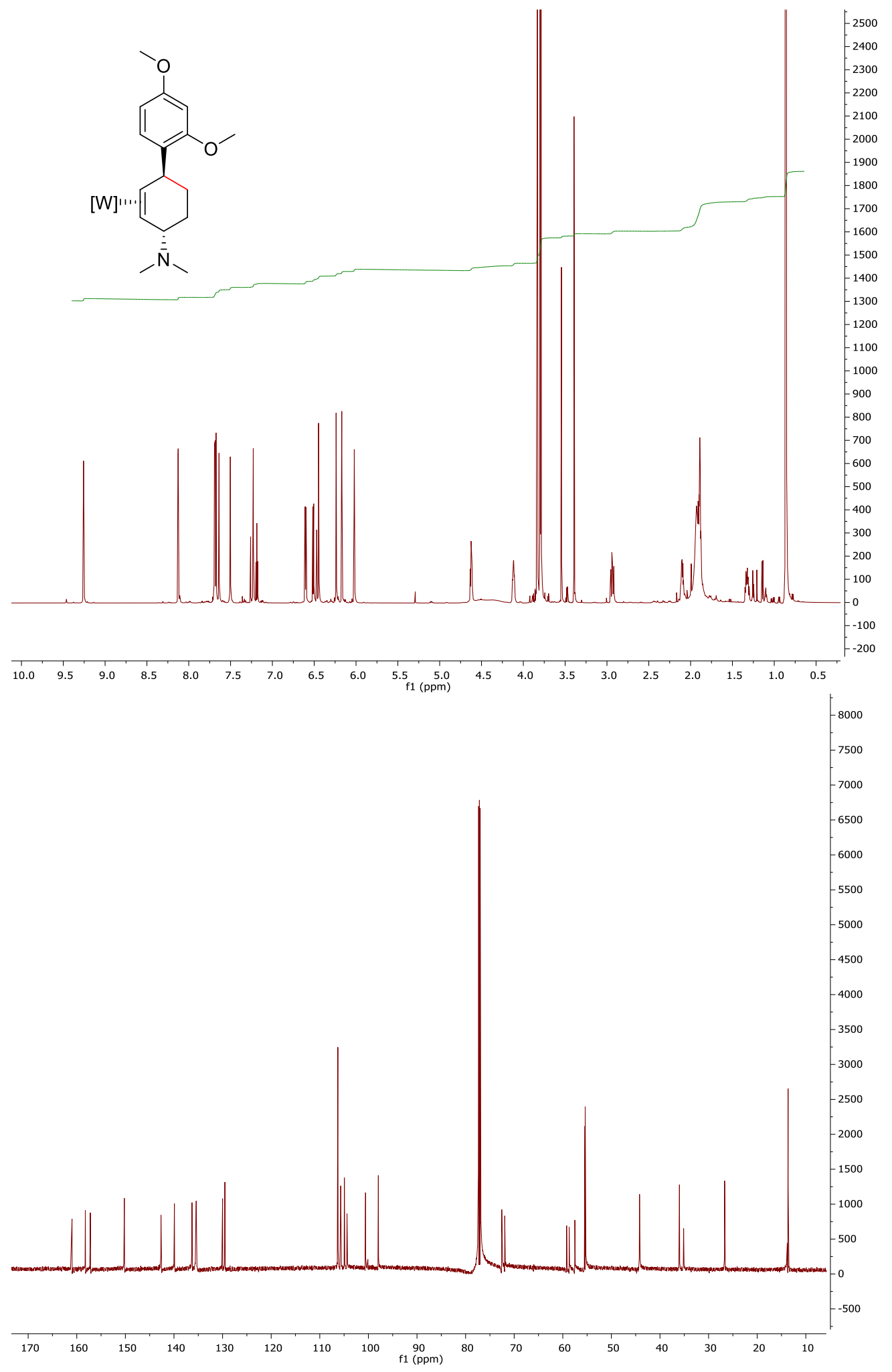


Compound 98:

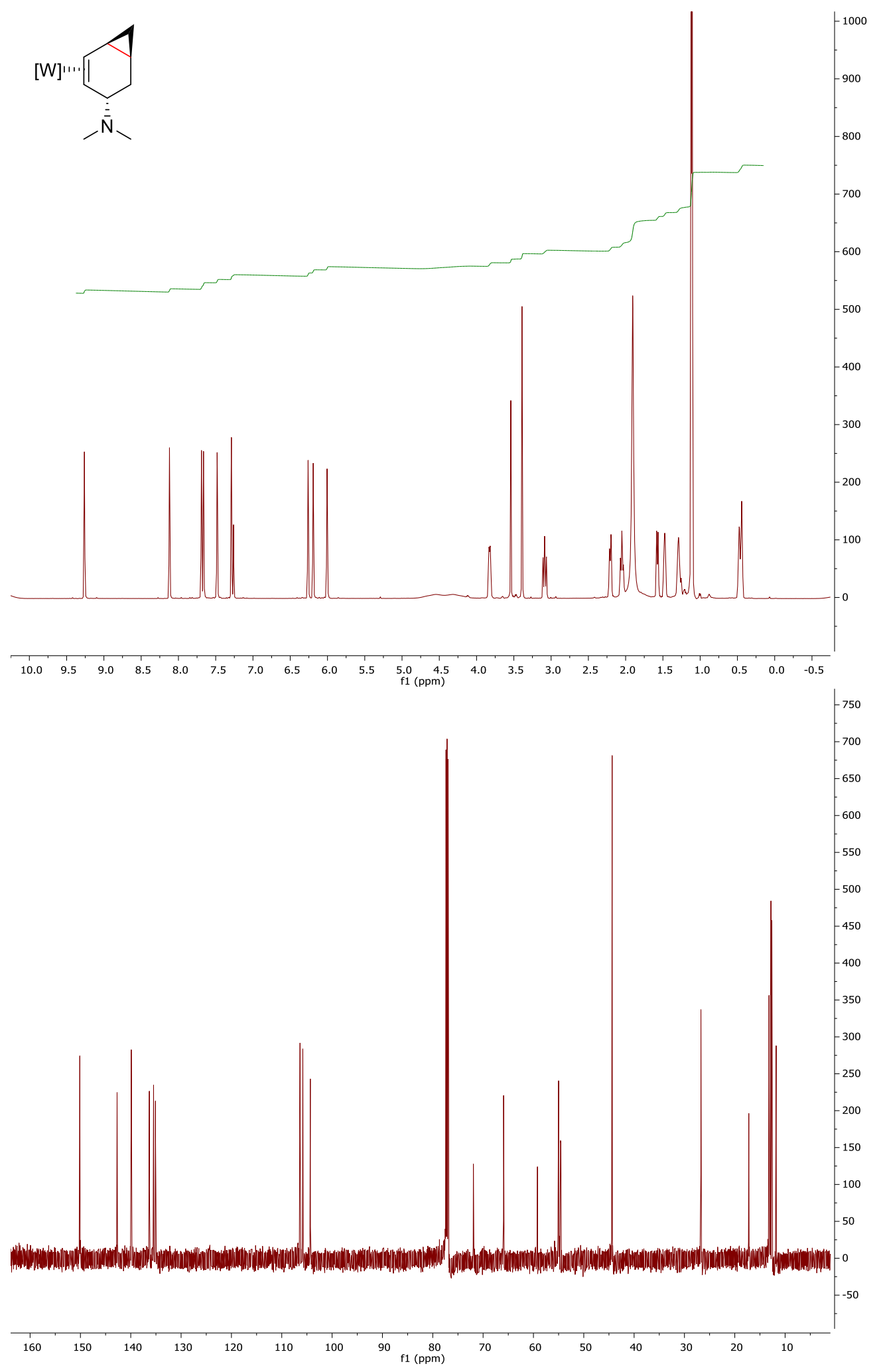


Compound 99:

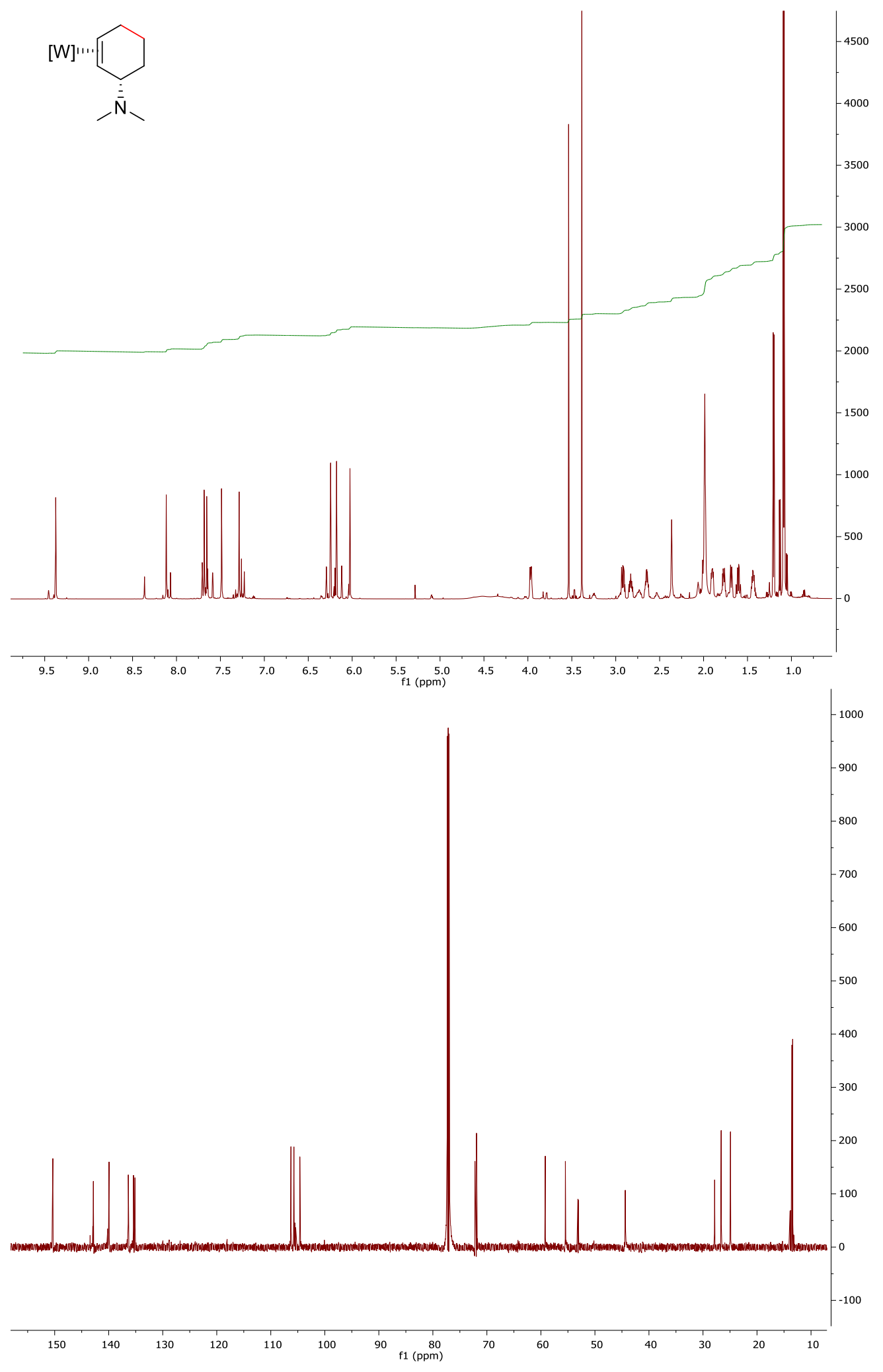


Compound 100:

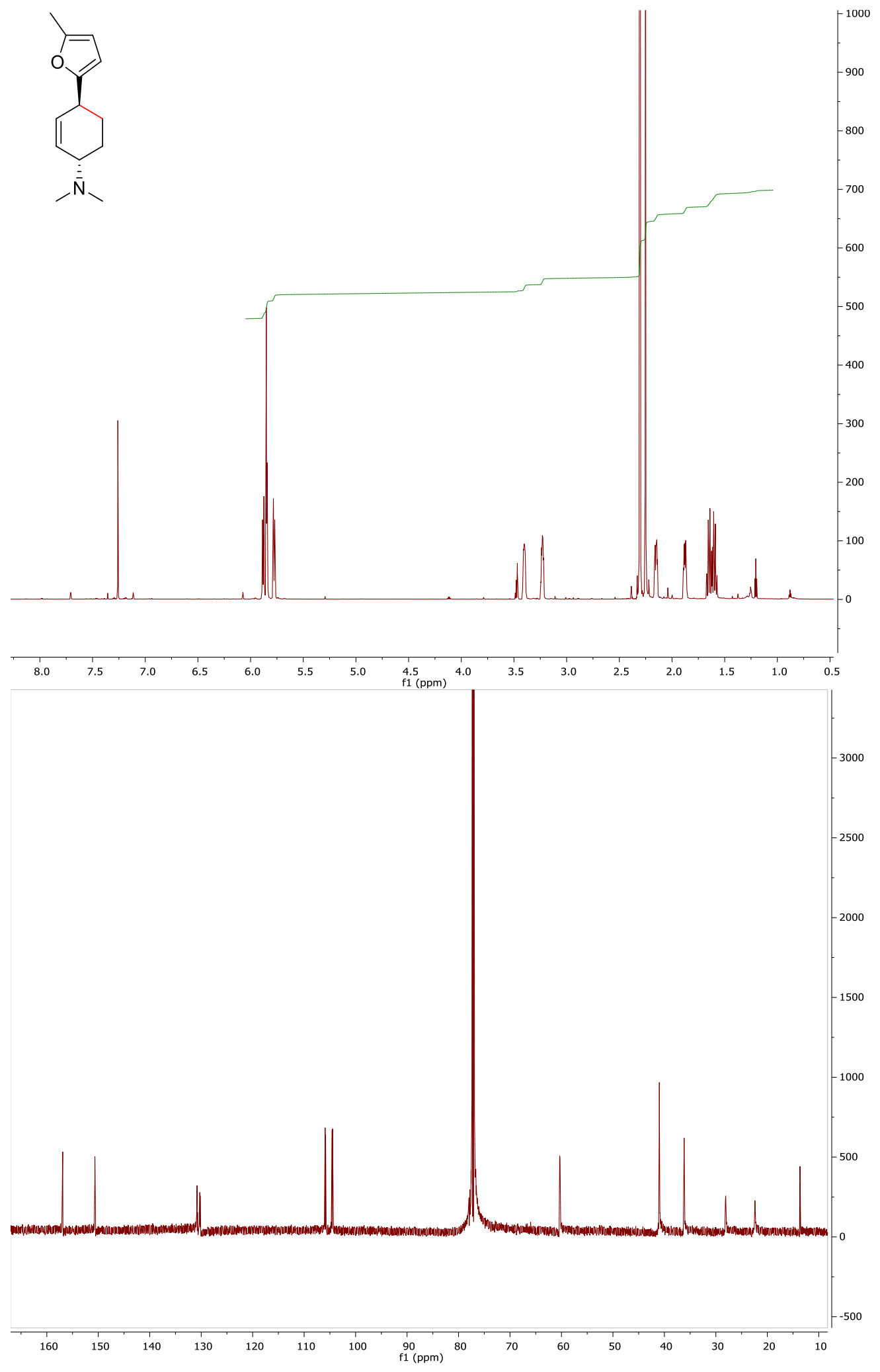




\section{Compound 101:}

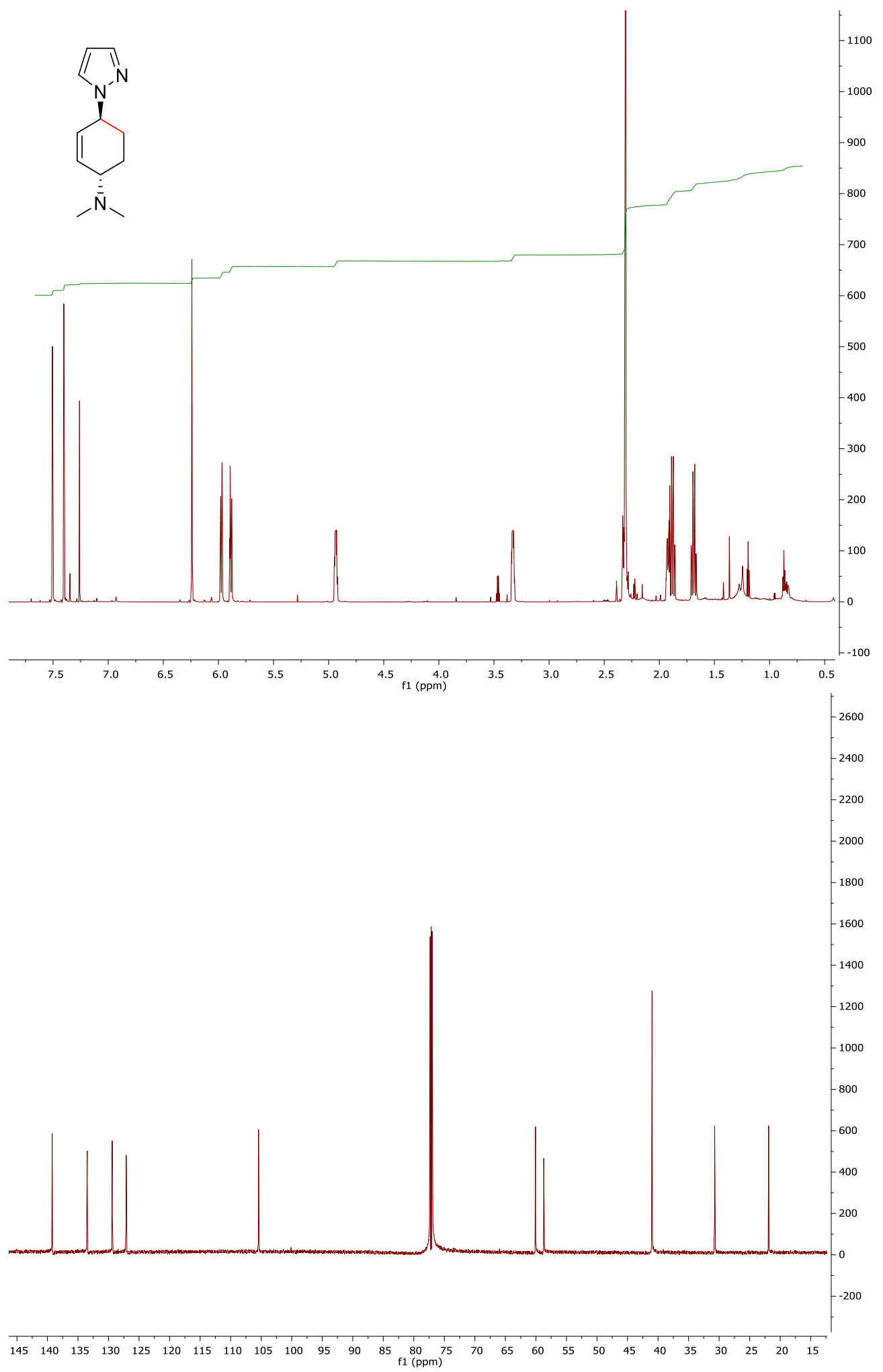


Compound 102:

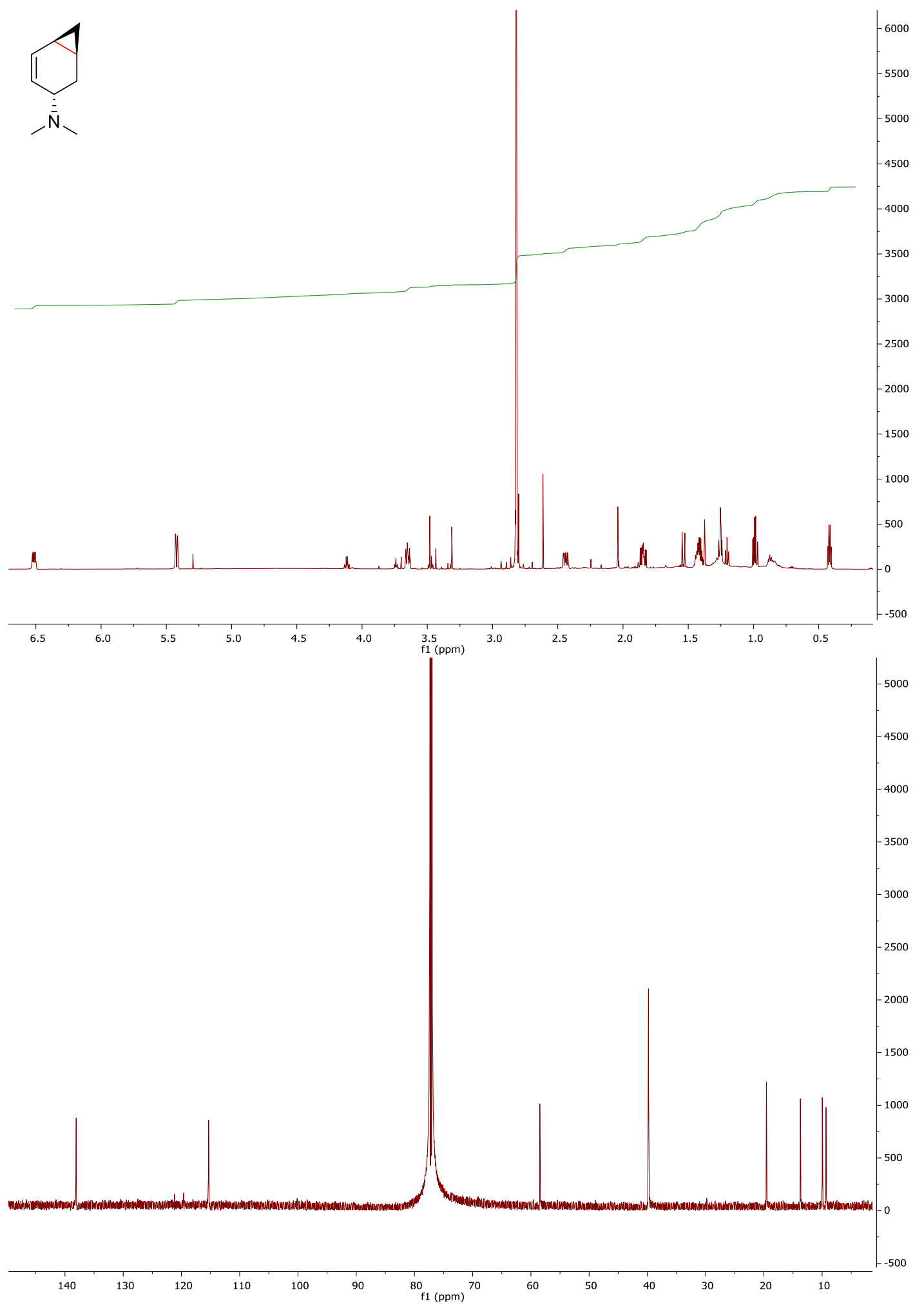


Compound 104:

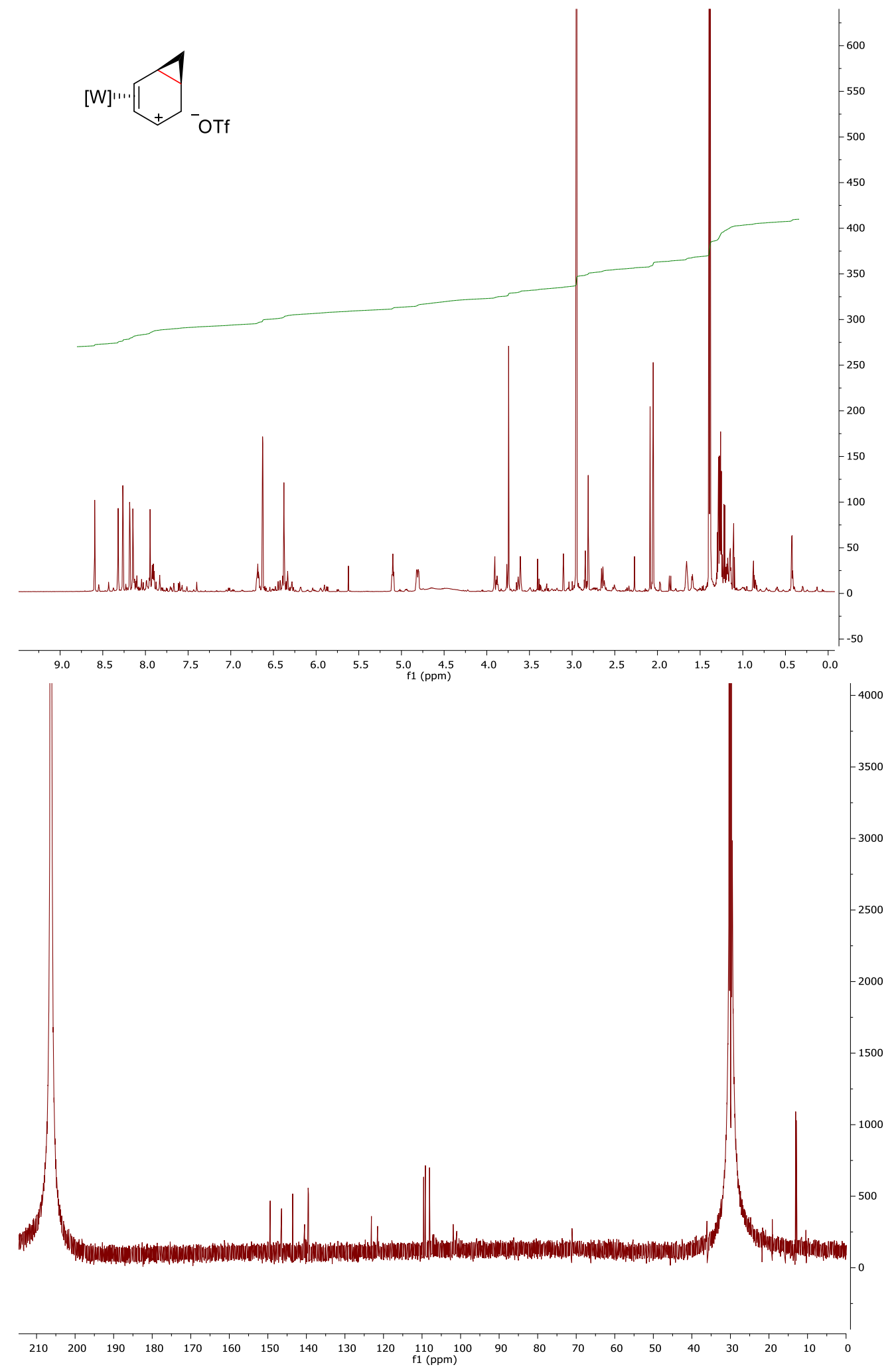

